\title{
Towards the treatment of Cantú syndrome
}

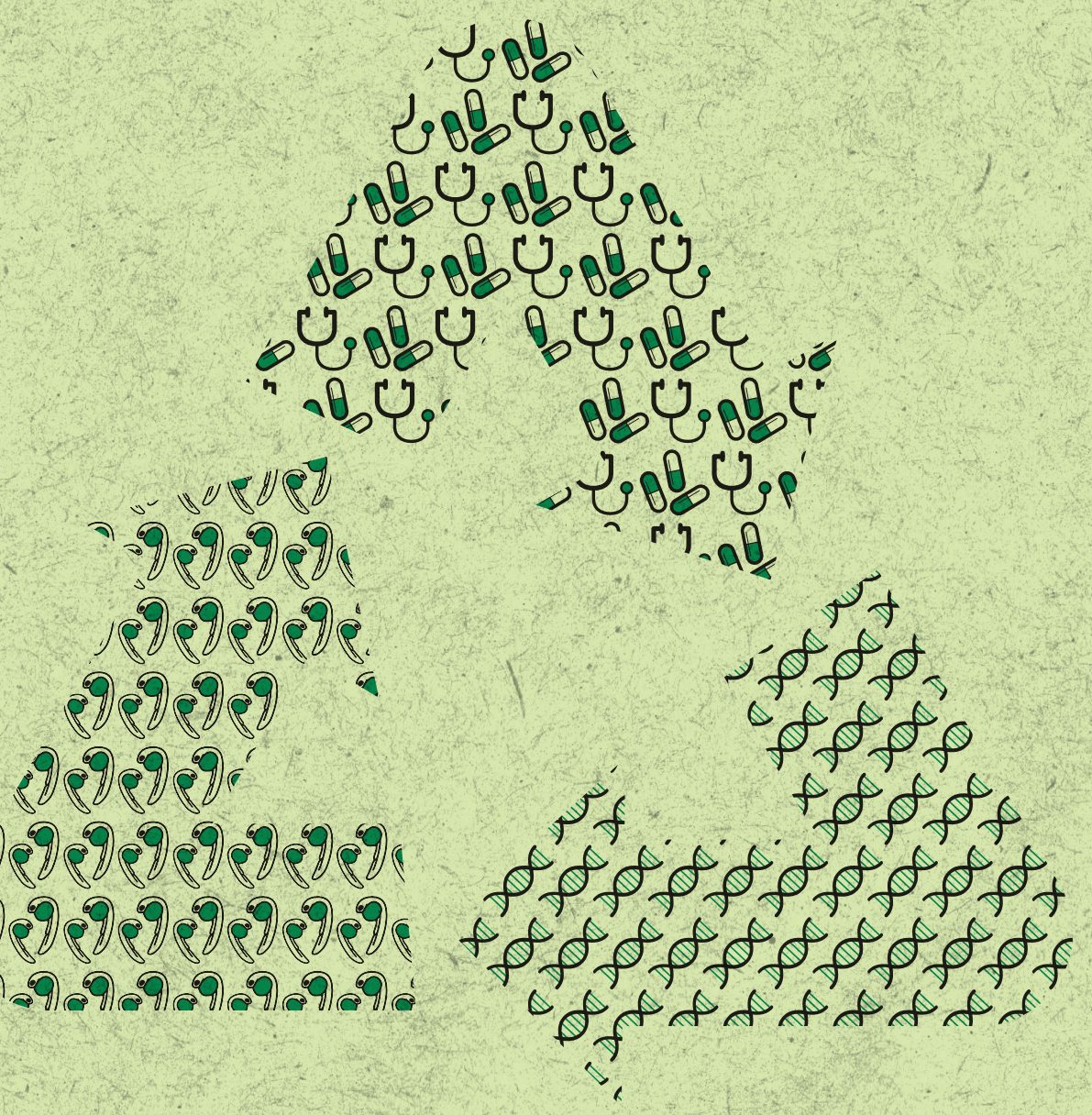





\section{Towards the treatment of Cantú syndrome \\ Drug repurposing for rare genetic diseases}

Helen Isabel Roessler 
Towards the treatment of Cantú syndrome

Drug repurposing for rare genetic diseases

PhD dissertation

(c) Helen I. Roessler

All rights reserved. No part of this thesis may be reproduced or transmitted, in any form or by any means, without prior written permission of the author and corresponding journal.

ISBN: 978-90-393-7376-7

Cover design by: Guus Gijben

Layout by: Guus Gijben

Printed by: Proefschrift-AlO

The work in this thesis was supported by the E-Rare Joint Transnational Cantú Treat Program to Gijs van Haaften (Grant Number: I-2101-B26). 


\title{
Towards the treatment of Cantú syndrome
}

\section{Drug repurposing for rare genetic diseases}

\author{
Op weg naar de behandeling van Cantú syndroom \\ Hergebruik van geneesmiddelen voor zeldzame genetische \\ ziekten \\ (met een samenvatting in het Nederlands)
}

Proefschrift

ter verkrijging van de graad van doctor aan de

Universiteit Utrecht

op gezag van de

rector magnificus, prof.dr. H.R.B.M. Kummeling, ingevolge het besluit van het college voor promoties

in het openbaar te verdedigen op

dinsdag 6 juli 2021 des middags te 2.15 uur

door

\section{Helen Isabel Roessler}

geboren op 20 juni 1992

te Heidelberg, Duitsland 
Promotor:

Prof. dr. V.V.A.M. Knoers

\section{Copromotoren:}

Dr. G.W. van Haaften

Dr. M.M. van Haelst 



\section{Content}

\section{Chapter 1}

Introduction: Towards treatment of rare genetic diseases.

\section{Part I - Clinical Characterization of Cantú syndrome}

\section{Chapter 2}

Cantú syndrome - a genetic syndrome reference guide.

\section{Chapter 3}

Cantú syndrome, the changing phenotype: a report of the two oldest Dutch patients.

\section{Chapter 4}

Behavioral and cognitive functioning in individuals with Cantú syndrome.

\section{Chapter 5}

Three-dimensional facial morphology in Cantú syndrome.

\section{Chapter 6}

Cantú syndrome: Findings from 74 patients in the International Cantú

Syndrome Registry.

\section{Part II - Modeling and Treatment of Cantú syndrome and Related Disorders in Zebrafish}

\section{Chapter 7}

Effective CRISPR/Cas9-based nucleotide editing in zebrafish to model human genetic cardiovascular disorders.

\section{Chapter 8}

Characterization and treatment of overactive $K_{\text {ATP }}$ channel in zebrafish 179 models for Cantú Syndrome. 


\section{Chapter 9}

ABCC9-related Intellectual disability Myopathy Syndrome is a $K_{A T P} 209$ channelopathy with loss-of-function mutations in ABCC9.

\section{Chapter 10}

General Discussion: A multi-disciplinary approach to treat rare genetic 273 diseases.

\section{Appendices}

$\begin{array}{ll}\text { Appendix } 1 \text { - References } & 301\end{array}$

Appendix 2 - Resources $\quad 310$

Appendix 3 - List of Abbreviations $\quad 312$

Appendix 4-Nederlandse samenvatting 316

Appendix 5 - Summary $\quad 320$

Appendix 6 - List of Publications $\quad 324$

Appendix 7 - Acknowledgments $\quad 326$

$\begin{array}{ll}\text { Appendix } 8 \text { - Curriculum vitae } & 331\end{array}$ 
-12
4
4
4

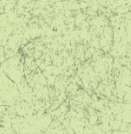

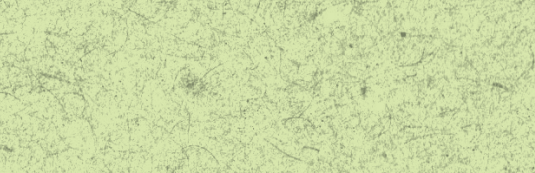

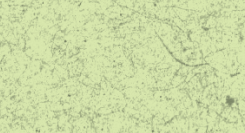

तr.

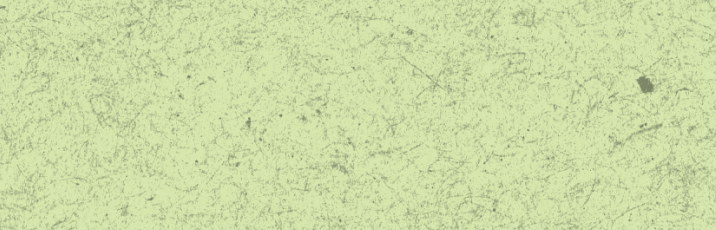

4

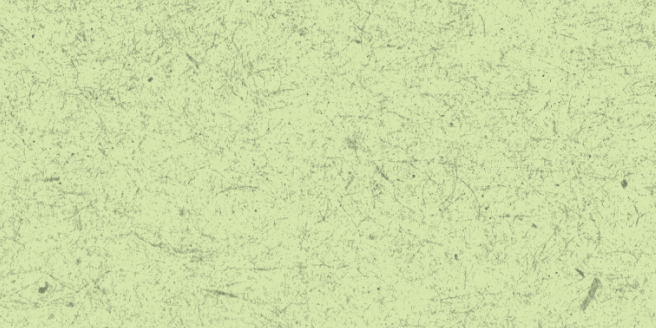




\section{1}

\section{Introduction: Towards treatment of rare genetic diseases}

Adapted and expanded from: Drug repurposing for rare diseases

Helen I. Roessler, Nine V.A.M. Knoers, Mieke M. van Haelst* and Gijs van Haaften*

*These authors contributed equally to this work.

Trends Pharmacol Sci (2021) Epub ahead of print DOI: 10.1016/j.tips.2021.01.003 


\section{From Gene to Treatment: Going full circle}

Nowadays, we live in a time of unprecedented opportunities to turn scientific discoveries into novel or improved treatments for genetic disorders. The contribution of modern genetics and genomics to the diagnosis of Mendelian genetic disorders has been remarkable. Advances in genomic technologies have produced an explosion of novel information about the genetic basis of human diseases, resulting in high expectations for new and optimized treatments for genetic disorders. Translating genetic discovery into therapeutic intervention, however, involves multiple laborious steps that cannot be accomplished within a short period of time. Starting with a detailed characterization of the phenotype and genotype, followed by investigating the effect of disease-causing gene variants in an appropriate model system all the way towards the identification of a treatment that can be tested and successfully approved in clinical trials.

Experience from recent years has shown that, despite scientific advances, a majority of genetic diseases still lack effective treatment options. Big data approaches and genetic technologies have led to the first transformational novel therapeutics for a handful of disorders. This motivates and challenges us as a society to accelerate progress so that no disease and no patient is ultimately left behind in getting access to safe and effective therapeutic interventions.

The objective of this thesis was to develop a novel therapeutic approach in treatment of the rare genetic disorder Cantú syndrome by successfully applying novel tools for clinical phenotyping of patients, in vivo modeling of patientspecific mutations and repurposing a compound already used in clinics for this new indication. Hence, we highlight a translational approach to go from gene discovery towards the investigation of a novel therapeutic strategy which could serve as a blueprint for tackling related rare conditions. 


\subsection{Rare genetic diseases: A unique group of disorders}

A rare disease (RD) can be any heterogeneous condition affecting a small percentage of the population (Europe: 1 person per 2000; USA: $<200.000$ individualsii). RDs are often chronic resulting in lifelong disability or early death; many RDs have a pediatric onset and about $30 \%$ of children with RD die before the age of 5 years (Sardana et al., 2011). Seventy percent of all RDs are genetic, caused by both germline and somatic gene mutations (Nguengang Wakap et al., 2020). Of the RDs with a genetic origin, many have a monogenic origin (Posey, 2019); they are caused by a defect single gene defect and follow a Mendelian inheritance pattern (dominant, recessive, X-linked). Additionally, RDs also show non-Mendelian inheritance which includes epigenetic changes (e.g. BeckwithWiedemann syndrome) and mitochondrial disorders (e.g. Rett syndrome) resulting from maternal transmission of variants in mitochondrial DNA (Rajput, Singh, \& Bhardwaj, 2015; Soejima \& Higashimoto, 2013). A minority of RDs are also caused by environmental, infectious or immunological factors (e.g. African trypanosomiasis) (Guillem, Cans, Robert-Gnansia, Ayme, \& Jouk, 2008) but will not be discussed here.

Around 7,000 RDs have been identified to date. While individually rare, they globally affect 300 million people (10\% of the population) worldwide with new diseases regularly being described in medical literature (Dawkins et al., 2018; Griggs et al., 2009; Nguengang Wakap et al., 2020). Prevalence rates vary widely among RDs where they can range from ultra-rare with only a few cases described globally to less rare for which diagnosis is dependent on the experience of the individual physicians (Brewer, 2009). For example, Hutchinson-Gilford Progeria Syndrome (HGPS), a genetic disorder resulting in premature aging, shows an incidence of merely 1 in 8 million live births (Prakash et al., 2018) whereas a relatively well-known rare condition, Huntington's disease, affects an estimated 3 to 7 per 100,000 people of European ancestry (Rawlins et al., 2016). Nevertheless, all RDs share similar clinical challenges, as they involve multisystem dysfunction and therefore require complex care (Ayme, Kole, \& Groft, 2008). Compared to common disorders, patients with a RD visit approximately twice as many specialists (Molster et al., 2016). 


\subsection{The path to diagnosis: An odyssey}

Without an accurate (molecularly confirmed) diagnosis, it is not only difficult to identify the cause but also to design an appropriate and effective treatment strategy to suppress or reverse the condition of interest. Moreover, a molecularly confirmed clinical diagnosis often allows a clinical geneticist to give accurate genetic counselling including providing information regarding patient's prognosis, inheritance pattern, prenatal investigation options and availability of (future) personalized treatment (Rabbani, Tekin, \& Mahdieh, 2014). Diagnosis of RDs, in particular, remains a challenge for patients, doctors and healthcare systems. Both patients and physicians have limited knowledge and experience with the disease they are dealing with due to insufficient characterization of the natural history of many RDs (Azie \& Vincent, 2012). Thus, a majority of (parents of) patients undergo a downright "diagnostic odyssey", which can be a long and frustrating journey for both patients and their families to obtain an accurate diagnosis. Typically, it can take 6 years from onset of symptoms to a correct diagnosis where patients tend to encounter 14 diagnostic procedures and 4.5 diagnoses (Bloss et al., 2017).

The identification of RD-associated gene variants is essential for diagnosis and disease prognosis. Next generation sequencing (NGS), in particular whole-exome sequencing (WES) and whole-genome sequencing (WGS), are established diagnostic tools to unravel the genetic background of RDs. Recent advances in these technologies make them affordable and indispensable. Although genetic tests are crucial to diagnose many rare genetic disorders, the initial stages of diagnosis still depend on basic clinical practices as physical examination, personal and family history, other laboratory tests and imaging. For instance, a detailed and complete family pedigree should be obtained as it can often assist in elucidating the inheritance pattern and may be helpful for differential diagnosis (Bennett, French, Resta, \& Doyle, 2008).

The identification of a causative gene variant in monogenic disease often requires a multi-step process, however eventually leads to a definitive diagnosis which is an important step for the patient (Figure 1). Moreover, a molecularly confirmed clinical diagnosis often allows a clinical geneticist to give accurate genetic counselling including providing information regarding patient's prognosis, inheritance pattern, prenatal investigation options and availability of (future) personalized treatment (Rabbani et al., 2014). Additionally, once the genetic basis of a Mendelian disorder has been determined, a patient with that 
suspected disorder can be genetically tested and the (parents of) the patient can receive a diagnosis and be counseled and managed accordingly.

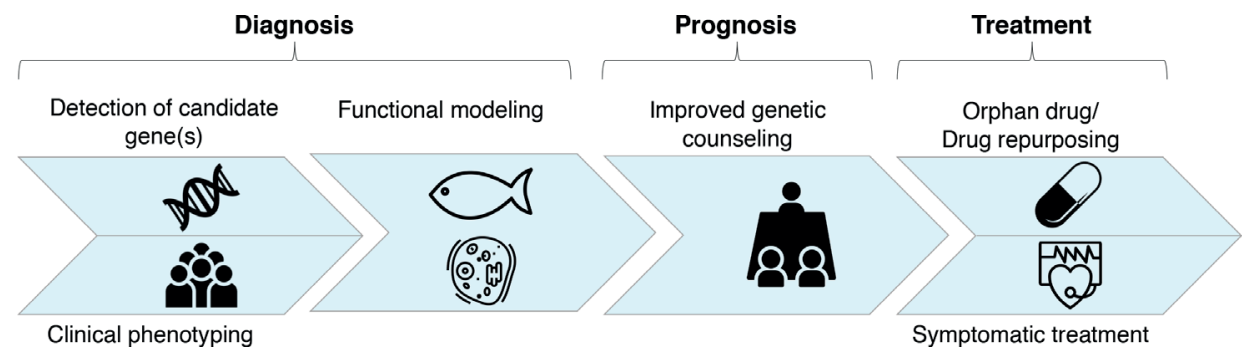

Figure 1 | From gene to treatment in rare disorders. The steps for rare disease diagnosis are essential, as with the diagnosis in hand, the healthcare professional can assess patient's prognosis and offer genetic counseling and potential personalized treatment options, which might be specific for the disease being treated and therefore provide a cure, or might be unspecific, focusing only on mitigating disease symptoms.

\subsection{Clinical phenotyping of rare diseases}

The clinical presentation, natural history, pathophysiology, and often mysterious nature of RDs have fascinated physicians for centuries. RDs provide opportunities to study human physiology and biomedical science from unique perspectives. Essential in this process are disease phenotypes which refer to the observable characteristics of an organism including behavior, function and other features, such as growth parameters, and enzyme activity. Having a complete overview of the clinical symptoms immensely helps to identify new patients more easily in the future. Moreover, in cases where genetic testing remains costly and is therefore an inefficient option, clinical phenotyping is a valuable resource for an accurate and faster diagnosis of rare diseases.

Deep phenotyping is typically the first step towards diagnosing a RD and greatly supports further measures in the process of diagnosis and treatment development. It is essential to collect a cohort with as many patients revealing an overlapping/ similar clinical phenotype as possible in order to maximize the chance of identifying the disease-causing gene variant after subjecting the clinical samples to NGS. Increased phenotypical knowledge can also be of advantage when prioritizing detected variants based on annotated gene function. After successfully identifying a candidate gene, further validation in model 
organisms is necessary. Detailed clinical characterization allows the researcher to pick a disease-related phenotypic readout most appropriate for the organism.

Clinical features can be recorded in a patient registry, an important tool for public health surveillance and RD research. Because RD patients are scattered across countries, registries need to be developed by multi-center collaborations. In that way, registries can generate a critical mass of patients in order to ensure a rapid progression towards clinical application (e.g. therapy) when preclinical studies are finished (Cavero-Carbonell et al., 2016).

Other possibilities to expand one's knowledge about a RD are European Reference Networks (ERNs) virtually connecting healthcare professionals around Europe with expertise in rare diseases. By centralizing knowledge and experience, these networks allow the discussion of a patient's diagnosis and treatment. After its' launch in 2017, more than 300 hospitals and 900 highly specialized teams are participating in the approved ERNs (Heon-Klin, 2017).

Lastly, one may use data collected in electronic health records and put effort in capturing and sharing self-reported patient data, in addition to data obtained in a research setting. Nowadays, patients are increasingly involved in reporting their features, outcomes and treatment histories applying social media and patientcentric research platforms. However, it is important to realize that such data collection can vary in quality due to variable data standards, privacy concerns or inaccurate reporting. Data standards are critical to understanding and using data and to ensure maximal utility in both research and for future product development (Southall et al., 2019). Therefore, it is advised to standardize data collection that should be in accordance with regulatory standards and created in consultation with patients and clinicians (Sherman et al., 2016).

\subsection{Discovering the genetic cause of rare diseases}

Over the last decade, progress in identifying disease genes has been spectacular. Since 2010, the number of new disease-causing gene variants identified per year has grown significantly due to advances in DNA sequencing technologies and big data analysis. This improvement is increasingly important for diagnosis of genetic disorders and has been transformative for rare Mendelian diseases (GonzagaJauregui, Lupski, \& Gibbs, 2012). Disease-gene associations are now being discovered at a rate of 300 per year (Figure 2). Currently, there are approximately 4000 genes responsible for RDs known (Online Mendelian Inheritance in Man) 
and recent estimates suggest that over 9000 monogenic disorders will ultimately be discovered and molecularly defined (Samuels, 2010).

Many relatively low-cost commercial and customized gene-sequencing panel analyses are available to identify specific RD associated gene variants, and directories of diagnostic laboratories offering these tests can be easily found online. For a more comprehensive analysis, WES has emerged as an effective tool for diagnosing patients who have already undergone Comparative Genomic Hybridization (CGH) or SNP-array techniques in order to rule out chromosomal aberrations (Need et al., 2012; Yang et al., 2013; Lee et al., 2014). WES limits sequencing mainly to the protein-coding regions of the human genome which contains 180.000 exons merely constituting 1.5 - 2\% of the whole genome. Nevertheless, the coding region reveals an estimated $85 \%$ of heritable Mendelian disease-causing mutations (Majewski, Schwartzentruber, Lalonde, Montpetit, \& Jabado, 2011). Within the last decade, WES has become technically feasible and more cost-effective, offering new diagnostic and research possibilities for Mendelian disease (Choi et al., 2009). Currently, the cost of sequencing an exome in a clinical setting is less than $1000 €$ (Schwarze, Buchanan, Taylor, \& Wordsworth, 2018). Additionally, its accuracy is remarkable with studies reporting a sensitivity of $98.3 \%$ for detecting previously identified mutations applying this technique (Boycott, Vanstone, Bulman, \& MacKenzie, 2013).

Nevertheless, the interpretation of WES data remains challenging and a large fraction of RD patients does still not get a molecular diagnosis. For instance, WES not only uncovers variants that have been reported previously or can be associated with a known disorder, it also identifies many rare variants whose functional impact still needs to be determined, termed variants of unknown significance (VUS). Each personal genome contains hundreds of such rare and unique variants that can potentially have dramatic functional significance, but mostly show no obvious effect. The immense number of such variants can complicate their interpretation resulting in apparent undiagnosed Mendelian disorders in many patients (Need et al., 2012). Thus, detecting disease-causing variants among thousands of benign variants, as well as the interpretation and validation of candidate variants remains a significant challenge, also because healthy individuals often carry heterozygous gene variants that would be deleterious or lethal when homozygous (Gao, Waggoner, Stephens, Ober, \& Przeworski, 2015). Algorithms applied as standard in exome data analyses give probability likelihoods of a variant being pathogenic. Eventually, detected variants get categorized based on novelty, predicted deleterious effect at the protein level or annotated gene function. Additionally, databases such as ClinVar 
and gnomAD are important tools listing pathological and normal population variants, respectively. Hence, detected variants can be filtered against such databases in order to prioritize or eliminate variants.
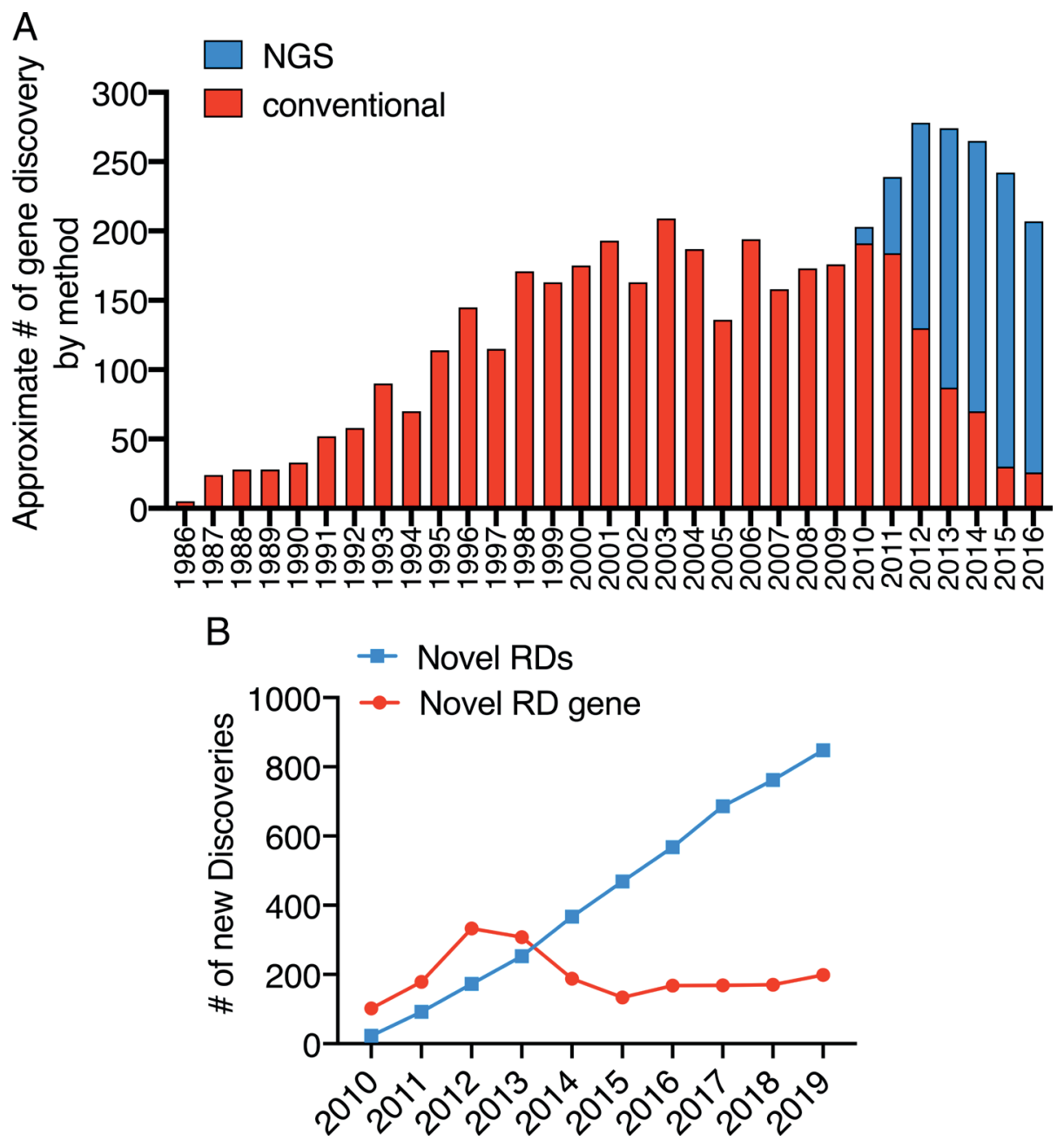

Figure 2 | Progress in identifying (RD) disease genes. (A) Approximate number of gene discoveries made by next generation sequencing (WES and WGS) compared to conventional approaches since 1986 according to OMIM data. (B) Cumulative number of new RDs and genes linked to RDs identified since 2010 according to Ophanet data. (A) adapted from by (Boycott 2017). 
WES is most powerful when not only samples of DNA from the patient but also the parents are taken as reference. This so-called trio-WES analysis can be applied for detecting variants that are merely present in the child and not in the parents. Such so-called de novo variants can be causal for a variety of rare genetic disorders (Mani, 2017). Additionally, trio-WES provides context for understanding gene variants that may not be common in the general population but that are also present in healthy family members and therefore unlikely to be pathogenic. Hence, research can focus elsewhere.

However, even though the application of WES represents a promising diagnostic option for RD patients, a diagnosis is not guaranteed. In over $70 \%$ of patients with a high degree suspicion of a monogenic RD, WES failed to provide a definitive molecular diagnosis (Boycott et al., 2019). Moreover, there are still cases of RDs that are caused by variants affecting regulatory and non-coding DNA regions which cannot be detected by WES (Sirmaci, Edwards, Akay, \& Tekin, 2012). Sequencing the whole genome (WGS) is a promising newer approach. Unlike WES, WGS produces sequence information of the coding exome as well as the other $98 \%$ of the genome which is non-coding but nevertheless might reveal functional relevance (Fresard \& Montgomery, 2018). Hence, interpretation of data originating from WGS is an even more considerable challenge than deciphering WES data.

Moreover, several novel technologies are currently underway to identify new RD-causing genes at remarkable speed. Computational approaches that focus on large-scale data integration across patients and within the single patient, and from healthy individuals, will enable the next frontiers of RD gene discovery (Boycott et al., 2019). Furthermore, the coupling of genomic data with phenotypes extracted from medical electronic health records enables largescale analysis of the clinical impact of rare variants (Abul-Husn et al., 2016; Dewey et al., 2016).

The identification of candidate variants or genes is merely the first step in diagnosis. Uncertainty remains until the same variant is found in independent patients with similar clinical characteristics, and/or functional tests are performed for further validation. Additionally, despite the significant increase in the speed of RD gene discovery, the delay in understanding the molecular and cellular mechanisms of rare disorders continues to increase. Hence, even though we have at least a basic knowledge of around 50 \% of human disease genes, our understanding of the entire catalog of highly penetrant, disease-causing mutations is rather limited. As a conclusion, there is an immediate need for 
functional modeling to not only validate but also put disease-causing genes into a biological context.

\subsection{Functional Modeling of Rare Diseases}

The diversity of life is immense. We simply assumed that this diversity arises from different developmental mechanisms with different underlying genes, signaling pathways and molecular events. However, knowing that for instance a fly or a fish share many genes and signaling pathways with humans, even though these organisms differ in so many morphological features, the explanation of the diversity of life is probably far more complex.

The sequencing of genomes eventually revealed the breadth of conservation of genes. Therefore, the architects of the Human Genome Project in the late 1980s had the foresight to include sequencing of the genomes of four key experimental organisms as part of the effort (Watson, 1990). Comparing the genome sequences of yeast, worm, fly, mouse and human confirmed the striking extent to which all organisms are built from the same set of genes (Dolinski \& Botstein, 2007). In the past 30 years, it was shown that genes are evolutionary conserved, and that many biological processes and physiological pathways identified in yeast, worms or flies can be directly related to vertebrate and human biology (Novick, Field, \& Schekman, 1980; Takeshige, Baba, Tsuboi, Noda, \& Ohsumi, 1992). Basic knowledge obtained from model organisms research is now frequently being translated to mammalian contexts.

The application of NGS technology has brought an unprecedented era for the identification of new common and RD genes and variants. The potential for clinical benefits is enormous, but how do we ensure we have identified the causal variant or gene? For instance, every individual harbors 0-5 de novo variants in the coding region of their genome. One of these variants could be causal for the disease of a patient, whereas others might not harm the typical function of the gene at all. Despite the development of various tools that help to prioritize detected variants, our ability to predict a variant's effect is far from flawless making it necessary to assess the effect of a variant on other levels. Functional modeling takes advantage of the conservation of genes and biological processes amongst organisms and is a way to experimentally show the effect of a variant at the molecular, cellular or phenotypic level as compared to the normal allele. Ideally, disrupting the candidate gene in a model organism should result in a phenotype that recapitulates the relevant pathology in humans and is unlikely to 
occur with disruption of genes selected at random (Boulding \& Webber, 2012; MacArthur et al., 2014).

In particular for RDs, functional modeling has turned out to be essential (Hmeljak \& Justice, 2019; Wangler et al., 2017). RDs as a group are common, however individual RDs often affect only a few people. This results in difficulties in diagnosing, understanding, and eventually treating affected individuals by studying the clinical phenotype alone. When a novel RD gene is discovered, there is typically minimal insight into its biological function, the pathogenic mechanisms of disease-causing variants and how therapy might be approached. Hence, there is a growing demand for the development of novel experimental models of rare genetic diseases to not only validate the disease-causing gene variant, but also to translate these gene discoveries into a mechanistic understanding of the target gene's function, to understand disease pathophysiology and to identify therapeutic intervention. In many cases, studying genes simultaneously in humans and model organisms provides additional significant insight into the cause of a particular disorder contributing to an understanding of the underlying pathogenic mechanisms.

Determining which model organism to use may depend on the cost, speed and tools available for assessing phenotypic features in the disease. Each model system shows unique advantages and limitations. The ability to generate many units as fast as possible and carry out genomic manipulations in a feasible manner are key factors in modeling disease. Especially for RDs, animal models need to be genetically and physically alike to humans with a fully sequenced genome, and well-suited to large-scale experimental manipulation on a whole organism scale, considering the vast number of novel variants that are being identified through NGS.

Cell lines are ideal for novel high-throughput technologies (Joung et al., 2017). However, they lack the physiological outcomes provided by whole organisms. This is especially important for complex disorders where interactions between multiple cell types or organ systems are required for a disease phenotype to manifest (Adamson, Sheridan, \& Grierson, 2018). Organoids are multidimensional culture systems and solve many problems that arise in monolayer cell cultures (Hibaoui \& Feki, 2020). Yeast reveals simple diploid genetics, a short generation time and can be grown on media applying nutritional supplements for selection and analysis at a single cell level (M. G. Smith \& Snyder, 2006). Invertebrates, in particular Drosophila melanogaster and Caenorhabditis elegans, are commonly applied as models in human genetic diseases (Moulton 
\& Letsou, 2016; Silverman et al., 2009). Even though they are well-suited for large-scale analysis, their evolutionary distance from humans and the resulting physical differences can result in a less relevant system making the investigation of physiological consequences of causal variants and treatments more difficult. Historically, the mouse (Mus musculus) has been considered as an excellent model organism for human genetic disorders (Dow \& Lowe, 2012). However, they would be impractical for validating the large number of candidate variants that are likely to be identified through large-scale RD programs, due to their high husbandry costs, small litter sizes and long generation times. Often a multisystem approach is ideal to model and understand a human genetic disease, and can be in particular valuable for RDs where human tissues are difficult to obtain.

\subsubsection{The zebrafish: an emerging model organism for rare genetic disorders}

The zebrafish (Danio rerio) has become a prominent and powerful model organism for research into vertebrate genetics, development, regeneration and toxicology over the past 40 years combining conserved vertebrate characteristics with a capacity for large-scale phenotypic and therapeutic screening (Veldman \& Lin, 2008). Zebrafish are common freshwater teleost fish and belong to the Cyprinidae family. They can be typically found in slow-moving or still waters in floodplains in India, Pakistan, Nepal and Bangladesh (Suriyampola et al., 2016). Unlike mice or rats, zebrafish can be housed in relatively large numbers in a small space making husbandry cost-effective and giving the possibility to keep multiple lines in one facility. Additionally, they are relatively easy to take care of due to small size. The life cycle of a zebrafish is rather fast as they reach sexual maturity after only 12 weeks. Zebrafish are able to generate hundreds of embryos per week. Embryonic development of zebrafish is not only rapid but also ex utero and the transparency of the cells and surrounding chorion facilitate easy observation and staging (Kimmel, Ballard, Kimmel, Ullmann, \& Schilling, 1995). These characteristics combined with the availability of reporter lines and imaging techniques make the zebrafish highly useful for exploring the developmental effects of RD-associated gene variants.

The zebrafish genome has been fully sequenced and duplicated chromosome segments suggest that a genome duplication occurred in ray-fin phylogeny (Postlethwait et al., 1998). Despite this duplication, the zebrafish genome possesses considerable homology with the human genome, with orthologues having been identified for roughly $70 \%$ of human genes and $84 \%$ of diseaserelated genes having a zebrafish counterpart (Howe et al., 2013). Notably, 140,000 genes have been mutated to study their function in development and 
disease in the zebrafish. Moreover, zebrafish are also highly genetically tractable and easy to genetically manipulate with novel techniques being developed and optimized constantly (Hwang et al., 2013). Anatomically and physiologically, the zebrafish is more distant from humans compared to the mouse. Hence, it can be more difficult to model genetic diseases affecting structures that are absent in fish. Nevertheless, the basic vertebrate architecture of zebrafish makes it possible to study disease in numerous organ systems and structures that are present in both fish and humans in a simplified manner (Adamson et al., 2018). The first human disease identified applying the zebrafish was a blood disorder involving a specific defect in hemoglobin production caused by mutations in ALAS2. Since then, many other mutants revealing phenotypic similarities to human genetic disorders have been identified. For instance, the zebrafish has successfully been applied to study genetic diseases affecting human cardiovascular (K. A. Smith et al., 2009), nervous (Chapman, Bennett, Ramesh, De Vos, \& Grierson, 2013), visual (Zhang et al., 2016), renal (Mangos et al., 2010) and muscular systems (Majczenko et al., 2012).

\subsubsection{Zebrafish development and embryonic applications}

Development of the zebrafish embryo starts with the external fertilization of the oocyte. Afterwards, a total of seven different stages of embryogenesis can be defined: zygote, cleavage, blastula, gastrula, segmentation, pharyngula and hatching, highlighting the changing spectrum of developmental processes occurring during the first three days post fertilization (dpf). The fish hatch from their chorion typically between 48 and 72 hours post fertilization (hpf) marking the end of the embryonic period (Kimmel et al., 1995). The swim bladder inflates resulting in upright swimming between 72 and $96 \mathrm{hpf}$ (Muller \& van Leeuwen, 2004) and the fish begin feeding shortly thereafter (Muto \& Kawakami, 2013). After the period of embryonic growth, zebrafish spend approximately four weeks in a larval state. In the meantime, growth varies drastically based on temperature, density and individual differences. Once the fish reach a size of 11 $\mathrm{mm}$, they are classified as juveniles, whereas $>17 \mathrm{~mm}$ mark adulthood (Parichy, Elizondo, Mills, Gordon, \& Engeszer, 2009).

The zebrafish embryo offers remarkable opportunities for the investigation of fundamental developmental processes. With the majority of RDs having a pediatric onset and about $30 \%$ of children with RDs dying before the age of 5 years (Sardana et al., 2011), developmental processes are likely to be implicated in the pathogenesis of multiple RDs and can be studied in the zebrafish. Many of the critical pathways that control development in vertebrates are highly conserved between human and zebrafish. All common vertebrate organs develop 
early on in zebrafish, with some as early as $24 \mathrm{hpf}$, such as the heart, eyes or kidneys. Thus, the development and onset of metabolic functions for these common organs can be observed within a few days after generation.

In particular at embryonic and larval level, zebrafish can be applied in large-scale screening processes. They can be housed in 96-well plates allowing phenotypic analysis applying high-throughput microscopy (Arslanova et al., 2010) or therapeutic screening with soluble compounds being directly dissolved into the medium (Kithcart \& MacRae, 2017). Several drugs have similar targets and therefore similar effects in both humans and zebrafish. Thus, the zebrafish has emerged as the model of choice for screening of chemical libraries for potential therapeutic compounds. Several drugs picked up in such screens have already made their ways into human clinical trials (Kaufman, White, \& Zon, 2009).

\subsubsection{Genomic manipulation of zebrafish}

Over time, a range of techniques have been used to model human genetic diseases in zebrafish including both strategies for generating transient (Nasevicius \& Ekker, 2000; Tessadori et al., 2017) and stable models (Moens, Donn, WolfSaxon, \& Ma, 2008; Sander et al., 2011; Hwang et al., 2013). Transient genetic manipulation of zebrafish is most useful when complete loss-of-function (LoF) of a gene can be excluded and has traditionally been accomplished applying morpholino-based knockdown of target genes or expression of human mRNA harboring variants of interest in zebrafish embryos.

Whereas traditional genetic manipulation of zebrafish has focused on untargeted mutagenesis using chemical mutagens like $\mathrm{N}$-ethyl- $\mathrm{N}$-nitrosourea (Rohner, Perathoner, Frohnhofer, \& Harris, 2011), more recently powerful techniques have been developed involving the use of engineered nucleases, like zinc finger nucleases (ZFNs), transcription activation-like effector nucleases (TALENs) and the clustered regularly interspaced repeat (CRISPR)/CRISPR-associated protein 9 (Cas9) system. The latter has become the tool of choice not only in zebrafish but also in other model organisms due to a cost efficient and less laborious process involved in generating all essential components. Most successes from CRISPR/Cas9-based gene modifications in zebrafish involve the generation of frameshift null alleles through non-homologous end-joining (NHEJ)-mediated repair of CRISPR-induced DNA breaks resulting in LoF. However, many (common and rare) genetic diseases are caused by missense mutations arising from single base pair substitutions either abolishing or altering protein function. In order to introduce these specific substitutions into the genome of the zebrafish, the knock-in of patient specific variants has more recently been achieved through 
both homology-independent (Auer, Duroure, De Cian, Concordet, \& Del Bene, 2014) and homology-directed repair (HDR) (Armstrong et al., 2016), however efficiency still requires improvement.

\subsubsection{Modeling cardiovascular disorders in zebrafish}

Zebrafish embryos are also largely utilized for investigating (genetic) cardiovascular diseases as they undergo similar morphogenetic processes and therefore reveal a similar embryonic heart structure as that of human embryos. Cardiac cells of the zebrafish begin as two lateral populations, migrating to the midline and forming the primitive heart tube. During embryo development, this tube undergoes cardiac looping and further separates into distinct cardiac chambers connected at the atrioventricular (AV) junction (Yelon, 2001). Ideally, the embryonic zebrafish heart is placed at a prominent position at the ventral side of the embryo allowing visual inspection of the function of the developing heart in live organisms (Figure 3). Furthermore, zebrafish larvae have the advantage to survive without adequate cardiac circulation for several days (Hill, Teraoka, Heideman, \& Peterson, 2005). Oxygen enters the embryo and reaches other tissues by passive diffusion. This unique feature helps the embryo develop from the initial developmental stages despite cardiovascular defects.

A large number of mutations affecting the contractility and/or rhythmicity of embryonic zebrafish hearts have been identified (J. N. Chen et al., 1996; Stainier et al., 1996). The majority of these zebrafish mutants reveal phenotypic characteristics of human cardiac disorders and multiple of these loci encode zebrafish homologues of genes related to human heart diseases. For example, mutations in essential myosin light chain, regulatory myosin light chain, titin, cardiac troponin $T$ and beta myosin heavy chain have been associated with cardiomyopathy in humans (Thierfelder et al., 1994; Poetter et al., 1996; Gerull et al., 2002; Klaassen et al., 2008; ). Moreover, the hearts of these mutant fish reveal decreased contractility, myofibril disarray and dysmorphic cardiac chambers further highlighting the conservation of cellular and molecular mechanisms underlying the contractile machinery among vertebrates (Berdougo, Coleman, Lee, Stainier, \& Yelon, 2003; Rottbauer et al., 2006; Sehnert et al., 2002; Xu et al., 2002). Next to the contractile machinery, mechanisms regulating cardiac rhythmicity seem to be conserved from fish to mammals. Similar to the mammalian heart, genes regulating ion flux are required for establishing and maintaining coordinated cardiac contractions (Langenbacher et al., 2005; Splawski et al., 2005). The conserved molecular mechanisms underlying contraction and rhythm in the heart as well as the phenotypic similarities between zebrafish mutants and 
human cardiac disorders indicate that zebrafish serve as a good model organism to investigate genetic cardiovascular disorders.
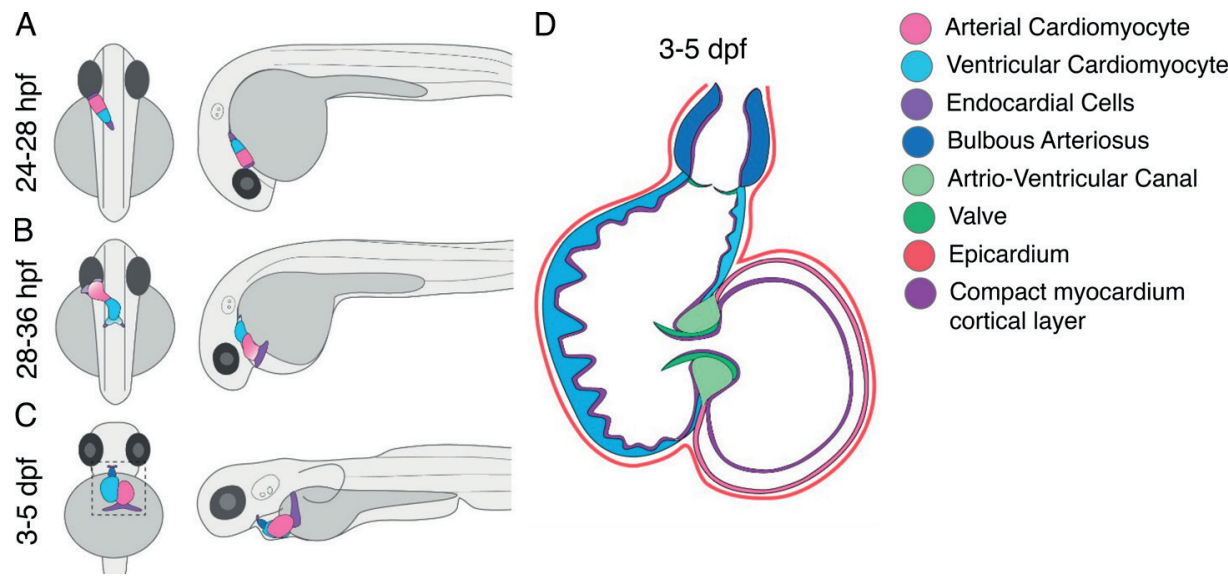

Figure 3 | Zebrafish heart development. (A-C) Heart development from $24 \mathrm{hpf}$ to $5 \mathrm{dpf}$ depicted in dorsal (left) and lateral (right) view. (D) Cross-section of embryonic heart from 3 to $5 \mathrm{dpf}$ with fully developed atrial and ventricular chambers separated by valves leaflets at the AV junction. Figure adapted from by (Brown 2016).

\subsection{Treating rare diseases}

In $95 \%$ of all RDs there is no licensed treatment or cure available (Kaufmann, Pariser, \& Austin, 2018) resulting in a drastic decrease in quality of life due to stress, anxiety, chronic pain, physical impairment or early mortality in the majority of patients (Bogart \& Irvin, 2017). Hence, the impact of therapeutic RD intervention is considerable, not only for the patients but also their family and healthcare providers. Currently, most patients merely receive symptomatic or comfort treatment which address second-order complications instead of the underlying disease cause. Current treatment is therefore aimed at the improvement of life quality of those affected, but does not prevent the inevitable decline in function. In Fabry disease, for example, previous interventions simply replaced the missing enzyme by a naturally occurring human lysosomal hydrolase enzyme resulting in a buildup of fat in various patient tissues and therefore not providing a cure per se. A novel treatment, Migalastat (Galafold), increases the enzyme activity by stabilizing the endogenous protein and supporting a proper folding and therefore significantly reduces associated symptoms (Lenders et al., 2020). 
This great unmet need makes it imperative that we find ways to accelerate the therapy development process so that we can help many RD patients who are in search of better treatments.

\subsubsection{Challenges in drug development for RDs}

One way to tackle the obvious gap in clinical management of RDs represents the development of novel orphan drugs. The development of such new drugs, however, is a major challenge with often only limited knowledge available regarding disease epidemiology, manifestations, heterogeneity, natural course and progression (Cote, $\mathrm{Xu}, \&$ Pariser, 2010). Taking into account that the process of developing a novel drug to treat any kind of disease is typically laborious, costly and failure-prone (Kola \& Landis, 2004), it is especially unappealing to do so for RDs that affect only a small number of individuals and therefore generate reduced profit. The introduction of a new compound to the market can cost as much as $\$ 2.5$ billion with further increasing numbers often including high development and manufacturing costs (DiMasi, Grabowski, \& Hansen, 2016; Nosengo, 2016). Notably, merely five out of 5,000 (0.1\%) experimental compounds that enter preclinical testing progress to evaluation in humans. Only one of these five compounds receives approval from the US Food and Drug Administration for use in humans highlighting the failure susceptibility of this process (Collins, 2011). Lastly, traditional drug discovery strategies are highly time-consuming often requiring 10 to 15 years of research and development efforts until a drug can finally enter the market (Figure 4). When large manufacturers invest in research and development of therapy options for RDs despite these challenges, market prices are typically extremely high resulting in higher profit but reduced access for patients. For instance, Nusinersen (Spinraza), the first approved drug for spinal muscular atrophy, is one of the most expensive drugs on the market, costing $\$ 750,000$ for the first year of treatment and $\$ 375,000$ every following year (Fitzgerald, Abel, Jones, \& Farrar, 2018).

Despite the monogenic nature of the majority of RDs, many variations or disease subtypes result in different clinical manifestations and disease progressions. Depending on RD heterogeneity a compound might work for only a subset of patients. The compound choice may depend on the type of mutation the patient has, as is exemplified in cystic fibrosis where mutation-specific therapies are already in development (Brewer, 2006; Villella et al., 2019). In addition, designing a clinical trial can be time consuming and complicated due to absence of structured databases and patient cohorts, and insufficient epidemiological and scientific data (Southall et al., 2019). Despite occasional success stories like novel therapy options for acute hepatic porphyria (Sardh et al., 2019), hereditary 
transthyretin-mediated amyloidosis (Solomon et al., 2019), cystic fibrosis (Burgener \& Moss, 2018) or spinal muscular atrophy (Farrar et al., 2017), these great challenges provide little hope for obtaining a product approval for most individuals with one of the nearly 7,000 RDs.

\subsubsection{Drug repurposing: a cheaper and faster option}

Drug repurposing (also known as drug repositioning or drug reprofiling) is the process of redeveloping a compound for use in a different disease (Simsek, Meijer, van Bodegraven, de Boer, \& Mulder, 2018) and is now becoming an increasingly important strategy for researchers in industry and academia (Naylor S, 2015). Although this strategy is far from new, repurposing success stories and companies leveraging repurposing strategies are increasing in number (Atkinson et al., 1975; Yonkman, 1959). They are based on the following core scientific principles: (i) single drugs often interact with multiple targets or pathways and (ii) various drugs may act on the same target or pathway (Boran \& Iyengar, 2010; Mele, Citro, Andreotti, \& Cubellis, 2015; Metz \& Hajduk, 2010). Growing evidence indeed suggests that any functional compound classified to be safe for human use is likely to have multiple therapeutic applications. Most repurposed drugs show little to no clear connection to their primary approved indications. For instance, compounds tend to show off-target effects triggering undesired adverse events (Guengerich, 2011). However, these effects might be of advantage for other indications. Lastly, a target involved in a certain disease is also often associated with other biological processes, pathways or phenotypes (Pujol, Mosca, Farres, \& Aloy, 2010) and pathogenetic mechanisms of rare and common disease often influence the function of more global molecular pathways and networks rather than merely the function of single genes (Chan \& Loscalzo, 2012; Piro, 2012).

Pharmaceutical companies search for inexpensive alternatives to compensate for the high costs and disappointing success rates associated with the drug discovery pipeline (Ashburn \& Thor, 2004). Hence, they turn to already approved, discontinued, shelved or experimental drugs and try to establish new medical applications. The process of drug repurposing is considered to be significantly faster and cheaper than traditional drug discovery and therefore offers hope to RD patients, for whom the conventional model is commercially unviable (Boguski, Mandl, \& Sukhatme, 2009). Specifically, development risk is reduced as repurposing candidates have already proven to be sufficiently safe in preclinical models and, at least, at early-stage trials in humans, thus being less likely to fail from a safety point of view in subsequent efficacy trials. Hence, after establishing dose compatibility, a majority of preclinical testing (e.g. in vitro and 


\section{Orphan drug discovery}

Drug discovery and research

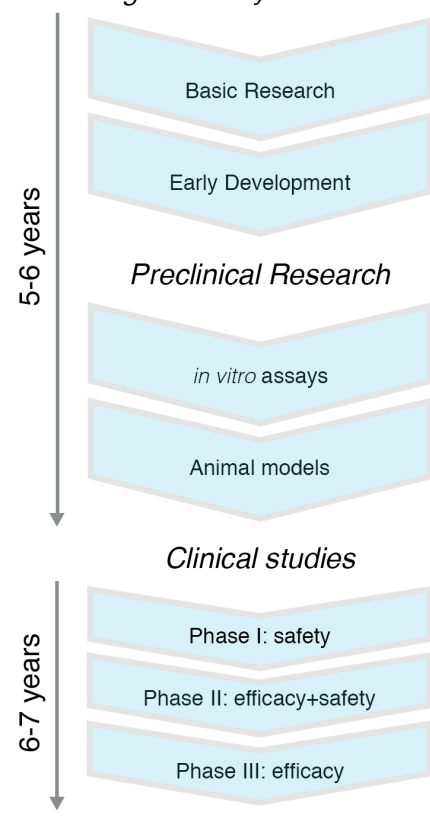

Marketing application review and approval

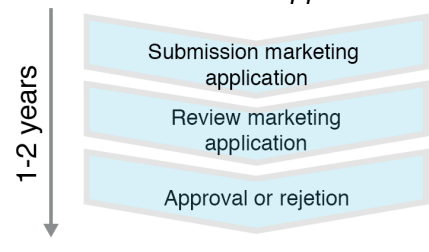

\section{Drug repurposing}

\section{Compound identification}

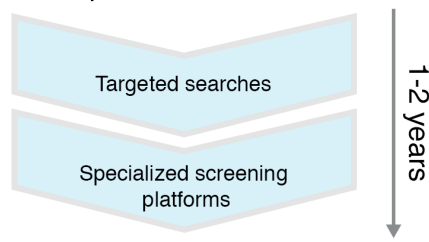

Compound validation and preclinical research

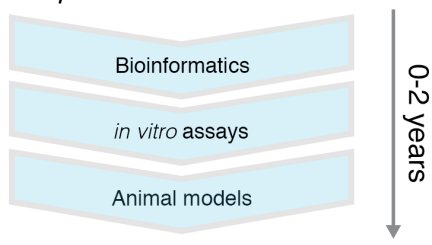

Clinical studies

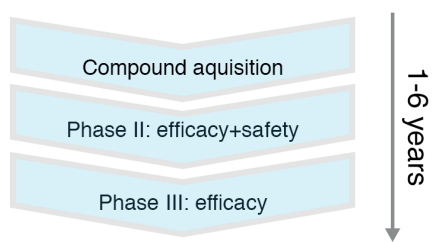

Compound registration

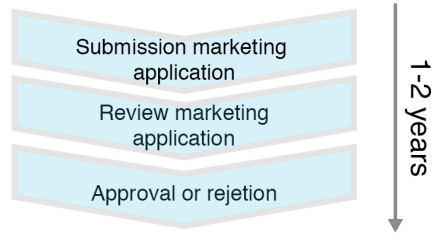

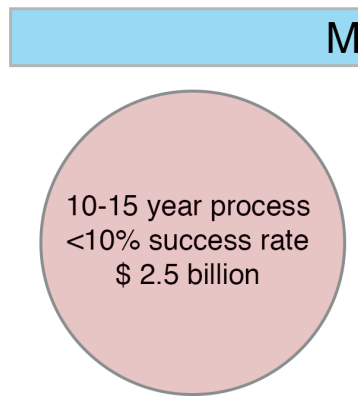

Market

Figure 4 | Advantages of drug repurposing over the traditional way of orphan drug development. Drug repurposing is shown to increase success rates, reduce development costs, and shorten time to the market, and therefore reduces the overall development risk compared to traditional drug development. Repurposing candidates has already proven to be sufficiently safe in preclinical models and, at least, at early-stage trials in humans, thus being less likely to fail from a safety point of view in subsequent efficacy trials. Often, the only step left to accomplish is to confirm efficacy for the new indication at preclinical and clinical levels. Hence, drug repurposing is an important alternative to orphan drugs especially in the field of rare diseases. 
in vivo screenings, chemical optimization, formulation development), safety assessment, bulk manufacturing and even phase I clinical trials can be bypassed. The only step left to accomplish is to confirm efficacy for the new indication at preclinical and clinical levels. On the other hand, developing a new formulation for already known drugs can also be considered an appealing strategy for drug developers, for instance resulting in obtaining new patent protection (Murteira, Ghezaiel, Karray, \& Lamure, 2013). Finasteride, for instance, was originally developed to treat benign prostatic hyperplasia, but eventually repurposed for male pattern baldness. Due to applying a new formulation, the novel method of use received a new patent despite the fact that the old one was still active (McClellan \& Markham, 1999).

Repurposed drugs can reach the patient as a marketed treatment in 3-12 years. On average, they cost $\$ 300$ million and have an estimated success rate ranging from 30\% (2014) to as high as a potential 75\% (Nosengo, 2016); five times higher than for developing new compounds (Figure 4). The approximately 3,000 drugs that have been approved by at least one country therefore represent a vast untapped resource if they can be used against another condition, especially a RD.

An example of such a drug repurposing effort has been with HGPS, the extremely rare and fatal premature aging disease caused by variants in the Lamin A/C gene ( LMNA) which activates a cryptic splice site and results in the production of a farnesylated mutant lamin A protein called progerin (Hennekam, 2006). Farnesyltransferase inhibitor (FTI) drugs which reduce the amount of permanently farnesylated progerin, are shown to hold therapeutic potential for this disorder. Based on observations from clinical trials, a new drug application has been submitted to the FDA and the process for approval of the FTI Lonafarnib (Sarasar), originally used for cancer treatment, as the first-ever treatment for HGPS has begun recently (Gordon et al., 2012; Gordon et al., 2014; Gordon et al., 2018). Another example where a drug was repurposed for a RD involves Muckle-Wells syndrome (MWS), an autoinflammatory disorder caused by increased interleukin-1 (IL-1). Canakinumab (Ilaris), a drug originally approved to treat rheumatoid arthritis, is a human IgG1 anti-IL-1 $\beta$ monoclonal antibody that provides selective and sustained blockage of IL-1 $\beta$ neutralizing the effect of excess IL-1 $\beta$. Various clinical studies suggested that Canakinumab results in a sustained control of disease activity and a rapid remission of associated symptoms in MWS patients (Kuemmerle-Deschner et al., 2013; Lachmann et al., 2009). It was approved by the FDA and by the European Commission in 2009 to treat patients with MWS. 
Instead of directly applying an existing drug for a new indication as shown in the previous examples, compounds identified as hits may also be subjected to further optimization. Even though this so-called lead optimization is a more complex endeavor as it requires full clinical trials due to the generation of new compounds, it also represent the opportunity to eliminate unwanted side-effects or off-target effects originating from the initial use. Moreover, this process can also result in the increase of potency of the chemical compound at the primary drug target protein (Hughes, Rees, Kalindjian, \& Philpott, 2011). In order to get closer to a treatment for Batten disease, a fatal nervous system RD, a library of derivates of a clinically available but due to side-effects restricted drug with neuroprotective activity was synthesized. Resulting compounds were shown to reveal physicochemical features desirable for disorders involved the central nervous system (Kinarivala, Patel, Boustany, Al-Ahmad, \& Trippier, 2017; Maalouf et al., 2020). These examples highlight that drug repurposing can be effective in finding a therapy to help RD patients in a time-efficient manner.

\subsubsection{Approaches to drug repurposing for RD}

Although drug repurposing is not a novel idea, the strategy to do it in a systematic and rational way is new. Many repurposed drugs have been found serendipitously in the past in the lab or by cautiously monitoring the effect of drugs in the clinic and performing subsequentially retrospective analysis of clinical observations (Demonaco, Ali, \& Hippel, 2006). This has been the case for one of the most popular repurposing success stories Minoxidil, originally investigated to treat ulcers; while conducting trials in dogs, it revealed a prolonged reduction in blood pressure. Later, while undergoing clinical trials to prove its efficacy as antihypertensive medication, the drug showed an unexpected positive effect on hair loss which subsequentially led to its application for male baldness (Kaler et al., 1987). However, as there are more than 7000 RDs in need of treatment, this approach clearly does not meet the requirements for these diseases. Thus, more systematic, organized and data-oriented searches for candidates have been launched profiting from technological advances like big data analytics, computer models and high-throughput screenings (Ekins, Williams, Krasowski, \& Freundlich, 2011; Ferreira \& Andricopulo, 2016; Pushpakom et al., 2019). Additionally, the availability of huge information on the genetic basis of many $\mathrm{RDs}$, on gene regulation, protein structure and drug-target interactions from the Human Genome Project has led to great opportunities to perform drug repurposing. Profiting from the improving availability of gene annotations for both human and model organisms, newly identified causative gene defects can now be checked to see whether they share same pathways or biological processes with common diseases. For example, genes associated with the RDs 
Neurofibromatosis Type-1, Cowden Disease and Retinoblastoma share common pathways that also play a role in various types of cancer (Gonzalez \& Riddle, 2016). Thus, a drug discovered to be useful in treating these cancer types might have undiscovered indications for RDs.

The current process of drug repurposing typically contains the following steps: (i) identification of a target and (ii) candidate molecule, (iii) mechanistic assessment of drug effect in preclinical models, (iv) preclinical drug development, ( $v$ ) evaluation of drug efficacy in phase II clinical trials (in case sufficient safety data from phase I studies as part of the original indication is available) and (vi) filing for marketing approval. During the process of identifying a candidate compound, various strategies that have already proven to be helpful in the field of drug repurposing can be applied. These can be broadly categorized into experimental and computational approaches. In the range of this thesis, merely experimental techniques have been applied which therefore will be described in more detail.

The drug discovery process has been revolutionized in the last two decades transitioning from low throughput animal model-based techniques to high throughput screens. The latter strategy takes advantage of the recent and rapid advances in screening technologies which allow to re-screen existing compounds against a variety of targets to identify possible therapeutic benefits or side-effects in an unbiased manner (O'Connor \& Roth, 2005). This approach can result in the discovery of novel interactions between approved drugs and previously unexplored- or incompletely explored targets like newly identified causative RD genes. The readout of such drug screens can either be based on known molecular targets or a phenotype associated with the disease. Phenotypic screens offer the opportunity to identify potential treatments for complex diseases where it might be challenging to find the primary therapeutic targets. On the other hand, screens based on a molecular-target can further expedite drug discovery for RDs by addressing several diseases that share a common molecular etiology within one high-throughput screening (Sun, Sanderson, \& Zheng, 2016).

A screening model resulting in recent advances in compound screening and evaluation of drug efficacy for neurological RDs are induced pluripotent stem cell (iPSC)-derived neurons. Whereas reliable in vitro models of human neurons are lacking and representation of many neurological RDs in animal models is often incomplete, patient-derived iPSCs represent a more relevant disease system in the appropriate setting (Farkhondeh et al., 2019; Shi, Inoue, Wu, \& Yamanaka, 
2017). Neuronal cells derived from iPSCs of Niemann-Pick disease type 1C, a rare lysosomal disorder, revealed cholesterol accumulation comparable to patients and responded to drug treatment (Yu et al., 2014). In cystic fibrosis, mutation-specific iPSC lines were established and applied to generate organoid models for the evaluation of drug efficacies (K. G. Chen et al., 2018; Gorshkov et al., 2019). Lastly, Kinarivala et al. successfully characterized the first Batten disease patient-specific iPSC-derived model of the blood-brain barrier resulting in the identification of small molecules modulating autophagy (Kinarivala et al., 2020).

\subsection{Thesis Outline}

Rare genetic disorders (RD) collectively impact a significant amount of the world's population. For many RDs there is limited information on pathophysiology and natural history available, and clinicians encounter challenges in differentiating between clinically similar conditions. This results in difficulties in genetic counseling and eventually clinical treatment (Part I). In contrast, technological advantages including NGS together with computer-aided tools and online resources allow a more thorough and detailed understanding of rare disorders. In particular, combining DNA sequencing technologies and functional modeling can help dramatically in diagnosing and establishing molecular understanding of RDs. Once diagnosed accurately, treatment possibilities open up. As the biomedical market of orphan drugs for RDs is significantly affected since pharmaceutical and biotechnological industries are deterred by high development costs and low return, the field of drug repurposing has dramatically increased in popularity in recent years (Sardana et al., 2011). In an attempt to repurpose drugs in a more systematic and rational manner compared to the past, multiple strategies have been launched from computer models to (highthroughput) screenings in model organisms (Part II).

The past years have seen a remarkable rate of discovery of (variants in) genes causing RDs and therefore an unprecedented increase in the number of diagnosable but nevertheless untreatable disorders. In the following chapters, I illustrate success stories where diagnosing and understanding Cantú syndrome (CS) and related disorders has opened up possible therapy options for patients.

In Part I, I highlight the importance of accurate and detailed clinical characterization of an RD. Establishing knowledge of the phenotypic spectrum, its heterogeneity and the natural history of a rare disorder is essential for a 
correct clinical diagnosis and gives patients the chance to avoid misdiagnoses, offer molecular testing and undergo adequate genetic consultation (Chapter 2 and 3). In Chapter 4, we assess cognitive profile, social functioning and psychiatric symptoms in a CS cohort systematically to ensure that (future) patients are treated with the appropriate intervention. In Chapter 5, we apply a novel technique for facial phenotyping in order to assess its' usefulness and functionality, establishing a clinical diagnosis and optimizing clinical counselling. In Chapter 6, we underline the importance of multi-center collaboration in $\mathrm{RD}$ research. By establishing an international patient registry, we provide information on both phenotype as well as genotype of a large cohort of CS patients and quantitative assessment of the penetrance of disease features.

In Part II, we progress to utilizing functional modeling in various model systems in order to validate and further investigate pathophysiology of RDs. In Chapter 7, we present a novel and effective approach to introduce patient-specific variants in the zebrafish genome and deep phenotyping of the resulting zebrafish embryos revealing key features of human genetic cardiovascular disorders. In Chapter 8, we apply these zebrafish models to further elucidate the pathophysiology of CS. Based on this knowledge, we use phenotype-based drug screening and identify two possible drugs that have the potential to be repurposed for this new indication. Finally, in Chapter 9, we determine the genetic and molecular basis of a novel RD by performing WES and validating the resulting candidate gene in two model organisms, the zebrafish and the mouse. 
Introduction: Towards treatment of rare genetic diseases 


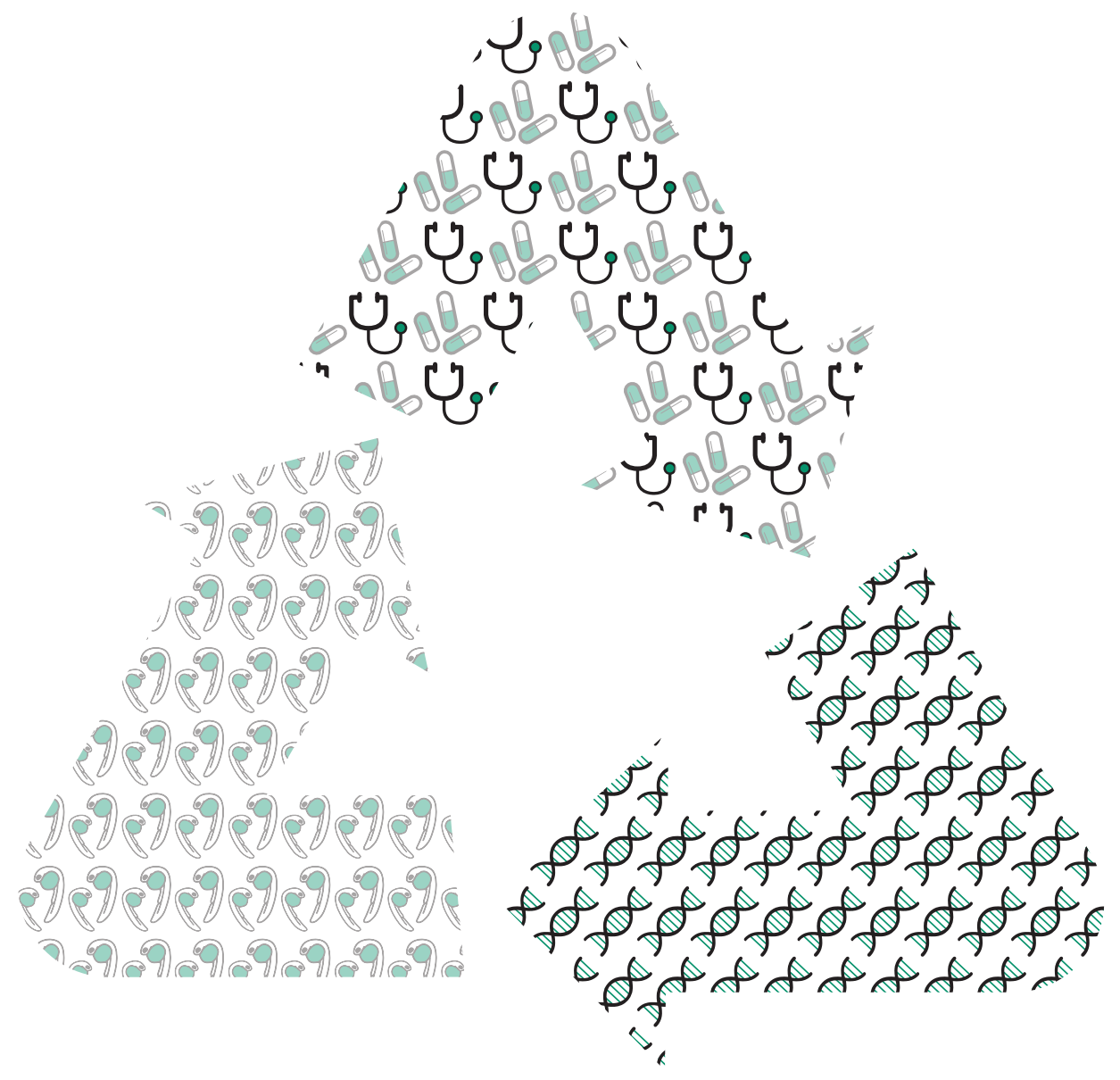




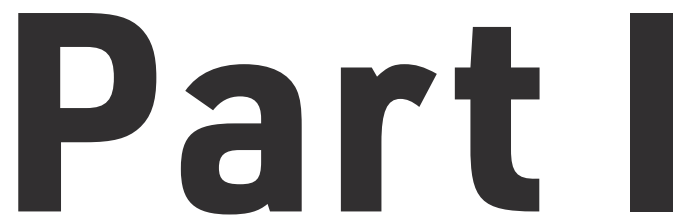

Clinical Characterization of Cantú syndrome 
-12
4
4
4

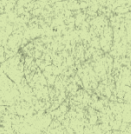

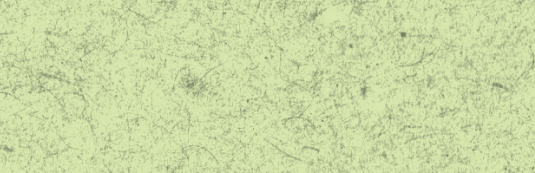

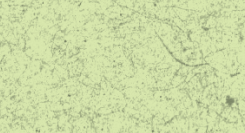

तr.

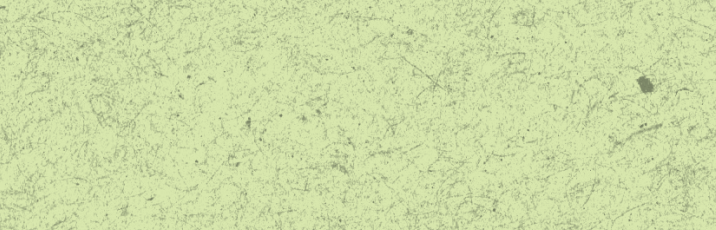

4

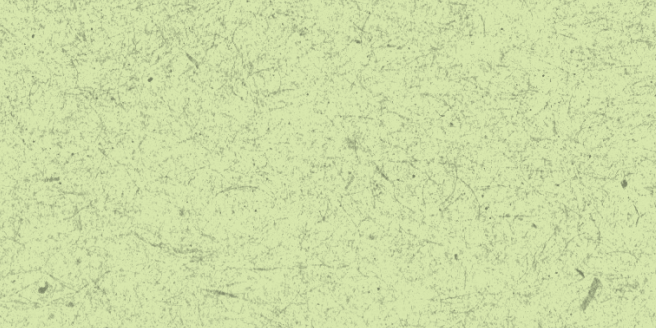




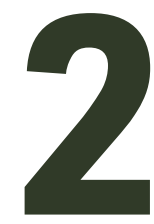

\section{Cantú syndrome - a genetic syndrome reference guide}

Helen I. Roessler, Mieke M. van Haelst 


\subsection{Gene defect}

Heterozygous gain-of-function mutations in ABCC9 or KCNJ8 (both 12p12.1)

OMIM: ABCC9, *601439; KCNJ8, *600935.

\subsection{Other names}

Hypertrichotic osteochondrodysplasia

hypertrichosis-osteochondrodysplasia-cardiomegaly syndrome

\subsection{Definition}

Cantú syndrome (CS) is a rare congenital multi-organ system genetic disorder that was first described by Cantú et al. in 1982 (Cantu, Garcia-Cruz, SanchezCorona, Hernandez, \& Nazara, 1982). Subsequent reports have described about 150 patients worldwide exhibiting variable clinical manifestations. CS is mainly characterized by congenital hypertrichosis, characteristic facial appearance (Figure 1), and a wide constellation of cardiovascular abnormalities, including an enlarged heart and patent ductus arteriosus (PDA) (Grange et al., 2019). The underlying genetic cause of CS was eventually discovered in 2012 being gain-offunction (GoF) mutations in ABCC9 and KCNJ8, which encode SUR2 and Kir6.1, respectively, both forming subunits of an ATP-sensitive potassium $\left(K_{A T P}\right)$ channel.

The overall incidence at birth is still unknown. However, increased awareness of the clinical phenotype will result in better recognition and higher documented prevalence.

\subsection{Etiology}

CS follows an autosomal dominant inheritance pattern and is caused by heterozygous pathogenic mutations in ABCC9 and less commonly KCNJ8 (Brownstein et al., 2013; Harakalova et al., 2012). A majority of reported CS patients show simple missense variants in either gene, which are often reported to be de novo. So far, only one individual harboring a deletion-insertion variant involving two nucleotides in ABCC9 was reported (Grange et al., 2019). Altogether, there about 35 variants in $A B C C 9$ and 3 variants in KCNJ8 known which all alter amino acids in or close to the transmembrane domains (TMD) of both proteins (Grange et al., 2019). A majority of variants in ABCC9 is reported to cluster around TMD2. Variants at p.Arg1154 and p.Arg1116 represent the most often observed variants in all confirmed cases. 
Together, ABCC9 and KCNJ8 encode proteins that form regulatory (SUR2) and pore-forming (Kir6.1) subunits of an ATP-sensitive potassium channel, a potassium-selective ion channel. These subunits are prominently expressed in cardiomyocytes and vascular smooth muscle cells.

An established model for the molecular consequences of CS variants is that the missense mutations in ABCC 9 and KCNJ8 reveal a GoF effect resulting in enhanced $K_{\text {ATP }}$ channel activity (McClenaghan et al., 2017). $K_{\text {ATP }}$ GoF is suggested to result in hyperpolarization of the membrane potential eventually reducing excitability in all cell types that show channel expression. In smooth muscles, such decreased excitability is suggested to cause relaxation. In case of blood vessels, this results in a decline in blood pressure and a consequential compensatory structural and electrical remodeling of the heart (e.g. cardiac hypertrophy, hypercontractility). Hence, it is suggested that cardiac enlargement arises independently of ventricular $\mathrm{K}_{\mathrm{ATP}}$ activity as a secondary consequence of vascular dysfunction (McClenaghan et al., 2019). How $K_{\text {ATP }}$ overactivity results in further CS-associated features remains largely unexplained and requires further investigation. 


\subsection{Clinical Manifestations}

Table 1 | Clinical manifestations of Cantú syndrome.

\begin{tabular}{|c|c|c|}
\hline Systems & Major (Signs/Symptoms) & Minor (Signs/Symptoms) \\
\hline $\begin{array}{l}\text { Integumentary } \\
\text { system }\end{array}$ & Hypertrichosis & $\begin{array}{l}\text { Loose/wrinkled skin, deep palmar/ } \\
\text { plantar creases }\end{array}$ \\
\hline $\begin{array}{l}\text { Cardiovascular } \\
\text { system }\end{array}$ & $\begin{array}{l}\text { PDA, cardiac enlargement, high } \\
\text { output state, pericardial effusion, } \\
\text { dilation of aortic root, dilated/ } \\
\text { tortuous cranial arteries, cardiac } \\
\text { arrhythmia, blood pressure } \\
\text { abnormalities, } \\
\text { heart failure }\end{array}$ & $\begin{array}{l}\text { valvular defects*, atrial septal } \\
\text { defects*, ventricular septal } \\
\text { defects, aortic coarctation* }\end{array}$ \\
\hline Nervous system & $\begin{array}{l}\text { Headaches/migraines, seizures, } \\
\text { hemiparesis*, stroke*, white } \\
\text { matter changes }\end{array}$ & \\
\hline $\begin{array}{l}\text { Visual/auditory } \\
\text { system }\end{array}$ & Myopia/hyperopia, hearing loss & \\
\hline Lymphatic system & (Lymph)edema in lower legs & \\
\hline $\begin{array}{l}\text { Skeletal system } \\
\text { (including } \\
\text { connective tissue) }\end{array}$ & $\begin{array}{l}\text { Macrocephaly, macrosomia, } \\
\text { osteochondrodysplasia/skeletal } \\
\text { dysplasia (e.g., broad ribs, pectus } \\
\text { carinatum, scoliosis) }\end{array}$ & $\begin{array}{l}\text { Osteoporosis/osteopenia*, } \\
\text { delayed bone age*, hypermobile } \\
\text { joints (hands), hernia (inguinalis/ } \\
\text { umbilicalis) }\end{array}$ \\
\hline Respiratory system & Pulmonary hypertension & $\begin{array}{l}\text { Respiratory tract malformations } \\
\text { (e.g., hemoptysis, } \\
\text { bronchomalacia)* }\end{array}$ \\
\hline Digestive system & $\begin{array}{l}\text { Intestinal dysfunction, gastro- } \\
\text { esophageal reflux }\end{array}$ & $\begin{array}{l}\text { Gastrointestinal bleeding*, pyloric } \\
\text { stenosis* }\end{array}$ \\
\hline Urinary system & & $\begin{array}{l}\text { Recurrent kidney or bladder } \\
\text { infections, hydronephrosis, vesico- } \\
\text { ureteral reflux }\end{array}$ \\
\hline Reproductive system & & Abnormal menstruation \\
\hline Immune system & $\begin{array}{l}\text { Immune dysfunction (e.g., } \\
\text { recurrent respiratory injections) }\end{array}$ & \\
\hline
\end{tabular}

*Frequency reported $<10 \%$.

\subsection{Diagnosis}

To date, no official diagnostic criteria for CS have been established. Typically, a diagnosis of CS is first established based on clinical findings and then molecularly confirmed by detection of a heterozygous pathogenic variant in ABCC9 or KCNJ8 either applying targeted Sanger sequencing of both genes or whole-exome sequencing (WES) or whole-genome sequencing (WGS). Key clinical indicators that should result in the suspicion of CS include a combination of congenital hypertrichosis, course facial features (Roessler, Volker-Touw, Terhal, van Haaften, \& van Haelst, 2018) and cardiovascular anomalies like cardiac enlargement or PDA (Grange et al., 2019). Due to the overall rarity and the limited experience of physicians with CS, cases can initially 
be misdiagnosed with metabolic disorders such as a mucopolysaccharidosis, Beckwith-Wiedemann syndrome, based on coarse facial features, or Pompe disease based on neonatal cardiomegaly (Grange et al., 2019). Thus far, there have been no prenatal diagnoses of CS. As the genes associated with CS have only recently been identified, especially older patients often lack a molecular diagnosis. Therefore, a vast majority of published cases were either diagnosed in early childhood or in adulthood. Interestingly, high intra-familial variability of CS-associated features has been reported, complicating clinical diagnosis. Sometimes, affected parents are diagnosed only after their more severely affected children received a molecular confirmed diagnosis of CS (Grange et al., 2019; Roessler et al., 2018).

\subsection{Management}

With the genetic cause of CS being discovered only recently, little is known about the progressive nature, especially as a majority of diagnosed individuals are children or young adults (Grange et al., 2019; Roessler et al., 2018). Thus, further longitudinal data needs to be generated to better understand the longterm effects and implications of CS pathophysiology. Although rare, CS is a debilitating syndrome, with no specific therapy available (as yet).

Currently, clinical management of CS involves symptomatic treatments to address secondary complications. If cardiovascular anomalies are present, annual echocardiograms are recommended. In case of frequent migraines, brain MRI/MRA should be considered to visualize possible neurological anomalies. Cosmetic treatment for generalized hirsutism may include daily shaving or laser hair removal. PDA often requires surgical closure in infancy and pericardial effusion may require pericardiocentesis and subsequent pericardial stripping. Additionally, peripheral edema is typically treated with compression stockings. Treatment of the associated heart problems mostly includes ACE inhibitors such as Quinapril or Enalapril to prevent heart failure, as well as beta-blockers like Atenolol or Bisoprolol. Notably, the majority of individuals that have been followed over multiple years have not developed heart failure, but they are still rather young in age. Many CS patients additionally receive diuretics including Furosemide and Spironolactone to treat edema and vasodilators such as Sildenafil to treat pulmonary hypertension (Grange et al., 2019).

It is postulated that $\mathrm{K}_{\text {ATP }}$ channel blockers could close the overactive potassium channel present in individuals affect with CS. For instance, $K_{\text {ATP }}$ channel inhibitors applied in the treatment of neonatal diabetes resulting from GoF in pancreatic SUR1- and Kir6.2-dependent channels could be repurposed to cure 
CS (McClenaghan et al., 2017). Recent studies in CS mice revealed reversal of cardiovascular abnormalities after treatment with glibenclamide, showing cardiac remodeling in CS is reversible (McClenaghan et al., 2019). Clinical trials in affected adolescent cases will be conducted to further test this hypothesis.
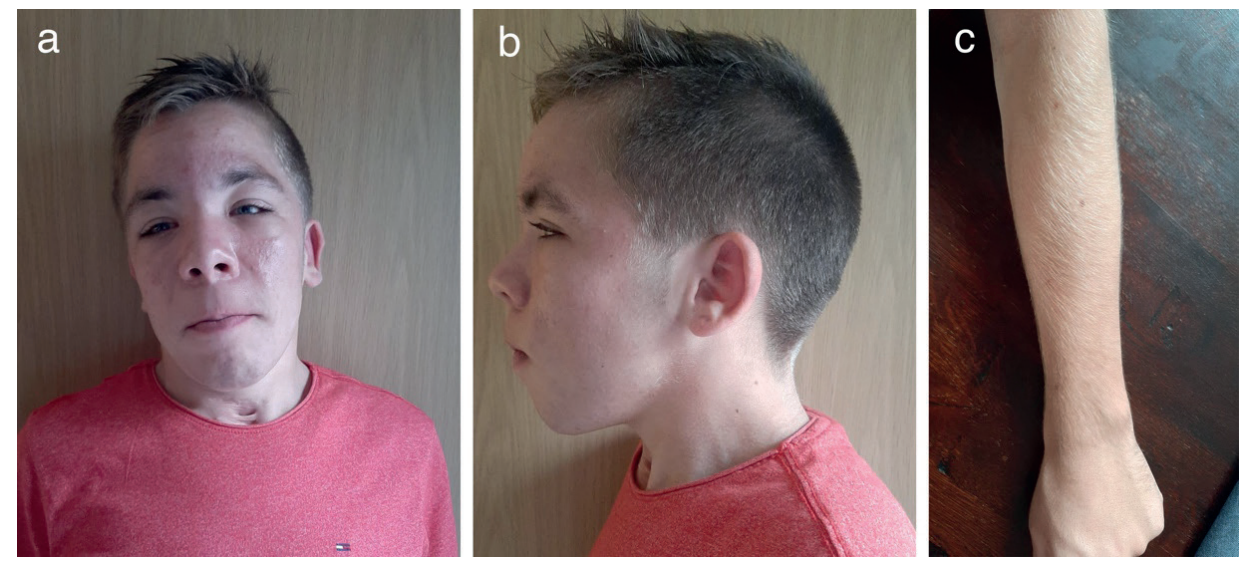

Figure 1 | Cantú syndrome features. (a, b) Facial phenotype of CS. Note edema of eyelids, epicanthal folds and widely spaced eyes. (c) Hypertrichosis of the limbs (not shaved). 
Cantú syndrome - a genetic syndrome reference guide

2 
-12
4
4
4

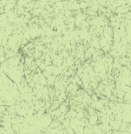

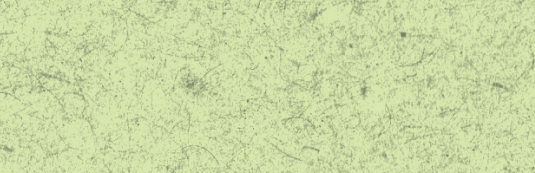

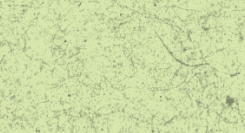

तr.

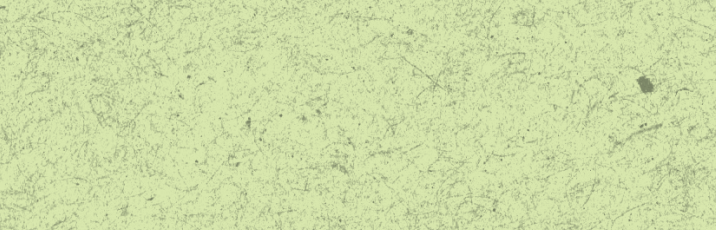

4

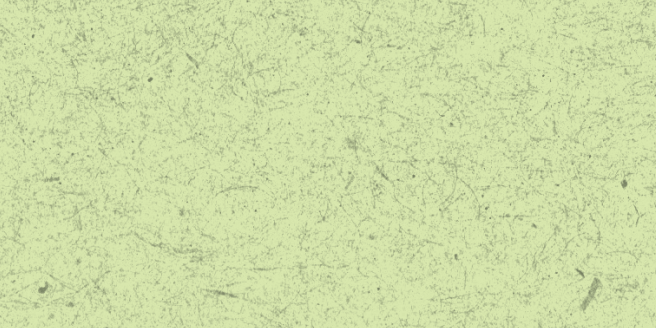




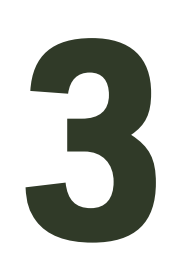

\section{Cantú syndrome, the changing phenotype: a report of the two oldest Dutch patients}

Helen I. Roessler, Catharina M.L. Volker-Touw, Paulien A. Terhal, Gijs van Haaften, Mieke M. van Haelst

Clin. Dysmorphol. 2018 July 27:78-83. DOI: 10.1097/MCD.0000000000000219 
Chapter 3

\section{List of key features}

Coarse facial features

Congenital hypertrichosis

Cardiomegaly

Osteochondrodysplasia

Cerebrovascular anomalies

Gain-of-function mutations in ABCC9 or KCNJ8 


\subsection{Introduction}

Cantú syndrome (CS, OMIM \#239850) is a rare but recognizable genetic disorder caused by gain-of-function mutations in $A B C C 9$ or $K C N J 8$, encoding SUR2 and Kir6.1, respectively, both forming subunits of ATP-sensitive potassium $\left(\mathrm{K}_{\mathrm{ATP}}\right)$ channels (Brownstein et al., 2013; Cooper et al., 2014; Harakalova et al., 2012; van Bon et al., 2012).

CS is characterized by variable clinical manifestations consisting of congenital hypertrichosis, cardiomegaly, and skeletal abnormalities that are observed evenly in nearly every patient (Grange, Lorch, Cole, \& Singh, 2006; Scurr et al., 2011). Coarse facial features are striking in younger patients, including a low frontal hairline, epicanthic folds, a flat nasal bridge, a prominent mouth, and thick lips, in many cases suggestive of a lysosomal storage disorder. With advancing age, however, these clinical features evolve considerably. Notably, cardiac manifestations such as cardiomegaly, patent ductus arteriosus (PDA), and pericardial effusions are present in the majority of cases. More recently, various neurologic manifestations, particularly cerebrovascular findings, have also been reported (Leon Guerrero et al., 2016).

Most of the reported patients (61\%) are still in their childhood at the time of examination. Consequently, patients between the age of 31 and 50 years have very rarely been reported in the current literature (8\%) (Figure 1). The oldest patient reported to date is 50 years of age (Scurr et al., 2011). These numbers highlight the relevance of focusing on the few known cases of older patients, with special attention to the progression of the condition. Knowledge of the phenotypic spectrum of CS and its changing phenotype with age is important with respect to follow-up and counseling of patients and their families. In addition, earlier recognition and molecular confirmation of this rare condition can be very useful and prevent an extensive metabolic work-up.

Here, we present two Dutch adult patients with CS (respectively 38 and 42 years of age), together with clinical data of their affected children, to highlight the possible intrafamilial phenotypic variability. We discuss the consistent clinical manifestations of CS at different ages and provide information on the phenotypic changes occurring over time. 


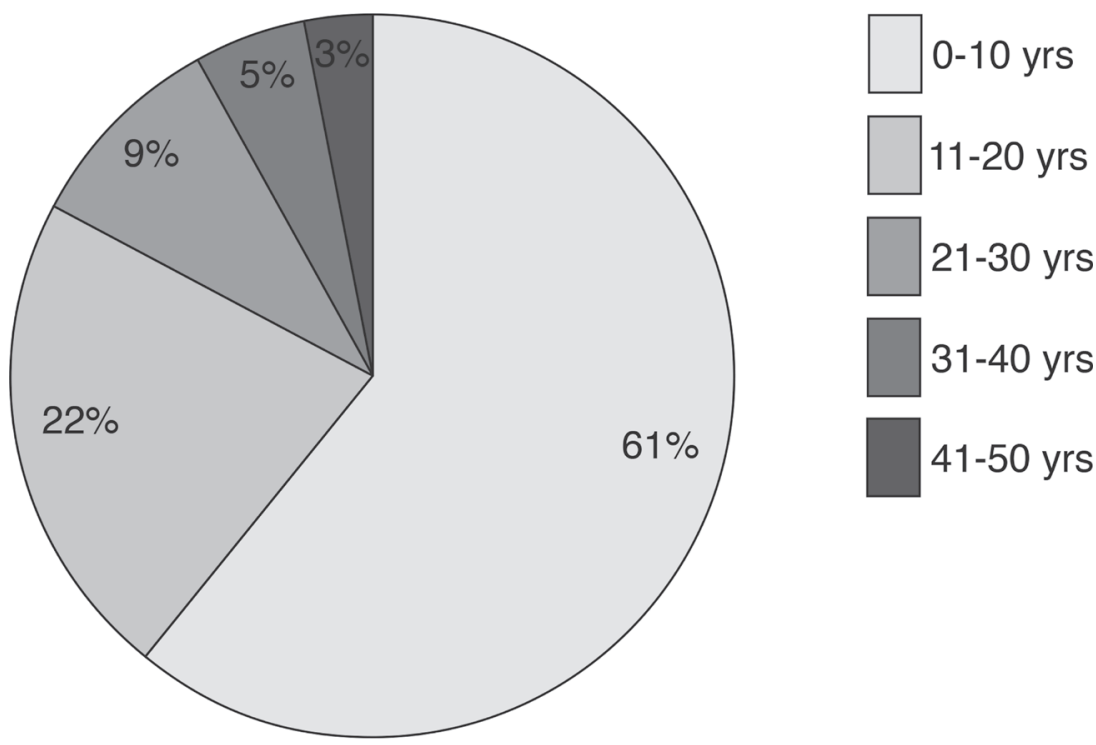

Figure 1 | Age spectrum of all 67 published Cantú syndrome patients. Notably, not every patient added in this graph had a molecular diagnosis (Afifi, Abdel-Hamid, Eid, Mostafa, \& Abdel-Salam, 2016; Brownstein et al., 2013; Cantu, Garcia-Cruz, Sanchez-Corona, Hernandez, \& Nazara, 1982; Concolino, Formicola, Camera, \& Strisciuglio, 2000; Cooper et al., 2014; Czeschik et al., 2013; Engels et al., 2002; Fryssira et al., 2017; Garcia-Cruz et al., 2011; Garcia-Gonzaleza et al., 2012; GarciaCruz et al., 1997; Grange et al., 2006; Graziadio et al., 2011; Harakalova et al., 2012; Hiraki et al., 2014; Kobayashi, Cook, \& Williams, 2010; Kurban et al., 2011; Lazalde, Sanchez-Urbina, NunoArana, Bitar, \& de Lourdes Ramirez-Duenas, 2000; Leon Guerrero et al., 2016; Nevin, Mulholland, \& Thomas, 1996; O'brien \& Ririe, 2008; Park, Koo, Jung, Lim, \& Chung, 2014; Robertson et al., 1999; Rosser et al., 1998; Scurr et al., 2011; Tan et al., 2005; van Bon et al., 2012).

\subsection{Clinical reports}

We present one female and one male patient as well as their affected children, all with a missense variant in ABCC9 (Table 1) .

\subsubsection{Patients 1 and 2}

Patient 1, a 39-year-old man, is the father of patient 2. Both have not been reported before. He is one of five children of healthy nonconsanguineous White parents. His father was also reported to have some coarse facial features. Otherwise, the family's history is unremarkable. He was born at term following an uncomplicated pregnancy. At birth, he was diagnosed with generalized hypertrichosis (Figure 2a) and a PDA. He developed normally and was reported to have normal intelligence, although when attending regular school, he was experiencing some learning difficulties requiring a special education program. 
Table 1 | Clinical presentation of the Cantú syndrome patients.

\begin{tabular}{|c|c|c|c|c|}
\hline Patient & 1 & 2 & 3 & 4 \\
\hline Sex & male & male & female & male \\
\hline Age at examination & 38 & 2 & 42 & 8 \\
\hline Previous publication & - & - & $\begin{array}{l}\text { Harakalova } \\
\text { et al, pt } 3\end{array}$ & $\begin{array}{l}\text { Harakalova } \\
\text { et al, pt } 2\end{array}$ \\
\hline \multicolumn{5}{|l|}{ Pregnancy and delivery } \\
\hline Polyhydramnios & ND & - & + & + \\
\hline Gestation (weeks) & 40 & 33 & 42 & 32 \\
\hline Birth weight (kg/centiles) & ND & $2.9(>98)$ & $4.1(>95)$ & $2.6(>99)$ \\
\hline \multicolumn{5}{|l|}{ Growth and development } \\
\hline Height (cm/centiles) & 197 (>98) & $89(>70)$ & ND & $134.5(>85)$ \\
\hline Weight (kg/centiles) & $130.1(>98)$ & $15.3(>95)$ & ND & $28.3(>70)$ \\
\hline $\begin{array}{l}\text { Head circumference }(\mathrm{cm} / \\
\text { centiles) }\end{array}$ & $61(>>98)$ & $54(>99)$ & ND & $57.5(>99)$ \\
\hline Developmental delay & - & + & - & $\begin{array}{l}\text { Feeding/ } \\
\text { speech/physical } \\
\text { therapy }\end{array}$ \\
\hline Learning difficulties & + & ND & - & + \\
\hline \multicolumn{5}{|l|}{ Face } \\
\hline Coarse face & + & + & + & + \\
\hline Epicanthic folds & + & + & + & + \\
\hline Brad/flat nasal bridge & + & + & - & + \\
\hline $\begin{array}{l}\text { Small nose/anteverted } \\
\text { nostrils }\end{array}$ & + & + & + & + \\
\hline Prominent mouth/full lips & + & + & + & + \\
\hline Long philtrum & + & + & + & + \\
\hline \multicolumn{5}{|l|}{ Skin and hair growth } \\
\hline Congenital hypertrichosis & + & + & + & + \\
\hline Wrinkled and/or loose skin & + & + & + & + \\
\hline Deep palmar/plantar creases & + & + & - & ND \\
\hline \multicolumn{5}{|l|}{ Heart } \\
\hline Congenital heart defects & PDA & PDA & - & PDA \\
\hline Cardiomyopathy & - & $\begin{array}{l}\text { biventricular } \\
\text { hypertrophy, } \\
\text { dilated } \\
\text { cardiomyopathy }\end{array}$ & $\begin{array}{l}\text { hypertrophic } \\
\text { cardiomyopathy } \\
\text { (left ventricle) }\end{array}$ & $\begin{array}{l}\text { hypertrophic } \\
\text { cardiomyopathy } \\
\text { (right ventricle) }\end{array}$ \\
\hline Pericardial effusion & - & + & + & - \\
\hline Cardiomegaly & + & + & + & + \\
\hline Pulmonary hypertension & - & + & - & - \\
\hline other cardiac features & $\begin{array}{l}\text { dilated aortic } \\
\text { root }\end{array}$ & $\begin{array}{l}\text { heart failure } \\
\text { diagnosed at } \\
\text { birth }\end{array}$ & $\begin{array}{l}\text { mitral valve } \\
\text { regurgitation }\end{array}$ & - \\
\hline
\end{tabular}


Table 1 | Continued

\begin{tabular}{|c|c|c|c|c|}
\hline \multicolumn{5}{|l|}{ Brain } \\
\hline Headaches & - & - & migraines & - \\
\hline Seizures & - & - & $\begin{array}{l}2 \text { seizures ( } 23 \\
\text { yrs) }\end{array}$ & - \\
\hline Blood vessel abnormalities & ND & ND & ND & ND \\
\hline Vision & $\mathrm{N}$ & $\mathrm{N}$ & myopia & $\begin{array}{l}\text { myopia, } \\
\text { nystagmus, } \\
\text { strabismus }\end{array}$ \\
\hline other cranial features & - & - & $\begin{array}{l}\text { calcifications } \\
\text { on CT/MRI }\end{array}$ & - \\
\hline \multicolumn{5}{|l|}{ Other } \\
\hline Joint laxity & + & + & + & + \\
\hline Lymphedema & - & - & lower legs & + \\
\hline Hernia & - & inguinal & $\begin{array}{l}\text { umbilical, } \\
\text { inguinal }\end{array}$ & $\begin{array}{l}\text { umbilical, } \\
\text { inguinal }\end{array}$ \\
\hline Immune dysfunction & - & $\begin{array}{l}\text { recurrent } \\
\text { infections }\end{array}$ & $\begin{array}{l}\text { recurrent ear } \\
\text { infections as } \\
\text { child }\end{array}$ & $\begin{array}{l}\text { recurrent } \\
\text { infections }\end{array}$ \\
\hline Genital anomalies & + & - & - & - \\
\hline Other features & - & $\begin{array}{l}\text { enlarged } \\
\text { kidneys }\end{array}$ & $\begin{array}{l}\text { Ménière's } \\
\text { disease, } \\
\text { conductive } \\
\text { deafness }\end{array}$ & $\begin{array}{l}\text { tracheobron- } \\
\text { chomalacia }\end{array}$ \\
\hline \multicolumn{5}{|l|}{ Mutation } \\
\hline ABCC9 Mutation (cDNA) & c. $1433 \mathrm{C}>\mathrm{T}$ & c. $1433 \mathrm{C}>\mathrm{T}$ & c. $3347 \mathrm{G}>\mathrm{A}$ & c. $3347 \mathrm{G}>\mathrm{A}$ \\
\hline Alteration (Protein) & p. (Ala478Val) & p. (Ala478Val) & p. (Arg1116His) & p. (Arg1116His) \\
\hline De novo status & de novo & $\begin{array}{l}\text { carried from } \\
\text { affected father } \\
\text { (pt } 1)\end{array}$ & de novo & $\begin{array}{l}\text { carried from } \\
\text { affected mother } \\
\text { (pt } 3 \text { ) }\end{array}$ \\
\hline
\end{tabular}

After his son was diagnosed with CS, he was first reviewed by a clinical geneticist at the age of 36 years. Clinical examination showed a height of $197 \mathrm{~cm} \mathrm{(>98th}$ centile), a weight of $130 \mathrm{~kg}$ (>98th centile), and a head circumference of $61 \mathrm{~cm}$ (>>98th centile). He had thick scalp hair and hypertrichosis predominantly on the arms, back, and forehead, which responded well to laser therapy. Furthermore, he had coarse facial features (Figure $\mathbf{2} \mathbf{h}-\mathbf{i}$ ) and gingival hyperplasia. Significant joint laxity was evident in the hands, with deep palmar creases and soft skin on the palms (Figure 2j).

After molecular confirmation of the diagnosis of CS at age 36 years, cardiac evaluation indicated a dilated aortic root and cardiomegaly. No ligation surgery was required. His neurological examination was unremarkable. 

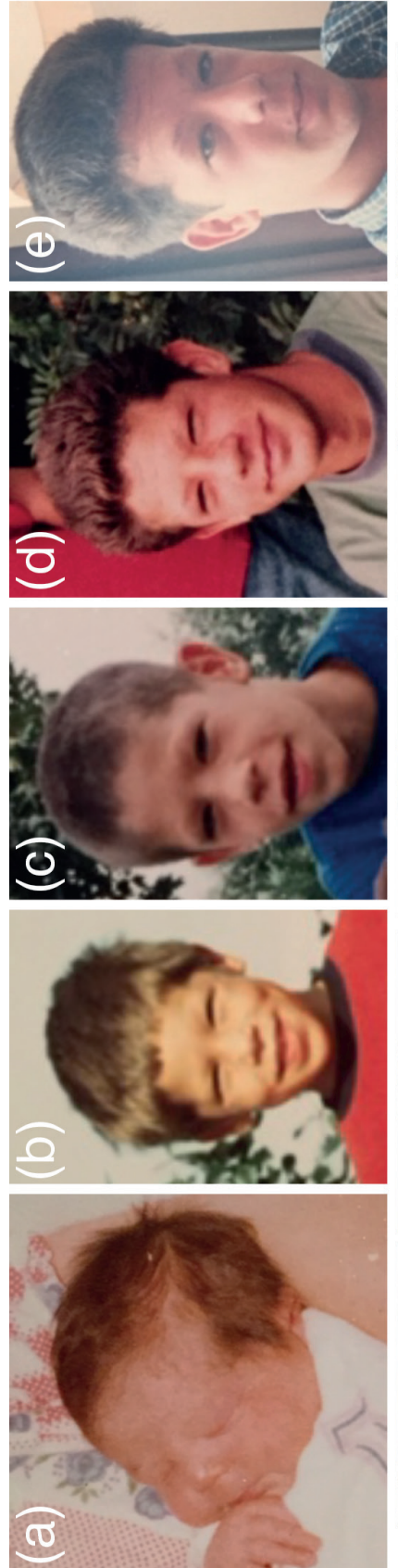
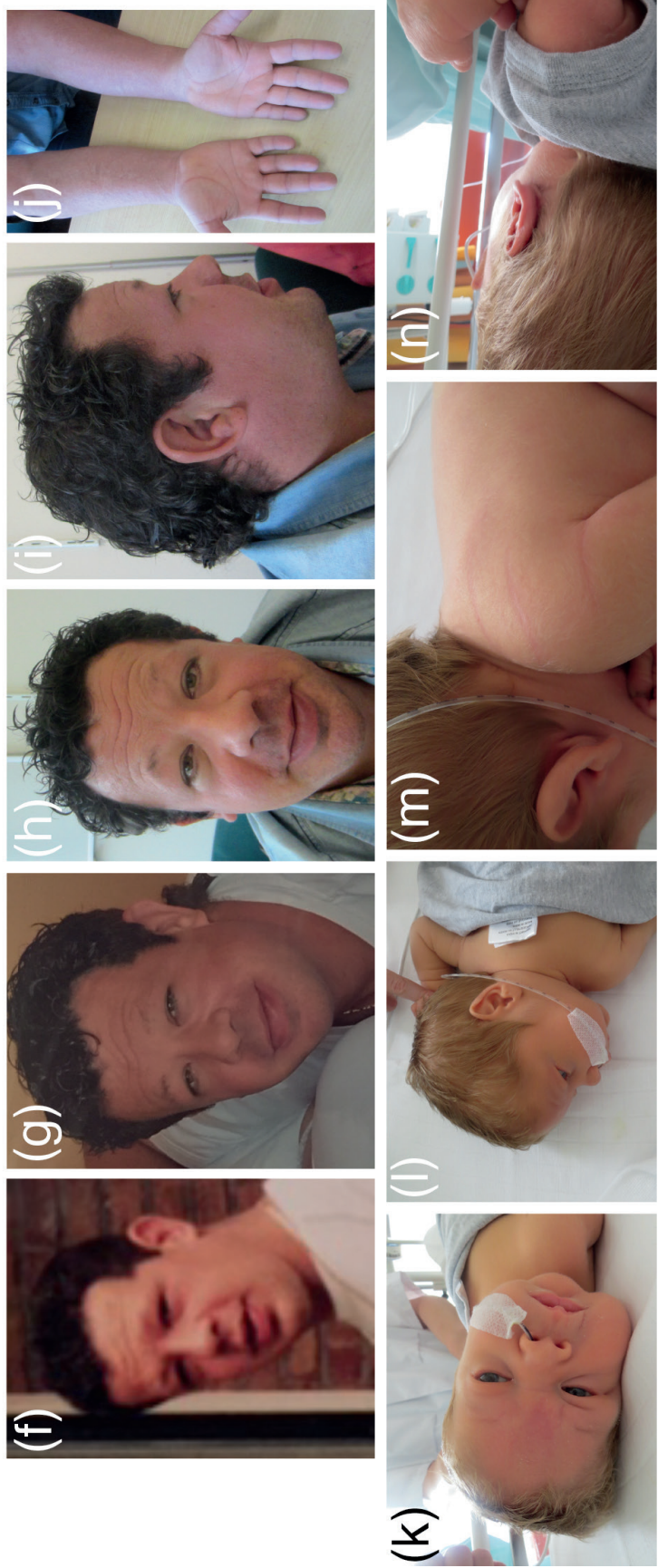

$\stackrel{ }{\Upsilon} \frac{0}{0} \cdot \frac{\pi}{4}$

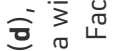

ज

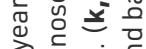
ก ก ल Uิ ก

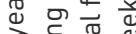
은 흔 뜬

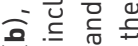
थ बं थ ธ ㄷำ 入 ब ฮ เ 0 는

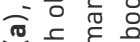
ज范 吉 品至 듀 ब 으. 뜨 등 - ᄃ 인 (1) 范 तु ज 임 $+\underset{0}{ }$ - 仓 는

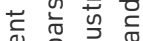
을

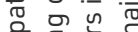

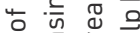
돈 믄 는 向 이요 응 등 흥

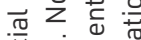
况 Ш ع ฉ 气 ง 은 응

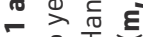
๓ 등ㅇㅇㅇ 尊祭 흐늠 응 ฮ ธั 닏ำ 苋

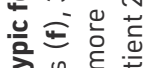
ก

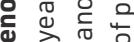
ธ - i் 믐 (4) 는 는 는 党 뮨 
Patient 2 is a 3-year-old boy who presented with generalized hypertrichosis as well as distinctive facial features. He is the fourth child of nonconsanguineous White parents. Ultrasound during pregnancy showed hydronephrosis and polyhydramnion. He was born prematurely at 33 weeks of gestation by cesarean section. He had a high birth weight ( $2977 \mathrm{~g}$, >98th centile). His facial appearance and hypertrichosis at 1 month of age are shown in Figure 2k-n. Notably, his cardiac abnormalities were more severe compared with his father. One month after birth, he was diagnosed with a constricting atrial septal defect and PDA requiring ligation surgery. During infancy, he was reported to have biventricular hypertrophy, dilated cardiomyopathy, pericardial effusion, cardiomegaly, and pulmonary hypertension. His development was delayed. An abdominal ultrasound showed enlarged kidneys after birth.

Both patients 1 and 2 carry a variant in ABCC9, c.1433C >T, p. (Ala478Val). Although the paternal grandfather was also suspected to have CS on the basis of his facial features, he was tested negative for variants in ABCC9 and KCNJ8, respectively.

\subsubsection{Patients 3 and 4}

Patient 3, a 45-year-old woman, is the mother of patient 4. Both have been reported previously (Harakalova et al., 2012). She is the first of two children of healthy nonconsanguineous White parents. She was born after 42 weeks of gestation, which was complicated by polyhydramnios. Birth weight was $4100 \mathrm{~g}$ (>95th centile). Hypertrichosis was evident at birth. Her facial appearance and low frontal hairline during childhood are shown in Figure 3. She was diagnosed with hypothyroidism as a child. At the age of 13 years, she started to develop recurrent episodes of lower limb swelling whose treatment with compression stockings was shown to be effective, especially at an older age. She has myopia and suffers from balance problems, Meniere's disease, and conductive deafness in her left ear since the age of 30 originating from recurrent ear infections in her childhood. Developmental milestones were all normal; she completed a PhD in Developmental Psychology and has a successful professional career.

She has low blood pressure and was reported to have left ventricular hypertrophy and mitral valve regurgitation. In addition, she developed pericardial effusion and cardiomegaly, for which medical management with an angiotensin converting enzyme inhibitor and diuretics is required. Consequently, she suffers from exercise intolerance. Strikingly, during her pregnancy at the age of 35 years, her blood pressure was markedly high. She has had frequent migraine episodes since early adolescence and had two generalized seizures when she 
was 23 years old. At the age of 44 years, a migraine episode consisted of left-side numbness of the face and speech difficulties. A cranial computed tomography and MRI of the brain showed calcifications, but no explanation for the acute onset of the numbness and speech difficulties.

She was first evaluated at the genetics outpatient clinic at the age of 36 years after her son was diagnosed with CS. Her coarse facial appearance including epicanthal folds, full lips, and long philtrum as well as generalized hypertrichosis with thick scalp hair extending to her forehead and cheeks, and excessive body hair on her arms, legs, and back were suggestive for CS.

Patient 4, a 10-year old boy, was reported to have right ventricular hypertrophy, cardiomegaly, and PDA, respectively. He presented with edema at birth and during childhood. At the age of 5 months, he required ventilation for 7.5 weeks because of a tracheal collapse that was eventually corrected by aortopexy. Notably, his motor skills were delayed, for which he required feeding, physical, and speech therapy. He attends a special school. Unlike his mother, he has no history of migraines.

Both patients 3 and 4 carry an ABCC9 variant, c.3347G>A, p. (Arg1116His). They are the only reported patients with this mutation so far.
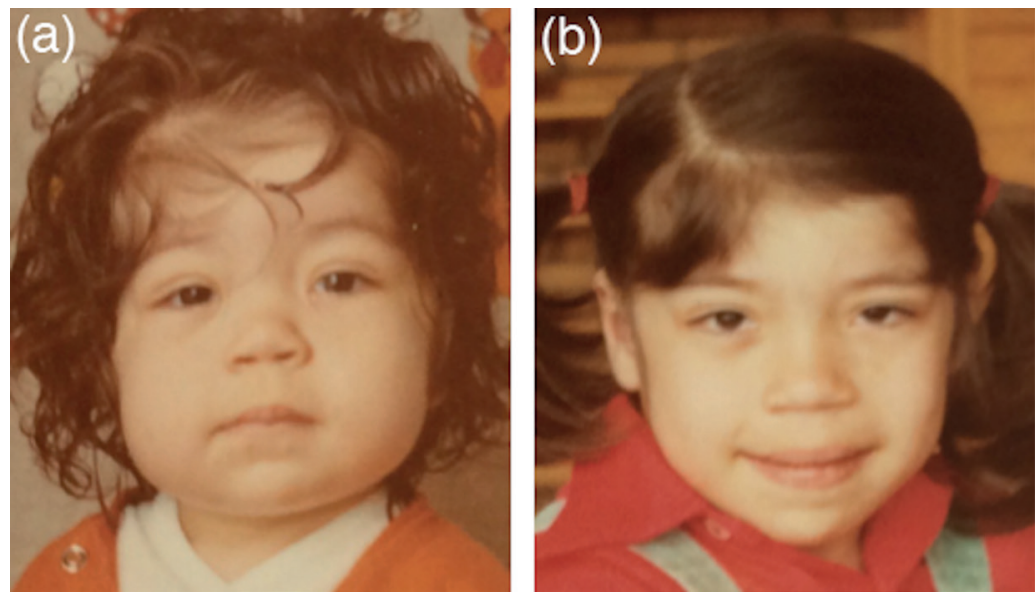

Figure 3 | Facial photographs of patient 3 at age 2 years (a) and 6 years (b). Note the further widening of the mouth. The patient did not consent to publishing of facial photographs at an older age. 


\subsection{Discussion}

CS is a rare multiorgan system genetic disorder. The genes associated with CS have only recently been identified (Brownstein et al., 2013; Cooper et al., 2014; Harakalova et al., 2012; van Bon et al., 2012). As a result, the majority of known affected patients are still in their childhood (Figure 1). In a subset of the cases, parents are diagnosed with CS after the diagnosis is made in their child, resulting in increasing knowledge of the changing phenotype throughout life. Hypertrichosis and dysmorphic facies are observed in all patients with CS (Table 1) and are usually evident at birth. These clinical manifestations are subject to consistent changes over time. Major changes in the face can be observed from infancy to adulthood, with the initial facial shape appearing round with full cheeks. The facial phenotype progresses to a lengthening of the face with a pointed chin and a more prominent forehead. In addition, the frontal hairline moves upwards (Figure 2a-i). As a result, the facial hair pattern presents as less striking compared with that at a younger age. This has been described previously (Scurr et al., 2011). The coarseness of the face increases including a further flattening of the nasal bridge with supraorbital ridges and fuller lips. The small nose takes on a more bulbous shape. The periorbital edema seems to be consistent (Figure $\mathbf{2 a - i}$ and $\mathbf{3}$ ). In addition, hypertrichosis appears to diminish on the extremities as well as the cheeks. Male patients seem to respond more effectively to hair removal treatment, suggesting a hormonal effect on hair growth.

Many affected newborns have a high birth weight and length. Adult height continues to be elevated in various patients including patient 1 of this study. A thin or even quite muscular appearance during childhood changes because of a weight gain that occurs after puberty, as is the case in our patients. Macrocephaly is often observed at birth and persists throughout life (Scurr et al., 2011).

Generalized edema at birth usually resolves spontaneously (Grange, Nichols, \& Singh, 2014). However, the development of lymphedema, especially in the lower limbs, often starts in adolescence or early adulthood. As reported previously (Cantu et al., 1982; Scurr et al., 2011), the swellings reappear continuously, as can be observed in patient 3 . Therefore, close monitoring for edema is essential. The contractility of smooth muscles lining lymphatic vessels is sensitive to $\mathrm{K}_{\text {ATP }}$ activation (von der Weid, Lee, Imtiaz, Zawieja, \& Davis, 2014). Therefore, we propose that the observed lymphedema is a direct result of the gain-of-function effects present in $\mathrm{K}_{\text {ATP }}$ channels of CS patients leading to vasodilation and consequently reduced fluid flow. 
CS is associated with various cerebrovascular findings, including dilated and torturous cerebral vessels. The associated increased blood flow in the brain could be a reason for the observed migraines in many CS patients (Leon Guerrero et al., 2016), including patient 3 in this study. These migraine headaches already present in late childhood and progress in frequency and severity over time, sometimes even accompanied by episodes of seizure. A brain MRI with magnetic resonance angiogram and magnetic resonance venography to evaluate persistent headaches or other neurological symptoms are therefore strongly suggested, in particular, in older patients.

It is important to realize that clinical manifestations vary among patients, and even among family members sharing the same ABCC9 variant. The heterogeneous clinical presentation of CS within both families described in this study confirms variability in brain and heart anomalies as well as developmental milestones (Table 1). The reason for this significant intrafamilial variation is unclear. We hypothesize that this is caused by environmental factors or modifier genes other than the currently known genes ABCC9 and KCNJ8, that seem to play a role in the phenotypic expressions of CS patients. It is noteworthy that this variability should also be considered when providing genetic counseling.

\subsection{Conclusion}

Characterization of the phenotypic changes in CS at different ages, as presented in this study, can enable an earlier clinical and molecular diagnosis and thus optimize genetic counseling. In addition, it can help to adjust expectations from the parents of younger patients and their health professionals as adequate information on the development of this disorder is still lacking.

We suspect that there are still a large number of undiagnosed or misdiagnosed cases, especially older patients. As the field of clinical genetics is a relatively new specialization, and the genes associated with CS have only recently been identified, not all patients might have had the opportunity to undergo an adequate genetic consultation yet. Moreover, specific features of CS, particularly hypertrichosis, are, apart from a cosmetic problem, not considered a medical issue. 
This report illustrates how older patients have only been diagnosed with CS after their children received the diagnosis. Considering the current efforts to develop a pharmacological treatment for CS, a correct diagnosis will be in the interest of every patient.

All patients participated in this study through a special annual Cantú syndrome research clinic at the University Medical Center Utrecht, The Netherlands. 
Cantú syndrome, the changing phenotype: a report of the two oldest Dutch patients 
-12
4
4
4

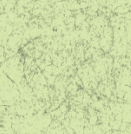

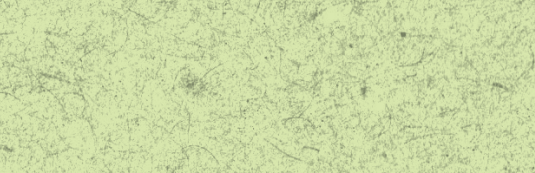

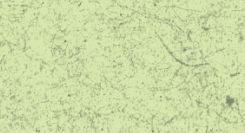

तr.

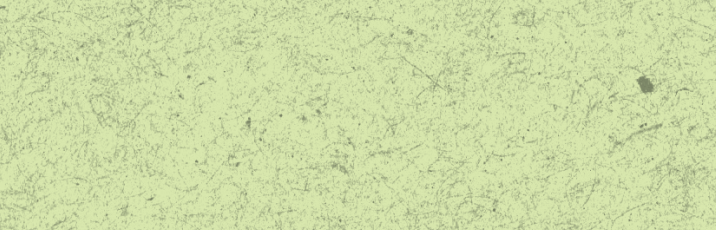

4

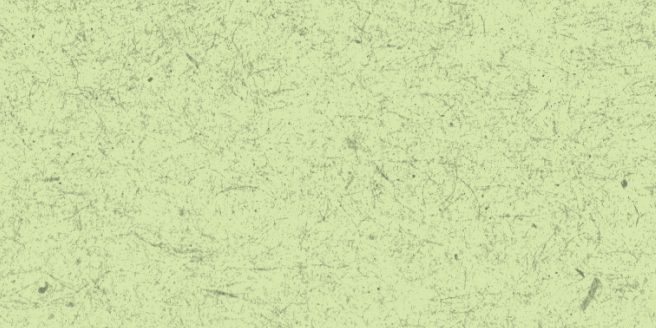




\section{4 \\ Behavioral and cognitive functioning in individuals with Cantú syndrome}

Helen I. Roessler, Lieke M. van der Heuvel, Kathleen Shields, Nine V.A.M. Knoers, Gijs van Haaften, Dorothy K. Grange, Mieke M. van Haelst 


\section{Abstract}

Cantú syndrome (CS) is caused by pathogenic variants in ABCC9 and KCNJ8 encoding regulatory and pore forming subunits of ATP-sensitive potassium $\left(\mathrm{K}_{\mathrm{ATP}}\right)$ channels. CS is characterized by congenital hypertrichosis, distinctive facial features, peripheral edema, cardiac- and neurodevelopmental abnormalities. Little is known about behavioural and cognitive functioning in CS individuals. To assess cognitive profile, social functioning and psychiatric symptoms in CS subjects systematically, we invited 35 individuals (1-69 years) with confirmed $A B C C 9$ variants and their relatives to complete various commonly applied standardized age-related questionnaires; the Kaufman Brief Intelligence Test 2 (KBIT-2), the Social Responsiveness Scale-2 (SRS-2) and the Achenbach System of Empirically Based Assessment (ASEBA). The majority of CS individuals revealed average verbal and nonverbal intelligence compared to the general population. Fifteen percent of cases showed social functioning strongly associated with a clinical diagnosis of autism spectrum disorder. Both externalizing and internalizing problems were also present in this cohort. In particular anxiety, ADHD and autism spectrum problems were predominantly observed in younger subjects of this cohort $(\geq 25 \%)$. These percentages decreased markedly in adolescents and adults. 


\subsection{Introduction}

Cantú syndrome (CS, OMIM \#239850) is a rare autosomal dominant genetic disorder that affects multiple organ systems with currently about 150 published cases worldwide. Individuals with CS have cardiac abnormalities, including an enlarged heart, pericardial effusion, blood pressure abnormalities and structural abnormalities, such as vessel (patent ductus arteriosus (PDA)) and heart valve defects. Additionally, CS subjects have non-cardiac manifestations that include large size at birth, hypertrichosis (thick/abundant scalp hair and excess body hair), distinctive facial features, skeletal abnormalities, peripheral edema, tortuous blood vessels in the brain and neurodevelopmental abnormalities like hypotonia, motor delays and exercise intolerance (Grange et al., 2006; Grange et al., 2019; Leon Guerrero et al., 2016; Roessler et al., 2020). CS is caused by gain-of-function (GoF) pathogenic variants in ABCC9 and, less commonly, in KCNJ8, which encode the regulatory (SUR2) and pore-forming (Kir6.1) subunits, respectively, of ATP-sensitive potassium $\left(\mathrm{K}_{\text {ATP }}\right)$ channels (Brownstein et al., 2013; Cooper et al., 2014; Harakalova et al., 2012; McClenaghan et al., 2017; van Bon et al., 2012). The majority of cases is considered to harbor de novo variants. Remarkably, there is considerable variation in the phenotypic spectrum, even within family members sharing the same ABCC9 variant (Grange et al., 2019; Roessler et al., 2018). The underlying reason for this heterogeneous clinical presentation is still unclear.

The majority of individuals with CS has normal intellect. Some cases show mild learning disabilities and/or developmental delay in early childhood, including delay in speech development and motor skills most likely due to hypotonia(Grange et al., 2019). Ultimately, most affected individuals attend regular schools and some are described as having a high IQ (Scurr et al., 2011). In the past, a few clinical reports have suggested behavioral abnormalities in CS subjects with an increased frequency of ADHD and autism spectrum features especially present in younger individuals (Grange, Nichols, \& Singh, 2020; Grange et al., 2019; Scurr et al., 2011). Scurr et. al additionally report mood swings, anxiety, obsessive-compulsive behavior and tics (Scurr et al., 2011). A few reported cases receive medication like diazepam and fluoxetine for treatment of anxiety (Jesani, Elangovan, \& Zaidi, 2019). No direct social and/or psychological consequences due to CS-associated impairments or appearance have been reported so far. Thus, the lack of evidence suggests more attention needs to be given to the characterization of the CS social and cognitive phenotype on a larger scale and in a standardized manner. Further characterization of the previously suggested behavioral features associated 
with CS might allow more targeted behavioral interventions in addition to additional information on CS associated phenotypes during the genetic counseling process.

This is the first study to assess behavior and cognitive profile as well as social functioning in CS individuals systematically. Using quantitative measures designed with specific focus on verbal and nonverbal intelligence, social behavior, autistic traits and psychiatric symptoms we aim to provide a more descriptive characterization of such deficits in this cohort.

\subsection{Results}

We assessed behavioral and intellectual functioning in 35 CS subjects, 14 males and 21 females, with an age range of 1-69 years. All individuals had confirmed genetic variants in ABCC9; in 27/35 cases this variant was de novo. Information regarding genotype, general features and previously (self-)reported behavioral abnormalities of all subjects is provided in Table 1. All individuals are part of the International Cantú Syndrome Registry (ICSR) (Grange et al., 2019) and thus have been published at least once before. Patient study numbers applied in this study can be traced back to the ICSR published by Grange et al., which documents clinical features of each patient in detail (Grange et al., 2019). All subjects participated in this study through a special Cantú syndrome research clinic in the Netherlands or the US. All participants were Caucasian and the majority identified as Non-Spanish/Hispanic/Latino (31/35, 89\%), reflecting the location of the participating sites.

\subsubsection{Reported psychiatric syndromes (total $n=22$ )}

Information regarding already established behavioral abnormalities is not complete in this cohort. Data is available for all cases assessed at Washington University St. Louis $(22 / 35,63 \%)$ (Table 1 ). Mood swings are reported in $7 / 22$ $(32 \%)$ subjects and depression in 6/22 (27\%). Four out of $22(18 \%)$ subjects are reported to have anxiety or attention deficit hyperactivity disorder (ADHD). Autism or autism spectrum disorder (ASD) and obsessive-compulsive disorder (OCD) are self-reported in 3/22 (14\%) individuals. These features either occur separately or in combination (Table 1 ). 


\subsubsection{Intelligence (total $n=13$ )}

To measure verbal and nonverbal intelligence in CS, 13 subjects seen at Washington University were administered the KBIT-2. Information on mean standard scores (SS) and associated clinical range can be seen in Table 2. Two out of $13(15 \%)$ assessed subjects scored an IQ composite SS below average, $2 / 13(15 \%)$ subjects showed a nonverbal SS below average and $1 / 13(7.5 \%)$ individuals revealed a verbal SS below average. As a group, the CS cohort presented with intellectual abilities merely 0.5 SD below the general population mean (IQ composite SS: 93.46 2.95 ). In order to compensate for the wide age range present in our cohort assessed by KBIT-2 (5-69 years), participating individuals were divided into two groups according to age for further analysis: (1) 4-17 years $(n=5)$ and (2) 18-90 years $(n=8)$. When comparing both groups, the mean IQ composite SS was considerably lower, but still within an average range,

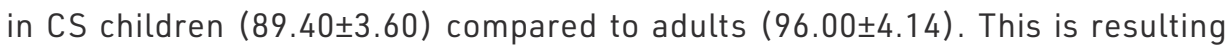
from a decreased nonverbal SS in younger individuals (4-17 years: $85.00 \pm 6.94$; 18-90 years: $94.13 \pm 4.63)$ which is $1 \mathrm{SD}$ below the general population mean. Verbal intelligence presented in a similar range between age groups. Notably, no gender-related differences could be observed in the assessed cohort. 
Table 1 | Patient ABCC9 variants and general features $(n=35)$. Due to age-dependency of the applied questionnaires, not every measure was administered to every individual. Additionally, sometimes questionnaires have been filled in at various ages of the patient. Therefore, we report a separate age for every administered questionnaire per patient. Patient identification numbers CS0001-CS0037 refer to US cohort, CS2001-CS2014 refer to Dutch cohort.

\begin{tabular}{|c|c|c|c|c|c|c|}
\hline Patient & Gender & $\begin{array}{l}\text { cDNA } \\
\text { variant }\end{array}$ & $\begin{array}{l}\text { Protein } \\
\text { alteration }\end{array}$ & $\begin{array}{l}\text { De novo } \\
\text { status }\end{array}$ & $\begin{array}{l}\text { Race/ethnic } \\
\text { background }\end{array}$ & $\begin{array}{l}\text { (Self-)Reported } \\
\text { behavioral } \\
\text { abnormalities }\end{array}$ \\
\hline CS0001 & $M$ & c. $3460 \mathrm{C}>\mathrm{T}$ & p.Arg1154Trp & de novo & $\mathrm{C}$ & $\begin{array}{l}\text { ADHD, mood } \\
\text { swings }\end{array}$ \\
\hline $\mathrm{CSOOO2}^{\dagger}$ & $F$ & c. $3461 \mathrm{G}>\mathrm{A}$ & p.Arg1154Gln & $\begin{array}{l}\text { Inherited } \\
\text { from } \\
\text { affected } \\
\text { mother }\end{array}$ & $\mathrm{C}$ & Depression \\
\hline $\mathrm{CSOO03}^{\dagger}$ & $\mathrm{F}$ & c. $3461 \mathrm{G}>\mathrm{A}$ & p.Arg1154Gln & $\begin{array}{l}\text { Inherited } \\
\text { from } \\
\text { affected } \\
\text { mother }\end{array}$ & $\mathrm{C}$ & - \\
\hline $\mathrm{CSOOO}^{\dagger}$ & $\mathrm{F}$ & c. $3461 \mathrm{G}>\mathrm{A}$ & p.Arg1154Gln & de novo & C & - \\
\hline CS0005 & $\mathrm{F}$ & c. $3460 \mathrm{C}>\mathrm{T}$ & p.Arg1154Trp & de novo & C & Depression \\
\hline CS0006 & $M$ & c. $3014 \mathrm{~A}>\mathrm{T}$ & p.His1005Leu & de novo & $\mathrm{C}$ & ADHD, ASD, OCD \\
\hline $\mathrm{CSOOOH}^{\dagger}$ & $\mathrm{F}$ & c. 3346 C $>T$ & p.Arg1116Cys & de novo & $\mathrm{C}$ & $\begin{array}{l}\text { Mood swings, } \\
\text { depression }\end{array}$ \\
\hline $\mathrm{CSOO09}^{\dagger}$ & $M$ & c. 3346 C>T & p.Arg1116Cys & $\begin{array}{l}\text { Inherited } \\
\text { from } \\
\text { affected } \\
\text { mother }\end{array}$ & C & - \\
\hline CS0010' & M & c.3346 C>T & p.Arg1116Cys & $\begin{array}{l}\text { Inherited } \\
\text { from } \\
\text { affected } \\
\text { mother }\end{array}$ & C & ASD \\
\hline CS0011 & $M$ & c. 3347 G > A & p.Arg1116His & de novo & $\mathrm{C}$ & - \\
\hline CS0013 & $M$ & c. $4469 \mathrm{~T}>\mathrm{A}$ & p.Va1490Glu & de novo & $\mathrm{C} / \mathrm{H}$ & - \\
\hline CS0015 & $M$ & c. $3796 \mathrm{G}>\mathrm{A}$ & p.Val1266Met & de novo & $\mathrm{C}$ & $\begin{array}{l}\text { ADHD, mood } \\
\text { swings, OCD }\end{array}$ \\
\hline CS0016 & $F$ & c. $2378 \mathrm{~A}>\mathrm{T}$ & p.Asp793Val & de novo & $\mathrm{C}$ & - \\
\hline CS0017 & $\mathrm{F}$ & c. $3014 \mathrm{~A}>\mathrm{T}$ & p.His1005Leu & de novo & $\mathrm{C} / \mathrm{H}$ & - \\
\hline CSO020 & $M$ & c. $3461 \mathrm{G}>\mathrm{A}$ & p.Arg1154Gln & de novo & $\mathrm{C}$ & $\begin{array}{l}\text { ASD, mood } \\
\text { swings, } O C D, \\
\text { anxiety }\end{array}$ \\
\hline $\mathrm{CSOO21}^{\dagger}$ & $\mathrm{F}$ & c. $881 \mathrm{G}>\mathrm{A}$ & p.Gly294Glu & $\begin{array}{l}\text { Inherited } \\
\text { from } \\
\text { affected } \\
\text { mother }\end{array}$ & $\mathrm{C}$ & $\begin{array}{l}\text { Depression, } \\
\text { anxiety, mood } \\
\text { swings }\end{array}$ \\
\hline $\mathrm{CSOO22}^{\dagger}$ & $F$ & c. $881 \mathrm{G}>\mathrm{A}$ & p.Gly294Glu & de novo & C & Depression \\
\hline CS0024 & $M$ & c. 4040 G > T & p.Arg1347Leu & de novo & $\mathrm{C}$ & Anxiety \\
\hline CS0028 & $\mathrm{F}$ & c. 3605 C>T & p.Thr1202Met & de novo & $\mathrm{C} / \mathrm{H}$ & $\begin{array}{l}\text { ADHD, mood } \\
\text { swings, anxiety }\end{array}$ \\
\hline
\end{tabular}




\begin{tabular}{llllll}
$\begin{array}{l}\text { Special } \\
\text { education during } \\
\text { school }\end{array}$ & $\begin{array}{l}\text { Highest } \\
\text { degree }\end{array}$ & $\begin{array}{l}\text { SRS-2 } \\
\text { (age } \\
\text { (years)) } \neq\end{array}$ & $\begin{array}{l}\text { ASEBA } \\
\text { (age } \\
\text { (years)) } \neq\end{array}$ & $\begin{array}{l}\text { KBIT2 } \\
\text { (age } \\
\text { (years)) } \neq\end{array}$ & $\begin{array}{l}\text { Previous } \\
\text { publication }\end{array}$ \\
\hline+ & Highschool & $+(16)$ & $+(18)$ & $+(15)$ & 1,4 \\
\hline- & Highschool & $+(21)$ & $+(21)$ & $+(20)$ & $1,2,3$ \\
& College & na & $+(29)$ & na & $1,2,3$
\end{tabular}

\begin{tabular}{|c|c|c|c|c|c|}
\hline+ & College & $+(52)$ & $+(52)$ & $+(49)$ & $1,2,3,4$ \\
\hline+ & College & $+(21)$ & $+(21)$ & $+(20)$ & $1,4,5$ \\
\hline+ & $\begin{array}{l}\text { Attends } \\
\text { school }\end{array}$ & $+(17)$ & $+(17)$ & $+(15)$ & 1,4 \\
\hline- & College & na & na & $+(34)$ & 1,4 \\
\hline+ & $\begin{array}{l}\text { Attends } \\
\text { school }\end{array}$ & na & na & $+(9)$ & 1,4 \\
\hline+ & $\begin{array}{l}\text { Attends } \\
\text { school }\end{array}$ & na & na & $+(8)$ & 1,4 \\
\hline+ & $\begin{array}{l}\text { Attends } \\
\text { school }\end{array}$ & $+(11)$ & $+(11)$ & na & $1,4,5$ \\
\hline- & - & $+(4)$ & $+(4)$ & na & 1,5 \\
\hline- & - & na & na & $+(5)$ & 1 \\
\hline- & - & $+(3)$ & $+(3)$ & na & 1,5 \\
\hline- & - & $+(3)$ & $+(3)$ & na & 1,5 \\
\hline+ & $\begin{array}{l}\text { Attends } \\
\text { college }\end{array}$ & $+(20)$ & $+(20)$ & $+(18)$ & 1 \\
\hline+ & College & $+(46)$ & $+(46)$ & $+(45)$ & 1 \\
\hline
\end{tabular}

$\begin{array}{lllll}\text { College } & \text { na } & \text { na } & +(69) & 1 \\ \begin{array}{l}\text { Attends } \\ \text { school }\end{array} & +(5) & +(7) & \text { na } & 1,5 \\ - & +(5) & +(5) & \text { na } & 1\end{array}$


Table 1 | Continued

\begin{tabular}{|c|c|c|c|c|c|c|}
\hline CS0030 & $F$ & c. $4480 \mathrm{G}>\mathrm{A}$ & p.Ala1494Thr & de novo & $\mathrm{C} / \mathrm{H}$ & $\begin{array}{l}\text { Mood swings, } \\
\text { depression }\end{array}$ \\
\hline CS0035 & $F$ & c. $3704 \mathrm{C}>\mathrm{T}$ & p.Ser1235Phe & de novo & C & - \\
\hline CS0037 & $\mathrm{F}$ & c. 3056 C > A & p.Thr1019Lys & de novo & C & - \\
\hline CS2001 & $F$ & c. $1295 \mathrm{C}>\mathrm{T}$ & p.Pro432Leu & de novo & C & na \\
\hline $\mathrm{CS}^{2002^{\dagger}}$ & $M$ & c. 1433 C > T & p.Ala478Val & de novo & C & na \\
\hline $\mathrm{CS2003}^{\dagger}$ & M & c. 1433 C > T & p.Ala478Val & $\begin{array}{l}\text { Inherited } \\
\text { from } \\
\text { affected } \\
\text { father }\end{array}$ & C & na \\
\hline CS2004 & $M$ & c. $178 \mathrm{C}>\mathrm{T}$ & p.His60Tyr & de novo & $C$ & na \\
\hline CS2005 & $\mathrm{F}$ & c. $3460 \mathrm{C}>\mathrm{T}$ & p.Arg1154Trp & de novo & C & na \\
\hline CS2006 & $\mathrm{F}$ & c. $3461 \mathrm{G}>\mathrm{A}$ & p.Arg1154Gln & de novo & C & na \\
\hline CS2008 & $\mathrm{F}$ & c. $3345 C>G$ & p.Arg.1116Gly & de novo & C & na \\
\hline CS2009 & $\mathrm{F}$ & c. $3461 \mathrm{G}>\mathrm{A}$ & p.Arg1154Gln & de novo & C & na \\
\hline CS2010 & $\mathrm{F}$ & c. $3460 \mathrm{C}>\mathrm{T}$ & p.Arg1154Trp & de novo & C & na \\
\hline $\mathrm{CS}^{2011^{\dagger}}$ & $F$ & c. $3345 \mathrm{G}>\mathrm{A}$ & p.Arg1116His & de novo & $C$ & na \\
\hline $\mathrm{CS}^{2012^{\dagger}}$ & M & c. $3345 \mathrm{G}>\mathrm{A}$ & p.Arg1116His & $\begin{array}{l}\text { Inherited } \\
\text { from } \\
\text { affected } \\
\text { mother }\end{array}$ & C & na \\
\hline CS2013 & $\mathrm{F}$ & c. $3460 \mathrm{C}>\mathrm{T}$ & p.Arg1154Trp & de novo & C & na \\
\hline CS2014 & M & c. $3461 \mathrm{G}>\mathrm{A}$ & p.Arg1154GIn & de novo & C & na \\
\hline
\end{tabular}

† Kindreds are grouped together: CS0002/CS0003/CS0004, CS0007/CS0009/CS0010, CS0021/ CS0022, CS2002/CS2003, CS2011/CS2012

₹ Questionnaires have been filled in at various ages of the patient

1: Grange et al (2019) Am J Med Genet C, 2: Grange et al. (2006) Am J Med Genet., 3: van Bon et al. (2012) Am J Human Genet., 4: Leon-Guerrero et al (2016) Neurology, 5: Roessler et al (2020) Am J Med Genet A, 6: Harakalova et al. (2012) Nat. Gen., 7: Roessler et al (2018) Clin. Dysm.

Abbreviations: ADHD: attention deficit hyperactivity disorder, ASD: autism spectrum disorder, C: Caucasian, H: Hispanic, na: not assessed, OCD: obsessive-compulsive disorder, $(+)$ :

present/assessed, (-): not present 


\begin{tabular}{|c|c|c|c|c|c|}
\hline- & $\begin{array}{l}\text { Graduate } \\
\text { school }\end{array}$ & $+(40)$ & na & na & 1 \\
\hline- & College & $+(58)$ & $+(58)$ & $+(58)$ & 1 \\
\hline- & - & $+(1)$ & $+(1)$ & na & 1 \\
\hline- & $\begin{array}{l}\text { Attends } \\
\text { school }\end{array}$ & $+(11)$ & $+(11)$ & na & $1,5,6$ \\
\hline+ & Highschool & $+(39)$ & $+(39)$ & na & 1,7 \\
\hline- & - & $+(3)$ & $+(3)$ & na & $1,5,7$ \\
\hline- & $\begin{array}{l}\text { Attends } \\
\text { school }\end{array}$ & $+(12)$ & $+(12)$ & na & 1,5 \\
\hline- & $\begin{array}{l}\text { Attends } \\
\text { school }\end{array}$ & $+(17)$ & $+(17)$ & na & 1,5 \\
\hline- & - & $+(5)$ & $+(5)$ & na & \\
\hline+ & $\begin{array}{l}\text { Attends } \\
\text { school }\end{array}$ & $+(15)$ & $+(15)$ & na & 1,5 \\
\hline- & Highschool & $+(22)$ & $+(22)$ & na & 1,5 \\
\hline- & $\begin{array}{l}\text { Attends } \\
\text { school }\end{array}$ & $+(17)$ & $+(17)$ & na & 1,5 \\
\hline- & $\begin{array}{l}\text { Graduate } \\
\text { school }\end{array}$ & $+(45)$ & $+(45)$ & na & $1,5,6,7$ \\
\hline+ & $\begin{array}{l}\text { Attends } \\
\text { school }\end{array}$ & $+(10)$ & $+(10)$ & na & $1,6,7$ \\
\hline- & $\begin{array}{l}\text { Attends } \\
\text { school }\end{array}$ & $+(10)$ & $+(10)$ & na & $1,5,6$ \\
\hline- & - & $+(3)$ & $+(3)$ & na & 1,5 \\
\hline
\end{tabular}


Table 2 | Descriptive Statistics for KBIT-2 Standard Scores in 4-17-year-old $(n=5)$ and 18-90-year-old $(n=8)$ individuals with Cantù syndrome.

\begin{tabular}{|c|c|c|c|c|c|c|}
\hline \multirow{3}{*}{$\begin{array}{l}\text { KBIT-2 } \\
\text { components }\end{array}$} & \multirow{3}{*}{$\begin{array}{l}\text { Mean } \\
( \pm \text { SEM) }\end{array}$} & \multicolumn{4}{|c|}{ 4-17 years $(n=5)$} & \\
\hline & & \multicolumn{5}{|c|}{ Descriptive Category } \\
\hline & & $\begin{array}{c}\text { Lower } \\
\text { extreme }\end{array}$ & $\begin{array}{c}\text { Below } \\
\text { average }\end{array}$ & Average & $\begin{array}{c}\text { Above } \\
\text { average }\end{array}$ & $\begin{array}{c}\text { Upper } \\
\text { extreme }\end{array}$ \\
\hline Age & $\begin{array}{c}9.80 \\
( \pm 2.18) \\
\end{array}$ & & & & & \\
\hline Verbal SS & $\begin{array}{c}96.40 \\
( \pm 3.74)\end{array}$ & $0 \%$ & $0 \%$ & $\begin{array}{c}100 \% \\
5 / 5 \\
\end{array}$ & $0 \%$ & $0 \%$ \\
\hline Nonverbal SS & $\begin{array}{c}85.00 \\
( \pm 6.94)\end{array}$ & $\begin{array}{c}20 \% \\
1 / 5\end{array}$ & $0 \%$ & $\begin{array}{c}80 \% \\
4 / 5\end{array}$ & $0 \%$ & $0 \%$ \\
\hline $\begin{array}{l}\text { IQ Composite } \\
\text { SS }\end{array}$ & $\begin{array}{c}89.40 \\
( \pm 3.60)\end{array}$ & $0 \%$ & $\begin{array}{c}20 \% \\
1 / 5\end{array}$ & $\begin{array}{l}80 \% \\
4 / 5\end{array}$ & $0 \%$ & $0 \%$ \\
\hline
\end{tabular}

Range: lower extreme (SS $\leq 69$ ), below average (SS 70-84), average (SS 85-115), above average (SS 116-130), upper extreme ( $S S \geq 131$ )

Abbreviations: SEM: Standard error mean, SS: Standard score

Table 3 | Social responsiveness derived from SRS-2 in 2.5-4.5-year-old ( $n=8), 5-17$-year-old $(n=9)$ and above 18-year-old $(n=10)$ individuals with Cantù syndrome.

\begin{tabular}{|c|c|c|c|c|c|c|c|c|}
\hline \multirow[b]{2}{*}{$\begin{array}{l}\text { Social } \\
\text { category }\end{array}$} & \multicolumn{4}{|c|}{ Preschool: $2.5-4.5$ years $(n=8)$} & \multicolumn{4}{|c|}{ School-age: $5-17$ years $(n=9)$} \\
\hline & $\begin{array}{c}\text { Mean } \\
( \pm \text { SEM) }\end{array}$ & mild & moderate & severe & $\begin{array}{c}\text { Mean } \\
( \pm \text { SEM) }\end{array}$ & mild & moderate & severe \\
\hline Age & $\begin{array}{c}3.75 \\
( \pm 0.37) \\
\end{array}$ & & & & $\begin{array}{r}13.78 \\
( \pm 1.06) \\
\end{array}$ & & & \\
\hline $\begin{array}{l}\text { Social } \\
\text { awareness }\end{array}$ & $\begin{array}{c}61.38 \\
( \pm 5.05) \\
\end{array}$ & $0 \%$ & $\begin{array}{c}24 \% \\
3 / 8 \\
\end{array}$ & $\begin{array}{c}12.5 \% \\
1 / 8 \\
\end{array}$ & $\begin{array}{c}51.22 \\
( \pm 4.46) \\
\end{array}$ & $0 \%$ & $0 \%$ & $\begin{array}{l}11 \% \\
1 / 9 \\
\end{array}$ \\
\hline $\begin{array}{l}\text { Social } \\
\text { cognition }\end{array}$ & $\begin{array}{c}59.5 \\
( \pm 6.44)\end{array}$ & $0 \%$ & $\begin{array}{c}12.5 \% \\
1 / 8\end{array}$ & $\begin{array}{c}25 \% \\
2 / 8\end{array}$ & $\begin{array}{c}48.56 \\
( \pm 3.03)\end{array}$ & $0 \%$ & $\begin{array}{l}11 \% \\
1 / 9\end{array}$ & $0 \%$ \\
\hline $\begin{array}{l}\text { Social } \\
\text { communication }\end{array}$ & $\begin{array}{c}63.38 \\
( \pm 6.84)\end{array}$ & $\begin{array}{c}12.5 \% \\
1 / 8\end{array}$ & $\begin{array}{c}12.5 \% \\
1 / 8\end{array}$ & $\begin{array}{c}37.5 \% \\
3 / 8\end{array}$ & $\begin{array}{c}52.44 \\
( \pm 5.50)\end{array}$ & $0 \%$ & $\begin{array}{l}11 \% \\
1 / 9\end{array}$ & $\begin{array}{l}11 \% \\
1 / 9\end{array}$ \\
\hline $\begin{array}{l}\text { Social } \\
\text { motivation }\end{array}$ & $\begin{array}{c}61.25 \\
( \pm 5.73) \\
\end{array}$ & $\begin{array}{c}12.5 \% \\
1 / 8\end{array}$ & $0 \%$ & $\begin{array}{c}25 \% \\
2 / 8\end{array}$ & $\begin{array}{c}52.56 \\
( \pm 3.00) \\
\end{array}$ & $\begin{array}{l}11 \% \\
1 / 9 \\
\end{array}$ & $\begin{array}{l}11 \% \\
1 / 9 \\
\end{array}$ & $0 \%$ \\
\hline $\mathrm{SCl}$ & $\begin{array}{c}58.75 \\
( \pm 8.81)\end{array}$ & $\begin{array}{c}25 \% \\
2 / 8\end{array}$ & $0 \%$ & $\begin{array}{c}37.5 \% \\
3 / 8\end{array}$ & $\begin{array}{c}52.44 \\
( \pm 4.77)\end{array}$ & $\begin{array}{l}11 \% \\
1 / 9 \\
\end{array}$ & $0 \%$ & $\begin{array}{l}11 \% \\
1 / 9 \\
\end{array}$ \\
\hline RRBI & $\begin{array}{c}64.5 \\
( \pm 6.77)\end{array}$ & $0 \%$ & $\begin{array}{c}25 \% \\
2 / 8\end{array}$ & $\begin{array}{c}37.5 \% \\
3 / 8\end{array}$ & $\begin{array}{c}56.00 \\
( \pm 4.94)\end{array}$ & $\begin{array}{l}11 \% \\
1 / 9\end{array}$ & $\begin{array}{l}11 \% \\
1 / 9\end{array}$ & $\begin{array}{l}11 \% \\
1 / 9\end{array}$ \\
\hline $\begin{array}{l}\text { SRS-2 overall } \\
\text { score }\end{array}$ & $\begin{array}{c}63.5 \\
( \pm 6.61))\end{array}$ & $\begin{array}{c}25 \% \\
2 / 8\end{array}$ & $0 \%$ & $\begin{array}{c}37.5 \% \\
3 / 8\end{array}$ & $\begin{array}{c}52.89 \\
( \pm 5.02)\end{array}$ & $\begin{array}{l}11 \% \\
1 / 9\end{array}$ & $0 \%$ & $\begin{array}{l}11 \% \\
1 / 9\end{array}$ \\
\hline
\end{tabular}

an informant report is not available for every individual.

Range: within normal limits ( $T$-score $\leq 59$ ), mild range ( $T$-score 60-65), moderate range (T-score 66-75), severe range (T-score $\geq 76$ ).

Abbreviations: IR: informant report, RRBI: Restricted interests \& Repetitive Behaviors Index,

SCI: Social Communication Index, SEM: Standard error mean, SR: self report 


\begin{tabular}{cccccc}
\hline \multirow{2}{*}{$\begin{array}{c}\text { Mean } \\
( \pm \text { SEM) }\end{array}$} & \multicolumn{7}{c}{$\mathbf{1 8 - 9 0}$ years $(\mathbf{n}=\mathbf{8})$} \\
\cline { 2 - 6 } & $\begin{array}{c}\text { Lower } \\
\text { extreme }\end{array}$ & $\begin{array}{c}\text { Below } \\
\text { average }\end{array}$ & Average & $\begin{array}{c}\text { Above } \\
\text { average }\end{array}$ & $\begin{array}{c}\text { Upper } \\
\text { extreme }\end{array}$ \\
\hline $39.13( \pm 6.79)$ & & & & \\
\hline $97.63( \pm 3.72)$ & $0 \%$ & $\begin{array}{c}12.5 \% \\
1 / 8\end{array}$ & $\begin{array}{c}87.5 \% \\
7 / 8\end{array}$ & $0 \%$ & $0 \%$ \\
\hline $94.13( \pm 4.63)$ & $0 \%$ & $\begin{array}{c}12.5 \% \\
1 / 8\end{array}$ & $\begin{array}{c}87.5 \% \\
7 / 8\end{array}$ & $0 \%$ & $0 \%$ \\
\hline $96.00( \pm 4.14)$ & $0 \%$ & $\begin{array}{c}12.5 \% \\
1 / 8\end{array}$ & $\begin{array}{c}87.5 \% \\
7 / 8\end{array}$ & $0 \%$ & $0 \%$ \\
\hline
\end{tabular}

\begin{tabular}{|c|c|c|c|c|c|c|c|}
\hline \multicolumn{8}{|c|}{ Adults: $\geq 18$ years $(S R, n=10 ; I R, n=4)^{a}$} \\
\hline \multicolumn{2}{|c|}{$\begin{array}{c}\text { Mean } \\
( \pm \text { SEM })\end{array}$} & \multicolumn{2}{|c|}{ mild } & \multicolumn{2}{|c|}{ moderate } & \multicolumn{2}{|c|}{ severe } \\
\hline SR & IR & SR & IR & SR & IR & SR & IR \\
\hline $36.40( \pm 4.53)$ & $31.75( \pm 6.05)$ & & & & & & \\
\hline $49.40( \pm 2.31)$ & $45.00( \pm 1.83)$ & $\begin{array}{l}10 \% \\
1 / 10\end{array}$ & $0 \%$ & $0 \%$ & $0 \%$ & $0 \%$ & $0 \%$ \\
\hline $52.10( \pm 3.22)$ & $50.75( \pm 1.89)$ & $0 \%$ & $0 \%$ & $0 \%$ & $0 \%$ & $\begin{array}{l}10 \% \\
1 / 10\end{array}$ & $0 \%$ \\
\hline $551.10( \pm 3.35)$ & $45.75( \pm 1.60)$ & $0 \%$ & $0 \%$ & $0 \%$ & $0 \%$ & $\begin{array}{l}10 \% \\
1 / 10\end{array}$ & $0 \%$ \\
\hline $58.90( \pm 3.53)$ & $53.00( \pm 2.38)$ & $\begin{array}{l}20 \% \\
2 / 10\end{array}$ & $0 \%$ & $\begin{array}{l}10 \% \\
1 / 10\end{array}$ & $0 \%$ & $\begin{array}{l}10 \% \\
1 / 10\end{array}$ & $0 \%$ \\
\hline $50.50( \pm 3.55)$ & $48.50( \pm 1.85)$ & $0 \%$ & $0 \%$ & $0 \%$ & $0 \%$ & $\begin{array}{l}10 \% \\
1 / 10\end{array}$ & $0 \%$ \\
\hline $54.90( \pm 3.80)$ & $49.50( \pm 5.97)$ & $\begin{array}{l}10 \% \\
1 / 10 \\
\end{array}$ & $\begin{array}{c}25 \% \\
1 / 4 \\
\end{array}$ & $0 \%$ & $0 \%$ & $\begin{array}{l}10 \% \\
1 / 10 \\
\end{array}$ & $0 \%$ \\
\hline $48.90( \pm 1.45)$ & $48.25( \pm 0.63)$ & $0 \%$ & $0 \%$ & $0 \%$ & $0 \%$ & $0 \%$ & $0 \%$ \\
\hline
\end{tabular}




\subsubsection{ASD-associated features (total $n=27$ )}

To assess whether CS subjects reveal criteria for ASD, participating Dutch and US subjects and/or their relatives were invited to complete the social responsiveness scale-2 (Constantino \& Gruber, 2012). Out of the 27 CS cases that provided a completed questionnaire (informant report only: $n=17$; self-report only: $n=6$; self-report and informant report: $n=4)$, three subjects (11\%) had SRS-2 total scores in the mild range, indicating mild interference with everyday social interactions, and four (15\%) in the severe range which is strongly associated with a clinical diagnosis of ASD. The remaining cases all scored within a normal range (74\%). In addition, $4 / 27$ (15\%) revealed social communication indices $(\mathrm{SCl})$ in the mild as well as the severe range, respectively. Restricted interests and repetitive behaviors indices (RRBI) were in the mild range for $2 / 27$ (7\%), in the moderate range for $3 / 27(11 \%)$ and in the severe range for $5 / 27(19 \%)$ subjects.

For further analysis, subjects were divided into three age-related groups according to the specific SRS-2 questionnaire they had to fill in: (1) preschool (2.5-4.5 years, $n=8)$, ( 2 ) school-age ( $5-17$ years, $n=9)$, and ( 3 ) adult ( $\geq 18$ years, $\mathrm{n}=10$ ). Mean SRS-2 T-scores and the percentage of individuals who received T-scores in the mild, moderate or severe range for each group are reported in Table 3. CS cases with an age of 2.5-4.5 years revealed the highest percentage of individuals with a total score in a clinically significant range $(5 / 8,62.5 \%)$, resulting from the mean $T$-score for $3 / 4$ assessed treatment subscales falling in the mild range. The remaining two age groups showed $22 \%(2 / 9)$ and $0 \%(0 / 10)$ of individuals in a clinically significant range, respectively. This observation indicates a mitigation of ASD-associated features in CS over time. Interestingly, ASD-associated features seem predominantly present in young and adolescent male CS cases in this cohort. Boys revealed a mean total score in the moderate range $(67.75 \pm 7.88, n=4)$ and male adolescents in the mild range $(61.25 \pm 10.00$, $\mathrm{n}=4$ ) while both female groups were represented in the normal range (2.44.5 years: $59.25 \pm 11.39,6-17$ years: $46.20 \pm 2.40$ ). No considerable differences between the self-report and informant report in adults could be observed. Interestingly, both individuals that self-reported a diagnosis of ASD revealed total scores in the severe range.

\subsubsection{Behavioral and emotional functioning (total $n=26$ )}

ASEBA assesses competencies, strengths, adaptive functioning, and behavioral, emotional, and social problems from age 1.5 to 59 years. Twenty-six CS subjects and/or their relatives completed the age-appropriate ASEBA questionnaire. Additionally to assessing the entire CS cohort, subjects were sorted into three 
age-related groups according to the specific ASEBA questionnaire they were asked to fill in: (1) 1.5-5 years ( $n=8, C B C L)$, (2) 6-17 years ( $C B C L, n=10$; YSR, $n=5)$, (3) 18-59 years ( $A B C L, n=8 ; A S R, n=11)$. In each assessed category, we indicate a mean $\mathrm{T}$-score and the percentage of individuals who received T-scores in the borderline clinical and clinical range (Table 4-6). For all groups, the questionnaire was divided in two parts: one assessing social competencies and another assessing behavior problems.

\section{Social and professional functioning}

Children (6-17 years) were assessed on competencies in terms of the child's functioning in activities, social relationships, and school, whereas adults (1859 years) were assessed on adaptive functioning for academic performance and favorable characteristics including friends, spouse/partner, family, job and education (Table 4). The mean total competence scores for group 2 (CBCL: 40.47 \pm 3.10 , YSR: $36.60 \pm 2.32$ ) and the mean adaptive functioning score for group 3 (ASR: 47.73 \pm 2.16 ) were in the normal range. In detail, all individuals from 6 years of age were (or had been) attending mainstream education schools, 11/21 $(52 \%)$ (had) required special education services. $3 / 10(30 \%)$ subjects currently attending school had to repeat at least one grade and 4/10 (40\%) were reported to perform below average. Eight out of twelve (67\%) eligible subjects (had) attended college after obtaining their high school degree, with two individuals receiving a graduate degree (Table 1 ). For adults, $7 / 12(58 \%)$ had a paid job at the time of assessment.

\section{Behavioral symptoms}

The list of items for psychiatric symptoms provides scores for eight empirical scales or syndromes, and for two second-order factors (internalizing disorders and externalizing disorders), in addition to the total score (Table 5). A total problem score in the clinical range was found in 7/26 (27\%) subjects according to the relative-rated ASEBA questionnaires, whereas the percentage was considerably lower when scored by subjects themselves $(1 / 21,5 \%)$. This was also evident when comparing the total mean scores between self and informant

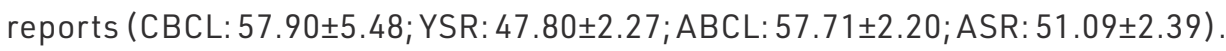
Internalizing problems in the clinical range were present in 10/26 (38\%) individuals and clinically recognizable externalizing problems were observed in $7 / 26(27 \%)$ subjects according to the relative-rated ASEBA questionnaires.

The following specific problem clusters contained four or more clinical cases: "withdrawn/depressed", "somatic complaints", "attention problems". Moreover, we observed that both clinical internalizing and externalizing problems seem to decrease over time. Merely clinical somatic complaints seemed to increase 
with age, reflecting the disease progression as has been reported in CS before (Grange et al., 2019).

When comparing psychiatric symptoms in male and female CS subjects from each age-specific group, young and adolescent male cases revealed a mean total (1.5-5 years: $63.25 \pm 4.05, n=4 ; 6-17$ years: $67.20 \pm 8.76, n=5)$ and internalizing problem score ( $1.5-5$ years: $68.50 \pm 7.00, n=4 ; 6-17$ years: $70.20 \pm 7.58, n=5)$ in the clinical range, whereas females presented in the normal range (internalizing problems: $1.5-5$ years: $52.75 \pm 7.01, n=4 ; 6-17$ years: $49.40 \pm 2.44, n=5$; total score: $1.5-5$ years: $53.75 \pm 8.42, n=4 ; 6-17$ years: $48.20 \pm 4.07, n=5)$.

Additionally, a list of six DSM-oriented scales derived through expert consensus are provided (Table 6). We observed that $8 / 26$ (31\%) subjects qualified for one or more DSM-diagnoses, according to relative-scored questionnaires. Notably, in patient-rated questionnaires only $2 / 16$ (13\%) revealed a clinical DSMdiagnosis. We observed that $\geq 25 \%$ of young children had T-scores in the clinical range on "anxiety", "autism spectrum problems" and "ADHD problems" DSMoriented scale. These percentages decreased markedly in adolescents $(\geq 10 \%$, CBCL, $n=10 ; Y S R, n=5)$ and adults $(0 \%, A B C L, n=8 ; A S R, n=11)$.

When comparing psychiatric symptoms and DSM-oriented problems between various family members with CS (familial cases, $\mathrm{n}=12$ ) a high variability was observed, confirming the known intra-familial variability of CS-associated features (Grange et al., 2019; Roessler et al., 2018). 
Table 4 | Social and professional functioning derived from ASEBA in 6-17-year-old $(n=10)$ and 18-59-year-old ( $n=11)$ individuals with Cantù syndrome.

\begin{tabular}{|c|c|c|c|c|c|c|c|c|c|c|c|c|}
\hline \multirow{3}{*}{$\begin{array}{l}\text { Social/ } \\
\text { professional } \\
\text { category }\end{array}$} & \multicolumn{6}{|c|}{ 6-17 years $(C B C L, n=10 ; Y S R, n=5)^{a}$} & \multicolumn{6}{|c|}{$18-59$ years $(A B C L, n=8 ; A S R, n=11)^{b}$} \\
\hline & \multicolumn{2}{|c|}{ Mean (£SEM) } & \multicolumn{2}{|c|}{ BR (\%) } & \multicolumn{2}{|c|}{ CR (\%) } & \multicolumn{2}{|c|}{ Mean ( } & \multicolumn{2}{|c|}{ BR (\%) } & \multicolumn{2}{|c|}{ CR (\%) } \\
\hline & CBCL & YSR & CBCL & YSR & CBCL & YSR & ABCL & ASR & $A B C L$ & ASR & ABCL & ASR \\
\hline Age & $\begin{array}{c}12.70 \\
( \pm 1.13) \\
\end{array}$ & $\begin{array}{c}14.40 \\
( \pm 1.25) \\
\end{array}$ & & & & & $\begin{array}{r}35.50 \\
( \pm 5.38) \\
\end{array}$ & $\begin{array}{r}33.733 \\
( \pm 4.42) \\
\end{array}$ & & & & \\
\hline Activities & $\begin{array}{c}40.67 \\
( \pm 2.54)\end{array}$ & $\begin{array}{c}37.40 \\
( \pm 2.93)\end{array}$ & $0 \%$ & $\begin{array}{c}20 \% \\
1 / 5 \\
\end{array}$ & $\begin{array}{c}11 \% \\
1 / 9\end{array}$ & $0 \%$ & na & na & na & na & na & na \\
\hline Social & $\begin{array}{r}45.00 \\
( \pm 2.78) \\
\end{array}$ & $\begin{array}{c}44.80 \\
( \pm 2.46) \\
\end{array}$ & $0 \%$ & $\begin{array}{c}20 \% \\
1 / 5 \\
\end{array}$ & $\begin{array}{l}10 \% \\
1 / 10 \\
\end{array}$ & $0 \%$ & na & na & na & na & na & na \\
\hline School & $\begin{array}{r}38.00 \\
( \pm 3.34) \\
\end{array}$ & na & $\begin{array}{c}25 \% \\
2 / 8 \\
\end{array}$ & na & $\begin{array}{c}25 \% \\
2 / 8 \\
\end{array}$ & na & na & na & na & na & na & na \\
\hline Friends & na & na & na & na & na & na & $\begin{array}{c}46.38 \\
( \pm 2.16) \\
\end{array}$ & $\begin{array}{r}43.45 \\
( \pm 2.69) \\
\end{array}$ & $0 \%$ & $0 \%$ & $0 \%$ & $\begin{array}{c}9 \% \\
1 / 11 \\
\end{array}$ \\
\hline $\begin{array}{l}\text { Spouse/ } \\
\text { Partner }\end{array}$ & na & na & na & na & na & na & $\begin{array}{c}47.20 \\
( \pm 4.75) \\
\end{array}$ & $\begin{array}{r}49.00 \\
( \pm 3.82) \\
\end{array}$ & $0 \%$ & $\begin{array}{c}14 \% \\
1 / 7 \\
\end{array}$ & $0 \%$ & $0 \%$ \\
\hline Family & na & na & na & na & na & na & na & $\begin{array}{c}48.45 \\
( \pm 2.01) \\
\end{array}$ & na & $0 \%$ & na & $0 \%$ \\
\hline Job & na & na & na & na & na & na & na & $\begin{array}{c}52.38 \\
( \pm 2.04) \\
\end{array}$ & na & $0 \%$ & na & $0 \%$ \\
\hline Education & na & na & na & na & na & na & na & $\begin{array}{c}52.00 \\
( \pm 8.00) \\
\end{array}$ & na & $0 \%$ & na & $0 \%$ \\
\hline $\begin{array}{l}\text { Personal } \\
\text { strengths }\end{array}$ & na & na & na & na & na & na & $\begin{array}{r}47.00 \\
( \pm 1.86) \\
\end{array}$ & $\begin{array}{c}50.64 \\
( \pm 1.78) \\
\end{array}$ & $0 \%$ & $0 \%$ & $0 \%$ & $0 \%$ \\
\hline $\begin{array}{l}\text { Total } \\
\text { competence } \\
\text { score }\end{array}$ & $\begin{array}{c}40.47 \\
( \pm 3.05)\end{array}$ & $\begin{array}{c}36.60 \\
( \pm 2.32)\end{array}$ & $\begin{array}{l}14 \% \\
1 / / 7\end{array}$ & $\begin{array}{c}20 \% \\
1 / 5\end{array}$ & $\begin{array}{l}14 \% \\
1 / / 7\end{array}$ & $0 \%$ & na & na & na & na & na & na \\
\hline $\begin{array}{l}\text { Adaptive } \\
\text { functioning } \\
\text { score }\end{array}$ & na & na & na & na & na & na & na & $\begin{array}{c}47.73 \\
( \pm 2.16)\end{array}$ & na & $\begin{array}{c}9 \% \\
1 / 11\end{array}$ & na & $0 \%$ \\
\hline
\end{tabular}

a Subjects from 6-10 years of age only required to fill in a CBCL, subjects from 11-17 years of age were additionally asked to fill in a YSR.

${ }^{b} \mathrm{An} A B C L$ is not available for every patient.

Range: Borderline range (BR) for syndrome scale, T-score 30-35; clinical range (CR) for syndrome scale, $\mathrm{T}$-score $\leq 30$.

Abbreviations: ABCL: Adult Behavior Checklist, ASR: Adult Self-Report, CBCL: Children Behavior Checklist, BR: borderline range, CR: clinical range, na: not assessed, SEM: Standard error mean, YSR: Youth Self-Report. 
Table 5: Psychiatric symptoms derived from ASEBA in 1.5-5-year-old $(n=8), 6-17$-year-old $(n=10)$ and 18-59-year-old $(n=11)$ individuals with Cantù syndrome.

\begin{tabular}{|c|c|c|c|c|c|c|c|c|c|}
\hline \multirow{3}{*}{$\begin{array}{l}\text { Psychiatric } \\
\text { symptoms }\end{array}$} & \multicolumn{3}{|c|}{$1.5-5$ years $(n=8)$} & \multicolumn{6}{|c|}{ 6-17 years $(C B C L, n=10 ; Y S R, n=5)^{a}$} \\
\hline & \multirow{2}{*}{$\begin{array}{c}\text { Mean } \\
( \pm \text { SEM) }\end{array}$} & \multirow{2}{*}{ BR } & \multirow{2}{*}{ CR } & \multicolumn{2}{|c|}{ Mean ( \pm SEM) } & \multicolumn{2}{|c|}{ BR (\%) } & \multicolumn{2}{|c|}{ CR (\%) } \\
\hline & & & & CBCL & YSR & CBCL & YSR & CBCL & YSR \\
\hline Age & $\begin{array}{c}3.25 \\
( \pm 0.45) \\
\end{array}$ & & & $\begin{array}{r}12.70 \\
( \pm 1.13) \\
\end{array}$ & $\begin{array}{c}14.40 \\
( \pm 1.25) \\
\end{array}$ & & & & \\
\hline $\begin{array}{l}\text { Internalizing } \\
\text { score }\end{array}$ & $\begin{array}{c}60.63 \\
( \pm 5.49) \\
\end{array}$ & $0 \%$ & $\begin{array}{c}50 \% \\
4 / 8 \\
\end{array}$ & $\begin{array}{c}59.80 \\
( \pm 5.11) \\
\end{array}$ & $\begin{array}{c}52.40 \\
( \pm 3.90)\end{array}$ & $0 \%$ & $0 \%$ & $\begin{array}{l}40 \% \\
4 / 10 \\
\end{array}$ & $\begin{array}{c}20 \% \\
1 / 5 \\
\end{array}$ \\
\hline $\begin{array}{l}\text { Anxious/ } \\
\text { depressed }\end{array}$ & $\begin{array}{c}60.38 \\
( \pm 4.88) \\
\end{array}$ & $\begin{array}{c}12.5 \% \\
1 / 8 \\
\end{array}$ & $\begin{array}{c}25 \% \\
2 / 8 \\
\end{array}$ & $\begin{array}{c}59.50 \\
( \pm 4.67) \\
\end{array}$ & $\begin{array}{c}52.40 \\
( \pm 2.40) \\
\end{array}$ & $\begin{array}{l}30 \% \\
3 / 10 \\
\end{array}$ & $0 \%$ & $\begin{array}{l}10 \% \\
1 / 10 \\
\end{array}$ & $0 \%$ \\
\hline $\begin{array}{l}\text { Withdrawn/ } \\
\text { depressed }\end{array}$ & $\begin{array}{c}63.88 \\
( \pm 4.47) \\
\end{array}$ & $\begin{array}{c}12.5 \% \\
1 / 8 \\
\end{array}$ & $\begin{array}{c}25 \% \\
2 / 8 \\
\end{array}$ & $\begin{array}{c}59.10 \\
( \pm 4.79) \\
\end{array}$ & $\begin{array}{r}54.00 \\
( \pm 2.50) \\
\end{array}$ & $\begin{array}{l}10 \% \\
1 / 10 \\
\end{array}$ & $0 \%$ & $\begin{array}{l}10 \% \\
1 / 10 \\
\end{array}$ & $0 \%$ \\
\hline $\begin{array}{l}\text { Somatic } \\
\text { complaints }\end{array}$ & $\begin{array}{r}60.50 \\
( \pm 4.17) \\
\end{array}$ & $\begin{array}{c}12.5 \% \\
1 / 8 \\
\end{array}$ & $\begin{array}{c}25 \% \\
2 / 8 \\
\end{array}$ & $\begin{array}{r}65.70 \\
( \pm 4.00) \\
\end{array}$ & $\begin{array}{c}60.00 \\
( \pm 2.92) \\
\end{array}$ & $\begin{array}{l}20 \% \\
2 / 10 \\
\end{array}$ & $\begin{array}{c}40 \% \\
2 / 5 \\
\end{array}$ & $\begin{array}{l}10 \% \\
1 / 10 \\
\end{array}$ & $0 \%$ \\
\hline $\begin{array}{l}\text { Emotionally } \\
\text { Reactive }\end{array}$ & $\begin{array}{c}62.38 \\
( \pm 4.91) \\
\end{array}$ & $\begin{array}{c}37.5 \% \\
3 / 8 \\
\end{array}$ & $\begin{array}{l}8 \% \\
1 / 8 \\
\end{array}$ & na & na & na & na & na & na \\
\hline $\begin{array}{l}\text { Externalizing } \\
\text { score }\end{array}$ & $\begin{array}{r}59.00 \\
( \pm 4.90) \\
\end{array}$ & $0 \%$ & $\begin{array}{c}50 \% \\
4 / 8 \\
\end{array}$ & $\begin{array}{r}51.90 \\
( \pm 6.19) \\
\end{array}$ & $\begin{array}{c}42.20 \\
( \pm 1.63)\end{array}$ & $0 \%$ & $0 \%$ & $\begin{array}{l}20 \% \\
2 / 10 \\
\end{array}$ & $0 \%$ \\
\hline $\begin{array}{l}\text { Aggressive } \\
\text { behavior }\end{array}$ & $\begin{array}{c}59.50 \\
( \pm 3.64) \\
\end{array}$ & $\begin{array}{c}37.5 \% \\
3 / 8 \\
\end{array}$ & $\begin{array}{c}12.5 \% \\
1 / 8 \\
\end{array}$ & $\begin{array}{c}57.10 \\
( \pm 5.08) \\
\end{array}$ & $\begin{array}{c}50.00 \\
( \pm 0.00) \\
\end{array}$ & $\begin{array}{l}10 \% \\
1 / 10 \\
\end{array}$ & $0 \%$ & $\begin{array}{l}10 \% \\
1 / 10 \\
\end{array}$ & $0 \%$ \\
\hline $\begin{array}{l}\text { Attention } \\
\text { problems }\end{array}$ & $\begin{array}{r}63.38 \\
( \pm 4.28) \\
\end{array}$ & $\begin{array}{c}25 \% \\
2 / 8 \\
\end{array}$ & $\begin{array}{c}37.5 \% \\
3 / 8 \\
\end{array}$ & $\begin{array}{r}61.60 \\
( \pm 4.72) \\
\end{array}$ & $\begin{array}{c}51.60 \\
( \pm 0.75) \\
\end{array}$ & $\begin{array}{l}10 \% \\
1 / 10 \\
\end{array}$ & $0 \%$ & $\begin{array}{l}20 \% \\
2 / 10 \\
\end{array}$ & $0 \%$ \\
\hline $\begin{array}{l}\text { Rulebreaking } \\
\text { behavior }\end{array}$ & na & na & na & $\begin{array}{r}58.50 \\
( \pm 4.86) \\
\end{array}$ & $\begin{array}{c}51.80 \\
( \pm 0.92)\end{array}$ & $0 \%$ & $0 \%$ & $\begin{array}{l}10 \% \\
1 / 10 \\
\end{array}$ & $0 \%$ \\
\hline Intrusive & na & na & na & na & na & na & na & na & na \\
\hline $\begin{array}{l}\text { Stress } \\
\text { problems }\end{array}$ & $\begin{array}{r}61.63 \\
( \pm 4.81) \\
\end{array}$ & $0 \%$ & $\begin{array}{c}37.5 \% \\
3 / 8 \\
\end{array}$ & na & na & na & na & na & na \\
\hline $\begin{array}{l}\text { Sleep } \\
\text { problems }\end{array}$ & $\begin{array}{c}59.13 \\
( \pm 4.00) \\
\end{array}$ & $0 \%$ & $\begin{array}{c}25 \% \\
2 / 8 \\
\end{array}$ & na & na & na & na & na & na \\
\hline $\begin{array}{l}\text { Thought } \\
\text { problems }\end{array}$ & na & na & na & $\begin{array}{r}64.10 \\
( \pm 4.78) \\
\end{array}$ & $\begin{array}{c}52.20 \\
( \pm 1.02) \\
\end{array}$ & $\begin{array}{l}10 \% \\
1 / 10 \\
\end{array}$ & $0 \%$ & $\begin{array}{l}30 \% \\
3 / 10 \\
\end{array}$ & $0 \%$ \\
\hline $\begin{array}{l}\text { Social } \\
\text { problems }\end{array}$ & na & na & na & $\begin{array}{r}62.70 \\
( \pm 5.01) \\
\end{array}$ & $\begin{array}{r}54.60 \\
( \pm 1.81) \\
\end{array}$ & $\begin{array}{l}20 \% \\
2 / 10 \\
\end{array}$ & $0 \%$ & $\begin{array}{l}10 \% \\
1 / 10 \\
\end{array}$ & $0 \%$ \\
\hline Critical items & na & na & na & na & na & na & na & na & na \\
\hline $\begin{array}{l}\text { Total problem } \\
\text { score }\end{array}$ & $\begin{array}{c}58.50 \\
( \pm 4.68)\end{array}$ & $0 \%$ & $\begin{array}{c}37.5 \% \\
3 / 8\end{array}$ & $\begin{array}{c}57.90 \\
( \pm 5.48)\end{array}$ & $\begin{array}{c}47.80 \\
( \pm 2.27)\end{array}$ & $\begin{array}{l}10 \% \\
1 / 10\end{array}$ & $0 \%$ & $\begin{array}{l}30 \% \\
3 / 10\end{array}$ & $0 \%$ \\
\hline
\end{tabular}

a Subjects from 6-10 years of age only required to fill in a CBCL, subjects from 11-17 years of age were additionally asked to fill in a YSR.

${ }^{\mathrm{b}} \mathrm{An} \mathrm{ABCL}$ is not available for every patient.

Range: Borderline range (BR) for syndrome scale, T-score 65-70; clinical range (CR) for syndrome scale, T-score $\geq 70$; BR for "internalizing, externalizing and total problems" scale, T-score 60-63; CR for "internalizing, externalizing and total problems" scale, T-score $\geq 64$. Abbreviations: ABCL: Adult Behavior Checklist, ASR: Adult Self-Report, CBCL: Children Behavior Checklist, BR: borderline range, CR: clinical range, na: not assessed, SEM: Standard error mean, YSR: Youth Self-Report. 


\section{8-59 years $(A B C L, n=8 ; A S R, n=11)^{b}$}

\begin{tabular}{|c|c|c|c|c|c|}
\hline \multicolumn{2}{|c|}{ Mean ( \pm SEM) } & \multicolumn{2}{|c|}{ BR (\%) } & \multicolumn{2}{|c|}{ CR (\%) } \\
\hline$A B C L$ & ASR & $A B C L$ & ASR & ABCL & ASR \\
\hline $\begin{array}{c}35.50 \\
( \pm 5.38) \\
\end{array}$ & $\begin{array}{l}33.733 \\
( \pm 4.42) \\
\end{array}$ & & & & \\
\hline $\begin{array}{c}61.14 \\
( \pm 4.13)\end{array}$ & $\begin{array}{c}54.64 \\
( \pm 2.69)\end{array}$ & $\begin{array}{c}12.5 \% \\
1 / 8\end{array}$ & $0 \%$ & $\begin{array}{c}25 \% \\
2 / 8\end{array}$ & $\begin{array}{c}18.2 \% \\
2 / 11\end{array}$ \\
\hline $\begin{array}{c}58.25 \\
( \pm 2.66) \\
\end{array}$ & $\begin{array}{c}54.18 \\
( \pm 2.00) \\
\end{array}$ & $\begin{array}{c}25 \% \\
2 / 8 \\
\end{array}$ & $0 \%$ & $0 \%$ & $\begin{array}{l}9.1 \% \\
1 / 11 \\
\end{array}$ \\
\hline $\begin{array}{c}56.88 \\
( \pm 3.02) \\
\end{array}$ & $\begin{array}{r}55.18 \\
( \pm 1.87) \\
\end{array}$ & $\begin{array}{c}12.5 \% \\
1 / 8 \\
\end{array}$ & $\begin{array}{l}9.1 \% \\
1 / 11 \\
\end{array}$ & $\begin{array}{c}12.5 \% \\
1 / 8 \\
\end{array}$ & $0 \%$ \\
\hline $\begin{array}{c}61.13 \\
( \pm 4.28) \\
\end{array}$ & $\begin{array}{c}59.73 \\
( \pm 2.50) \\
\end{array}$ & $0 \%$ & $0 \%$ & $\begin{array}{c}25 \% \\
2 / 8 \\
\end{array}$ & $\begin{array}{c}18.2 \% \\
2 / 11 \\
\end{array}$ \\
\hline na & na & na & na & na & na \\
\hline $\begin{array}{c}54.00 \\
( \pm 2.72)\end{array}$ & $\begin{array}{c}44.00 \\
( \pm 2.53)\end{array}$ & $\begin{array}{c}12.5 \% \\
1 / 8\end{array}$ & $0 \%$ & $\begin{array}{c}12.5 \% \\
1 / 8\end{array}$ & $0 \%$ \\
\hline $\begin{array}{c}55.13 \\
( \pm 2.56) \\
\end{array}$ & $\begin{array}{c}52.64 \\
( \pm 1.25) \\
\end{array}$ & $0 \%$ & $0 \%$ & $\begin{array}{c}12.5 \% \\
1 / 8 \\
\end{array}$ & $0 \%$ \\
\hline $\begin{array}{c}58.63 \\
( \pm 1.81) \\
\end{array}$ & $\begin{array}{c}55.27 \\
( \pm 2.32) \\
\end{array}$ & $0 \%$ & $0 \%$ & $0 \%$ & $\begin{array}{l}9.1 \% \\
1 / 11 \\
\end{array}$ \\
\hline $\begin{array}{c}55.38 \\
( \pm 6.32)\end{array}$ & $\begin{array}{c}50.73 \\
( \pm 0.30)\end{array}$ & $\begin{array}{c}12.5 \% \\
1 / 8\end{array}$ & $0 \%$ & $0 \%$ & $0 \%$ \\
\hline $\begin{array}{c}52.63 \\
( \pm 0.80) \\
\end{array}$ & $\begin{array}{c}52.09 \\
( \pm 4.42) \\
\end{array}$ & $0 \%$ & $0 \%$ & $0 \%$ & $0 \%$ \\
\hline na & na & na & na & na & na \\
\hline na & na & na & na & na & na \\
\hline $\begin{array}{c}52.13 \\
( \pm 0.81)\end{array}$ & $\begin{array}{c}54.09 \\
( \pm 1.93)\end{array}$ & $0 \%$ & $\begin{array}{l}9.1 \% \\
1 / 11 \\
\end{array}$ & $0 \%$ & $0 \%$ \\
\hline na & na & na & na & na & na \\
\hline $\begin{array}{c}55.86 \\
( \pm 2.02)\end{array}$ & $\begin{array}{c}52.55 \\
( \pm 1.32) \\
\end{array}$ & $\begin{array}{c}12.5 \% \\
1 / 8 \\
\end{array}$ & $\begin{array}{l}9.1 \% \\
1 / 11 \\
\end{array}$ & $\begin{array}{c}12.5 \% \\
1 / 8 \\
\end{array}$ & $0 \%$ \\
\hline $\begin{array}{c}57.71 \\
( \pm 2.20) \\
\end{array}$ & $\begin{array}{c}51.09 \\
( \pm 2.39) \\
\end{array}$ & $\begin{array}{c}25 \% \\
2 / 8 \\
\end{array}$ & $0 \%$ & $\begin{array}{c}12.5 \% \\
1 / 8 \\
\end{array}$ & $\begin{array}{l}9.1 \% \\
1 / 11 \\
\end{array}$ \\
\hline
\end{tabular}


Table 6: DSM-oriented scales derived from ASEBA in 1.5-5-year-old $(n=8), 6-17$-year-old $(n=10)$ and 18-59-year-old $(n=11)$ individuals with Cantù syndrome.

\begin{tabular}{|c|c|c|c|c|c|c|c|c|c|}
\hline \multirow{3}{*}{$\begin{array}{l}\text { DSM-oriented } \\
\text { scales }\end{array}$} & \multicolumn{3}{|c|}{$1.5-5$ years $(n=8)$} & \multicolumn{6}{|c|}{ 6-17 years $(C B C L, n=10 ; Y S R, n=5)^{a}$} \\
\hline & \multirow{2}{*}{$\begin{array}{c}\text { Mean } \\
( \pm \text { SEM) }\end{array}$} & \multirow{2}{*}{ BR } & \multirow{2}{*}{ CR } & \multicolumn{2}{|c|}{ Mean (土SEM) } & \multicolumn{2}{|c|}{ BR } & \multicolumn{2}{|c|}{ CR } \\
\hline & & & & CBCL & YSR & CBCL & YSR & CBCL & YSR \\
\hline $\begin{array}{l}\text { Depressive } \\
\text { problems }\end{array}$ & $\begin{array}{c}60.50 \\
( \pm 4.13)\end{array}$ & $\begin{array}{c}12.5 \% \\
1 / 8\end{array}$ & $\begin{array}{c}12.5 \% \\
1 / 8\end{array}$ & $\begin{array}{c}65.20 \\
( \pm 4.58)\end{array}$ & $\begin{array}{c}55.60 \\
( \pm 1.78)\end{array}$ & $\begin{array}{l}10 \% \\
1 / 10\end{array}$ & $0 \%$ & $\begin{array}{l}20 \% \\
2 / 10\end{array}$ & $0 \%$ \\
\hline $\begin{array}{l}\text { Anxiety } \\
\text { problems }\end{array}$ & $\begin{array}{r}63.75 \\
( \pm 5.64) \\
\end{array}$ & $\begin{array}{c}12.5 \% \\
1 / 8 \\
\end{array}$ & $\begin{array}{c}37.5 \% \\
3 / 8 \\
\end{array}$ & $\begin{array}{c}60.50 \\
( \pm 4.58) \\
\end{array}$ & $\begin{array}{c}53.80 \\
( \pm 3.07) \\
\end{array}$ & $\begin{array}{l}20 \% \\
2 / 10 \\
\end{array}$ & $\begin{array}{c}20 \% \\
1 / 5 \\
\end{array}$ & $\begin{array}{l}20 \% \\
2 / 10 \\
\end{array}$ & $0 \%$ \\
\hline $\begin{array}{l}\text { Autism } \\
\text { spectrum } \\
\text { problems }\end{array}$ & $\begin{array}{c}64.38 \\
( \pm 4.24)\end{array}$ & $\begin{array}{c}12.5 \% \\
1 / 8\end{array}$ & $\begin{array}{c}25 \% \\
2 / 8\end{array}$ & na & na & na & na & na & na \\
\hline $\begin{array}{l}\mathrm{AD} / \mathrm{H} \\
\text { problems }\end{array}$ & $\begin{array}{c}60.25 \\
( \pm 4.52) \\
\end{array}$ & $\begin{array}{c}12.5 \% \\
1 / 8\end{array}$ & $\begin{array}{c}25 \% \\
2 / 8 \\
\end{array}$ & $\begin{array}{c}58.20 \\
( \pm 3.42)\end{array}$ & $\begin{array}{c}53.60 \\
( \pm 1.44) \\
\end{array}$ & $\begin{array}{l}10 \% \\
1 / 10 \\
\end{array}$ & $0 \%$ & $\begin{array}{l}20 \% \\
2 / 10 \\
\end{array}$ & $0 \%$ \\
\hline $\begin{array}{l}\text { Oppositional } \\
\text { Defiant } \\
\text { Problems }\end{array}$ & $\begin{array}{c}58.88 \\
( \pm 3.53)\end{array}$ & $\begin{array}{c}12.5 \% \\
1 / 8\end{array}$ & $\begin{array}{c}12.5 \% \\
1 / 8\end{array}$ & $\begin{array}{c}54.90 \\
( \pm 3.01)\end{array}$ & $\begin{array}{c}50.40 \\
( \pm 0.245)\end{array}$ & $0 \%$ & $0 \%$ & $\begin{array}{l}10 \% \\
1 / 10\end{array}$ & $0 \%$ \\
\hline $\begin{array}{l}\text { Conduct } \\
\text { problems }\end{array}$ & na & na & na & $\begin{array}{r}57.80 \\
( \pm 5.02) \\
\end{array}$ & $\begin{array}{c}51.20 \\
( \pm 1.20) \\
\end{array}$ & $\begin{array}{l}10 \% \\
1 / 10 \\
\end{array}$ & $0 \%$ & $\begin{array}{l}10 \% \\
1 / 10 \\
\end{array}$ & $0 \%$ \\
\hline $\begin{array}{l}\text { Somatic } \\
\text { problems }\end{array}$ & na & na & na & $\begin{array}{c}63.40 \\
( \pm 4.13)\end{array}$ & $\begin{array}{c}60.00 \\
( \pm 2.85)\end{array}$ & $\begin{array}{l}20 \% \\
2 / 10\end{array}$ & $\begin{array}{c}20 \% \\
1 / 5\end{array}$ & $\begin{array}{l}10 \% \\
1 / 10\end{array}$ & $0 \%$ \\
\hline $\begin{array}{l}\text { Avoidant } \\
\text { personality }\end{array}$ & na & na & na & na & na & na & na & na & na \\
\hline $\begin{array}{l}\text { Antisocial } \\
\text { personality }\end{array}$ & na & na & na & na & na & na & na & na & na \\
\hline
\end{tabular}

a Subjects from 6-10 years of age were only required to fill in a CBCL, subjects from 11-17 years of age were additionally asked to fill in a YSR.

${ }^{b}$ An $A B C L$ is not available for every patient.

Range: Borderline range (BR) for DSM-oriented scale, T-score 65-70 (93th to 97th percentile); clinical range (CR) for DSM-oriented scale, T-score $\geq 70$ (percentiles of 98 and higher).

Abbreviations: ABCL: Adult Behavior Checklist, AD/H: Attention Deficit/ Hyperactivity, ASR:

Adult Self-Report, BR: borderline range, CBCL: Children Behavior Checklist, CR: clinical range, na: not assessed, SEM: Standard error mean, YSR: Youth Self-Report 


\section{8-59 years ( $A B C L, n=8 ; A S R, n=11)$}

\begin{tabular}{cccccc}
\multicolumn{2}{c}{ Mean ( \pm SEM) } & \multicolumn{2}{c}{ BR } & \multicolumn{2}{c}{ CR } \\
ABCL & ASR & ABCL & ASR & ABCL & ASR \\
\hline 59.50 & 56.00 & $12.5 \%$ & $18.2 \%$ & $0 \%$ & $0 \%$ \\
$( \pm 1.60)$ & $( \pm 2.01)$ & $1 / 8$ & $2 / 11$ & & \\
\hline 54.13 & 54.09 & $12.5 \%$ & $9.1 \%$ & $0 \%$ & $0 \%$ \\
$( \pm 1.91)$ & $( \pm 1.65)$ & $1 / 8$ & $1 / 11$ & & \\
\hline na & na & na & na & na & na
\end{tabular}

\begin{tabular}{cccccc}
\hline 57.13 & 55.18 & $0 \%$ & $0 \%$ & $0 \%$ & $9.1 \%$ \\
$( \pm 1.53)$ & $( \pm 2.22)$ & & & & $1 / 11$ \\
\hline na & na & na & na & na & na
\end{tabular}

\begin{tabular}{cccccc}
\hline na & na & na & na & na & na \\
& & & & & \\
\hline 60.25 & 59.64 & $0 \%$ & $9.1 \%$ & $25 \%$ & $18.2 \%$ \\
$( \pm 3.79)$ & $( \pm 3.47)$ & & $1 / 11$ & $2 / 8$ & $2 / 11$ \\
\hline 60.25 & 55.91 & $12.5 \%$ & $9.1 \%$ & $12.5 \%$ & $9.1 \%$ \\
$( \pm 3.32)$ & $( \pm 2.59)$ & $1 / 8$ & $1 / 11$ & $1 / 8$ & $1 / 11$ \\
\hline 56.50 & 51.27 & $12.5 \%$ & $0 \%$ & $0 \%$ & $0 \%$ \\
$( \pm 2.78)$ & $( \pm 0.68)$ & $1 / 8$ & & & \\
\hline
\end{tabular}




\subsection{Discussion}

In this study, we investigated the presence of behavioral, social and intellectual abnormalities in a large cohort of Cantù syndrome individuals. The majority of cases revealed an average verbal and nonverbal intelligence suggesting that both children and adults with CS, as a group, develop at a consistent rate relative to the KBIT-2 normative sample and no intellectual deficit was observed. The mean IQ composite SS of the described CS cohort was merely 0.5 SD below the general population mean confirming published literature (Scurr et al., 2011). Children and adolescents with CS revealed a decreased non-verbal SS, 1 SD below the general population mean. Hence, minor deficits in reasoning and problem solving with patterns and relationships, pictorial analogies, and categories could be observed in this group potentially explaining the increased need for special education services observed in this CS cohort 11/21 (52\%). This might indicate that minor intellectual abilities as measured by the KBIT-2 potentially reveal age-dependency in CS. However, a larger cohort subjected to the same questionnaire will be necessary, to confirm this observation in the future.

Moreover, this study was designed to measure strengths and weaknesses in social functioning of CS subjects by applying the SRS-2, often used to assess ASD-related features (Constantino \& Gruber, 2012). Results suggest that especially young children have clinically recognizable difficulties with social behavior that may be indicative of ASD, in particular expressive social communication and the ability to pick up on social cues. These deficits seem to almost disappear in adolescents and adults, again indicating age-dependency. In adults, observations are similar when comparing self-reports $(n=10)$ and the few available informant reports $(n=4)$. Notably, it has been suggested that SRS scores are highly influenced by behavioral, non-ASD-related symptoms (Hus, Bishop, Gotham, Huerta, \& Lord, 2013). Thus, SRS scores may also be interpreted as a subjects' or informants' perception of the subjects' overall level of impairment which may for instance be affected by developmental difficulties, behavioral problems and ASD features. In the age group of 1 to 5 years, 7/9 (78\%) assessed CS subjects were reported to have developmental delay (e.g. speech delay) requiring occupational, physical and speech therapy which potentially contributes to the difficulties in social functioning and therefore the yielded SRS-2 scores (Grange et al., 2019). Interestingly, ASD-associated features seem predominantly present in young and adolescent male CS cases in this cohort. However, sample sizes in these subgroups are very restricted and 
further research would be necessary to confirm the observed gender-specific differences in the assessed cohort.

Similarly, various psychiatric symptoms assessed by ASEBA questionnaires might show an age- and/or gender-related progression in this cohort. In particular, anxiety, ADHD and autism spectrum problems were observed in $\geq 25 \%$ of young individuals, whereas such symptoms seemed to lessen in older subjects. Interestingly, internalizing problems such as anxiety/depression and emotional reactiveness, seemed to be predominantly present in male CS subjects prior to the age of 17 in our small sample.

Interpreting whether mildly impaired social functioning and observed psychiatric symptoms, especially in young CS individuals, is directly caused by CS-associated ABCC9 variants and resulting $\mathrm{K}_{\text {ATP }}$ channel dysfunction or is rather influenced by psychological and emotional distress due to CS symptoms (e.g. congenital hypertrichosis or distinctive facial features) is not straightforward. So far, no connection between ABCC 9 and ASD-related phenotypes has been observed. In order to further investigate and pinpoint the reason for the observed symptoms in this cohort, more specific questionnaires and/or psychological analyses should be applied in the future. In addition, the majority of CS individuals in this cohort who presented with ASD and other psychological features are most likely too young to have enough self-awareness to be able to recognize that they look different compared to other children their age therefore most likely not causing changes in social functioning. The improvement in symptoms over age might be explained by therapy services and special education interventions applied by these younger CS subjects.

Recently, we described a novel ABCC9-related Intellectual disability Myopathy Syndrome (AIMS) resulting from loss-of-function (LoF) mutations in ABCC9, in which patients exhibit mild to moderate intellectual disability, low IQ and anxiety (Smeland et al., 2019). Neuronal $K_{\text {ATP }}$ channels predominantly consist of Kir6.2 and SUR1 subunits; nevertheless transcripts for all $K_{A T P}$ channel subunits have been observed in multiple neuronal tissues(Liss \& Roeper, 2001) and SUR2 is reported to be expressed in central and peripheral neurons (Kawano et al., 2009; Nelson, Jicha, et al., 2015; Zoga et al., 2010). However, so far it is not obvious how $\mathrm{K}_{\text {ATP }}$ channel dysfunction could lead to cognitive impairment or anxiety.

The potential presence of additional causal, but CS-independent variants in this cohort resulting in neurodevelopmental disorders such as ADHD or ASD cannot be ruled out. Psychiatric disorders are highly heritable and can be influenced 
by many single nucleotide polymorphisms (Brikell, Kuja-Halkola, \& Larsson, 2015; Tick, Bolton, Happe, Rutter, \& Rijsdijk, 2016). Hence, the presence of such symptoms should also be examined in unaffected family members of affected CS cases. Moreover, non-genetic influences should be considered; for instance, it is known that parents of children with congenital disorders tend to overprotect children which can interfere with their otherwise normal development.

Standardized questionnaires as applied in this study furthermore offer a way to investigate genotype-phenotype correlations in terms of behavioral and intellectual functioning. However, one would have to significantly increase cohort size in order to perform a statistical analysis in individuals with CS and draw reliable conclusions. An additional drawback of this study is the varying numbers of participating subjects per applied questionnaire. As not all questionnaires were introduced during the same CS research clinic and some individuals were not able to attend each annual clinic, not every form has been completed by every participating subject, potentially resulting in a bias. Moreover, the outcome of informant questionnaires might also be affected by a parent's own anxiety and stress caused by their child's CS diagnosis rather than reflect the presence of behavioral features in the CS subject itself. Thus, further investigations might include questionnaires directed towards parents' attitudes to examine and later on exclude these possible anxieties from the analysis.

Nevertheless, results of this study contribute to increased awareness regarding the presence of social and psychiatric difficulties in CS subjects. Especially psychiatric symptoms such as anxiety are treatable with the appropriate intervention. Even though no treatment has been shown to cure ASD or ADHD, several interventions have been developed and studied. These interventions may reduce symptoms, improve cognitive ability and daily living skills, and maximize the ability of the individual to function and participate in the community (Shier, Reichenbacher, Ghuman, \& Ghuman, 2013; Weitlauf et al., 2014). Currently, there are no targeted therapies for CS available. Therefore, clinical management involves symptomatic treatments to address secondary complications. However, considerable efforts are being invested in the development of a pharmacological treatment for CS subjects mainly focusing on the reversibility of clinical features such as cardiovascular anomalies, hypertrichosis and edema (Ma et al., 2019; McClenaghan et al., 2019). If successful, such a therapy might also contribute to a decrease in the described behavioral symptoms; even though no clarification for these features based on knowledge of underlying CS pathogenesis is currently available. 


\subsection{Subjects and Methods}

\subsubsection{Study participants}

All data reported here was collected after consent of the patient or the parents or legal guardians for individuals younger than 18 years old.

Participants were 35 individuals (14 males; 21 females) aged 1-69 years (due to age-dependency not every questionnaire was administered to all subjects). All participants had been molecularly diagnosed with CS either by Sangersequencing of the $A B C C 9$ gene or whole exome or genome sequencing. All study participants are part of the International Cantú Syndrome Registry (ICSR) and have been reported previously at least once (Grange et al., 2019). Patient demographics are provided in Table 1. Questionnaires were filled in during special annual Cantú syndrome research clinics at the Utrecht University Medical Center in the Netherlands $(n=13)$ and Washington University St. Louis, Missouri, USA $(n=22)$.

\subsubsection{Data collection}

Data were collected using three standardized questionnaires: the Kaufman Brief Intelligence Test 2 (KBIT-2) (A. S. Kaufman \& Kaufman, 2004), the Social Responsiveness Scale - Second Edition (SRS-2) (Constantino \& Gruber, 2012) and the Achenbach System of Empirically Based Assessment (ASEBA) (Achenbach \& Rescorla, 2001, 2003). All questionnaires were available in the native language of the patient or informant. Notably, not every individual completed every questionnaire. As not all questionnaires were introduced during the same CS research clinic and some individuals were not able to attend every annual clinic, not every form has been completed by every participating subject. The KBIT-2 has exclusively been filled in by the CS cohort at Washington University St. Louis. (Figure 1) As a result, the total number of individuals per questionnaire vary throughout the study. Additionally, a few subjects filled in the questionnaires over the span of multiple CS research clinics, hence their age deviates between the different assessments. Table 2 shows the type of questionnaires that have been completed by every participating individual per study site and the age at the time of examination. 

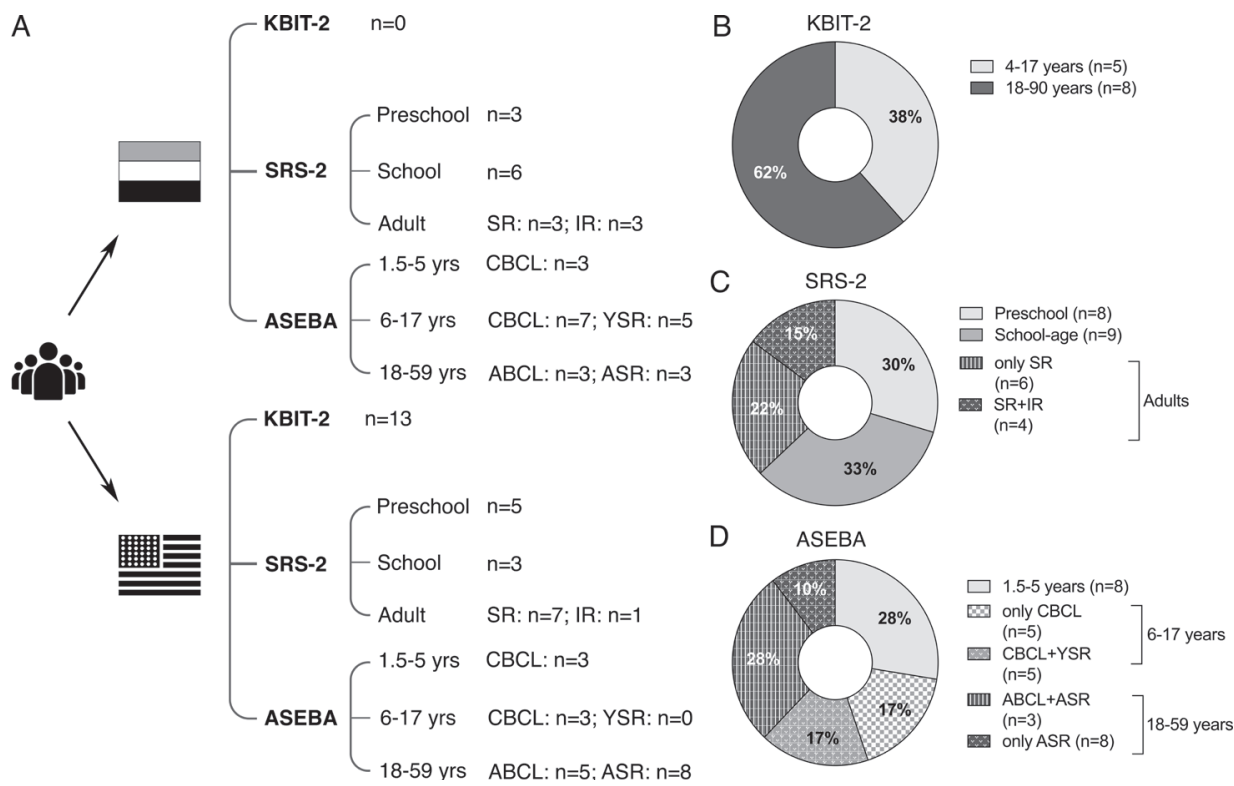

Figure 1 | (A) Overview indicating the number of completed KBIT-2, SRS-2 and ASEBA questionnaires (and their subtypes) in both participating countries. (B-D) Distribution of agerelated groups and questionnaire subtypes in this study.

ABCL: Adult Behavior Checklist, ASR: Adult Self-Report, CBCL: Children Behavior Checklist, IR: informant report, SR: self-report, YSR: Youth Self-Report.

\subsubsection{Kaufman Brief Intelligence Test 2: verbal and non-verbal intelligence}

The KBIT-2 was designed to measure verbal and non-verbal intelligence of individuals ranging from 4 to 90 years of age and consists of a verbal, non-verbal and IQ composite scale (add reference). The verbal scale contains two subtests which assess verbal knowledge (measuring receptive language and general information) and riddles (measuring verbal reasoning and comprehension), respectively. The nonverbal scale measures fluid intelligence (i.e. the ability to think logically and solve novel problems. The IQ composite measures general intelligence and is determined from an individual's performance on the verbaland nonverbal scales.

Raw scores were converted to standard scores. All standard scores have a mean of 100 for the general population, with a range from 40 to 160 . 


\subsubsection{Social Responsiveness Scale-2: social impairment intrinsic to ASD}

SRS-2 identifies impairment associated with Autism Spectrum Disorders (ASD) (Constantino \& Gruber, 2012). It is designed to identify social impairment intrinsic to ASD and quantify its severity across the lifespan. SRS-2 forms contain 65 items in a Likert-like (1-4) scale format with total scores ranging from 0 to 195; higher scores indicate higher degrees of social impairment. Depending on age of the individual, three SRS-2 questionnaires were used: preschool (2.5-4.5 years), school-age (5-17 years) and adult ( $\geq 18$ years). The first two questionnaires were filled in by the parent or other relative and address developmental milestones, the educational history of the child, family supports, preventive health monitoring, and socioeconomic status. SRS-2 questionnaires for adults included a self-report and an informant (e.g. spouse, relative) form. A total score, four treatment subscale scores (Social Awareness, Social Cognition, Social Communication, Social Motivation) and two indices (Social Communication Index (SCl), Restricted interests \& Repetitive Behaviors Index (RRBI) were obtained. Raw scores were converted in T-scores, with T-score $\leq 59$ indicating a normal degree of social responsiveness), T-score 60-65 indicating mild shortcomings in social responsiveness, T-scores 66-75 indicating moderate shortcomings in social responsiveness, and T-scores $\geq 76$ indicating serious shortcomings in social responsiveness.

\subsubsection{Achenbach System of Empirically Based Assessment: behavioral and emotional problems}

Additionally, CS subjects and their relatives were asked to complete ASEBA questionnaires. The ASEBA questionnaires are highly reliable and valid selfadministered measures of behavioral and emotional problems in children and adults (Achenbach \& Rescorla, 2001, 2003). Depending on the age of the patient, the forms had to be filled in by either a parent/spouse or the patient themselves (Achenbach \& Rescorla, 2001, 2003). All ASEBA questionnaires include two empirically-derived broadband scales representing internalizing and externalizing problems, eight empirically-derived syndrome scales representing different patterns of co-occurring emotional and behavioral problems, and six DSM-oriented scales derived through expert consensus. The latter are derived from the Diagnostic and Statistical Manual of Mental Disorders, 5th edition, which is the mental health field's predominant method of categorizing supposedly psychopathological conditions. Each DSM-oriented scale reflects a broad emotional or behavioral problem that corresponds to a broad DSM diagnostic category. Additionally, competence and adaptive functioning were assessed. For each profile, normalized T-scores $(X=50$, standard deviation $(S D)=10$ ) 
were assigned to total scores according to the percentiles for raw scores in a normative sample. The scores are set to discriminate between normal and clinical levels (Achenbach \& Rescorla, 2001, 2003). An overview of the applied age-related ASEBA questionnaires and associated scales and T-scores is shown in Table 7.

Table 7 | Overview of age-related ASEBA questionnaires and associated scales and T-scores.

\begin{tabular}{|c|c|c|c|}
\hline Age & $\begin{array}{l}\text { Type of } \\
\text { questionnaire }\end{array}$ & Scales included & $\begin{array}{l}\text { Range and associated } \\
\text { T-scores }\end{array}$ \\
\hline \multirow[t]{3}{*}{$1.5-5$ years } & $\mathrm{CBCL}$ & $\begin{array}{l}\text { Internalizing, externalizing and total } \\
\text { problems scale }\end{array}$ & $\begin{array}{l}\text { BR: T-score 60-63 } \\
\text { CR: T-score } \geq 64\end{array}$ \\
\hline & & Syndrome scale & $\begin{array}{l}\text { BR: T-score } 65-70 \\
\text { CR: T-score } \geq 70\end{array}$ \\
\hline & & DSM-oriented scale & $\begin{array}{l}\text { BR: T-score } 65-70 \\
\text { CR: T-score } \geq 70\end{array}$ \\
\hline \multirow[t]{3}{*}{$6-10$ years } & CBCL & $\begin{array}{l}\text { Internalizing, externalizing and total } \\
\text { problems scale }\end{array}$ & $\begin{array}{l}\text { BR: T-score } 60-63 \\
\text { CR: T-score } \geq 64\end{array}$ \\
\hline & & Syndrome scale & $\begin{array}{l}\text { BR: T-score } 65-70 \\
\text { CR: T-score } \geq 70\end{array}$ \\
\hline & & DSM-oriented scale & $\begin{array}{l}\text { BR: T-score } 65-70 \\
\text { CR: T-score } \geq 70\end{array}$ \\
\hline $11-17$ years & CBCL, YSR & $\begin{array}{l}\text { Competitive functioning scale } \\
\text { Internalizing, externalizing and total } \\
\text { problems scale } \\
\text { Syndrome scale } \\
\text { DSM-oriented scale }\end{array}$ & $\begin{array}{l}\text { BR: T-score } 30-35 \\
\text { CR: T-score } \leq 30\end{array}$ \\
\hline $18-59$ years & $A S R, A B C L$ & $\begin{array}{l}\text { Professional functioning scale } \\
\text { Internalizing, externalizing and total } \\
\text { problems scale } \\
\text { Syndrome scale } \\
\text { DSM-oriented scale }\end{array}$ & $\begin{array}{l}\text { BR: T-score } 30-35 \\
\text { CR: T-score } \leq 30\end{array}$ \\
\hline
\end{tabular}

Abbreviations: ABCL: Adult Behavior Checklist, ASR: Adult Self-Report, BR: Borderline range, CBCL: Children Behavior Checklist, CR: Clinical range, YSR: Youth Self-Report

\subsubsection{Data analysis}

Descriptive statistics were applied to describe the data. Standard error of mean was calculated using Prism (GraphPad).

No statistical tests have been performed due to low patient numbers. 
Behavioral and cognitive functioning in individuals with Cantú syndrome 
-12
4
4
4

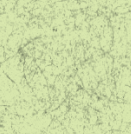

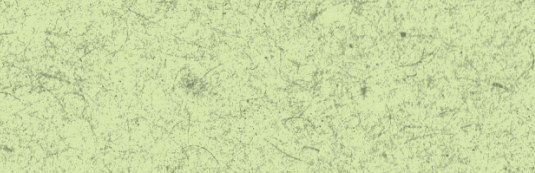

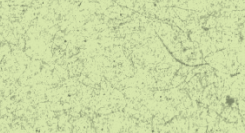

तr.

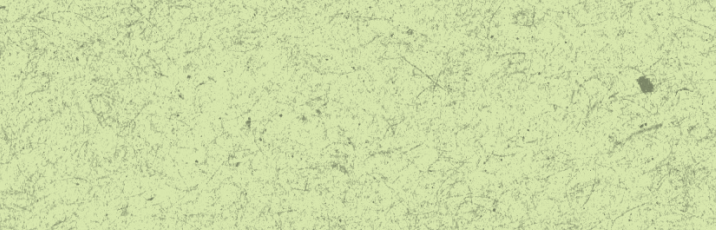

4

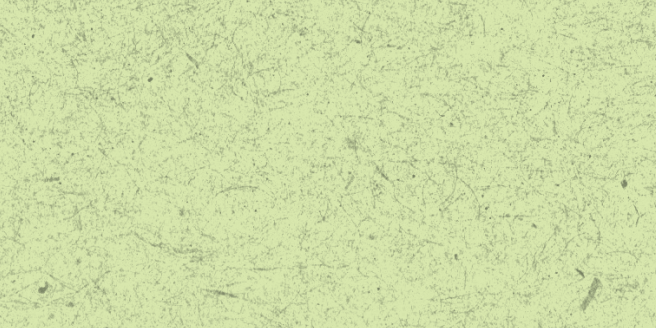




\section{5 Three-dimensional facial morphology in Cantú syndrome}

Helen I. Roessler, Kathleen Shields, Dorothy K. Grange, Nine V.A.M. Knoers, Gijs van Haaften, Peter Hammond*, Mieke M. van Haelst*

*These authors contributed equally to this work.

Am J Med Genet A. 2020 May;182 (5):1041-1052

DOI: 10.1002 /ajmg.a.61517. 


\section{Abstract}

Cantú syndrome (CS) was first described in 1982, and is caused by pathogenic variants in ABCC9 and KCNJ8 encoding regulatory and pore forming subunits of ATP-sensitive potassium $\left(\mathrm{K}_{\mathrm{ATP}}\right.$ ) channels, respectively. It is characterized by congenital hypertrichosis, osteochondrodysplasia, extensive cardiovascular abnormalities and distinctive facial anomalies including a broad nasal bridge, long philtrum, epicanthal folds, and prominent lips. Many genetic syndromes, such as CS, involve facial anomalies that serve as a significant clue in the initial identification of the respective disorder before clinical or molecular diagnosis are undertaken. However, an overwhelming number of CS patients receive misdiagnoses based on an evaluation of coarse facial features. By analyzing three-dimensional images of CS faces, we quantified facial dysmorphology in a cohort of both male and female CS patients with confirmed ABCC9 variants. Morphometric analysis of different regions of the face revealed genderspecific significant differences in face shape. Moreover, we show that 3D facial photographs can distinguish between CS and other genetic disorders with specific facial dysmorphologies that have been mistaken for CS-associated anomalies in the past, hence assisting in an earlier clinical and molecular diagnosis. This optimizes genetic counseling and reduces stress for patients and parents by avoiding unnecessary misdiagnosis. 


\subsection{Introduction}

Cantú syndrome (CS; OMIM \#239850) is a rare autosomal dominant condition characterized by a wide constellation of clinical features, namely coarse facial features, congenital hypertrichosis, osteochondrodysplasia and extensive cardiovascular anomalies including cardiomegaly, patent ductus arteriosus (PDA), pericardial effusion and dilated and torturous cerebral blood vessels (Grange et al., 2006; Grange et al., 2019; Leon Guerrero et al., 2016; Scurr et al., 2011). CS is caused by gain-of-function (GoF) pathogenic variants in ABCC9 and, less commonly, in KCNJ8, which encode the regulatory (SUR2) and poreforming (Kir6.1) subunits, respectively, of ATP-sensitive potassium ( $\left.\mathrm{K}_{\text {ATP }}\right)$ channels (Brownstein et al., 2013; Cooper et al., 2014; Harakalova et al., 2012; McClenaghan et al., 2017; van Bon et al., 2012). Notably, a majority of cases are considered to harbor de novo variants.

Remarkably, there is considerable variation in the phenotypic spectrum, even within family members sharing the same ABCC9 variant (Roessler, VolkerTouw, Terhal, van Haaften, \& van Haelst, 2018). The underlying reason for this heterogeneous clinical presentation is still unclear.

Dysmorphic facial features, on the other hand, are observed in every CS patient and are usually evident at birth. In younger patients, features include a low frontal hairline, epicanthal folds, flat nasal bridge, anteverted nares, long philtrum, macroglossia, prominent mouth, and thick lips. Additionally, the palate may be high arched and/or narrow, the gingiva may be thickened and an anterior open bite may be present (Grange et al., 2019). With advancing age, however, these clinical features are subject to considerable phenotypic changes. Whereas the initial facial shape appears round with full cheeks, the facial phenotype progresses to a lengthening of the face with a pointed chin and a more prominent forehead (Scurr et al., 2011). Progressive coarsening of the face results in further flattening of the nasal bridge with prominent supra-orbital ridges and fuller lips. The small nose becomes more bulbous. Periorbital fullness seems to be consistent (Roessler et al., 2018).

Despite the identification of $A B C C 9$ and KCNJ8 pathogenic variants as being causal, an explanation for variability of CS-associated features remains unknown. The correlation between GoF mutations in $\mathrm{K}_{\text {ATP }}$ channels and the coarse facial appearance is currently still not understood. Additionally, in a recent report describing 74 patients in the International Cantú Syndrome Registry 
(ICSR) we were unable to correlate genotype to specific phenotypic features in CS individuals including facial appearance (Grange et al., 2019).

Facial dysmorphism plays an important role in the clinical diagnosis of genetic conditions such as CS since it often presents as a preliminary clue before clinical examination and molecular genotyping are undertaken. Since the genes associated with CS have only recently been identified and not all patients have had adequate genetic counseling and investigation, we suspect that there is still a large number of undiagnosed or misdiagnosed cases. Additionally, the low incidence of CS limits exposure during training of clinical geneticists and inhibits the development of skills in recognizing the facial features characteristic of this syndrome. An overwhelming number of CS patients initially received other diagnoses, often based on coarse facial features with mucopolysacharidosis being most common; an unnecessary and stressful process for parents (Grange et al., 2019). Hence, quantitative analysis of the described facial morphology is of vital importance in characterizing the phenotype, which will assist in achieving earlier clinical and molecular diagnosis and thus optimizing appropriate medical care and genetic counseling.

Three-dimensional (3D) surface imaging systems have already been successfully applied in a range of clinical situations such as burns (Kovacs et al., 2005), dermatology (Ardehali et al., 2007) and orthodontics (Hajeer, Millett, Ayoub, \& Siebert, 2004). Rapid, noninvasive 3D imaging enables the face to be viewed from any angle and at closer proximity than most children, or even adults, would tolerate with a human observer. Consequently, 3D face analysis using dense surface modeling (DSM) has proven successful in delineating facial morphology in Williams syndrome (WS; OMIM \# 194050) (Hammond \& Suttie, 2012), Noonan syndrome (NS; OMIM \#163950) (Hammond, 2007), Wolf-Hirschhorn syndrome (WHS; OMIM \#194190) (Hammond \& Suttie, 2012) and Smith-Magenis syndrome (SMS; OMIM \#182290) (Tomona, Smith, Guadagnini, \& Hart, 2006), establishing accurate discriminating characteristics or assisting the determination of phenotype-genotype correlations. In addition, computer-based models of 3D facial morphology can assist in dysmorphology training (Hammond, 2007).

In the present study, we investigated the effect of CS-associated mutations in ABCC9 on craniofacial structures using DSM based analysis of 3D photographs of 20 clinically affected and molecularly proven CS patients. Additionally, we compared facial dysmorphism in CS with genetic disorders that can be a differential diagnosis of CS because of overlapping facial features. Our study 
assembles the second largest cohort of CS patients studied so far, considering the overall rareness of the disorder (Grange et al., 2019).

We demonstrate that DSM-based analysis provides a very accurate instrument to classify faces or facial regions along the CS-control spectrum. In addition, we highlight specific facial differences between CS and genetic disorders with similar dysmorphisms to improve initial diagnosis of these patients. As a result, our findings demonstrate the utility of $3 \mathrm{D}$ imaging during a genetic diagnostic process.

\subsection{Results}

We present 3D imaging analysis in 20 CS patients. All patients have confirmed genetic variants in ABCC9 (Figure S2); in 17/20 patients this variant is de novo. Information regarding genotype and general features of all 20 patients is provided in Table 1. Clinical phenotype among patients was similar to those reported in previous studies. Table $\mathbf{S} 1$ lists major clinical features of CS found in the study participants. All patients are part of the ICSR (Grange et al., 2019) and thus have been published at least once before. Patient study numbers applied in this study can be traced back to the ICSR published by Grange et al. (2019), which documents clinical features of each patient in detail.

\subsubsection{Patient demographics}

The 20 patients (10 females and 10 males) range in age from 2.7 to 22.0 years (mean: 11.46 years) (Table 1 ). Patients were recruited by two clinical genetic centers, in the Netherlands and the United States. All participants are Caucasian and most (17/20) identify as non-Spanish/Hispanic/Latino, reflecting the location of the participating sites. 
Patient

\begin{tabular}{|c|c|c|c|c|c|c|c|c|c|c|}
\hline 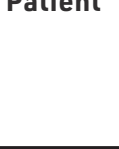 & 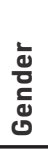 & 芩 & 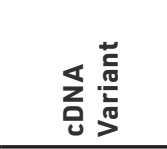 & . & 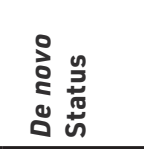 & 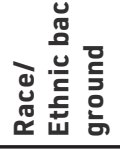 & 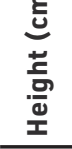 & 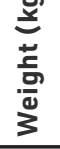 & 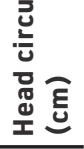 & $\frac{0}{3}$ \\
\hline CSO001 & M & 16 & c. $3460 \mathrm{C}>\mathrm{T}$ & p.Arg1154Trp & de novo & $C$ & 152 & 45 & 56 & 1,2 \\
\hline CSO002 & $\mathrm{F}$ & 21 & c. $3461 \mathrm{G}>\mathrm{A}$ & p.Arg1154Gln & de novo & $\mathrm{C}$ & 157 & 44 & 58 & $1,2,3$ \\
\hline CSO005 & $\mathrm{F}$ & 22 & c. $3460 \mathrm{C}>\mathrm{T}$ & p.Arg1154Trp & $\begin{array}{l}\text { inherited } \\
\text { from } \\
\text { affected } \\
\text { mother }\end{array}$ & $\mathrm{C}$ & 157 & 68 & 56 & 1,2 \\
\hline CS0011 & M & 11 & c. $3347 \mathrm{G}>\mathrm{A}$ & p.Arg1116His & de novo & C & 150 & 47 & 58 & 1,2 \\
\hline CS0013 & $M$ & 2 & c. $4468 \mathrm{~T}>\mathrm{A}$ & p.Val1490Glu & de novo & $\mathrm{C} / \mathrm{H}$ & 107 & 18 & 53 & 1 \\
\hline CS0016 & $\mathrm{F}$ & 3 & c. $2378 \mathrm{~A}>\mathrm{T}$ & p.Asp793Val & de novo & $\mathrm{C}$ & 74 & 10 & 51 & 1 \\
\hline CS0017 & $\mathrm{F}$ & 3 & c. $3014 A>T$ & p.His1005Leu & de novo & $\mathrm{C} / \mathrm{H}$ & 71 & 11 & 48 & 1 \\
\hline CSO020 & M & 20 & c. $3461 \mathrm{G}>\mathrm{A}$ & p.Arg1154Gln & de novo & C & 193 & 69 & 56 & 1 \\
\hline CSO024 & $M$ & 5 & c. $4040 G>T$ & p.Arg1347Leu & de novo & $C$ & 114 & 20 & 53 & 1 \\
\hline CS0028 & $\mathrm{F}$ & 5 & c. $3605 C>T$ & p.Thr1202Met & de novo & $\mathrm{C} / \mathrm{H}$ & 114 & 20 & 53 & 1 \\
\hline CS2001 & $\mathrm{F}$ & 11 & c. 1295 C>T & p.Pro432Leu & de novo & $\mathrm{C}$ & 138 & 33 & na & 1,4 \\
\hline CS2003 & $M$ & 3 & c. $1433 \mathrm{C}>\mathrm{T}$ & p.Ala478Val & $\begin{array}{l}\text { inherited } \\
\text { from } \\
\text { affected } \\
\text { father }\end{array}$ & C & 69 & 15 & 53 & 1,5 \\
\hline CS2004 & M & 12 & c. $178 \mathrm{C}>\mathrm{T}$ & p.His60Tyr & de novo & C & 137 & 36 & na & 1 \\
\hline CS2005 & M & 17 & c. $3460 \mathrm{C}>\mathrm{T}$ & p.Arg1154Trp & de novo & C & na & 50 & na & 1 \\
\hline CS2008 & $\mathrm{F}$ & 15 & c. $3346 C>G$ & p.Arg1154Gly & de novo & $\mathrm{C}$ & na & na & na & 1 \\
\hline CS2009 & F & 20 & c. $3461 \mathrm{G}>\mathrm{A}$ & p.Arg1154Gln & de novo & C & na & na & na & 1 \\
\hline CS2010 & $\mathrm{F}$ & 17 & c. $3460 \mathrm{C}>\mathrm{T}$ & p.Arg1154Trp & de novo & C & 183 & 57 & 57 & 1 \\
\hline CS2012 & $M$ & 10 & c. $3347 \mathrm{G}>\mathrm{A}$ & p.Arg1116His & $\begin{array}{l}\text { inherited } \\
\text { from } \\
\text { affected } \\
\text { mother }\end{array}$ & C & na & na & na & $1,4,5$ \\
\hline CS2013 & $\mathrm{F}$ & 10 & c. $3460 \mathrm{C}>\mathrm{T}$ & p.Arg1154Trp & de novo & $\mathrm{C}$ & 127 & 26 & na & 1,4 \\
\hline CS2014 & M & 3 & c. $3461 \mathrm{G}>\mathrm{A}$ & p.Arg1154Gln & de novo & C & 91 & 15 & 51 & 1 \\
\hline
\end{tabular}

Abbreviations: C, Caucasian; H, Hispanic; na, not assessed

${ }^{1}$ Grange et al (2019) Am J Med Genet; ${ }^{2}$ Leon-Guerrero et al (2016) Neurology; ${ }^{3}$ Grange et al (2006) Am J Med Genet; ${ }^{4}$ Harakalova et al (2012) Nat. Gen; ${ }^{5}$ Roessler et al (2018) Clin. Dysm. 


\subsubsection{Facial dysmorphology in CS}

Using software developed in house, a dense surface model (DSM) was constructed from the landmarked images including 20 CS patients, 272 individuals with a syndrome other than CS (203 age-matched) and 356 controls (145 age-matched). Approximately 25,000 points on each face were used in the dense correspondence. The corresponding DSM contained 40 principal components covering $99 \%$ of all shape variation.

Comparison of the mean faces of the CS and control group confirms many of the previously published facial features. In Figure 1, the mean face of the 20 CS patients (b) and an age-matched control (a) are shown in portrait and profile. A signature heat map of the mean CS face reflects location differences between corresponding points on the mean age-matched control along a surface normal (Figure 1c). Points on the CS mean face are shown in green if they are coincident with their corresponding points on the unaffected mean surface. They are colored red/blue if they are displaced inward/outward relative to the matched mean control surface on a -2 to +2 SD scale. The abundance of blue especially on the nose, lips and mandible of the mean CS face shows clear differences compared to controls. Signature heat maps of all 20 CS patients can be found in Figure 1d.

When compared with the age-matched control mean, the mean CS face shows general lengthening of the face, whereas width seems to be normal. The nasal bridge appears broader and the nasal tip turns upwards resulting in anteriorly facing nostrils. The philtrum length is increased and the lips show general thickening as well as concave curling of the upper lip. Additionally, epicanthic folds are present resulting from narrowing of both the supra-orbital arch and notch. Periorbital fullness is vaguely present. Notably, due to presence of eyelashes and wetness of eye surfaces, 3D camera devices often lead to image capture errors, such as holes in the surface or even spikes, or do not obtain an accurate surface around the eyes, at all.

All DSM analyses were performed for a reduced face patch since ears were not included during 3D image capture. However, from previous studies we know that no dysmorphologies of the ears are expected (Grange et al., 2019). 
(a)

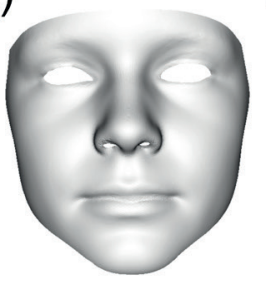

(b)

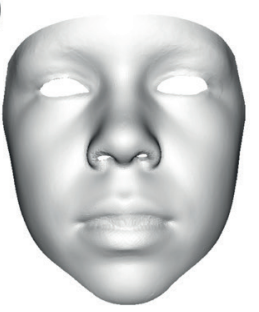

(c)
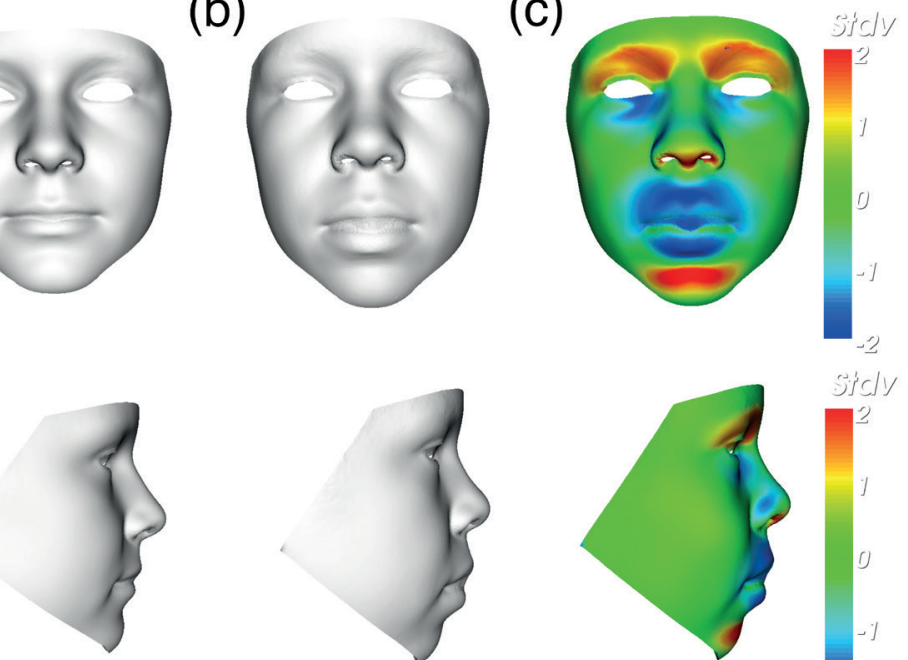

Control
Cantú syndrome
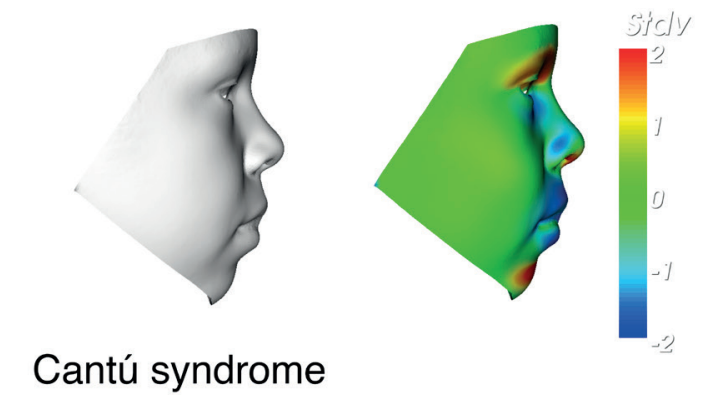

(d)

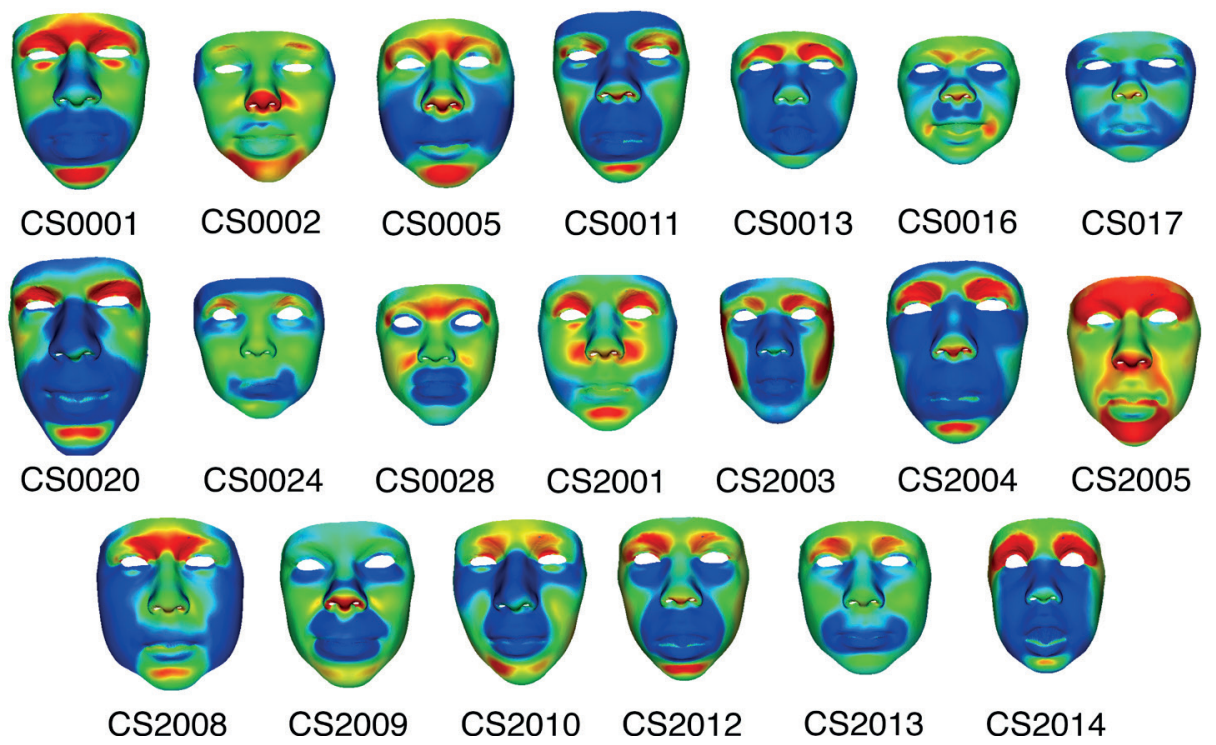

Figure 1 | Three-dimensional images of 20 Cantú syndrome patients. Portrait and profile of average faces of 20 individuals with Cantú syndrome (b) and 143 healthy controls (a) with same mean age. (c) Heat-mapped face signatures showing normalized face shape differences (displacement of surface points orthogonal to face surface and parallel to three axes) compared with matched control mean. The red/green/blue coloring reflects displacement orthogonal to the surface in the first signature (red/green/blue = contraction/coincidence/expansion) and in the three axial signatures parallel to the colored arrows with green = no displacement and maximal red-blue hues reflecting two SDs or more. (d) Heat mapped face asymmetry signatures of all investigated 20 Cantú patients. 


\subsubsection{Mean male and female faces and dynamic morphs}

Figures 2 and $\mathbf{3}$ show static portrait and profile views signature heat map comparisons between all CS males $(n=10)$ (Figure 2 ) or females $(n=10)$ (Figure 3) and an age- and sex-matched average of appropriate controls. Dynamic morphs of portrait and lateral views between the control and CS mean faces very clearly demonstrate the important differences in facial characteristics in both males and females (see Supplementary Movie S1 and S2).

When compared to the mean face of the male controls, the color-distance-coded comparison of the mean CS male suggests a longer and wider nose, a longer philtrum, retrognathia, fuller lips, a more bulbous nose, a more prominent and pointed chin and lifting of supra-orbital ridges (Figure 2a). By viewing the dynamic comparisons of the mean faces these face-shape differences are shown in a more dramatic manner. Additionally, a lengthening of the entire face becomes visible. Figure $\mathbf{2} \mathbf{b}$ shows the results of a double-tailed t-test comparison where this is appropriate. Statistically significant differences between male CS patients and age- and gender-matched controls confirm the wider nose $(p=.0003)$, longer philtrum $(p=.0004)$ and increased nose length $(p=.011)$, whereas there is no obvious increase in mouth width.

The static comparison (Figure 3a) as well as the dynamic morphs in the Supplementary Material show similar but less pronounced facial differences in the female cases. Whereas, patients show an upward/backward displacement of the nose resulting in anteriorly facing nostrils compared to the control group, both the lengthening of the face and therefore nose as well as thickening of the lips are less significant. Additionally, the chin seems less pointed. Both male and female individuals show no obvious increase in mouth width. The nose width $(p=.0003)$ is statistically significant when compared to age- and gendermatched controls, whereas philtrum length $(p=.032)$, nose length $(p=.325)$ and mouth width $(p=.092)$ are not (Figure $\mathbf{3 b})$.

In order to compensate for the wide age range (2-22 years) and the changes in morphometric analysis according to age, we additionally investigated differences in facial features in the following age- and gender-specific CS subgroups compared to respectively matched controls: Male subgroup 1 (age 2-5 years), male subgroup 2 (age 10-22 years), female subgroup 3 (age 2-5years) and female subgroup 4 (age 10-22 years). Signature heat map comparisons between all four subgroups and controls suggest that the pointiness of the chin develops at a later age (>10 years) whereas periorbital fullness decreases over time (Figure S4a-S7a). 
(a)
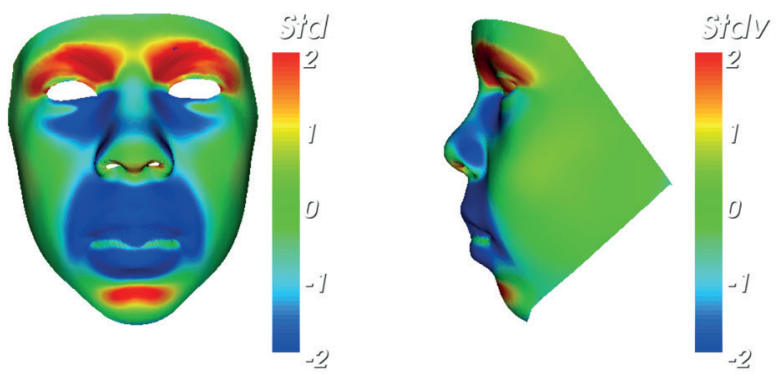

(b)

nasion to pronasale

\section{nose width}
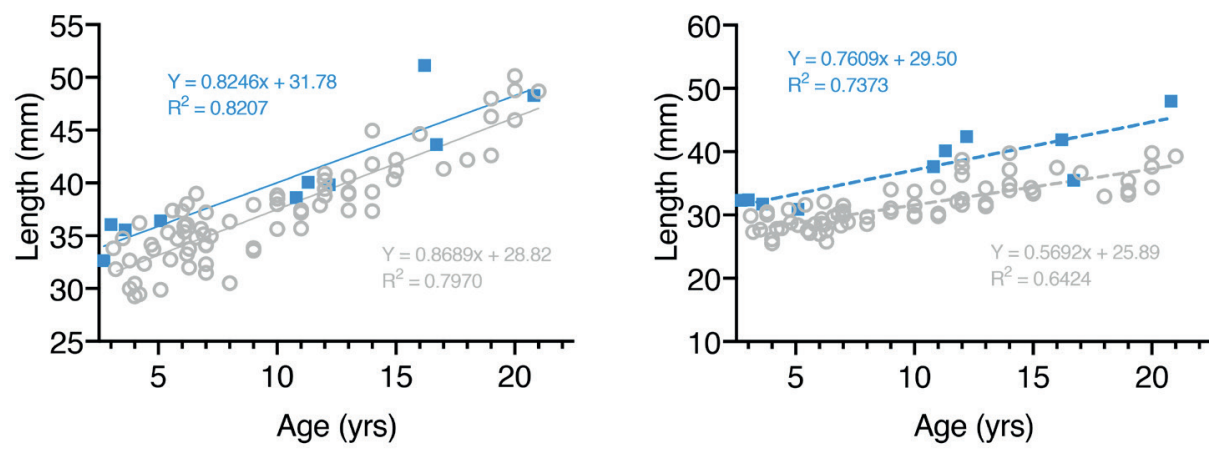

philtrum length
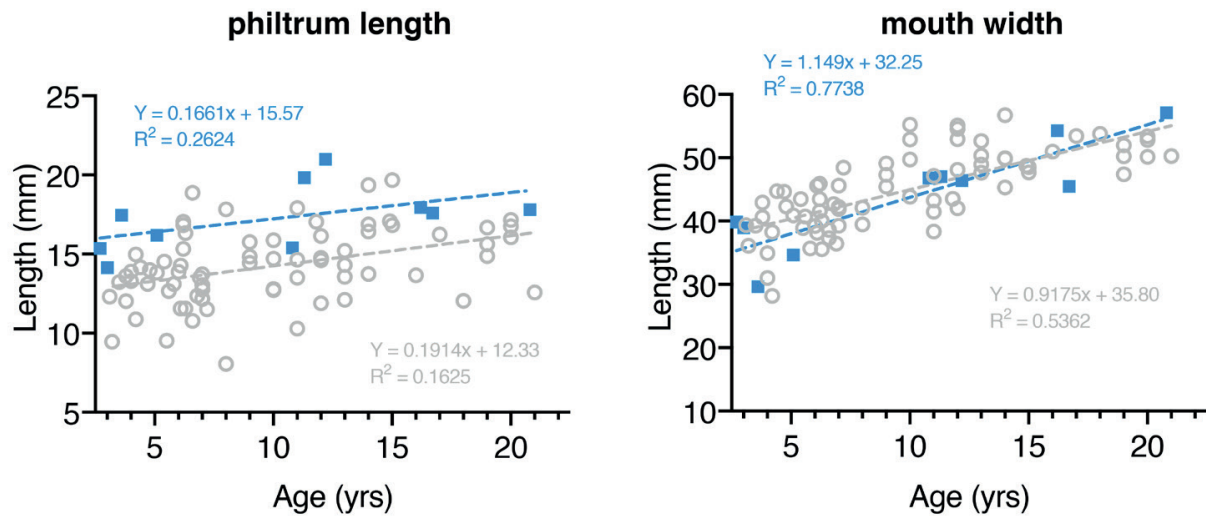

- Controls - CS

Figure 2 | Male specific facial abnormalities in CS patients. (a) Portrait and profile views of the color coded comparison of male CS patients with the average of the male control group ( $n=76$, age range: 3.1-21 years). The color scale of all depicted heatmaps is equal to Figure 1. (b) A scatter of age against different facial features is shown for a single DSM for a reduced face patch (no ears) for both age matched controls and male CS patients. Statistically significant differences between male CS patients and age and gender matched controls confirm a wider nose $(p=.0003)$, longer philtrum $(p=.0004)$ and increased nose length $(p=.011)$. DSM, dense surface model. 
(a)
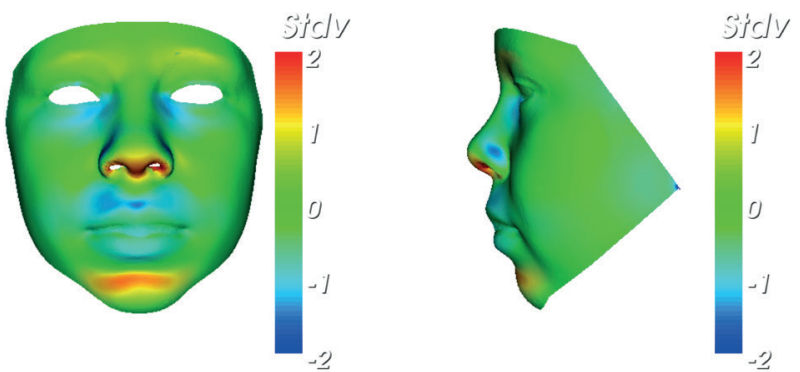

(b)

nasion to pronasale

nose width

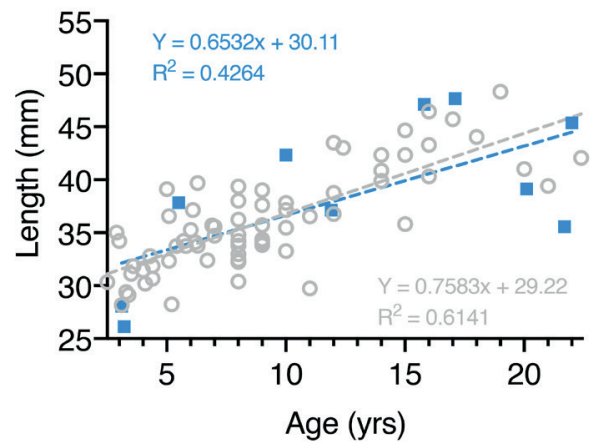

philtrum length
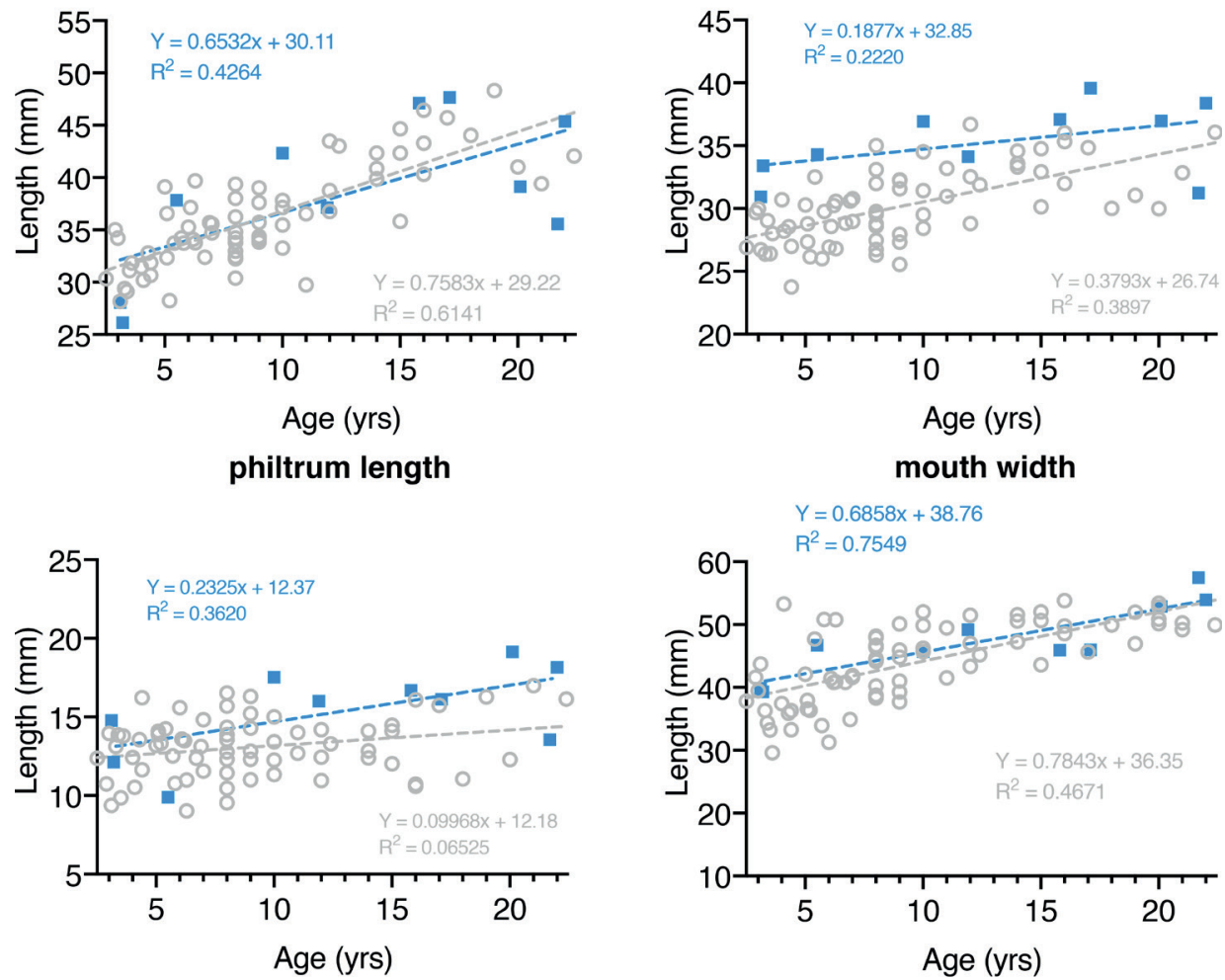

mouth width

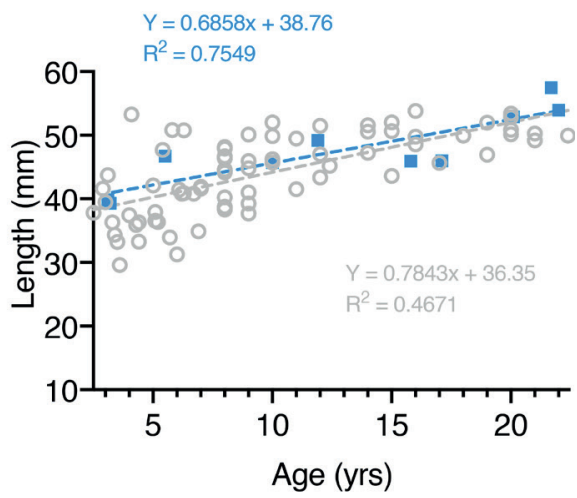

- - Controls - - CS

Figure 3 | Female specific facial abnormalities in CS patients. (a) Portrait and profile views of the color coded comparison of female CS patients with the average of the female control group ( $n$ $=69$, age range: $2.4-22.4$ years). The color scale of all depicted heatmaps is equal to Figure 1. (b) A scatter of age against different facial features is shown for a single DSM for a reduced face patch (no ears) for both age matched controls and female CS patients. Statistically significant differences between female CS patients and age and gender matched controls confirm a wider nose $(p=.0003)$. DSM: Dense surface model. 
Retrognathia, lifting of supra-orbital ridges and bulbousness of the nose develop early on. Whereas younger male CS patients show a significantly wider nose $(p=.0026)$, longer philtrum $(p=.0087)$ and increased nose length ( $p=.0079$ ) compared to healthy individuals, older male CS patients only reveal a statistically significant difference in nose width $(p=.0061)$ and philtrum length $(p=.0112)$ when compared with age- and gender-matched controls suggesting a slight decrease or at least stagnation in overall dysmorphism over age (Figure S4b and S5b). Similary, nose width of female CS patients in young age shows statistical significance $(p=.0101)$, but becomes more equal to healthy individuals in older age $(p=.079)$. Philtrum length, on the other hand, is only significantly increased in older female CS patients $(p=.0036)$ (Figure S6b and S7b). Notably, sample sizes in these subgroups are restricted and results should therefore only be seen as indications of progression of facial anomalies in CS.

\subsubsection{Facial growth and overall dysmorphism in CS}

In a face DSM with mixed age range, the first principal component (PC1) typically reflects growth and is highly correlated with age. Separate linear regression of PC1 against age for gender-specific control and CS subgroup (2-22 years) suggests face size is significantly greater in patients (males: $p<.0001$; female: $\mathrm{p}=.0325$ ) (Figure 4a). However, care must be taken in this interpretation as the retrognathia and open bite may exaggerate facial length in CS patients. On the other hand, a majority of CS patients show increased body size and macrocephaly at birth, hence an increased facial growth compared to controls is to be expected. Additionally, this reflects observations stated when comparing the average face of CS patients and controls (Figure $\mathbf{1} \mathbf{a}, \mathbf{b}$ ).

When calculating PC1 for all four age- and gender-specific subgroups facial growth is still significantly increased in both male subgroups (age range 2-5 years: $p=.0004$, age range $10-22$ years: $p=.001$ ), whereas facial growth seems to become more similar to healthy controls in female CS patients (age range 2-5 years: $p=.0713$, age range 10-22 years: $p=.0776)$ (Figure S8a,b).

The overall dysmorphism score was calculated as the square root of the sum of squared differences of displacements in the signature heat map comparison with the mean of age- and sex-matched controls. Firstly, this score suggests that facial dysmorphism does not increase much over time in CS patients (Figure 4b). However, a comparison between female and male patients shows significantly more dysmorphism across all ages in male patients $(p=.0151)$. Calculation of 
signature weight in all four age- and gender-specific subgroups confirms these observations (Figure S8c).

\section{(a) males \\ - Controls - CS \\ females}

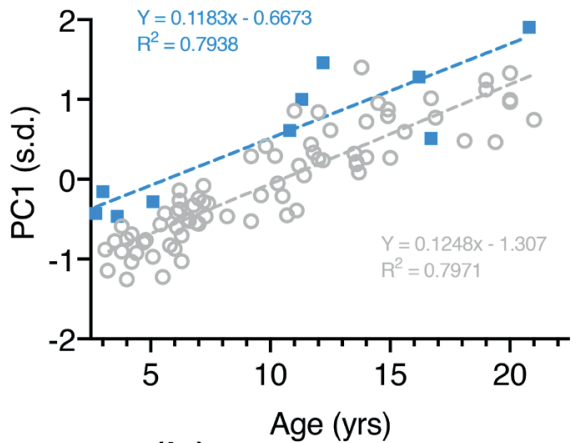

(b)

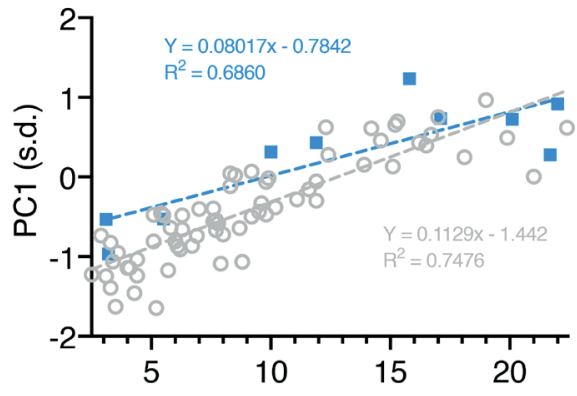

Age (yrs)

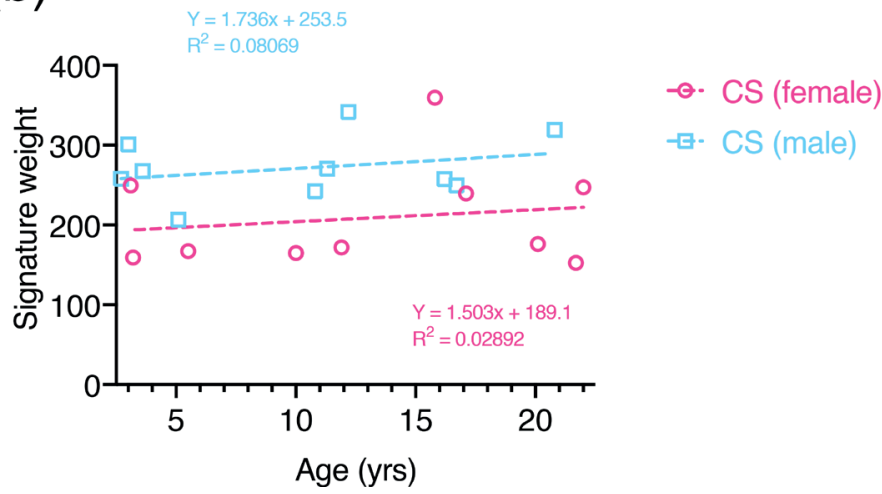

Figure 4 | Facial growth and overall dysmorphism in CS. (a) A scatter of age against PC1 is shown for a single DSM for a reduced face patch (no ears) for both the control and CS subgroups. The scatter is annotated by separate linear regression lines for each subgroup. PC1 reflects facial growth. Male control group includes $n=76$ individuals with an age range of 3.1-21.0 years; female control group includes $n=69$ subjects ranging from 2.5 to 22.4 years of age. (b) A scatter of age against signature weight is shown for a single DSM for a reduced face patch (no ears) for male and female CS subgroups. Signature weight is a rough estimate of facial dysmorphism. DSM, dense surface model; PCA, principal component.

\subsubsection{DSM analysis results in high levels of discrimination between CS and other disorders with similar facial dysmorphologies}

According to previous publications (Grange et al., 2019), many CS patients initially received other diagnoses, often based on coarse facial features. This has been extremely stressful for parents who were informed about a bad prognosis based on a wrong diagnosis. To evaluate whether $3 \mathrm{D}$ imaging analysis 
can be successfully applied to discriminate between disorders and thus avoid misdiagnoses, we compared facial features of CS patients with readily available cohorts of other genetic disorders with facial anomalies that have been mistaken with CS-related anomalies in the past; namely Fabry disease (FD) ( $n=28$ ) (CoxBrinkman et al., 2007), Rasopathies ( $n=33$ ) (Hammond et al., 2004), Williams syndrome (WS) ( $n=83)$ (Hammond \& Suttie, 2012) and Rubinstein-Taybi syndrome (RTS) ( $n=34$ ) (Menke et al., 2018). Notably, mucopolysacharidosis is the most common misdiagnosis in CS based on similar facial appearance (Grange et al., 2019), however there was no collection of 3D images from a patient cohort available for comparison. For this purpose, subgroups of individuals with other syndromes were selected in terms of age-range compatibility with the CS patients. Any individual's face can be compared to variation between the average face of two subgroups.

Figure 5 shows a simultaneous comparison along three axes: mean control to mean CS; mean FD to mean Rasopathy and mean RTS to mean WS. No overlap could be observed between the CS, FD and RTS subgroups, but CS does share small similarities with Rasopathy and WS patients.

Separate linear regressions of PC1 against age for CS and the remaining disorder subgroups suggest both delay and diminished rate of facial growth in all syndromes but CS. The difference in intercept was highly statistically significant (CS/Rasopathies and CS/RTS, $p<.0001$; CS/FD, $p=.0021$ ). When comparing CS to WS the slopes differ significantly $(p=.0014)$, but it was not possible to test whether the intercepts differ significantly (Figure S9). These results are to be expected considering the universal clinical observation of generalized macrosomia, with large birth weight and length, as well as persistent macrocephaly commonly reported in CS. In contrast, individuals with WS (Carrasco, Castillo, Aravena, Rothhammer, \& Aboitiz, 2005), FD (Colon et al., 2017) and Rasopathies (Tajan, Paccoud, Branka, Edouard, \& Yart, 2018) generally show low birth weight. Similar observations could be made when comparing age-specific CS subgroups (subgroup 1: age range 2-5, subgroup 2: age range 10-22) to age-matched disease cohorts (Figure S10).

Calculation of the overall dysmorphism score for each individual and separate linear regressions against age additionally suggest that facial dysmorphism increases with age in all disorders but CS. The difference in slope was highly statistically significant when comparing CS and Rasopathies ( $p=.0057)$. No statistical significance was found in all other cases (CS/WS, $p=.3$; CS/ RTS, $p=.0862$; CS/FD, $p=.2780$ ) (Figure S11). When separating CS patients 
in subgroups according to age, overall dysmorphism of young CS patients is significantly decreased compared to individuals with WS (Figure S12).

(a)

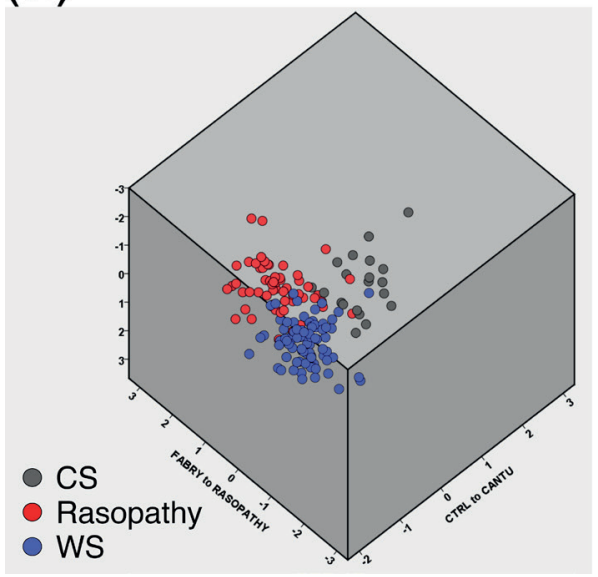

(b)

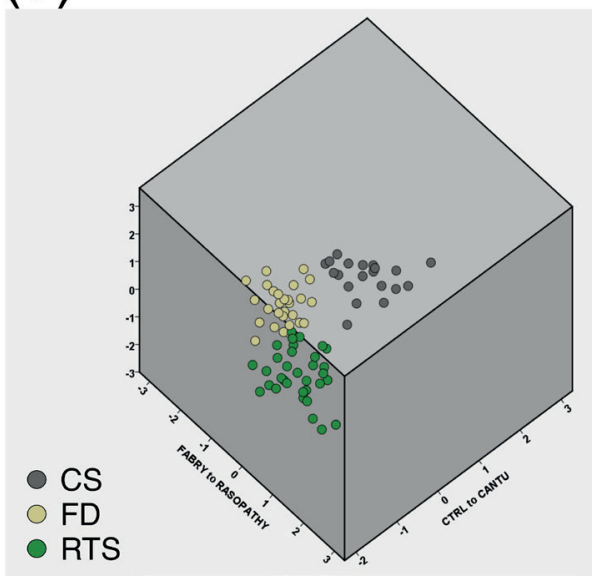

Figure 5 I Comparison of Mean Faces Along 3 Axes. Comparison between mean control to mean CS; mean FD to mean Rasopathy and mean RTS to mean WS. (a) CS, Rasopathy and WS are depicted. (b) CS, FD and RTS are depicted. Sample size, CS, $n=20$ (age range: $2.7-22.0$ years); Rasopathies, $\mathrm{n}=33$ (age range: $2.3-21.9$ years); WS, $\mathrm{n}=83$ (age range: $2.1-19.7$ years); RTS, $\mathrm{n}=59$ (age range: 2.0-21.6 years); $F D, n=28$ (age range: $3.4-22.8$ years). CS, Cantú syndrome; FD, Fabry disease; RTS, Rubinstein-Taybi syndrome; WS, Williams syndrome.

\subsection{Discussion}

In this study, facial dysmorphism in patients with CS is objectively assessed by $3 \mathrm{D}$ dense surface modeling and anthropometric analysis, revealing facial dysmorphic features in male and female patients. The most prominent facial anomalies shared by both genders are a broadened nasal bridge and wider nose, narrow supra-orbital arches, epicanthal folds, a longer philtrum, thick lips, and retrognathia. Male patients additionally reveal lengthening of the face and nose which is less pronounced in females who, on the other hand, show an upward and backward displacement of the nose, anteverted nares, and lifting of supra-orbital ridges. The majority of all CS-related facial features seem to develop early on. Merely, the pointed chin and the longer philtrum in female patients are only revealed in older age. Facial anomalies tend to remain until the age of at least 22 with only the nose width of female CS patients normalizing after childhood. 
Face size and growth in CS patients, interpreted by PC1 in the model, is always greater compared to age-matched controls. Overall facial dysmorphism, is significantly higher in male CS patients compared to females but unlike the other syndromes investigated, it barely increases or even stagnates with age in all studied subgroups. Notably, we would like to point out that all analysis performed in the four age- and gender-specific CS subgroups can merely be seen as an indication of facial development in CS since the subject numbers are small (3-7 patients) and therefore miss statistical robustness. Thus, we strongly suggest to primarily focus on data aquired from CS subgroups containing all male or female patients as shown in Figures $\mathbf{2}$ and 3. In order to properly assess changes in facial features in different CS age groups one would require a cohort with significantly more subjects.

Our results are mostly in line with the features described in various clinical reports that assessed facial characteristics of CS patients based on medical photography (Roessler et al., 2018; Scurr et al., 2011). However, increase in mouth width which has been previously reported in the literature (Grange et al., 2014; Roessler et al., 2018) was not observed applying 3D image analysis. Hence, we demonstrate that 3D imaging recognizes common facial anomalies observed in CS patients. Since the results generated in this study are produced by objective quantitative analysis and are thus not hampered by inter-observer variability, the applied approach enables a more reliable assessment and quantification of facial anomalies observed in CS patients. Moreover, we include comparison of both male and female CS patients with respective controls as well as evaluation of facial growth and overall dysmorphism.

Notably, our study cohort only includes Caucasians, originating from both the Netherlands and the United States with an age range of 2.7-22.0 years, which resembles the overall patient demographic reported in literature so far (Grange et al., 2019). Even though all applied control subjects in this study are also Caucasian, they originate from two European populations of close phylogenetic and geographic proximity, the UK and The Netherlands (Figure S2). Even though previous studies have shown facial differences in different adult Caucasian populations (Hopman, Merks, Suttie, Hennekam, \& Hammond, 2014), we do not expect these to be present in younger individuals.

The uneven age distribution of patients, which can be observed in our CS cohort, might be seen as a limitation when making simple anthropometric comparisons with controls but for shape specific considerations like face signature each 
individual is normalized against a sex-and-age matched set of controls which is more reliable.

It would be of interest to perform a similar study in CS patients with an increased age as well as from non-European ancestry, which would require a larger cohort as is identified currently. Additionally, 3D face morphometry of CS patients could be compared to healthy siblings as similarity and variability between healthy and diseased siblings might highlight the real face morphometry of the disorder. This, however, would require capturing 3D images of healthy siblings of the same sex and a similar age which might be a challenge given the overall rarity of CS.

Another interesting prospect of 3D face shape models is the appliance in genotype-phenotype studies. So far, no obvious differences in frequency or degree of craniofacial dysmorphology, hypertrichosis, or skin appearance depending on the site of the ABCC9 variant could be observed in CS individuals. Also, cardiovascular and neurological anomalies were similar across the ABCC 9 variant locations (Grange et al., 2019). Even within families containing multiple affected members, there is highly variable expression of CS features, comparable with other autosomal dominant conditions (Grange et al., 2019; Roessler et al., 2018). 3D imaging technologies offer a way to investigate genotype-phenotype correlations in terms of facial features. However, one would have to increase cohort size in order to a perform reliable analysis.

Notably, it would be interesting to acquire and analyze 3D facial photographs of patients harboring a variant in KCNJ8 because they may potentially have phenotypic differences that can only be distinguished by quantitative evaluation instead of by eye. However, since at the moment only two patients with a CSassociated variant in KCNJ8 are known (Brownstein et al., 2013; Cooper et al., 2014) this would be of interest once a larger cohort has been acquired in the future. Notably, it is speculated that such variants might frequently be lethal (Grange et al., 2019).

Clinical geneticists often refer to facial differences and features that offer clues to diagnosis. Articulating those differences can be challenging, especially if an individual's face shows a mild facial phenotype or if morphological characteristics are manifested in only part of the face. These complications can lead to misdiagnosis of patients. According to a recent report on 74 patients from the ICSR, $41 \%$ of participating CS patients initially received other diagnoses based on various primarily assessed features, including a lysosomal storage disorder such as mucopolysaccharidosis or Pompe disease based on neonatal 
cardiomegaly (Grange et al., 2019). The majority of misdiagnoses were based on facial dysmorphology and hypertrichosis and could however not be confirmed by additional genetic of metabolic testing. Since these misdiagnoses implicate a completely different prognosis and life expectancy, earlier CS face recognition could prevent unnecessary stress for parents.

By comparing facial characteristics of CS with patient cohorts whose facial anomalies have been mistaken with CS in the past we demonstrate that $3 \mathrm{D}$ imaging analysis can be successfully applied to delineate between syndromes and thus avoid further misdiagnoses. It would be of interest to perform a similar comparison between CS and MPS patients in the future. Notably, congenital hypertrichosis, also observed in all CS patients (Grange et al., 2019), can sometimes be applied as clear discriminator between CS and other mentioned syndromes, if severe enough.

Most importantly, the described 3D imaging technique reveals multiple advantages including practicality, availability and low costs. 3D cameras and appropriate computer software for analysis are available in every clinic that makes assessing facial morphology via 3D imaging quick and cheap. Hence, the implementation of this in general practice for clinical diagnosis of CS patients but also other disorders with a facial component is feasible.

Recent innovations based on 2D technologies combined with computer vision and deep learning algorithms also hold promise to be worthwhile in diagnosis of genetic disorders with facial components (Gurovich et al., 2019; van der Donk et al., 2019). Although 2D images are more readily available, they are subject to greater variation in quality and pose due to projection distortion, and more sophisticated analysis is required to extract the discriminating features. Thus, a significantly increased number of images is required to train the pattern recognition algorithms. Therefore, 2D technologies may represent a considerable alternative in clinics to successfully diagnose CS and other genetic disorders with facial anomalies in the future, but it still remains to be seen which approach has more potential to be established in a stable fashion. 


\subsection{Conclusion}

In conclusion, we report a valid method for quantification of facial dysmorphic features in CS. Although facial dysmorphism in CS can be clinically recognizable, an objective, quantitative evaluation is especially valuable when assessing phenotypically or genotypically unusual cases.

The genes associated with CS have only recently been identified. Hence, we suspect there may be more affected individuals-particularly older patientswho have not received genetic testing and therefore remain undiagnosed. We demonstrate that 3D imaging analysis can be confidently applied to unravel characteristic facial features of CS and successfully discriminate between the syndrome and other genetic disorders it has been confused with in the past due to facial similarities. This will help in diminishing parental anxiety related to misdiagnoses.

In the future, 3D-based analysis will enable the recognition of CS facial characteristics in patients who have not had an adequate clinical diagnosis. Subsequent molecular analysis of such patients may identify mutations and in combination with detailed facial analysis enable unequivocal identification of causative genes. Considering the current efforts to develop a pharmacological treatment for CS (Ma et al., 2019; McClenaghan et al., 2019), 3D technology additionally may enable the evaluation and quantitative measurement of effective response to therapy options in the future which will possibly lead to a decrease in coarseness of the face. 


\subsection{Subjects and Methods}

\subsubsection{Study participants}

All data reported here were collected after informed written consent of the patient or the parents or legal guardians for patients younger than 18 years old.

The CS patients group comprised 10 male patients $2.7-16.2$ years old and 10 female patients 3.1-22.0 years old. Figure S1 shows distribution of country of origin over age in CS patients. All patients had been molecularly diagnosed with CS either by Sanger-sequencing of the ABCC9 gene or whole exome sequencing. All study participants are part of the ICSR and have been reported previously at least once (Grange et al., 2019). 3D face images of individuals with CS were collected during special annual Cantú syndrome research clinics at the Utrecht University Medical Center, the Netherlands, and at Washington University, St. Louis, MO. The accuracy of such 3D imaging devices has been shown to be highly reliable (Aldridge, Boyadjiev, Capone, DeLeon, \& Richtsmeier, 2005; Camison et al., 2018). Some images were unusable because of incomplete face coverage or poor subject cooperation. Details of the specific ABCC 9 variants and body weight and height were obtained from the ICSR (Grange et al., 2019).

Images of healthy subjects as well as patients diagnosed with Williams syndrome (WS), Fabry disease (FD), Rubinstein-Taybi syndrome (RTS) and Rasopathies were selected from an existing collection. Dense surface models (DSM) were constructed with the following number of individuals to get the fullest face shape variation possible: Healthy controls included 183 unrelated male patients 0.2-66.5 years old and 173 unrelated female patients $0.3-62.2$ years old; WS cohort included 51 unrelated male patients 1-17 years old and 41 unrelated female patients 1.1-19.7 years old; FD cohort included 30 unrelated male patients 3.4-64.4 years old and 38 unrelated female patients 5.8-71.5 years old; RTS cohort included 18 unrelated male patients 2-31 years old and 23 unrelated female patients 1.2-31 years old; Rasopathies cohort included 31 unrelated male patients 1.7-26.4 years old and 40 unrelated female patients 0.9-47.6 years old. However, only age- and sex-compatible subgroups were used during analysis.

For comparison with all male and female CS patients the sample size and age range was as follows: healthy male controls, $n=76$ (age range: 3.1-21.0 years); healthy female controls, $n=69$ (age range: $2.4-22.4$ years old); male WS patients, $n=45$ (age range: $2.1-17$ years); female WS patients, $n=38$ (age range: 2.3-19.7 years); male FD patients, $n=10$ (age range: $3.4-22.7$ years); female FD patients, $\mathrm{n}=18$ (age range: $5.8-22.8$ years); male RTS patients, $\mathrm{n}=15$ (age 
range: $2.0-17.9$ years); female RTS patients, $n=19$ (age range: $2.4-21.6$ years); male Rasopathies patients, $n=15$ (age range: 2.4-22.4 years); female Rasopathies patients, $n=18$ (age range: 2.3-21.9 years old). For comparison with age-specific male and female CS subgroups the sample size and age range was as follows: male CS subgroups, $\mathrm{n}=4$ (age range: $2.7-5.1$ years); $\mathrm{n}=6$ (age range: $10.8-20.8$ years); female CS subgroups, $n=3$ (age range: $3.1-5.5$ years); $\mathrm{n}=7$ (age range: 10.0-22.0 years); male healthy controls, $\mathrm{n}=17$ (age range: $3.1-5.8$ years); $n=36$ (age range: $10.0-21.0$ years); female healthy controls, $n$ $=20$ (age range: $2.5-5.8$ years); $n=25$ (age range: $10.0-22.4$ years); $W S, n=26$ (age range: $2.1-5.9$ years), $n=35$ (age range: $10.5-19.7$ years); $F D, n=3$ (age range: $3.4-5.8$ years), $\mathrm{n}=19$ (age range: $10.1-22.8$ years); RTS, $\mathrm{n}=11$ (age range: $2.0-5.5$ years), $n=12$ (age range: 10.2-21.6 years); Rasopathies, $\mathrm{n}=18$ (age range: $2.3-5.8$ years), $\mathrm{n}=28$ (age range: $10.5-22.4$ years).

All individuals used in this study are Caucasian but from different countries of origin. Figure $\mathbf{S 2}$ shows distribution of country of origin of controls and patients with another disorder used in this study.

Notably, the mutation profile of all age-matched rasopathies patients is as follows: HRAS $(n=12), \operatorname{BRAF}(n=11)$, PTNP11 $(n=6)$, MEK1 $(n=2)$, KRAS $(n=1)$, sos1 $(n=1)$.

\subsubsection{D image capture and preparation}

A total of 203 D face images of individuals with CS were captured with commercial photogrammetric devices (3dMDface System [3dMD Inc.] and Canfield Vectra 3D system [Canfield Scientific Inc.]). All images were manually annotated by a single operator $(\mathrm{PH})$ with 22 facial landmarks previously shown to be accurate and reproducible (Toma, Zhurov, Playle, Ong, \& Richmond, 2009).

\subsubsection{DSM building and closest mean classification}

A dense surface model of a set of landmarked face surfaces, described in detail elsewhere (Hammond, 2007; Hutton, Buxton, Hammond, \& Potts, 2003), comprises shape variation modes arising from a principal component analysis (PCA) of differences of the surface points' positions from those of a comparator average face. Prior to the PCA, using a base mesh (whole face or patch) and aligned sparse landmarks, a dense correspondence of surface points across all faces is induced with no manual interaction. The proportion of face shape variance covered by each DSM mode is computed, and typically the modes are ordered in terms of decreasing variance coverage. For a DSM of a collection of faces of children and adults, the first mode typically reflects facial growth and 
correlates strongly with age (Hammond, 2007). We retained sufficient modes to cover $99 \%$ of shape variance in each constructed DSM.

Each face surface captured has as many as 30,000 mesh points. The signature of a face surface is the set of position differences at constituent image mesh points from corresponding points on the mean of age/sex-matched healthy controls, normalized against the variation in controls. The signature weight of a surface is the square root of the sum of the squared normalized differences across all of the densely corresponded points. Signature weight is a rough estimate of facial dysmorphism. A signature heat map visualizes the significance of localized differences using a red-green-blue spectrum with, for example, red and blue reflecting extreme opposite displacements and green coincidence with the mean of the matched controls. Thus, an axis normal to the face surface reflects inward/outward displacement.

In order to investigate differences in face shape, we used multi-folded discrimination testing (closest mean; support vector machines; linear discriminant analysis) to determine baseline discrimination rates between controls and syndromes. Anthropometric comparisons were made against appropriate age-range matched controls.

\subsubsection{Comparison of linear regressions}

Linear regressions were undertaken for various facial measurements and DSMbased markers against age. Prism (Graphpad) was used to determine significant differences in slope and/or intercept in comparisons of separate regressions for control, CS and other disorder cohorts.

\subsubsection{Statistical analysis}

Significance values when comparing gender-specific facial features of CS patients and controls were calculated using an unpaired Student's t-test throughout the article. Statistical robustness was further enhanced through applying a Bonferroni correction that divides the $p$-value cut-off by the number of tests run. To find statistically significant differences in facial features between male and female CS patients and age- and gender-matched controls, 5\% p-value cut-off was divided by 4, resulting in a threshold p-value of .0125 . 


\subsection{Supplemental information}


Supplementary Table 1 | Major Clinical Features of Cantú syndrome found in all 20 studied subjects and compared with findings from ICSR.

\begin{tabular}{|c|c|c|c|c|c|c|c|c|}
\hline 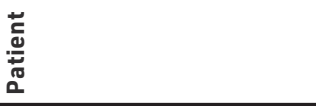 & 용 & $\begin{array}{l}\text { 옹 } \\
\text { 옹 }\end{array}$ & 옹 & Г & $\begin{array}{l}m \\
\text { 옹 }\end{array}$ & 옹 & 동 & 옹 \\
\hline \multicolumn{9}{|l|}{ Pregnancy/delivery } \\
\hline Polyhydramnios & - & - & - & + & + & + & + & + \\
\hline Gestation (weeks) & 36 & $\mathrm{FT}$ & $\mathrm{FT}$ & 29 & 34 & 35 & $\mathrm{FT}$ & 37 \\
\hline \multicolumn{9}{|l|}{ Skin/hair growth } \\
\hline Cong. hypertrichosis & + & + & + & + & + & + & + & + \\
\hline Wrinkled and/or loose skin & - & - & + & + & - & - & + & + \\
\hline \multicolumn{9}{|l|}{ Cardiovascular } \\
\hline PDA & + & + & + & + & + & - & + & - \\
\hline Aortic root dilation & + & - & - & + & + & + & + & + \\
\hline Enlarged heart & + & + & + & + & + & + & + & + \\
\hline Pericard. Eff. & - & + & - & + & - & + & - & + \\
\hline Arrhythmia & - & + & - & - & - & + & - & + \\
\hline Pulmonary hypertension & - & - & - & + & + & - & - & - \\
\hline Blood pressure abnorm. & - & - & - & + & - & - & - & + \\
\hline Lymphedema & - & + & + & - & - & - & - & - \\
\hline Abnorm. blood vessels & + & + & + & + & - & + & - & + \\
\hline Edema & - & + & + & - & + & + & + & + \\
\hline Other & - & - & $\begin{array}{l}A P C, M V \\
\text { regurg. }\end{array}$ & - & - & - & PFO & MV regurg. \\
\hline
\end{tabular}

Neurological

\begin{tabular}{|c|c|c|c|c|c|c|c|c|}
\hline Headaches/migraine & M & $\mathrm{H}$ & M & $\mathrm{H} / \mathrm{M}$ & - & - & 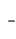 & M \\
\hline Seizures & - & - & Febrile & - & + & $\begin{array}{l}\text { Convulsions during } \\
\text { sleep }\end{array}$ & - & - \\
\hline Brain abnorm. & - & - & $\begin{array}{l}\text { White } \\
\text { matter } \\
\text { lesions }\end{array}$ & $\begin{array}{l}\text { Decreased } \\
\text { white } \\
\text { matter }\end{array}$ & $\begin{array}{l}\text { External } \\
\text { hydroceph- } \\
\text { alus }\end{array}$ & $\begin{array}{l}\text { Dysgenesis of corpus } \\
\text { callosum, diffuse } \\
\text { leptomeningeal } \\
\text { enhancement }\end{array}$ & 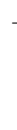 & $\begin{array}{l}\text { Periventricular } \\
\text { leukomalacia }\end{array}$ \\
\hline
\end{tabular}

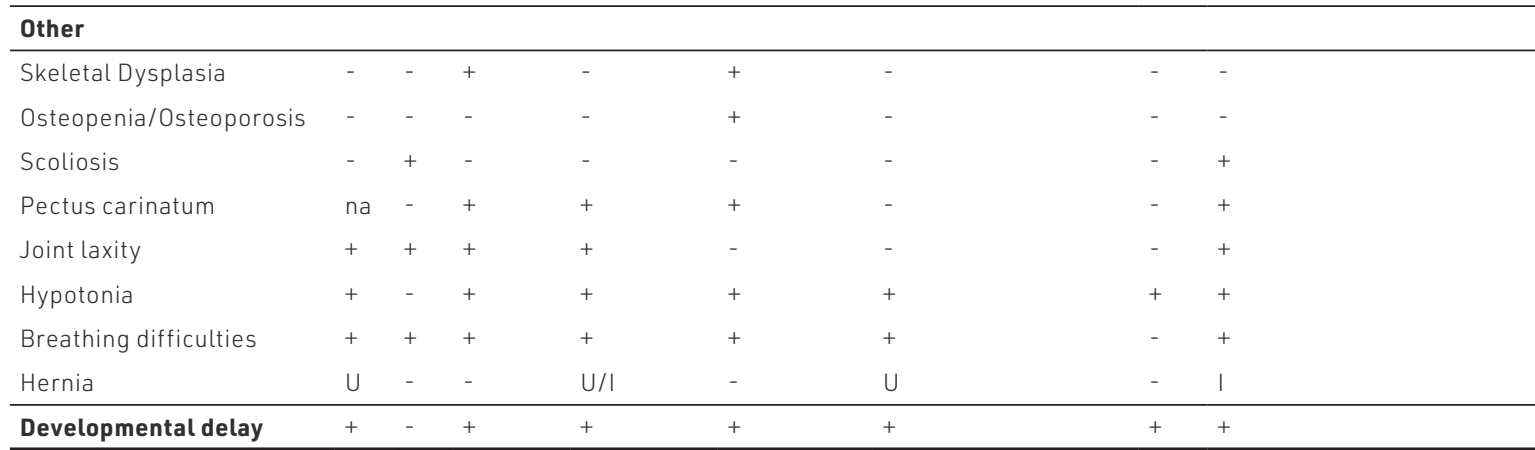

Abbreviations: Aort. Coarct., aortic coarctation; APC, aorto-pulmonary collateral; FT, full term; $\mathrm{H}$, headache; ICSR, International Cantú syndrome registry; I, inguinal; M, migraine; MV regurg., mitral valve regurgitation; na, data not available; PFO, patent foramen ovale; U, umbilical

a Numbers derive from International Cantú syndrome registry (Grange et al., 2019) 


\begin{tabular}{|c|c|c|c|c|c|c|c|c|c|c|c|c|c|}
\hline 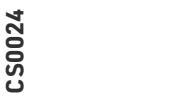 & $\begin{array}{l}\stackrel{\infty}{\mathbb{S}} \\
\text { O } \\
\text { ஸ }\end{array}$ & $\begin{array}{l}\text { S } \\
\text { ్ㅗ }\end{array}$ & $\begin{array}{l}\text { ̊̊ } \\
\text { ஸ̆ }\end{array}$ & 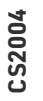 & 옹 & $\begin{array}{l}\infty \\
\text { O } \\
\text { N } \\
\text { U }\end{array}$ & $\begin{array}{l}\stackrel{\circ}{8} \\
\text { ํㅗ }\end{array}$ & $\begin{array}{l}0 \\
\text { 옹 } \\
\text { ํํ }\end{array}$ & 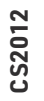 & $\begin{array}{l}\frac{m}{0} \\
\text { N } \\
\text { N }\end{array}$ & 몽 & 혼 를 & $\frac{\approx}{\alpha}$ \\
\hline - & + & na & - & na & na & na & na & na & na & na & + & $7 / 12$ & $34 / 60$ \\
\hline 38 & FT & $\mathrm{FT}$ & 33 & 38 & FT & na & FT & $\mathrm{FT}$ & 32 & 37 & FT & $\begin{array}{l}37.3 \\
\text { weeks }\end{array}$ & $\begin{array}{l}33.9 \\
\text { weeks }\end{array}$ \\
\hline+ & + & + & + & + & + & + & + & + & + & + & + & $20 / 20$ & $68 / 72$ \\
\hline+ & + & + & + & + & + & - & + & + & + & - & - & $13 / 20$ & $42 / 69$ \\
\hline - & - & - & + & + & + & - & + & - & + & - & + & $12 / 20$ & $44 / 73$ \\
\hline+ & + & na & - & - & - & na & na & - & na & na & - & $8 / 16$ & $21 / 66$ \\
\hline+ & + & + & + & + & + & na & + & na & + & na & - & $17 / 18$ & $42 / 69$ \\
\hline - & - & na & + & + & - & na & na & - & - & na & - & $6 / 16$ & $17 / 69$ \\
\hline+ & - & na & - & + & - & na & na & - & - & na & - & $5 / 16$ & $12 / 65$ \\
\hline - & - & na & + & - & - & na & na & - & na & - & - & $3 / 16$ & $16 / 67$ \\
\hline - & + & + & - & - & - & + & na & + & - & - & - & $6 / 19$ & $16 / 71$ \\
\hline - & - & - & - & - & - & na & + & - & - & - & - & $3 / 19$ & $8 / 72$ \\
\hline - & - & - & - & na & - & - & na & na & na & na & - & $6 / 15$ & $13 / 68$ \\
\hline- & - & + & - & + & - & na & + & + & + & + & - & $12 / 19$ & $27 / 63$ \\
\hline $\begin{array}{l}\text { Enlarged left } \\
\text { heart, bicuspid } \\
\text { aortic valve }\end{array}$ & - & - & - & - & $\begin{array}{l}\text { MV } \\
\text { regurg. }\end{array}$ & - & $\begin{array}{l}\text { Valve does } \\
\text { not close } \\
\text { properly }\end{array}$ & $\begin{array}{l}\text { MV } \\
\text { regurg. }\end{array}$ & - & - & - . & $7 / 20$ & $21 / 74$ \\
\hline
\end{tabular}

\begin{tabular}{|c|c|c|c|c|c|c|c|c|c|c|c|c|c|}
\hline $\mathrm{H}$ & - & - & - & M & - & $M$ & M & - & - & $\mathrm{H}$ & - & $10 / 20$ & $27 / 68$ \\
\hline Febrile & Febrile & - & - & + & - & + & - & - & - & + & - & $8 / 20$ & $17 / 72$ \\
\hline- & - & - & - & - & - & - & $\begin{array}{l}\text { Abnormally } \\
\text { small pitu- } \\
\text { itary }\end{array}$ & - & - & na & - & $6 / 19$ & $11 / 49$ \\
\hline
\end{tabular}

\begin{tabular}{|c|c|c|c|c|c|c|c|c|c|c|c|c|c|}
\hline - & - & - & - & na & na & - & - & na & na & na & - & $2 / 15$ & $12 / 64$ \\
\hline - & - & na & - & na & + & - & na & na & na & na & - & $2 / 14$ & $4 / 64$ \\
\hline - & - & - & - & na & + & - & - & - & - & - & - & $3 / 19$ & $10 / 70$ \\
\hline - & - & + & - & + & + & - & + & - & + & + & + & $11 / 19$ & $17 / 69$ \\
\hline - & - & + & - & + & + & + & + & + & + & - & + & $13 / 20$ & $39 / 70$ \\
\hline - & + & + & - & - & + & + & + & na & + & + & + & $15 / 19$ & $42 / 65$ \\
\hline+ & + & - & + & + & + & + & - & + & + & - & + & $16 / 20$ & $43 / 71$ \\
\hline U & - & $U$ & I & U & - & - & - & - & $U / I$ & - & - & $9 / 20$ & $25 / 72$ \\
\hline - & + & - & + & + & - & + & + & - & + & - & + & $14 / 20$ & $45 / 71$ \\
\hline
\end{tabular}

For supplementary figures please see the online version of this article. 
-12
4
4
4

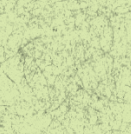

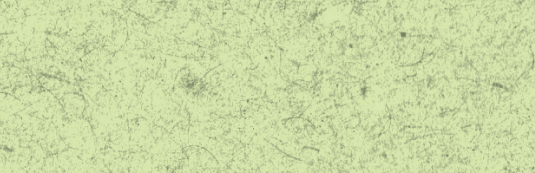

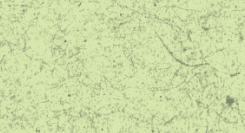

तr.

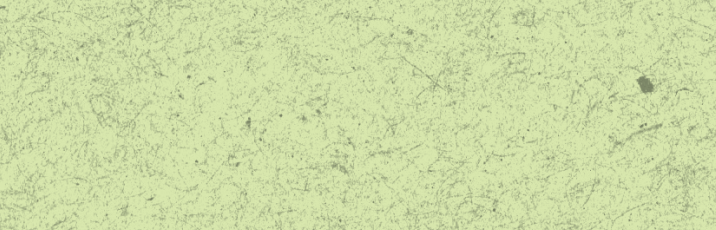

4

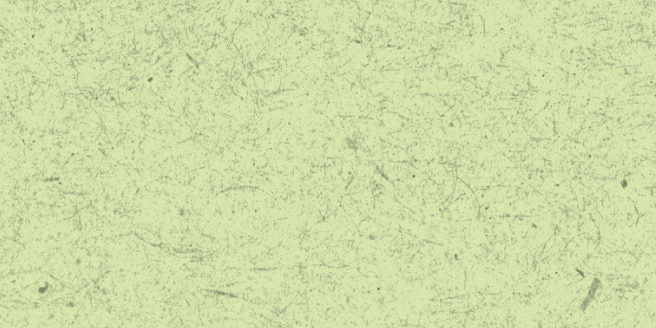




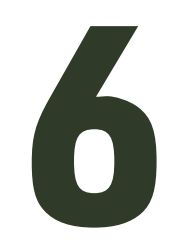

\section{Cantú syndrome: Findings from 74 patients in the International Cantú Syndrome Registry}

Dorothy K. Grange*, Helen I. Roessler*, Conor McClenaghan*, Karen Duran, Kathleen Shields, Maria S. Remedi, Nine V.A.M. Knoers, Jin-Moo Lee, Edwin P. Kirk, Ingrid Scurr, Sarah H. Smithson, Gautam K. Singh, Mieke M. van Haelst, Colin G. Nichols, Gijs van Haaften

*These authors contributed equally to this work.

Am J Med Genet C Semin Med Genet. 2019 Dec;181 (4):658-681

DOI: 10.1002 /ajmg.c.31753 


\section{Abstract}

Cantú syndrome (CS), first described in 1982, is caused by pathogenic variants in ABCC9 and KCNJ8, which encode the regulatory and pore forming subunits of ATP-sensitive potassium $\left(\mathrm{K}_{\text {ATP }}\right.$ ) channels, respectively. Multiple case reports of affected individuals have described the various clinical features of CS, but systematic studies are lacking. To define the effects of genetic variants on CS phenotypes and clinical outcomes, we have developed a standardized REDCap-based registry for CS. We report phenotypic features and associated genotypes on 74 CS subjects, with confirmed ABCC9 variants in 72 of the individuals. Hypertrichosis and a characteristic facial appearance are present in all individuals. Polyhydramnios during fetal life, hyperflexibility, edema, patent ductus arteriosus (PDA), cardiomegaly, dilated aortic root, vascular tortuosity of cerebral arteries, and migraine headaches are common features, although even with this large group of subjects, there is incomplete penetrance of CSassociated features, without clear correlation to genotype. 


\subsection{Introduction}

Cantú syndrome (CS) (OMIM\#239850) is an autosomal dominant condition caused by gain-of-function (GoF) pathogenic variants in ABCC9 and, less commonly, in KCNJ8, which encode the regulatory (SUR2) and pore-forming (Kir6.1) subunits, respectively, of ATP-sensitive potassium $\left(\mathrm{K}_{\text {ATP }}\right)$ channels (Brownstein et al., 2013; Cooper et al., 2014; Harakalova et al., 2012; McClenaghan et al., 2017; van Bon et al., 2012). A wide constellation of clinical features have been described in previous CS case reports (Grange et al., 2006; Grange et al., 2014; Scurr et al., 2011). Many pregnancies in which the fetus has CS are complicated by polyhydramnios, leading in some instances to repeated amniotic fluid reductions as well as preterm labor and delivery. Congenital hypertrichosis and coarse facial features, including a low frontal hairline, epicanthal folds, flat nasal bridge, long philtrum, macroglossia, prominent mouth, and full lips are defining features described in every affected individual and are usually evident at birth.

Generalized macrosomia, with large birth weight and length, as well as persistent macrocephaly, are also commonly reported (Cantu, Garciacruz, Sanchezcorona, Hernandez, \& Nazara, 1982; Grange et al., 2014), as well as generalized edema at birth and lymphedema/peripheral edema (Grange et al., 2014). Affected individuals have extensive cardiovascular anomalies, including cardiomegaly, patent ductus arteriosus (PDA) and other congenital cardiac anomalies, pericardial effusion, pulmonary hypertension, dilated aortic root, and dilated and torturous cerebral blood vessels (Grange et al., 2006; Nichols, Singh, \& Grange, 2013). Skeletal abnormalities including osteochondrodysplasia and generalized mild osteopenia are usually asymptomatic, although clinically significant scoliosis may occur (Concolino et al., 2000; Rosser et al., 1998). Symptoms of a connective tissue abnormality with loose skin and hyperextensible joints are often present (Grange et al., 2014). Additional reported features include developmental delays, ADHD and autism spectrum features, without intellectual disability (Grange et al., 2014).

To date, 60 individuals with CS have been reported in the literature, with approximately half being reported based on a clinical diagnosis. In the previously reported CS patients, genetic testing in $\sim 35$ individuals identified heterozygous pathogenic variants in ABCC9 (Harakalova et al., 2012; van Bon et al., 2012), and two cases thus far of heterozygous pathogenic variants in KCNJ8 (Brownstein et al., 2013; Cooper et al., 2014). The overall incidence is still unknown, but increased awareness of the clinical phenotype will undoubtedly lead to improved recognition and, hence, higher documented prevalence. There is considerable 
variation in the phenotypic spectrum, even within family members sharing the same ABCC9 variant (Roessler et al., 2018).

Despite the identification of $A B C C 9$ and KCNJ8 variants as the underlying cause of $C S$, pathophysiologic mechanisms resulting in the clinical manifestations remain poorly understood, and there is no specific therapy. Development of effective targeted therapies will require well-characterized patient cohorts, with standardized data collection. Unlike common diseases, for which patient resources are routinely available and large patient cohorts can be built at a single site, such studies in rare diseases require multi-site collaboration to maximize cohort size. Appropriately designed and executed patient registries that collate such data can then provide a real-world view of common patient characteristics and outcomes, as well as a platform to facilitate clinical trials. Registries are especially valuable for rare disorders without appropriate treatments (CaveroCarbonell et al., 2016) such as CS, for which data are generally only available in scattered case reports. Here, we present clinical and genetic findings on $74 \mathrm{CS}$ subjects enrolled in an international REDCap-based CS registry. This represents the largest cohort of CS subjects studied thus far, permitting estimates of penetrance for disease features and providing data on inter-familial and intrafamilial variability and genotype-phenotype correlation. 


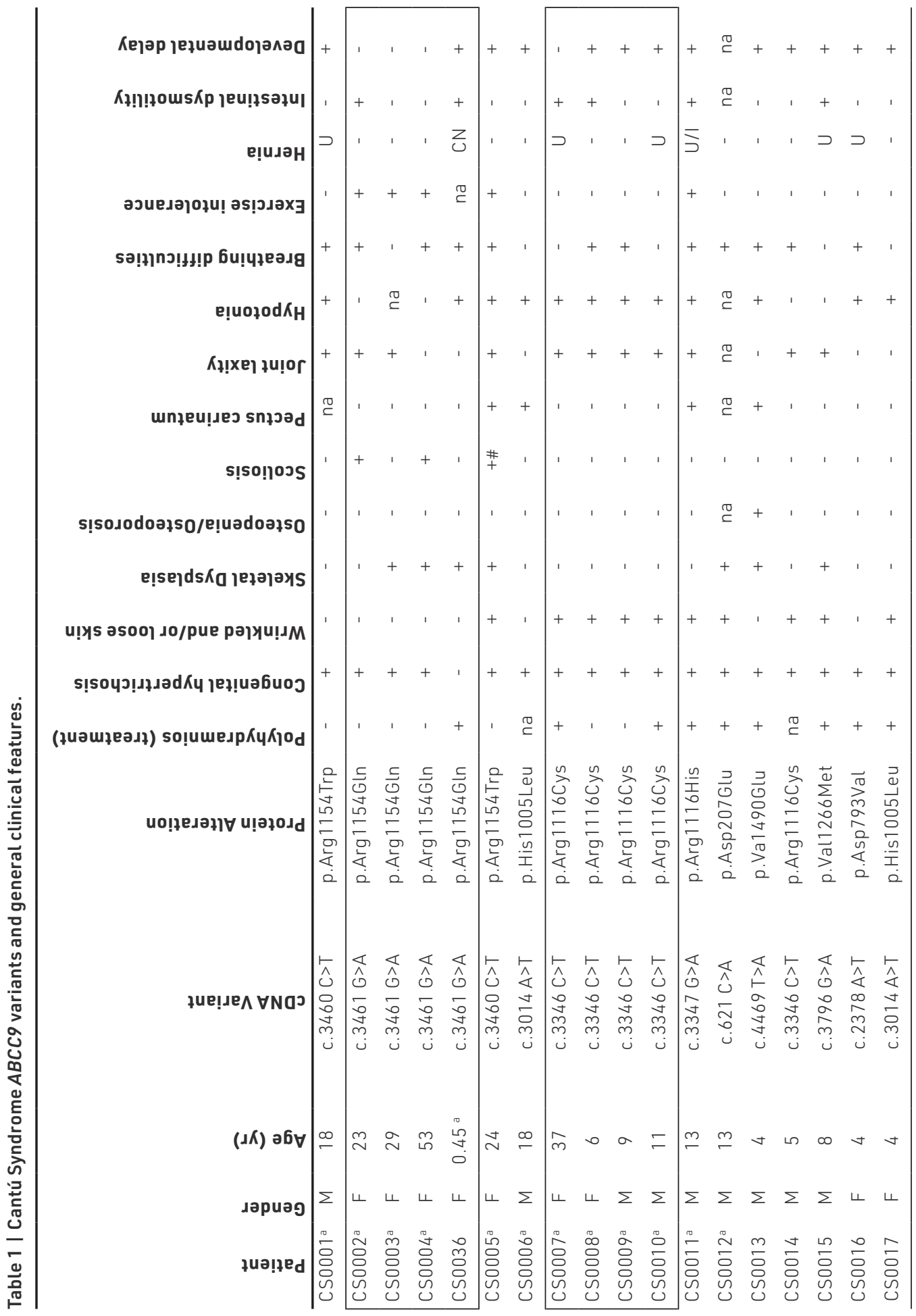




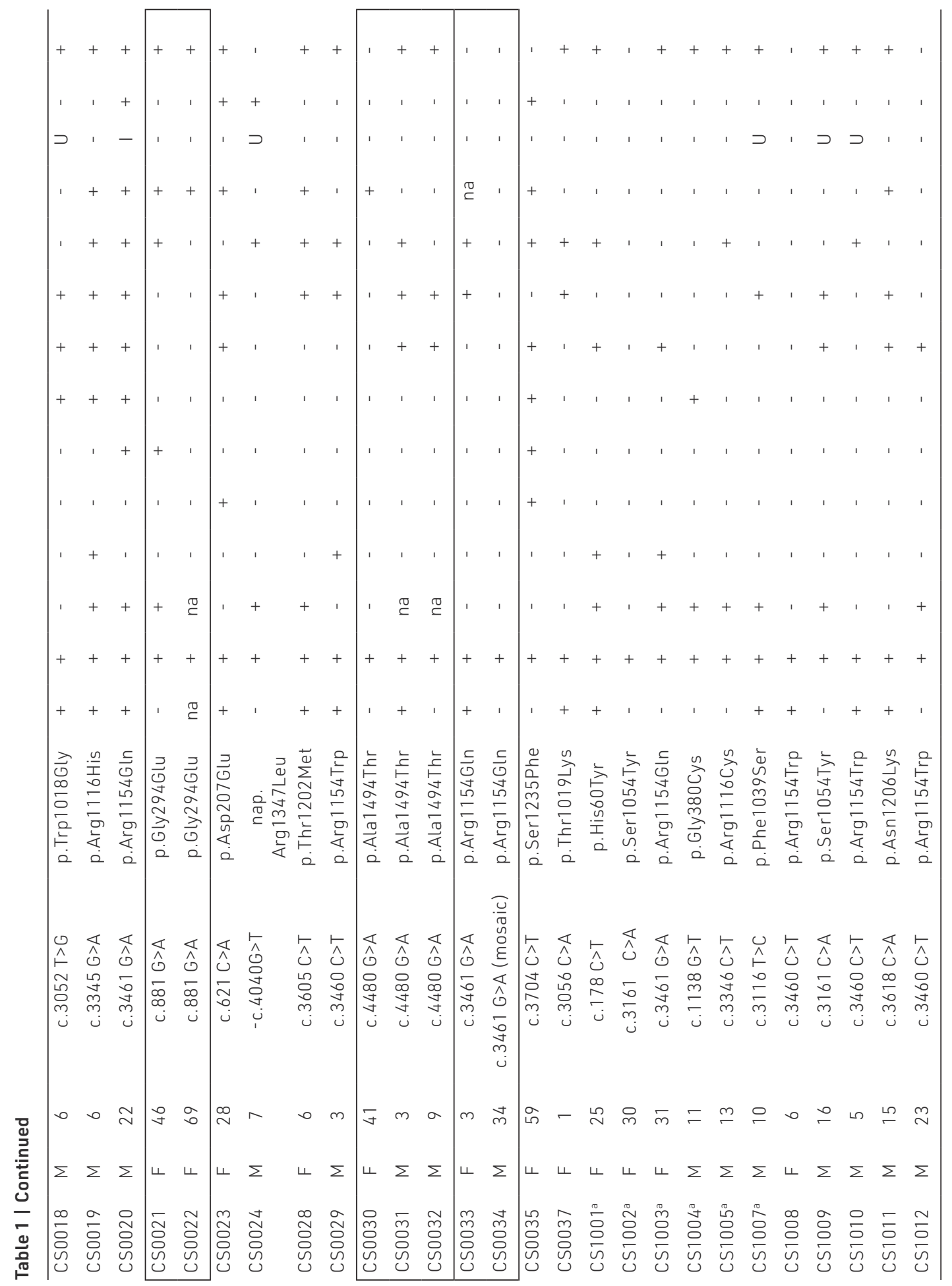




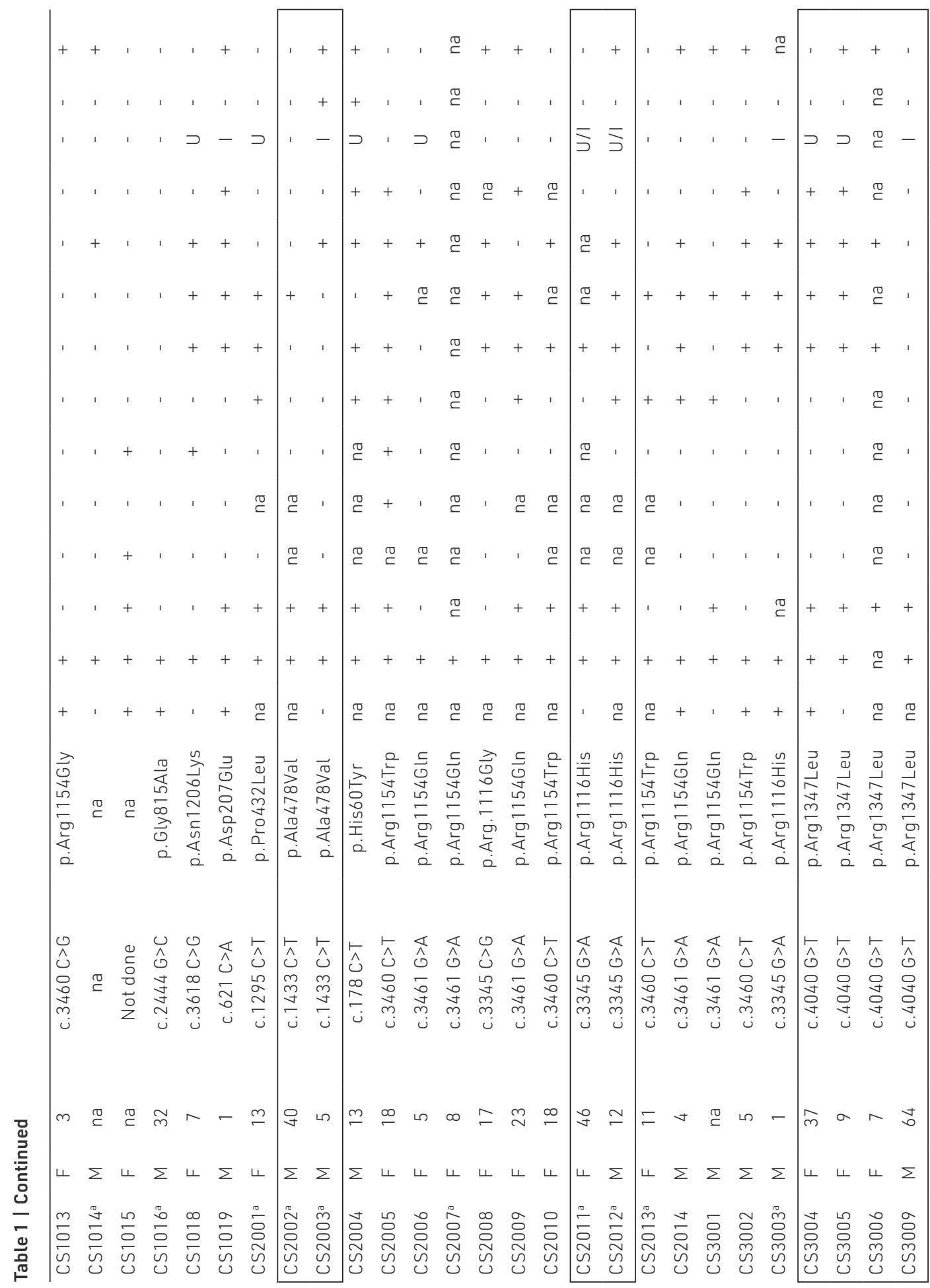




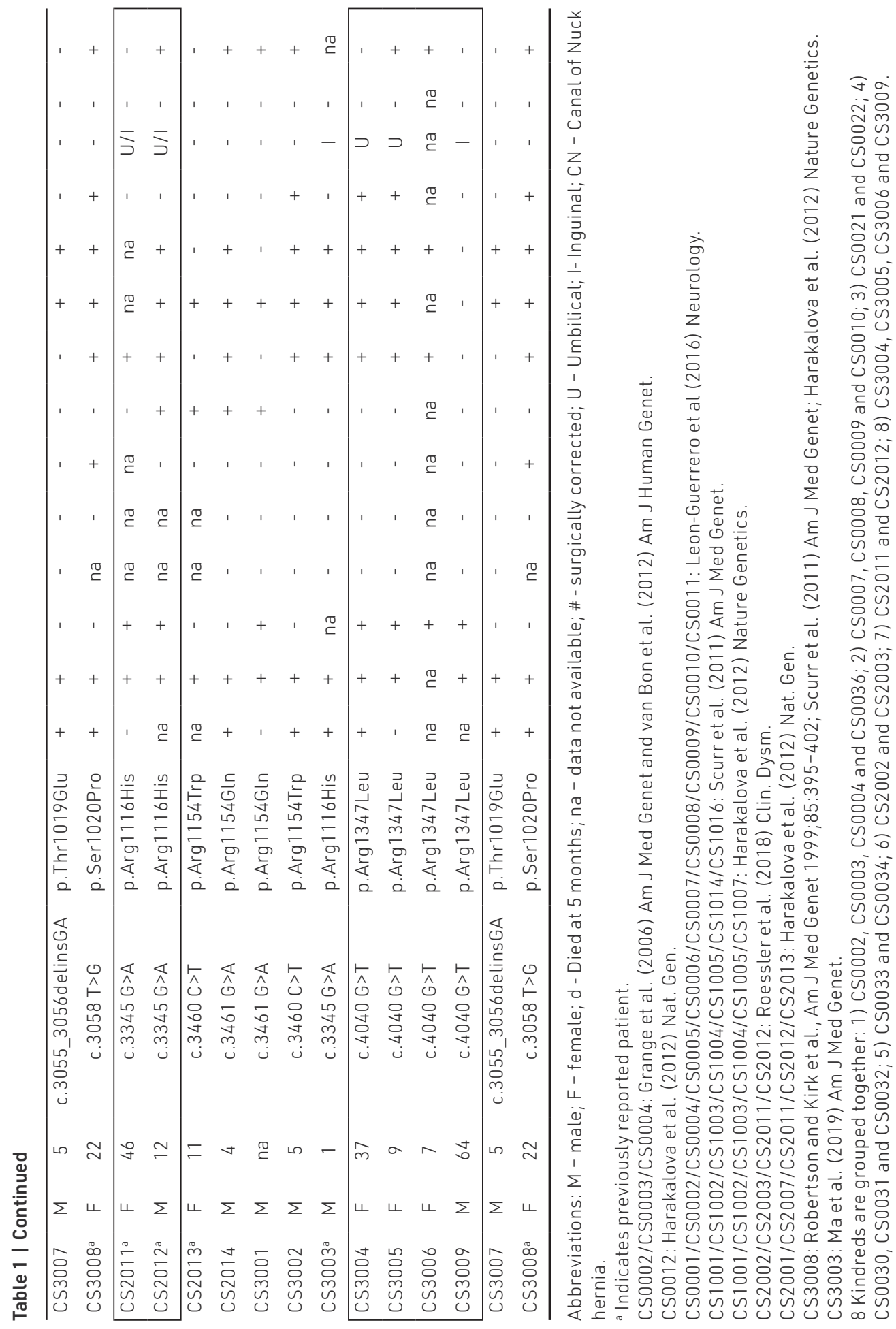




\subsection{Results}

We present results from 74 CS subjects, including 45 new subjects and 29 subjects that have been previously reported (Grange et al., 2006; Harakalova et al., 2012; Leon Guerrero et al., 2016; Ma et al., 2019; Robertson et al., 1999; Roessler et al., 2018; Scurr et al., 2011). Seventy-two of the patients have confirmed variants in ABCC9 while one has a convincing clinical diagnosis of CS but has not had molecular testing and one has had testing, but the ABCC9 variant result is not available. Information regarding genotype and general features of all 74 subjects are provided in Table $\mathbf{1 .}$

\subsubsection{Patient demographics}

The 74 subjects (36 females [49\%] and 38 males [51\%]) range in age from 2 months to 67 years (Figure 1a, Table 2). Subjects were recruited by four centers, in the United States, the Netherlands, the United Kingdom, and Australia. The overwhelming majority of participants (73/74) are Caucasian and most (64/74) identify as Non-Spanish/Hispanic/Latino, reflecting the location of the participating sites. Ages at the time of diagnosis are shown in Figure $\mathbf{1} \mathbf{b}$.

Table 2 | Demographics of CS Subjects.

\begin{tabular}{lc}
\hline Descriptive characteristics & All $(\mathbf{n}=\mathbf{7 4})$ \\
\hline Age & \# Patients* $\%)$ \\
\hline 55 year & $15(21.4 \%)$ \\
$>5$ to 10 years & $18(25.7 \%)$ \\
$>10$ to 20 & $15(21.4 \%)$ \\
$>20$ years & $23(31 \%)$ \\
\hline Gender & \\
\hline Female & $36 / 74(48.6 \%)$ \\
Male & $38 / 74(51.3 \%)$ \\
\hline Race & $73 / 74(98.6 \%)$ \\
\hline Caucasian & $1 / 74(1.4 \%)$ \\
\hline Black/African American & $10 / 71^{\star *}(14 \%)$ \\
\hline Ethnicity & $61 / 71 * \star(86 \%)$ \\
\hline Not Hispanic/Latino & \\
\hline
\end{tabular}

Abbreviations: a Current age available for only $71 / 74$ patients, ${ }^{b}$ Question not answered in three patients, but all these three indicated that they are Caucasian. 


\subsubsection{Pregnancy and birth}

Half of the mothers of affected individuals reported prenatal abnormalities during their pregnancies, primarily abnormal ultrasound findings (Figure 1c). Macrosomia and macrocephaly were evident during third trimester ultrasounds in eight patients. Polyhydramnios was observed during gestation in 34/60 (57\%) pregnancies and in multiple cases, repeated amniotic fluid reduction procedures were required (Figure 1c). Polyhydramnios is reported in both inherited and de novo CS individuals (i.e., non-CS mothers) and thus likely originates from fetal $\mathrm{K}_{\text {ATP }}$ channel dysfunction. Gestational age at birth ranged from 24 to 39 weeks with an average gestation period of $33.9 \pm 3.4$ weeks $(n=39)$ and $42 / 72(58 \%)$ babies were born preterm (i.e., less than 37 weeks gestational age). Hence, children with CS were born significantly more prematurely than normal (Figure 1c).

Babies with CS had macrosomia with increased length and weight, as well as macrocephaly (Figure 1e). The average birth weight for all infants reported was $3.7 \pm 1.0 \mathrm{~kg}(\mathrm{n}=68)$, and $4.1 \pm 0.7 \mathrm{~kg}$ for full term infants $(n=28) .12 \%$ of CS infants had a low birth weight (i.e., $<2,500 \mathrm{~g}$ ) and $38 \%$ had a high birth weight (i.e., $>4,000 \mathrm{~g}$ ). As shown in Figure 1e, birth length and weight are consistently above average, irrespective of gestational age.

At birth, the most consistent clinical features were excessive body hair in essentially all subjects $(67 / 68,99 \%)$, edema in $27 / 63(43 \%)$ and wrinkled and/ or loose skin in 43/69 (62\%) (Figure 1c). Multiple subjects reported congenital cardiac defects, as discussed below. Before or after the pregnancy with the affected CS participant, 2/41 (5\%) of mothers (one of whom had CS) who responded to the question experienced a stillbirth, which is higher than the estimated risk of stillbirth in the general population ( $1 \%$ of pregnancies greater than 20 weeks gestation), although the numbers are too small to be certain whether this is a genuine association or a chance observation. Of 40 women who responded to the question, miscarriages were reported by five $(13 \%)$, comparable to $10-20 \%$ incidence in the general population. 

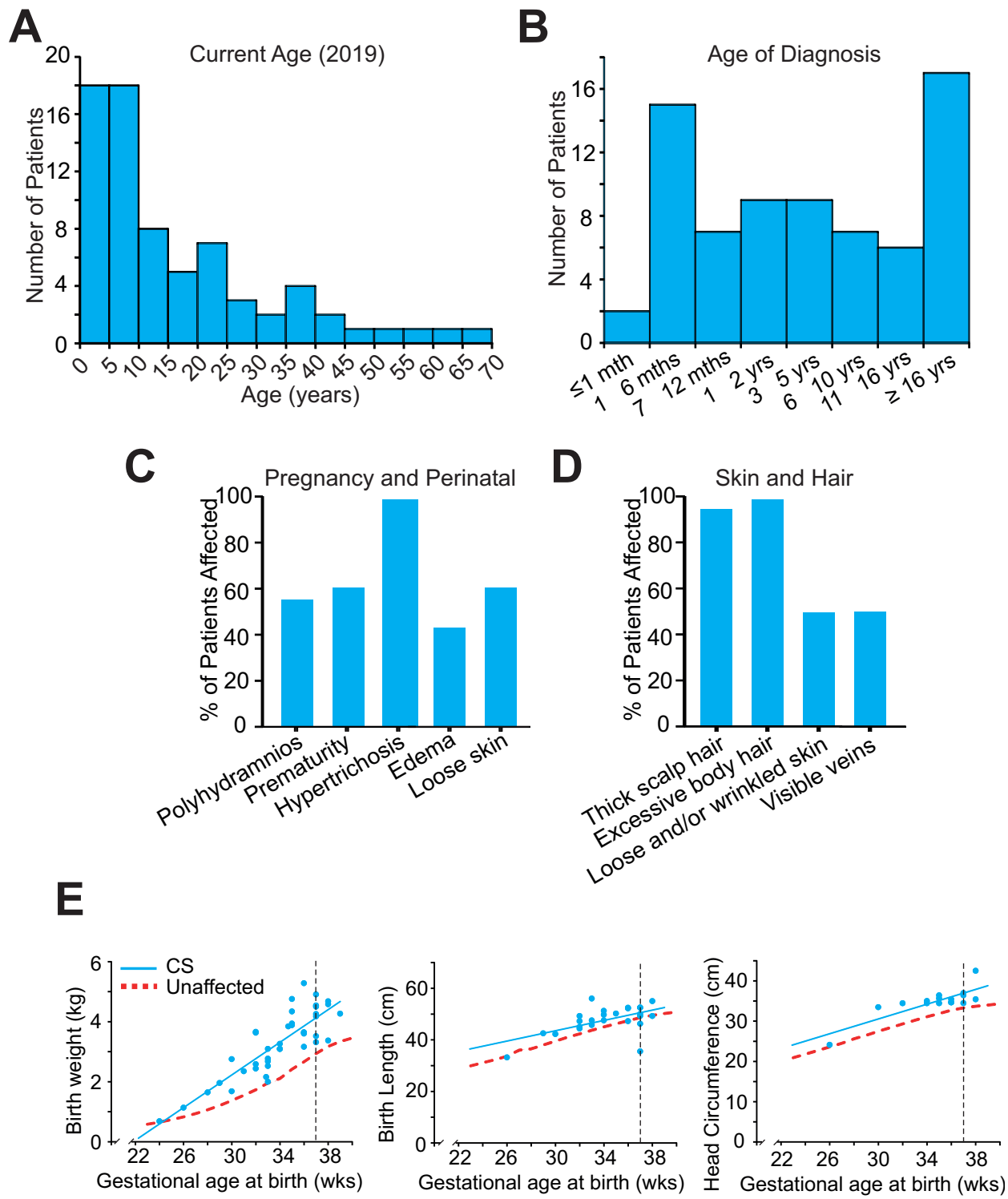

Figure 1 | Subject characteristics. (a) Current subject ages, (b) Age at diagnosis, (c) Pregnancy and perinatal features, (d) Skin and hair features, (e) Growth parameters in newborns with Cantú syndrome (Left) Birth weights of CS newborn infants plotted versus gestational age, showing elevated birth weights. (Center) Birth lengths of CS newborn infants plotted versus gestational age, showing increased birth length. (Right) Birth head circumference of CS newborn infants plotted versus gestational age, showing macrocephaly. The 50th centile for normal infants is shown in red. Vertical dashed line indicates 37 weeks. CS, Cantú syndrome. 


\subsubsection{Growth}

At the most recent assessments, subjects exhibited an average BMI of 23.2 \pm 8.4 (range $15.9-58.0, n=36$ ). A thin or even quite muscular physique was most commonly evident during childhood, while adults were more likely to be overweight due to weight gain after puberty. Mean adult height in males was at the 95th centile for the general population, although data on height measurements were available in only a limited number of men in the registry (Table 3). Females were mostly within the normal range for height for adult women (50-75th centile). However, macrocephaly persists throughout life in both men and women; mean head circumference was $>98$ th centile ( +2.5 SD) in women and the 98 th centile (+2 SD) in men (Table 3 ).

Table 3 | Growth parameters in adults with Cantú syndrome.

\begin{tabular}{ll}
\hline Characteristics & \\
\hline Mean height $(\mathrm{cm})$ adult female & $167.0 \mathrm{~cm}\left(70^{\text {th }} \%\right.$ ile) $\mathrm{N}=11$ \\
& Normal range $151-175 \mathrm{~cm}$ \\
\hline Mean height $(\mathrm{cm})$ adult male & $187.7 \mathrm{~cm}\left(95^{\text {th }} \%\right.$ ile) $\mathrm{N}=5$ \\
& Normal range $163-190 \mathrm{~cm}$ \\
\hline Mean head circumference $(\mathrm{cm})$ adult female & $58.7 \mathrm{~cm}\left(>98^{\text {th }} \%\right.$ ile) $\mathrm{N}=9$ \\
& Normal range $52-57.5 \mathrm{~cm}$ \\
\hline Mean head circumference $(\mathrm{cm})$ adult male & $57.8 \mathrm{~cm}\left(98^{\text {th }} \%\right.$ ile $) \mathrm{N}=4$ \\
& Normal range $53-58 \mathrm{~cm}$ \\
\hline
\end{tabular}

\subsubsection{Development and behavior}

Developmental delays in childhood were reported in 45/71 (63\%) subjects and $29 / 64$ (45\%) reported some degree of special educational support. A majority of individuals were reported to have hypotonia $(42 / 65,65 \%)$ and speech delays in early childhood, requiring occupational, physical and speech therapy. Persistent intellectual impairment was not evident, however, and most individuals clearly attain normal educational levels. About $18 \%$ of the subjects in the registry currently attend first to eighth grade (or equivalent) and $12 \%$ are in high school, $21 \%$ have achieved their high school diploma or equivalent, $18 \%$ have a college degree, and $6 \%$ have a graduate degree.

With regard to behavioral abnormalities, the ICSR data are not complete for the entire cohort, but systematic information is available for the patients assessed through the Washington University site. Of these, ADHD is reported in $6 / 32$ (19\%), autism or autism spectrum disorder in 6/31 (16\%), mood swings in 10/31 $(32 \%)$, obsessive compulsive disorder (OCD) in 4/31 (13\%), anxiety in 4/31 $(13 \%)$, and depression in 6/32 (19\%) of respondents. Many of the subjects selfreported more than one of these behavioral abnormalities. Four subjects had 
two concomitant behavioral concerns (ADHD and mood swings in one subject; depression and mood swings in one subject; ADHD and autism in two subjects), three subjects had three behavioral concerns ( $A D H D$, mood swings and OCD in one subject; mood swings, anxiety and depression in one subject; ADHD, mood swings and anxiety in one subject) and three subjects had four behavioral concerns (ADHD, autism, OCD and mood swings in two subjects; autism, mood swings, $O C D$, and anxiety in one subject).

\subsubsection{Craniofacial dysmorphology, hypertrichosis, and skin appearance}

All affected individuals have a distinctive facial appearance with coarse features, including a low frontal hairline, epicanthal folds, puffy eyelids, flat nasal bridge with broad nasal tip, long philtrum, macroglossia, and prominent mouth with full lips (Figure 2). Hypertrichosis is a highly penetrant feature, with almost all subjects reporting thick scalp $(68 / 72,94 \%)$ and body hair $(72 / 73,99 \%)$. In $65 / 68(96 \%)$ subjects, hypertrichosis was present within the first year of life, in particular, abnormal hair coverage was reported on the arms, back, cheeks, chin, feet, forehead, legs, and hands of infants (Figure 1d, 3, 1d, 3) . 21/68 (31\%) subjects report variable success at managing hypertrichosis using approaches such as shaving, waxing and laser therapy.

Loose and/or wrinkled skin with deep palmar/plantar creases are present in a majority of infants with CS $(42 / 69,61 \%)$ and frequently in adults. Prominent veins or visible small blood vessels, predominantly on the lower extremities, chest and back, are reported in 33/71 (46\%) cases (see Figure 31, m). Many adults have larger varicosities on the lower extremities, occurring at a younger age than is typical in the general population. 


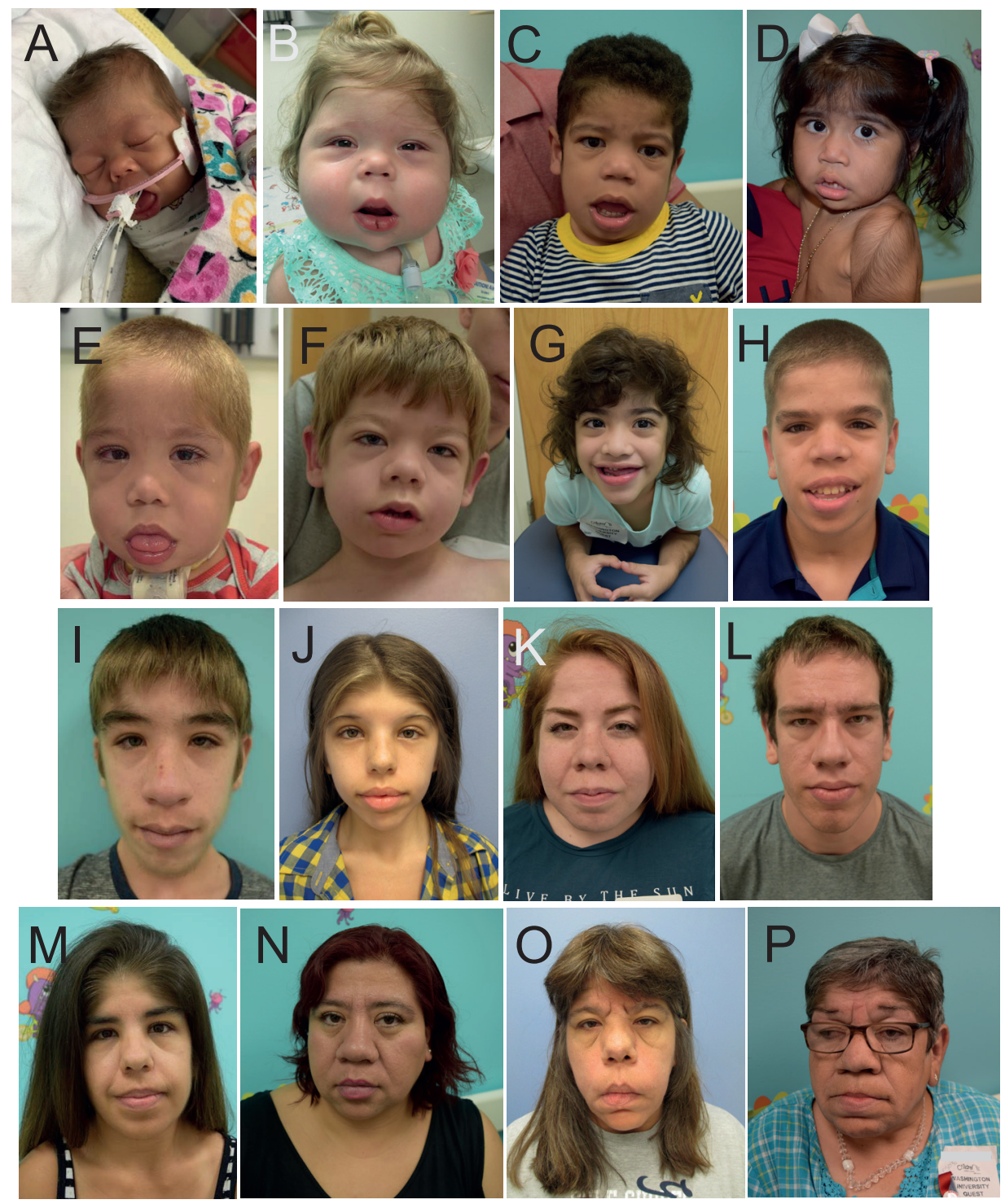

Figure 2 | Facial phenotype of Cantú syndrome subjects throughout life. (a) 2 months; (b) 16 months; (c) 22 months; (d) 26 months; (e) 2.5 years; (f) 3 years; (g) 4 years; (h) 12 years; (i) 15 years; (j) 20 years; (k) 23 years; (l) 20 years; (m) 26 years; (n) 40 years; (o) 50 years: (p) 57 years. Note edema of eyelids in many of the younger subjects, epicanthal folds, widely spaced eyes, full lips, and macroglossia. Hypertrichosis of the forehead tends to decrease with age, but facial features become more prominent over time. 

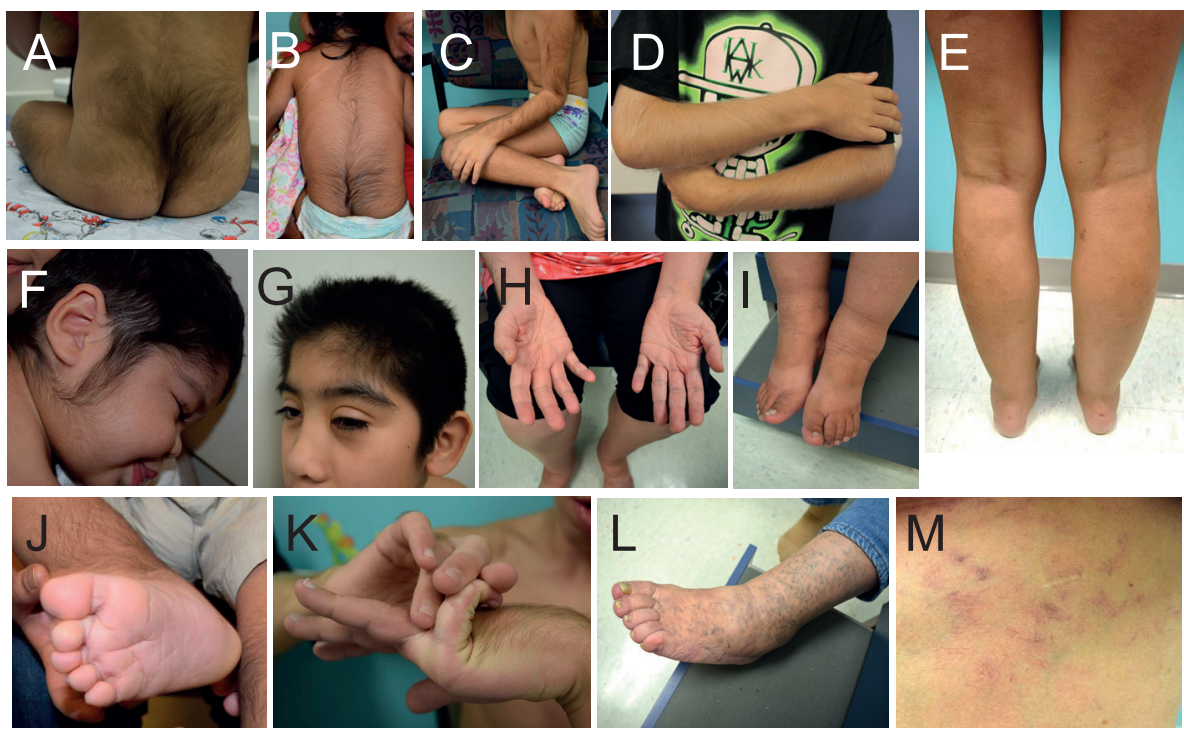

Figure 3 | Physical characteristics. (a) Hypertrichosis over the sacral area; (b) hypertrichosis of the upper and lower back; (c and $\mathbf{d}$ ) hypertrichosis of the limbs; (e) asymmetry of lower extremities due to edema of the right leg; ( $\mathbf{f}$ and $\mathbf{g}$ ) hypertrichosis of the cheek and forehead; (h) wrinkled skin and deep palmar creases in an adult; (i) edema of the ankles showing indentation from socks; (j) deep plantar crease in a young child; (k) hyperflexibility of the digits; (l) prominent superficial veins and small varicosities in an older individual; $(\mathbf{m})$ prominent small blood vessels and visible capillaries on the torso of an adult.

\subsubsection{Cardiovascular abnormalities}

Cardiovascular features of all 74 subjects are presented in Table 4 and Figure 4a-c. Congenital heart defects are the most commonly documented CS cardiovascular abnormality, present in 56/73 (77\%) subjects in our CS cohort. Persistent PDA was reported most frequently (44/73, 58\%) with a variety of other defects observed including atrial septal defects (6\%), ventricular septal defects $(6 \%)$, and aortic coarctation (3\%) (Figure 4a). Surgical closure of PDA was required in $28 / 52$ subjects ( $54 \%$ ). $18 \%$ of subjects reported valvular defects including mitral valve regurgitation (9\%), aortic stenosis (2\%), and bicuspid aortic valve (10\%) (Figure $4 \mathrm{c}$ ). Dilation of the aortic root was reported in $21 / 66$ (32\%) subjects, with $1 / 56$ subjects reporting aortic aneurysm. Cardiac enlargement was reported by $64 \%$ of subjects, variably described as ventricular hypertrophy, hypertrophic cardiomyopathy, or dilated cardiomyopathy, based on information provided to them by their primary cardiologist or other physician (Figure 4b). However, in many of these cases, specific echocardiographic data are not available through the ICSR, and therefore cardiac features may be poorly defined; in a subset of 
subjects extensively characterized at the Washington University Cantú Syndrome Research Clinic between 2013 and 2018, cardiac enlargement was specifically confirmed as ventricular dilation (left ventricular end diastolic volume index $>2$ $Z$ score) and increased left ventricular mass index (partially reported in Levin et al., 2016), with increased left ventricular systolic function, in 29/32 (90\%) subjects. Thus, cardiac enlargement was accompanied by a high output state in the great majority of subjects in whom cardiac output was noninvasively measured.

Pericardial effusion was reported for $17 / 69$ (25\%) participants. In three severe cases that needed invasive treatment, pericardiocentesis was unsuccessful and pericardial stripping was required; in one individual, pericardiocentesis alone was required. In the remaining cases, medication was given, or no treatment was needed, presumably because the effusion was small and asymptomatic (Table 4). Although baseline ECG analyses typically do not reveal significant electrical defects (Levin et al., 2015), 12/65 (18\%) subjects reported a history of cardiac arrhythmia. Additionally, $14 \%$ of subjects reported a history of heart failure (Figure 4b). In most cases for which information was available (8/9 patients), the heart failure was identified in infancy, and potentially related to untreated or unrepaired PDA.

In this CS cohort, 16/71 (23\%) subjects reported blood pressure abnormalities, with $10 / 16$ responders reporting high blood pressure (half receiving medical treatment) and 6/16 reporting low blood pressure (Table 4). Interestingly, previous analysis demonstrated consistently low blood pressure for age (Levin et al., 2016), although this decrease did not reach clinical threshold for a hypotension diagnosis, and postural hypotension is not reported for any CS subjects.

\subsubsection{Pulmonary hypertension and respiratory abnormalities}

A history of pulmonary hypertension (PHN) was reported in 16/67 (24\%) subjects. This was first identified in infancy in $8 / 10$ patients for whom information is available, and was treated with sildenafil in 3 patients (Table 4 ). In 3/13 reporting subjects, PHN spontaneously resolved. PHN and PDA were co-incidental findings in 10/13 of these subjects. Bidirectional flow across the PDA indicated the presence of PHN. Resolution of PHN occurred with treatment (chemical or surgical) of the PDA in all 10 subjects. 
Four individuals reported respiratory tract malformations. One had bronchomalacia requiring a tracheostomy. One individual developed recurrent hemoptysis at age 20 and further investigation via pulmonary angiogram revealed a large bronchial collateral artery (aorto-pulmonary collateral artery, APC) that required coiling. Another individual had hemoptysis and arterial magnetic resonance imaging (MRA) showed multiple tortuous AV communications, requiring ligation, in both lungs; this individual also had bronchiectasis on CT scan. In a third individual, bleeding from abnormal vessels in the lungs required stapling. In addition to a relatively high incidence of respiratory tract infections, exercise intolerance and difficulty breathing were reported by a large fraction of subjects (Figure 4b). 


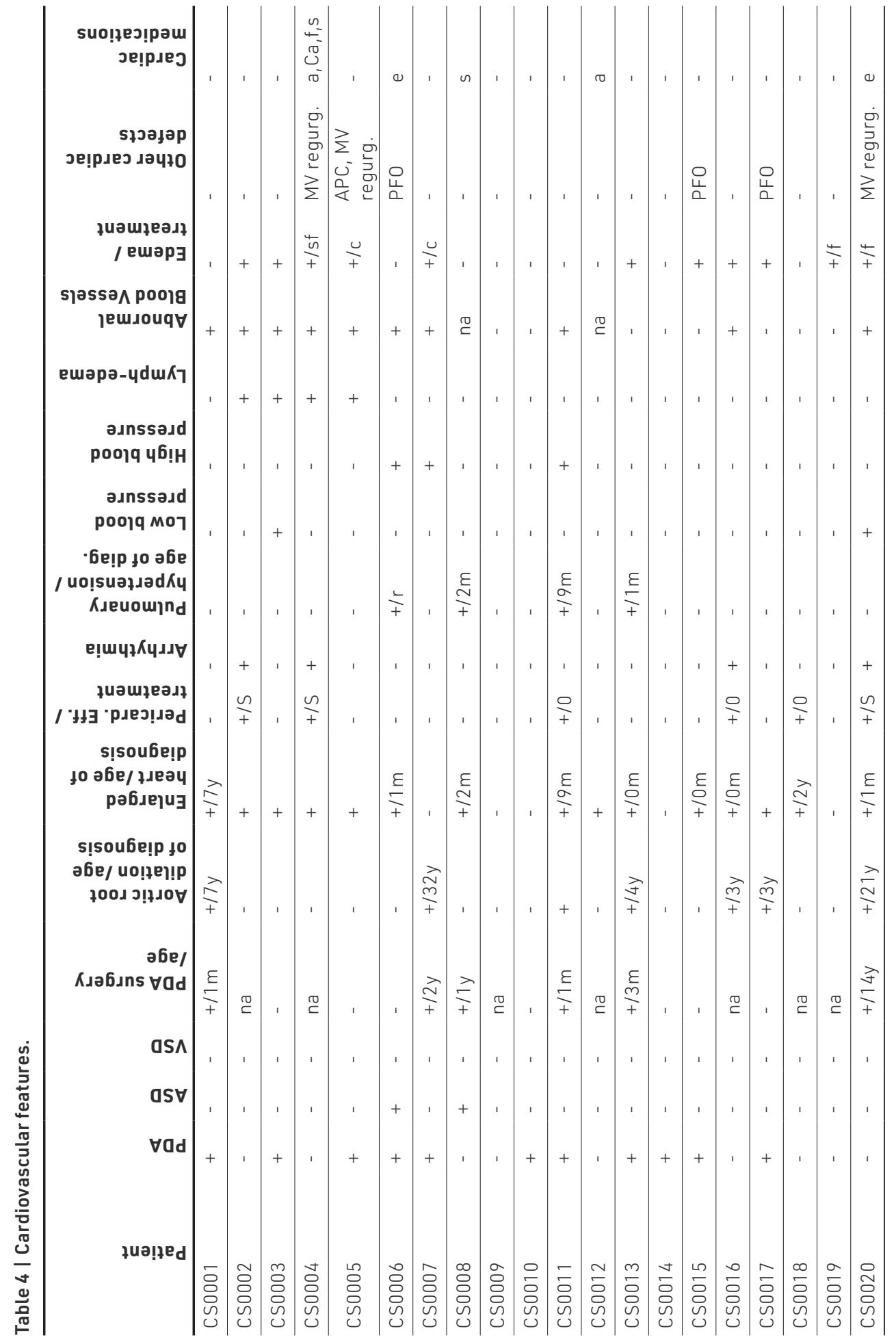




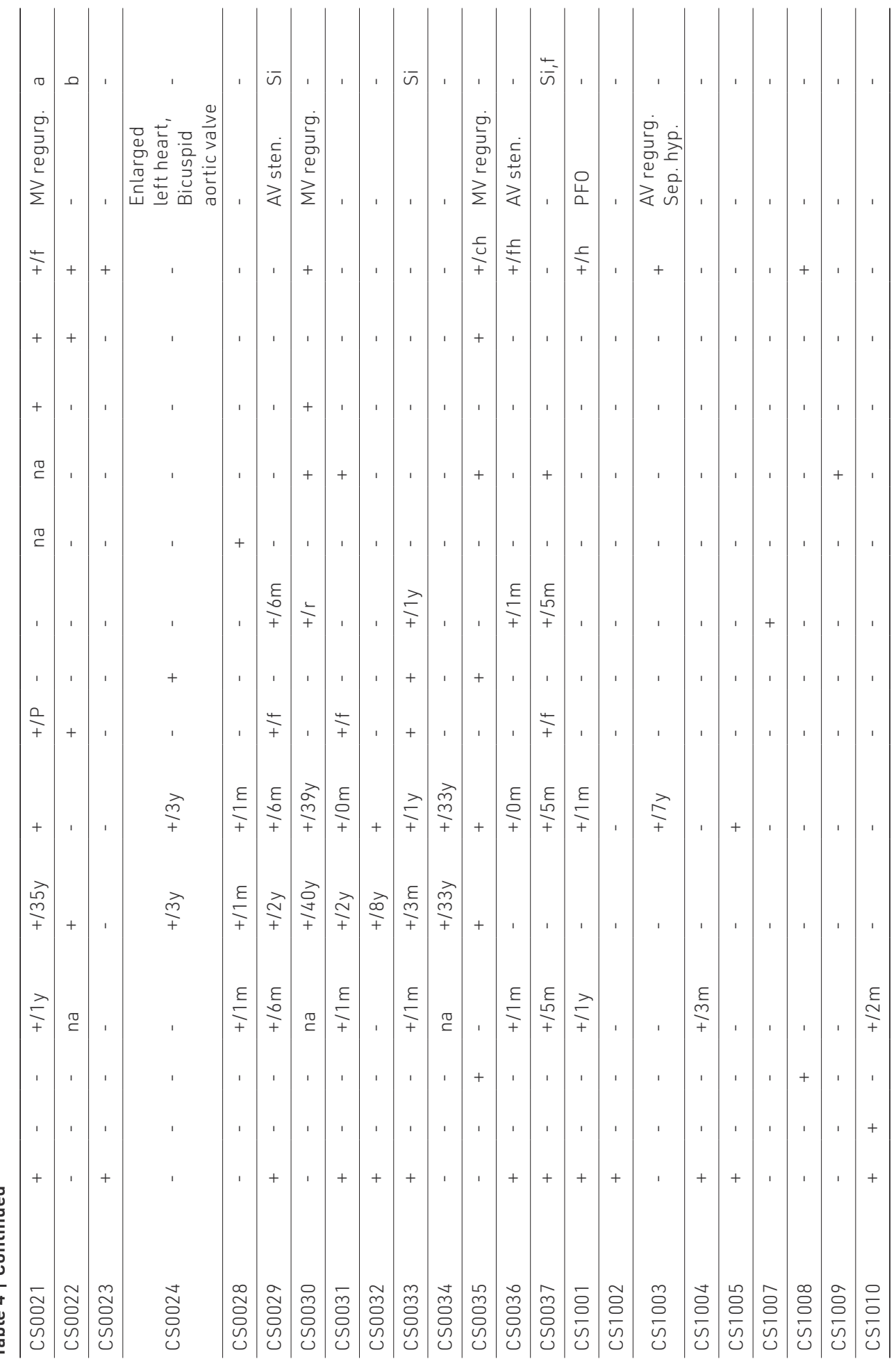




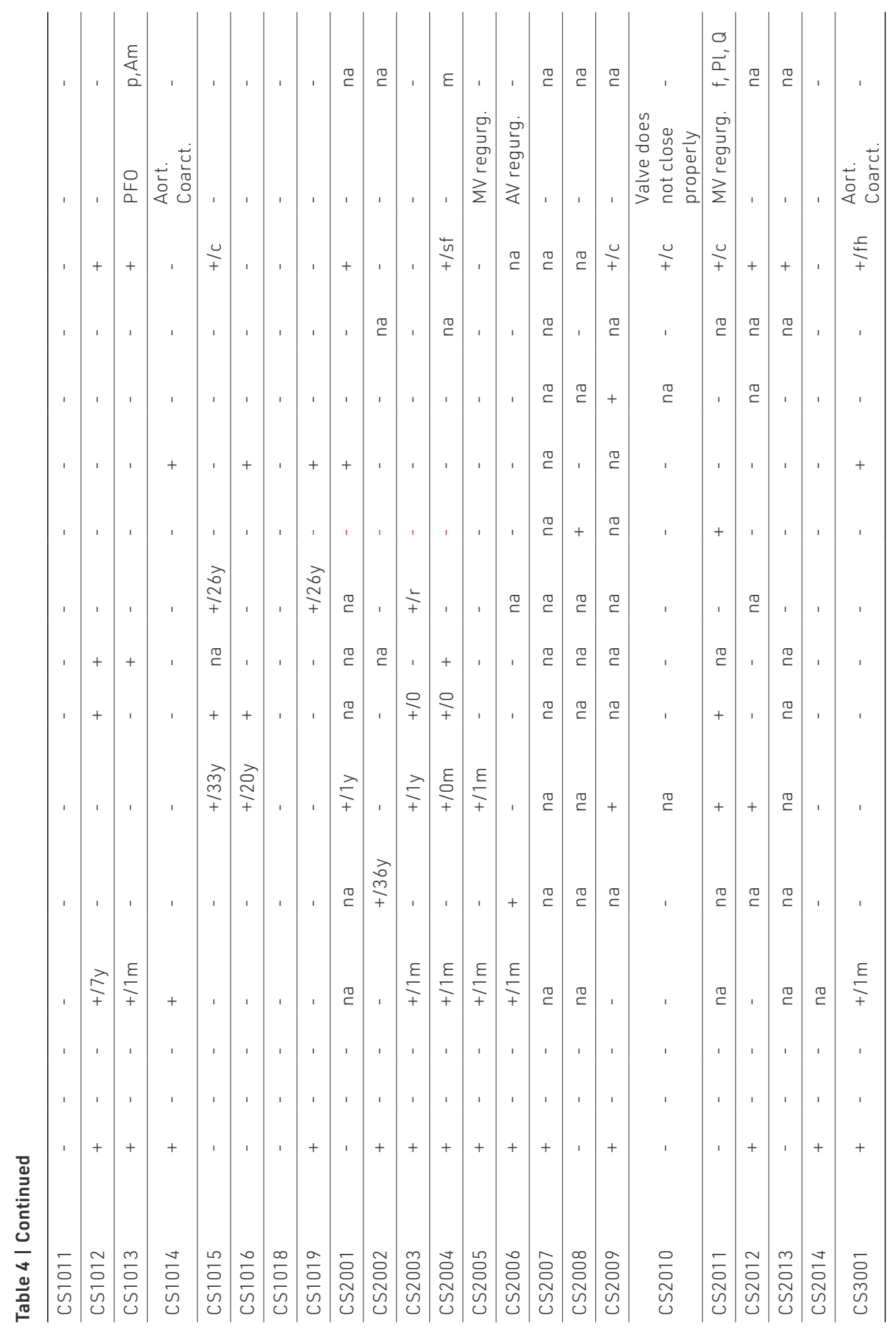




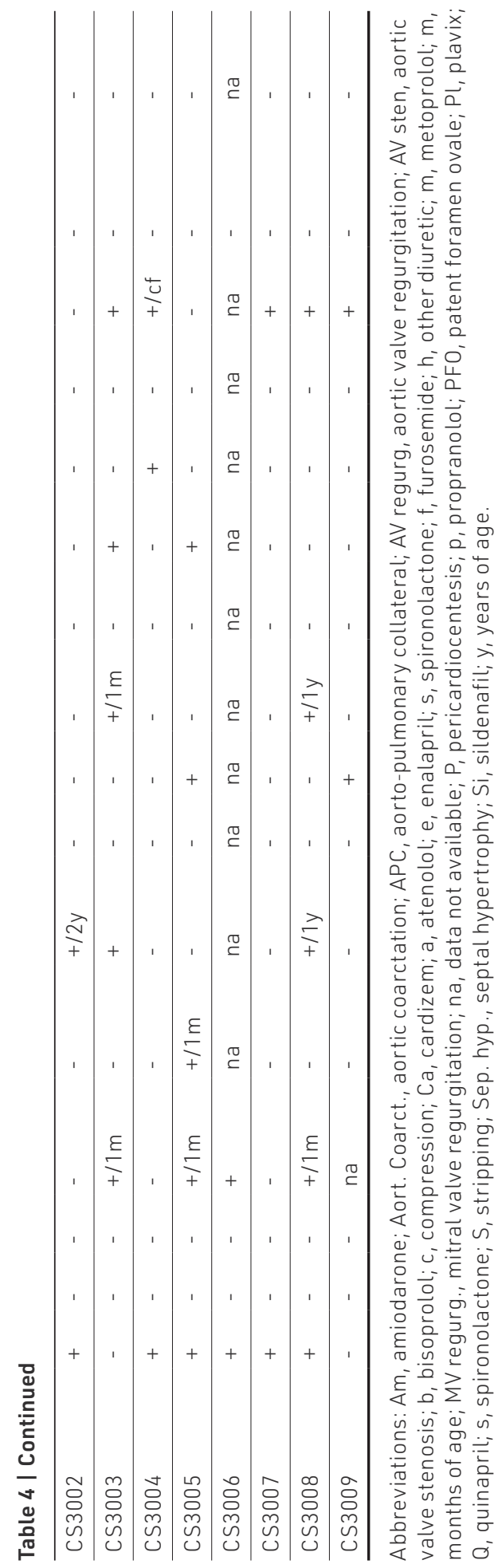




\subsubsection{Edema and lymphedema}

$36 / 70(51 \%)$ subjects report a history of edema, variously reported as edema or lymphedema, with lower legs most commonly affected (Figure 4d). The swelling in the lower legs was typically first recognized in adolescence or young adulthood, and was usually noted to spare the tops of the feet. Interestingly, edema was reported by women more frequently than men, the diagnosis being specified as lymphedema in eight female subjects, but not in any of the male subjects. Many subjects report attempting to manage edema using compression stockings as well as diuretic medications (Table 4) with varying success.
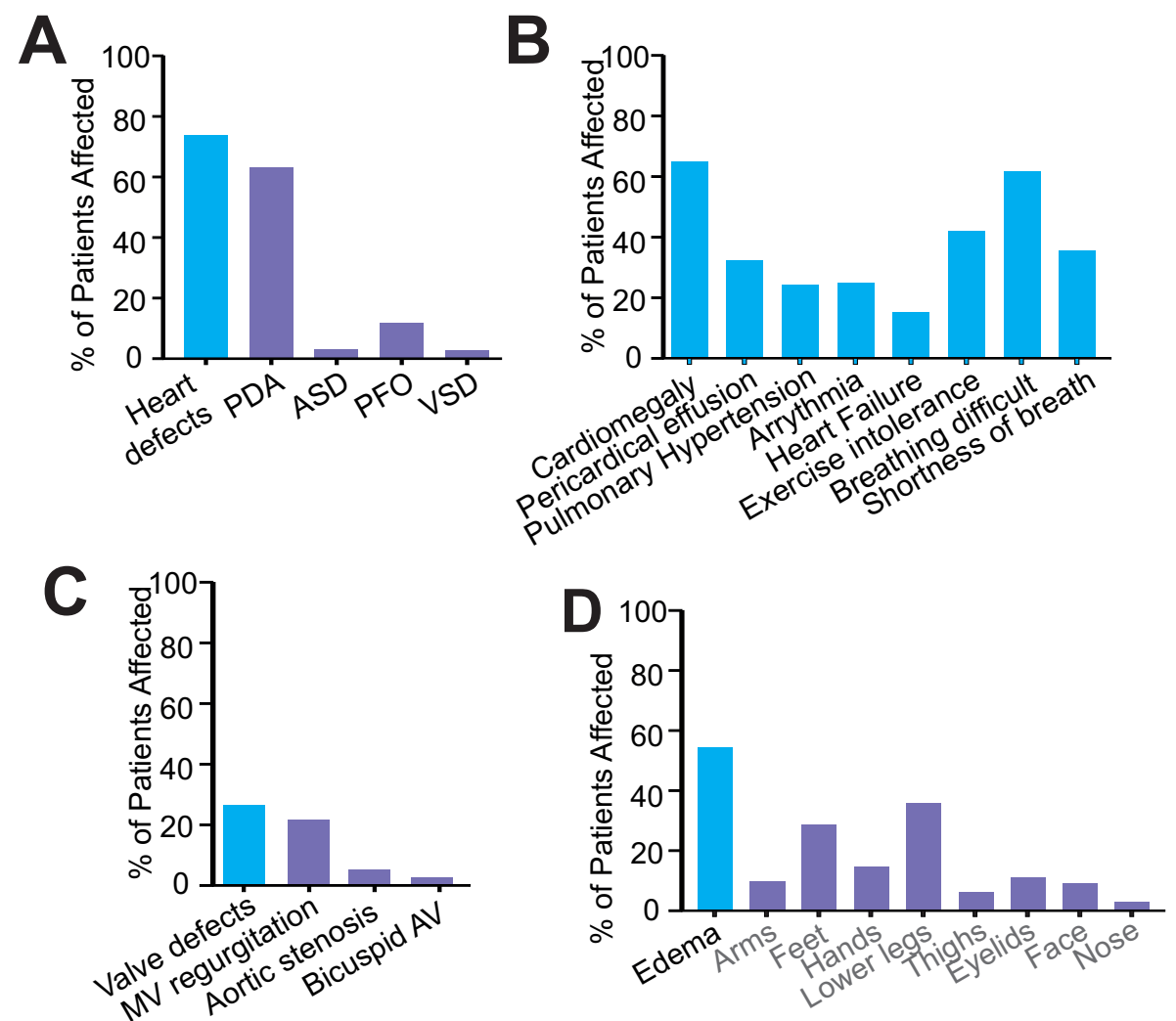

Figure 4 | Cardiovascular and edema features. (a-c) Incidence of cardiovascular abnormalities, and pulmonary and respiratory abnormalities. (d) Reported incidence of edema and location in CS subjects. CS, Cantú syndrome. 


\subsubsection{Skeletal and connective tissue abnormalities}

Osteochondrodysplasia has previously been associated with CS (Grange et al., 2014), including thickening of the calvaria, broad ribs, platyspondyly, ovoid vertebral bodies, narrow thorax and shoulders, pectus carinatum, scoliosis, and Erlenmeyer-flask-like long bones with metaphyseal flaring. In this registry cohort, $12 / 64(19 \%)$ subjects reported skeletal dysplasia or osteochondrodysplasia, $24 \%$ reported pectus carinatum, $21 \%$ reported scoliosis, $6 \%$ reported osteoporosis/osteopenia and $6 \%$ reported delayed bone age (Figure 5a). It should be noted that not all subjects have had a complete set of skeletal radiographs, so again these findings may be underreported.

Hyperflexible ("loose") joints were reported in 40/72 (56\%) of subjects (Figure 5b), in addition to loose and/or wrinkled skin with deep palmar/plantar creases, consistent with previous reports (Grange et al., 2014). Hernias were also reported, umbilical hernia $(28 \%)$ being most common, followed by inguinal hernia (16.9\%) (Table 1, Figure 5b).

\subsubsection{Gastrointestinal features}

Intestinal dysfunction was reported by $12 / 71$ (17\%) subjects, generally severe constipation or slow intestinal motility. Gastro-esophageal reflux was present in $15 / 36$ (42\%), gastrointestinal bleeding was reported in $7 \%$, and pyloric stenosis in infancy in $4 \%$ of subjects. $22 \%$ of subjects required a feeding tube as infants or young children (Figure $\mathbf{5 c}$ ).

\subsubsection{Renal features}

Renal problems were reported in $14 \%$ of subjects, and if present, consisted of recurrent kidney or bladder infections, hydronephrosis and vesico-ureteral reflux. Polycystic kidney disease was reported by one individual, and therefore it is unclear if this is related to CS, or represents a separate problem.

\subsubsection{Reproductive system features}

$74 \%$ of adult subjects reported that they reached puberty at the expected time. Abnormal menstruation was reported in 10/22 adult females, with heavy menses frequently noted. Male reproductive system defects were reported in only 1/34 responding subjects, who required surgery for an undescended left testicle. 


\subsubsection{Immune function}

Immune dysfunction was reported by $30 / 74$ (41\%) subjects. Recurrent respiratory infections are also cited, but specific information is not available in all cases. Low immunoglobulin levels were reported by several subjects, and five CS subjects in the cohort have been treated with IVIG. However, immunoglobulin levels checked in the CS Research Clinic at Washington University have all been essentially normal. Therefore, the relevance of this finding is unclear at this time, and further investigation is needed.

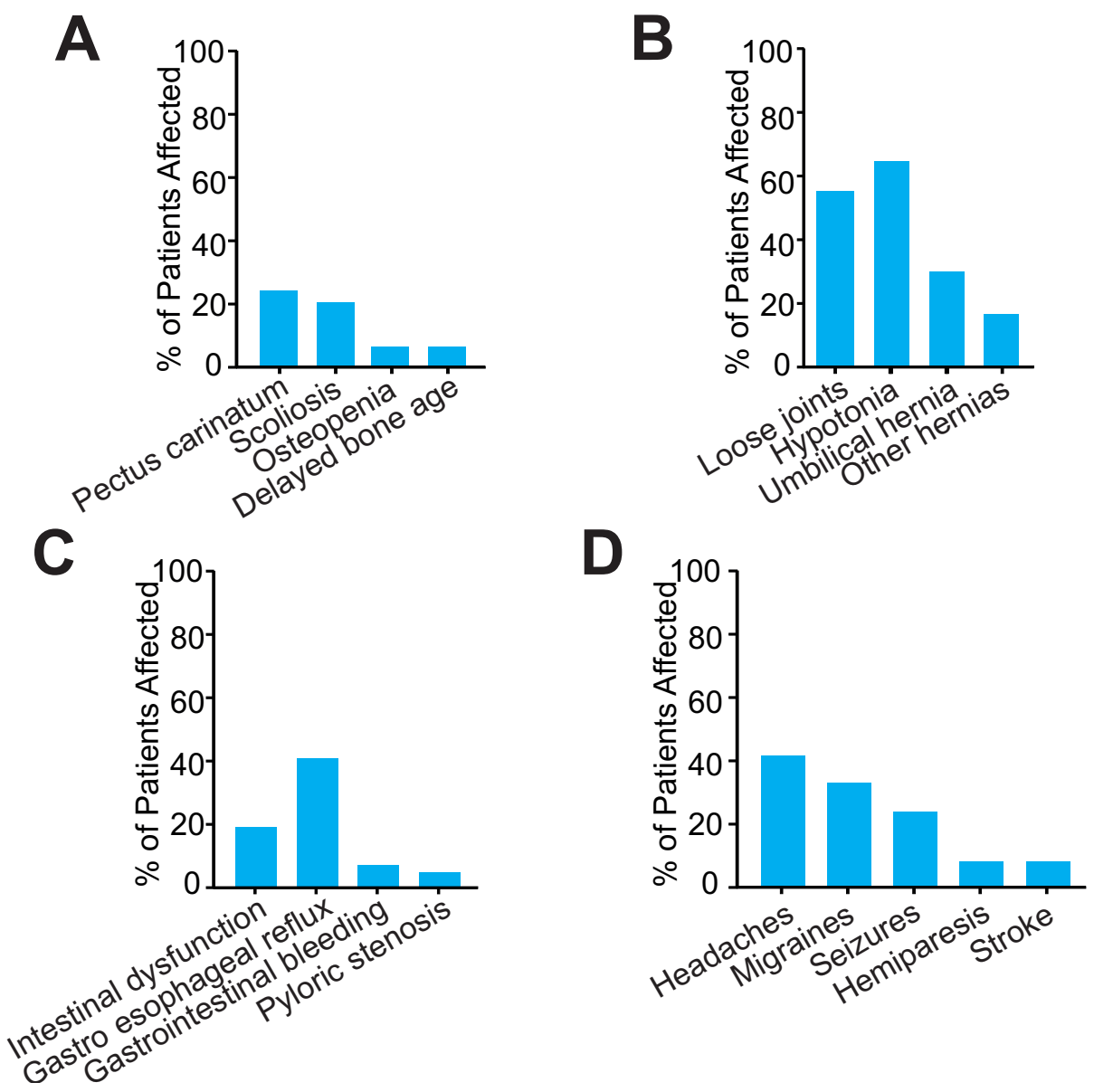

Figure 5 | Musculoskeletal (a) and connective tissue (b) abnormalities, (c) gastrointestinal features, and (d) neurological abnormalities. 


\subsubsection{Neurological and neurovascular features}

There were a variety of associated abnormal neurological features (Table 5) consistent with those reported previously (Leon Guerrero et al., 2016). 27/68 $(40 \%)$ subjects report frequent headaches, with 16 of these $27(60 \%)$ reporting migraine type headaches, often with associated aura, photophobia, phonophobia, and occasionally with transient hemiparesis (Figure 5d). In addition, 17/72 $(24 \%)$ subjects report a history of various seizures types including febrile, tonic-clonic, and absence seizures, and temporal lobe epilepsy. 8\% report hemiparesis, sometimes transient and associated with migraines, and $6 \%$ report a history of stroke (Figure $\mathbf{5} \mathbf{d}$ and Table $\mathbf{5}$ ). One individual had a stroke in infancy and has persistent unilateral weakness and two adults had strokes in their fourth and fifth decades. One adult had evidence of a prior stroke on brain imaging but the age at which it occurred is unknown and she has no current clinical symptoms.

Neuroimaging of the brain was available for $61 \%$ of subjects and revealed a variety of abnormal findings (Figure 6). Head and neck MR angiography revealed dilated and tortuous arteries, including internal carotids and vessels of the circle of Willis, in $10 / 10(100 \%)$ patients assessed at the Washington University site(Leon Guerrero et al., 2016). Abnormal or tortuous blood vessels were also observed in the retina of $34 \%$ of participants. Various additional brain abnormalities were reported in $11 / 49(22 \%)$ subjects, including hydrocephalus, "extra ventricle," dysgenesis of the corpus callosum, diffuse leptomeningeal enhancement, FLAIR hyperintensities in the subcortical white matter, intraventricular hemorrhage, mild lateral ventricular dilatation, bilateral thalamostriate vasculopathy, calcifications, and pituitary abnormalities. 

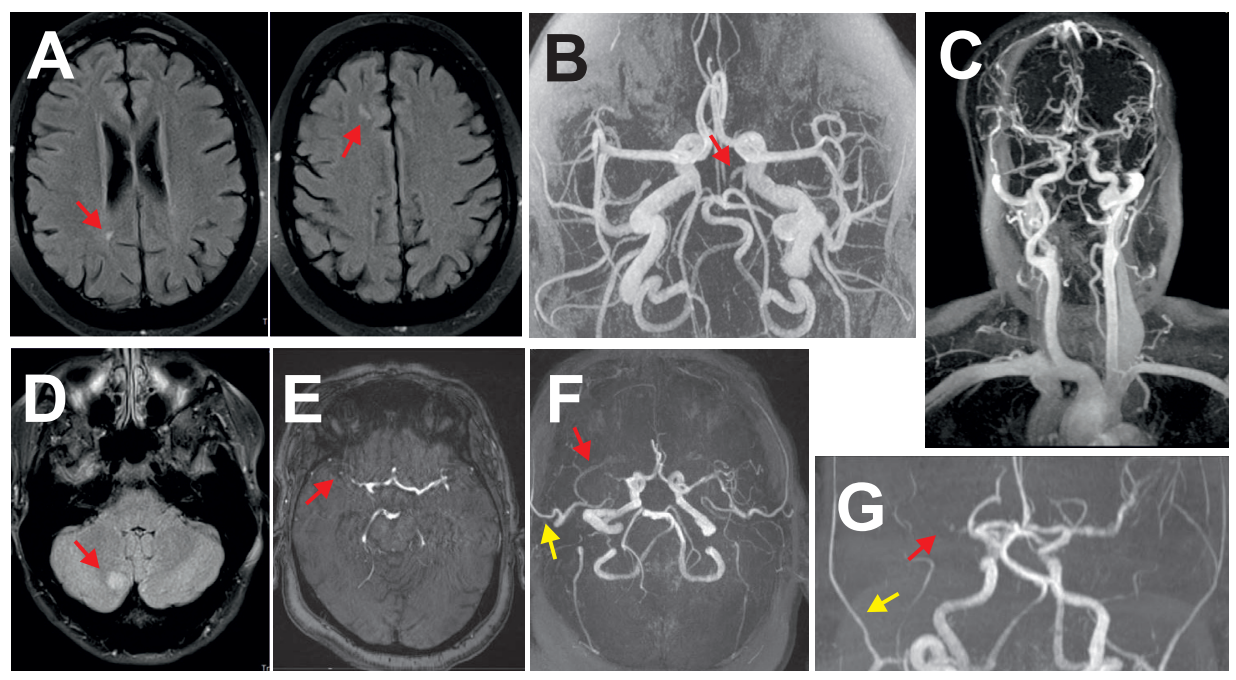

Figure 6 | Neuroimaging features. (a) MR images from a 49 year old female with CS. Nonspecific white matter lesions are frequently seen on MR imaging in CS and may be due to ischemia or demyelination, but etiology is unclear; ( $b$ and $\mathbf{c}$ ) Diffusely dilated and tortuous cerebral arteries have been identified in essentially all CS subjects undergoing imaging at Washington University; the red arrow indicates a persistent trigeminal artery in a 20 year old female with CS; (d) right cerebellar lesion without enhancement, mass effect or diffusion restriction, possibly due to ischemia or demyelination in a 33 year old female with CS; $(\mathbf{e}-\mathbf{g})$ MR images from a 57 year old female with CS showing mildly dilated cerebral arteries, occlusion of right MCA consistent with old stroke (red arrows), as well as right temporal artery collateral (yellow arrows). CS, Cantú syndrome. 


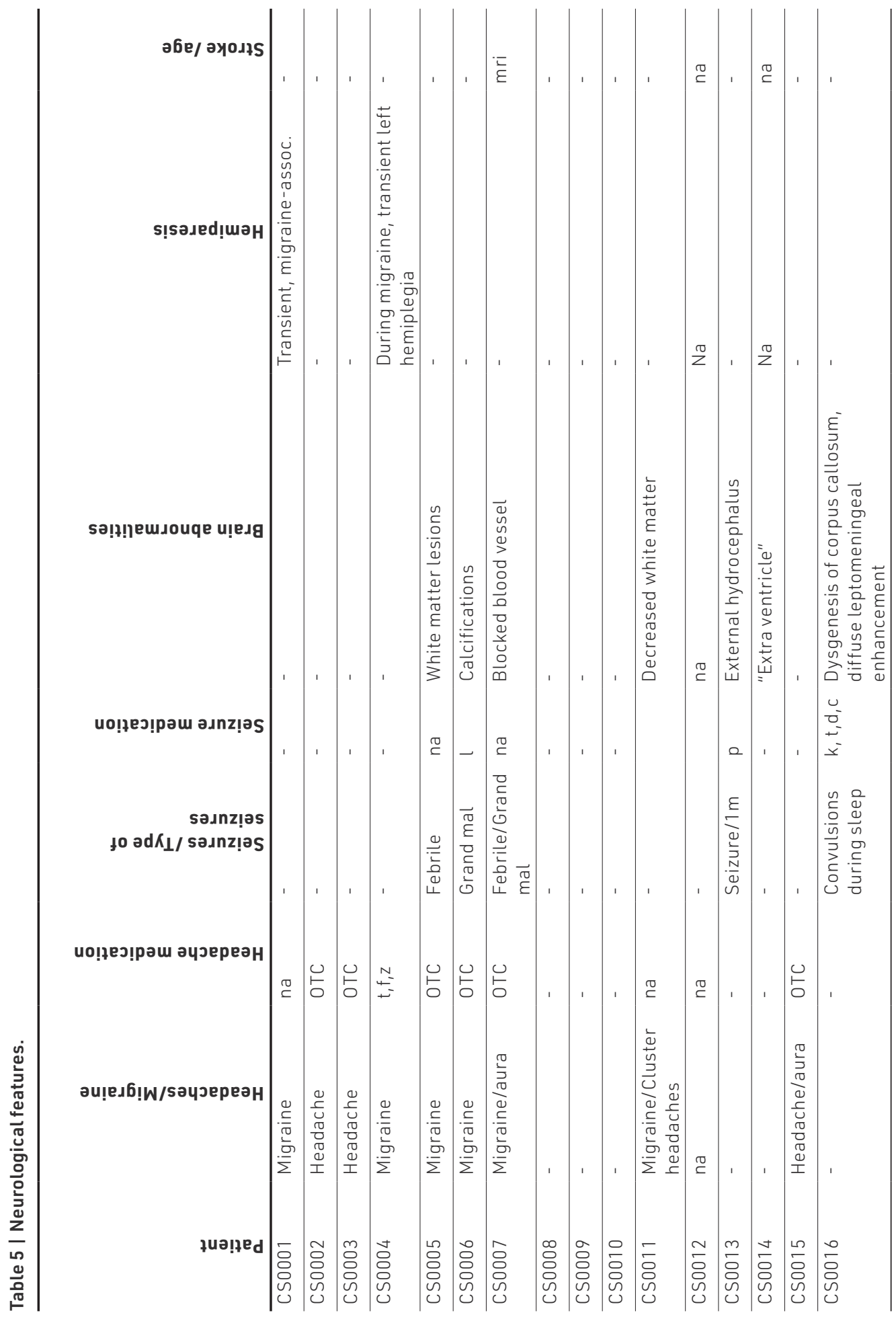




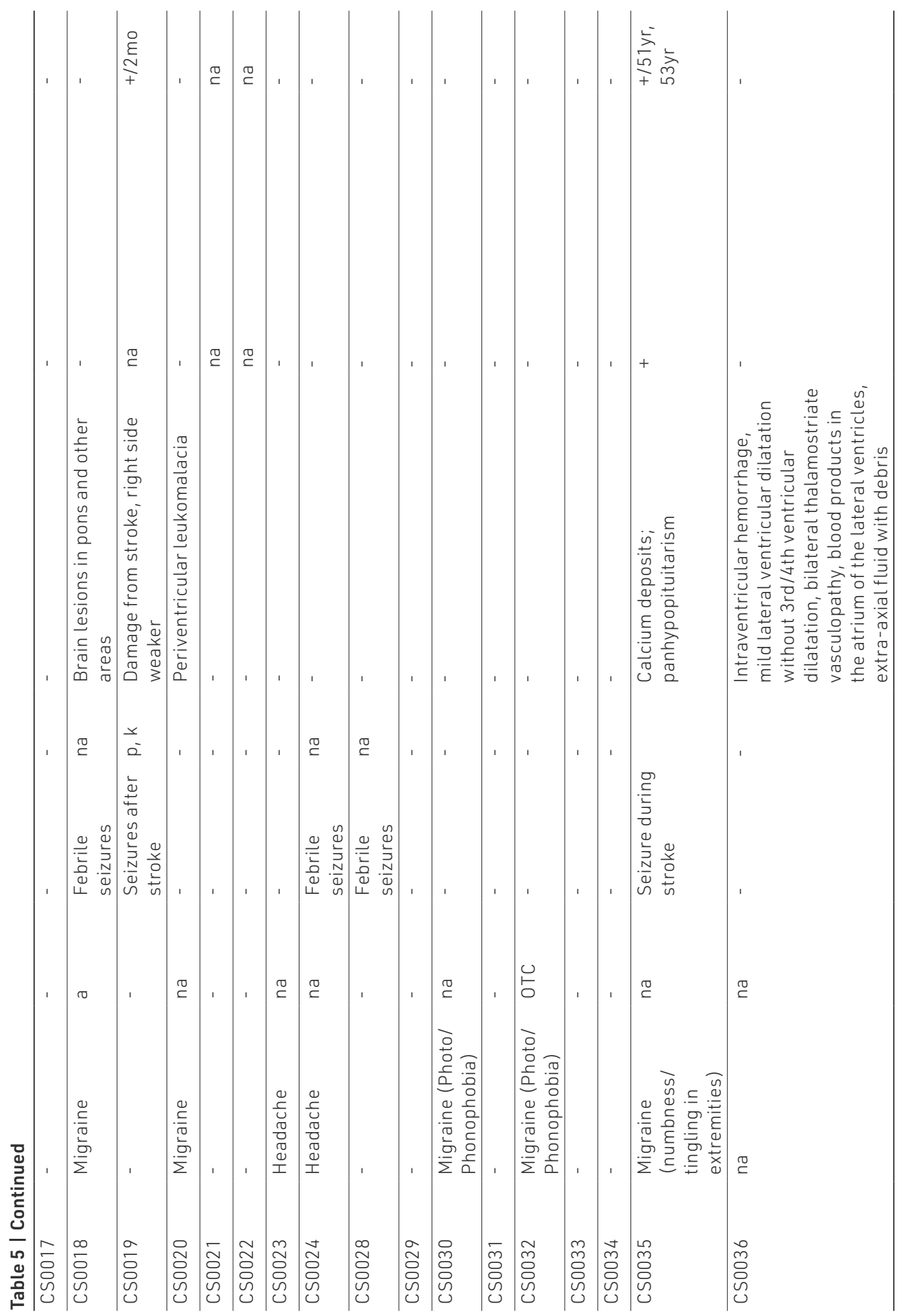




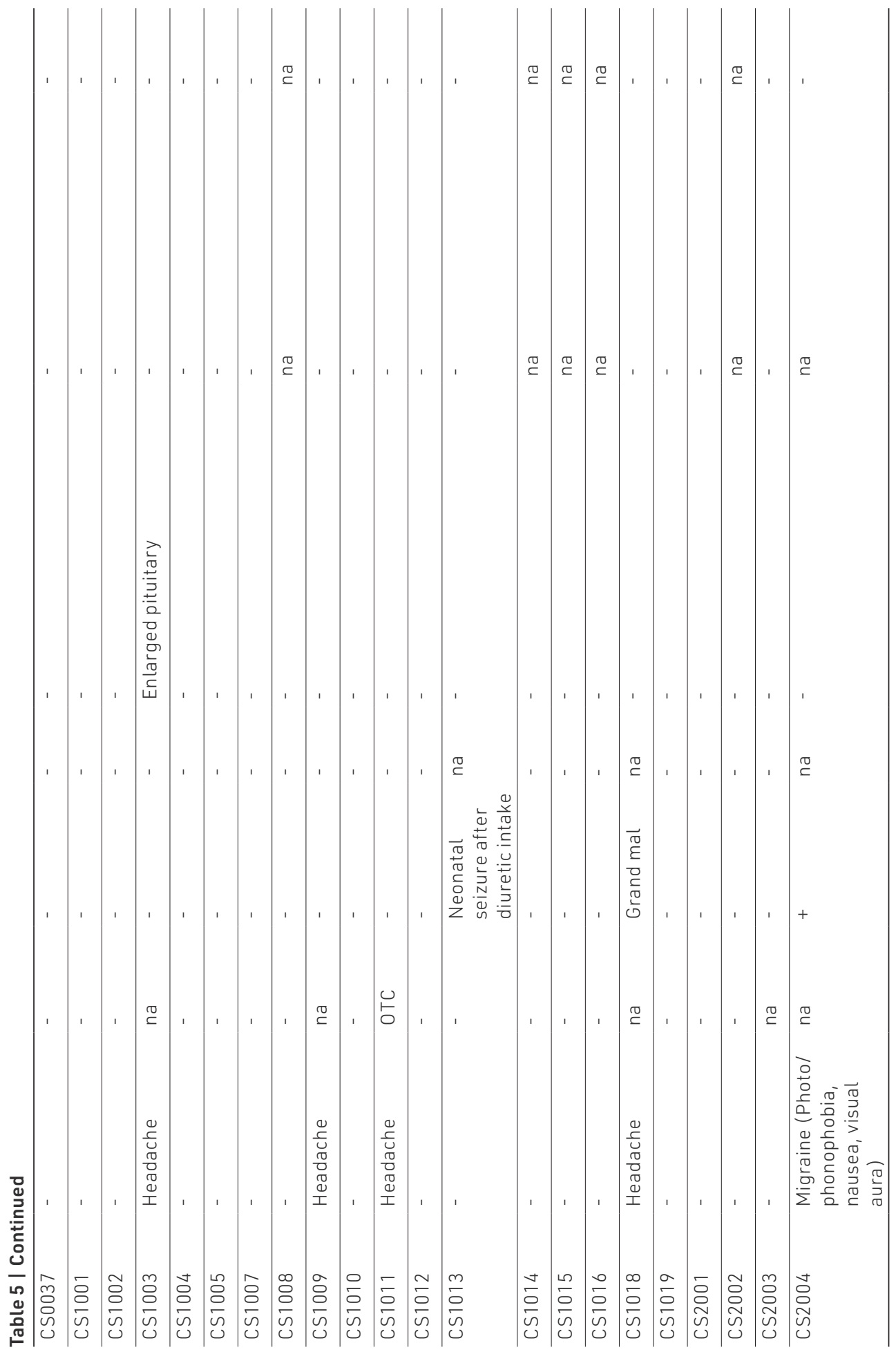




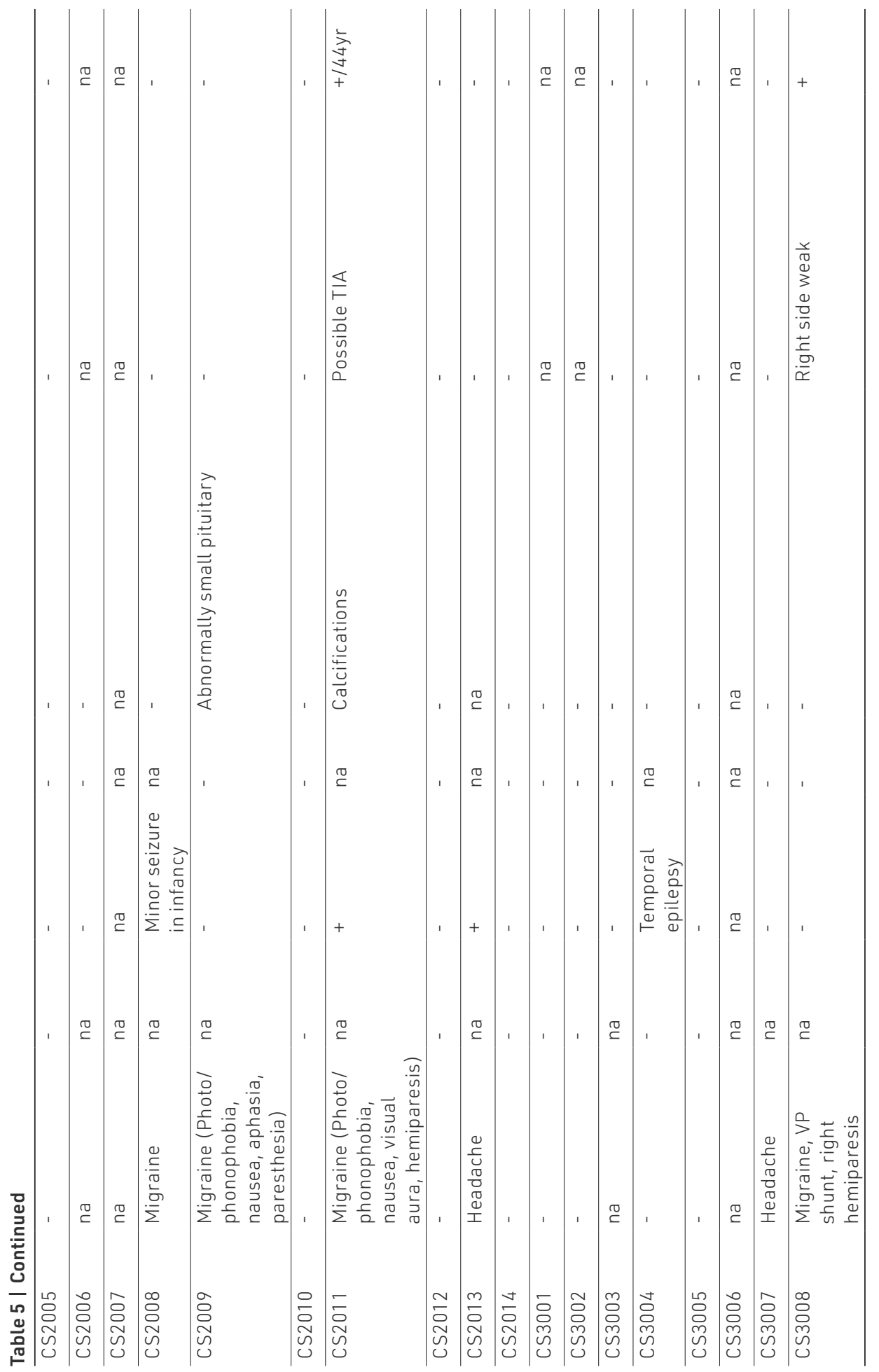




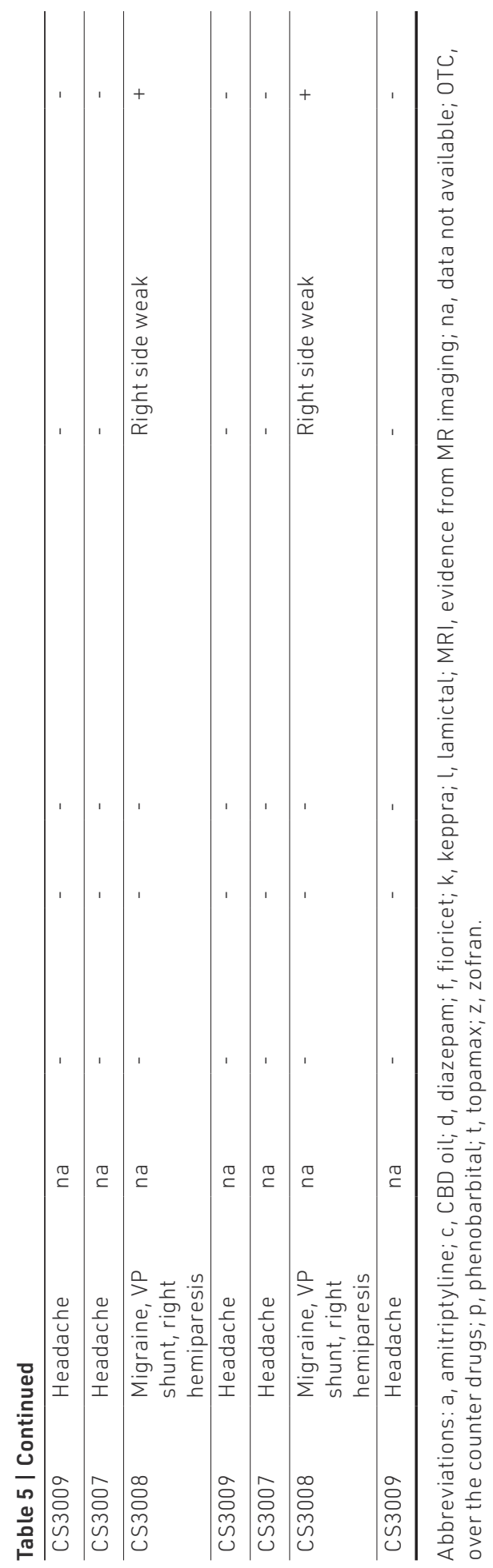




\subsubsection{Vision}

Myopia and hyperopia are the most frequent vision disturbances in CS subjects; $36 \%$ of patients report problems with eyesight and $32 \%$ wear glasses. Infrequently, nystagmus, strabismus, retinal, or optic nerve anomalies were reported.

\subsubsection{Hearing and ENT}

Five of 15 subjects reporting hearing loss (33\%) were diagnosed with conductive deficits, 1/14 (7\%) was diagnosed with sensorineural deficits, and 9/15 (60\%) were of unknown origin. Thirty-four subjects at the Washington University site responded to questions about ENT concerns and prior surgery; $8 / 34$ (24\%) subjects reported laryngomalacia. Three of $31(8.8 \%)$ subjects seen in the Washington University clinic had a tracheostomy, which was eventually removed in one case. Five of $32(14 \%)$ reported sleep apnea, and either adenoidectomy alone or both tonsillectomy and adenoidectomy was required.

\subsubsection{Endocrine problems}

A single individual reported panhypopituitarism, with multiple pituitary hormone deficiencies, including ACTH, TSH, FSH, and LH. One individual has diabetes insipidus. One individual has been treated for hypothyroidism since infancy. One individual was diagnosed with growth hormone deficiency and was on replacement therapy.

\subsubsection{Medications}

Eleven of $64(17 \%)$ affected subjects report taking cardiac medications, including ACE inhibitors, such as Quinapril and Enalapril, and beta-blockers such as Atenolol and Bisoprolol (Table 4), either currently or in the past. Of the subjects reporting hypertension, $63 \%$ are currently being treated with ACE inhibitors, and $60 \%$ with edema report current or previous medication treatment with diuretics, including furosemide, spironolactone and amiloride. Three subjects reported previous treatment for pulmonary hypertension with sildenafil (See cardiovascular section).

\subsubsection{Genetic testing and CS genotype}

ABCC9 variants were identified in 72/74 (97\%) participants in the ICSR cohort (Table 1, Table 6) and none harbor a variant in KCNJ8. Most subjects (60/74; 81\%) were initially given a clinical diagnosis of $C S$, subsequently confirmed by $A B C C 9$ sequencing or research or targeted exome sequencing in 58/60 cases. However, in 12 subjects $(12 / 72 ; 16.6 \%)$, the diagnosis was arrived at through exome sequencing, without CS being clinically suspected. In 16/72 (22\%) subjects 
with a molecular diagnosis, the $A B C C 9$ variant was inherited from an affected parent (16\% mother, 6\% father). Of 54 reporting participants, 11 have one or more affected sibling(s). This cohort includes eight families with two or three generations of affected individuals; five of these families have been evaluated at the Washington University CS clinic, two families are from the Netherlands and one is from Australia (See Table 1). In one of the families, a father was found to have mosaicism for the ABCC9 variant in blood, consistent with somatic and germline mosaicism in him. Clinically, he was only mildly affected in segments of his body, predominantly hypertrichosis, without evidence of systemic features, and he was only identified after diagnosis of a severely affected daughter. In the remaining individuals with negative family history, the ABCC9 variant was presumed to be de novo.

In every case, the diagnosis of CS was made after birth, with no instances of prenatal diagnosis. Since the two genes associated with CS have only recently been identified, older individuals often lack a molecular diagnosis. Hence, a majority of confirmed subjects were diagnosed either within the first year of life or after age 17, and some parents participating in this study were only diagnosed with CS after their affected children received the diagnosis. Interestingly, $41 \%$ of participants initially received other diagnoses, including lysosomal storage disorders such as a mucopolysaccharidosis, or Beckwith-Wiedemann syndrome, based on coarse facial features, or Pompe disease based on neonatal cardiomegaly.

We identified 28 different $A B C C 9$ variants in 72 participants (Table 1, Table 6), applying targeted Sanger sequencing of the ABCC9 gene, or exome sequencing (ES) or genome sequencing (GS). Fifteen variants have not previously been reported. The majority of reported subjects show simple missense variants in $A B C C 9$, and only 1 individual harbors a deletion-insertion variant involving two nucleotides. Figure 7 shows the protein architecture of the ABCC9-encoded $\mathrm{K}_{\text {ATP }}{ }^{-}$ channel SUR2 subunit, including all documented variants and their distribution across different protein domains. A majority of variants $(68 \%)$ cluster around transmembrane domain 2 (TMD2). Variants at p.Arg1154 (identified in 24 subjects, 33\%) and p.Arg1116 (identified in 11 subjects, 15\%), represent the most commonly observed (Table 6).

\subsubsection{Comparison of phenotypes by genetic variants}

We evaluated the correlation of $A B C C 9$ variants with syndromic features. We have not identified any obvious difference in frequency or degree of craniofacial dysmorphology, hypertrichosis or skin appearance depending on the site of 
the gene variant, and cardiovascular and neurological anomalies were similar across the various variant sites (Tables 4 and $\mathbf{5}$ ). Even within families having multiple affected members, there is highly variable expression of CS features, in common with other autosomal dominant conditions. In one family (Figure 8), in which the mother was diagnosed only after her children, the 8-year-old boy (Figure 8b) has autism spectrum disorder and developmental delays, the 6-yearold boy (Figure $\mathbf{8 c}$ ) has typical features of CS, without evidence of autism, while the 3.5 year old girl (Figure $\mathbf{8 d}$ ) had esophageal atresia and a PDA that required surgery, as well as pulmonary hypertension and a requirement for oxygen supplementation and gastrostomy tube feedings for the first 2 years of life.

Table 6 | ABCC9 variant distribution in 72 subjects with Cantú syndrome.

\begin{tabular}{|c|c|}
\hline Pathogenic $A B C C 9$ variant & $\begin{array}{c}\text { \# of Patients } \\
\text { (\# of Kindreds) }\end{array}$ \\
\hline p.His60Tyr & $2(2)$ \\
\hline p.Asp207Glu & $3(3)$ \\
\hline p.Gly294Glu & $2(1)$ \\
\hline p.Gly380Cys & $1(1)$ \\
\hline p.Pro432Leu & $1(1)$ \\
\hline p.Ala478Val & $2(1)$ \\
\hline p.Asp793Val & $1(1)$ \\
\hline p.Gly815Ala & $1(1)$ \\
\hline p.His1005Leu & $2(2)$ \\
\hline p.Trp1018Gly & $1(1)$ \\
\hline p.Thr1019Glu & $1(1)$ \\
\hline p.Thr1019Lys & $1(1)$ \\
\hline p.Ser1020Pro & $1(1)$ \\
\hline p.Phe1039Ser & $1(1)$ \\
\hline p.Ser1054Tyr & $2(2)$ \\
\hline p.Arg1116Cys & $6(3)$ \\
\hline p.Arg1116Gly & $1(1)$ \\
\hline p.Arg1116His & $5(4)$ \\
\hline p.Arg1154Gln & $13(9)$ \\
\hline p.Arg1154Gly & $1(1)$ \\
\hline p.Arg1154Trp & $10(10)$ \\
\hline p.Thr1202Met & $1(1)$ \\
\hline p.Asn1206Lys & $2(2)$ \\
\hline p.Ser1235Phe & $1(1)$ \\
\hline p.Val1266Met & $1(1)$ \\
\hline p.Arg1347Leu & $5(3)$ \\
\hline p.Val1490Glu & $1(1)$ \\
\hline p.Ala1494Thr & $3(1)$ \\
\hline Unknown & 2 \\
\hline
\end{tabular}




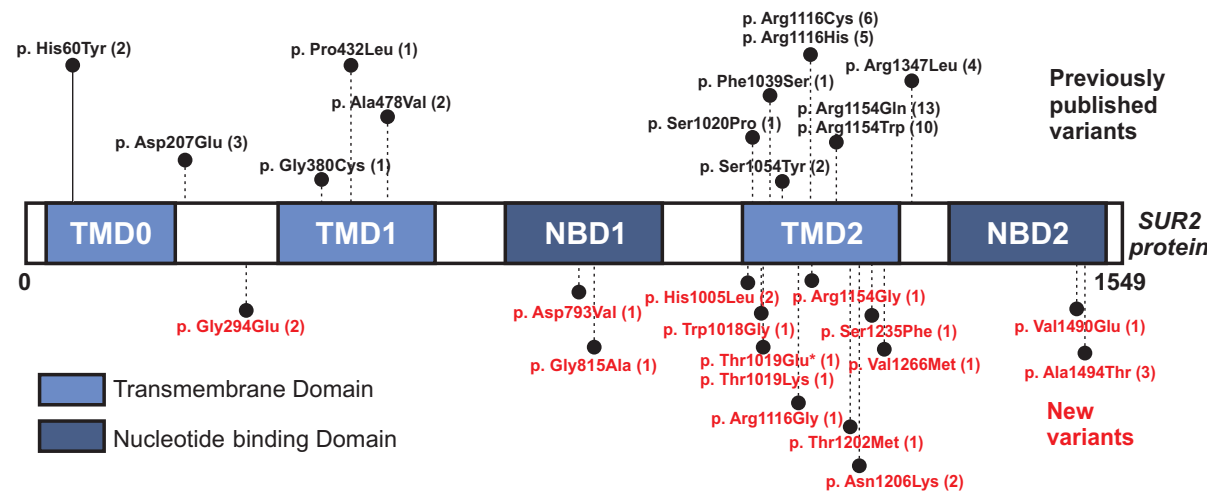

Figure 7 I Location of CS variants in the SUR2 protein. Schematic of SUR2 protein domain architecture: TMDO, transmembrane domain 0 , with five predicted transmembrane helices; TMD1, with six transmembrane helices; NBD1, nucleotide-binding domain 1; TMD2, with six transmembrane helices; and NBD2. Amino acid changes identified in CS subjects are indicated. The number of subjects harboring each variant is indicated in brackets. Previously published variants are shown in black and new variants in orange. Transmembrane domains (TMDs) are highlighted in light blue, nucleotide-binding domains (NBDs) in dark blue. Deletion-insertion variants are indicated by an asterisk. CS, Cantú syndrome.
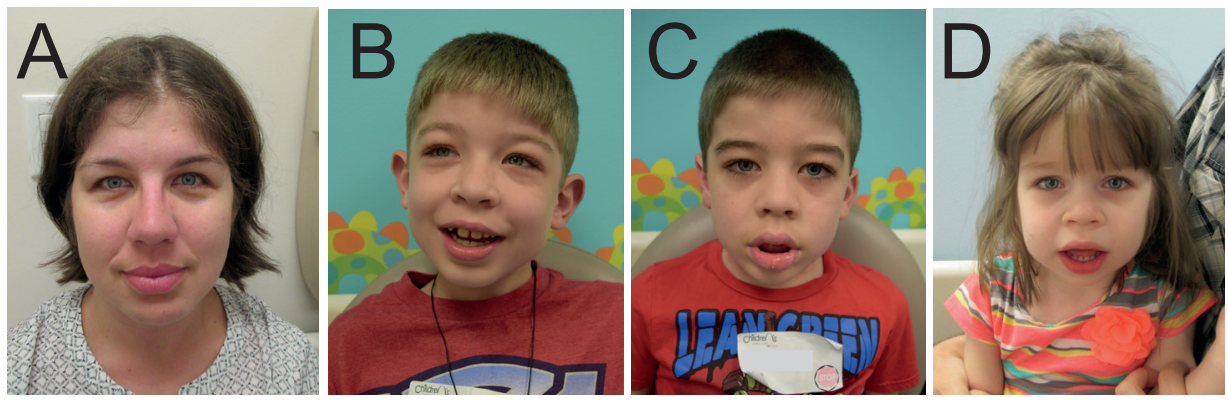

Figure 8 | Intra-family presentation of Cantú syndrome. Mildly affected mother (a) and her three children who are variably affected (see text). 


\subsection{Discussion}

The identification of pathogenic variants in ABCC9 and KCNJ8 as the molecular genetic basis of CS has led to the diagnosis of numerous additional patients, as well as increased awareness of the syndrome in the field of clinical genetics. This study reports multiple novel CS-associated ABCC9 variants, further defines the genetic basis of the disease, and provides quantitative assessment of the penetrance of disease features.

Notably, the majority of subjects in our cohort are children or young adults, with $59 \%$ below the age of 15 at the most recent examinations (Table 2, Figure 1), consistent with a previous analysis of all reported cases of CS in which $61 \%$ of patients were children (Roessler et al., 2018). Since the genes associated with CS have only recently been identified, we suspect there may be many affected individuals, particularly older individuals, who have not received genetic testing and therefore remain undiagnosed.

\subsubsection{The spectrum of CS features}

Abnormal growth is common in CS, and may be identified both prenatally and postnatally. Birth length and weight are consistently above average, irrespective of gestational age, which suggests the possibility that the increased incidence of prematurity may be related to excessive in utero growth. Polyhydramnios is a known risk factor for premature delivery, but we did not observe a correlation between presence of polyhydramnios and early delivery. Our analysis shows that macrocephaly is typically present at birth and persists throughout life (Figure 1, Table 3). Both birth weight and length are increased, with some adult males continuing to show elevated height (Table 3 ). A thin or even quite muscular appearance during childhood changes due to weight gain that occurs after puberty.

Previous reports have noted developmental delay in CS patients (Grange et al., 2014). It is clear that developmental milestones are delayed in a majority of CS patients, and motor development may be impaired due to hypotonia or joint laxity. There is also an increased frequency of reported behavioral problems, including ADHD and autism spectrum features in young patients. However, despite early developmental delays, most patients with CS attain normal cognition and intellectual function by adulthood; speech develops normally in most patients and assessed intelligence tends to be normal, with most children attending regular schools. Cerebrovascular abnormalities have been recognized in CS patients, including dilated and tortuous cerebral vessels, as well as white 
matter changes and persistent fetal circulation (Figure 6) (Brownstein et al., 2013; Leon Guerrero et al., 2016). Additional neuroimaging studies in our larger cohort reveal these features to be common. Although further studies are required, increased blood flow, associated with vascular dilation in the brain, may be causally associated with the increased incidence of headaches and migraines in CS patients (Table 5). There appears to be an increased frequency of stroke in this CS cohort, but the long term risk is unclear at this time. Brain imaging, including evaluation of the cerebral vasculature, should be considered for all patients with CS, especially those with headaches and other neurological symptoms.

Previous analyses of CS patients have revealed multiple cardiovascular abnormalities, both developmental and pathophysiological (Grange et al., 2014). PDA was reported most frequently and required surgical closure in almost half of affected individuals. Cardiomegaly, due to ventricular enlargement, with ventricular systolic function either normal or increased, is a common finding. The resultant increased cardiac output (high output state) is distinct from dilated cardiomyopathy, in which the ventricles are dilated and have decreased systolic function. It is also distinct from hypertrophic cardiomyopathy in which ventricular walls are thickened but cardiac chamber cavity is either normal or smaller than normal. There is no evidence of diastolic dysfunction in the majority of patients. The majority of subjects that have been followed long term ( $>5$ years) have not developed heart failure, but they are still relatively young. Two older individuals have developed high output heart failure, potentially exacerbated by inappropriate treatment with ACE-inhibitors. Thus, while the "high output state" may ultimately lead to cardiac dysfunction, long-term natural history, and cardiac outcomes are yet to be determined.

Dilated and tortuous large arteries, as well as persistent fetal arteries, are common findings in CS (Hiraki et al., 2014; Leon Guerrero et al., 2016). PDA is disproportionately associated with pulmonary hypertension, suggesting a common origin due to increased blood volume. The consistent finding of cardiomegaly with preserved cardiac function (Levin et al., 2016) is replicated in mice (Huang et al., 2018) and zebrafish (Tessadori et al., 2018) carrying CRISPR/Cas9 engineered CS variants in ABCC9. These animal studies suggest that cardiac enlargement and enhanced cardiac output is a secondary response to decreased vascular resistance and lowered blood pressure (Huang et al., 2018). In this case, as with persistent PDA, increased pulmonary blood flow may contribute to the development of pulmonary hypertension in the long term due to risk of the development of pulmonary vascular occlusive disease, and therefore, 
treatment with phosphodiesterase type 5 inhibitors such as sildenafil might not be appropriate.

Edema has been reported previously in CS subjects (Grange et al., 2014), and is a common finding in the present cohort; generalized edema is often present at birth and resolves, but reappears later in life, usually in adolescence or young adulthood. In some subjects, this is reported specifically as lymphedema, and it is unclear at this juncture whether lymphatic dysfunction is the underlying basis more broadly. As discussed below, it is possible that both vasodilation and lymphatic dysfunction may share a common origin in CS, related to decreased smooth muscle activity. A high frequency of pericardial effusion, clearly detected in the present cohort, may also be related to increased blood volume or perhaps to reduced cardiac lymphatic drainage. Treatment is often needed, including pericardial stripping in extreme cases.

\subsubsection{Genotype-phenotype correlation and CS}

Since the initial reports of an association of CS with variants in ABCC9 (Harakalova et al., 2012; van Bon et al., 2012), and KCNJ8 (Brownstein et al., 2013; Cooper et al., 2014), it has become clear that pathogenic variants in one or the other of these two genes is the primary cause of $C S$, but the expression of disease features is clearly quite variable. A sub-set of patients with ABCC 9 variants, but without evidence of cardiovascular or other features beyond hypertrichosis and characteristic facial features, have previously been defined as distinct syndromes-hypertrichosis with acromegaloid facial features (HAFF) or acromegaloid facial appearance (AFA) (Afifi et al., 2016; Harakalova et al., 2012). The present study further emphasizes the variability of clinical features, even with the same gene variant, and in multiple individuals within a single family. Patients reported as having HAFF or AFA clearly fall within the milder end of the CS spectrum, and therefore, we strongly suggest that such alternative terms should no longer be used.

We attempted to correlate severity of clinical features with genotype for the most common variants (p.Arg1116 and p.Arg1154), but it is clear that there is essentially as much variability of features among patients carrying these variants as between genotypes. While it remains important to draw quantitative phenotype-to-genotype comparisons where possible, a bigger cohort may be required. In the present study, there are several families with multiple affected family members in whom variability is demonstrated (Figure 8). Variability is present in multiple organ systems, and especially notable in cardiac-related abnormalities. In some family members, recognition of CS would have been 
difficult if the proband had not been diagnosed and available for comparison. These observations confirm findings of intrafamilial variability previously described in the literature (Czeschik et al., 2013; Hiraki et al., 2014; Roessler et al., 2018).

It is important to note that even in this extended cohort no individual has been recognized to carry a pathogenic missense variant in ABCC9 without showing a clinically relevant phenotype. One individual in this cohort who was only mildly affected was found to have somatic and germline mosaicism for the ABCC9 variant, suggesting that mosaicism might account for milder clinical features in some cases. Since not all relatives of individuals with CS have been evaluated for the presence of the variant found in the proband, we cannot rule out nonpenetrance; however, the present data do not suggest this. So far, only two individuals harboring variants in KCNJ8 have been identified (Brownstein et al., 2013; Cooper et al., 2014). In human neonatal diabetes resulting from equivalent mutations in the paralogous KCNJ11 (Kir6.2) and ABCC8 (SUR1) genes, heterozygous activating variants in KCNJ11 account for approximately three times as many cases as variants in ABCC8 (Naylor, Greeley, Bell, \& Philipson, 2011). Both of the identified individuals with a KCNJ8 variant have key clinical features of CS including congenital hypertrichosis, macrosomia at birth, macrocephaly, coarse facial appearance, cardiomegaly, skeletal abnormalities, and developmental delay, with reported severity equal to or perhaps greater than that seen in ABCC9 related CS. Mouse models of CS in which human CS variant equivalents have been introduced to the endogenous $A B C C 9$ and KCNJ8 loci (Huang et al., 2018) indicate that, at least for the Kir6.1 (p.V65M) variant, the features are more severe than for the introduced ABCC9 (SUR2 [p.A478V]) variant. It is therefore unclear why the incidence of KCNJ8 related CS is so low, and raises the possibility that such mutations might frequently be lethal. Regulation of Kir6.2/SUR1 and Kir6.1/SUR2-dependent $\mathrm{K}_{\text {ATP }}$ channel activity by cytoplasmic nucleotides is qualitatively similar (in both cases, ATP inhibits the channel by binding to the Kir6 subunit, Mg nucleotides activate by interaction with the SUR subunit), but there are important quantitative differences (Nichols, 2006), and tissue dependent modulation by second messengers may be very different (Flagg, Enkvetchakul, Koster, \& Nichols, 2010). 


\subsection{Conclusion and Prospectus}

The current study represents the largest survey of individuals $(n=74)$ with CS published to date. The most notable diagnostic features are hypertrichosis and facial dysmorphology, which are typically evident at birth. Additionally, we confirm a high incidence of PDA, cardiomegaly, edema and migraines or severe headache in CS patients. More than half of the mothers of CS babies exhibit polyhydramnios, and babies with CS are found to be at a high risk for preterm delivery (<37 weeks). Macrocephaly is typically observed at birth and persists throughout life. Adult females fall within the normal range for height, but adult males may be taller than average, although data are limited in this cohort. Developmental delay is commonly reported in young children, but intellectual disability is not common, and adult intelligence is typically normal. The complex clinical presentation of CS may be attributed to the presence of SUR2/Kir6.1 channels in multiple tissues throughout the body (Nichols et al., 2013), as well as the variable severity of features resulting from any given gene variant, even the same variant within the same family. This variability points to yet unidentified genetic or other modifiers that influence the phenotypic outcome.

The ICSR has been built not only in order to generate a critical mass of affected individuals to identify new clinical characteristics of the disorder, but also to ensure rapid progression toward clinical application of current genetic and scientific study results. Moving forward, the hope is that the registry will continue to expand to include as many individuals as possible from around the world and to generate longitudinal data, essential to understanding the longterm effects and implications of CS pathophysiology, as well as to provide essential data and a large well-characterized patient cohort for development of a directed pharmacological treatment for CS. Currently, there are no targeted therapies for CS available and it is not known to what extent associated features can be reversed once manifest. Hence, clinical management at this time involves symptomatic treatments to address secondary complications.

There are undoubtedly still numerous undiagnosed or misdiagnosed cases of CS, especially individuals of older ages. While commercial gene sequencing is available for clinical genetic diagnostic purposes in most advanced countries, such facilities are not available to all. The authors welcome contact from patients/caregivers, both regarding inclusion in the ICSR, and for potential inclusion in annual clinics. 


\subsection{Subjects and Methods}

\subsubsection{Study overview and patient identification}

Development of the International Cantú Syndrome Registry (ICSR) was initiated in 2012, and resulted from the coordinated efforts of four CS clinics (at Washington University in St. Louis, Missouri in the United States, University Medical Center Utrecht in the Netherlands, the University of Bristol in the United Kingdom and the University of New South Wales in Sydney, Australia) that had already identified groups of CS subjects in their respective countries. The REDCap-based registry is maintained at Washington University in St. Louis, and the ICSR is now established as an international multicenter registry to collate clinical and genetic data on CS subjects. By effectively gathering data on CS subjects from all over the world, with data collected during recurring annual Cantú research clinics to provide longitudinal information, it is hoped that the ICSR will provide a full characterization of the clinical phenotype and mutational spectrum of CS, and a critical mass of well-phenotyped patients for rapid progression toward future interventional studies.

Clinical information, molecular data and blood samples from CS subjects and their families worldwide are collected through interaction with physicians treating CS subjects, by contacting physicians who have published on CS, and by receiving inquiries from physicians treating possible CS subjects. Individuals who present with key diagnostic criteria such as hypertrichosis, typical facial features, and cardiac abnormalities undergo sequencing for variants in ABCC 9 and KCNJ8, applying targeted Sanger sequencing, whole exome sequencing (WES) or whole genome sequencing (WGS).

A questionnaire asking all subjects to self-report currently known clinical features observed in CS is completed either during the visit to a participating facility or is sent to the referring physician and/or subject directly. Additionally, subjects are invited to participate in special annual CS research clinics, which take place at the participating institutions to document clinical features and to gather data regarding the progression of CS over time. Seventy-one of the subjects reported here have been evaluated at least once through one of the CS research clinics, while the remaining three subjects consented to participate in the registry through the Washington University site and completed the questionnaire, but were not clinically evaluated in person. Some clinical and genetic findings for 29 of the 74 subjects have previously been published (see Table 1 and footnotes). 


\subsubsection{Data management}

Research personnel at individual institutions are responsible for obtaining and maintaining institutional review board or ethics approval, performing recruitment and enrollment procedures, and collecting and entering data into the database.

Study data are collected and managed using the Research Electronic Data Capture (REDCap) (Harris et al., 2009) database, a secure, web-based application designed to support data capture for research studies, hosted at Washington University, St. Louis. The research coordinator at each site holds the code to link anonymous patient code numbers to identifiable patient details. Pooling de-identified data from all participating institutions enables data to be analyzed with statistical power that no single clinic could achieve on its own. Data are updated or re-evaluated after follow-up visits.

\subsubsection{Patient eligibility and enrollment}

The ICSR is a voluntary study open to subjects of all ages who have a clinical and, in most cases, molecular diagnosis of CS. The host institution, as well as each participating health center, is granted institutional review board or ethics approval before proceeding with subject recruitment. Consent and recruitment procedures vary among participating centers, according to each clinic's setting, available resources, and institutional policies.

\subsubsection{Data collection}

Data are primarily collected in questionnaire form, establishing the subject's background and medical history, as well as by review of known phenotypic features of CS. Prior medical records, including results of molecular genetic testing, are requested after the subject or parent/guardian signs a release form. The questionnaire is revised and re-evaluated as data collection continues and new phenotypic features are discovered. Data reported in this study were collected over a period of 6 years from 2012 to 2018, most during routine visits as part of standard care for subjects with CS or during annual CS research clinics. All but three of the subjects enrolled in the ICSR have attended one of the clinics. Data on these three subjects were obtained through the questionnaire from the subject and his or her attending physician and may involve review of medical records. All data reported here were collected after consent of the subject or the parents or legal guardians for patients younger than 18 years old. 
To date, 21 data modules have been developed to collect data on demographics, physicians, and specialists caring for the subject, birth and basic medical history, hair, skin, cardiovascular abnormalities, upper airway and ear, nose, and throat (ENT) concerns, pulmonary, bone and joint abnormalities, neurological and brain abnormalities, vision, hearing, gastrointestinal system, genitourinary system, reproductive system, endocrine system, immune function, learning and cognition, behavioral issues, and family history. In order to ensure standardization of information collection, checklists tracking data collection are maintained and updated for all participants.

\subsubsection{Data analysis and statistics}

Given the limited data available, data are presented as individual values without statistical analysis, unless otherwise noted. 

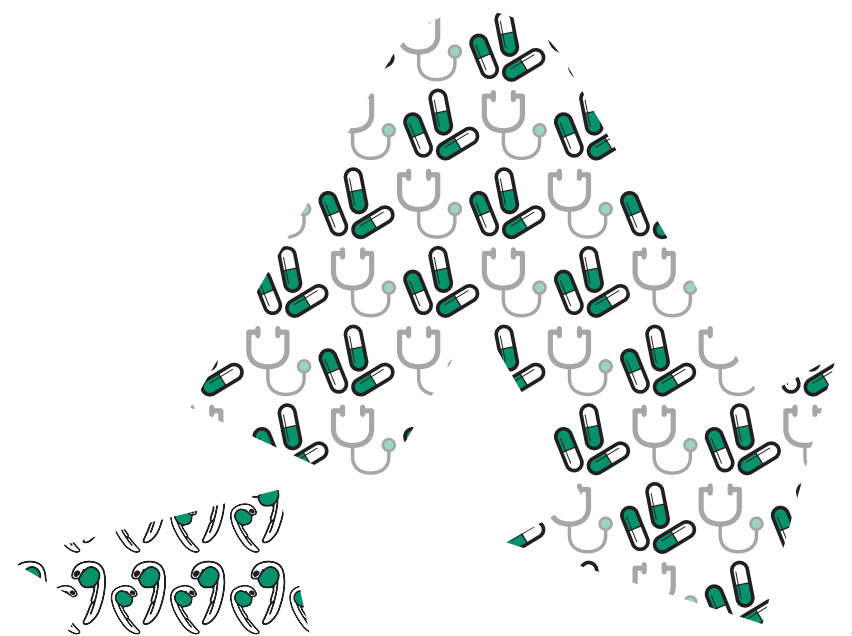

$18^{\circ} r^{\circ} r^{\circ} r^{\circ} r^{\circ}$

(8) $598^{\circ}$

(2) 9898.

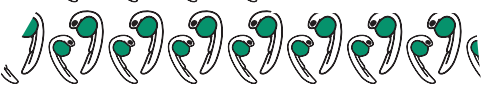

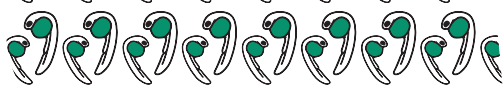
จ จ

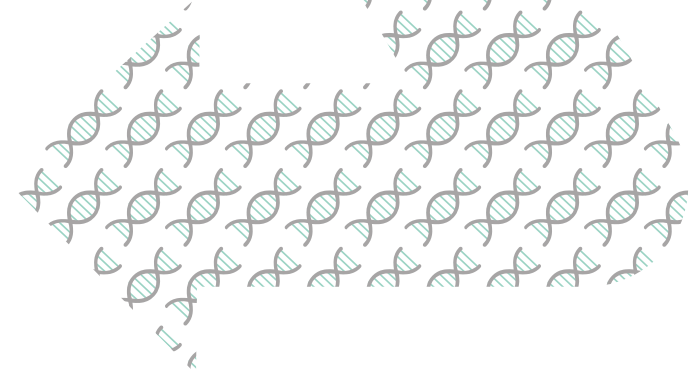




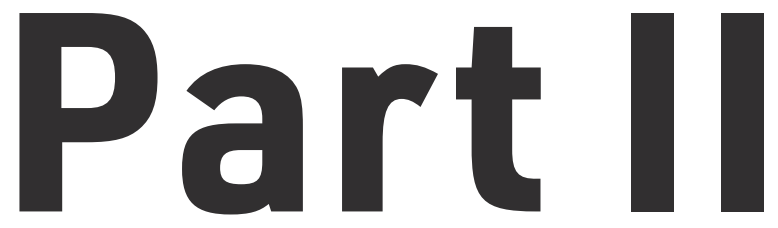

Modeling and Treatment of Cantú syndrome and Related Disorders in Zebrafish 
-12
4
4
4

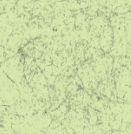

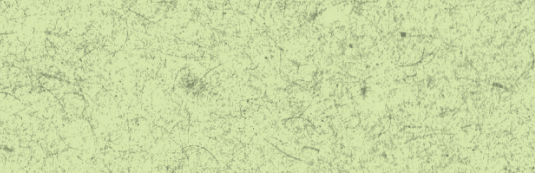

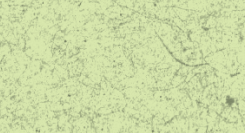

तr.

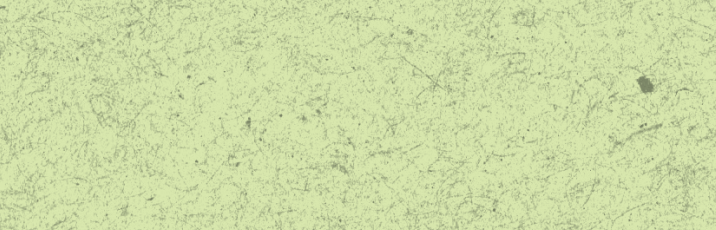

4

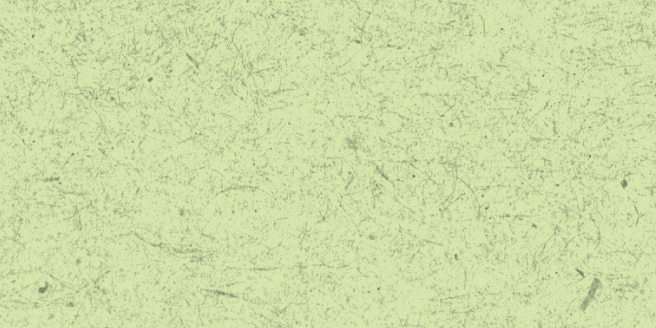




\section{Effective CRISPR/Cas9-based nucleotide editing in zebrafish to model human genetic cardiovascular disorders}

Federico Tessadori*, Helen I. Roessler*, Sanne M.C. Savelberg*, Sonja Chocron, Sarah M. Kamel, Karen Duran, Mieke M. van Haelst, Gijs van Haaften, Jeroen Bakkers

*These authors contributed equally to this work.

Dis Model Mech. 2018 Oct 18;11(10):dmm035469

DOI: $10.1242 / \mathrm{dmm} .035469$ 


\section{Abstract}

The zebrafish (Danio rerio) has become a popular vertebrate model organism to study organ formation and function due to its optical clarity and rapid embryonic development. The use of genetically modified zebrafish has also allowed identification of new putative therapeutic drugs. So far, most studies have relied on broad overexpression of transgenes harboring patient-derived mutations or loss-of-function mutants, which incompletely model the human disease allele in terms of expression levels or cell-type specificity of the endogenous gene of interest. Most human genetically inherited conditions are caused by alleles carrying single nucleotide changes resulting in altered gene function. Introduction of such point mutations in the zebrafish genome would be a prerequisite to recapitulate human disease but remains challenging to this day. We present an effective approach to introduce small nucleotide changes in the zebrafish genome. We generated four different knock-in lines carrying distinct human cardiovascular-disorder-causing missense mutations in their zebrafish orthologous genes by combining CRISPR/Cas9 with a short template oligonucleotide. Three of these lines carry gain-of-function mutations in genes encoding the pore-forming (Kir6.1, KCNJ8) and regulatory (SUR2, ABCC9) subunits of an ATP-sensitive potassium channel $\left(\mathrm{K}_{\text {ATP }}\right)$ linked to Cantú syndrome (CS). Our heterozygous zebrafish knock-in lines display significantly enlarged ventricles with enhanced cardiac output and contractile function, and distinct cerebral vasodilation, demonstrating the causality of the introduced mutations for CS. These results demonstrate that introducing patient alleles in their zebrafish orthologs promises a broad application for modeling human genetic diseases, paving the way for new therapeutic strategies using this model organism. 


\subsection{Introduction}

Cardiovascular disorders are multifactorial conditions in which genetics plays an important role in pathogenicity. For example, mutations in $>50$ genes have been associated with the development of dilated cardiomyopathy (DCM), a condition characterized by dilatation and impaired contraction of heart muscle, and the most common form of non-ischemic cardiomyopathy worldwide (Cahill, Ashrafian, \& Watkins, 2013). For instance, DCM can be caused by an in-frame deletion in the phospholamban (PLN) gene (PLN R14del) (van der Zwaag et al., 2012). Cardiovascular disorders can also be part of rare genetic syndromes: Cantú syndrome (CS), an autosomal dominant condition, is characterized by congenital hypertrichosis, distinctive facial features and extensive cardiovascular abnormalities, including an enlarged, hypercontractile heart, pericardial effusions and diffusely dilated and tortuous blood vessels (Grange et al., 2006; Levin et al., 2016) (Table S1). Gain-of-function (GoF) missense mutations in genes encoding the pore-forming (Kir6.1, KCNJ8) and regulatory (SUR2, ABCC9) subunits of the predominantly cardiovascular isoforms of an ATP-sensitive potassium channel $\left(\mathrm{K}_{\text {ATP }}\right)$ have been identified in CS patients (Brownstein et al., 2013; Cooper et al., 2014; Harakalova et al., 2012; van Bon et al., 2012). How these missense mutations contribute to the pathogenesis in CS is poorly understood. To improve our knowledge of gene-phenotype relations and to develop an in vivo model that can be used to screen or test potential therapeutic strategies, we set out to generate zebrafish models incorporating the underlying genetic cause of the diseases.

\subsection{Results}

To introduce specific mutations in the zebrafish abcc9, kcnj8 or pln genes, we designed CRISPR/Cas9 single-guide RNAs (sgRNAs) following previously published guidelines (Gagnon et al., 2014) and designed the sgRNAs according to the online tool ChopChop (http://chopchop.cbu.uib.no/; Figure 1A; Figure S1). Additionally, we provided a single-stranded 50 bp DNA template (Bedell et al., 2012) encompassing not only the site of the mutation that was being introduced (single base pair changes in the case of CS, deletion of three base pairs for $p(n)$, but also the protospacer adjacent motif (PAM) sequence targeted by the sgRNA (Figure 1B; Figure S2). Since it has been demonstrated that Cas9 cleavage occurs around 3 bp upstream of the PAM sequence (Hwang et al., 2013; Jinek et al., 2012), we selected specific mutations as close as possible to a potential PAM sequence, as we reasoned that this would maximize efficiency of the introduction 
of the chosen mutation. For all four mutations presented in this study, this was possible within $4 \mathrm{bp}$ of a potential PAM sequence. Furthermore, the template oligonucleotide was not only designed to introduce the patient-specific mutation(s), but also carried silent mutations that removed the PAM recognition sequence to prevent targeting of Cas 9 after introduction of the mutation in the genome. For Pln_R14Del, the mutation introduced a Bgll restriction site, which was used for genotyping purposes (Figure S2). We provided Cas9 nuclease to the microinjection mixture as mRNA or purified protein (Table 1).

Figure 1 presents an overview of the steps to generate knock-in (KI)-specific zebrafish lines. After microinjection of the CRISPR/Cas9 without oligo template was carried out in the first cell of zebrafish embryos, these were let to grow until 24 hours post-fertilization (hpf). Healthy microinjected embryos were assayed for efficiency of the guide RNA, using an adapted method for detecting simple sequence-length polymorphisms (Knapik et al., 1998) (SSLPs; Figure S3). For the sgRNAs we tested, we observed relatively high indel (insertion/deletion) introduction efficiencies, as they varied from between 71.4 and 100\% [ABCC9 G989E: 21/28 (75\%); ABCC9 C1043Y: 21/28 (75\%); KCNJ8 V65M: 28/28 (100\%); PLN R14Del: 20/28 (71.4\%); Figure S3). For all tested sgRNAs for CS and PLN R14Del, we proceeded to microinjections with addition of template oligonucleotide and tested their incorporation by Sanger sequencing or, for PLN R14Del, by using a Bgll restriction site (Figure S2 and S3). Microinjected siblings (F0) of the tested embryos were grown to adulthood, including embryos microinjected without the template oligonucleotide, in order to obtain adult mosaic zebrafish founders (FO) for the $\mathrm{KI}$ and knockout (KO) lines. To determine successful introduction of indels or specific mutations, we carried out sequencing of the progeny of FO fish (Figure 1C; Figure S4). For KO lines, we obtained positive results in 67-100\% of the analyzed larvae (not shown). For KI lines, mosaic founders carried various non-specific indels or unmodified genomic loci in addition to precise introduction of the mutated sequences, the efficiency of which ranged from 3.8 to $21.4 \%$ (Table 1; Tables S1 and S2). We also checked for mutational events at genomic sites displaying sequence similarity with the used sgRNAs, and did not observe any off-target effect (Figure S5). For all lines, sequencing analysis of the progeny of F1 fish after outcross to a WT line confirmed that truncating indels or specific nucleotide mutations were introduced and segregate, as expected, in a Mendelian fashion. As displayed in Figure 1, our approach allows generation of stable KI heterozygous zebrafish lines in as little as 22 weeks. Although somewhat costlier and less efficient than the generation of KO mutants (Figure S6), generation of $\mathrm{KI}$ lines could be achieved in the same time frame as the corresponding KO lines, which were also generated for phenotypic comparison. 
A

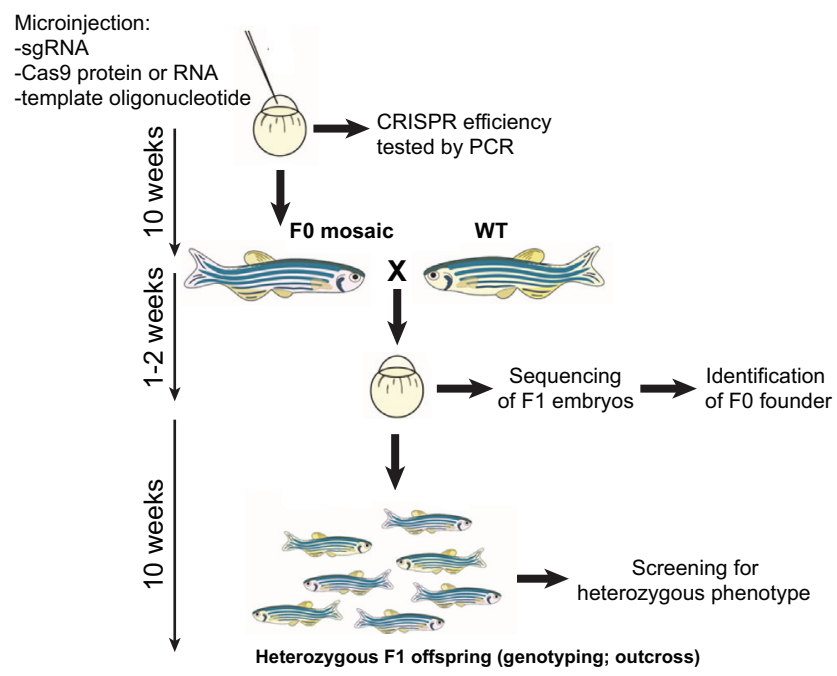

B

Template oligo

CCTGCAGGACGTCTTCACCACTCTGATGGATCTGAAGTGGCGCTTCACGCT

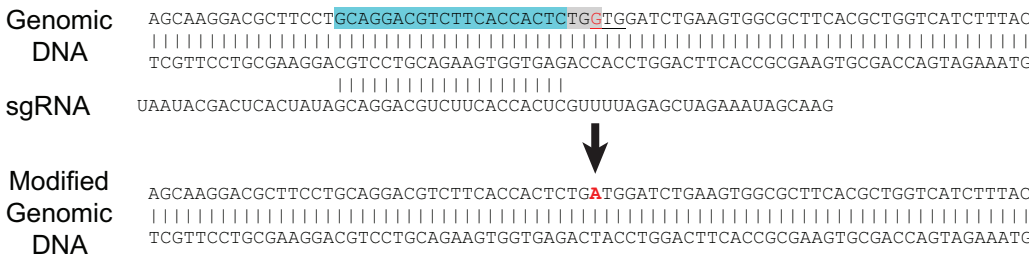

C

E Q G R F L Q D I F T T L V D L K W R H

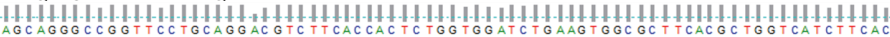

kcnj8 $8^{+/+}$

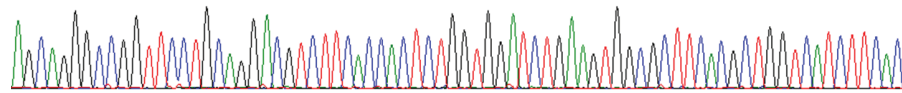

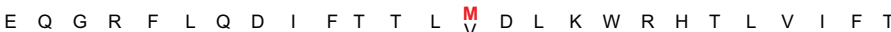
AGCAGGCCOGTTCTOCAGGACGTCTCACCACTCTGGTGGACTGAAGTGOGCTTCACGCTGGTCATTTCAC

kcnj8 $8^{+/ N 65 M}$

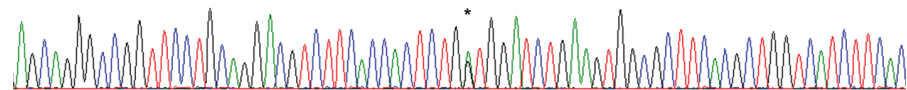

Figure 1 | Generation of patient-specific KI lines in zebrafish. (A) Stepwise procedure followed to establish the KI lines described in this study with corresponding minimal timeline for each step. (B) Schematics showing the targeted genomic sequence for the introduction of the c.A193G substitution resulting in the p.V65M in the kcnj8 genomic sequence. The PAM sequence is highlighted in gray, the specific section of the sgRNA is highlighted in cyan and the modified codon is underlined. Red text indicates substituted nucleotide. Note that in this case the substitution is located on the PAM sequence. (C) Sequencing traces for wild-type and heterozygous $\mathrm{kcnj}^{+/ N 65 \mathrm{M}} z e b r a f i s h$. Asterisk denotes the substituted nucleotide in the heterozygous $\mathrm{kcnj}^{+/ \mathrm{N} 65 \mathrm{M}}$ sequencing trace. 
Table 1 | Efficiency and screening results for the zebrafish abcc9, kcnj8 and pln KI lines.

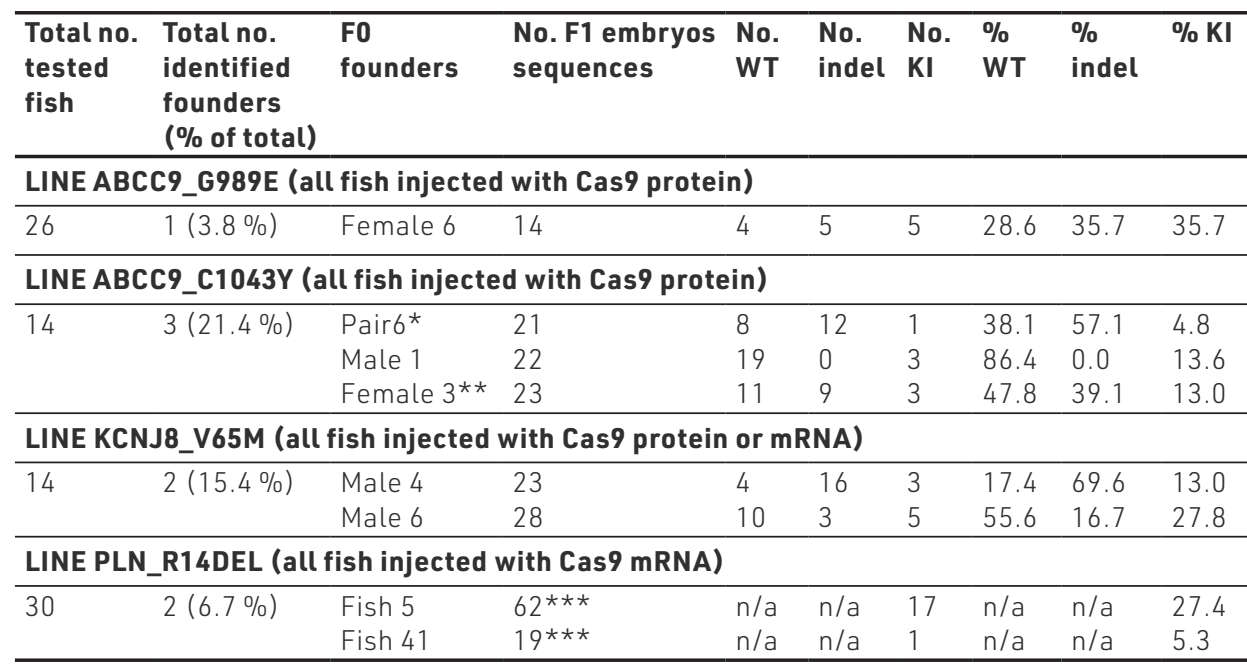

A more detailed version of this table can be found as Table S2.

No. WT: number of embryos displaying a wildtype sequence at the sgRNA-targeted locus.

No. indel: Number of embryos displaying a non-specific indel at the sgRNA-targeted locus.

No. KI: Number of embryos displaying precise sequence replacement at the sgRNA-targeted

locus.

*: male 6 died before rescreening. Female 6 did not carry the Abcc9_C1043Y mutation.

**: female 3 displayed correct sequence replacement for Abcc9_C1043Y but no PAM modification.

$\star \star \star$ : testing of the PIn_R14Del was carried out by Bgll restriction. Sequencing was only carried out on the fish carrying the Pln_R14Del mutation subsequently to their identification.

We then proceeded to systematically test the functional validity of both our heterozygous and homozygous CRISPR-generated lines. Both heterozygous and homozygous larvae were morphologically inconspicuous (Figure 2A; Figure S7A, S10A and S11A), viable through adulthood and fertile. Thanks to its optical clarity and simple yet functionally relevant heart structure, the zebrafish embryo provides an excellent model for studying pathophysiological cardiovascular development in vivo. We applied live high-speed video imaging of the beating embryonic heart, cardinal vein and dorsal aorta of zebrafish to quantify cardiac function (Hoage, Ding, \& Xu, 2012) in the KI lines described in this study (Figure 2A). In $p / n^{+/ R 14 D e l}$ larvae, we could not detect any anomalous cardiac function (not shown). This did not come as a surprise since, in contrast to CS, DCM is not a congenital condition and may hence have no effect on embryos. We therefore continued functional characterization exclusively on CS larvae. Remarkably, analogous to CS patients (Brownstein et al., 2013; Cooper et al., 2014; Levin et al., 2016), heterozygous $\mathrm{kcnj} 8^{+/ \mathrm{V} 65 \mathrm{M}} \mathrm{KI}$ larvae showed significantly elevated mean end-diastolic (mEDV) and end-systolic (mESV) volumes with strikingly 
enhanced cardiac output (53\%) (Figure 2B) due to equally increased stroke volume $(P<0.0001)$ at 5 days post-fertilization (dpf) (Figure S7). Additionally, contractile function was elevated and an increased amount of pericardial edema $(P<0.05$ ) was observed (Figure 2B; Figure S7 and S8B, Movies 1 and $2)$. A significantly reduced cardinal vein and dorsal aorta blood flow velocity (Figure 2C, P<0.0001; Figure S8C, P<0.0062; Movies 3 and 4) was measured in heterozygous $k c n j 8^{+/ V 65 M}$ larvae, and can be associated with low blood pressure and diminished vascular tone reported in CS patients (Levin et al., 2016). Homozygous kcnj8 ${ }^{\mathrm{V} 65 \mathrm{M} / \mathrm{V} 65 \mathrm{M}}$ embryos presented with similar features (Figure S7 and S8) .

Additionally, cardiomegaly was studied in 3-month-old kcnj8 V65M fish and respective wild-type siblings. Here, hearts were sectioned and stained with Hematoxylin and Eosin (H\&E; Figure 2E, Figure S9A, B). For individual assessment of ventricular and atrial size, tissue sections revealing the largest chamber area were selected and area was measured. In accordance with our findings in $5 \mathrm{dpf}$ larvae and in CS patients (Levin et al., 2016), heterozygous adult fish reveal a significantly enlarged ventricular chamber volume $(P=0.0096$; Figure 2E), whereas atrial area remains similar to wild-type size (Figure S9B). The same was observed in homozygous kcnj8 $8^{\mathrm{V} 65 \mathrm{M} / \mathrm{V} 65 \mathrm{M}}$ mutants (Figure S9A, B). To account for variations in heart size, overall body length was measured, revealing no difference in size between all studied genotypes (Figure S9C).

Quantification of cardiac function in heterozygous and homozygous abcc 9 G989E $\mathrm{KI}$ larvae revealed a similar phenotypic spectrum (Figure S10B-E), whereas abcc9 C1043Y KI embryos showed no distinct CS-related cardiac pathology (Figure S11B-E). Notably, this recapitulates the features of the only patient known with a C1043Y variant, who is reported to lack cardiomegaly as well as other cardiac features (van Bon et al., 2012). Additionally, CS presents as a strikingly heterogeneous condition with only hypertrichosis and coarse facial appearance present in all cases. Further features, such as pericardial effusions, enlarged hearts and hypercontractile hearts, are only exhibited by a certain number of cases, illustrating the fact that the penetrance of CS-associated features is inconsistent, without clear correlation to genotype. Therefore, respective phenotypical variability in zebrafish is appropriately in line with the observed inconsistent distribution of features in patients. 
A

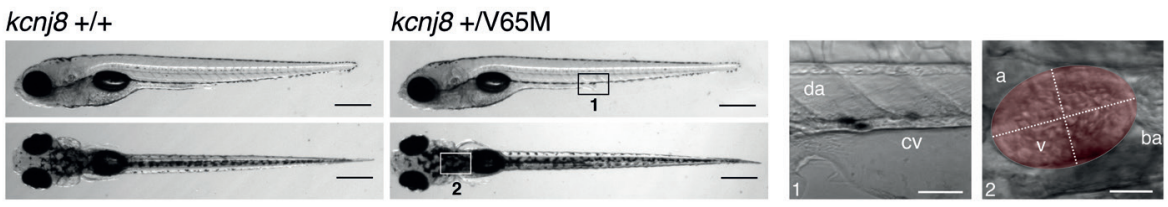

B
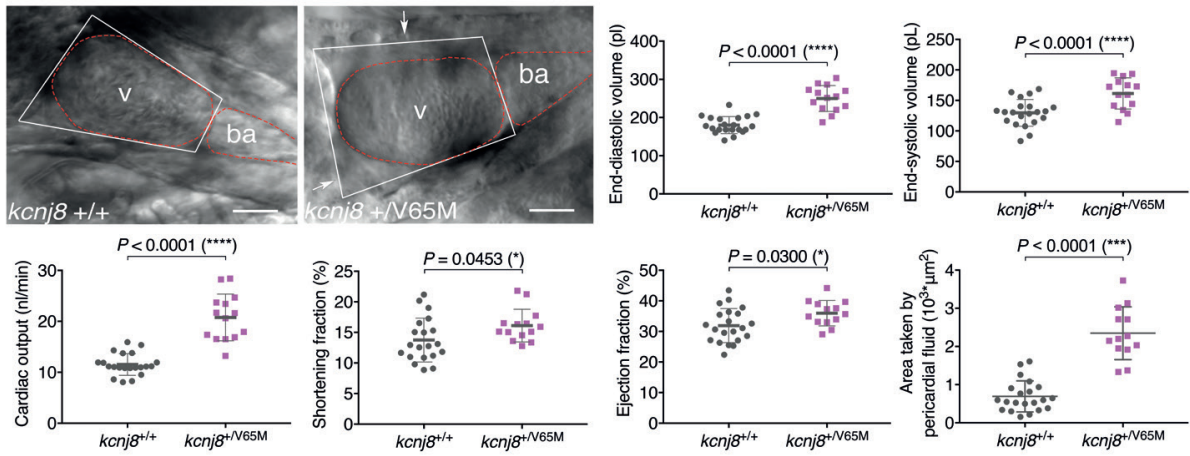

\section{c}
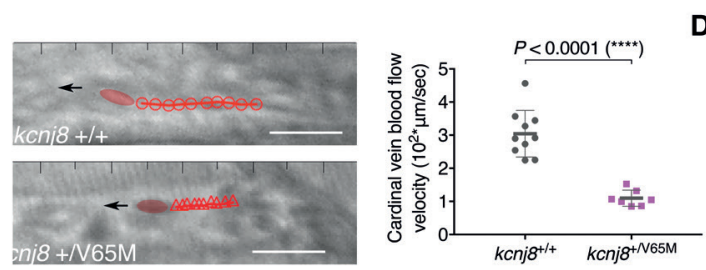

D
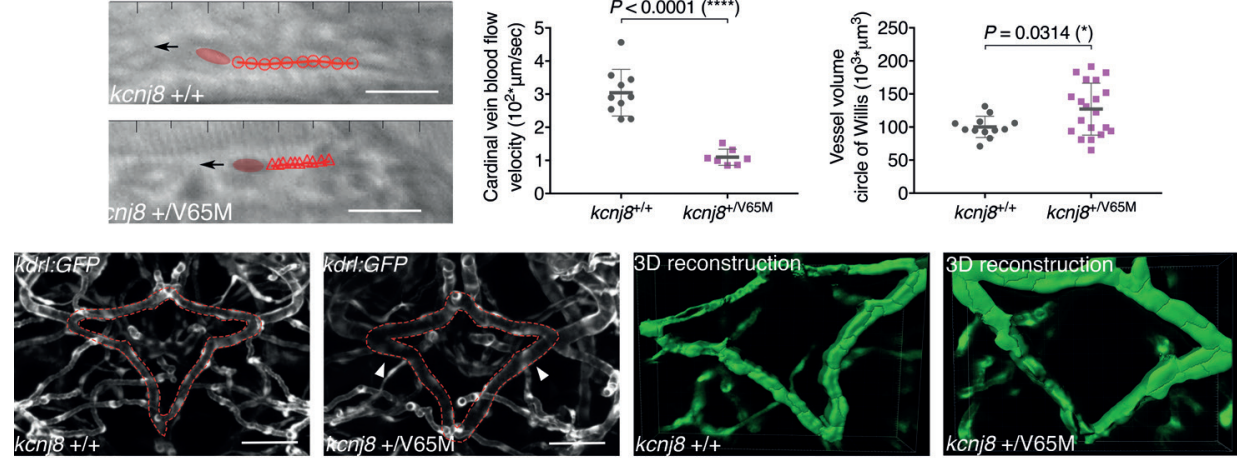

E
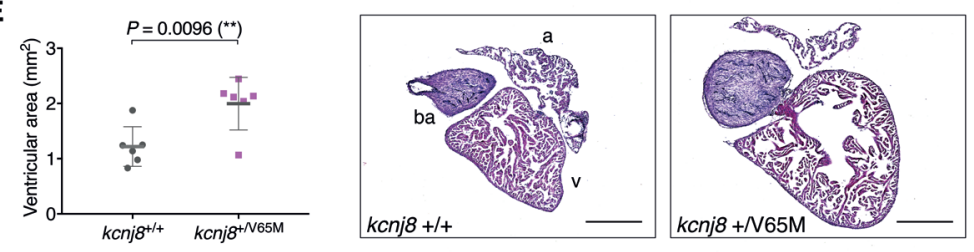

Figure 2 | See next page for legend. 
To characterize aberrant blood vessels, we examined the entire vasculature of live $5 \mathrm{dpf}$ mutants in a $\mathrm{Tg}(k d r l: G F P)$ transgenic background for dilations and tortuosity by applying confocal microscopy. Given that CS patients predominately present with cerebral vascular anomalies (Leon Guerrero et al., 2016), we focused on the same area in all CRISPR-generated CS lines. An initial systematic examination of larvae from heterozygous $k c n j 8^{+/ V 65 M}$ incrosses revealed a diffusely dilated circular structure comprising the basal communicating artery (BCA) and posterior communicating segments (PCS). This structure resembles the human circle of Willis, a prominent area of vascular anomalies in CS patients (Brownstein et al., 2013; Leon Guerrero et al., 2016). A quantification of the vascular volume of this structure identified significant $(P<0.05)$ cerebral vasodilation displayed in heterozygous fish (Figure 2D). The observed variation is in line with intra-familial variability for the different features reported in CS patients (Roessler et al., 2018). Homozygous mutants show similar vasodilation (Figure S6D). No further abnormalities of the vasculature in whole embryos could be observed during the initial examination.

Figure 2 | Heterozygous $\mathbf{k c n j}^{+/ \mathrm{N} 65 \mathrm{M}}$ mutation induces CS-related cardiac anomalies and cerebral vasodilation in zebrafish. (A) Representative images illustrating the morphology of $5 \mathrm{dpf}$ wild-type and $\mathrm{kcnj}^{\mathrm{t}}{ }^{\mathrm{N} 65 \mathrm{M}}$ mutants as seen from a left lateral (top) and dorsal (bottom) view. Boxes designate imaged areas that were used to assess cardiac function: the cardinal vein (1) and the heart (2). The ventricular area of the heart is highlighted, with the long axis and short axis of the ventricle indicated by dashed lines. a, atrium; ba, bulbous ateriosus; cv, cardinal vein; da, dorsal aorta; v, ventricle. (B) Quantification of cardiac function using individual characteristic confocal sections from a time series of the embryonic cardiac cycle at $5 \mathrm{dpf}$. Pericardial edema was quantified by measuring pericardial area using striking morphological landmarks, indicated by white boxes. Ventricular area was subtracted. Arrows show accumulation of fluid in $k c n j 8^{+/ N 65 M}$ mutants. Dotted red lines indicate ventricle ( $v$ ) and bulbous arteriosus (ba). (C) Tracking of individual red blood cells (RBCs) measuring blood flow velocity in the cardinal vein. RBCs were tracked for ten frames using ImageJ (NIH) and the plugin MTrackJ (Meijering, Dzyubachyk, \& Smal, 2012). One representative image of each genotype is shown. Black arrow indicates the direction of RBC movement. (D) Quantification of vascular dilations in a $\mathrm{Tg}(\mathrm{kdrl}: G F P)$ background. Representative confocal images of the circular structure comprising the BCA and PCS in wild-type and heterozygous $5 \mathrm{dpf}$ fish are outlined in red. The arrowheads indicate distinct regions of vasodilation. 3D reconstruction of vascular structure in

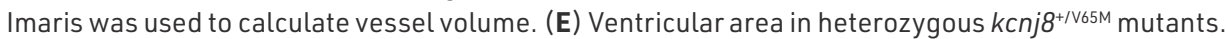
Representative heart histology of adult $\mathrm{kcnj}^{+/ \mathrm{V} 65 \mathrm{M}}$ mutants and respective wild-type siblings after H\&E staining. Exemplary depiction of one WT and one $k c n j 8^{+/ N 65 M}$ heart. For assessment of ventricular chamber size, tissue sections showing the largest ventricular area were selected and area was quantified using Image $(\mathrm{NIH})$. For all graphs, significance was determined by two-tailed unpaired Student's t-test or Mann-Whitney two-tailed U-test: ${ }^{*} P \leq 0.05$; ${ }^{* *} P \leq 0.01$; ${ }^{* * *} P \leq 0.001$; $* * * * P \leq 0.0001$. The black horizontal bar indicates the mean value for each condition. Sample sizes: (B) $k c n j 8^{+/+}, \mathrm{n}=21 ; k c n j 8^{+/ N 65 M}, \mathrm{n}=14 ;$ (C) $k c n j 8^{+/+}, \mathrm{n}=10 ; k c n j 8^{+/ V 65 M}, \mathrm{n}=7$; (D) $k c n j 8^{+/+}, \mathrm{n}=12 ; k c n j 8^{+/ v 65 M}$, $\mathrm{n}=20 ;(\mathbf{E}) k c n j 8^{+/+}, \mathrm{n}=6 ; k c n j 8^{+/ V 65 \mathrm{M}}, \mathrm{n}=6$. Scale bars: (A) $1 \mathrm{~mm}$ (top and middle) and $50 \mu \mathrm{m}$ (bottom); (B) $50 \mu \mathrm{m}$; (C) $10 \mu \mathrm{m}$; (D) $50 \mu \mathrm{m}$; (E) $500 \mu \mathrm{m}$. All embryos analyzed originated from group matings of adult zebrafish. 
The same strategy was applied to larvae from either abcc $9^{+/ 6989 E}$ or $a b c c 9^{+1}$ c1043Y heterozygous parents, which likewise revealed vascular abnormalities exclusively in the BCA/PCS vessel structure. Whereas both heterozygous and homozygous abcc9 G989E KI fish showed comparable vasodilation ( $P>0.05$; Figure S10F) but no tortuosity, abcc 9 C1043Y larvae presented with tortuous but normally sized BCA and PCS (Figure S11F).

Next to cardiovascular anomalies, kcnj8 V65M mutants were assessed for craniofacial phenotypes by Alcian Blue staining at $5 \mathrm{dpf}$ (Figure S12), revealing no cartilage deformations in both heterozygous and homozygous larvae. Notably, the origin of craniofacial features in CS patients is not fully determined yet. For instance, acromegaloid facial appearance so far observed in all CS cases may not descend from bone deformities but rather facial swellings due to underlying edema. This possibility is supported by an observed overlapping facial and edematous phenotype in individuals with CS and individuals treated with Minoxidil, a $\mathrm{K}_{\text {ATP }}$ channel opener (Kaler et al., 1987; Mehta, Mamdani, Shansky, Mahurkar, \& Dunea, 1975).

Additionally, all CRISPR-generated KI lines were assessed for macrocephaly and macrosomia, respectively - two features frequently observed in CS patients at birth. However, no difference between KI larvae and wild-type controls was observed (not shown). The absence of these non-cardiovascular CS features in the CRISPR-generated KI lines at $5 \mathrm{dpf}$ may be due to the lack of bone formation at this developmental time point in zebrafish or differences between zebrafish and human physiology. Further studies are required to resolve this question.

\subsection{Discussion}

In the study presented here, we were successful in generating four different $\mathrm{KI}$ lines by introducing four distinct missense mutations causing human cardiovascular disorders. This was achieved by combining the CRISPR/Cas 9 system with a specific template oligonucleotide, which allowed us to introduce the disease-causing missense mutations into the corresponding zebrafish orthologous genes. It is important to note that parameters such as a high initial efficiency of the sgRNA and the proximity of the PAM with the desired site of mutations are paramount to the success of the method (Figure S3 and S4). Indeed, our study presents successful small nucleotide changes located within $4 \mathrm{bp}$ of the corresponding PAM site. This parameter, which constitutes a limitation of our approach, should become less stringent in the close future, as 
evolved Cas9 proteins with a broader range of PAM requirements for targeting DNA sequences have very recently been published ( $\mathrm{Hu}$ et al., 2018). These promise improved capacity for the CRISPR/Cas9 system to model patientspecific mutations in animal or in vitro models.

Our work demonstrates that CS-associated variants in both kcnj8 and abcc9 lead to a remarkable combination of cardiovascular anomalies in zebrafish, such as enlarged, hypercontractile ventricles with increased cardiac output, elevated occurrence of pericardial edema, reduced blood vein flow velocity, and cerebral vasodilation and tortuosity. Importantly, we observed that larvae heterozygous for kcnj8 or abcc 9 mutations display these anomalies, which is in good agreement with the inheritance mode of CS (autosomal dominant). Animals with the $\mathrm{kcnj}^{+1}$ v65M allele displayed stronger phenotypes than both abcc9 KI lines. Notably, clinical hallmarks in patients harboring a missense mutation in KCNJ8 are generally considered to be more severe than in patients with an ABCC9 mutation (Brownstein et al., 2013; Cooper et al., 2014). Hence, our novel zebrafish models recapitulate characteristic hallmarks clinically observed in CS patients, and thereby confirm the causality of KCNJ8 and ABCC9 mutations for CS (Grange et al., 2006; Leon Guerrero et al., 2016; Levin et al., 2016). The presence of CS key features in all CRISPR-generated KI lines further demonstrates that initial molecular consequences must originate from tissues expressing both Kir6.1 and SUR2 subunits.

$\mathrm{K}_{\text {ATP }}$ channels are hetero-octameric complexes, with ABCC9 (SUR2) and KCNJ8 (Kir6.1) prominently expressed in cardiomyocytes, vascular smooth muscle and vascular endothelial cells (Flagg, Enkvetchakul, Koster, \& Nichols, 2010), suggesting that cardiovascular features may predominate in CS (Armstrong et al., 2016). Naturally, gain of $K_{\text {ATP }}$ channel function would be expected to cause reduced contractility and vasodilation in blood vessels and action potential shortening resulting in reduced cardiac contractility and output in the heart (Nichols, 2006). Although our vascular findings are consistent with these expected consequences of $\mathrm{K}_{\text {ATP }}$ GoF, both $k c n j 8^{+/ V 65 M}$ and abcc $9^{+/ G 989 E} \mathrm{KI}$ fish manifest a high output state with enhanced cardiac function, a pathophysiology opposite to the prediction. In mice with constitutive or tamoxifen-induced cardiac-specific Kir6.1 GoF subunit expression, this counter-observation can be explained by a compensatory increase in basal L-type $\mathrm{Ca}^{2+}$ current, paralleled by changes in phosphorylation of the pore-forming 1 subunit of the cardiac voltagegated calcium channel Cav1.2 resulting in remodeling of cardiac excitationcontraction coupling, and hence hypercontractility and high cardiac output 
(Levin et al., 2016). Further studies are necessary to confirm the alteration of this mechanism in the described CS zebrafish mutants.

With cardiac output being directly proportional to blood pressure, we additionally propose that the observed high-output state compensates for hypotension due to vasodilation in CS patients. At the moment, there is no treatment for CS available. However, the CS $K_{\text {ATP }}$ channel can be pharmaceutically targeted by several approved drugs, such as second-generation sulfonylureas, which are already used in clinical setups to inhibit GoF in the pancreatic $\mathrm{K}_{\text {ATP }}$ isoforms involved in neonatal diabetes mellitus (Ashcroft, 2010; McClenaghan et al., 2017). The successful validation of our CS KI zebrafish may provide the opportunity of phenotype-based screening to test the efficiency of these and further potential therapeutic compounds. Consequently, future studies in our CS zebrafish models will help to shed light on underlying molecular mechanisms and contribute to the development of a treatment for the disorder, insights that may improve understanding and therapeutic management of CS.

In conclusion, we have improved a CRISPR/Cas9-based single-nucleotideediting approach with which we established novel zebrafish KI lines that closely model cardiovascular features of human disease. Beyond the application illustrated here, our approach offers a versatile tool to edit the zebrafish genome at the nucleotide level and provide broad possibilities for modeling of patientspecific mutations. 


\subsection{Materials and Methods}

\subsubsection{Fish maintenance and preparation}

All animal experiments were conducted under the guidelines of the animal welfare committee of the Royal Netherlands Academy of Arts and Sciences (KNAW). Adult zebrafish (Danio rerio) were maintained and embryos raised and staged as previously described (Westerfield, 1993). The zebrafish lines used in this study were Tübingen longfin (wild type) and Tg(kdrl:GFP) (Choi et al., 2007).

Adding phenylthiourea (PTU) at a concentration of $0.003 \%(\mathrm{v} / \mathrm{v})$ to the E3 embryonic raising medium at the Prim-5 stage (about $24 \mathrm{hpf}$ ) blocked pigmentation for high-speed imaging and confocal analysis.

\subsubsection{Cas9 mRNA expression plasmid and Cas9 protein}

Plasmid pCS2-nCas9n (Addgene \#47929) was used as template for synthesis of capped mRNA (see below for a more detailed description). Cas9 nuclease protein was purchased at NEB (catalog number NEBM0386M).

\subsection{3 sgRNA and template oligonucleotide}

Design and synthesis of sgRNAs used in this study were fundamentally carried out following the guidelines given in previous literature (Chari, Mali, Moosburner, \& Church, 2015; Gagnon et al., 2014), with minor modifications. All singlestranded DNA oligonucleotides (sgRNA template, constant oligonucleotide, template oligonucleotide) were purchased at Integrated DNA Technologies (IDT) as standard desalted same-day oligos. A table detailing all ssDNAs used in this study can be found in Figure $\mathbf{S 2}$.

\subsection{4 sgRNA and Cas9 mRNA synthesis}

Synthesis of the sgRNA specific to each mutation was carried out using the Ambion MEGAscript T7 or SP6 kits (Ambion). Cas9 mRNA was synthesized by linearizing pCS2-nCas9n (Addgene \#47929) with the restriction enzyme Notl and subsequently using the linearized plasmid as template for the SP6 mMessage Machine kit (Ambion) to produce capped mRNA. Purification of the in vitro synthesized mRNA was achieved with the RNeasy Mini Kit (Qiagen).

\subsubsection{Microinjection in zebrafish embryos}

One-cell-stage zebrafish embryos were microinjected with an injection mixture consisting of (final concentrations): $150 \mathrm{ng} / \mu \mathrm{l}$ nuclear Cas9 (nCas9) mRNA or $5 \mu \mathrm{M}$ nCas9 protein, 20-40 ng/ $\mu \mathrm{l}$ sgRNA, 10\% (v/v) Phenol Red and $25 \mathrm{ng} / \mu \mathrm{l}$ template oligo. nCas 9 mRNA and protein microinjections were carried in the cell. 
At $24 \mathrm{hpf}$, genomic DNA was extracted from microinjected, healthy embryos and sequenced for assessment of CRISPR/Cas9 introduction of genomic editing.

\subsubsection{PCR and electrophoresis-based genotyping}

PCR primers encompassing the CRISPR sites (see Figure S2 for primer sequences) were used to produce PCR products of approximately $250 \mathrm{bp}$. The PCR products were then loaded and migrated by electrophoresis on a high percentage (2-4\%) Tris-borate-EDTA (TBE) agarose gel supplemented with ethidium bromide. The minimal differences in migration speed of the PCR products caused by the CRISPR/Cas 9 genome editing can be visualized on the electrophoresis gel. Deletions and insertions can be easily visualized as distinct bands or smears on a $4 \%$ agarose gel after sufficient migration time (Figure S3). Restriction for genotyping of PLN_R14Del was carried out with Bgll (NEB).

\subsubsection{Sequencing}

CRISPR site-specific PCR primers were used to amplify the flanking genomic regions of the CRISPR target sites. Taq Gold polymerase (Applied Biosystems, Thermo Fisher Scientific, Waltham, MA, USA) was used [1 $\mu$ isolated DNA, $2.5 \mathrm{mM} \mathrm{MgCl}$, $0.2 \mathrm{mM}$ deoxynuclotides (dNPTs), 1× Taq buffer, 20 pmol of both primers, $1 \mathrm{U}$ Taq polymerase) in combination with a touch down PCR program: initial denaturation at $95^{\circ} \mathrm{C}$ for $5 \mathrm{~min}$, followed by 15 cycles of denaturation at $95^{\circ} \mathrm{C}$ for $30 \mathrm{~s}$, annealing at $65^{\circ} \mathrm{C}$ to $57.5^{\circ} \mathrm{C}$ for $30 \mathrm{~s}$ by decreasing $0.5^{\circ} \mathrm{C}$ steps cycle-wise ('touchdown' PCR) and extension at $72^{\circ} \mathrm{C}$ for $1 \mathrm{~min}$, followed by 27 cycles of denaturation at $95^{\circ} \mathrm{C}$ for $30 \mathrm{~s}$, annealing at $58^{\circ} \mathrm{C}$ for $30 \mathrm{~s}$ and extension at $72^{\circ} \mathrm{C}$ for $1 \mathrm{~min}$, followed by a final extension step at $72^{\circ} \mathrm{C}$ for $7 \mathrm{~min}$ and infinite hold at $8^{\circ} \mathrm{C}$. Amplicons of $\mathrm{KO}$ fish were visualized on a $3 \%$ agarose gel and specific $\mathrm{KI}$ mutations were confirmed by Sanger sequencing.

\subsubsection{In vivo high-speed imaging}

Image acquisition was conducted using a Hamamatsu C9300-221 high-speed CCD camera (Hamamatsu Photonics) at 150 frames per second (fps) mounted on a Leica DM IRBE inverted microscope (Leica Microsystems) using Hokawo 2.1 imaging software (Hamamatsu Photonics). Image analysis was subsequently carried out with ImageJ (http://rsbweb.nih.gov/ij/, last accessed November 2017). For analysis of cardiac function, adult zebrafish heterozygous for the respective mutations in kcnj8 or abcc 9 were interbred, and genotyped by Sanger sequencing post-imaging. High-speed brightfield image sequences of the embryonic zebrafish heart were acquired for zebrafish at $5 \mathrm{dpf}$ using a 20-fold magnification. Zebrafish were anesthetized in 16 mg/ml tricaine (MS222; SigmaAldrich) in E3 medium and mounted in dorsal position in small microscopic 
chambers filled with $0.25 \%(\mathrm{w} / \mathrm{v}$ ) agarose (Sigma-Aldrich) prepared in the same concentration of anesthetic. Zebrafish hearts were imaged for $10 \mathrm{~s}$ (approximately 30 cardiac cycles) at $28 \pm 0.3^{\circ} \mathrm{C}$.

\subsubsection{Measurement of ventricular volume, cardiac output, shortening fraction, fractional area change and ejection fraction}

The time interval between three heartbeats was measured and the heart rate (bpm) was calculated. Images from high-speed movies were used to outline the perimeter of the ventricle. Measurement analysis was carried out using the 'fit-to-ellipse' algorithm, which worked on calculating the center of mass and subsequently the best-fitting ellipse. The long axis length (a) and short axis length (b) at diastole and systole were determined and used to calculate ventricular end-systolic (ESV) and diastolic (EDV) volumes applying the formula: $V=4 / 3 \varpi(b / 2)^{2}(a / 2)$. The stroke volume (SV) was calculated as the difference between three ventricular EDVs and ESVs. Cardiac output was obtained by multiplying the heart rate with stroke volume. The percent shortening fraction (SF) was calculated using the formula: $S F(\%)=$ (length at diastole-length at systole)/(length at diastole) $\times 100$. Ejection fraction $(E F)$ can be calculated with the following formula: $E F(\%)=(S V / E D V) \times 100$. Fractional area change $(F A C)$ was calculated as follows: FAC $(\%)=$ (area at diastole-area at systole) $/$ (area at diastole) $\times 100$. Every cardiac parameter was calculated in triplicate.

\subsubsection{Blood flow velocity}

Zebrafish were mounted in lateral position at $5 \mathrm{dpf}$ and the region behind the cloaca showing both cardinal vein and dorsal aorta was imaged for $10 \mathrm{~s}$ at $28 \pm 0.3^{\circ} \mathrm{C}$. Blood flow velocity was calculated by assessing frame-by-frame motion of three single erythrocytes per fish (at least seven fish per group) determined from high-speed images to assess mean erythrocyte cell velocity ( $\mu \mathrm{m} / \mathrm{second}$ ) using ImageJ. Cardinal blood flow velocity was measured over ten frames at a video frame rate of $150 \mathrm{fps}$ as non-pulsatory venous flow allows frame-by-frame analysis. In contrast, dorsal aorta blood flow velocity was analyzed over the whole imaged area to account for pulsatory blood flow.

\subsubsection{Quantification of pericardial edema}

Images at cardiac diastole with focus on ventricular perimeter were taken from high-speed movies to outline pericardial area. Striking morphological landmarks, such as the ventriculo-bulbar valves and the inner pericardial wall, were used to obtain the same area in every imaged embryo. In order to correct for possible enlarged ventricular size in mutants, ventricular area was subtracted. 


\subsubsection{Heart extraction and H\&E staining}

Zebrafish hearts were dissected and fixed in $4 \%$ paraformaldehyde in PBS at $4^{\circ} \mathrm{C}$ overnight and subjected to paraffin embedding and sectioning at $10 \mu \mathrm{m}$ intervals. Heart sections were stained with $\mathrm{H} \& \mathrm{E}$ following standard procedures. Six fish were used for each genotype.

\subsubsection{Quantification of heart chamber size}

To assess ventricular and atrial chamber size, tissue sections showing the largest ventricular and atrial area were selected and area was measured using ImageJ $(\mathrm{NIH})$.

\subsubsection{In vivo confocal microscopy and image quantification}

Confocal imaging of cerebrovasculture was carried out on a Leica SPE confocal microscope (Leica Microsystems) using a 10× and 20x objective. Adult zebrafish heterozygous for kcnj8 V65M, abcc9 G989E and abcc9 C1043Y and carrying the kdrl:GFP transgene were interbred, and genotype identified by Sanger sequencing post-imaging. Zebrafish at $5 \mathrm{dpf}$ were anesthetized with $16 \mathrm{mg} /$ $\mathrm{ml}$ tricaine and mounted in dorsal position in $0.25 \%$ agarose. $3 \mathrm{D}$ quantification of cerebral vasculature was carried out with Imaris software (Bitplane, Oxford Instruments). A Leica SPE confocal system (Leica Microsystems) was used to generate confocal stacks of approximately $250 \mu \mathrm{m}$ with a slice interval of $1 \mu \mathrm{m}$ under identical imaging conditions for all samples in an experiment. Stacks were aligned and reconstructed into a 3D volume using Imaris. The volume of the structure resembling the human circle of Willis was measured after eliminating any interfering vessels.

\subsubsection{Whole-embryo brightfield imaging}

In vivo phenotypic assessment for whole-embryo imaging were carried out on a Leica M165FC stereomicroscope (Leica Microsystems) with transmitted light. Images were captured with a DFC420 digital microscope camera (Leica Microsystems).

\subsubsection{Cartilage staining (Alcian Blue)}

Zebrafish larvae at $5 \mathrm{dpf}$ were incubated overnight at $4^{\circ} \mathrm{C}$ in fixative solution (76\% ethanol; $20 \%$ acetic acid; $4 \%$ formaldehyde supplemented with $15 \mathrm{mg}$ Alcian Blue). Subsequently, larvae were washed in $70-100 \%$ ethanol, briefly transferred to $100 \%$ methanol and finally imaged in Murray's solution (v/v: 2:1 benzylbenzoate:benzylalcohol). 


\subsubsection{Measurement of macrocephaly and body length}

To assess macrocephaly in heterozygous and homozygous mutants, wholeembryo brightfield images were applied to measure the distance between the convex tip of the eyes (interorbital distance) in $5 \mathrm{dpf}$ larvae using ImageJ ( $\mathrm{NIH}$; https://imagej.nih.gov/ij/). Overall, larval and adult body length was measured from the tip of the head to the end of the trunk (before the caudal fin).

\subsubsection{Statistical analysis}

Sample size was not predetermined by statistical analysis. In all experiments involving zebrafish embryos, selection was random for scoring. Exact numbers of analyzed embryos are reported at relevant locations in the main text or the supplementary information. Statistical analysis was carried out with Prism (GraphPad). Normal distribution of the data sets was confirmed by D'AgostinoPearson omnibus normality test. Significance values were calculated using an unpaired Student's t-test throughout the manuscript. All values are expressed as mean \pm s.d. 


\subsection{Supplemental information}

Supplementary Table 1 | Major clinical features of Cantú syndrome.

\begin{tabular}{l}
\hline Neonatal features \\
\hline Macrosomia \\
Macrocephaly \\
Maternal polyhydramnios \\
\hline Craniofacial dysmorphology \\
\hline Epicanthal folds \\
Broad nasal bridge \\
Small nose/anteverted nostrils \\
Long philtrum \\
Prominent mouth/full lips \\
Macroglossia \\
Gingival hyperplasia
\end{tabular}

\section{Hair}

Thick scalp hair

Excessive body hair on forehead, face, back and limbs

\section{Skin and joints}

Loose and/or wrinkled skin

Deep palmar and plantar creases

Hyperflexibility of joints

\section{Cardiovascular}

Cardiomegaly

Pericardial effusions

Patent ductus arteriosus

Pulmonary hypertension

Increased cardiac output

Ventricular hypercontractility

Reduced vascular tone

Tortuosity and dilation of cranial blood vessels

\section{Brain}

Headaches

Seizures

\begin{tabular}{l}
\hline Skeletal abnormalities \\
\hline Narrow thorax \\
Pectus carinatum \\
Broad ribs \\
Delayed bone age \\
\hline Other reported features \\
\hline Lymphedema \\
Immune dysfunction or recurrent infections \\
Umbilical hernia \\
Developmental delay
\end{tabular}


Effective CRISPR/Cas9-based nucleotide editing in zebrafish to model human genetic cardiovascular disorders

For supplementary figures please see the online version of this article. 
-12
4
4
4

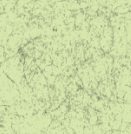

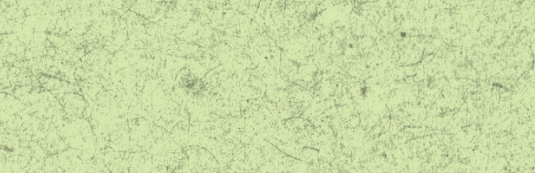

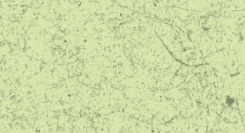

तr.

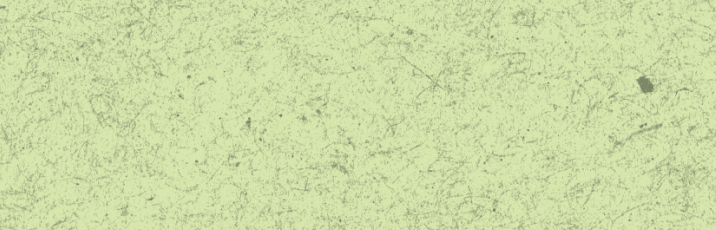

4

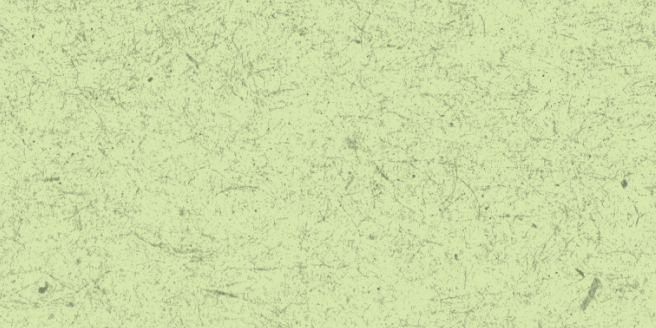




\section{8}

\section{Characterization and treatment of overactive $K_{\text {ATP }}$ channels in zebrafish models of Cantú syndrome}

Helen I. Roessler*, Soma S. Singareddy*, Conor McClenaghan, Sanne M.C. Savelberg, Rob Tyron, Federico Tessadori, Teun P. de Boer, Jeroen Bakkers, Nine V.A.M. Knoers, Mieke M. van Haelst, Colin G. Nichols* and Gijs van Haaften*

* These authors contributed equally to this work. 


\section{Abstract}

Cantú Syndrome (CS) is a rare genetic disorder caused by gain-of-function (GoF) mutations in genes encoding the pore-forming (Kir6.1, KCNJ8) and regulatory (SUR2, ABCC9) subunits of cardiovascular $\mathrm{K}_{\text {ATP }}$ channels. CS patients consistently present with hypertrichosis and facial anomalies alongside a varying range of cardiovascular (CV) abnormalities. CS is debilitating with no targeted therapy available. To elucidate the complex pathophysiology of CS and to perform therapeutic drug screening, we introduced patient-specific point mutations in the germline of zebrafish orthologues of ABCC9 and KCNJ8. Applying patchclamping of isolated ventricular cardiomyocytes (VCMs) and vascular smooth muscle cells (VSMCs), we validate CS-related GoF in mutant fish which is reflected by a shift in ATP sensitivity in VCMs and a greater basal $\mathrm{K}^{+}$conductance in VSMCs. We further show that $\mathrm{K}_{\text {ATP }}$ GoF initially results in reduced $\mathrm{Ca}^{2+}$ entry and contractility in the heart, subsequently compensated by $\beta$-adrenergic intervention causing an increase in $\mathrm{Ca}^{2+}$ signaling and establishment of key $\mathrm{CS}$ features of hypercontractility and high-output state. Next, we tested the in vivo efficacy of glibenclamide and repaglinide, which are clinically used to block pancreatic GoF $K_{\text {ATP }}$ channels involved in neonatal diabetes. Glibenclamide resulted in significant correction of CS cardiac phenotypes in Sur [G989E] knockin larvae, whereas repaglinide revealed similar effects in Kir6.1 [V65M] mutants. These results indicate that (1) zebrafish represent a suitable model to study pathophysiology and therapy options for CS and other disorders with aberrant $\mathrm{CV} \mathrm{K}_{\text {ATP }}$ channels and (2) $\mathrm{K}_{\text {ATP }}$ channel blockers are promising candidates for drug repurposing in CS. 


\subsection{Introduction}

The rare multi-organ system genetic disorder Cantú syndrome (CS, OMIM \#239850) is characterized by congenital hypertrichosis, craniofacial dysmorphology, multiple cardiovascular (CV) abnormalities including cardiomegaly with high-output state, pericardial effusion and low blood pressure (BP), and diffusely dilated and tortuous cerebral blood vessels (Grange et al., 2019; Leon Guerrero et al., 2016; Levin et al., 2016). CS results from mutations in either $A B C C 9$ or KCNJ8 encoding the regulatory (SUR2) and pore-forming (Kir6.1) subunits, respectively, of ATP-sensitive potassium $\left(\mathrm{K}_{\mathrm{ATP}}\right)$ channels (Brownstein et al., 2013; Cooper et al., 2014; Harakalova et al., 2012; van Bon et al., 2012).

$\mathrm{K}_{\text {ATP }}$ channels are potassium-selective ion channels composed of four poreforming Kir6.x subunits (Kir6.1 or Kir6.2 encoded by KCNJ8 and KCNJ11, respectively) and four regulatory sulfonylurea receptor SURx subunits (subfamily C: SUR1, SUR2 encoded by $A B C C 8$ and $A B C C 9$, respectively) (Martin et al., 2017). SUR2 can be spliced variably increasing molecular heterogeneity of $\mathrm{K}_{\text {ATP }}$ channels; particularly prominent are two essential splice isoforms of SUR2: SUR2A and SUR2B (Inagaki et al., 1996; Nichols, 2006). $\mathrm{K}_{\text {ATP }}$ channels are nucleotide-gated metabolic sensors which couple cellular metabolism to electrical excitability (Nichols, 2006). Hence, physiological activity of $\mathrm{K}_{\text {ATP }}$ channels is determined primarily by the relative concentrations of ATP and ADP: by binding to Kir6.x subunits ATP inhibits $\mathrm{K}_{\text {ATP }}$ function (Tucker, Gribble, Zhao, Trapp, \& Ashcroft, 1997), whereas $\mathrm{Mg}^{2+}$-nucleotide complexes (MgADP and MgATP) bind to SURx subunits to stimulate channel activity (Nichols et al., 1996). $\mathrm{K}_{\text {ATP }}$ channels are widely distributed and present in a number of tissues including cardiac and vascular smooth muscle cells (VSMCs). In the heart, SUR2A subunits co-assemble with Kir6.2 and lead to significant shortening of the ventricular action potential (AP) (Cole, McPherson, \& Sontag, 1991). In VSMCs, $\mathrm{K}_{\text {ATP }}$ channels are primarily formed of Kir6.1 and SUR2B regulating vascular tone and BP (Figure 1) (Aziz et al., 2014).

Recent reports show that CS variants result in enhanced activity of recombinant $\mathrm{K}_{\text {ATP }}$ channels mediated by various molecular mechanisms such as augmented magnesium-nucleotide activation and increased intrinsic stability of the open state resulting in decreased sensitivity to ATP inhibition (Cooper, McClenaghan, Chen, Stary-Weinzinger, \& Nichols, 2017; Cooper et al., 2014; Harakalova et al., 2012; McClenaghan et al., 2017). A gain-of-function (GoF) effect in vivo was successfully shown in distinct CS mouse models (Huang et al., 2018). However, 
this conclusion does not provide a direct explanation for the complex and diverse pathophysiology of CS. As the same CS disease features arise from mutations in either of the two $\mathrm{K}_{\text {ATP }}$ subunits and Kir6.1 is merely expressed in VSMCs but not in cardiac myocytes, it is suggested that the disorder primarily arises from increased $\mathrm{K}_{\text {ATP }}$ channel activity in vascular smooth muscle (VSM) directly leading to the chronically dilated vasculature observed in patients. Cardiac features, on the other hand, are proposed to result from secondary consequences (Huang et al., 2018; McClenaghan et al., 2019).

Currently, there is no specific therapy available for CS. It is possible that $\mathrm{K}_{\text {ATP }}$ channel inhibitors such as those used in the treatment of neonatal diabetes resulting from GoF in pancreatic SUR1- and Kir6.2-dependent channels, might close overactive $K_{\text {ATP }}$ channels in CS patients (McClenaghan et al., 2017; Nichols et al., 2013). Recent studies in CS mice revealed reversal of CV abnormalities after treatment with glibenclamide (GLB) showing that cardiac remodeling in CS is reversible (McClenaghan et al., 2019).

As an alternative model system in which to assess cellular and organ-level mechanisms resulting from CS-associated $\mathrm{K}_{\text {ATP }}$ variants, we previously introduced three patient-specific point mutations in the germline of zebrafish orthologues of ABCC9 and KCNJ8. A phenotypic characterization revealed distinct CS-related CV features (Tessadori et al., 2018). We now set out to (1) thoroughly characterize the electrophysiological effect of these CS mutations on $\mathrm{K}_{\text {ATP }}$ channels in VCM and VSMCs and (2) to further elucidate secondary consequences of increased $K_{\text {ATP }}$ channel activity in the heart. Lastly, we have (3) used these models to perform phenotype-based drug screening to assess efficacy of various $\mathrm{K}_{\text {ATP }}$ channel blockers on the development of CV abnormalities in CS larvae. 


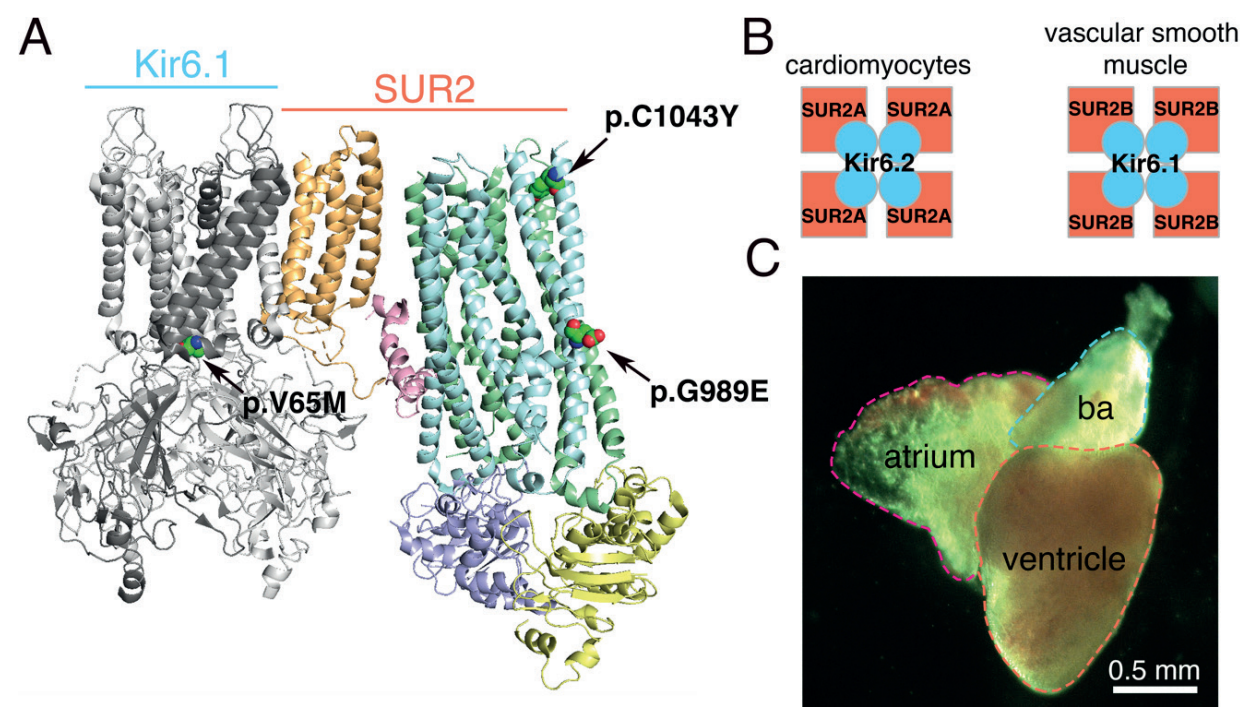

Figure 1 | Structure of $\mathbf{K}_{\text {ATP }}$ channels and CS zebrafish. (A) Structural representation of the Kir6.2/ SUR1 $\mathrm{K}_{\text {ATP }}$ channel expressed in the pancreas (PDB ID: 5WUA). $\mathrm{K}_{\text {ATP }}$ channels form hetero-octamers of four pore-forming Kir6.x subunits and four regulatory SURx subunits. Expected positions of Sur2[G989E], Sur2 [C1043Y] and Kir6.1[V65M] are highlighted. TMD0: orange, L0: pink, TMD1: green, TMD2: blue, NBD1: purple, NDB2: yellow. TMD: Transmembrane domain, NBD: Nucleotide binding domain, L: Linker. (B) Predominant isoform composition of $\mathrm{K}_{\text {ATP }}$ channels in cardiomyocytes (Kir6.2/SUR2A) and vascular smooth muscle (Kir6.1/SUR2B). (C) Zebrafish cardiovascular tissues used for isolation of ventricular cardiomyocytes (ventricle) and vascular smooth muscle cells (bulbous arteriosus, ba).

\subsection{Results}

\subsubsection{Mutation of Sur2 results in GoF of native ventricular myocyte $\mathrm{K}_{\mathrm{ATP}}$ in zebrafish}

All three CS zebrafish models, namely Sur2[G989E], Sur2[C1043Y] and Kir6.1 [V65M], established and characterized previously (Tessadori et al., 2018) were examined for the direct effect of CS variants on $\mathrm{K}_{\text {ATP }}$ channel function in the heart applying excised, inside-out patch-clamp electrophysiology on acutely dissociated ventricular myocytes (Figure 1). Various distinct nucleotidesensing mechanisms can be responsible for increased $K_{\text {ATP }}$ channel activity. These can include increased $\mathrm{Mg}$-nucleotide activation or reduced ATP inhibition either resulting from decreased binding affinity or decreased efficacy due to stabilization of the channel in the open state thereby increasing the open probability (McClenaghan et al., 2017). To investigate the direct effects of CS mutations on ATP sensitivity, we prepared a plot of ATP concentration vs $\mathrm{K}_{\text {ATP }}$ current at that concentration normalized to the maximal $K_{\text {ATP }}$ current observed 
(at 0 ATP). As shown in Figure 2 B and D, a decreased sensitivity to ATP was observed in Sur2 [G989E] and Sur2[C1043Y] mutant VCMs represented by an increase in $\mathrm{IC}_{50}$ values ( 4- and $\sim 2.5$-fold increases in Sur2 ${ }^{\mathrm{CY} / \mathrm{CY}}$ and Sur2 ${ }^{\mathrm{GE} / \mathrm{GE}}$, respectively).

There was no significant increase in $\mathrm{IC}_{50}$ values due to the Kir6.1 p.V65M mutation which is consistent with the prevailing evidence from mammals that cardiac $\mathrm{K}_{\text {ATP }}$ channels are composed of primarily Kir6.2/SUR2A subunits (Figure 2 F).

Whereas the inhibitory ATP binding site is situated in the pore-forming Kir6.x subunit, activating nucleotides interact with Sur in a magnesium-dependent manner. Thus, to further investigate the effects of the introduced mutations on Sur2-dependent nucleotide interactions, MgATP dose-responses were assessed by adding $\mathrm{Mg}^{2+}$ in equal concentrations of ATP (Figure 3 ).

In Sur2[G989E] mutant channels, the presence of $\mathrm{Mg}^{2+}$ resulted in increased channel activity for a given ATP concentration, indicating that the mutation primarily increases channel activity by enhancing $\mathrm{Mg}^{2+}$-nucleotide activation. This effect is reflected in a significant right-shift in the MgATP dose-response curve relative to $\mathrm{Mg}^{2+}$-free ATP ( 2.5-fold increase in $\mathrm{IC}_{50}$ in Sur2 ${ }^{\mathrm{GE} / \mathrm{GE}}$ ) (Figure 3 D versus 2 D). Sur2 [C1043Y] channels, on the other hand, showed no change in $\mathrm{IC}_{50}$ values in the presence of $\mathrm{Mg}^{2+}$ suggesting that sensitivity activating $\mathrm{Mg}^{2+}$ was unaffected by this mutation and that GoF most likely results from decreased sensitivity to inhibition by ATP (Figure 3 B versus 2 B). Notably, the GoF effect induced by each mutation in the clinically relevant heterozygous state was evident but quite subtle.

In conclusion, heterozygous and homozygous Sur2[C1043Y] and [G989E] mutations resulted in a progressive increase in GoF in zebrafish cells. This confirms the expected molecular consequences of the Sur2 substitutions resulting in GoF of $\mathrm{K}_{\text {ATP }}$ channels in cardiomyocytes, whereas the Kir6.1 [V65M] substitution has no significant effect on cardiac $K_{\text {ATP }}$ channels. 


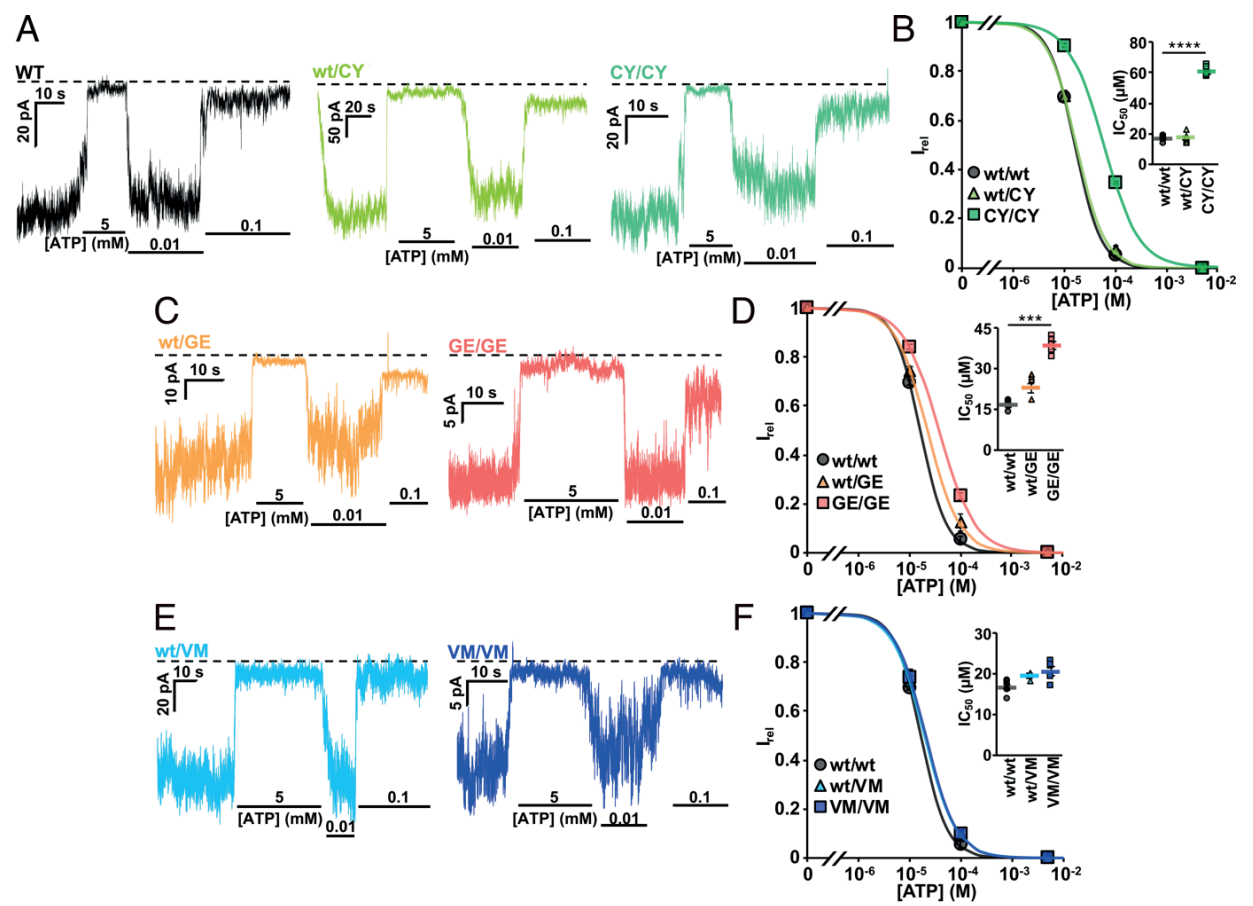

Figure 2 I $\mathrm{K}_{\text {ATP }}$ channels in Sur2 ventricular myocytes reveal GoF due to introduction of CS variants. (A) Sample inside-out excised patch-clamp recordings showing $\mathrm{K}_{\mathrm{ATP}}$ channel activity in response to ATP from acutely dissociated ventricular cardiomyocytes from WT (left, black), Sur2 ${ }^{\text {wt/CY }}$ (middle, light green) and Sur2 ${ }^{\text {cy/CY }}$ (right, dark green) zebrafish. (B) [ATP]-response curves with individual $I C_{50}$ values from separate experiments shown in inset. (C) Sample inside-out excised patch-clamp recordings showing $\mathrm{K}_{\text {ATP }}$ channel activity in response to ATP from acutely dissociated ventricular cardiomyocytes from acutely dissociated ventricular myocytes from Sur2 ${ }^{\text {wt/GE }}$ (left, orange) and Sur2 ${ }^{\text {GE/GE }}$ (right, red) zebrafish. (D) [ATP]-response curves with individual IC ${ }_{50}$ values from separate experiments shown in inset. (E) Sample inside-out excised patch-clamp recordings showing $\mathrm{K}_{\text {ATP }}$ channel activity in response to ATP from acutely dissociated ventricular myocytes from Kir6. $1^{\text {w/VM }}$ (left, light blue) and Kir6. $1^{\mathrm{VM} / \mathrm{VM}}$ (right, dark blue) zebrafish. (F) [ATP]-response curves with individual $\mathrm{IC}_{50}$ values from separate experiments shown in inset. For all graphs, significance was determined by one-way ANOVA: ${ }^{*} p<0.05 ;{ }^{* *} p<0.01 ;{ }^{* * *} p<0.001 ;{ }^{* * * *} p<0.0001$. Sample size: (B) $W T, n=6 ; w t / C Y, n=8 ; C Y / C Y, n=5$; (D) $W T, n=6$; wt/GE, n=6; GE/GE, n=5; (F) WT, n=6; wt/VM, n=3; VM/VM, $\mathrm{n}=5$. 
A
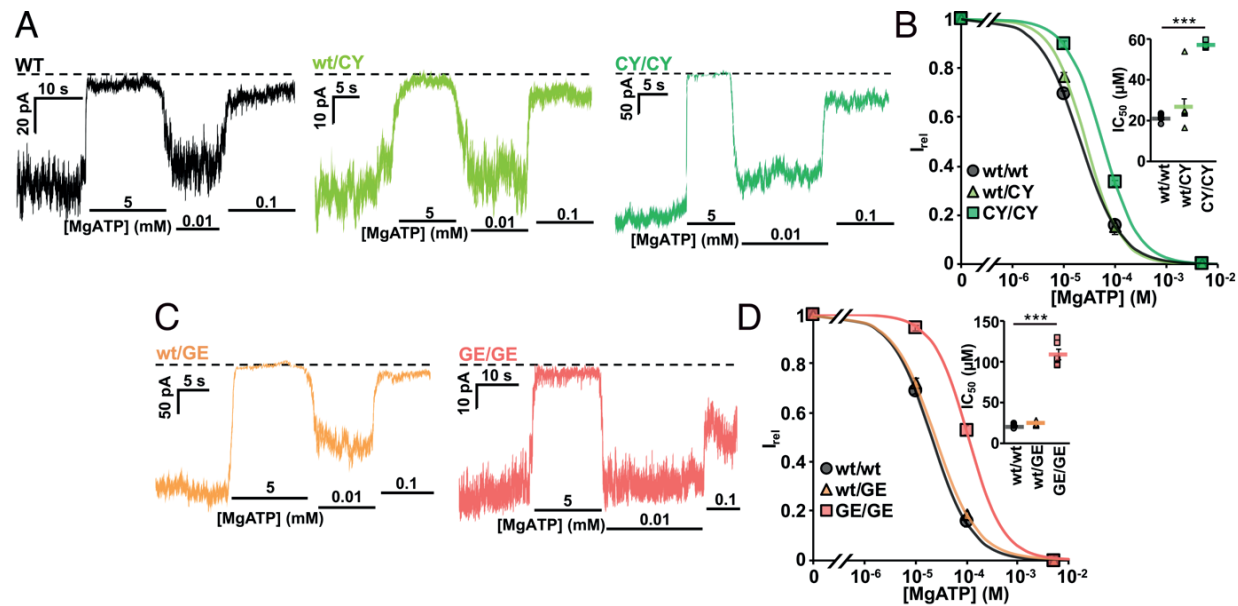

Figure 3 | Sur2 [G989E] causes $\mathrm{K}_{\text {ATP }}$ GoF by enhancing $\mathrm{Mg}^{2+}$-nucleotide activation. (A) Sample inside-out excised patch-clamp recordings showing $\mathrm{K}_{\text {ATP }}$ channel activity in response to MgATP from acutely dissociated ventricular myocytes from WT (left, black), Sur2 ${ }^{\text {wt/CY }}$ (middle, light green) and Sur2 ${ }^{\text {CY/CY }}$ (right, dark green) zebrafish. (B) [MgATP]-response curves with individual $I_{50}$ values from separate experiments shown in inset. (C) Sample inside-out excised patch-clamp recordings showing $\mathrm{K}_{\mathrm{ATP}}$ channel activity in response to MgATP from acutely dissociated ventricular myocytes from Sur2 ${ }^{\text {wt/GE }}$ (left, orange) and Sur2 ${ }^{\text {GE/GE }}$ (right, red) zebrafish. (D) [MgATP]-response curves with individual $\mathrm{IC}_{50}$ values from separate experiments shown in inset. For all graphs, significance was determined by one-way ANOVA: ${ }^{*} p<0.05 ;{ }^{* *} p<0.01 ;{ }^{* * *} p<0.001 ;{ }^{* \star \star *} p<0.0001$. Sample size: (B) WT, n=6; wt/CY, n=8; CY/CY, n=4; (D) WT, n=6; wt/GE, n=8; GE/GE, n=5.

\subsubsection{Mutation of Kir6.1 results in GoF of native vascular smooth muscle $K_{A T P}$ channels in zebrafish}

Since p.V65M is present in the Kir6.1 domain, any likely GoF caused by this variant would be expected to be present in VSMCs (Figure 1). Whole-cell patchclamping was performed on VSMCs isolated from the bulbous arteriosus (ba) of Kir6.1 [V65M] mutants to characterize channel activity. Using an intracellular pipette solution with no ATP, only very small $\mathrm{K}^{+}$currents were observed in WT VSMCs. However, both Kir6. $1^{\mathrm{wt} / \mathrm{VM}}$ and Kir6.1 ${ }^{\mathrm{VM} / \mathrm{VM}}$ revealed large $\mathrm{K}^{+}$currents (Figure 4 A and $\mathbf{C}$ ) that were essentially abolished when $100 \mu \mathrm{M}$ ATP was included in the patch pipette (Figure $\mathbf{4} \mathbf{B}$ and $\mathbf{C}$ ), confirming that the observed conductances in fact resulted from $\mathrm{K}_{\mathrm{ATP}}$ channel activity, and indicating a marked $\mathrm{GoF}$ in the pore-forming domain. 
A no ATP in Pipette solution
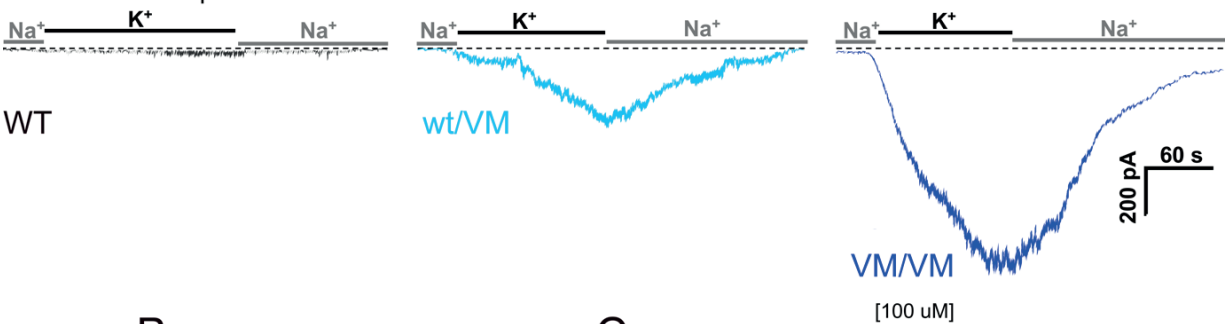

B [100uM] ATP in Pipette solution
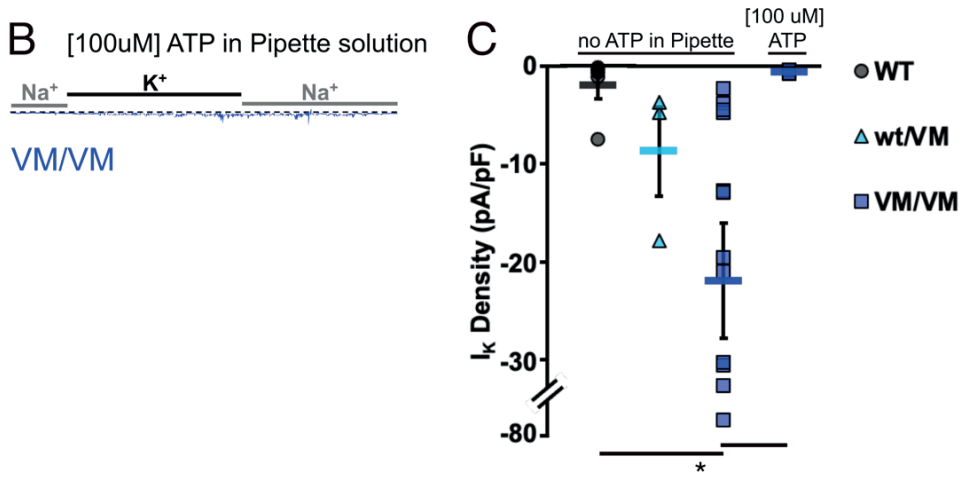

Figure 4 | Kir6.1 [V65] causes GoF in vascular smooth muscle $K_{\text {ATP }}$ channels. (A) Sample wholecell patch-clamp recordings from acutely isolated VSMCs originating from the bulbous arteriosus of WT (left, black), Kir6.1 wt/VM (middle, light blue) and Kir6.1 ${ }^{\text {wt/VM }}$ (right, dark blue) zebrafish. Cells were voltage clamped at $-70 \mathrm{mV}$ in a high- $\mathrm{Na}^{+}$bath solution before switching to a high- $\mathrm{K}^{+}$bath solution enabling the determination of $\mathrm{K}^{+}$currents. (B) Sample whole-cell patch-clamp recording

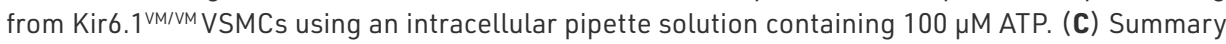
of whole-cell current densities from patch-clamp recordings of Kir6. $1^{\mathrm{w} / \mathrm{VM}}$ and Kir6. $1^{\mathrm{VM} / \mathrm{VM}}$ VSMCs. A significant increase in basal $K_{A T P}$ conductance was observed. For all graphs, significance was determined by one-way ANOVA: * $p<0.05 ;{ }^{* *} p<0.01 ;{ }^{* *} p<0.001$. Data shown as mean \pm SEM. Sample size: (C) WT, $n=5 ;$ wt/VM, $n=3 ; V M / V M, n=12 ; V M / V M[100 \mu M]$ ATP, $n=3$.

\subsubsection{Sur2 [G989E] zebrafish demonstrate $\beta$-adrenergic intervention as secondary consequence to $\mathrm{K}_{\text {ATP }} \mathrm{GoF}$ in the heart}

The confirmed GoF effect of patient-specific CS variants in CV $K_{\text {ATP }}$ channels, however, does not provide a direct explanation for the complex and diverse pathophysiology of CS. In the heart, $\mathrm{K}_{\text {ATP }}$ GoF mutations are predicted to shorten $\mathrm{AP}$ and subsequently reduce contractility and cardiac output. However, we have previously shown that $5 \mathrm{dpf}$ CS zebrafish larvae display increased ejection fraction (EF) and high-output state in the ventricle (Tessadori et al., 2018) reflecting the findings in CS mice and in patients (Huang et al., 2018; Levin et al., 2016).

We hypothesized that the in zebrafish observed increased cardiac contractility may result from enhanced $\mathrm{Ca}^{2+}$ entry via L-type $\mathrm{Ca}^{2+}$ channels (LTCC) as a consequence of phosphorylation of $\beta$-adrenergic receptors ( $\beta A R s$ ) in 
cardiomyocytes. To investigate the intracellular $\mathrm{Ca}^{2+}$ dynamics in larval CS zebrafish hearts in a spatiotemporal manner, we crossed Sur2 [G989E] fish with a line expressing a genetically encoded fluorescent calcium sensor (GCaMP6f) in the heart specifically and injected embryos with a silent heart morpholino to inhibit cardiac contractions (Figure 5 A) (van Opbergen et al., 2018). After confirming a previously published observation that $\beta$-adrenergic regulation in WT zebrafish is established around $4 \mathrm{dpf}$ (Figure 5 B) (Schwerte, Prem, Mairosl, \& Pelster, 2006), we investigated $\mathrm{Ca}^{2+}$ signaling in the ventricle of 3 and $5 \mathrm{dpf}$ Sur2 [G989E] zebrafish in the absence and presence of the $\beta$-adrenergic antagonist propranolol (PR). As shown in Figure 5 C, without functional $\beta A R s, \mathrm{Ca}^{2+}$ transient frequency was decreased in $3 \mathrm{dpf}$ mutant fish (Sur2 ${ }^{\text {wt/GE }}$ and Sur2 ${ }^{\mathrm{GE} / \mathrm{GE}}$ ), but at $5 \mathrm{dpf}$, when $\beta$-adrenergic stimulation was active, mutant $\mathrm{Ca}^{2+}$ transient amplitude was higher than control and was reduced by acute PR treatment (Figure 5 D). Similarly, we applied live high-speed imaging to examine EF in 3 and $5 \mathrm{dpf}$ zebrafish incubated in a placebo medium or PR, respectively (Figure 5 E). Consistent with the proposed mechanism, mutant fish revealed significantly reduced EF at 3 dpf compared to WT (Figure $\mathbf{5} \mathbf{F}$ ) but increased EF at $5 \mathrm{dpf}$, and this was reversed by PR exposure (Figure $\mathbf{5} \mathbf{G}$ ).

These experiments confirm remodeling of contractility in the heart in response to Sur2 GoF and further suggest that these events occur quite rapidly in zebrafish larvae.

\subsubsection{Pharmacological correction of CS-associated cardiac abnormalities in zebrafish larvae}

We next tested whether overactive $\mathrm{K}_{\text {ATP }}$ channels in zebrafish larvae may be pharmacologically inhibited by various $\mathrm{K}_{\text {ATP }}$ channel blockers, such that CSassociated cardiac abnormalities observed previously (Tessadori et al., 2018) might be mitigated.

Taking advantage of zebrafish embryos as a high-throughput model for drug screening assays, we exposed CS fish to various $\mathrm{K}_{\text {ATP }}$ channel blockers (dissolved in DMSO), namely glibenclamide (GLB), repaglinide (RPG) and PNU-37883, for a period of 96 hours starting at $1 \mathrm{dpf}$ (Figure $\mathbf{6} \mathbf{A}$ ). Next to control embryos kept in E3 embryonic raising medium (for simplification not shown in figures), a vehicle control was taken along to ensure no cardiac modifications were being caused by DMSO. Thanks to its optical clarity and simple yet functionally relevant heart structure, cardiac function is easily monitored in zebrafish larvae. $5 \mathrm{dpf}$ larvae were randomly selected from control and treatment groups and subjected to live high-speed video imaging to capture at least three cardiac cycles of the beating 
zebrafish larval heart (Hoage et al., 2012). CS zebrafish lines Sur[G989E] and Kir6.1 [V65M] were used and cardiac output (CO), EF and diastolic end volume (DEV) (in case of Kir6.1 [V65M] fish) were assessed and applied as readout for drug efficacy.

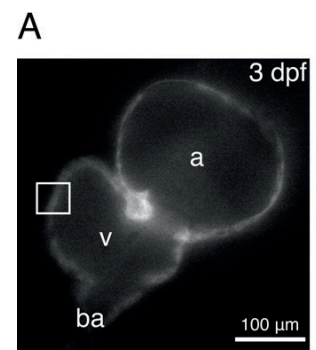

$E$

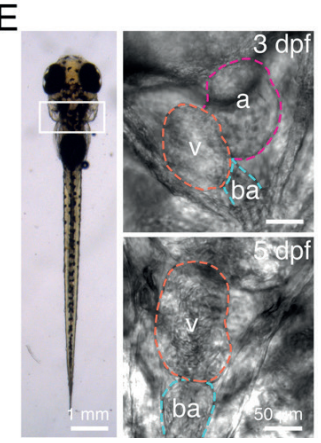

$\mathrm{B}$

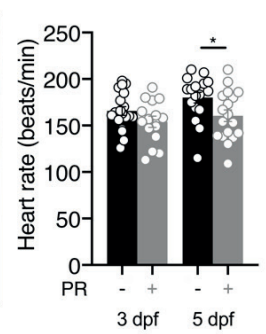

C

$\mathrm{F}$
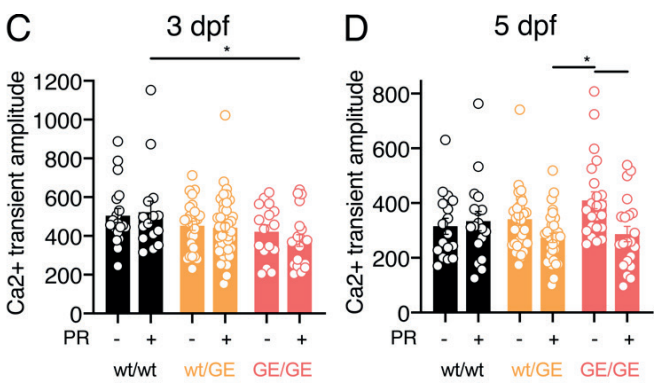

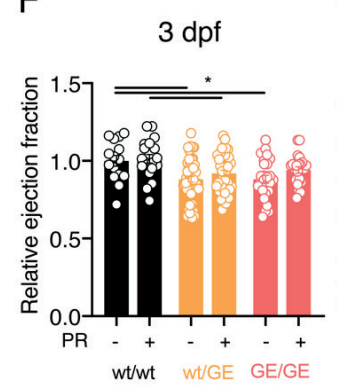

G

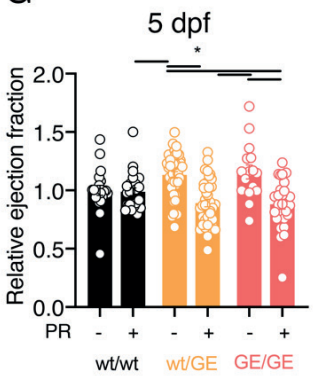

Figure 5 I Adrenergic intervention as effect of CS-related gain-of-function in embryonic CS GCaMP6f fish. (A) CS GCaMP6f fish were injected with a silent heart morpholino and movies of noncontracting embryonic hearts were recorded with a high-speed fluorescence camera. $\mathrm{Ca}^{2+}$ signaling was examined in the ventricle (v) of 3 and $5 \mathrm{dpf}$ Sur2 [G989E] zebrafish larvae with and without acute treatment with $\beta$-adrenergic receptor antagonist propranolol (PR) for $30 \mathrm{~min}$. Experiments were recorded at $28{ }^{\circ} \mathrm{C}$. (B) Changes in heartrate of 3 and $5 \mathrm{dpf}$ WT fish after application of 100 $\mu \mathrm{M}$ propranolol. (C-D) $\mathrm{Ca}^{2+}$ transient amplitudes were averaged from $20 \mathrm{Ca}^{2+}$ transitions per fish. (E) Applying live high-speed video imaging, ventricular function was examined in 3 and $5 \mathrm{dpf}$ Sur2 [G989E] zebrafish larvae with and without acute treatment with propranolol. (F-G) Images from high-speed movies were used to calculate relative ejection fraction (average from three separate cycles). For all graphs, significance was determined by one-way ANOVA: * $p<0.05$; * $\mathrm{p}<0.01 ;{ }^{* * *} \mathrm{p}<0.001 ;{ }^{* * \star *} \mathrm{p}<0.0001$. Data shown as mean \pm SEM. Sample sizes: (B) $3 \mathrm{dpf}(-), \mathrm{n}=19$; $3 \mathrm{dpf}(+), \mathrm{n}=15 ; 5 \mathrm{dpf}(-), \mathrm{n}=18 ; 5 \mathrm{dpf}(+), \mathrm{n}=19 ;$ (C) wt/wt(-), n=19; wt/GE(-), n=27; GE/GE(-), n=17; wt/wt(+), n=15; wt/GE(+), n=41; GE/GE(+), n=21; (D) wt/w(-), n=18; wt/GE(-), n=37; GE/GE(-), $\mathrm{n}=23 ; \mathrm{wt} / \mathrm{wt}(+), \mathrm{n}=19 ; \mathrm{wt} / \mathrm{GE}(+), \mathrm{n}=31 ; \mathrm{GE} / \mathrm{GE}(+), \mathrm{n}=20 ;(\mathbf{F})$ wt/wt $(-), \mathrm{n}=15 ; \mathrm{wt} / \mathrm{GE}(-), \mathrm{n}=54 ; \mathrm{GE} /$ GE(-), n=26; wt/wt(+), n=20; wt/G (+), n=40; GE/GE(+), n=22; (G) wt/wt(-), n=20; wt/GE(-), n=43; GE/GE(-), n=16; wt/wt(+), n=24; wt/GE(+), n=37; GE/GE(+), n=27. All embryos analyzed originated from group matings of adult heterozygous Sur2 [G989E]:GCaMP6f CS zebrafish. 
Remarkably, Sur2 ${ }^{\text {wt/GE }}$ fish showed significantly decreased EF ( $25 \%$ ) and CO ( $25 \%$ ) in the presence of GLB due to equally lowered stroke volume with stable heart rate, almost restoring wildtype conditions (Figure $\mathbf{6} \mathbf{B}-\mathbf{D}$ and Figure S1 A). Notably, exposure to $1 \%$ DMSO vehicle did not modify cardiac function and resulted in similar values to those in untreated fish. This observation is in accordance with a study undertaken in adult CS mice revealing reversal of cardiac features after chronic GLB administration (McClenaghan et al., 2019) indicating that CS zebrafish larvae and the screening setup can be successfully used for testing the efficacy of further $\mathrm{K}_{\text {ATP }}$ channel inhibitors as potential CS therapy options.

So far, there are three CS patients with mutations in the pore-forming Kir6.1 (KCNJ8) subunit (Brownstein et al., 2013; Chihara, Asahina, \& Itoh, 2020; Cooper et al., 2014). However, GLB failed to significantly rescue any of the elevated cardiac parameters in Kir6. $1^{\text {wt/VM }}$ fish (Figure 6 E-G). Notably, p.V65M results in a drastic $\mathrm{K}_{\text {ATP }}$ GoF and markedly decreases GLB efficacy in recombinant channels (Cooper et al., 2017) and CS mice and this is also linked to a reduced ability to reverse the cardiac features in these mice (McClenaghan et al., 2019).

Next, we exposed both CS zebrafish models to RPG. Whereas no beneficial effect of RPG on CS-related aberrant cardiac function was observed in Sur2 ${ }^{\text {wt/GE }}$ fish (Figure 7 A-C), Kir6. $1^{\mathrm{wt} / \mathrm{VM}}$ larvae revealed complete correction of otherwise elevated CO ( $32 \%)$, EF ( $17 \%)$ and diastolic end volume (DEV) ( $18 \%$ ) (Figure 7 D-F).

Notably, PNU-37883 did not result in correction of CS features in either of the CS zebrafish models (Figure $\mathbf{S 2}$ ).

These results illustrate the in vivo efficacy of RPG in addition to GLB as pharmacological correctors of cardiac abnormalities in CS, but also importantly illustrate how $\mathrm{K}_{\text {ATP }}$ blocker treatment efficacy may depend on the severity and/or position of the underlying mutation in the $\mathrm{K}_{\text {ATP }}$ channel subunits. 


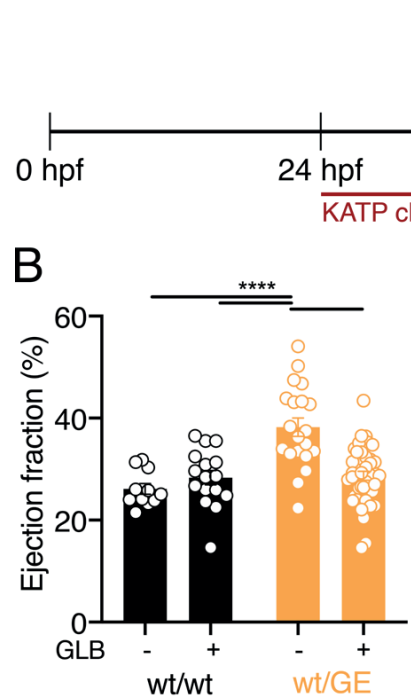

E
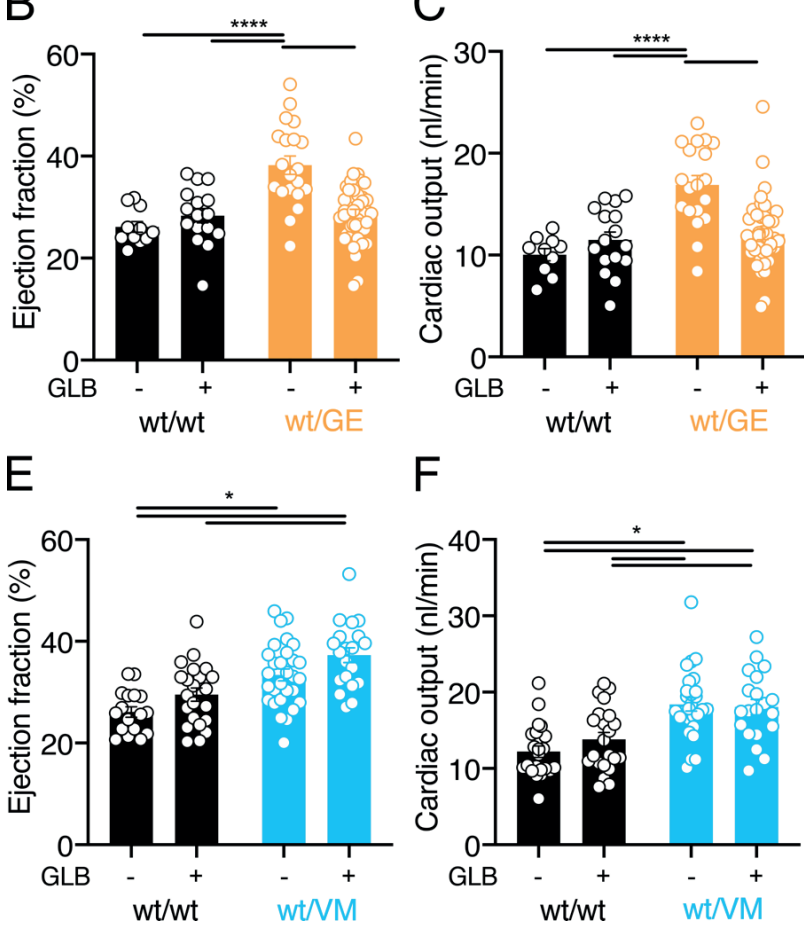

F

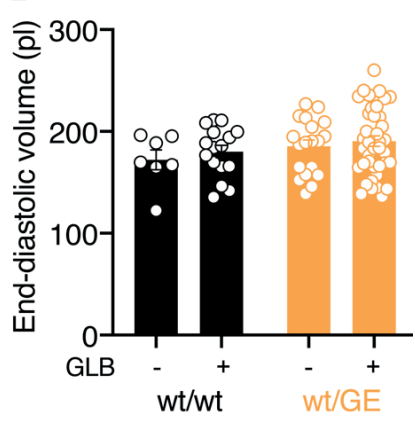

$\mathrm{G}$
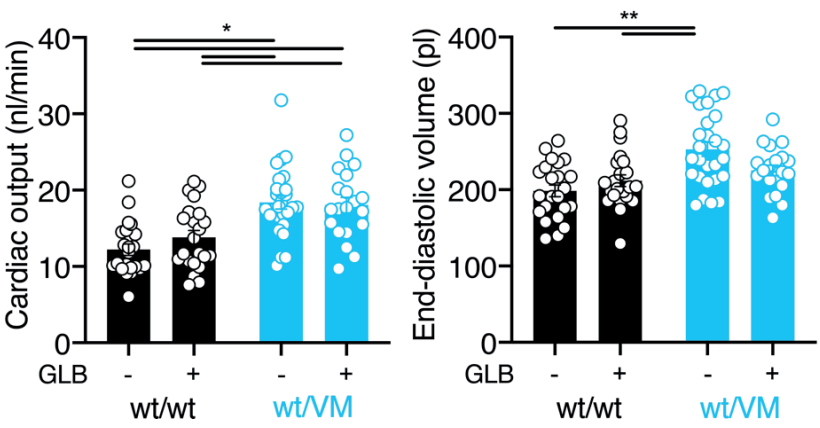

Figure 6 I Glibenclamide corrects CS-related cardiac phenotype in Sur2 ${ }^{\text {wt/GE }}$ larvae. (A) Sur2 ${ }^{\text {wt/GE }}$ and Kir6. $1^{\text {wt/VM }}$ larvae were exposed to various $K_{\text {ATP }}$ inhibitors over a period of 96 hours starting at $1 \mathrm{dpf}$. Larvae treated with DMSO were used as a vehicle control. (B-D) CS-associated cardiac features, namely ejection fraction, cardiac output and end-diastolic volume were assessed in Sur2 ${ }^{\text {wt/GE }}$ larvae after GLB treatment using high-speed video imaging. (E-G) CS-associated cardiac features, namely ejection fraction, cardiac output and end-diastolic volume were assessed in Kir6. $1^{\text {wt/VM }}$ larvae after GLB treatment using high-speed video imaging. For all graphs, significance was determined by one-way ANOVA and subsequent post-hoc Tukey's test for pairwise comparison: ${ }^{*} p<0.05 ;{ }^{* *} p<0.01 ;{ }^{* * *} p<0.001 ;{ }^{* * *} p<0.0001$. Data shown as mean \pm SEM. Sample size: (B-D) wt/wt(-), n=10; wt/wt(+), n=16; wt/GE(-), n=19; wt/GE(+), n=41; (E-G) wt/wt(-), n=23; wt/wt(+), $\mathrm{n}=22 ; w t / V M(-), n=26 ; w t / V M(+), n=20$. All embryos analyzed originated from group matings of adult Sur2 ${ }^{\mathrm{w} / \mathrm{GE}}$ and Kir6. $1^{\mathrm{w} / \mathrm{MM}}$ fish. 

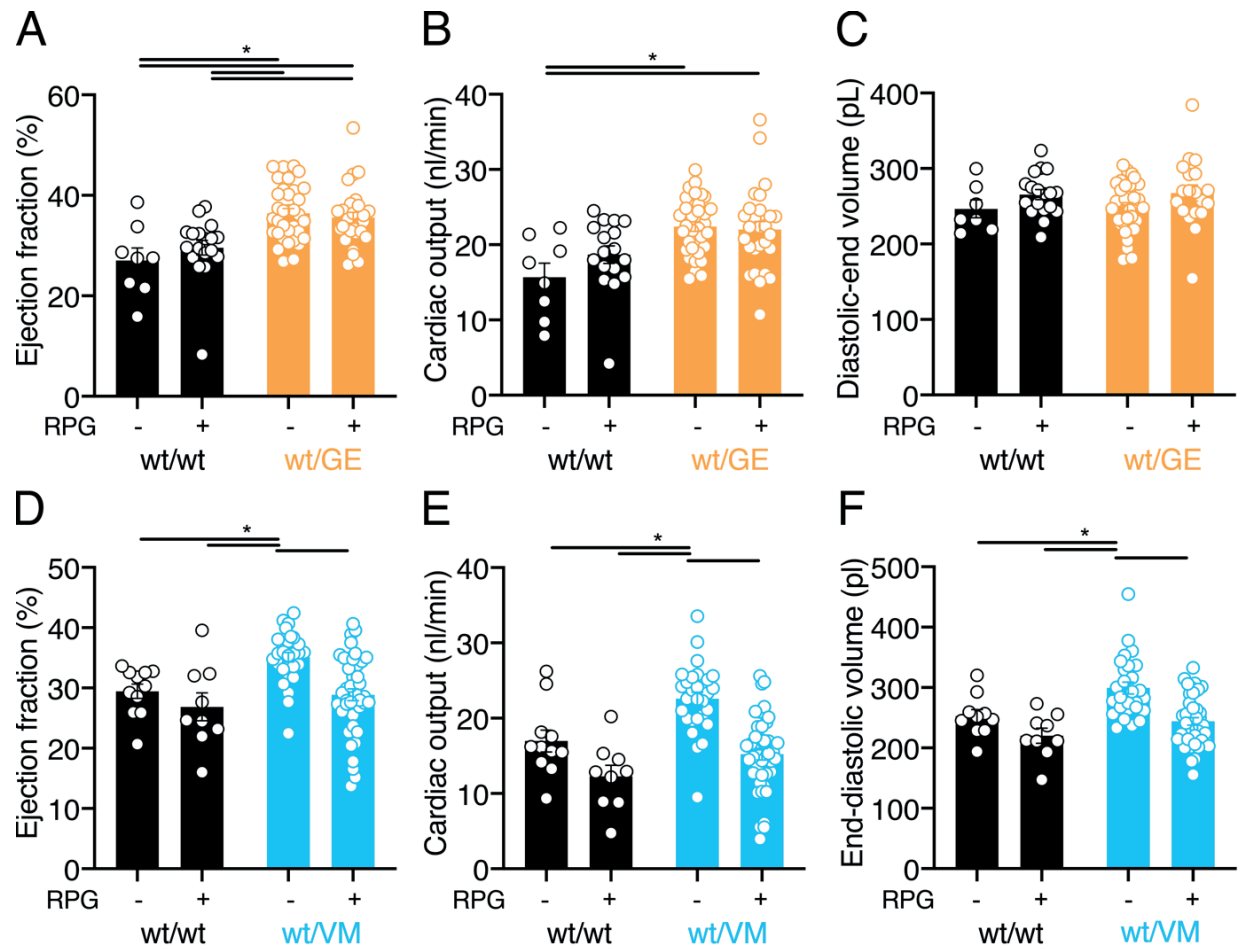

Figure 7 | Repaglinide corrects CS-related cardiac enlargement in Kir6. ${ }^{\text {wt/VM }}$ larvae. (A-C) Sur2 ${ }^{\text {wt/ }}$ ${ }^{G E}$ larvae were exposed to RPG over a period of 96 hours starting at $1 \mathrm{dpf}$ as shown in Fig. 6 A. Larvae treated with DMSO were used as a vehicle control. CS-associated cardiac features, namely ejection fraction, cardiac output and end-diastolic volume were assessed afterwards using high-speed video imaging. (D-F) Kir6. $1^{\mathrm{w} / \mathrm{VM}}$ larvae were exposed to RPG and ejection fraction, cardiac output and enddiastolic volume were measured. For all graphs, significance was determined by one-way ANOVA and subsequent post-hoc Tukey's test for pairwise comparison: ${ }^{*} p<0.05 ;{ }^{* *} p<0.01 ;{ }^{* *} p<0.001$. Data shown as mean \pm SEM. Sample size: (A-C) wt/wt(-), n=8; wt/wt $(+), n=18 ; w t / G E(-), n=34 ;$ wt/ $\mathrm{GE}(+), \mathrm{n}=28$; (D-F) wt/wt(-), n=10; wt/wt(+), n=9; wt/VM(-), n=29; wt/VM(+), n=42. All embryos analyzed originated from group matings of adult Sur2 ${ }^{\mathrm{wt} / \mathrm{GE}}$ and Kir6. $1^{\mathrm{w} t / \mathrm{MM}}$ fish.

\subsubsection{Hypoglycemia as side effect of GLB and RPG treatment in zebrafish}

Both GLB and RPG are already successfully used in clinical setups to inhibit GoF in pancreatic $\mathrm{K}_{\text {ATP }}$ isoforms Kir6.2 and SUR1, which underlie the pancreatic $\mathrm{K}_{\text {ATP }}$ channels involved in neonatal diabetes mellitus and which exhibit a significantly higher sensitivity towards both compounds than CV Kir6.1/SUR2 channels (Ashcroft, 2010). Hence, high doses of both compounds, as are required to correct CS-related phenotypes, would not only block CS $\mathrm{K}_{\text {ATP }}$ channels but also pancreatic channels, leading to elevated insulin secretion and decreased blood glucose. Consequently, hypoglycemia is predicted as the most likely adverse 
effect of both compounds when applied in non-diabetic patients, potentially limiting clinical application for CS. Thus, we assessed the effects of chronic GLB and RPG exposure in Sur2 ${ }^{\mathrm{wt} / \mathrm{GE}}$ and Kir6. $1^{\mathrm{wt} / \mathrm{VM}}$ larvae. We used a highly sensitive glucose assay to measure absolute glucose levels in larval lysates as a proxy for blood glucose (Jung et al., 2016). Control larvae revealed whole body glucose (WBG) levels in accordance with previously published studies demonstrating a peak at $1 \mathrm{dpf}$ followed by a significant decrease at $3 \mathrm{dpf}$ due to the formation of pancreatic $\beta$-cell islets and an associated increase in pancreatic insulin expression (Jurczyk et al., 2011; Rocha et al., 2014). GLB exposure resulted in an obvious but not significant decrease in WBG that failed to fully normalize after day 4 of treatment although an upwards trend was visible (Figure 8 A). RPG also led to a less severe drop in WBG (Figure $8 \mathbf{B}$ ).

A
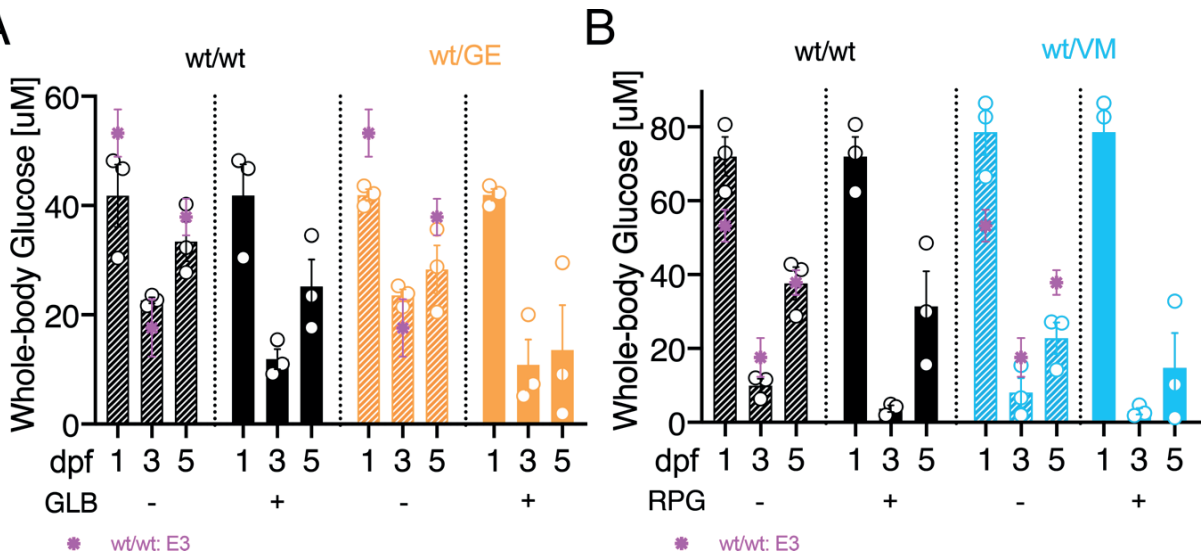

- wt/wt: E3

Figure 8 | Chronic exposure to GLB and RPG reduces whole-body glucose levels in CS zebrafish larvae. Absolute glucose levels in larval lysates consisting of a pool of 10 larvae each were measured applying a highly sensitive glucose assay kit. Glucose was measured in WT fish and fish prior to GLB and RPG exposure at $1 \mathrm{dpf}$ and after at 3 and $5 \mathrm{dpf}$. (A) Mean whole-body glucose measurements from WT and Sur2 ${ }^{\text {wt/GE }}$ control and GLB treatment group. Additionally, absolute glucose levels of WT fish grown in E3 medium are shown. (B) Mean whole-body glucose measurements from WT and Kir6. $1^{\mathrm{w} / \mathrm{MM}}$ control and RPG treatment group. Data shown as mean \pm SEM. Sample size: glucose assay was performed in triplicates. All embryos analyzed originated from group matings of adult zebrafish.

\subsection{Discussion}

\subsubsection{Zebrafish as a model organism for studying pathophysiology of Cantú syndrome}

Autosomal dominant GoF variants in ABCC9 (SUR2) and KCNJ8 (Kir6.1) have been established as the genetic cause of CS (Cooper et al., 2014; Harakalova et al., 2012; McClenaghan et al., 2017). To date, a variety of CS-associated variants 
in both genes have been shown to result in GoF of $\mathrm{K}_{\text {ATP }}$ channels due to multiple mechanisms either involving decreased ATP sensitivity or augmented activation by $\mathrm{Mg}^{2+}$-bound nucleotides (McClenaghan et al., 2017). Other than the Kir6.1 variant p.V65M, and SUR2 p.A478V and p.R1154Q, the functional consequences of most CS-associated variants have been tested only by patch-clamping recombinant channels. In this study we provide the first in vivo characterization of Sur2 CS variants p.G989E and p.C1043Y in zebrafish. By comparing the sensitivity of WT and mutant channels to ATP in the absence and presence of $\mathrm{Mg}^{2+}$, we examined the inhibitory effect of ATP independent of $\mathrm{Mg}^{2+}$ separately from the activating effect of MgATP. This investigation showed that p.C1043Y reduces ATP inhibition itself, whereas p.G989E increases activity by enhancing MgATP activation confirming previous observations in recombinant channels (Cooper, Sala-Rabanal, Lee, \& Nichols, 2015; McClenaghan et al., 2017).

Notably, the GoF induced by each mutation in the clinically relevant heterozygous state was evident but quite subtle; reports of GoF mutations in Kir6.2 and SUR1 resulting in neonatal diabetes suggest that even minor electrophysiological changes can lead to disease (Vedovato et al., 2016).

Previous studies in the zebrafish indicate that $\mathrm{K}_{\text {ATP }}$ channels are physiologically relevant in zebrafish (Capiotti et al., 2014; Emfinger et al., 2017; Pascoe et al., 2015; C. Zhang et al., 2006), although direct analysis of $K_{\text {ATP }}$ expression and functional characterization has been performed in $\beta$-cells (Emfinger et al., 2017). Our study provides definitive analysis of $\mathrm{K}_{\text {ATP }}$ channel composition in the heart and vasculature of zebrafish. Similarly to mammals, zebrafish cardiomyocytes seem to express Kir6.2/Sur2 channels, as the introduction of p.V65M in Kir6.1 did not result in a visible GoF in ventricular myocytes (Cole et al., 1991). Whole-cell patch-clamping of vascular smooth muscle cells, on the other hand, confirmed GoF due to the introduction of this Kir6.1 mutation (Figure 1 B).

\subsubsection{Primary and secondary consequences of Cantú syndrome in the heart}

Because GoF mutations in either ABCC9 (SUR2) or KCNJ8 (Kir6.1) result in similar pathophysiological features in CS patients (Brownstein et al., 2013; Grange et al., 2019) and zebrafish (Tessadori et al., 2018), it is clear that the CS phenotype primarily originates from changes in the Kir6.1/SUR2 $\mathrm{K}_{\text {ATP }}$ channel complex, which is prominently expressed in smooth, but not cardiac muscle, such that structural and functional changes of the heart are likely to result from secondary consequences (Aziz et al., 2014; McClenaghan et al., 2019). GoF in

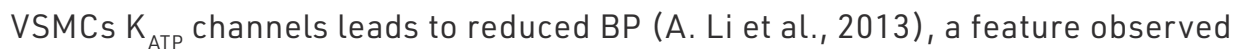


in many CS patients (Grange et al., 2019). Since this will otherwise result in underperfusion, a compensatory cardiac hypertrophy and high-output state are established (McClenaghan et al., 2019). We found that zebrafish embryos reveal enhanced EF and CO (Figure 5) with DEV being increased in adult fish (Tessadori et al., 2018), potentially reflecting the manifestation of heart enlargement after persistent exposure to volume overload.

In contrast to effects of VSMCs GoF, little is clear regarding the contribution of $\mathrm{K}_{\text {ATP }}$ GoF in the heart itself to CS pathophysiology. $\mathrm{K}_{\text {ATP }}$ GoF in the heart is predicted to shorten AP thereby reducing $\mathrm{Ca}^{2+}$ entry, contractility and $\mathrm{CO}$. The high-output state in CS is counter to this prediction resulting in the hypothesis of a feedback response to the heart which remodels excitation-contraction coupling and subsequently establishes hypercontractility as a secondary consequence. In CS zebrafish embryos, measurements of $\mathrm{Ca}^{2+}$ dynamics and $\mathrm{EF}$ are consistent with such a compensation indeed occurring in the form of a $\beta$-adrenergic intervention: whereas ventricles from $3 \mathrm{dpf}$ CS zebrafish embryos revealed the predicted decrease in $\mathrm{Ca}^{2+}$ signaling and $\mathrm{EF}$ as a primary effect of $\mathrm{K}_{\text {ATP }}$ GoF, increased $\beta$-adrenergic signaling in $4 \mathrm{dpf}$ embryos initiates a remodeling of the heart causing increased contractility as secondary consequence (Figure $\mathbf{5}$ and $\mathbf{9}$ ).

Hence, the zebrafish embryo represents an elegant visualization of primary and secondary consequences of $\mathrm{K}_{\text {ATP }}$ GoF in the heart. Our data suggests that two distinct effects originating from VSMCs and VCM contribute to increased CO and long-term cardiac enlargement in CS. 


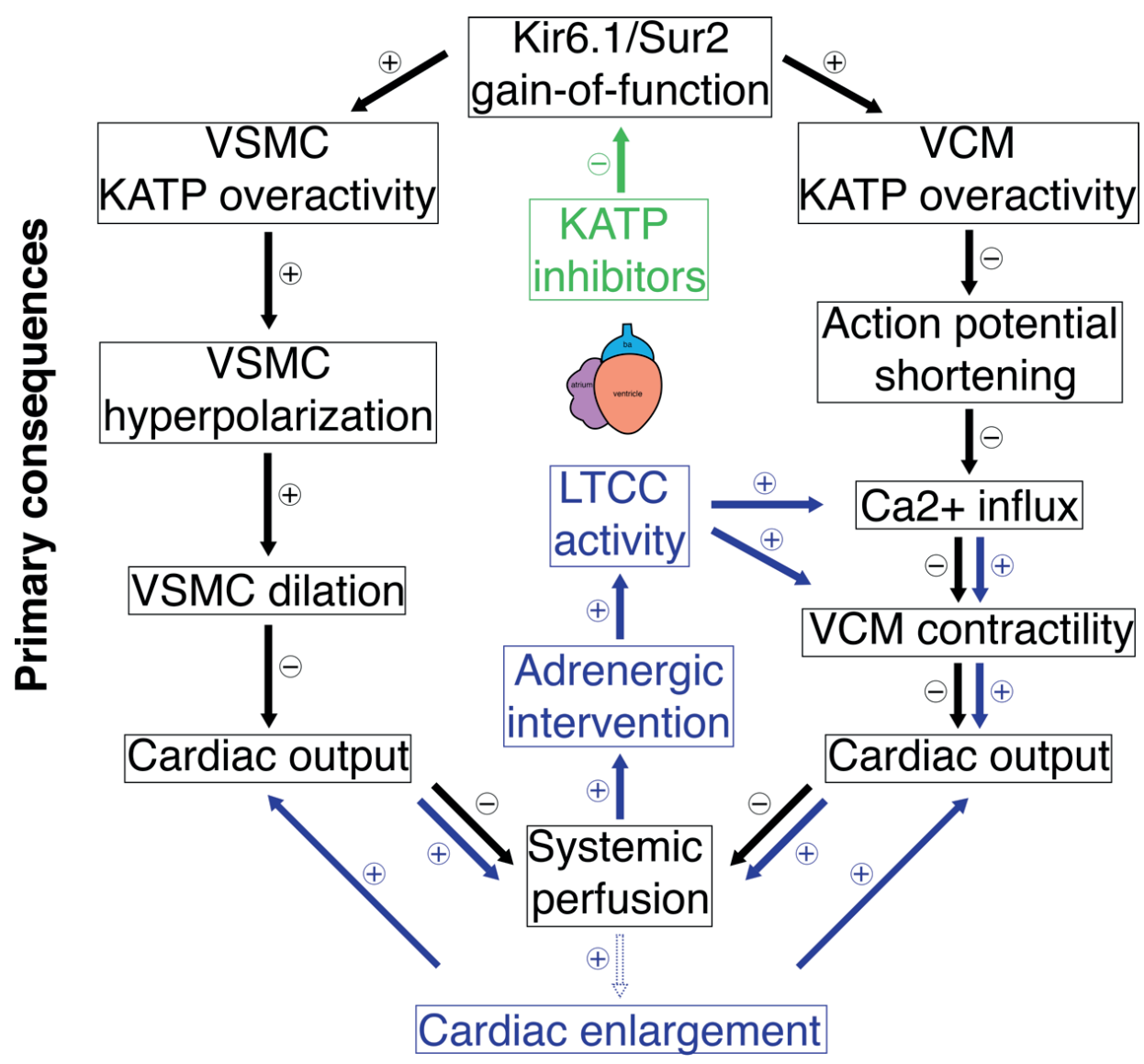

\section{Secondary consequences}

Figure 8 I Primary and secondary consequences of CS-associated GoF in the cardiovascular system. Gain-of-function associated with CS variants in both $\mathrm{K}_{\text {ATP }}$ channel subunits Kir6.1 and SUR2 results in channel overactivity. In VSMC, this gives rise to hyperpolarization, vessel dilation and subsequent reduced cardiac output and systemic perfusion. In the heart, $\mathrm{K}_{\mathrm{ATP}}$ overactivity leads to shortening of the action potential, reduced $\mathrm{Ca}^{2+}$ influx via LTCC, reduced contractility and similarly decreased cardiac output and systemic perfusion. Combined, these primary consequences trigger $\beta$-adrenergic intervention which will enhance $\mathrm{Ca}^{2+}$ uptake by increased phosphorylation of LTCC resulting in elevated contractility, cardiac output and systemic perfusion. Eventually, this chronic volume overload gives rise to cardiac enlargement as another long-term secondary consequence.

\subsubsection{Repurposing $\mathrm{K}_{\text {ATP }}$ blockers as a strategy to treat CS}

While the genetic basis of CS was established almost a decade ago, targeted treatment has remained elusive. By directly targeting over-active $\mathrm{K}_{\text {ATP }}$ channels using $K_{\text {ATP }}$ channel inhibitors, it may be possible to prevent or reverse 
consequences of $\mathrm{K}_{\text {ATP }}$ GoF. Thus, we tested the in vivo efficacy of GLB, RPG and PNU-37883 for correction of CS-associated cardiac abnormalities in two CS zebrafish models, Sur2[G989E] and Kir6.1[V65M]. In Sur2wt/Ge larvae, GLB significantly corrected hypercontractility and abnormal high-output phenotypes otherwise seen in the control group. This observation aligns with findings from our previously published study showing successful reversal of cardiac enlargement and vascular resistance in CS SUR2 knock-in mice (McClenaghan et al., 2019). Our data not only validates CS zebrafish larvae as an appropriate model organism in which to test $\mathrm{K}_{\text {ATP }}$ inhibitors as potential therapies for CS but also strengthens the evidence for in vivo efficacy of GLB to correct cardiac phenotypes resulting from CS-associated mutations in ABCC9. Additionally, our findings suggest that the development of cardiac anomalies can be prevented altogether by treatment at an early timepoint.

In accordance with findings by McClenaghan et al., chronic GLB exposure did not result in full correction of cardiac features in Kir6.1[V65M] larvae. Clinical hallmarks in patients and animal models harboring a mutation in KCNJ8 may be more severe than in organisms with an ABCC9 mutation (Brownstein et al., 2013; Cooper et al., 2014; Huang et al., 2018; McClenaghan et al., 2019; Tessadori et al., 2018). Whereas p.G989E did not significantly affect GLB sensitivity (McClenaghan et al., 2017), p.V65M markedly decreased GLB efficiency in recombinant channels (Cooper et al., 2017). Thus, GLB might not be appropriate for targeting channels including Kir6.1[V65M] subunits. Alternatively, reversal of a more severe phenotype might require longer exposure times.

Taking advantage of the zebrafish as a model organism ideal for high-throughput drug screening, we continued to investigate the in vivo efficacy of two further $K_{\text {ATP }}$ channel blockers, RPG and PNU-37883. Whereas RPG, structurally unrelated to GLB or other sulfonylureas, is also applied in clinics to treat diabetes due to its inhibitory effect on pancreatic Kir6.2/SUR1-dependent $K_{\text {ATP }}$ channels, PNU-37883 is a reputedly Kir6.1-specific inhibitor, not clinically approved. RPG successfully corrected cardiac enlargement and further CSrelated cardiac anomalies that are otherwise observed in heterozygous Kir6. $1^{\text {wt/ }}$ VM larvae, but surprisingly failed to show a comparable effect in Sur2 ${ }^{\text {wt/GE }}$ fish. Electrophysiological analysis suggested that p.G989E acts by increasing $\mathrm{K}_{\text {ATP }}$ channel activation by MgATP, whereas the p.V65M mutation is shown to reduce ATP inhibition itself (Figure 2 and 3) (Cooper et al., 2017). The RPG binding site in SUR1 partially overlaps with the GLB binding site (Ding, Wang, Wu, Kang, \& Chen, 2019) possibly explaining differences found in drug efficacy in both zebrafish models. As the position of RPG is incompatible with the $\mathrm{Mg}$-nucleotide- 
bound state of SUR1 due to steric clash (Ding et al., 2019), we hypothesize that RPG only blocks CS channels with mutations that do not increase the affinity to MgATP. As discussed above, GLB and RPG are used clinically for the treatment of neonatal diabetes, and since pancreatic $\mathrm{K}_{\text {ATP }}$ channels exhibit markedly higher sensitivity to these drugs than $\mathrm{CV} \mathrm{K}_{\mathrm{ATP}}$ channels, high doses required to inhibit CV $K_{\text {ATP }}$ channels will also unavoidably inhibit pancreatic $K_{\text {ATP }}$ channels and would be expected to provoke increased insulin secretion and consequently induce hypoglycemia. The only reported human CS case thus far treated with GLB revealed transient hypoglycemia (Ma et al., 2019). GLB and RPG exposure in zebrafish larvae did lead to some decrease in whole-body glucose. Notably, GLB-related hypoglycemia in CS mice normalized after a few days of treatment (McClenaghan et al., 2019). This transient effect has been demonstrated before and likely involves long-term compensatory down-regulation of insulin secretion from pancreatic $\beta$-cells despite continued $K_{\text {ATP }}$ channel inhibition (Remedi \& Nichols, 2008). Our measurements in zebrafish larvae were performed during development, a crucial phase where small changes in environment can have a great impact. Additionally, there is no food intake to keep absolute glucose levels in balance. Hence, we suggest that blood glucose measurements in CS mice represent the effect of treating patients with GLB more accurately.

\subsection{Conclusions and Future perspectives}

In conclusion, we show that zebrafish CV K ${ }_{\text {ATP }}$ channels have similar composition and functional properties to those in humans, and that the zebrafish thus represents a suitable model organism for studying pathophysiology and possible therapy options for disorders involving aberrant CV $\mathrm{K}_{\text {ATP }}$ channels.

In this study, we provide proof for the in vivo efficacy of two already available $\mathrm{K}_{\mathrm{ATP}}$ channel blockers, and hence promising candidates for drug repurposing, to treat cardiac abnormalities associated with CS.

Further potential side-effects of chronic GLB and RPG exposure, in addition to hypoglycemia, will have to be investigated in the future; e.g., inhibitory actions on skeletal and cardiac muscle. There is a long-standing debate regarding potential cardiovascular side effects of sulfonylureas like GLB. It is suggested that blockade of cardiac $\mathrm{K}_{\text {ATP }}$ channels may be harmful in conditions like myocardial ischemia, during which these channels can open and are presumed protective. This debate is still on-going (Gore \& McGuire, 2011; Schramm et al., 2011). 
Finally, we show that it may be essential to determine $\mathrm{K}_{\text {ATP }}$ channel blocker sensitivity for specific mutations for future individualized therapy. Notably, numerous $\mathrm{K}_{\text {ATP }}$ GoF mutations that reduce inhibitor sensitivity, including neonatal diabetes mutations in Kir6.2/SUR1 as well as CS mutations, have already been reported (Cooper et al., 2017; Koster, Remedi, Dao, \& Nichols, 2005; Proks, 2013). While this can be done in recombinant channels as it has been done in the past, such studies can also be performed in native channels in zebrafish or mouse models, or originating from human-induced pluripotent stem cell-derived cardiovascular cells allowing a more precise measurement of the potential effect of CS-associated mutations on the pharmacological response.

\subsection{Materials and Methods}

\subsubsection{Fish maintenance}

All animal experiments were conducted under the guidelines of the animal welfare committee of the Royal Netherlands Academy of Arts and Sciences (KNAW) and the Washington University Institutional Animal Care and Use Committee. Adult zebrafish (Danio rerio) were maintained and embryos raised and staged as previously described (Westerfield, 1993). All CrispR/Cas9 injections were performed in the wild-type strain Tübingen longfin (Tessadori et al., 2018).

\subsubsection{Zebrafish models of Cantú syndrome}

The CS zebrafish were generated as previously described (Tessadori et al., 2018). In brief, CRISPR-Cas9 genome editing was combined with a short template oligonucleotide to introduce a single nucleotide mutation into abcc 9 (c.2969 GC>AA; Z.Gly983Glu and c.3176 G>A; Z.Cys1052Tyr) and kcnj8 (c.204 G>A; Z.Val65Met) to mimic the human disease-causing amino acid substitutions, H.Gly989Glu and H.C1043Y in Sur2 and H.V65M in Kir6.1.

\subsubsection{Isolation of zebrafish ventricular cardiomyocytes}

Previously published protocols for isolation of zebrafish cardiomyocytes have been adapted as follows (Brette et al., 2008; Louch, Sheehan, \& Wolska, 2011; V. Sander, Sune, Jopling, Morera, \& Belmonte, 2013). Animals were anaesthetized by transfer into ice water before they were decapitated. After removal of the skin and opening of the pericardial sac, the hearts were harvested and both bulbous arteriosus and atrium were removed. Ventricles from 3-4 fish were pooled together and placed in perfusion buffer: (mM) 10 HEPES, 30 Taurine, 5.5 Glucose, 10 2,3-Butanedione monoxime in 1xPBS. Ventricles were gently torn open to 
drain out blood. Ventricular myocytes were obtained by enzymatic dissociation (perfusion buffer supplemented with $12.5 \mathrm{uM} \mathrm{CaCl}_{2}, 5 \mathrm{mg} / \mathrm{ml}$ collagenase II and IV (Worthington), $5 \mathrm{ng} / \mathrm{ml}$ Insulin in perfusion buffer) at $32{ }^{\circ} \mathrm{C}$ and $800 \mathrm{rpm}$ for $40 \mathrm{~min}$. Dissociation was ended by transfer to stopping buffer (perfusion buffer supplemented with 10\% (vol/vol) FBS, $12.5 \mathrm{uM} \mathrm{CaCl}, 10 \mathrm{mg} / \mathrm{ml} \mathrm{BSA}, 5$ ng.ml Insulin in perfusion buffer). After 15 min incubation at room temperature, stopping buffer was replaced by perfusion buffer and cells were dispersed by gentle trituration using a Pasteur pipette. Cells were viable for physiological measurements at room temperature for up to 12 hours.

\subsubsection{Inside-out patch-clamp recordings}

Upon successful isolation of zebrafish cardiomyocytes, inside-out excised patch-clamping was performed to characterize $\mathrm{K}_{\text {ATP }}$ channel expression and activity, using a perfusion chamber with piezo-regulated flow control (Cannell \& Lederer, 1986; Lederer \& Nichols, 1989). The chamber consisted of four inflow lanes. A drop of isolated cells was placed in lane 1 and patches of membrane were excised from these cells. The electrode tip can be moved from one lane to another, thereby exposing the excised patch to different solutions flowing through the lanes.

Micropipettes for patch-clamping were pulled from soda lime glass microhematocrit tubes (Kimble-Chase 2502) using a P-97 puller (Sutter Instruments). The pipette tips were coated with a molten mix of Parafilm ${ }^{\text {TM }}$ in mineral oil to reduce the capacitance and had a resistance of 1-2 $\mathrm{MW}$ when filled with pipette solution. The pipette and lane solutions for excised patching were typically $\mathrm{K}_{\mathrm{INT}}$ buffer (140 mM KCl, $10 \mathrm{mM}$ HEPES and $1 \mathrm{mM} \mathrm{EGTA}$ at $\mathrm{pH}$ 7.4) with or without additional nucleotides/drugs/ $\mathrm{Mg}^{2+}$ added depending on the study. With or without additional nucleotides/drugs $/ \mathrm{Mg}^{2+}$. The pipette solution for whole- cell patching was internal cell solution (110 Potassium Aspartate, 30 $\mathrm{KCl}, 10 \mathrm{NaCl}, 1 \mathrm{MgCl}_{2}, 10 \mathrm{HEPES}, 0.5 \mathrm{CaCl}_{2}, 4 \mathrm{~K}_{2} \mathrm{HPO}_{4}$ and 5 EGTA in mM at pH 7.2 adjusted with $\mathrm{KOH}$ ).

A characteristic feature of $\mathrm{K}_{\text {ATP }}$ channels is rapid inhibition by intracellular ATP $(\sim 5 \mathrm{mM})$. To measure $\mathrm{K}_{\text {ATP }}$ channel activity and sensitivity to ATP via insideout excised patching, the lanes were perfused with different concentrations of ATP ( $0 \mathrm{mM}, 5 \mathrm{mM}, 10 \mu \mathrm{M}$ and $100 \mu \mathrm{M}$ respectively in lanes 1-4). Excised-patch membrane currents were recorded at a constant holding potential of $50 \mathrm{mV}$, using an Axopatch-1D amplifier and Axon pCLAMP software from Molecular Devices. Experiments were performed at room temperature. Triplicates of recordings were obtained for statistical significance. The excised-patch channel 
currents in solutions of varying nucleotide concentrations were normalized to the basal currents in the absence of nucleotides for respective recordings and a dose-response curve was plotted using a four parameter Hill fit according to the following equation

Normalized current $=I_{\min }+\left(I_{\max }-I_{\min }\right) /\left(1+\left([X] / I C_{50}\right) H\right)$

where the current in $\mathrm{K}_{\mathrm{INT}}=\mathrm{I}_{\max }=1, \mathrm{I}_{\min }$ is the normalized minimum current observed

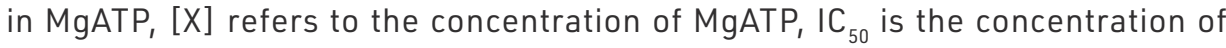
half-maximal inhibition, and $\mathrm{H}$ denotes the Hill coefficient.

\subsubsection{Isolation of zebrafish vascular smooth muscle cells}

The middle layer cells of the bulbous arteriosus (ba) of teleost fish are predominantly comprised of 'arterial-like' vascular smooth muscle cells (Icardo, Colvee, Cerra, \& Tota, 2000). 4 bas were excised from anaesthetized zebrafish and placed in Solution 1 (S1; 0.1\% BSA (w/v), $145 \mathrm{NaCl}, 4 \mathrm{KCl}, 10 \mathrm{HEPES}, 10$ Glucose, $0.05 \mathrm{CaCl}_{2}, 1 \mathrm{MgCl}_{2}$ in $\mathrm{mM}$, adjusted to $\mathrm{pH} 7.4$ using $\mathrm{NaOH}$ ) on ice. $\mathrm{S} 1$ was then replaced by $400 \mu$ Solution 2 (S2; 2 ml S1, 3 mg Papain (Worthington), $2 \mathrm{mg}$ DTT (Sigma)). The bas were digested in $\mathrm{S} 2$ at $32^{\circ} \mathrm{C}, 800 \mathrm{rpm}$ for 25 minutes. After that, S2 was replaced by $500 \mu$ Solution 3 (S3; $2 \mathrm{~mL} \mathrm{S1,} 3$ mg Collagenase Type H (Sigma), 2 mg Trypsin Inhibitor (Worthington), 1 mg Elastase (Worthington)) and digested for 5 minutes. Digestion was ended by replacing S3 with $500 \mu \mathrm{l}$ S1 in which the tissue was triturated using a Pasteur pipette and the cells were plated onto coverslips, and then left to attach for at least 1 hour before further experiments.

\subsubsection{Whole-cell patch-clamp recordings}

For whole-cell patch-clamping, only lane 1 of the perfusion chamber mentioned in 8.5.4 was used. The inflow line was connected parallelly to different bath solutions, whose individual flow could be controlled using solenoid valves (LFA series, The Lee Company). Recordings were sampled at $3 \mathrm{kHz}$ and filtered at $1 \mathrm{kHz}$. Currents were initially measured at a holding potential of $-70 \mathrm{mV}$ in a high- $\mathrm{Na}^{+}$bath solution containing $136 \mathrm{NaCl}, 6 \mathrm{KCl}, 2 \mathrm{CaCl}_{2}, 1 \mathrm{MgCl}_{2}, 10 \mathrm{HEPES}$, and 10 glucose in $\mathrm{mM}$, with $\mathrm{pH}$ adjusted to 7.4 with $\mathrm{NaOH}$ before switching to a high- $\mathrm{K}^{+}$bath solution $\left(140 \mathrm{KCl}, 2 \mathrm{CaCl}_{2}, 1 \mathrm{MgCl}_{2}, 10 \mathrm{HEPES}\right.$, and 10 glucose, with $\mathrm{pH}$ adjusted to 7.4 with $\mathrm{KOH}$ ). The pipette solution contained 110 potassium aspartate, $30 \mathrm{KCl}, 10 \mathrm{NaCl}, 1 \mathrm{MgCl}_{2}, 10 \mathrm{HEPES}, 0.5 \mathrm{CaCl}_{2}, 4 \mathrm{~K}_{2} \mathrm{HPO}_{4}$, and 5 EGTA in $\mathrm{mM}$, with $\mathrm{pH}$ adjusted to 7.2 with $\mathrm{KOH}$. 


\subsubsection{Calcium imaging}

For calcium imaging, CS zebrafish lines were crossed with $\operatorname{tg}(m y l 7: G a l 4 F F$; UAS:GCaMP6f) (van Opbergen et al., 2018). A morpholino (MO) oligomer targeted against tnnt2a (5'-CATGTTTGCTCTGATCTGACACGCA-3') was used to uncouple contraction from excitation in embryos, thereby preventing contraction artifacts in our recordings of cardiac electrophysiology and $\mathrm{Ca}^{2+}$ dynamics. This 'silent heart' ATG morpholino was applied as described previously (Sehnert et al., 2002). To block pigmentation for imaging, E3 embryonic raising medium with phenylthiourea (PTU) in a concentration of $0.003 \%(\mathrm{v} / \mathrm{v})$ was used from $24 \mathrm{~h}$ postfertilization (hpf). Recordings were performed using a custom-build upright widefield microscope (Cairn research, Kent, UK) equipped with a 20x $1.0 \mathrm{NA}$ objective (Olympus XLUMPLFLN20X W). White LED excitation light was filtered using a 438/24 nm filter (Semrock FF02-438/24-25) and reflected towards the objective using a $458 \mathrm{~nm}$ dichroic mirror (Semrock FF458-Di02-25×36). Emitted fluorescence was directed to an emission splitter unit (OptoSplit II ByPass Image Splitter) fitted with a $509 \mathrm{~nm}$ dichroic mirror (Semrock FF509-FDi01-25×36) and 483/32 nm and 514 long-pass emission filters (Semrock FF01-483/32-25 and LP02-514RU-25, respectively). Images were projected on a high-speed camera (Andor Zyla 4.2 plus sCMOS). Recordings were performed at $100 \mathrm{fps}$, for 1000 s frames. Basal parameters were recorded first. Subsequently, propranolol hydrochloride (Sigma) was diluted in $28^{\circ} \mathrm{C}$ E3-Tricaine medium to $100 \mu \mathrm{M}$ and the medium was mixed vigorously to assure a homogeneous concentration of the drug. Embryos were incubated for $30 \mathrm{~min}$ at $28^{\circ} \mathrm{C}$ in normal E3-Tricaine medium (Placebo experiments) or E3-Tricaine-drug mixture and parameters were measured again.

All embryos analyzed originated from group matings of adult heterozygous Sur2 [G989E]:GCaMP6f CS zebrafish. Genotyping was performed by Sanger sequencing post imaging analysis. Recordings were analyzed blindly using ImageJ and Matlab (Version R2015a, Mathworks, Natick, MA, USA).

\subsubsection{Drug treatment in zebrafish larvae}

Drug delivery was achieved by soaking. Glibenclamide (Sigma), Repaglinide (RPG) (Sigma) and PNU-37883 (4-morpholinecarboximidine-N-1-adamantyl$\mathrm{N}^{\prime}$-cyclohexyl-hydrochloride (Nichols lab, WashU) stock solutions were prepared in 100\% dimethylsulphoxide (DMSO; Sigma) and serial dilutions were made before each experiment. Embryos originated from group matings of adult zebrafish heterozygous for respective mutations in abcc 9 and kcnj8. Genotyping was performed by Sanger sequencing post imaging. At $24 \mathrm{hpf}$ embryos were dechorionated and treated for period of $96 \mathrm{~h}$. Here, drug stocks were made in 
E3/PTU medium (GLB $50 \mu \mathrm{M}$, RPG $25 \mu \mathrm{M}$, PNU-37883 $75 \mu \mathrm{M}$ ). Final $\mathrm{K}_{\text {ATP }}$ blocker concentrations were determined by establishing concentration-dependent survival curves in WT fish over 4 days of exposure. Drug concentrations that resulted in $>70 \%$ of healthy fish were selected for treatment of CS fish. Zebrafish treated with DMSO (GLB: 1\%, RPG: 0.5\%, PNU-37883: 0.75\%) were used as a vehicle control (Zhu et al., 2014). Untreated zebrafish were used to confirm that the vehicle solvent did not have an adverse effect. Experiments were carried out at a constant temperature $\left(28^{\circ} \mathrm{C}\right)$. After treatment, cardiac function of $5 \mathrm{dpf}$ zebrafish was examined using high-speed imaging.

\subsubsection{In vivo high-speed imaging}

Image acquisition was conducted as previously described (Tessadori et al., 2018). Briefly, zebrafish embryos were anaesthetized in $16 \mathrm{mg} / \mathrm{ml}$ tricaine (MS222; Sigma-Aldrich) in E3 medium and mounted in dorsal position in small microscopic chambers filled with $0.25 \%(\mathrm{w} / \mathrm{v})$ agarose prepared in the same concentration of anaesthetic. Zebrafish hearts were imaged for $10 \mathrm{~s}$ (approximately 30 cardiac cycles) at $28^{\circ} \mathrm{C} \pm 0.2^{\circ} \mathrm{C}$ using a Hamamatsu C9300221 high speed CCD camera (Hamamatsu Photonics) at $150 \mathrm{fps}$ mounted on a Leica DM IRBE inverted microscope (Leica Microsystems) applying Hokawo 2.1 imaging software (Hamamatsu Photonics). High-speed brightfield image sequences of the larval zebrafish heart were acquired for zebrafish at age of 5 dpf using a 20-fold magnification. All embryos originated from group matings of adult heterozygous CS zebrafish. Genotyping was performed by Sanger sequencing post imaging analysis. Image analysis was carried out blindly with ImageJ (http://rsbweb.nih.gov/ij/, last accessed January 2020).

\subsubsection{Measurement of ventricular volume, cardiac output, shortening fraction and ejection fraction}

Images from high-speed movies were used to outline the perimeter of the ventricle. Measurement analysis was carried out by "fit-to-ellipse" algorithm, which worked on calculating the centre of mass and subsequently the best fitting ellipse. The long axis length (a) and short axis length (b) at diastole and systole were determined and used to calculate ventricular systolic-end (SEV) and diastolic volumes (DEV) applying the formula:

$V=4 / 3 \pi(b / 2) 2(a / 2)$

The stroke volume (SV) was calculated as the difference between three ventricular EDVs and ESVs. The time interval between three heart beats was measured and the heart rate (bpm) was calculated. Cardiac output was obtained 
by multiplying the heart rate with stroke volume. Ejection fraction (EF) can be calculated with the following formula:

$E F(\%)=(S V / E D V) \times 100$

Every cardiac parameter represents an average of triplicates.

\subsubsection{Whole-body glucose measurements in zebrafish larvae}

Quantitative analysis of glucose levels was performed from whole body lysates using a highly sensitive glucose assay kit (Biovision). Reactions were assembled on ice in black, flat bottom 96-well plates. Briefly, 10 zebrafish larvae in each experimental group were frozen on crushed ice and $100 \mu \mathrm{l}$ of cold glucose assay buffer was added. To measure glucose in larval extracts, $9 \mu$ of sample was added to $36 \mu \mathrm{l}$ assay buffer. To this, $2.5 \mu \mathrm{l}$ of glucose assay buffer, $0.5 \mu \mathrm{l}$ PicoProbe, $1 \mu$ l Glucose Enzyme Mix and $1 \mu$ Glucose Substrate Mix were added. Standard curves were generated using glucose standard solution (according to instructions) and were included in each assay. Reactions were incubated for 30 min at $37^{\circ} \mathrm{C}$ in the dark. Fluorescence (excitation $535 \mathrm{~nm}$; emission, $587 \mathrm{~nm}$ ) was measured using a Pherastar microplate reader.

\subsubsection{Statistical analysis}

Sample size was not predetermined by statistical analysis. In all experiments involving zebrafish larvae, selection was random for scoring. Exact numbers of analyzed larvae are reported at relevant locations in the main text or the supplementary information. Statistical analysis was carried out with Prism (GraphPad). Normal distribution of the data sets was confirmed by D'AgostinoPearson omnibus or Shapiro-Wilk test normality test. Significance values were calculated using one-way ANOVA and subsequent post-hoc Tukey's test for pairwise comparison throughout the manuscript. All values are expressed as mean \pm SEM. Results were considered significant at $p<0.05$. 


\subsection{Supplemental information}
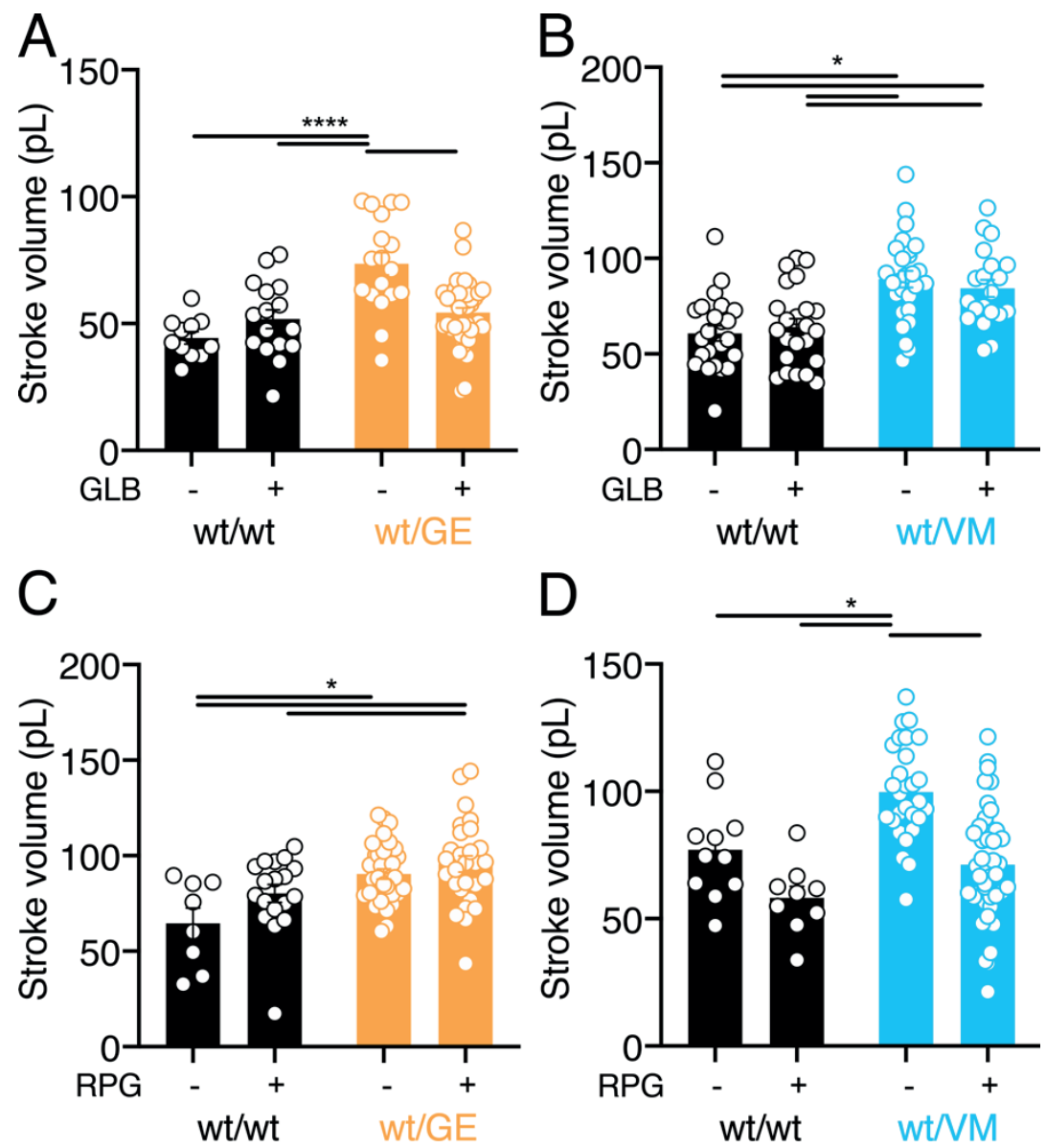

\footnotetext{
Supplementary Figure 1 I Effect of GLB and RPG on stroke volume of Sur2 ${ }^{\mathrm{wt} / G \mathrm{E}}$ and Kir6. ${ }^{\mathrm{wt} / \mathrm{VM}}$ zebrafish larvae. Sur2 ${ }^{\mathrm{w} / \mathrm{GE}}$ and Kir6. $1^{\mathrm{wt} / \mathrm{VM}}$ larvae were exposed to GLB and RPG over a period of 96 hours starting at $1 \mathrm{dpf}$. Larvae treated with DMSO were used as a vehicle control. (A-D) Stroke volume was measured. WT controls and mutant fish are the same as in Fig. 6 and 7. For all graphs, significance was determined by one-way ANOVA and subsequent post-hoc Tukey's test for pairwise comparison: ${ }^{*} p<0.05 ;{ }^{* *} p<0.01 ;{ }^{* * *} p<0.001 ;{ }^{* * * *} p<0.0001$. Data shown as mean \pm SEM. Sample size: (A) wt/wt(-), $n=10 ; w t / w t(+), n=16 ; w t / G E(-), n=19 ; w t / G E(+), n=41 ;(B) w t / w t(-), n=23 ;$ wt/ wt(+), n=22; wt/VM(-), n=26; wt/VM(+), n=20; (C) wt/wt(-), n=8; wt/wt(+), n=18; wt/GE(-), n=34; wt/GE(+), n=28; (D) wt/wt(-), n=10; wt/wt(+), n=9; wt/VM(-), n=29; wt/VM(+), n=42. All embryos analyzed originated from group matings of adult Sur2 ${ }^{\mathrm{wt} / \mathrm{GE}}$ and Kir6. $1^{\mathrm{wt} / \mathrm{VM}}$ zebrafish.
} 
A

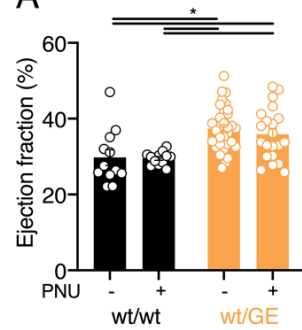

$\mathrm{E}$

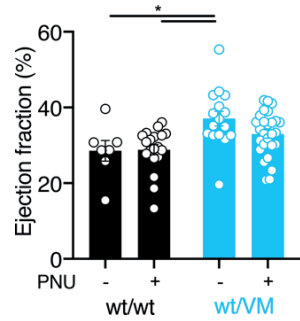

B

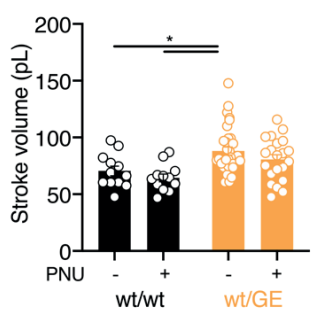

$\mathrm{F}$

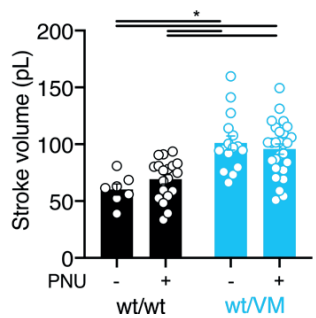

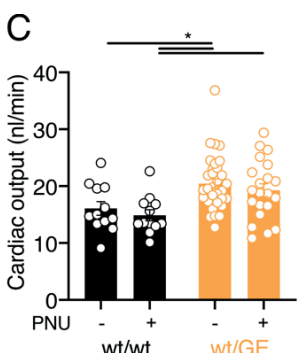

G

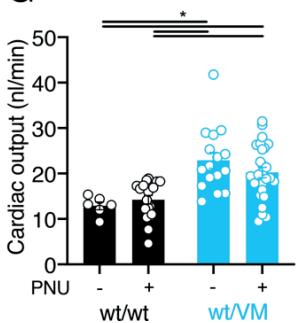

D

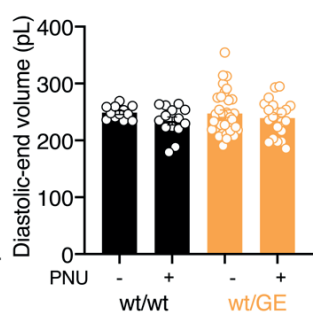

$\mathrm{H}$

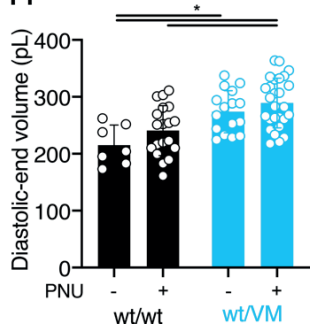

Supplementary Figure 2 I PNU-37883 has no effect on CS-related cardiac phenotype in Sur2 ${ }^{\text {wt/GE }}$ and Kir6. wt/VM $^{\text {tarvae. Sur2 }}{ }^{\text {wt/GE }}$ (A-D) and Kir6. $1^{\text {wt/VM }}$ larvae (E-H) were exposed to PNU37883 over a period of 96 hours starting at $1 \mathrm{dpf}$. Larvae treated with DMSO were used as a vehicle control. CS-associated cardiac features, namely ejection fraction, stroke volume, cardiac output and end-diastolic volume were assessed in treatment using high-speed video imaging. For all graphs, significance was determined by one-way ANOVA and subsequent post-hoc Tukey's test for pairwise comparison: ${ }^{*} p<0.05 ;{ }^{* *} p<0.01 ; * * * p<0.001 ; * * * * p<0.0001$. Data shown as mean \pm SEM. Sample size: (A-D) wt/wt(-), $n=12 ;$ wt/wt(+), $n=12 ; w t / G E(-), n=33 ; w t / G E(+), n=21$. (E-H) wt/wt(-), n=7; $w t / w t(+), n=16 ; w t / V M(-), n=19 ; w t / V M(+), n=27$. All embryos analyzed originated from group matings of adult Sur2 ${ }^{\mathrm{w} / \mathrm{GE}}$ and Kir6. ${ }^{\mathrm{wt} / \mathrm{MM}}$ zebrafish. 
Characterization and treatment of overactive $K_{\text {ATP }}$ channels in zebrafish models of Cantú syndrome 
-12
4
4
4

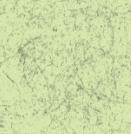

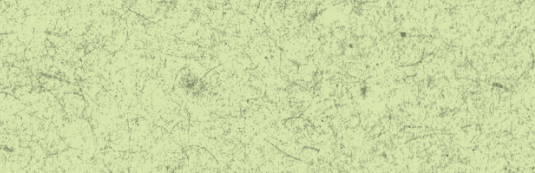

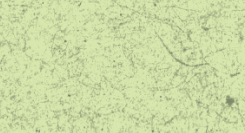

तr.

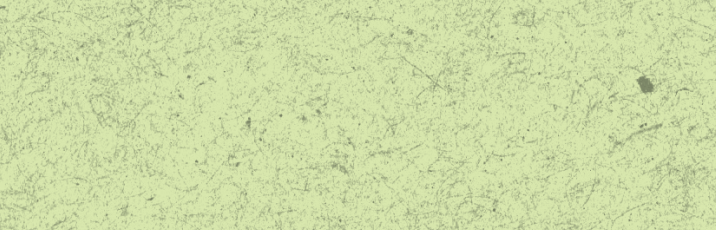

4

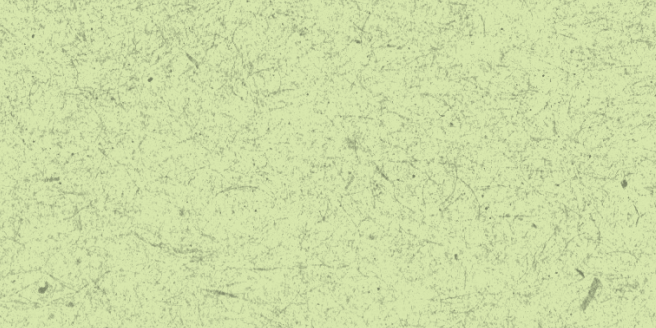




\section{9}

\section{ABCC9-related Intellectual disability Myopathy Syndrome is a $K_{A T P}$ channelopathy with loss-of- function mutations in $A B C C 9$}

Marie F. Smeland*, Conor McClenaghan*, Helen I. Roessler*, Sanne M.C. Savelberg, Geir A.M. Hansen, Helene Hjellnes, Kjell A. Arntzen, Kai I. Müller, Andreas Rosenberger Dybesland, Theresa Harter, Monica Sala-Rabanal, Chris H. Emfinger, Yan Huang, Soma S. Singareddy, Jamie Gunn, David F. Wozniak, Attila Kovacs, Maarten Massink, Federico Tessadori, Sarah M. Kamel, Jeroen Bakkers, Maria S. Remedi, Marijke Van Ghelue*, Colin G. Nichols*, Gijs van Haaften*

*These authors contributed equally to this work. 


\section{Abstract}

Mutations in genes encoding $\mathrm{K}_{\text {ATP }}$ channel subunits have been reported for pancreatic disorders and Cantú syndrome. Here, we report a syndrome in six patients from two families with a consistent phenotype of mild intellectual disability, similar facies, myopathy, and cerebral white matter hyperintensities, with cardiac systolic dysfunction present in the two oldest patients. Patients are homozygous for a splice-site mutation in ABCC9 (c.1320+1 G>A), which encodes the sulfonylurea receptor 2 (SUR2) subunit of $K_{\text {ATP }}$ channels. This mutation results in an in-frame deletion of exon 8 , which results in non-functional $\mathrm{K}_{\text {ATP }}$ channels in recombinant assays. SUR2 loss-of-function causes fatigability and cardiac dysfunction in mice, and reduced activity, cardiac dysfunction and ventricular enlargement in zebrafish. We term this channelopathy resulting from loss-of-function of SUR2-containing $\mathrm{K}_{\text {ATP }}$ channels ABCC9-related Intellectual disability Myopathy Syndrome (AIMS). The phenotype differs from Cantú syndrome, which is caused by gain-of-function ABCC 9 mutations, reflecting the opposing consequences of $\mathrm{K}_{\text {ATP }}$ loss-versus gain-of-function. 


\subsection{Introduction}

$\mathrm{K}_{\text {ATP }}$ channels are nucleotide-gated potassium channels formed by the obligate co-assembly of pore-forming Kir6.x subunits and regulatory sulfonylurea receptors (SURx), which couple the membrane potential to metabolic state in multiple cell types (Martin et al., 2017; Nichols, 2006). In mammals, two Kir6.x isoforms are encoded by the paralogous KCNJ8 (Kir6.1; [OMIM: 600935]) and KCNJ11 (Kir6.2; [OMIM: 600937]) genes, which are each co-located with genes encoding two SUR isoforms, ABCC9 (SUR2; [OMIM: 601439]) and ABCC8 (SUR1; [OMIM: 600509]), respectively, on chromosomes 12 and 11 . Molecular heterogeneity is further increased by alternative splicing of ABCC9 mRNA, yielding two major splice variants, SUR2A and SUR2B - while multiple other splice variants have also been reported (Chutkow, Makielski, Nelson, Burant, \& Fan, 1999; Foster \& Coetzee, 2016; Inagaki et al., 1996; Isomoto et al., 1996; Nichols, 2006; Shi, Ye, \& Makielski, 2005; Ye et al., 2009). Pancreatic and neuronal $K_{\text {ATP }}$ channels are predominantly formed by Kir6.2 and SUR1, smooth muscle $K_{\text {ATP }}$ channels are comprised of Kir6.1 and SUR2B, and the predominant combination in striated muscle is Kir6.2 and SUR2A (Foster \& Coetzee, 2016).

The causative role of gain-of-function (GoF) or loss-of-function (LoF) mutations in the Kir6.2/SUR1-dependent pancreatic $\mathrm{K}_{\text {ATP }}$ channels in neonatal diabetes and congenital hyperinsulinism, respectively, was established nearly two decades ago (Gloyn et al., 2004; Koster, Marshall, Ensor, Corbett, \& Nichols, 2000; P. Thomas, Ye, \& Lightner, 1996; P. M. Thomas et al., 1995). Recently, it has been demonstrated that dominant GoF mutations in KCNJ8 and ABCC9 underlie Cantú syndrome (CS [OMIM: 239850]) (Cooper et al., 2014; Harakalova et al., 2012; van Bon et al., 2012). CS is characterized by hypertrichosis, coarse facial features, and multiple cardiovascular abnormalities, including cardiomegaly and tortuous, dilated vasculature (Harakalova et al., 2012; Leon Guerrero et al., 2016; van Bon et al., 2012). Behavioral problems and mild developmental delay have been reported in CS, but intellectual function is typically normal (Grange et al., 2014).

The human consequences of LoF in Kir6.1 and SUR2 remain uncertain. In a single report, two heterozygous LoF mutations in an exon found only in SUR2A were associated with dilated cardiomyopathy (DCM [MIM: 608569]) (Bienengraeber et al., 2004). A missense mutation in the same exon was reported as predisposing to paroxystic adrenergic atrial fibrillation (AF [MIM: 614050]), but only in one 53-year-old female patient (Olson et al., 2007). The pathophysiological consequences of complete SUR2 LoF are unclear. 
We report six patients from two non-consanguineous families from Northern Norway who exhibit a shared pathological constellation including similar facies, intellectual disability and developmental delay, anxiety, myopathy with hypotonia, muscle weakness, and fatigability. Cardiac systolic dysfunction is found in the two oldest patients. All have cerebral white matter hyperintensities, and hyperreflexia is found in the oldest four. The families are investigated by comprehensive clinical exome sequencing, a powerful tool for identifying the genetic basis of rare and complex syndromes, both in patients with de novo mutations and in families with suspected recessive inheritance (Najmabadi et al., 2011; Vissers \& Veltman, 2015).

We identify a homozygous ABCC9 splice site mutation (c.1320+1G>A) in all affected individuals. We show that the mutation causes the in-frame deletion of exon 8, resulting in SUR2 protein lacking 52 amino acids, and loss of plasmalemmal $K_{\text {ATP }}$ function. Using CRISPR/Cas9 genome engineering, we introduce frameshift mutations into $A B C C 9$ that result in premature protein truncation, in both zebrafish and mice. These animals lack functional SUR2 protein and myocyte $\mathrm{K}_{\text {ATP }}$ channels and recapitulate the myopathy and cardiac dysfunction observed in patients. We conclude that SUR2 LoF results in a recessive syndrome: $A B C$ C9-related Intellectual disability Myopathy Syndrome (AIMS).

\subsection{Results}

\subsubsection{Patient descriptions}

Four siblings from Family 1 (aged 12-21 years) and two siblings from Family 2 (aged 29, 33) (Figure 1a) are described. The families are not known to be related, but are from the same area of Northern Norway. Genetic investigations had earlier been performed with normal results in several of the patients, including G-banding, high-resolution Single-Nucleotide Polymorphism (SNP) array to look for genomic deletions and duplications, FMRI CGG repeat analysis, DMPK PCR, sequencing of multiple neuromuscular disease genes, screening for mitochondrial DNA sequence variants/deletions, and screening for inborn errors of metabolism. Clinical photographs of the patients are presented in Figure $\mathbf{1} \mathbf{b}$, c. MRI images are presented in Figure 1d. Clinical features are summarized in Tables 1 and 2 . 


\section{Family 1}

Patient 1-1 is the first child of healthy parents from Northern Norway with probable Finnish ancestry. Pregnancy and birth were uneventful. Weight and length were below the 2.5 percentile in childhood. Hearing loss was reported in childhood, but a recent hearing evaluation was normal. Early psychomotor development was described as normal, but at age 2, in-toeing, toe-walking and reduced fine motor skills, generalized hypotonia, lumbar lordosis, and delayed development were noted. At age 11, neuropsychological testing identified mild intellectual disability. From age 15, she experienced episodes of tonic/tonicclonic seizures, but epileptic activity was absent in repeated EEGs. Lamotrigine treatment was partly effective. She continues to report muscular pain, fatigue, and anxiety. Recently, hyperprolactinemia was found (720 mlU L-1 (ref: 102$496 \mathrm{~m}(U \mathrm{~L}-1))$. An MRI of the pituitary, however, did not disclose pituitary adenomas. She lives in her own home, but requires frequent supervision and help. For neuromuscular evaluation of all patients, including cerebral MRI with white matter changes, see Tables 1 and $\mathbf{2}$.

Patient 1-2 is the younger brother of 1-1. Pregnancy and birth were uneventful. Delayed psychomotor development and lumbar lordosis was noted early alongside eating difficulties and low body weight. In childhood, he displayed left lower extremity weakness, hyperreflexia, and limping. Muscle biopsy showed unspecific changes of mitochondrial aggregation and muscle fiber caliber variation. Mild intellectual disability was diagnosed by neuropsychological testing in teenage years.

Patient 1-3 is the younger brother of 1-1. Pregnancy and birth were unremarkable. He showed delayed early psychomotor development with generalized hypotonia and toe-walking. Hyperreflexia was noted in the lower extremities from age 5 . Eating difficulties, nausea, abdominal pain, and low body weight were present from childhood. Neuropsychological testing at 5 years showed mild intellectual disability. Profound muscular pain and stiffness are reported after physical exercise. Mild bilateral high-frequency sensorineural hearing loss was found at age 12.

Patient 1-4 is the youngest brother of 1-1. Pregnancy and birth were unremarkable. Eating difficulties and low body weight were observed in early years, with weight and length at the 2.5 percentile. Delayed psychomotor development, in-toeing, lumbar lordosis, generalized muscular hypotonia, and strabismus were noted. At age 7, he experienced a cerebral episode with coma and tetraplegia preceded directly by repeated vomiting, and a few days earlier by high fever. 

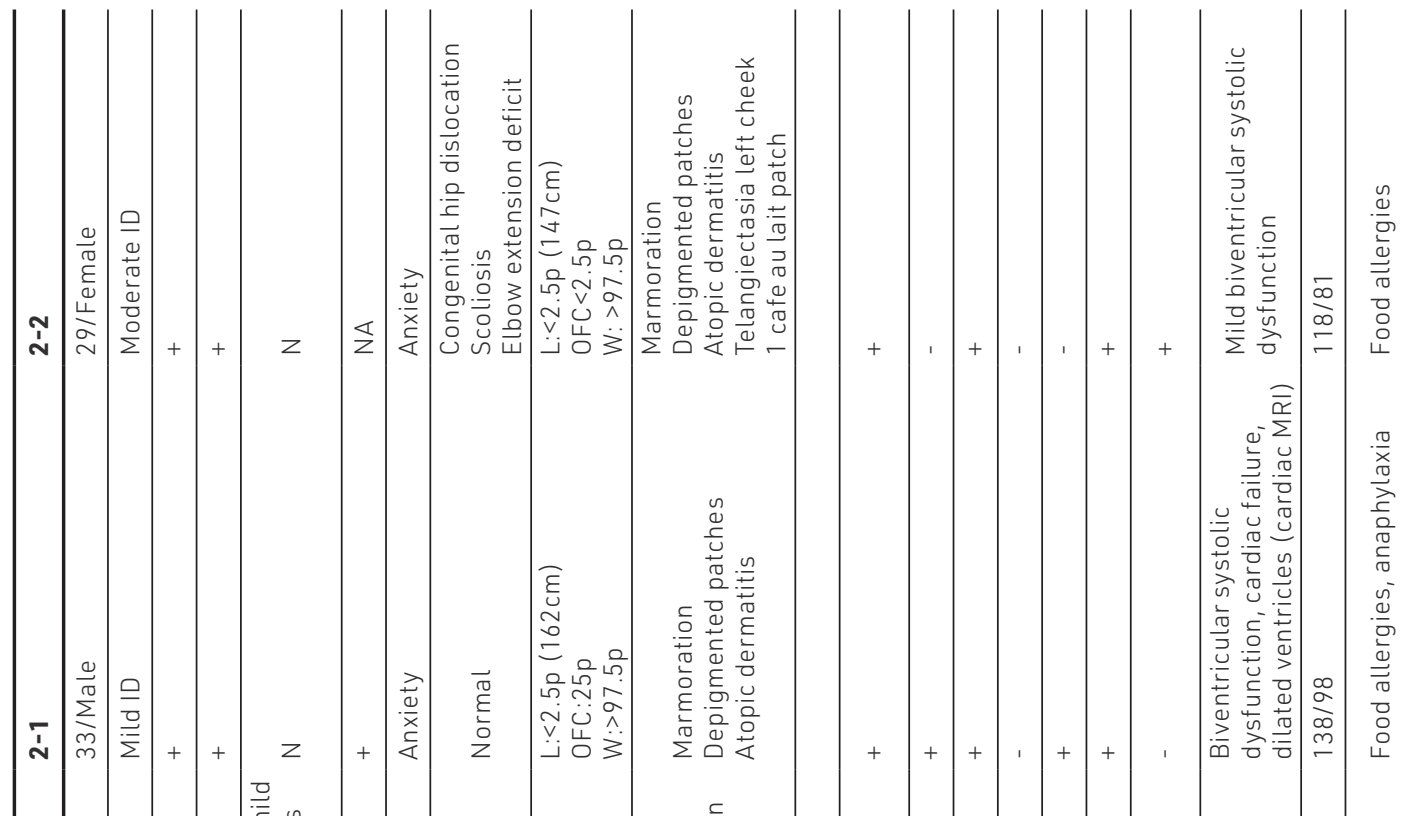

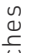

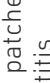

离

है

है. 흠 응

\begin{tabular}{ll|l|l}
$\Sigma$ & - & \\
& $=$
\end{tabular}

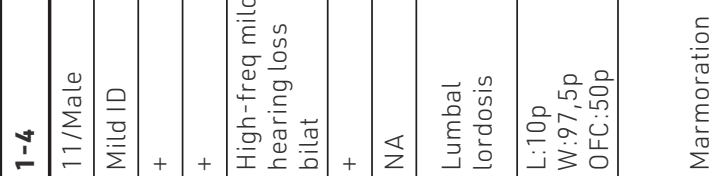

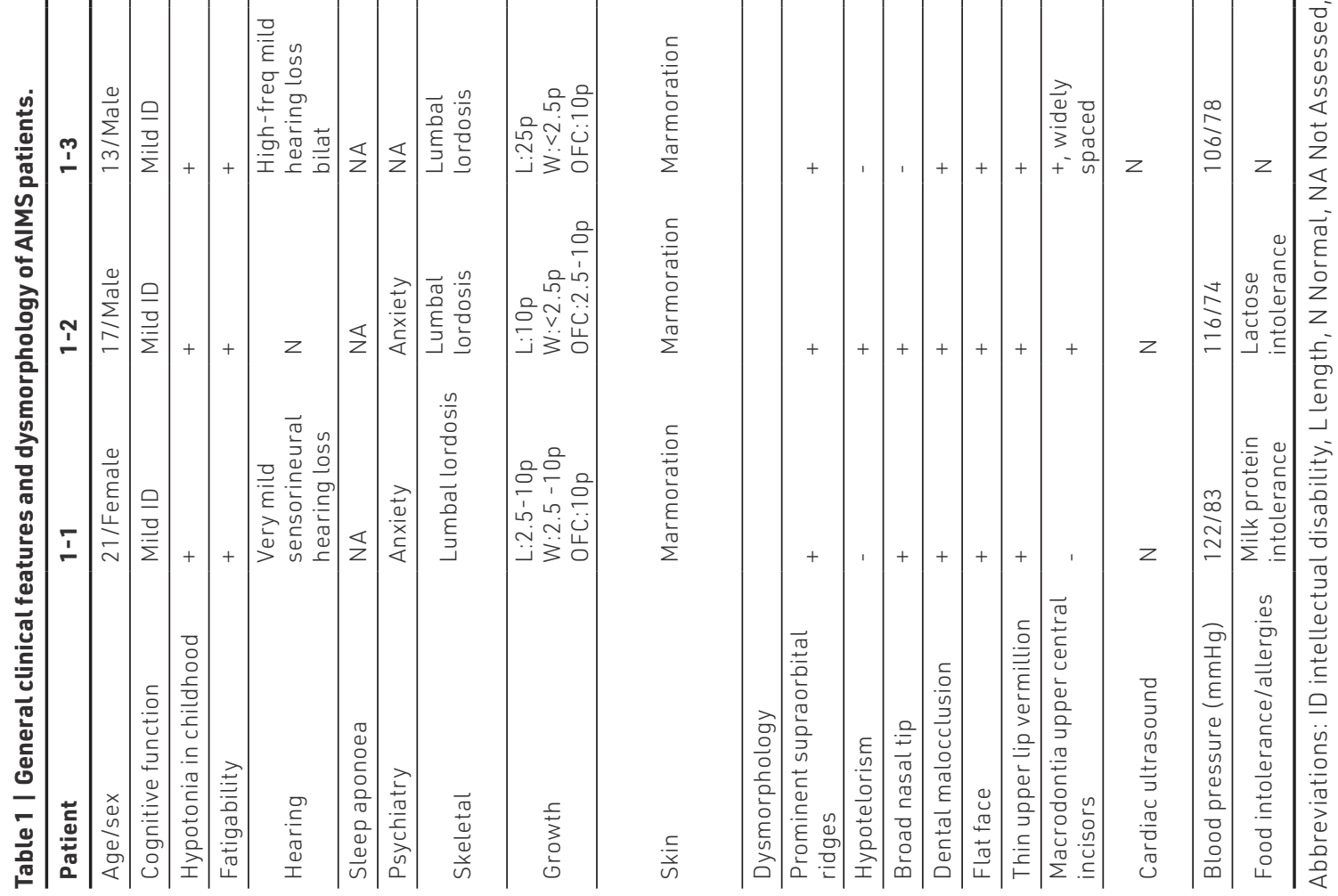




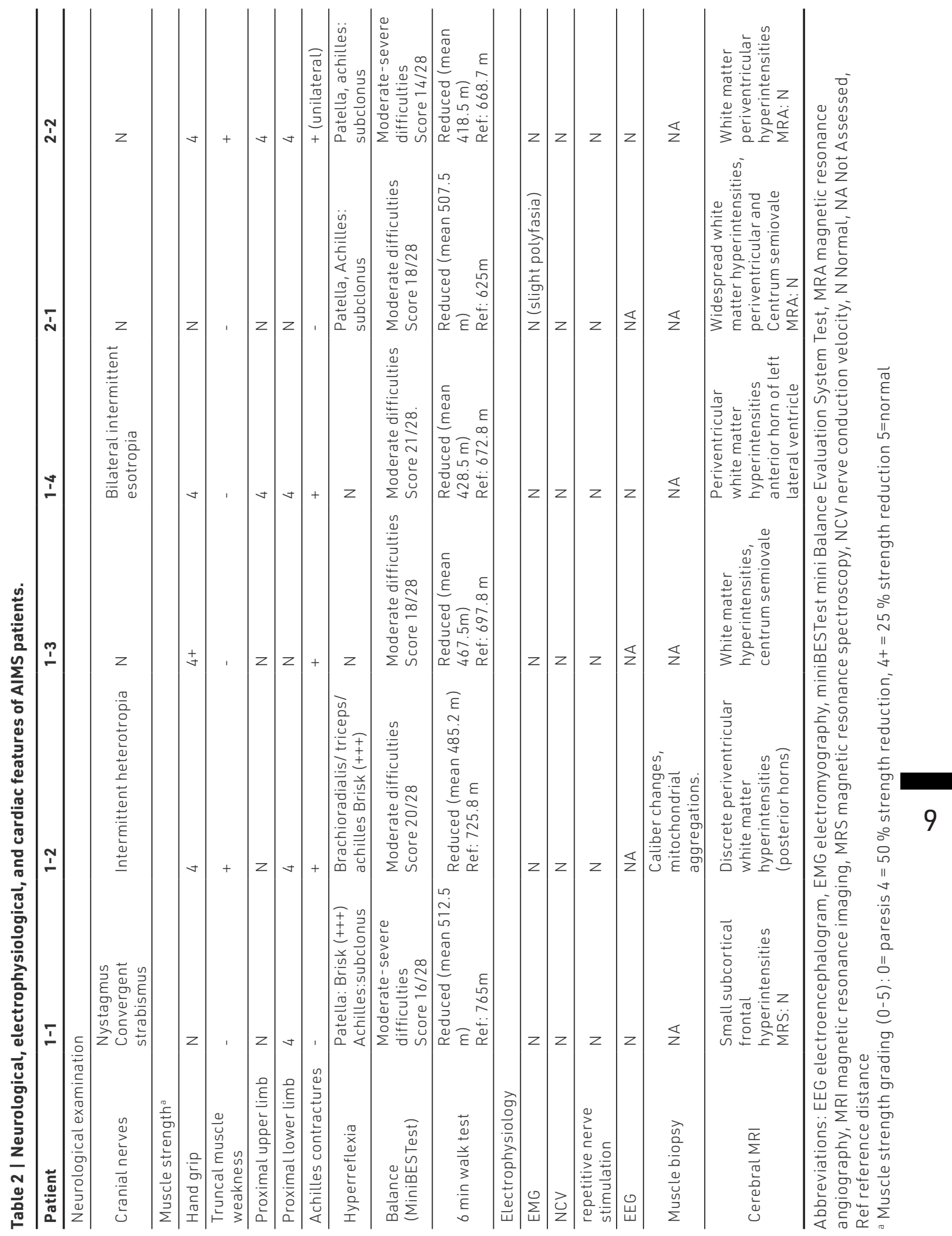


Serum measurements showed a metabolic acidosis with S-lactate 6, normal S-potassium (measured after intravenous infusion), and elevated S-creatin kinase (738) at hospital admission. Cerebral MRI revealed multiple lesions in both hemispheres periventricularly, subcortically, in the pons and the basal ganglia, in both gray and white matter. Acute disseminated encephalomyelitis was discussed, and steroid treatment was given. He regained consciousness over the following few days. Re-evaluation of MRI findings concluded with an "inflammatory perivascular reaction". MRI lesions were normalized a few weeks later, except for a white matter lesion by the anterior horn of the left ventricle. He regained the same psychomotor level as before. Weight increased after that episode and is currently $>97.5$ percentile. Neuropsychological testing at age 9 resulted in a diagnosis of mild intellectual disability. Obstructive sleep apnea, probably related to being overweight, a large tongue and hanging soft palate were diagnosed at age 11, and operative treatment was recommended. Mild bilateral high-frequency hearing loss was found at age 11 .

Individual II.3 was a female fetus, conceived in between patients 1-2 and 1-3. The parents elected to terminate pregnancy in pregnancy week 21 , due to suspected thanatophoric dysplasia with micromelia and a narrow thorax.

\section{Family 2}

Patient 2-1 is the first child of healthy parents. Pregnancy and birth were unremarkable. Delayed psychomotor development and muscular hypotonia were noted in toddler years. Due to similar findings in him and his sister, with a few depigmented skin patches and white matter lesions on cerebral computed tomography scan, a diagnosis of tuberous sclerosis (TS) was suggested, but later abandoned. The MRI pattern is not the same as in TS. Neither sibling has experienced epileptic seizures. Feeding was difficult in childhood, with low body weight, but he gained weight in adulthood, and is now overweight, with short stature. Severe atopic eczema is currently managed by cyclosporine, and he has multiple food allergies.

Recent neuropsychological testing led to a diagnosis of mild intellectual disability. He has been medicated for anxiety with a selective serotonin reuptake inhibitor for the last few years. He reports frequent dizziness attacks. Cardiac ultrasound at age 33 revealed biventricular systolic dysfunction, moderate left ventricle dysfunction, left ventricle ejection fraction (EF) of $35-40 \%$, and raised NT-proBNP. Left ventricle diameter was within the normal range, although, cardiac MRI shows left ventricle dilatation, without obvious pathology of the myocardium-compatible with early-stage dilated cardiomyopathy. Treatment 
with an ACE-antagonist and a beta-blocker was started. Hypertension was diagnosed before treatment initiation. A cardiac ultrasound in teenage years was normal. Cerebral MRIs at ages 16 and 33 showed widespread confluent white matter signal changes, described as similar to leucodystrophy. Lesions have increased significantly since the first investigation. MR angiography is normal, with normal cerebral vasculature calibers. Point lesions are found in the basal ganglia, pons, and white matter, representing possible mineral depositions. He lives in a sheltered home with daily help and supervision. He attends a sheltered work program, but is easily exhausted, and needs rest during the day. He is cheerful and social. Muscle strength is normal.

Patient 2-2 is the younger sister of 2-1. Pregnancy and birth were unremarkable. She was treated for congenital hip dysplasia. Psychomotor development was delayed. Cerebral MRI in childhood showed periventricular white matter changes, and a diagnosis of tuberous sclerosis was considered (see above). Hypotonia, reduced muscle strength, and exhaustibility are reported since childhood. She has short stature and microcephaly. She had a thin build in childhood, but is now overweight. Recent neuropsychological testing indicates mild-to-moderate intellectual disability. She lives in her own flat with assistance. She has anxiety and has been followed by a local outpatient psychiatry service. Cardiac ultrasound at age 29 revealed biventricular systolic dysfunction, mildly reduced left ventricle ejection fraction (EF) of $48 \%$, and a hypokinetic left ventricle with normal diameter. ProBNP is normal and there are no clinical signs of cardiac failure at this time. Cerebral MRI performed recently shows an increase in periventricular white matter signal changes compared with the last MRI at age 13. MR angiography of cerebral vessels is normal.

Individual II-2 is the mother of patients 2-1 and 2-2. Recent cardiac ultrasound, ECG and bicycle stress echocardiogram were normal at age 51.

Individual II-3 is the younger sister of patients 2-1 and 2-2. She is healthy, and is the mother of two healthy children. Recent cardiac ultrasound, ECG, and bicycle stress echocardiogram were normal at age 25 . 

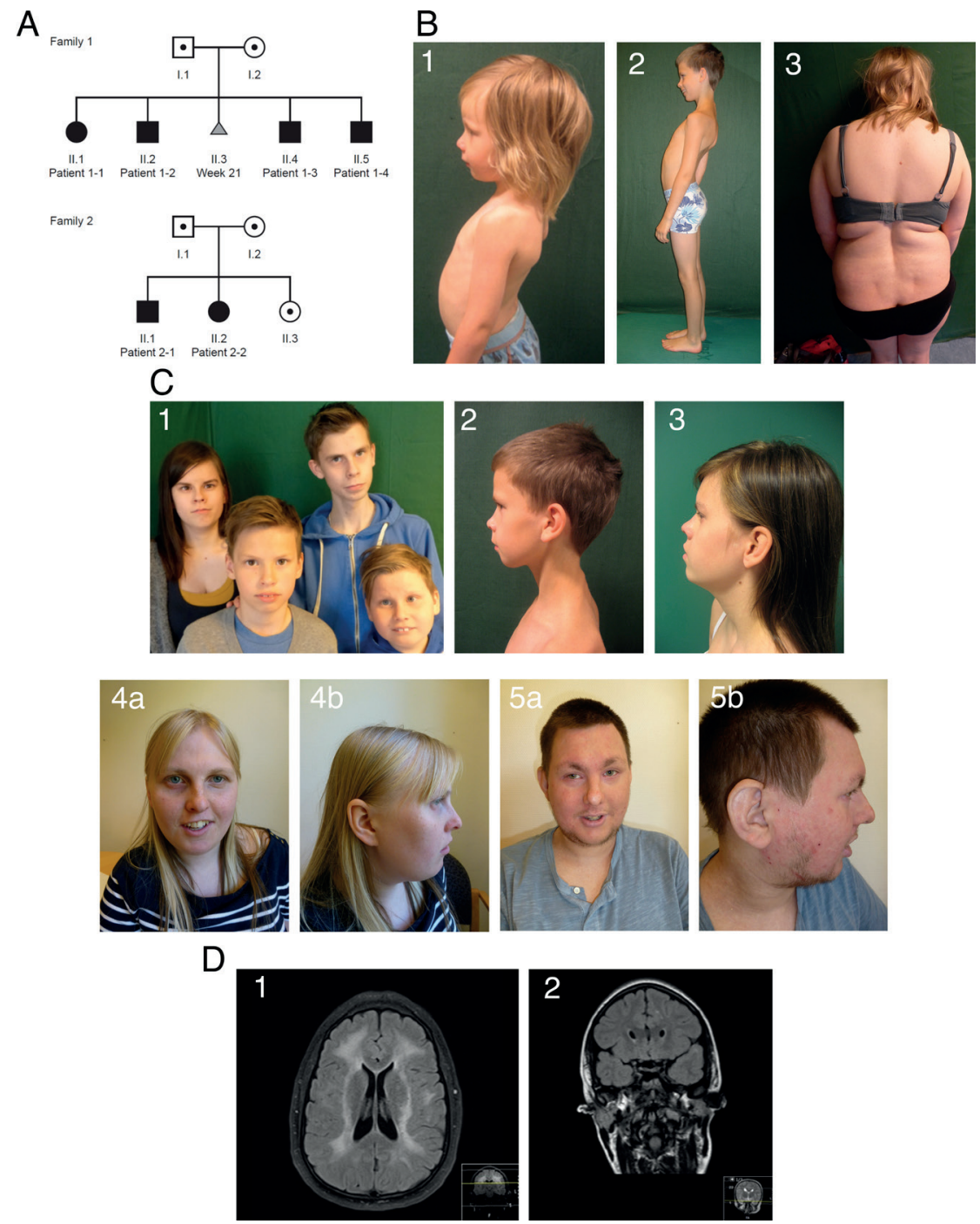

Figure 1 | Clinical features and pedigrees of AIMS patients. a Pedigrees of both affected families. Black defines patients homozygous for the $A B C C 9$ c. $1320+1 \mathrm{G}<\mathrm{A}$ mutation. Black dots indicate individuals heterozygous for the ABCC9 variant. Gray triangle represents a fetus affected by probable thanatophoric dysplasia and terminated pregnancy. b Musculoskeletal features in AIMS patients. (1) Lumbar lordosis in patient 1-4 at age 4 ; (2) lumbar lordosis, thin habitus in patient 1-2 at age 10; (3) thoracolumbar scoliosis in patient 2-2 at age 28. c Facial features with prominent orbital ridges, hypotelorism, thin upper lip, flat midface in several of the patients. (1) Family 1. Upper left: patient 1-1 at age 20. Upper right: patient 1-2 at age 16. Lower left: patient 1-3 at age 11. Lower right: patient $1-4$ at age 10 ; (2) profile of patient $1-2$ at age $10 ;(3)$ profile of patient 1-1 at age 15 ; (4) patient 2-2 at age 28 ; (5) patient 2-1 at age 32 . $\mathbf{d}$ Cerebral MRI of AIMS patients. (1) Magnetic resonance imaging (MRI) of the brain of patient 2-1. T2-weighted fluid-attenuated inversion recovery (FLAIR), coronal section shows widespread white matter hyperintensities. (2) MRI of the brain of patient 1-1. T2-weighted FLAIR, axial section shows juxtacortical white matter hyperintensities. 


\subsubsection{Identification of a splice site mutation in ABCC9}

We performed sequencing of one affected individual from each family (patients 1-2 and 2-1), plus their respective healthy parents, using a targeted panel of $>4800$ disease-associated genes. The samples were analyzed for recessive variants (homozygous and compound heterozygous) and non-Mendelian inheritance. The two trios were analyzed independently, and from a total of $\sim 10,000$ variants in each of the families, the homozygous variant ABCC9 c. $1320+1 \mathrm{G}>\mathrm{A}$ was the only remaining causal candidate after filtering against quality, region of interest, coding effect, minor allele frequency, and manual review of $\sim 20$ variants in each of the two families. Next-generation sequencing (NGS) and subsequent Sanger sequencing revealed that all six patients were homozygous for ABCC9 c. $1320+1 \mathrm{G}>\mathrm{A}$. Analysis of DNA from the four parents and one unaffected sibling from family 2 showed that they were each heterozygous for the same mutation.

To exclude distant relatedness between the two families and thus exclude the possibility of the presence of more shared rare variants with an effect on protein function, we performed whole-genome sequencing on one affected individual from each family (patients 1-2 and 2-1), and determined a kinship coefficient of 0.0403 . A kinship coefficient of $\sim 0.05$ is expected for unrelated samples, confirming non-relatedness between our families.

Since the original NGS analysis was performed on a gene panel, we analyzed the WGS data sets for candidate causal recessive and dominant variants. The focus of the analysis was the identification of shared variants or different variants in shared genes with possible damaging, but not identical variants leading to the same clinical phenotype, among the two cases. After the initial analysis, 67 shared variants were identified, of which 66 were heterozygous and 1 was homozygous (Table S2). The single homozygous variant remaining after the filtering was the above variant in ABCC9:NC_000012.11:g.22063090 C > T; NM_020297.2:c.1320+1G > A (Figure S2). This variant lies in a shared homozygous block of 3.8 MB (chr12:18.326.590-22.176.010 hg19). Notably, gene interactions with the homozygous $A B C C 9$ c. $1320+1 \mathrm{G}>\mathrm{A}$ variant cannot be excluded as participating in the syndrome. A list of all shared variants including allele frequencies is provided in Table S2. For a dominant mode of inheritance, we observed that, in 842 genes, one or more variants pass the filtering criteria in both cases. Again, the ABCC9 variant was the only variant seen in homozygous state in both samples (Figure S3). Compound heterozygous gene candidate analysis identified seven genes with two or more variants shared between the two cases. None of these genes are likely to contribute to the phenotype (Figure 
S4). Thus, after gene panel and WGS, we identified the homozygous ABCC9 c. $1320+1 \mathrm{G}>\mathrm{A}$ variant as the most likely causal variant. The variant is reported at very low allele frequency in the heterozygous state in the European population (Finnish: 3/6586; European: (Non-Finnish) 4/66386, Exac, June 2018). According to gnom $A D$, the variant is reported at surprisingly high frequency in heterozygous state in the Finnish population with an allele frequency of 0.0007 (18/24850). It is less common in non-Finnish Europeans (5/128232, allele frequency 0.00004 ) and absent in Asian or African populations (May 2019). Considering the probable Finnish ancestry of all patients, the syndrome might be more prevalent in the Finnish population than others. The homozygous state is absent in gnomAD.

The ABCC9 c. $1320+1 \mathrm{G}>\mathrm{A}$ variant is predicted to disrupt a splice donor site of exon 8 (Figure 2a). To study the effect of the mutation, we performed cDNA analysis on fibroblasts from members of both families (Figure $\mathbf{2 b}$ ). Sequence analysis of the homozygous cDNA samples revealed that the sequence variant caused an in-frame deletion of exon 8 (r.1165_1320del) in SUR2 cDNA, and predicts a 52 amino acid (p.Ala389_Gln440del) deletion within the SUR2 protein. Thus, all patients are homozygous for a splice variant in ABCC9 which results in an in-frame deletion.

\subsubsection{The effect of exon 8 deletion on $\mathrm{K}_{\text {ATP }}$ channel function}

Deletion of exon 8 is predicted to disrupt multiple transmembrane helices in SUR2 and thus have a profound effect on structure and function (Figure 3a). We deleted exon 8 in SUR2 cDNA (SUR2A $\triangle 8$ ) and performed expression analyses on Cosm6 cells transiently transfected with Kir6.2 alongside either Flagtagged SUR2A-WT or SUR2A $\triangle 8$. Western blot of whole-cell lysates shows that exon 8 deletion results in a $\sim 50 \%$ decrease in SUR2 protein expression (Figure 3b). We used a radioactive rubidium $(86 \mathrm{Rb}+)$ efflux assay to determine effects on $\mathrm{K}_{\text {ATP }}$ channel function. Cells expressing SUR2A-WT with Kir6.2 exhibited robust rubidium efflux rates when $\mathrm{K}_{\text {ATP }}$ channels were activated by metabolic inhibition (Figure $\mathbf{3 c}$ ). In contrast, cells expressing Kir6.2/SUR2A $\triangle 8$ showed no rubidium efflux above the background levels observed in GFP-transfected cells. When Kir6.2 was co-expressed with a heteromeric 1:1 ratio of SUR2A-WT and SUR2A $\triangle 8, K_{A T P}$-dependent efflux rate was similar to WT rates, indicating that SUR2A $\triangle 8$ has no marked dominant-negative effect on functional $K_{A T P}$ expression. The effect of the exon 8 deletion in SUR2B was also determined and, again, no significant efflux was observed in cells expressing SUR2B $\Delta 8$ (Figure 3d). 
A

1 abcc9 c. $1320+1 G>A$

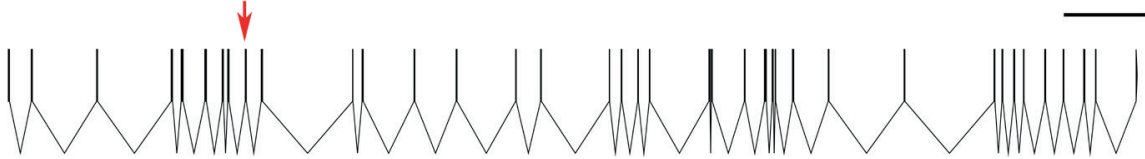

2

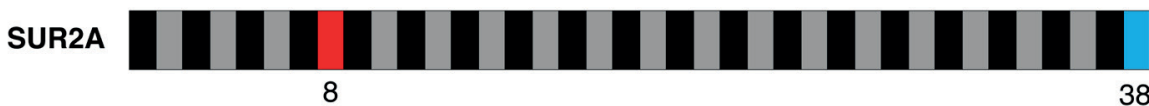

SUR2B

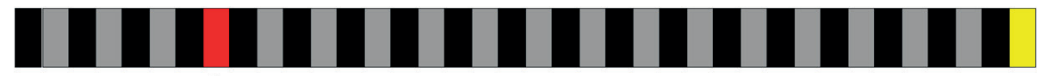

8

B

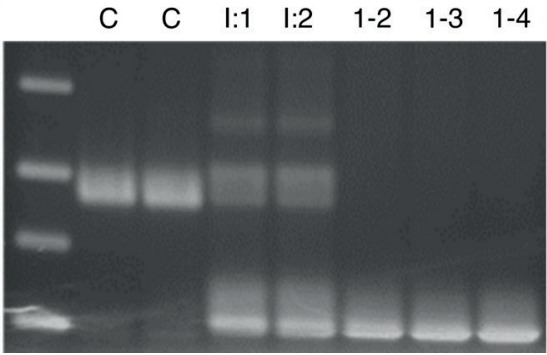

Figure 2 I Molecular analysis in AIMS patients. a Genomic organization of the ABCC9 gene: (1) basic genomic structure of $A B C C 9$ includes at least 39 potential exons, excluding untranslated regions (UTRs). The c. $1320+1 \mathrm{G}>\mathrm{A}$ mutation predicted to disrupt the splice donor site of exon 8 is indicated by a red arrow. Scale bar, $5000 \mathrm{bp}$. (2) The mutation impacts both the SUR2A and SUR2B splice forms, which differ only in the last exon. Affected exon 8 in patients is marked in red. Oddnumbered exons are presented as black boxes, even-numbered exons as gray boxes. b Analysis of the effect of the mutation at the cDNA level in Family 1 . Two control cDNA samples (indicated by a $C$ ) show the wild-type PCR product containing exon 8 , parents $(I: 1,1: 2)$ show heterozygosity for the wt and a lower band lacking exon 8 , whereas patient cDNA $(1-2,1-3,1-4)$ only yielded the lower band.

A complete absence of functional $K_{A T P}$ channels was observed in inside-out patch clamp recordings from cells co-transfected with Kir6.2/SUR2A $\triangle 8$, in contrast to robust expression in cells transfected with SUR2A-WT or a 1:1 mix of SUR2A-WT and SUR2A $\triangle 8$ (Figure $3 \mathbf{e}, \mathbf{f}$ ). Therefore, homomeric deletion of exon 8 results in a significant decrease in protein expression and complete loss of $\mathrm{K}_{\text {ATP }}$ channel function. The decrease in functional expression of SUR $2 \Delta 8$ containing channels may arise due to either the absence of surface-expressed channels or the expression of nonfunctional channels, which requires more detailed study for elucidation. Co-expression of SUR2A-WT and SUR2A $\triangle 8$ did not affect channel regulation by ATP or pharmacological activation by pinacidil, suggesting that in the heterozygous context, the c. $1320+1 \mathrm{G}>\mathrm{A}$ mutation is without significant effect (Figure 3g; Figure S5). 


\subsubsection{Fatigability and cardiac dysfunction in SUR2-STOP mice}

To model the effects of SUR2 LoF in vivo, we used a mouse line in which a frameshift mutation, resulting in a premature stop codon at position Y1148 (SUR2STOP), was introduced using CRISPR/Cas9 (Figure 4a). Inside-out patch clamp recordings from ventricular myocytes and aortic smooth muscle cells showed that functional $K_{\text {ATP }}$ channels were essentially absent in homozygous SUR2-STOP mice (Figure 4b, c; Figure S6), thus the SUR2-STOP mice recapitulate the key functional channel consequences of the exon 8 deletion.
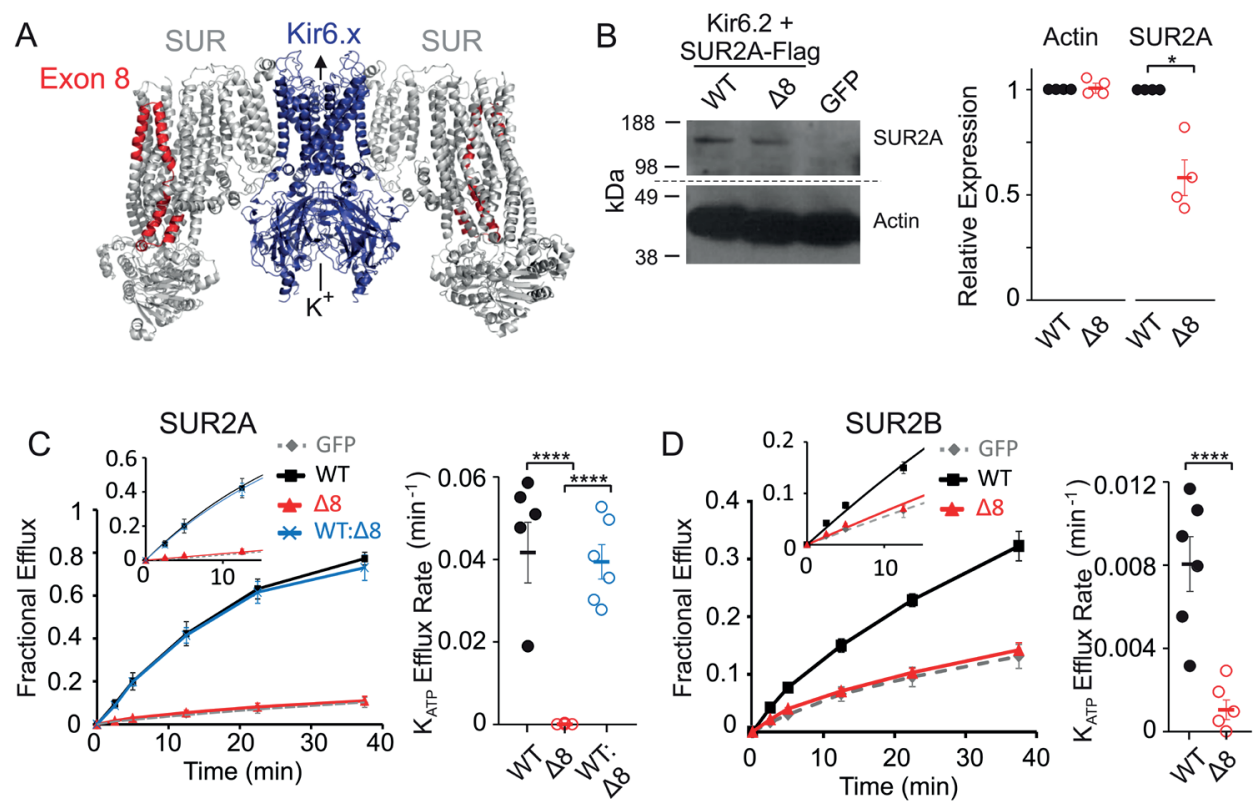

E

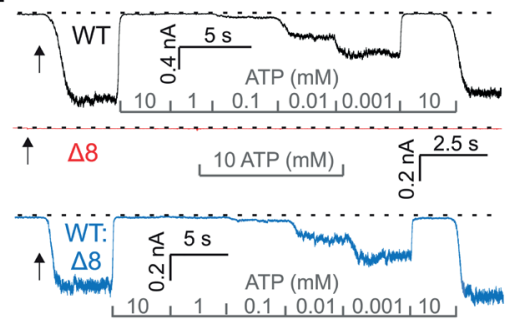

$\mathrm{F}$

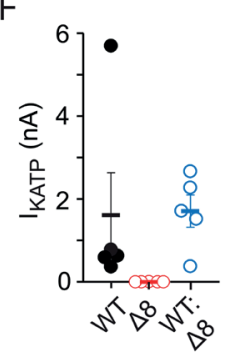

G

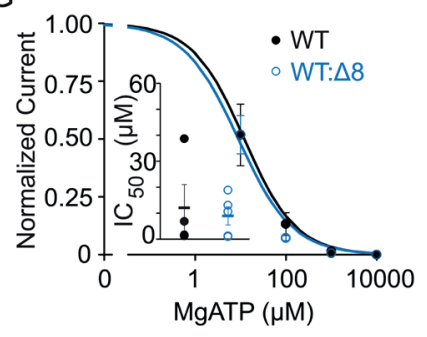

Figure 3 | See next page for legend. 
SUR2-STOP mice and WT littermate controls were evaluated on a multiple-trial inverted screen test to assess strength and fatigability. SUR2-STOP and WT mice performed comparably in the first trial, suggesting no significant initial deficits in strength per se. However, in subsequent repeated trials, SUR2-STOP mice exhibited clear decreases in performance, whereas WT mice performance remained high (Figure 4d). Significant genotype effects were observed, as well as genotype $x$ trial and genotype $x$ session interactions (Table S3). These findings indicate that SUR2-STOP mice exhibited significant performance deficits, dependent on the specific session and trial. The total time the mice remained inverted across the six trials was calculated, and a significant decrease in performance was observed in SUR2-STOP mice (Figure 4e). Thus, global loss of SUR2 results in decreased physical performance, suggestive of increased fatigability, which may be related to the clinically observed myopathy.

Echocardiographic assessment revealed that left ventricle fractional shortening was significantly decreased in SUR2-STOP mice (Figure 4f, g), consistent with the findings in older AIMS patients, and in previously reported SUR2-deficient mice (Stoller et al., 2007). A small, but statistically significant increase in left ventricular internal dimension in diastole (normalized to body length) was observed in SUR2-STOP mice, also mirroring the mild dilatation observed

Figure 3 I Exon 8 deletion results in $K_{\text {ATP }}$ channel loss-of-function. a $K_{A T P}$ channel structure showing pore-forming Kir6.x subunits (blue) associated with two of four SUR subunits (gray) (PDB 5WUA [doi 10.2210/pdb5WUA/pdb]), with the equivalent position of the SUR2 amino acids encoded by ABCC9 exon 8 in red (Ala389-Gln440). b Western blot of whole-cell lysate from Cosm6 cells transiently transfected with GFP or Kir6.2 alongside either wild-type SUR2A-Flag (WT) or SUR2AFlag $\Delta 8$. Left: Immunoblots using a primary antibody targeting the Flag-tag of SUR2A-Flag $\Delta 8$ (top) and actin control (bottom). Right: Normalized expression for actin and SUR2A-Flag from cells transfected with WT SUR2A-Flag or SUR2A-Flag $\Delta 8$. The data from four independent experiments, * denotes $p<0.05$ according to Mann-Whitney U Test. A representative example blot is included in the associated Source Data File. c Left: 86Rb+ efflux from Cosm6 cells transfected with GFP alone, Kir6.2 alongside WT SUR2A, SUR2A $\triangle 8$, or a 1:1 ratio of WT SUR2A and SUR2A $\triangle 8$ (WT: $\triangle 8$ ). Inset: early time efflux/time data points used to derive efflux rate constants. Right: Efflux rate constants for cells transfected with WT SUR2A, SUR2A $\triangle 8$, and 1:1 ratio of WT: $\triangle 8$. The data from six replicates in three independent experiments, ${ }^{* \star * *} \mathrm{p}<0.0001$ (Mann-Whitney U Test). d Left: $86 \mathrm{Rb}+$ efflux experiment from Cosm6 cells transfected with GFP alone, with Kir6.2 alongside WT SUR2B or SUR2B 8 . Right: Efflux rate constants for cells transfected with WT SUR2B or SUR2B $\triangle 8$. The data from six replicates in three independent experiments, ${ }^{* \star \star *} \mathrm{p}<0.0001$ (Mann-Whitney U Test). e Example inside-out patch clamp recordings from Cosm6 cells transfected with Kir6.2 alongside SUR2A-WT (black), SUR2A $\triangle 8$ (red), or a 1:1 mix of SUR2A-WT and SUR2A $\triangle 8$ to mimic heterozygous expression (blue). The membrane potential was held at $-50 \mathrm{mV}$ in symmetrical KINT solutions, and ATP was applied as indicated. Arrows indicate the point of patch excision. $\mathbf{f} \mathrm{K}_{\text {ATP }}$ currents from excised patches. $\mathbf{g}$ ATP dose-response relationship for SUR2A-WT or 1:1 SUR2A-WT:SUR2A 8 channels. Inset: summary of ATP IC50 values. The data from individual experiments shown as dots alongside mean \pm SEM. Source data are provided as a Source Data file. 
our eldest patient (patient 2-1), and the dilated cardiomyopathy previously associated with SUR2 mutations (Bienengraeber et al., 2004).

Blood pressure was significantly increased in SUR2-STOP mice (Figure S6c, d), consistent with the known role of SUR2-containing vascular smooth muscle $\mathrm{K}_{\text {ATP }}$ channels in the regulation of vascular tone (Chutkow et al., 2002).
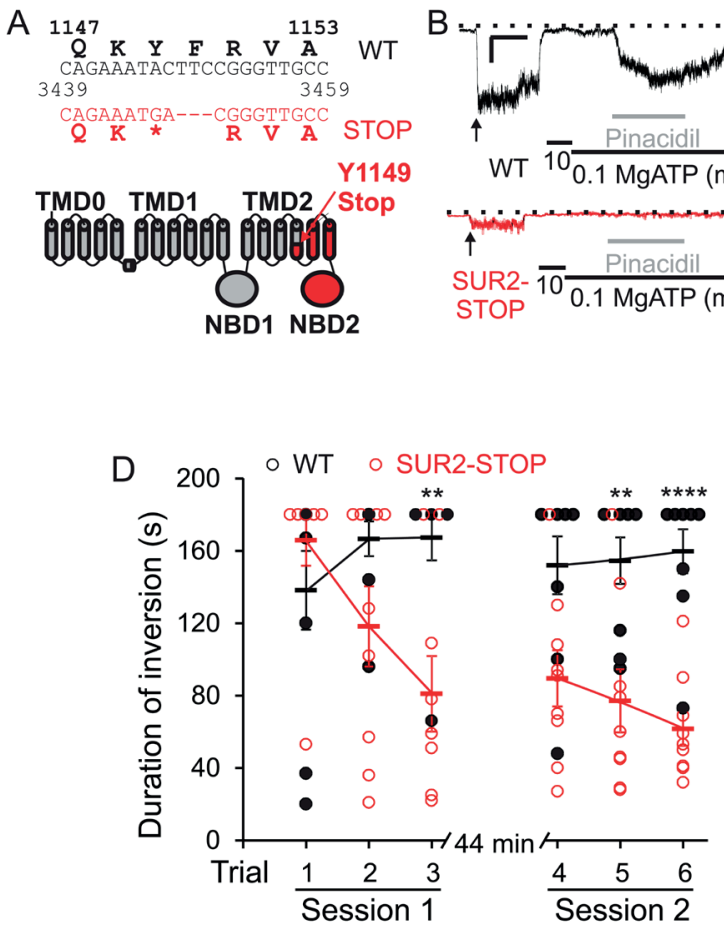
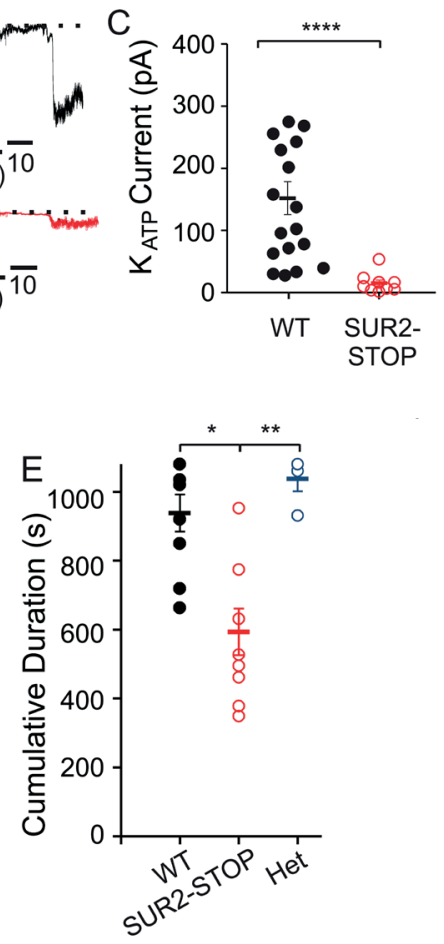
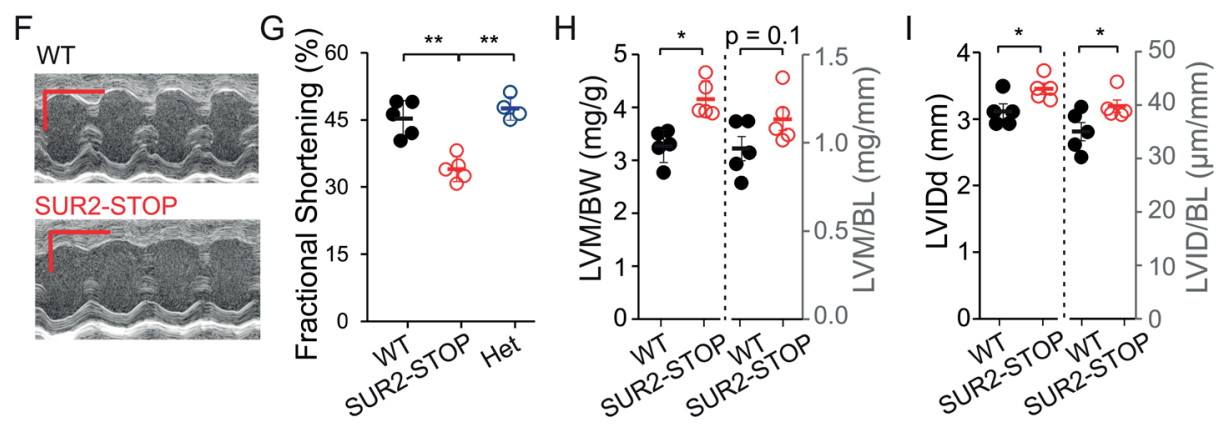

Figure 4 | See next page for legend. 


\subsubsection{Absence of cognitive or behavioral defects in SUR2-STOP mice}

SUR2-STOP and WT mice were evaluated on a battery of cognitive and behavioral tests. During a 1-h locomotor activity test, SUR2-STOP mice displayed trends toward decreased total ambulations (whole-body movements) and vertical rearing frequency, and increased rest time, but there were no significant overall genotype effects (Figure 5a-c). No differences were observed in the distance traveled in the center or peripheral zones of the test field, indices of emotionality in mice (Figure 5d, e). In addition, neither significant effects were found in a battery of sensorimotor measures designed to assess balance, coordination, strength, and speed of movement (ledge test, platform test, pole test, inclined screen test, Figure 5f-i) nor in tests of spatial learning and memory, evaluated using a Morris water maze (Figure $\mathbf{5} \mathbf{j}-\mathbf{l}$ ). Finally, an elevated plus maze was used to assess anxiety-like behaviors, which involves quantifying the reluctance of mice to move from the "protected areas" of the enclosed arms to open arms. No significant differences were observed between genotypes in terms of distance traveled, time spent or entries made into the open arms, or total distance traveled throughout the entire maze (Figure 5m-p). Collectively, the behavioral findings suggest that the SUR2-STOP mice do not exhibit marked deficits in learning and memory nor show any obvious anxiety-like behaviors.

Figure 4 I SUR2-STOP mice exhibit cardiac dysfunction and fatigability. a Top: The c.3446_3450delACTTCinsGA indel in ABCC9 and consequent premature stop codon following K1148 (p.Y1149Stop). Bottom: schematic of SUR2 with the site of the introduced Y1149Stop mutation in TM15 indicated and the downstream region in red. b Example current traces from inside-out voltage clamp recordings from ventricular myocytes of WT (black) or SUR2-STOP (red) mice ( $-50 \mathrm{mV}$ holding potential in the presence and absence of MgATP and pinacidil as indicated). Scale bar shows $5 \mathrm{~s}$ (x-axis) and $25 \mathrm{pA}$ (y-axis). $\mathrm{c} \mathrm{K}_{\text {ATP }}$ channel current amplitudes from excised patches from mouse ventricular myocytes. The data shown from 18 patches for WT, and 10 patches for SUR2-STOP from $\geq 3$ mice. ${ }^{* * *} p<0.0001$ (two-tailed t test). $\mathbf{d}$ Duration mice remained inverted during the multiple-trial inverted screen test. The data were analyzed using a repeated measures ( $\mathrm{rm})$ ANOVA model that contained one between-subjects variable (genotype) and two withinsubjects variables (trials and sessions; see Supplementary Table 3 for summary statistics). The results from the rmANOVA revealed a significant genotype effect, as well as genotype $x$ trial and genotype $x$ session interactions. The data from nine WT and nine SUR2-STOP mice, ${ }^{*} p$-values for the pairwise comparisons exceeded Bonferroni correction ( $p<0.008[0.05 / 6] ;{ }^{*} p<0.05 ;{ }^{* *} p<0.01$; $\left.{ }^{* * *} p<0.001\right)$. e Cumulative inversion time, ${ }^{*} p<0.05$ and ${ }^{* *} p<0.01$ according to one-way ANOVA and post hoc Tukey test. $\mathbf{f}$ Example M-mode echocardiography recordings from WT (top) and SUR2STOP (bottom) mice. Scale bar shows $0.1 \mathrm{~s}$ (x-axis) and $1 \mathrm{~mm}$ (y-axis). g Ventricular fractional shortening measured from echocardiographic imaging (all echocardiographic data from five WT and five SUR2-STOP mice), ${ }^{* \star} p<0.01$ (one-way ANOVA and post hoc Tukey test). $\mathbf{h}$ Left ventricular mass (LVM) as determined from echocardiography imaging normalized to body (LVM/BW) and body length (LVM/BL). ${ }^{*} p<0.05$ (student's $t$ test). $\mathbf{i}$ Left ventricular internal diameter in diastole as measured from echocardiographic imaging. ${ }^{*} p<0.05$ (student's $t$ test). The data from individual experiments shown as dots alongside mean \pm SEM. Source data are provided as a Source Data file. 


\subsubsection{Decreased motility in SUR2-STOP zebrafish}

Assessment of a SUR2-STOP zebrafish model (Figure $\mathbf{6 a}$, b) also demonstrated clear phenotypic overlap with the clinical syndrome and mouse model. cDNA was analyzed by qPCR, to assess the effect of the $\$ 985$ truncation, introduced via CRISPR/Cas9, on abcc9 mRNA expression in SUR2-STOP zebrafish larvae. This revealed fourfold abcc 9 mRNA decrease in mutant fish compared with wild-type controls (Figure 6c), consistent with aberrant mRNA being eliminated by nonsense mediated decay. Inside-out patch clamp recordings revealed the complete absence of functional $K_{\text {ATP }}$ channels in ventricular myocytes from SUR2-STOP zebrafish (Figure 6d). Hence, the zebrafish model also recapitulates the key channel consequences of the exon 8 deletion.

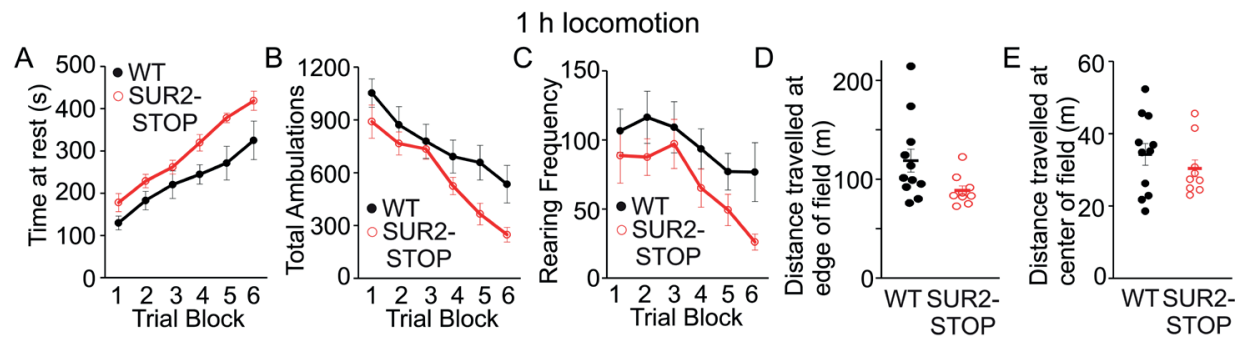

Sensorimotor Battery
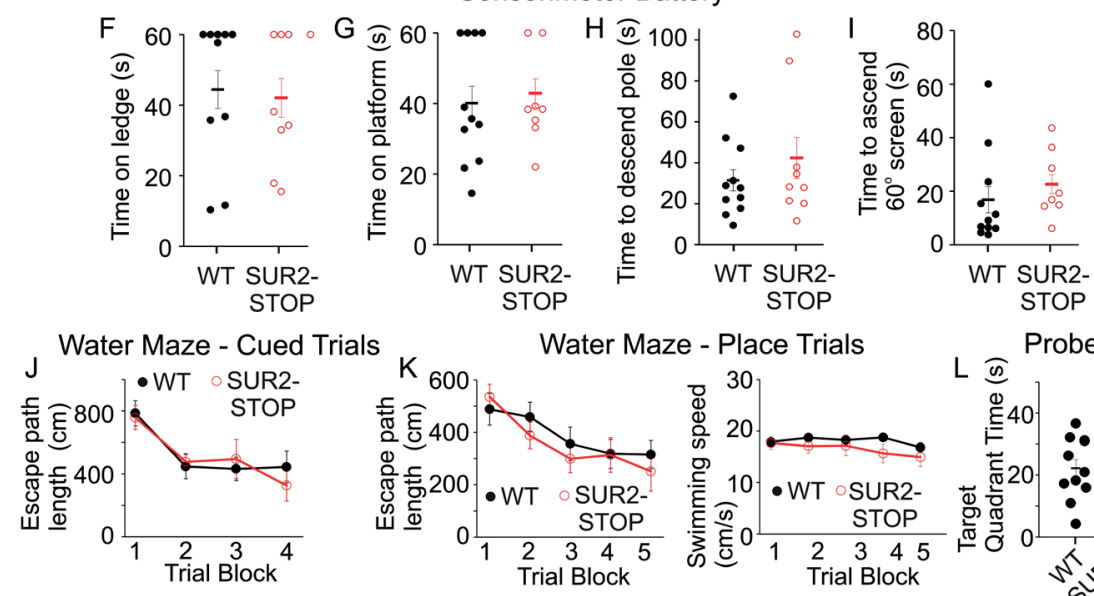

$L$ (s) Probe Trial

Elevated Plus Maze
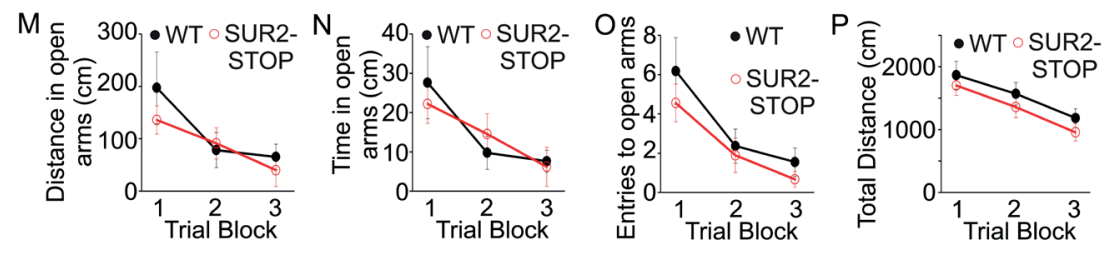

Figure 5 | See next page for legend. 
Consistent with hypotelorism observed in multiple AIMS patients, significantly decreased interorbital distance, normalized to overall larval body length, was observed in SUR2-STOP zebrafish (Figure 6d, e). In contrast, no significant difference was observed between the inter-eye distance in WT and SUR2-STOP mice. Visual inspection of SUR2-STOP zebrafish revealed no other striking dysmorphic features (Figure 6b). We used a behavioral tracking system to quantify locomotor activity in zebrafish larvae (Figure 6f). SUR2-STOP larvae displayed significantly decreased spontaneous total movement and decreased total swimming distance compared with control larvae (Figure $\mathbf{6 g}, \mathbf{h}$ ). Despite shorter swimming distances, SUR2-STOP embryos move for a similar period of time as WT fish (Figure 6i). Analysis of the duration of high-speed movements revealed a significant decrease in SUR2-STOP larvae (Figure 6j). Notably, SUR2STOP embryos hatched normally from their chorion, a process that also requires muscle contraction.

\subsubsection{Cardiac abnormalities in SUR2-STOP zebrafish}

We performed high-speed video imaging24 of the hearts of wild-type and SUR2STOP larvae to examine cardiac function (Figure 7a; Supplementary Movies 1, 2 ). Analysis revealed systolic dysfunction-resembling the cardiac phenotype in older patients. Both fractional shortening (FS) and ejection fraction (EF) are significantly reduced in SUR2-STOP mutants (FS: 29\%, EF: 25\%) (Figure 7b, c). Consequently, cardiac output is dramatically lower $(28 \%)$ (Figure 7d) due to equivalently reduced stroke volume (Figure S7A). Figure S8 illustrates the assessment of ventricular contractility via high-speed video microscopy in zebrafish embryos. Ventricular end-diastolic volume (VEDV) and end-systolic volume (VESV) were unchanged in SUR2-STOP larvae (Figure 7e).

Figure 5 I No major behavioral or cognitive deficits observed in SUR2-STOP mice. a Total time at rest $\mathbf{b}$ The total number of ambulations (whole-body movements), $\mathbf{c}$ the number of incidents of rearing, $\mathbf{d}$ distance traveled at edge of the observational field, and $\mathbf{e}$ distance traveled within center of observational field in $1 \mathrm{~h}$ locomotion observation, $\mathrm{n}=11$ for WT and 9 for SUR2-STOP for all behavioral tests. $\mathbf{f}$ Time mice spent on elevated ledge, $\mathbf{g}$ time mice remained on elevated circular platform, $\mathbf{h}$ time taken to descend during the pole test, and $\mathbf{i}$ time taken to ascend a wire-mesh screen maintained at 600 angle for WT and SUR2-STOP mice in sensorimotor battery. $j$ The escape path length taken to find the platform by WT and SUR2-STOP mice in Morris Water Maze Cued trials. Note, no significant difference in swimming speed was observed between genotypes. $\mathbf{k}$ The mean escape path length (left) taken to find the platform, and average swimming speed (right) by WT and SUR2-STOP mice in Morris Water Maze Place trials. I The time spent within the target quadrant (the quadrant in which the platform had previously been positioned during Cued and Place trials) was measured. $\mathbf{m}$ Distance traveled within open arms, $\mathbf{n}$ time spent in open arms, $\mathbf{o}$ entries into open arms, and $\mathbf{p}$ total distance traveled in the Elevated Plus Maze test for WT and SUR2-STOP mice. The data were analyzed using a repeated measures $(\mathrm{rm})$ ANOVA model that contained two betweensubjects variable (genotype and sex) and one within-subjects variable (trial block). The data from individual experiments shown as dots alongside mean \pm SEM. Source data are provided as a Source Data file. 
Blood flow velocity of WT and SUR2-STOP larvae was assessed by high-speed video imaging of the cardinal vein. An increased velocity of red blood cells in SUR2-STOP fish (Figure 7f) can be associated with high blood pressure found in SUR2-STOP mice.

No cardiac abnormalities were observed in larvae heterozygous for the SUR2STOP mutation (Figure S9).

A

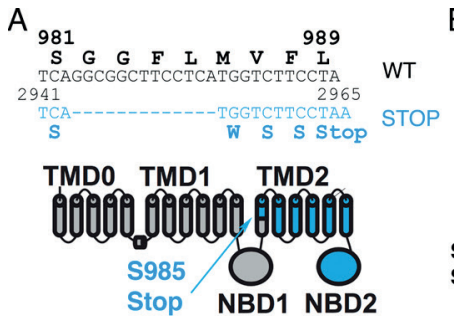

D

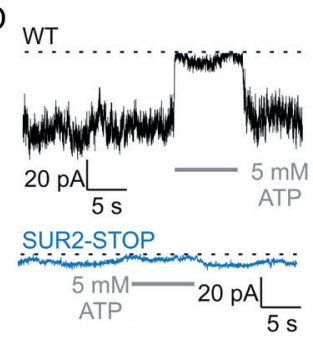

B

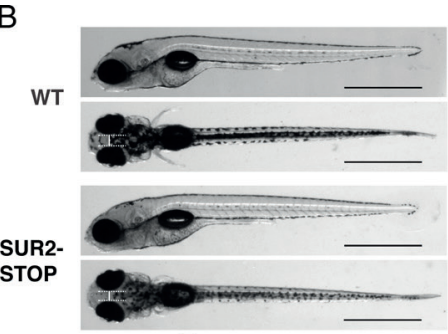

$\mathrm{F}$
$\mathrm{E}$

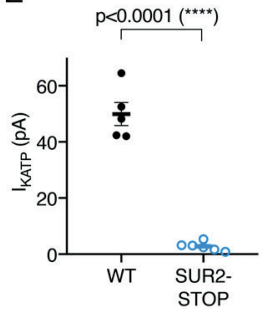

C

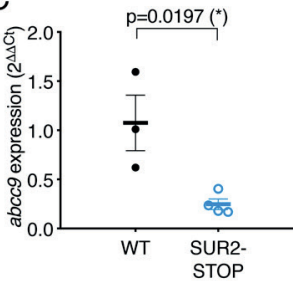

G
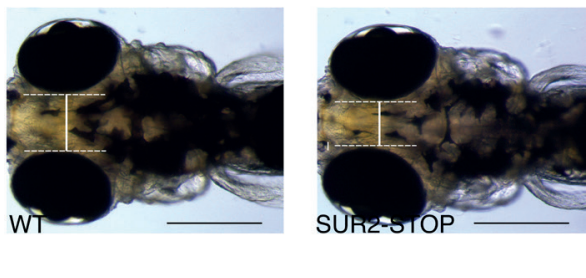

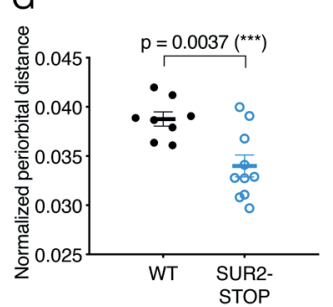

$\mathrm{H}$

WT

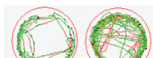

SUR2-STOP

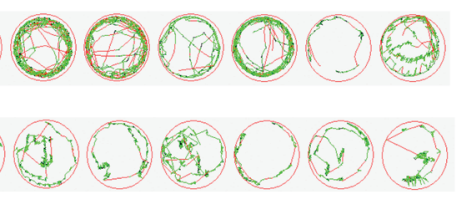

।

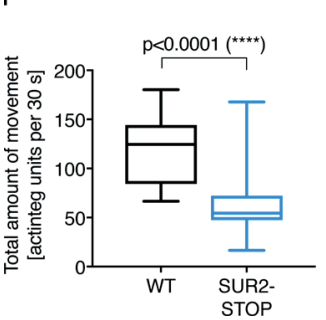

J

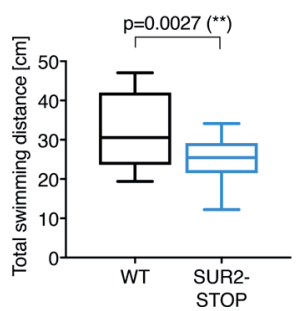

K

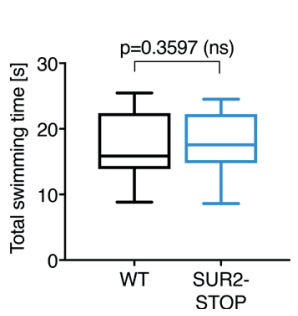

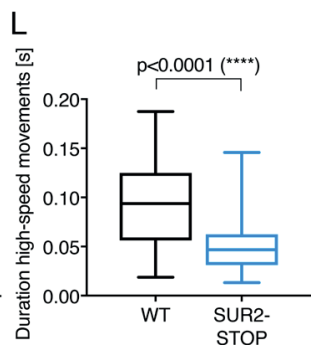

Figure 6 | See next page for legend. 
Adult zebrafish hearts were analyzed after sectioning and $\mathrm{H} \& \mathrm{E}$ staining (Figure $\mathbf{7 g}$. For assessment of ventricular size, tissue sections revealing the largest chamber area were selected. In five out of six SUR2-STOP fish, ventricular area was strikingly enlarged with abnormal morphology compared with control siblings. The atrial area shows similar morphology (Figure 7f; Figure S10). We stained cryosections of WT and SUR2-STOP hearts with Acid Fuchsin Orange G (AFOG), which labels myocardium orange, collagen blue, and fibrin red. This revealed no visible fibrosis in SUR2-STOP hearts (Figure S11). TUNEL (TdTmediated nick end labeling) analysis revealed very few apoptotic cells in WT hearts, while a sizable number of cells were TUNEL-positive in both cardiac chambers of SUR2-STOP fish (Figure 7h). Myofiber structure in SUR2-STOP hearts is not different from WT as determined by immunohistochemistry staining for tropomyosin (Figure S12).

Figure 6 | Hypotolerism and decreased locomotor behavior in SUR2-STOP zebrafish larvae. a The c.2944_2957del13 indel in abcc9 and consequent frameshift premature stop codon following S984 (p.S985Stop) and schematic of SUR2 with the site of the introduced S985Stop mutation in TM12 indicated, downstream region in blue. b Representative images illustrating the morphology of $5 \mathrm{dpf}$ wild-type and SUR2-STOP mutants as seen from a left lateral (top) and dorsal view (bottom). Scale bars, $1 \mathrm{~mm}$. c Quantitative RT-PCR to assess abcc9 expression in pools of 60 WT (three pools) and SUR2-STOP (four pools) embryos. d Representative current traces from inside-out patch clamp recordings from ventricular myocytes of WT (black; five patches) or SUR2-STOP (blue; six patches) zebrafish ( $-50 \mathrm{mV}$ holding potential, ATP applied as indicated). e $\mathrm{K}_{\text {ATP }}$ channel currents from excised patches from zebrafish ventricular myocytes. The data from five patches (WT), and six patches (SUR2-STOP) from $\geq 3$ zebrafish. $\mathbf{f}, \mathbf{g}$ To assess hypotelorism, the distance between the convex tips of the eyes was measured and normalized to body length $(W T, n=8 ; H O M, n=10)$. Scale bars, $200 \mu \mathrm{m}$. $\mathbf{h}$ Examples of movement traces shown in red, green, and black representing high-speed, intermediate, and slow movements, respectively. i-l Five-minute video recordings ( $n=62$ larvae per genotype) of $5 \mathrm{dpf}$ homozygous SUR2-STOP fish and wild-type controls were analyzed for total amount of movement $\mathbf{i}$, total swimming distance (TSD) $\mathbf{j}$, total swimming duration $\mathbf{k}$, and duration of high-speed movements land compared with respective wild-type larvae. The data on the y-axis refer to the respective average value per $30-\mathrm{s}(\mathrm{s})$ intervals. The data from four independent experiments (16 larvae per experiment) ${ }^{*} p \leq 0.05 ;{ }^{* *} p \leq 0.01 ;{ }^{* * *} p \leq 0.001 ;{ }^{* * *} p \leq 0.0001$ (two-tailed unpaired Student's t test or Mann-Whitney $\mathrm{U}$ test). The black horizontal bar indicates the mean value for each condition. Sample size, $W T, n=3$; SUR2-STOP, $n=4$ in $c ; W T, n=5$; SUR2-STOP, $n=5$ in e; WT, $n=8$; SUR2-STOP, $n=10$ in $\mathbf{g}$; $W T, n=62$; SUR2-STOP, $n=62$ in $\mathbf{i}-\mathbf{l}$. The data from individual experiments shown as dots alongside mean \pm SEM. Source data are provided as a Source Data file. 

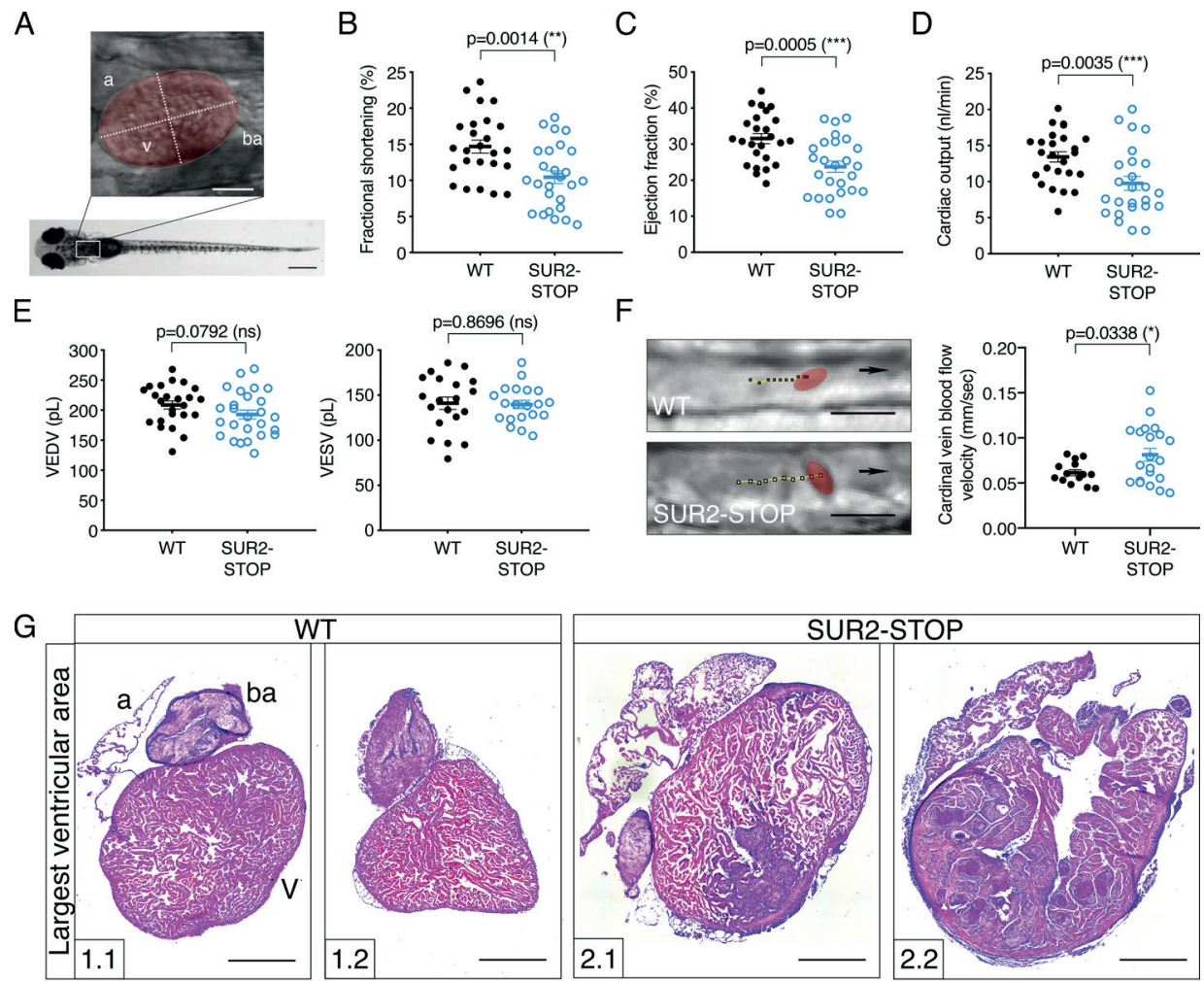

$\mathrm{H}$
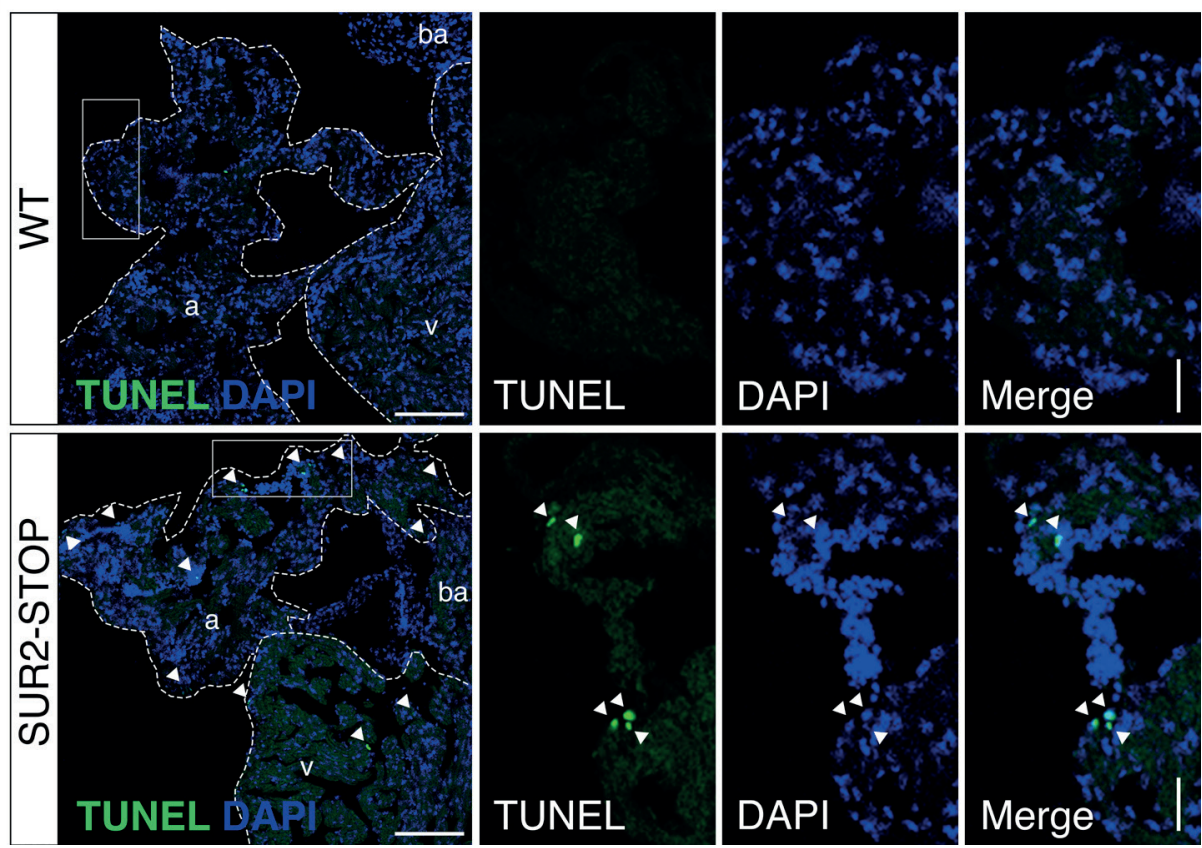

Figure 7 | See next page for legend. 


\subsection{Discussion}

The major clinical features observed in our patients consist of delayed psychomotor development with intellectual disability, anxiety, muscle weakness and fatigability, and some shared dysmorphic features. Cerebral MRI revealed white matter abnormalities in all subjects. Cardiac systolic dysfunction is found in the two oldest, possibly as an early stage of dilated cardiomyopathy. All patients were found to be homozygous for a previously unreported splice site mutation in ABCC9 (c.1320+1 G > A), while unaffected parents are healthy heterozygous carriers of the variant (Figure 2). No other shared recessive mutations were identified in the affected individuals using either next-generation gene panel analysis or whole-genome sequencing.

We show that this splice site mutation leads to the complete in-frame exclusion of exon 8 (SUR2 $\triangle 8$ ) and consequent deletion of 52 amino acids within the TMD1 domain of the resultant SUR2 protein (Figure 3 ). SUR2 is a regulatory subunit of $\mathrm{K}_{\text {ATP }}$ channels expressed in various tissues, including striated and smooth muscle (Foster \& Coetzee, 2016; Inagaki et al., 1996; Isomoto et al., 1996). Deletion of exon 8 results in complete loss of functional channel activity in recombinant SUR2/Kir6.2 $\mathrm{K}_{\text {ATP }}$ channels, indicating that AIMS represents the human consequence of loss of SUR2 (Figure 3). We show that key features of myopathy and cardiac dysfunction in AIMS are recapitulated in novel SUR2-STOP mouse

Figure 7 | Systolic dysfunction and enlarged heart size in SUR2-STOP zebrafish. a Box designates imaged area to assess cardiac function. The ventricular area of the heart is highlighted, with the long axis and short axis of the ventricle indicated by dashed lines. b-e Quantification of cardiac function using individual characteristic confocal sections from a time series of the embryonic cardiac cycle at $5 \mathrm{dpf}$. $\mathbf{f}$ Tracking of individual red blood cells (RBCs) measuring cardinal vein blood flow velocity. RBCs were tracked for ten frames using ImageJ $(\mathrm{NIH})$ and the plugin MTrackJ(Meijering et al., 2012). One representative image of each genotype is shown. Black arrow indicates the direction of RBC movement. g Heart histology of adult SUR2-STOP mutants and respective siblings after H\&E staining. Exemplary depiction of 2 WT and 2 SUR2-STOP hearts. For assessment of ventricular chamber size, tissue sections showing the largest ventricular area were selected. $\mathbf{h}$ TUNEL assay on adult hearts to detect apoptotic cells (white arrowheads) in WT and SUR2-STOP fish. Heart chambers are indicated by white dashed line. Nuclei are stained with DAPI. All experiments were performed comparing SUR2-STOP and its WT siblings. For all graphs, significance was determined by two-tailed unpaired Student's $t$ test or Mann-Whitney two-tailed $U$ test. Asterisks indicate statistical significance $\left({ }^{*} p \leq 0.05 ;{ }^{* *} p \leq 0.01 ;{ }^{* *} p \leq 0.001 ;{ }^{* * * *} p \leq 0.0001\right)$. The black horizontal bar indicates the mean value for each condition. Sample size, WT, $n=20$; SUR2-STOP, $n=20$ in b-e; WT, $\mathrm{n}=14$; SUR2-STOP, $\mathrm{n}=21$ in $\mathbf{f}$; WT, $\mathrm{n}=6$; SUR2-STOP, $\mathrm{n}=6$ in $\mathbf{g}$ and $\mathbf{h}$. Scale bars, $1 \mathrm{~mm}$ and $50 \mu \mathrm{m}$ in a; $10 \mu \mathrm{m}$ in $\mathrm{f}, 500 \mu \mathrm{m}$ in $\mathrm{g} ; 100 \mu \mathrm{m}$ (overview) and $50 \mu \mathrm{m}$ (close up) in h. All embryos analyzed originated from group matings of adult zebrafish. a atrium, ba bulbous arteriosus, v ventricle. The data from individual experiments shown as dots alongside mean \pm SEM. Source data are provided as a Source Data file. 
(Figure 4, 5) and zebrafish models (Figure 6, 7). The animal models used in this study do not recapitulate the genetic defect identified in the AIMS patients, but were chosen as the functional effects of the frameshift mutations introduced into SUR2-STOP mice and fish mirror the functional effect of the SUR2 $\Delta 8$ mutation. Future studies of CRISPR/Cas9 genome edited animal models in which humandisease-associated AIMS mutations are introduced may provide further insights into the severity and variety of phenotypes arising from specific mutations.

How loss of SUR2-dependent $\mathrm{K}_{\text {ATP }}$ channel function may result in the complex pathophysiology observed in AIMS is discussed below.

Facial features: Affected individuals from the two families share some similar facial features, including prominent supraorbital ridges, flat face, and thin upper lip vermilion, as well as macrodontia and/or widely spaced upper central incisors and dental malocclusion. Since the affected individuals are from two siblingships, more patient observations will be necessary in order to establish whether these facial features are consistent. Some of the individuals have hypotelorism, consistent with significantly decreased intraorbital distance found in SUR2-STOP zebrafish (Figure $\mathbf{6 f}, \mathbf{g}$ ). This combination of facial features is markedly different from the acromegaloid facial features that characterize Cantú syndrome and the associated conditions within the same spectrum: Acromegaloid Facial Features (AFF [MIM: 102150]) (Kini \& ClaytonSmith, 2004) and hypertrichosis acromegaloid facial features disorder (HAFF) (Afifi et al., 2016), which all arise from GoF mutations in ABCC9. None of our patients have hypertrichosis, unlike Cantú syndrome patients. Therefore, Cantú syndrome and AIMS, arising from opposing molecular mechanisms, are both dysmorphologically and mechanistically distinct.

Neuromuscular manifestations: All patients report fatigability, present with generalized hypotonia, and muscle strength is below normal in all but one. Lumbar lordosis is found in all but one, and scoliosis in one. The miniBEST test shows moderate to severe deficits in balance in all patients, and walking distance in the 6-min walk test is reduced in all individuals. S-CK, B-lactate, nerve conduction velocities, and electromyography, including repetitive nerve stimulation, are normal in all individuals, except for discrete myopathic discharges in patient 2-2. In skeletal muscle, $\mathrm{K}_{\text {ATP }}$ channels are typically closed at rest but open in response to metabolic stress or fatigue (Tricarico et al., 2016). Channel activation results in action potential shortening and stabilization of the resting membrane potential during the development of fatigue, which serves to reduce intracellular calcium, decrease resting tension and protect myocytes 
from damage. Therefore, loss of $\mathrm{K}_{\text {ATP }}$ function might be expected to result in failure to recover from fatigue, myofiber degeneration, and excessive calcium influx, as reflected in the inverted screen tests in the mice. In addition, lower limb hyperreflexia was observed in the four oldest individuals, which might be caused by the white matter abnormalities and decreased inhibition or increased activation of upper motor neurons.

Although the expression pattern of Kir6.2 is not identical to that of SUR2, it is notable that previous studies of Kir6.2-null mice, as well as mice in which an internal deletion in SUR2 was engineered (resulting in loss of full length SUR2 but persistent expression of a mitochondria-limited short form), have reported that the consequent decrease of myocyte $\mathrm{K}_{\text {ATP }}$ activity results in impaired exercise capacity and response, and myofiber damage(Kane et al., 2004; Stoller et al., 2007). Only relatively minor histological abnormalities were observed in a singleskeletal muscle biopsy from patient 1-2. This is consistent with previous reports which showed that SUR2 knockout mice only exhibit significant histological abnormalities when subjected to significant and chronic exercise (Thabet, Miki, Seino, \& Renaud, 2005). Here, we show that novel mouse and zebrafish models, in which CRISPR/Cas9 was used to introduce frameshift mutations resulting in premature stop codons and nonfunctional subunits, also exhibit decreased performance. Specifically, SUR2-STOP mice showed a diminished ability to hang upside down during the multiple-trial inverted screen test, suggesting decreased strength resulting from increased fatigability (Deacon, 2013; Grady, Wozniak, Ohlemiller, \& Sanes, 2006) (Figure 4d, e), while SUR2-STOP zebrafish show decreased total overall movement and swimming speed in tracking studies (Figure 6i-l).

Intellectual disability and neurological abnormalities: Neuropsychological testing revealed mild-to-moderate intellectual disability in all affected individuals, and anxiety was also reported in several patients. It is not obvious how myocyte $\mathrm{K}_{\text {ATP }}$ dysfunction could explain the intellectual disability or anxiety, and there are no reports of cognitive impairment in previous SUR2 mutant animal models. Neuronal $K_{\text {ATP }}$ channels are predominantly formed of Kir6.2 with SUR1 subunits, although transcripts for all $\mathrm{K}_{\text {ATP }}$ channel subunits have been identified in various neuronal populations (Liss \& Roeper, 2001) and SUR2 is reportedly expressed in both central and peripheral neurons (Kawano et al., 2009; Nelson, Jicha, et al., 2015; Zoga et al., 2010), where it has been implicated in hippocampal sclerosis in aging and amyotrophic lateral sclerosis (Nelson et al., 2014; Nelson, Jicha, et al., 2015; Nelson, Wang, et al., 2015; Vidal-Taboada et al., 2018). 
However, recent data demonstrate that SUR2-containing $\mathrm{K}_{\text {ATP }}$ channels play a critical role in regulation of cerebral vascular architecture (Leon Guerrero et al., 2016). In Cantú syndrome, MRI reveals diffusely dilated and tortuous cerebral blood vessels and white matter changes as multiple T2 subcortical or scattered hyperintensities. Transient white matter changes suggestive of a reversible posterior encephalopathic syndrome are reported in one patient. Several Cantú syndrome patients have migraine, and some have epilepsy. Developmental delay is common, although seeming to improve with age (Leon Guerrero et al., 2016). Since $K_{\text {ATP }}$ channel GoF results in chronic vasodilation and altered neurovascular coupling (Leon Guerrero et al., 2016), it is conceivable that $\mathrm{K}_{\text {ATP }}$ loss of function may impact the cerebral vasculature in a way that results in impaired dynamic coupling of blood flow to match neuronal metabolic demand.

Patient 1-1 has a diagnosis of epilepsy with episodes of unconsciousness and tonic-clonic seizures, but no definitive epileptic activity has been demonstrated on EEGs. Patient 1-4 experienced an episode of coma with transient widespread white and gray matter abnormalities, which is left unexplained. Possibly, these incidents could be caused by focal or generalized circulatory alterations. Interestingly, white matter hyperintensities are observed in both Cantú syndrome and AIMS. In both cases, they could result from ischemic events due to dysregulated cerebral blood flow, although the cognitive phenotype seems to be more definite in AIMS than in Cantú syndrome. Tests for cognitive deficits and anxiety did not reveal significant behavioral impairments in SUR2-STOP mice (Figure 5). Additional testing for specific deficits (such as an assessment of working memory, fear conditioning, or other nonspatial forms of learning and memory) would be necessary to more fully characterize the behavioral phenotype of the SUR2-STOP mice. Notably, mild cognitive impairment, as seen in humans, can be difficult to recognize in animals (Pepeu, 2004). Wholegenome sequencing and copy number analysis did not identify other causes of intellectual disability in our patients.

Cardiovascular manifestations: While the younger patients (aged 11-21) displayed no clear cardiovascular abnormalities, the two oldest patients display cardiac biventricular systolic dysfunction, with only slightly decreased ejection fractions. No clinical signs of heart failure were observed in patient 2-2, but decreased ejection fraction and heart failure was observed in her brother, patient 2-1. Cardiac MRI in patient 2-1 showed dilated ventricles, and together with other clinical findings this may suggest early stages of dilated cardiomyopathy. In a previous study by Bienegraber et al., two individuals with dilated cardiomyopathy (DCM) were found to have heterozygous, LoF, missense 
mutations in exon 38 of $A B C C 9$, an exon only included in the SUR2A splice variant, and not in SUR2B (Bienengraeber et al., 2004). In that case, $K_{\text {ATP }}$ LoF would be expected in cardiomyocytes and skeletal muscle but not smooth muscle, where SUR2B is expressed. The DCM patients reported in that study were older than the patients we report and displayed drastic reductions in ejection fraction (to 15-23\%). This may suggest a progressive cardiomyopathy which will require longitudinal analysis in AIMS.

In previously reported mouse models, SUR2 knockout resulted in cardiac hypertrophy with LV dilation and decreased fractional shortening (Gao et al., 2014; Stoller et al., 2007). Here, we show that SUR2-STOP mice also display decreased fractional shortening and LV dilation (Figure $\mathbf{4 f}, \mathbf{g}$ ), while SUR2-STOP zebrafish show marked cardiac enlargement with reduced ejection fraction

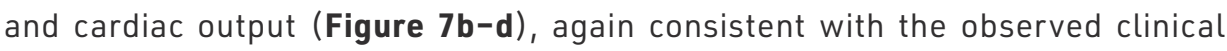
phenotype in the older AIMS patients. Significant cardiomegaly, with elevated cardiac output, is observed in Cantú syndrome. This cardiac remodeling may arise as a secondary compensation to counter the vasodilatory effect of $\mathrm{K}_{\text {ATP }}$ GoF in vascular smooth muscle cells (Huang et al., 2018; Levin et al., 2016). SUR2 LoF, in contrast, would be expected to increase vascular contractility and blood pressure. However, blood pressure was normal in AIMS patients, except for elevated systemic pressures in the oldest patient. SUR2 and Kir6.1-null mice are hypertensive (Chutkow et al., 2001; Miki et al., 2002), which is again a feature of the SUR2-STOP mice, but in humans, effects on blood pressure may be more subtle and subject to tighter regulation through homeostatic feedback, or perhaps not yet manifesting in our still relatively young patients.

Other features: Mild hearing loss was found in $3 / 6$ patients. This is not straightforward to explain, as there is no known function for SUR2 in auditory neurons. One explanation might be altered vascular tone and ischemic stress of these neurons. Strabismus and myopia were found in several individuals while one also displayed nystagmus. Sensory assessment was not performed in our animal models but should be the subject of future study. Feeding difficulties in childhood were reported in all individuals. $\mathrm{K}_{\text {ATP }}$ channels are expressed in gastrointestinal smooth muscle (Koh et al., 1998), and thus LoF may affect GI contractility and motility. The two patients in family 2 have short stature and microcephaly. In Cantú syndrome, there is a range of skeletal manifestations. Our patients are not thoroughly evaluated radiologically, but do not display obvious skeletal changes, except for scoliosis, congenital hip dysplasia, and elbow extension deficits in patient 2-2. Lastly, patient 1-1 presents with hyperprolactinemia lacking pituitary adenomas. Interestingly, pituitary adenomas 
and elevated levels of prolactin are recently reported in a family with Cantú syndrome (Marques et al., 2018).

Disease progression: As discussed above, the two oldest patients exhibit decreased myocardial contractility, which may reflect the onset of a progressive cardiomyopathy, and hypertension is found in the oldest patient. It will be important to chronicle the long-term effects of $\mathrm{K}_{\text {ATP }}$ LoF in all the patients to elucidate the natural history of AIMS, which may also be informed by longitudinal studies in the animal models presented here. White matter hyperintensities were found to have increased with age in the two oldest patients, where MRIs have been repeated.

Heterozygous carriers: Lack of phenotype in the healthy heterozygous carriers of the ABCC9 c. $1320+1 \mathrm{G}>\mathrm{A}$ variant is consistent with functional studies of recombinant $K_{\text {ATP }}$ channels reported here, which show that SUR2 $\triangle 8$ subunits do not exert obvious dominant-negative effects in heterozygous expression with WT subunits. Indeed, essentially normal Rb fluxes in this case may reflect a rescue by WT subunits (Figure 3c).

Possible additional molecular consequences: In addition to its canonical role as a regulatory subunit of plasmalemmal $K_{\text {ATP }}$ channels, short-form variants of SUR2 have been identified in the mitochondria and reported to form functional $\mathrm{K}_{\mathrm{ATP}}$ channels6, although the role of these truncated channel complexes remain poorly understood. It is also conceivable that SUR2 proteins play additional noncanonical (i.e., non-channel regulatory) roles, which may or may not be impaired by the exon 8 deletion. Importantly, the animal models employed here, in which premature stop codons were engineered into ABCC9, are not a bona fide recapitulation of the human genetics of the AIMS patients, and accordingly may not reiterate specific novel consequences of the truncated SUR2 $\Delta 8$ protein.

Potential pharmacotherapy: In principle, $\mathrm{K}_{\text {ATP }}$ channel LoF could be countered using $K_{\text {ATP }}$ channel activators, such as cromakalin, pinacidil, diazoxide or minoxidil, which are used clinically as vasodilatory agents (Mannhold, 2004). In the case of the AIMS patients presented here, however, where the exon 8 deletion results in a complete loss of functional channel expression, these drugs would likely be ineffective. Interestingly, in each of the myocyte tissues in which SUR2 is expressed, $\mathrm{K}_{\text {ATP }}$ forms part of an electrical "brake", where $\mathrm{K}_{\text {ATP }}$ activation results in membrane potential hyperpolarization, decreased intracellular calcium, and promotes myocyte relaxation. Loss of $\mathrm{K}_{\text {ATP }}$ function is therefore expected to 
increase intracellular calcium and modulating myocyte calcium handling might prove beneficial.

In the future, it is expected that AIMS patients will be identified with additional mutations in $A B C C 9$. Such mutations may prove to be autosomal dominant or may exhibit only partial LoF, as is observed in the analogous pancreatic $\mathrm{K}_{\text {ATP }}$ disorder, congenital hyperinsulissm (CHI [MIM: 256450]), which arises from LoF mutations in $A B C C 848$. Depending on the mutational consequence, some $\mathrm{CHI}$ patients are responsive to treatment by the potassium channel opener diazoxide, and $\mathrm{K}_{\mathrm{ATP}}$ -openers may indeed prove beneficial in some AIMS patients. 


\subsection{Methods}

\subsubsection{Patients}

Four siblings from Family 1 and two siblings from Family 2 (Figure 1a, pedigrees) were initially referred for assessment of developmental delay and/ or neuromuscular impairment, and were investigated in a clinical diagnostic setting. Written informed consent for Next-Generation-Sequencing in both a diagnostic and research setting was obtained from the patients' parents. The authors affirm that human research participants provided informed consent for publication of the images in Figure $\mathbf{1} \mathbf{b}-\mathbf{d}$. Whole-genome sequencing on patients was approved by the Medical Ethical Committee of the University Medical Center Utrecht. All study participants, or their legal guardian, provided informed written consent prior to publication.

\subsubsection{Clinical evaluation by the Neuromuscular Centre team}

The clinical diagnostic evaluation included patient history, a structured neurological examination and physiotherapist examinations including the miniBalance Evaluation System Test (miniBEST) (Franchignoni, Horak, Godi, Nardone, \& Giordano, 2010) and 6-min walk test. The miniBEST is an assessment of dynamic balance and postural control. There are no normative values for children or people with intellectual disability, but the test was modified for our patients. The 6-min walking test- 6MWT (Enright \& Sherrill, 1998; Geiger et al., 2007) assesses distance walked over 6 min as a sub-maximal test of aerobic capacity/ endurance. For adults, normative values can be calculated by an equation developed by Enright and Sherill (1998) (Deacon, 2013). The calculations are: men: $\quad 6 M W D=(7.57 \times$ height $\mathrm{cm})-(5.02 \times$ age $)-(1.76 \times$ weight $\mathrm{kg})-309$. Women: 6 MWD $=(2.11 \times$ height $\mathrm{cm})-(2.29 \times$ weight $\mathrm{kg})-(5.78 \times$ age $)+667 \mathrm{~m}))$. Normative values for children and adolescents are described by Geiger et al. (Geiger et al., 2007). Normative values are not given for intellectually disabled people. The neurological examination comprised the following: cranial nerve examination, motor function (strength, tempo, tone), deep tendon reflexes (biceps, brachioradialis, triceps, patella, achilles) and plantar reflexes, superficial skin sensation, vibration, proprioception, cerebellar function tests, and gait. In addition, the following supplementary electrophysiological investigations were performed: electromyography (EMG), nerve conduction studies (NCS), repetitive nerve stimulation. All patients had previously undergone cerebral magnetic resonance imaging (MRI). Blood samples were analyzed for serum levels of CK and lactate. In addition, all patients had audiometry, electrocardiograms (ECG), and electrocardiography done. 


\subsubsection{Illumina TruSight One gene panel analysis}

DNA libraries for sequencing of 4813 genes with known associated clinical phenotypes were generated for patients 1-2 and 2-1 (Figure 1) with their respective parents for trio analyses.

Genomic DNA was extracted from whole blood using MagAttract DNA midi 48 kit (Qiagen, katalognr 951356) on a Biorobot M48 (Qiagen) according to the manufacturer's protocol. Sequencing libraries were prepared from $50 \mathrm{ng}$ of gDNA using the TruSight One Sequencing Panel (Illumina, San Diego, CA, USA) according to the manufacturer's instructions, Paired-end 150 bp sequencing was performed on a MiSeq (Illumina). Desktop Sequencer, targeting a mean region coverage depth $>100 x$ and $>97 \%$ of the region at $>10 \times$. Analysis of sequencing data was performed by applying a MiSeq Reporter Enrichment Workflow (v.2.4.60.8) including a Burrows-Wheeler Aligner (v.0.6.1-r104-tpx) and a Genome Analysis Toolkit (v.1.6-22-g3ec78bd), with the target region defined as the exome part with flanking $10 \mathrm{bp}$ of 4813 disease-associated genes as defined by Illumina TruSight One Sequencing panel (http://www.illumina.com/products/ trusight-one-sequencing-panel.html).

Cartagenia Bench Lab NGS (v.4) was used for variant annotation and prioritization, considering a recessive inheritance model using Cartagenia Inheritance analysis with the index patients defined as affected and the parents as unaffected. The focus of the analysis was to identify potentially damaging homozygous or compound heterozygous variants inherited in a recessive pattern from both the parents, or variants showing non-Mendelian inheritance, indicating a de novo event. Variants were filtered based on quality criteria (LowGQX $<10$, LowVariantFreq $<0.20$, LowGQ $<30.00$, R8 $>8$, SB $>-10$, LowDP $<20$ ) and their presence in population frequency databases with a maximum minor allele frequency of $1 \%$ (ExAC, 1000 genomes, dbSNP. Splicesite analysis was restricted to 8 intronic bp positions from the nearest intronexon boundary and 3 exonic bp positions from the nearest intron-exon boundary. Findings in patients 1-2 and 2-1 were validated by Sanger sequencing. Sanger sequencing for the $A B C C 9$ sequence variant was performed for both healthy and affected family members.

\subsubsection{Whole-genome sequencing}

DNA libraries for Illumina sequencing were generated for patients 1-2 and 2-1 using standard protocols (Illumina) from $200-1 \mu \mathrm{g}$ of genomic DNA. The libraries were sequenced with paired-end $(2 \times 150 \mathrm{bp})$ runs using Illumina HiSeq $X$ Ten sequencers with a target depth of $30 \times$ base coverage. The Illumina data 
were processed with our in-house developed pipeline v 2.5.1 (https://github. com/UMCUGenetics/IAP) including Genome Analysis Toolkit (GATK) v3.4-46 (McKenna et al., 2010) according to the best practices guidelines(Van der Auwera et al., 2013). Briefly, sequence reads were mapped against the human reference genome GRCh37 using Burrows-Wheeler Aligner v0.7.5a mapping tool(Li \& Durbin, 2009). Sequence reads were marked for duplicates using Sambamba v0.5.855 and recalibrated per sample using GATK BaseRecalibrator. Next, GATK Haplotypecaller was used to call SNPs and indels to create gVCF's. These gVCF's were genotyped with GATK. Variants are flagged as PASS only if they do not meet the following criteria: $Q D<2.0, M Q<40.0, F S>60.0$, HaplotypeScore $>13.0$, MQRankSum <-12.5, ReadPosRankSum <-8.0, snpclusters $>=3$ in $35 \mathrm{bp}$. For indels: $Q D<2.0, F S>200.0$, ReadPosRankSum $<-20.0$. WGS statistics are provided in Table $\mathbf{S 1}$.

Full analysis was performed on a merge of the reference coding regions obtained from Ensembl (v.75) (http://www.ensembl.org/) and UCSC (https:// genome.ucsc.edu/) with \pm 100 bp flanks. Cartagenia Bench Lab NGS v.4.3.5 was used for variant interpretation and prioritization. Variant analyses were made considering a recessive inheritance model using the Cartagenia cohort analysis tool. The focus of the analysis was to identify shared variants or shared genes with possible damaging, but not identical variants, amongst the two cases. Lenient quality parameters were used to determine high quality variant calls (read depth $>4$ reads, filter:pass, genotype quality $>98$ ). Variants were filtered based on presence in population frequency databases with a maximum MAF of 1\% (ExAC, 1000 genomes, dbSNP, and GoNL). Variants are categorized as LoF (frameshift, stopgain, startloss, stoploss), nonsynonymous, synonymous, and inframe variants. Furthermore, splice-site analysis was restricted to 8 intronic bp positions from the nearest intron-exon boundary and 3 exonic bp positions from the nearest intron-exon boundary. The Kinship coefficient was calculated from WGS data. Notably, due to multi-sample calling and noise present in the data, the kinship coefficient of unrelated samples is expected to be around 0.05 . Hence, this is usually applied as proximate to calculate relatedness.

\subsection{5 cDNA analysis}

RNA was isolated from fibroblast cultures generated from skin biopsiesusing an RNeasy minikit (Qiagen) according to the manufacturers protocol. cDNA

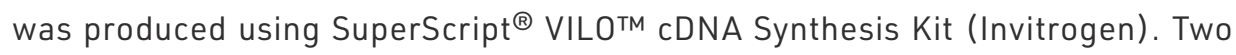
microliters of cDNA were used in a PCR reaction amplifying ABCC9 exon 6-9 using primers as described in the Supplementary Methods. PCR products were subsequently verified by Sanger sequencing. 


\subsubsection{Molecular biology and recombinant expression}

For recombinant protein expression and functional characterization, a 52 amino acid deletion (Ala386 to $G \ln 437 ; \Delta 8$ ) equivalent to the human exon 8 coding region was engineered into either a $\mathrm{N}$-terminal Flag-tagged rat SUR2A construct (pcDNA_rSUR2A-Flag; GenBank accession No. D83598.1), a wild-type rat SUR2A construct ( $p C M V \_S U R 2 A-W T$; GenBank accession No. D83598.1) or a wild-type rat SUR2B construct ( $p c D N A \_r S U R 2 B-W T ;$ GenBank accession No. AF019628.1). The rat SUR2 sequence is $97 \%$ identical to human SUR2 and was used to allow for comparison with an extensive number of previous studies of recombinant SUR2 (Cooper, McClenaghan, Chen, Stary-Weinzinger, \& Nichols, 2017; Cooper et al., 2014; Cooper, Sala-Rabanal, Lee, \& Nichols, 2015; Harakalova et al., 2012; McClenaghan et al., 2017). SUR2 cDNA $(1 \mu \mathrm{g})$ was transiently transfected into Cosm6 cells59 (RRID: CVCL_8561) using Fugene 6 (Roche) alongside wild-type Kir6.2 (0.6 $\mu$ g; pcDNA3.1_mKir6.2; GenBank accession No. D50581.1). To mimic heterozygous expression, Kir6.2 was co-transfected with a 1:1 ratio of SUR2AWT and SUR2A $\triangle 8(0.5 \mu \mathrm{g}: 0.5 \mu \mathrm{g})$. Western blot analysis and radioactive rubidium efflux assays were performed 48-72 $\mathrm{h}$ after transfection.

\subsubsection{Western blot analysis of recombinant SUR2}

Cosm6 cells were transfected with GFP alone, Kir6.2 with Flag-tagged SUR2AWT or Kir6.2 with Flag-tagged SUR2A $\triangle 8$ ( $N$-terminal Flag tag with amino acid sequence MDYKDDDDKGAP was inserted prior to the SUR2A start codon). After $48 \mathrm{~h}$, cells were harvested in phosphate buffered saline (PBS; Gibco), pelleted, and lysed in $50 \mu$ l of PBS containing $1 \%$ Triton X-100 (Sigma) by mixing for $1 \mathrm{~h}$ at $4{ }^{\circ} \mathrm{C}$. Cell lysate was mixed with $2 \times$ SDS loading buffer, and was separated by electrophoresis using a precast $4-12 \%$ Bis-Tris Gel (Nupage). Gel contents were transferred to membranes using an iBlot 2 (Thermo-Fisher). Membranes were first incubated in a blocking solution (PBS/1\% TWEEN 20/0.4\% milk; PBSTM) for $1 \mathrm{~h}$ at room temperature (RT) with gentle shaking, before being washed ( $3 \times$ using $\mathrm{PBS} / \mathrm{TWEEN}$ ) and incubated in the primary horseradish peroxidase-conjugated anti-Flag antibody (Anti-Flag M2-HRP-conjugate Sigma \# A8592; 1 in 5000 in PBSTM), or an anti- actin antibody (clone C4 MilliporeSigma \# MAB1501;1 in 3000 in PBSTM) for $1 \mathrm{~h} / \mathrm{RT}$. Anti-actin blots were washed ( $3 \times$ in PBSTM) and incubated with horseradish peroxidase-conjugated secondary antibody (Goat anti-mouse IgG antibody-HRP conjugate, Santa Cruz Biotechnology \# sc-2005; 1 in 5000 in PBSTM) for $1 \mathrm{~h}$ at room temperature. A Pierce ECL Western Blotting Substrate kit was used for chemiluminescence detection; blots were analyzed and protein density quantified using Image $(\mathrm{NIH})$ and Excel (Microsoft). Actin and SUR2A $\triangle 8$ expression was normalized to values observed in WT SUR2A-Flag transfected cells in each independent experiment. 


\subsubsection{Rubidium (86Rb+) efflux assay}

Cosm6 cells were plated and transfected in 12-well plates at sufficient density to allow for $\sim 75 \%$ confluence on the day of experimenting. Cells were loaded with radioactive $86 \mathrm{Rb}+$ by incubation ( $>6 \mathrm{~h}$ ) with DMEM media spiked with $1 \mu \mathrm{Ci}$ $\mathrm{ml}-186 \mathrm{RbCl}$ (PerkinElmer) at $37^{\circ} \mathrm{C} / 5 \% \mathrm{CO2}$. After isotope loading, cells were washed in Ringer's solution which contained (in mM): $118 \mathrm{NaCl}, 10 \mathrm{HEPES}, 25$ $\mathrm{NaHC0}, 4.7 \mathrm{KCl}, 1.2 \mathrm{KH} 2 \mathrm{PO} 4,2.5 \mathrm{CaCl} 2$, and $1.2 \mathrm{MgSO} 4$, and was supplemented with $2.5 \mathrm{mg} / \mathrm{ml}$ oligomycin and $1 \mathrm{mM} 2$-deoxy-D-glucose to induce metabolic inhibition (MI), and incubated at room temperature for a further $10 \mathrm{~min}$. Cells were then washed and Ringer's solution was added to each well, before being collected and replaced at the defined time points $(2.5,5,12.5,22.5$, and $37.5 \mathrm{~min}$ ). Cells were then lysed with $2 \%$ SDS to liberate remaining intracellular $86 \mathrm{Rb}+$ and sample radioactivity was determined by scintillation counting.

Cumulative $86 \mathrm{Rb}+$ efflux was calculated from the total counts from each time point. A time-dependent divergence from a mono-exponential efflux was observed, as noted previously (McClenaghan et al., 2017), and thus rate constants were derived from exponential functions fit to early time points only (2.5-12.5 min). $\mathrm{K}_{\text {ATP }}$-independent efflux rate constants ( $\mathrm{k} 1$ ) were obtained from GFP-only transfected cells using the equation:

Efflux $=1-e^{-k 1 . t,}$

The $K_{\text {ATP }}$-dependent efflux rate constant ( $k 2$ ) was obtained from $K_{\text {ATP }}$ -transfected cells using the equation:

Efflux $=1-e^{((-k 1 . t)+(-k 2 . t))}$,

where k1 was obtained from GFP-transfected cells (Eq. (1)), and the number of active channels was assumed to be proportional to k2. Efflux-time data shown represent the mean \pm SEM from at least three independent experiments with multiple internal replicates $(N \geq 3, n \geq 4)$. The Mann-Whitney $U$ test was used to determine statistical significance at a $p$-value $<0.05$.

\subsubsection{Patch clamp recording of recombinant channels}

Cosm6 cells were transfected with WT Kir6.2 $(0.6 \mu \mathrm{g})$ and either WT SUR2A $(1 \mu \mathrm{g})$, SUR2A $\triangle 8(1 \mu \mathrm{g})$, or a 1:1 ratio of WT and SUR2A $\triangle 8(0.5 \mu \mathrm{g}: 0.5 \mu \mathrm{g})$, and $0.1 \mu \mathrm{g}$ pcDNA3.1-eGFP to identify transfected cells. In all, 48-72 h after transfection, excised inside out patch clamp recordings were made. The membrane potential was voltage clamped at $-50 \mathrm{mV}$. Symmetrical KINT solution which contained (in 
$\mathrm{mM}$ ) : $140 \mathrm{KCl}, 10 \mathrm{HEPES}, 1 \mathrm{EGTA}(\mathrm{pH} 7.4$ with $\mathrm{KOH}$ ) was used for pipette and bath solutions. ATP and pinacidil were applied (in the presence of $0.5 \mathrm{mM}$ free $\mathrm{Mg} 2+$, calculated using CaBuf (Katholieke Universiteit Leuven) using a Dynaflow Resolve perfusion chip (Cellectricon). Experiments were performed at $20-22^{\circ} \mathrm{C}$. $\mathrm{K}_{\text {ATP }}$ channel currents in the absence and presence of nucleotides and pinacidil were normalized to the basal current in KINT, and dose-response data were fit with a four-parameter Hill fit according to Eq. (3), using the Data Solver Function in Microsoft Excel, where the current in $\mathrm{KINT}=\mathrm{Imax}=1$; Imin is the normalized minimum current observed in ATP; $[X]$ refers to the concentration of ATP; IC50 is the concentration of half-maximal inhibition; and $\mathrm{H}$ denotes the Hill coefficient.

Normalized current $=I \min +(I \max -I \min ) /\left(1+([X] / I C 50)^{H}\right)$.

\subsubsection{Generation of the SUR2 LoF mouse}

All mouse studies were performed in compliance with the standards for the care and use of animal subjects defined in the NIH Guide for the Care and Use of Laboratory Animals, and were reviewed and approved by the Washington University Institutional Animal Care and Use Committee. SUR2-STOP mice were generated using CRISPR/Cas9-mediated genome engineering (Ran et al., 2013). In mice in which an attempt was made to generate a Cantú syndromeassociated missense mutation, an artefactual indel mutation in ABCC 9 (c.3446_3450delACTTCinsGA) was identified in one founder individual (B6CBA F1/J background), which results in the introduction of a premature stop codon (p.Y1148Stop). This founder (termed heterozygous SUR2-STOP) was viable and fertile and was crossed with C57BL/6J mice to generate F1 SUR2wt/STOP mice. gDNA was isolated from F1 mouse tails, and PCR was used to verify the presence of the indel mutation in positive offspring. One positively identified SUR2wt/ STOP F1 mouse was selected, and further crossed against C57BL/6J to generate F2 SUR2wt/STOP, which were in turn intercrossed to generate F3 homozygous SUR2STOP/STOP (hereafter referred to as SUR2-STOP) and wild-type and heterozygous littermates, which were used for experiments.

\subsubsection{Electrophysiological recordings of isolated myocytes}

Ventricular myocytes were isolated from adult mice as previously described (Zhang et al., 2011). Inside-out patch clamp recordings were made in symmetrical pipette and bath $\mathrm{K}_{\mathrm{INT}}$ solutions which contained (in $\mathrm{mM}$ ): $140 \mathrm{KCl}$, 10 HEPES, 1 EGTA ( $p H$ 7.4). Solution exchange was achieved using a Dynaflow Resolve perfusion chip (Fluicell). MgATP and pinacidil (both Sigma) were applied as indicated. Free $\mathrm{Mg}^{2+}$ was maintained at $0.5 \mathrm{mM}$ by supplementing ATP-containing solutions with $\mathrm{MgCl}_{2}$, where necessary. Recording electrodes 
were formed from sodalime hematocrit glass (Kimble) and had a resistance of 1-1.4 $\mathrm{M} \Omega$ when filled. Currents were recorded at $-50 \mathrm{mV}$, sampled at $3 \mathrm{kHz}$, and filtered at $1 \mathrm{kHz}$ using an Axopatch 700B and Digidata 1200 (Molecular Devices). $\mathrm{K}_{\text {ATP }}$ current was calculated as the difference between the current in the absence of nucleotides and in the presence of a fully inhibiting concentration of $10 \mathrm{mM}$ MgATP.

Vascular smooth muscle cells were acutely isolated form the descending thoracic aorta, as previously described (Huang et al., 2018). Whole-cell patch clamp recordings were made in an initial $\mathrm{Na}+$ bath solution (which contained, in $\mathrm{mM}$ ): $\mathrm{NaCl} 136, \mathrm{KCl} 6, \mathrm{CaCl}_{2} 2, \mathrm{MgCl}_{2}$ 1, HEPES 10, and glucose 10 ( $\mathrm{pH} 7.4$ ) before switching to a $\mathrm{K}+$ bath solution which contained (in $\mathrm{mM}$ ) $\mathrm{KCl} 140, \mathrm{CaCl}_{2} 2, \mathrm{MgCl}_{2}$ 1, HEPES 10, and glucose $10 \mathrm{pH} \mathrm{7.4,} \mathrm{in} \mathrm{the} \mathrm{absence} \mathrm{and} \mathrm{presence} \mathrm{of} \mathrm{pinacidil} \mathrm{and}$ glibenclamide as indicated. The pipette solution contained (in $\mathrm{mM}$ ) potassium aspartate 110, $\mathrm{KCl} 30, \mathrm{NaCl} 10, \mathrm{MgCl}_{2} 1$, HEPES 10, $\mathrm{CaCl}_{2} 0.5, \mathrm{~K}_{2} \mathrm{HPO}_{4}$ 4, and EGTA 5 , with $\mathrm{pH}$ adjusted to 7.2 with $\mathrm{KOH}$. The glibenclamide sensitive current density at a holding potential of $-70 \mathrm{mV}$ was taken as a measure of $\mathrm{K}_{\text {ATP }}$ channel activity.

The data were analyzed using Clampfit (Molecular Devices) and Excel (Microsoft) and are presented as individual replicates in scatter plots, alongside mean \pm SEM. The two-tailed t test was used to determine statistical significance at a $p$-value $<0.05$ using the RealStatistics add-in for Microsoft Excel.

\subsubsection{Mouse echocardiography}

Echocardiography was performed on adult (5-month-old) mice, as previously described (Razani et al., 2011). Images were acquired using the Visual Sonics Vevo 770 Imaging System (Visual Sonics Inc., Toronto, Canada) and analyzed with Visual Sonics software. Mice were lightly anesthetized using Avertin and $\mathrm{M}$-mode images of the parasternal long axis were obtained, from which measurements of fractional shortening, left ventricular mass, and left ventricular internal dimension in diastole (LVIDd) were calculated. Left ventricular mass and LVIDd were normalized to the mouse body weight and length (tip of nose to base of tail) for statistical comparisons. One-way ANOVA followed by post hoc Tuket test or Two-tailed t tests were used to determine statistical significance using the RealStatistics add-in for Microsoft Excel, as described.

\subsubsection{Multiple-trial inverted screen test}

The procedure involved a modification of a previously published single-trial method that had been used to demonstrate impaired strength in a mutant mouse model of muscular dystrophy31. This methodology was adapted to evaluate 
deficits in strength related to decreases in physical endurance, which involved conducting multiple test trials administered in a single day. The apparatus was a wire-mesh screen (16 squares per $10 \mathrm{~cm}$ ) that was elevated $\sim 50 \mathrm{~cm}$. A trial consisted of placing an adult (2-3-month-old) SUR2-STOP mouse, heterozygote (Het) SUR2-STOP mouse, or a wild-type littermate control on the screen that was inclined to $60^{\circ}$ with its head oriented down toward the base of the screen. After the mouse was stabilized on the screen, it was slowly inverted resulting in the mouse hanging upside down from the screen. Soft bedding was placed underneath the mouse to help protect it from injury. The time a mouse remained hanging upside down following inversion of the screen was recorded during an initial 3-min test trial. After the first trial, a mouse was placed into a holding cage for 5 min after which two more trials were conducted with 5 min being spent in the holding cage between trials. After completing the three trials, a mouse was placed back into the holding cage where it remained for $44 \mathrm{~min}$, which was followed by the same $3 \times 3$-min test trial procedure described above. If a mouse remained hanging from the screen for the full 3 min duration, it was removed from the screen and assigned a value of $180 \mathrm{~s}$. The time a mouse remained hanging upside from the screen (3-min maximum) was analyzed for each of the six trials as well as the total duration summed across all six trials.

\subsubsection{Behavioral and cognitive testing of SUR2-STOP mice}

Cognitive and other behavioral functions were assessed in adult (4-5-monthold) SUR2-STOP mice and wild-type littermates by conducting a battery of tests to evaluate ambulatory activity, emotionality, sensorimotor capabilities, learning and memory, and anxiety-like behaviors. The battery included the following tests (in order): 1-h locomotor activity; a series of seven simple sensorimotor measures; the Morris water maze, object recognition; and elevated plus maze, respectively. Full descriptions of these procedures are provided in the Supplementary Methods.

\subsubsection{Blood pressure recordings in SUR2-STOP mice}

Arterial blood pressures were measured in 12-month-old SUR2-STOP mice and WT littermates anesthetized with $1.5 \%$ isoflurane. The left carotid artery was catherized and a Millar pressure transducer advanced to the ascending aorta. Pressures were recorded using Powerlab data acquisition (ADInstruments), and mean arterial pressure (MAP) was calculated using LabChart 7 (ADInstruments). 


\subsubsection{Zebrafish maintenance}

All zebrafish experiments were conducted under the guidelines of the animal welfare committee of the Royal Netherlands Academy of Arts and Sciences (KNAW) and were approved by the local ethics committee at the Royal Dutch Academy of Sciences (KNAW). Adult zebrafish (Danio rerio) were maintained at $28^{\circ} \mathrm{C}$ and subjected to the 14-h light/10-h dark cycle. Embryos were produced by natural mating and kept in a humidified incubator at $28^{\circ} \mathrm{C} 63$. All CrispR/Cas 9 injections were performed in the wild-type strain Tübingen longfin.

\subsubsection{CRISPR/Cas9 design and embryo injections}

A target site within the abcc9 open-reading frame was chosen using CHOPCHOP (http://chopchop.cbu.uib.no/). sgRNA template was purchased at Integrated DNA Technologies (IDT) as standard desalted same-day oligos and synthesis was carried out using the Ambion MEGAscript T7 or SP6 kits (Ambion). Purification of the in vitro synthesized mRNA was achieved with the RNeasy Mini Kit (Qiagen) (Gagnon et al., 2014). gRNA and Cas9-encoding mRNA were co-injected into one-cell stage zebrafish embryos. Each embryo was injected with $2 \mathrm{nl}$ of solution containing $\sim 12.5 \mathrm{ng} \mu \mathrm{l}-1$ of sgRNA and $~ 300 \mathrm{ng} \mu \mathrm{l}-1$ of Cas 9 mRNA. Injected embryos were grown to adulthood to generate "founder" fish (FO), and screened for germline transmission of CRISPR-induced indel mutations using an adapted method for detecting simple sequence-length polymorphisms (SSLPs). Each putative founder adult fish was crossed with a wild-type adult fish (F1). Sequencing analysis confirmed successful introduction of $13 \mathrm{n}$ deletion and segregation in a Mendelian fashion. Homozygous fish (F2) were generated by inbreeding heterozygous mutant carriers.

\subsubsection{Quantitative RT-PCR}

Zebrafish larvae at $5 \mathrm{dpf}$ were pooled in groups of 60 (three different groups for WT and four groups for homozygous mutants) and homogenized. RNA was purified using Trizol following standard procedures and cDNA synthesized using High-Capacity cDNA Reverse Transcription Kit. qPCR was performed using a StepOne Real Time PCR System (Applied Bioscience) and PrimeTime qPCR primer/probe assays, as detailed in the Supplementary Methods (Integrated DNA Technologies). Relative gene expression (2- $\Delta \Delta \mathrm{Ct}$ ) was calculated by normalizing abcc 9 expression to the mean expression observed in WT pools using Gapdh as a reference gene and the Pfaffl Method65. 


\subsubsection{Isolation of adult zebrafish ventricular myocytes}

For isolation of cardiomyocytes, 1-year-old animals were anaesthetized by transfer into ice water before they were decapitated. After removal of the skin and opening of the pericardial sac, the hearts were harvested and both bulbous arteriosus and atrium were removed. Ventricles from three to four fish were pooled together and placed in an epi containing perfusion buffer: (mM) 10 HEPES, 30 Taurine, 5.5 Glucose, 10 BDM in PBS. Ventricular myocytes were obtained by enzymatic dissociation (perfusion buffer supplemented with $12.5 \mu \mathrm{M} \mathrm{CaCl}$, $5 \mathrm{mg} / \mathrm{ml}$ collagenase II and IV) at $32{ }^{\circ} \mathrm{C}$, and $800 \mathrm{rpm}$ for $40 \mathrm{~min}$. Dissociation was ended by transfer to stopping buffer (perfusion buffer supplemented with $10 \%$ (vol/vol) FBS, $12.5 \mu \mathrm{M} \mathrm{CaCl}_{2}, 5 \mathrm{mg} / \mathrm{ml} \mathrm{BSA}$ ). Cells were dispersed by gentle trituration using a Pasteur pipette.

\subsubsection{Electrophysiological recordings of isolated myocytes}

Pipettes were made from soda lime glass microhematocrit tubes (Kimble) and had a resistance of 1-2 $M \Omega$ when filled with pipette solution. All recordings were made in symmetrical $\mathrm{K}_{\mathrm{INT}}$ solution ( (mM) $140 \mathrm{KCl}, 10 \mathrm{HEPES}, 0.5 \mathrm{EGTA}$ ( $\mathrm{pH} 7.4$ with $\mathrm{KOH})$ ). ATP (Sigma) was applied as indicated. Free $\mathrm{Mg}^{2+}$ was maintained at $0.5 \mathrm{mM}$ by supplementing ATP-containing solutions with $\mathrm{MgCl} 2$ where necessary. Currents were recorded at $-50 \mathrm{mV}$, sampled at $3 \mathrm{kHz}$, and filtered at $1 \mathrm{kHz}$ using an Axopatch 1D and Digidata $1322 \mathrm{~A}$ (Molecular Devices). $\mathrm{K}_{\text {ATP }}$ current was calculated as the difference between the current in the absence of nucleotides and in the presence of a fully inhibiting concentration of $5 \mathrm{mM}$ ATP. The data were analyzed using Clampfit (Molecular Devices) and Excel (Microsoft), and are presented as individual replicates in scatter plots, alongside mean \pm SEM.

\subsubsection{Larval locomotor assay}

The Viewpoint Zebrabox system (Viewpoint Behaviour Technology, Lyon, France) was used to evaluate larval locomotor activity in $5 \mathrm{dpf}$ zebrafish larvae. Larvae were transferred to a 48 -well plate with $500 \mu$ of embryo medium, placed into the Zebrabox and subjected to a $30 \mathrm{~min}$ acclimatization period to the plate and Zebrabox environment. A total of 24 replicates (one embryo placed individually in each plate well per replicate) were used per genotype. Behavior was monitored at $28.5^{\circ}$ using a ZebraBox system (ViewPoint Behaviour Technology) consisting of a soundproof chamber with an infrared camera capable of recording 60 frames per second; analyses were performed using Zebralab locomotion tracking software (ViewPoint Behavior Technology). The integration period was set to $30 \mathrm{~s}$ for the 10-min duration of the experiment. A nontransparent background mode with a detection threshold of 20 was used and a minimum velocity of $2 \mathrm{~mm} \mathrm{~s}^{-1}$ was defined as record threshold of inactivity in order to remove 
system noise. A movement was considered "small" when individuals moved $<10 \mathrm{~mm} / \mathrm{s}$. The larval locomotor assay consisted of a $5 \mathrm{~min}$ baseline recording. The total movement was recorded, then, quantified using ZebraLab software (Viewpoint, Lyon, France) and plotted in "actinteg" units, which is the sum of all pixel changes detected during the experimental period (Afrikanova et al., 2013). In addition, from the data recorded, the ZebraLab software calculated several parameters such as total swimming distance (TSD) and total swimming time (TST). The data were compiled and mean activity, TSD or TST for every genotype during each integration period calculated. The data were pooled together from three independent experiments at the same conditions. All experiments were performed comparing SUR2-STOP and its WT siblings. All embryos analyzed originated from group matings of adult zebrafish.

\subsubsection{In vivo high-speed imaging}

Image acquisition was conducted using a Hamamatsu C9300-221 high-speed CCD camera (Hamamatsu Photonics) at $150 \mathrm{fps}$ mounted on a Leica DM IRBE inverted microscope (Leica Microsystems) using Hokawo 2.1 imaging software (Hamamatsu Photonics). Image analysis was subsequently carried out with ImageJ (http://rsbweb.nih.gov/ij/, last accessed November 2017). For analysis of cardiac function, adult zebrafish heterozygous for abcc 9 KO were interbred, and genotype identified by sanger sequencing post imaging. High-speed brightfield image sequences of the embryonic zebrafish heart were acquired for zebrafish at age of 5 days post fertilization (dpf). To inhibit pigmentation, 0.003 $\%(v / v)$ 1-phenyl-2-thiourea was added to the embryo medium at the Prim-5 stage (about $24 \mathrm{~h}$ post fertilization (hpf)). After being anaesthetized in $16 \mathrm{mg} /$ $\mathrm{ml}$ tricaine (MilliporeSigma) in E3 medium zebrafish embryos were mounted in dorsal position in small microscopic chambers filled with $0.25 \%(w / v)$ agarose (Invitrogen) prepared in the same concentration of anesthetic. Zebrafish hearts were imaged for $10 \mathrm{~s}(\sim 30$ cardiac cycles $)$ at $28^{\circ} \mathrm{C} \pm 0.2^{\circ} \mathrm{C}$.

\subsubsection{Cardiac dimensions and function in SUR2-STOP zebrafish}

The time interval between three heart beats was measured, and the heart rate (bpm) was calculated in triplicates. Sequential still frames from high-speed movies were used to outline the perimeter of the ventricle. Measurement analysis was carried out by "fit-to-ellipse" algorithm, which calculates the center of mass and subsequently the best fitting ellipse. The long axis length (a) and short axis length (b) at diastole and systole were determined and used to calculate ventricular end-systolic (ESV) and diastolic volumes (EDV) in triplicates applying the formula: $V=4 / 3 \varpi a b 2$. The stroke volume was calculated as the difference between three ventricular EDVs and ESVs. Cardiac output 
was obtained by multiplying the heart rate with stroke volume. The percent shortening fraction (SF) was calculated using the formula: SF= (length at diastole - length at systole)/(length at diastole) $\times 100$. Fractional area change (FAC) was calculated as follows: FAC = (area at diastole - area at systole $) /($ area at diastole) $\times 100$. Measurements were performed blindly.

\subsubsection{Cardinal vein blood flow velocity}

Zebrafish larvae were mounted in lateral position at $5 \mathrm{dpf}$ and the cardinal vein region behind the cloaca was imaged for $5 \mathrm{~s}$ at $28 \pm 0.3^{\circ} \mathrm{C}$. Vein blood flow velocity was calculated by assessing frame-by-frame motion of three single erythrocytes per fish determined from high-speed images to assess mean erythrocyte cell velocity ( $\mathrm{mm} / \mathrm{second}$ ) using ImageJ. Cardinal blood flow velocity was measured over ten frames at a video frame rate of $150 \mathrm{fps}$ as non-pulsatory venous flow allows frame-by-frame analysis (Tessadori et al., 2018).

\subsubsection{Heart extraction and hematoxylin and eosin (H\&E) staining}

Adult zebrafish hearts were dissected and fixed in $4 \%$ paraformaldehyde (in phosphate buffer with $4 \%$ sucrose) at $4{ }^{\circ} \mathrm{C}$ overnight and subjected to paraffin embedding and sectioning at $10 \mu \mathrm{m}$ intervals. Heart sections were stained with H\&E following standard procedures. Six fishes were used for each genotype. Image acquisition was conducted using a Leica DM4000 B LED upright automated microscope.

\subsubsection{TUNEL assay}

TUNEL apoptosis staining was performed on $10 \mu \mathrm{m}$ cryosections and detected using the in situ cell death detection kit from Roche (Mannheim, Germany) according to the manufacturer's instructions. Nuclei are shown by DAPI (4',6-diamidino-2-phenylindole) staining. Confocal images were acquired using a Leica Sp8 confocal microscope.

\subsubsection{Statistical analysis}

Rubidium flux, patch clamp electrophysiology and echocardiography data were analyzed using two-tailed t test or Mann-Whitney $U$ test as described. For the inverted screen test of mice, data were analyzed using a repeated measures ( $\mathrm{rm}$ ) ANOVA model that contained one between-subjects variable (genotype) and two within-subjects variables (trials and sessions), followed by pairwise comparison by two-tailed t test with Bonferroni correction. Behavioral tests in mice were analyzed using a repeated measures ( $\mathrm{rm}$ ) ANOVA model that contained two between-subjects variable (genotype and sex) and one within-subjects variable (trial block). The data are presented as mean \pm SEM. 
For zebrafish studies sample size was not predetermined by statistical analysis. In all experiments involving zebrafish embryos, selection was random for scoring. Exact numbers of analyzed embryos are reported at relevant locations in the main text or figure legends. Statistical analysis was carried out with Prism (GraphPad). Distribution of the data sets was tested by D'Agostino-Pearson omnibus normality test. Depending on the outcome of this test, comparison of two conditions with each other was carried out using a Student's two-tailed test or Mann-Whitney two-tailed $U$ test throughout the manuscript. All values are expressed as mean \pm SEM.

\subsection{Supplemental Material and Methods}

\subsubsection{Mouse model}

\section{Behavioral, emotionality and cognitive testing of SUR2-Stop mice}

1-hour locomotor activity, exploratory behavior and sensorimotor battery: Baseline locomotor activity was assessed using a transparent polystyrene enclosure $(47.6 \times 25.4 \times 20.6 \mathrm{~cm})$ and computerized photobeam instrumentation as described previously (Dougherty et al., 2013; Wozniak et al., 2004). Total ambulatory time and vertical rearings were taken as measures of activity whilst the time spent in the center of the field $(33 \times 11 \mathrm{~cm})$ and the edges of the field $(5.5 \mathrm{~cm}$ from walls) were used as indices of emotionality. The next day, mice were subjected to a series of sensorimotor tests selected to assess coordination, balance (ledge, platform, pole and inclined screen tests), strength (screen tests), and initiation of movement (walking initiation), as described previously (Grady et al., 2006; Wozniak et al., 2004). For the balance tests, mice were placed on a Plexiglass ledge $(0.75 \mathrm{~cm}$ wide, $30 \mathrm{~cm}$ elevation) or a small circular platform ( $3 \mathrm{~cm}$ in diameter, $47 \mathrm{~cm}$ elevation) and the time the mice remaining on the ledge/platform was recorded. For the pole test mice were placed headupwards with forepaws on top of a textured rod ( $8 \mathrm{~mm}$ diameter, $55 \mathrm{~cm}$ height) and the time taken for the mouse to turn and descend the pole was recorded. The screen tests were conducted by placing a mouse head-downwards upon a mesh screen ( 16 squares per $10 \mathrm{~cm}$, elevated $47 \mathrm{~cm}$ and angled at 60 or 900 ), the time the mouse took to turn and climb to the top of the screen was recorded. The times of two trials for each test were averaged for analysis.

Morris Water Maze Navigation: The day after the sensorimotor battery we employed the MWM using a computerized tracking system (ANY-maze; Stoelting) to assess spatial learning and memory as described previously (Dougherty et al., 2013; Wozniak et al., 2004). Cued trials were performed in which a visible 
platform was variably placed in the water maze with visible cues. Four trials were conducted per day (60 s maximum time) for two consecutive days with the platform being moved to different locations for each new trial using a $30 \mathrm{~min}$ inter-trial interval (ITI) and with limited, distal spatial cues being present to limit spatial learning. The time, distance and swimming speed for mice to find the platform was recorded across four blocks of trials (two trials/block). Three days later, place trials were initiated in which the platform was submerged and hidden but remained in a constant location, to determine spatial learning. Mice were required to learn the single location of a submerged platform in relation to spatial cues. Place trial data were recorded from over five blocks of trials (four trials/block), in which each block included the performance level for each of five consecutive days of testing. Finally, a probe trial wherein a mouse was released into the water maze where the platform had been removed was administered $\sim 1$ $\mathrm{h}$ after the final Place Trial (on day 5 of place trial testing). The time spent in the various pool quadrants, including the target quadrant (where the platform had previously been placed), was recorded.

Object recognition test: Elevated Plus Maze: Anxiety-like behavior was measured using the Elevated Plus Maze as previously described (Dougherty et al., 2013; Schaefer et al., 2000). The black Plexiglass EPM apparatus consisted of two opposed open arms (without walls) and two opposed closed (walled) arms (36 x $6.1 \times 15 \mathrm{~cm}$ ) which extended in a + shape from a central square platform $(5.5 \mathrm{x}$ $5.5 \mathrm{~cm}$ ). Behavior in the maze was recorded using an automated, computerized recording set up (Kinder Scientific). Beam break data was recorded and analyzed using MotorMonitor software (Kinder Scientific), distance traveled, time spent in each area and entries into open and closed arms were recorded. Test sessions were performed in a dimly lit room (lighting with 13-watt blacklight bulbs; Ecobulbs, Feit) where each session began by placing a mouse in the center of the maze allowing for free exploration of the maze. Each session lasted 5 min and the mice were tested over 3 consecutive days.

\section{Statistical analyses}

Data was analyzed as previously reported (Stein et al., 2014). Numerical data were presented as mean \pm SEM. ANOVA models were used to test for statistical significance. Repeated-measures ANOVA (rmANOVA) models containing two between-subjects variables (genotype and sex) and once within-subject (repeated measures) variable (e.g. blocks of trials) were typically used to analyze the MWM and EPM data. The Huynh-Fledt adjustment of levels was used for all within-subject effects containing more than two levels to protect against violations of sphericity/compound symmetry assumptions underlying rmANOVA 
models. We used one-way ANOVA models to test for between-group differences in the $1 \mathrm{~h}$ locomotor activity and sensorimotor tests.

\subsubsection{Zebrafish model}

\section{Whole-embryo brightfield imaging and measurement of interorbital distance and body length}

In vivo phenotypic assessment for whole-embryo imaging were carried out on a Leica M165FC stereomicroscope (Leica Microsystems) with transmitted light. Images were captured with a DFC420 digital microscope camera (Leica Microsystems). Images were applied to measure the distance between the convex tip of the eyes (interorbital distance) in $5 \mathrm{dpf}$ larvae using Image $(\mathrm{NIH})$. To account for variations in size periorbital distance was normalized to overall larval body length. Body length was measured from the tip of the head to the end of the trunk (before the caudal fin).

\section{AFOG staining}

Adult zebrafish hearts were dissected and fixed in $4 \%$ paraformaldehyde (in Phosphate buffer with $4 \%$ sucrose) at $4^{\circ} \mathrm{C}$ for 4 hours, incubated in $\mathrm{P} 04+30 \%$ sucrose at $4^{\circ} \mathrm{C}$ overnight and subjected to embedding in tissue freezing medium (Leica) and sectioning at $10 \mu \mathrm{m}$ intervals. Acid Fuchsin Orange-G (AFOG) staining was performed as described previously (Poss, Wilson, \& Keating, 2002). Image acquisition was conducted using a Leica DM4000 B LED upright automated microscope.

\section{Immunofluorescence}

Immunofluorescence was performed in cryosections. PEMTx buffer $(80 \mathrm{mM}$ Na-PIPES, $5 \mathrm{mM} \mathrm{EGTA,} 1 \mathrm{mM} \mathrm{MgCl}_{2}$, pH 7.4; 0.2\% TritonX-100) was used for immunohistochemistry. Epitopes were retrieved by heating in citrate buffer ( $\mathrm{pH}$ 6.0) for 10 minutes at $85^{\circ} \mathrm{C}$. Non-specific binding sites were saturated by incubation for at least 1 hour in blocking solution ( $5 \%$ BSA, 0.2\% TritonX-100). Primary antibodies used was monoclonal mouse anti-tropomyosin (1:200; T9283, Sigma). Cy3-conjugated goat anti-mouse secondary antibody (1:500; 115-165146, Jackson ImmunoR) was used to reveal primary antibody signal. Nuclei were stained with DAPI (4',6-diamidino-2-phenylindole) and slides were mounted in Vectashield (Vector, Burlingame, CA, USA). 


\subsection{Supplemental Data}

Supplementary Table 1 | Whole genome sequencing quality statistics for patient 1-2 and 2-1.

\begin{tabular}{lcc}
\hline & Coverage statistics & \\
\hline & NGS150131_dedup & NGS170003_dedup \\
\hline Total number of reads & 836441988 & 713843771 \\
Percentage reads mapped & $98.69 \%$ & $99.01 \%$ \\
GENOME_TERRITORY & 2858674662 & 2858674662 \\
MEAN_COVERAGE & 3.238 .531 & 2.783 .482 \\
SD_COVERAGE & 1.130 .788 & 1.057 .191 \\
MEDIAN_COVERAGE & 32 & 28 \\
MAD_COVERAGE & 6 & 6 \\
PCT_EXC_MAPQ & 0.052223 & 0.049995 \\
PCT_EXC_DUPE & 0.137133 & 0.133782 \\
PCT_EXC_UNPAIRED & 0.004893 & 0.005329 \\
PCT_EXC_BASEQ & 0.003871 & 0.003954 \\
PCT_EXC_OVERLAP & 0.021847 & 0.024628 \\
PCT_EXC_CAPPED & 0.01043 & 0.01012 \\
PCT_EXC_TOTAL & 0.230398 & 0.227809 \\
PCT_5X & 0.986623 & 0.985449 \\
PCT_10X & 0.980767 & 0.974341 \\
PCT_15X & 0.960780 & 0.932274 \\
PCT_20X & 0.913031 & 0.825773 \\
PCT_25X & 0.809961 & 0.641745 \\
PCT_30X & 0.628778 & 0.419392 \\
PCT_4OX & 0.215735 & 0.093175 \\
PCT_50X & 0.032871 & 0.010065 \\
PCT_60X & 0.004915 & 0.002882 \\
PCT_70X & 0.002727 & 0.002092 \\
PCT_80X & 0.002146 & 0.001379 \\
PCT_90X & 0.001759 & \\
PCT_100X & 0.001484 & \\
\hline & & \\
& &
\end{tabular}




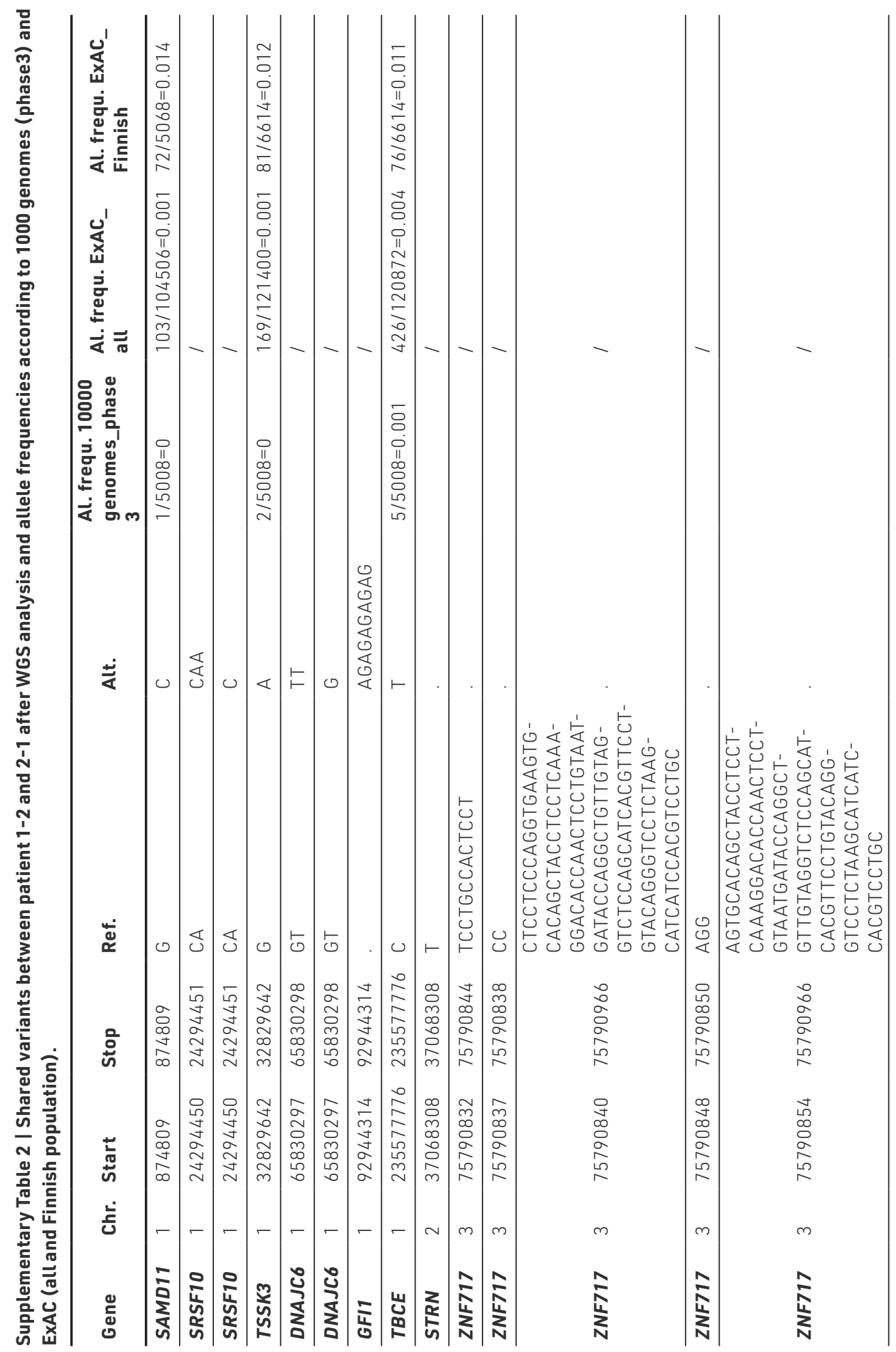




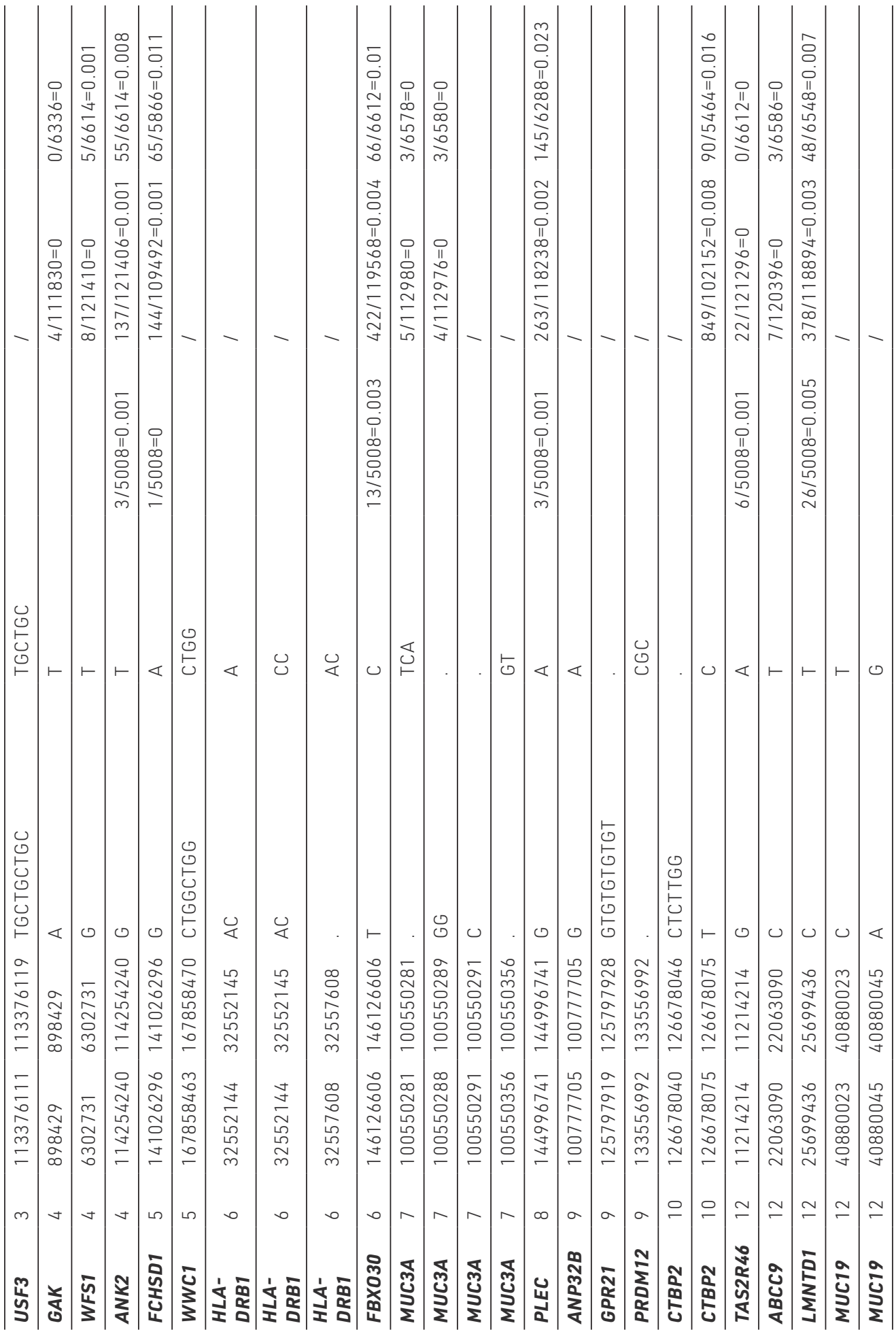




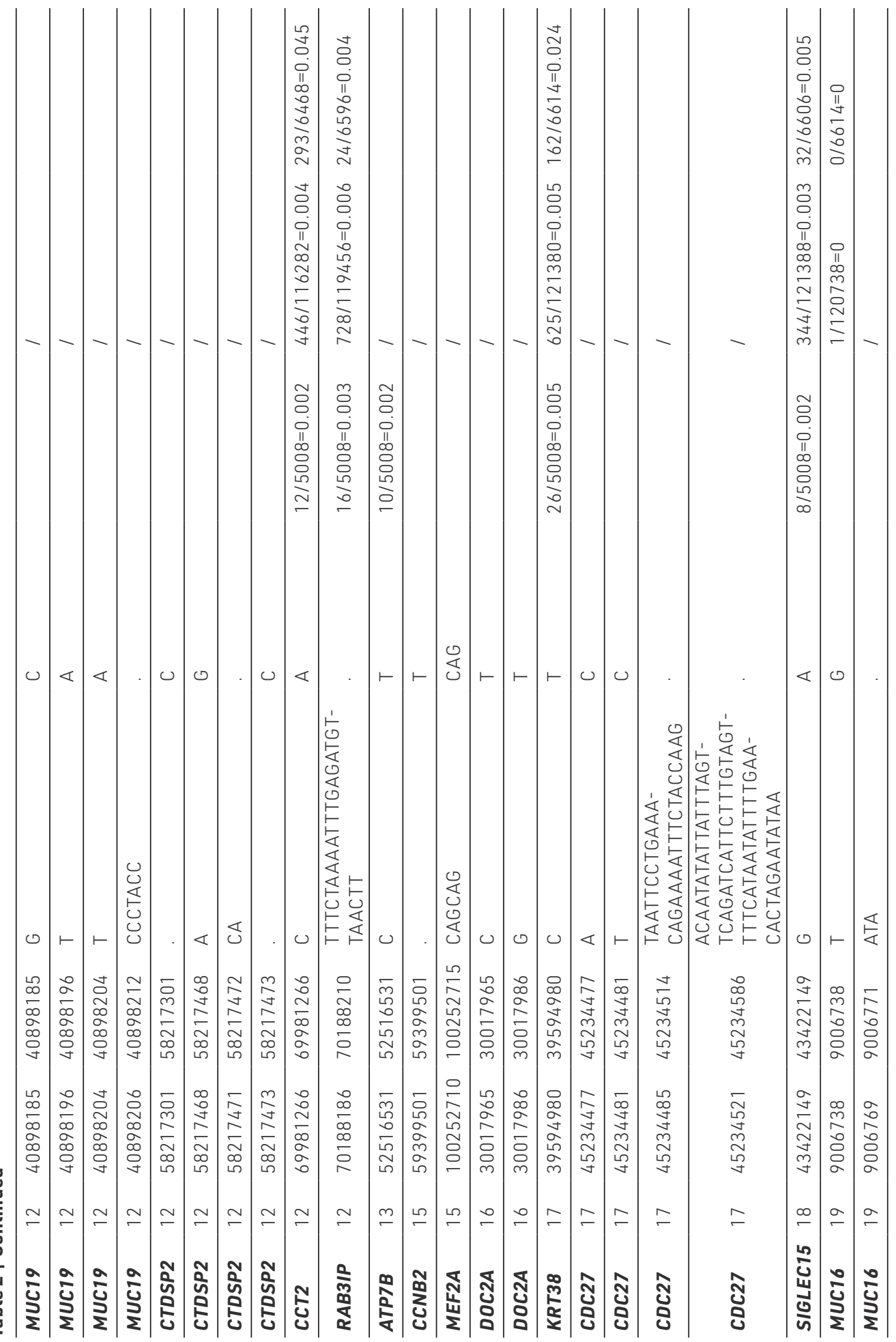




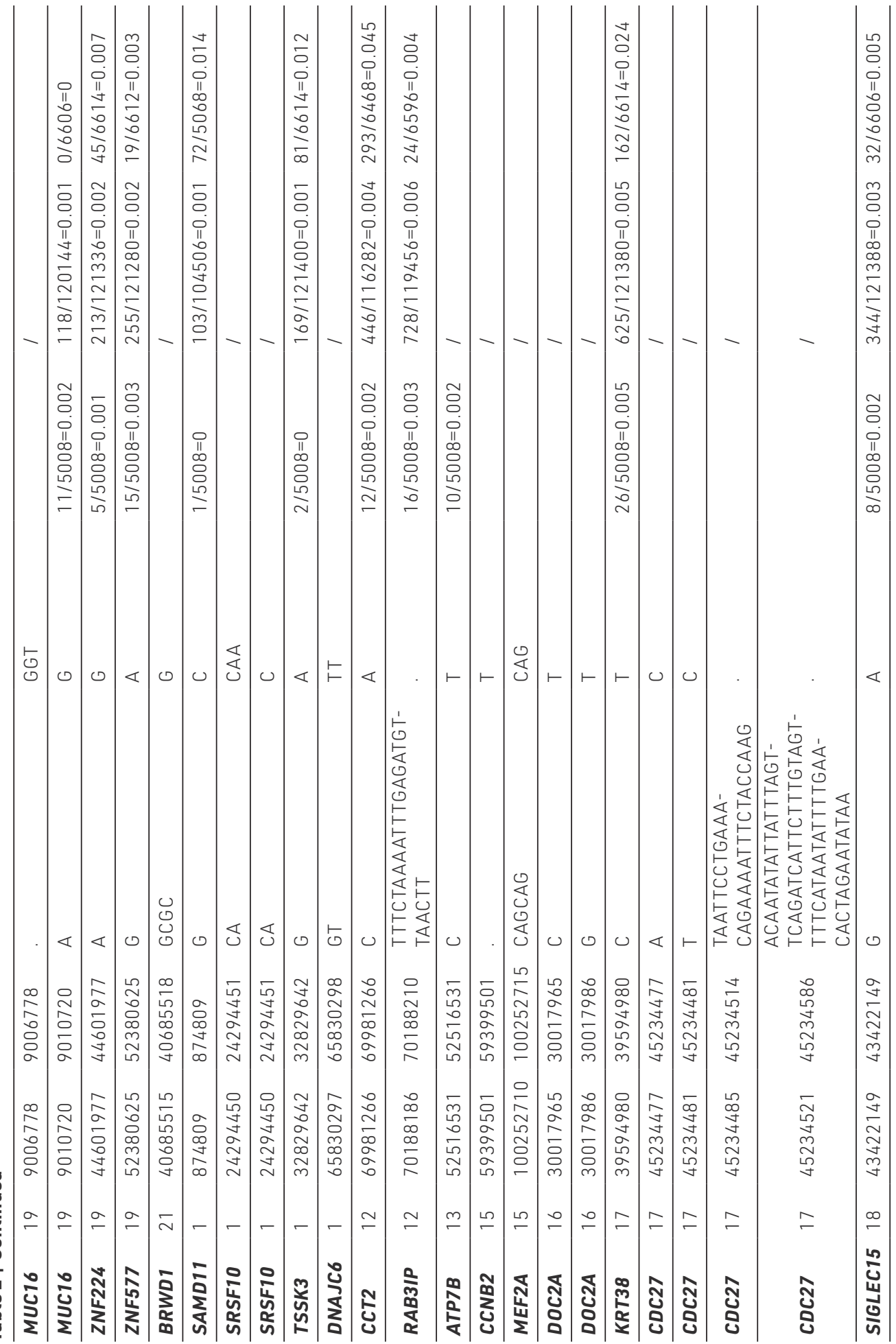




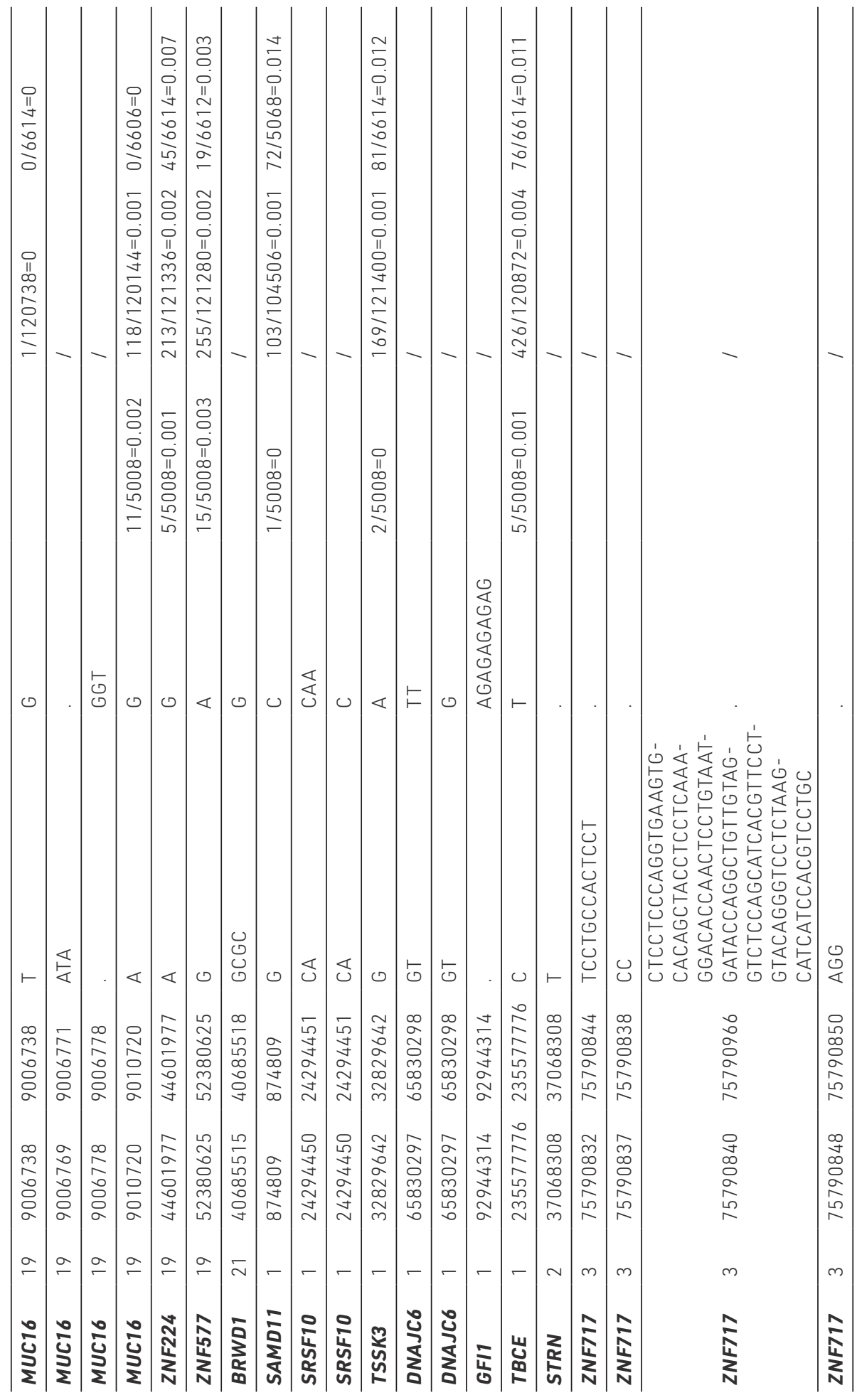




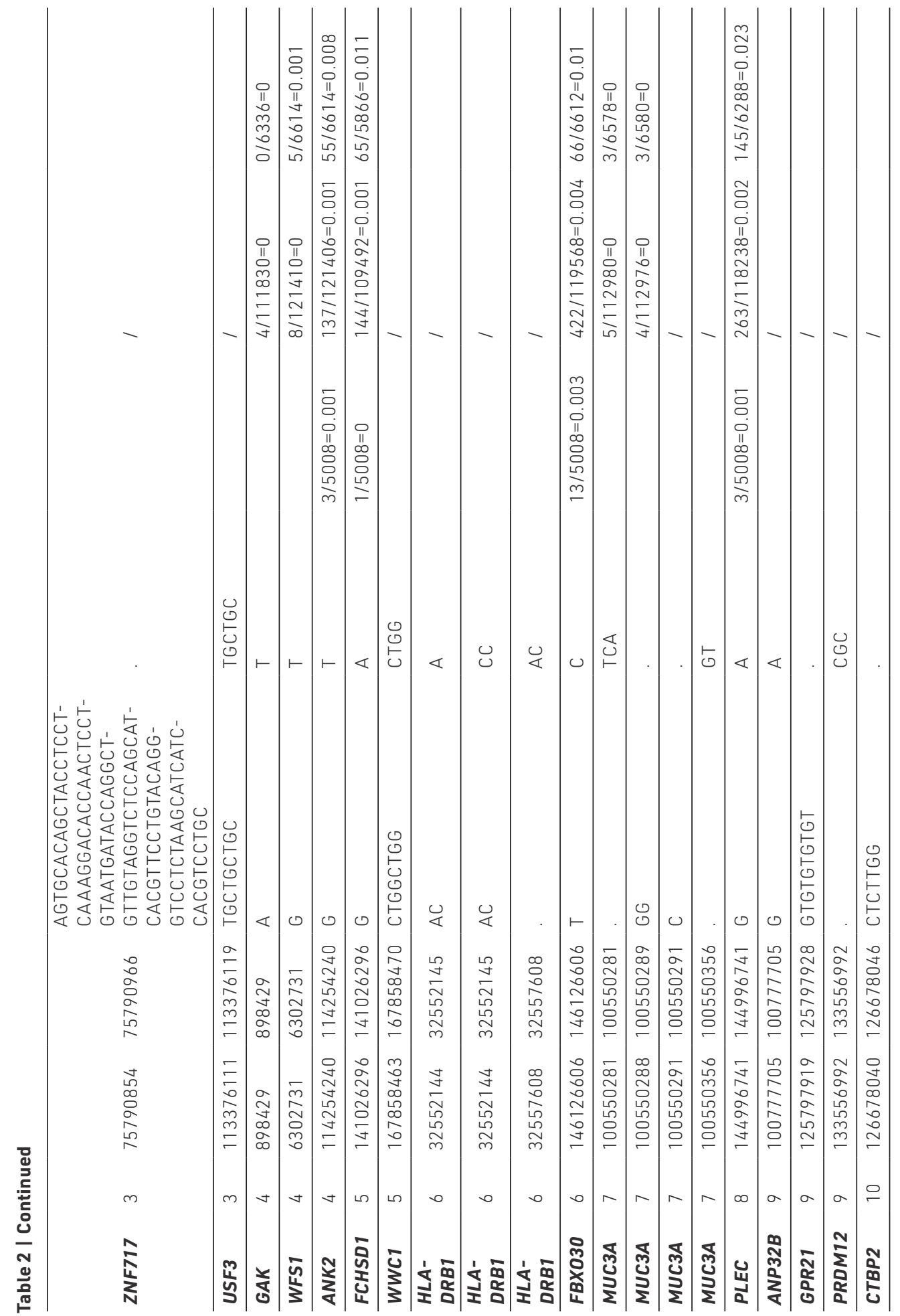




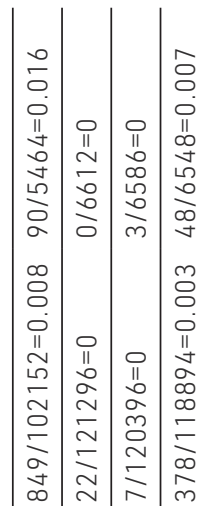

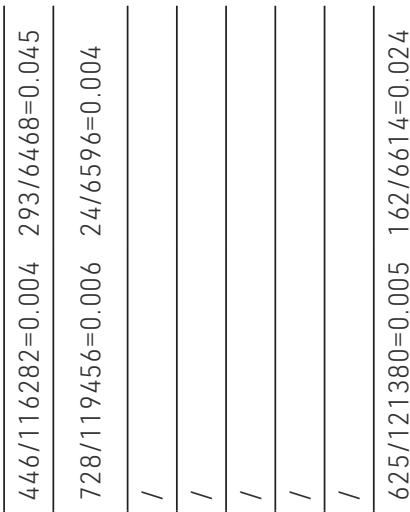

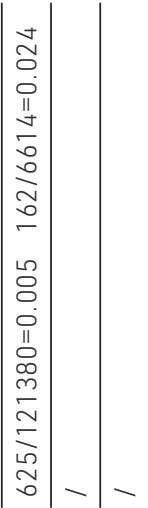

$\begin{array}{ll} & - \\ 0 \\ 0 \\ 0 \\ 11 \\ 00 \\ 0 \\ 0 \\ 0 \\ 0 \\ 0\end{array}$

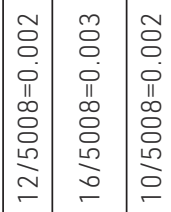

2
0
0
0
11
0
0
0
0
0
$\sim$

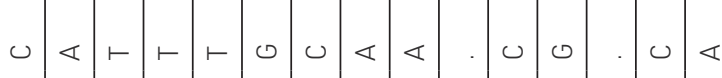

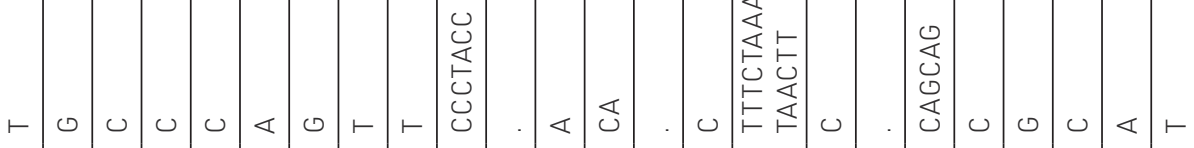

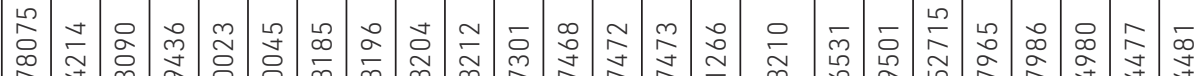

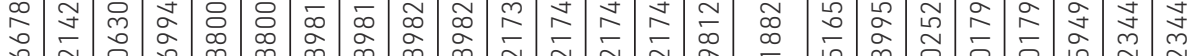

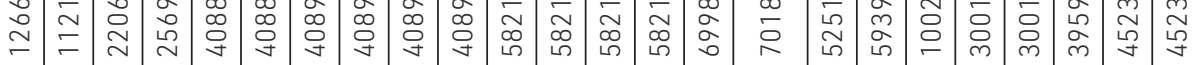

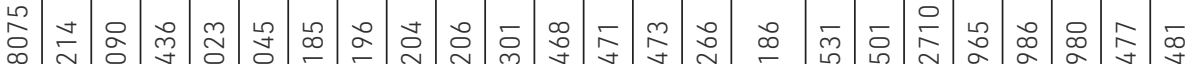

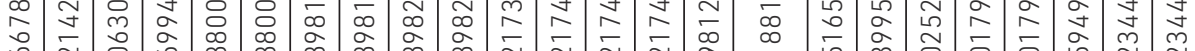

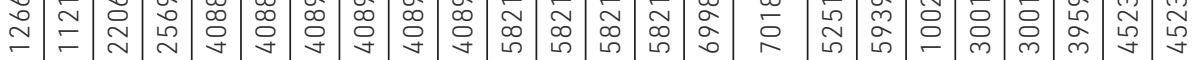

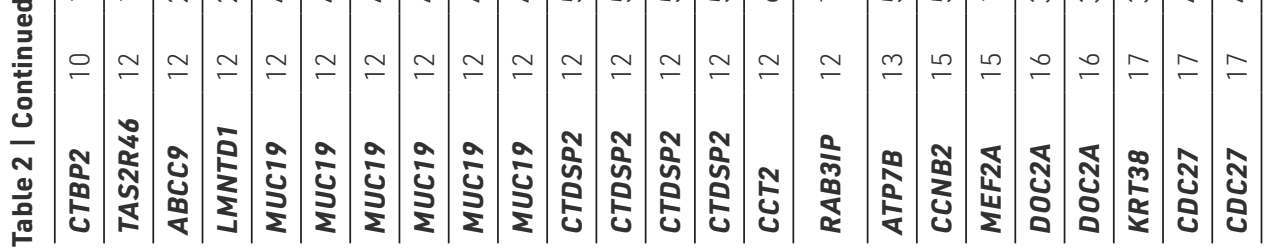




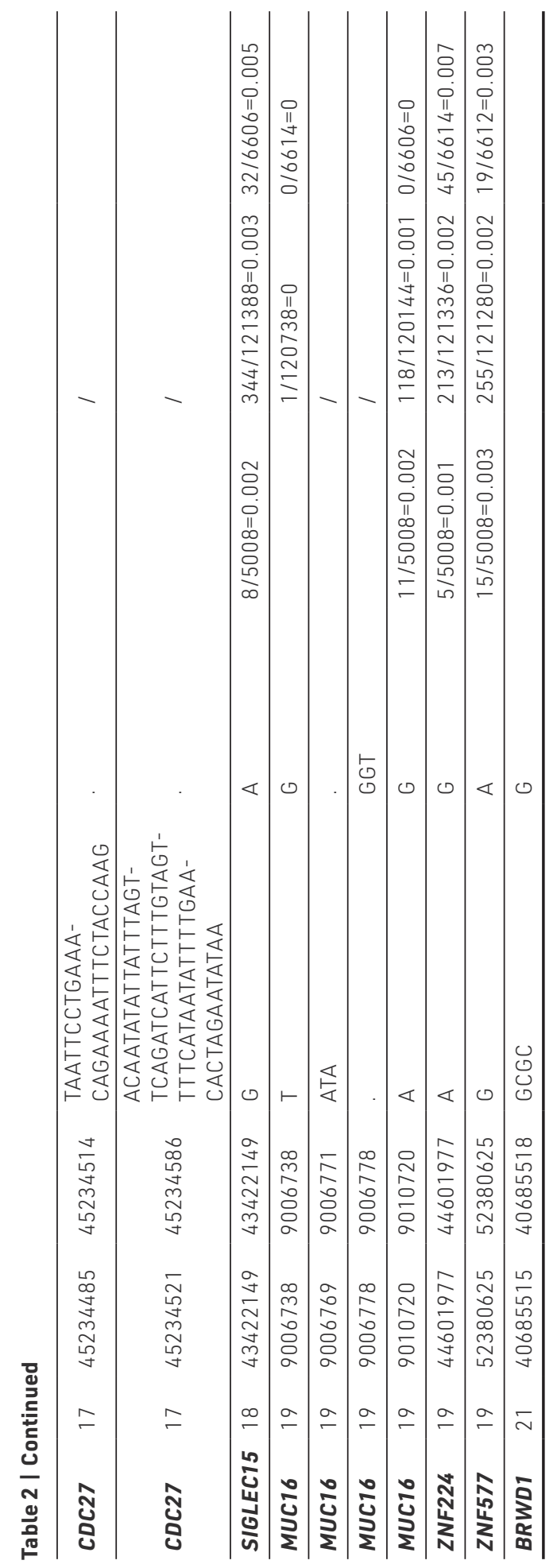




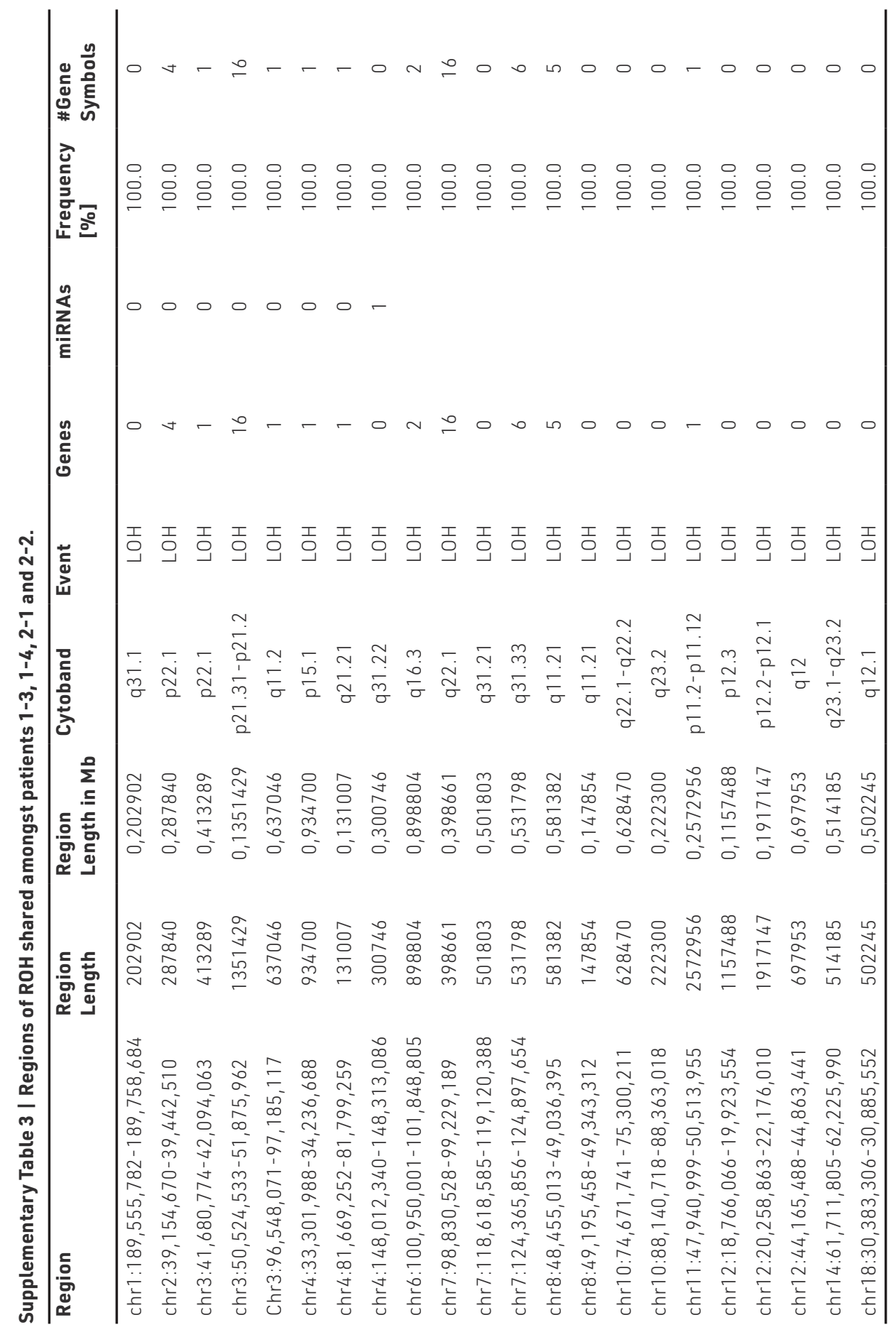


Supplementary Table 4 | ANOVA effects for multiple trial inverted screen test.

\begin{tabular}{ll}
\hline Overall effects & F Statistics \\
\hline Genotype & $F(1,16)=15.84, p=0.001$ \\
Session & $F(1,16)=8.46, p=0.010$ \\
Genotype x Session & $F(1,16)=7.13, p=0.017$ \\
Trial & $F(2,32)=2.30, \mathrm{~ns}(p=0.12)$ \\
Genotype x Trial & $F(2,32)=8.93, p=0.0008$ \\
Session x Trial & $F(2,32)=0.44, \mathrm{~ns}(p=0.61)$ \\
Genotype x Trial x Session & $F(2,32)=2.19, \mathrm{~ns}(p=0.14)$ \\
\hline Pair-wise comparisons & \\
\hline Session 1, Trial 1 & $F(1,16)=1.14, \mathrm{~ns}(p=0.30)$ \\
Session 1, Trial 2 & $F(1,16)=3.99, \mathrm{~ns}(p=0.063)$ \\
Session 1, Trial 3 & $F(1,16)=12.46, p=0.003$ \\
Session 2, Trial 1 & $F(1,16)=7.83, p=0.013$ \\
Session 2, Trial 2 & $F(1,16)=12.79, p=0.003$ \\
Session 2, Trial 3 & $F(1,16)=40.61, p<0.00005$ \\
\hline
\end{tabular}




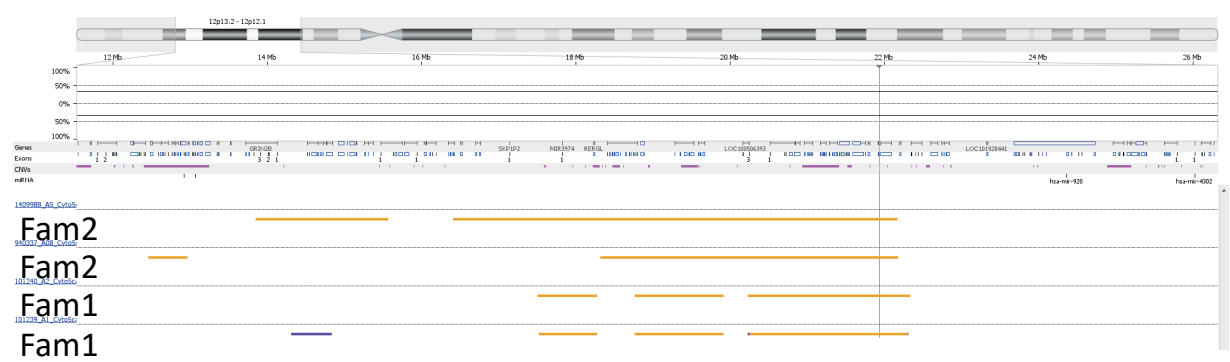

Supplementary Figure 1 | Regions of homozygosity in the ABCC9 gene regions of patients 1-3, 1-4, 2-1 and 2-2.

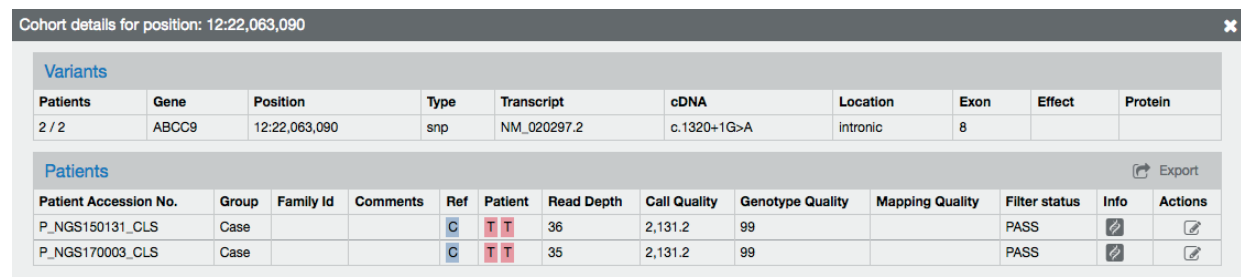

\section{Supplementary Figure 2 | Shared homozygous variant in patients 1-2 and 2-1.}

\begin{tabular}{|c|c|c|c|c|c|c|c|c|c|c|c|c|c|c|c|}
\hline & Gene & $=$ & Cases & Controls & Difference - & Position & $t$ & Ref & Alt: & Read Depth & Type & Transcript & CDNA & & Location : \\
\hline 0 & GF11 & 즐 & - & 0 & 2 & 1::22,944,314 & Q & & $A G A G \cdots G$ & 1 & insertion & NM_005263.3 & c.925-5.925-4irs CTCTCTCTCT & Q & intronic \\
\hline 0 & NTSDC2 & & 0 & 0 & 1 & $3.52,567,792-52,567,799$ & a & $C C A G \ldots G$ & c & I & deletion & - NM_001134231.1 & c.-46_-40delCGGGCTG & a & UTR5 \\
\hline a & NTSDC2 & & 0 & 0 & 1 & 3.52.567,792-52,567,799 & a & $C C A G \ldots G$ & $\operatorname{coc} \theta \cdots \bar{a}$ & i & substtution & INM_001134231.1 & c. 46 -39delCGGGCTGGins CGGGCTGCGGGCGGCGGGCGGG & a & UTR5 \\
\hline ㅁ & HLA-DRB1 & 줍 & 0 & 0 & 2 & 6:32,552,144-32,552,145 & a & AC & $\overline{\mathrm{A}}$ & i & deletion & NM_.002124.3 & c.111dela & a & exonic \\
\hline a & ADAM12 & 젭 & 0 & 0 & 1 & 10:128,019,025 & a & c & $\overline{\mathrm{A}}$ & $i$ & snp & IN NM_003474.5 & $0.142 \mathrm{Q}>\mathrm{T}$ & a & exonio \\
\hline$\square$ & $\operatorname{CCDC} 15$ & & (1) & 0 & 1 & 11:124,875,006 & a & G & $\bar{A}$ & i & snp & NM_.025004.2 & $.2309 G \times A$ & a & exonic \\
\hline a & PIK3C2G & - & (1) & 0 & 1 & 12:18,719,887 & a & c & T & $i$ & snp & 1. NM_001288772.1 & c.3907CST & $\hat{Q}$ & exonic \\
\hline 0 & $\mathrm{ABCC} 9$ & 站 & $\bullet$ & 0 & 2 & 12:22,063,090 & a & c & $\bar{T}$ & i & snp & NM_020297.2 & c. $1320+16>A$ & a & intronic \\
\hline
\end{tabular}

Supplementary Figure 3 | Shared Homo-, Hemi- and VarX-VarY variants.

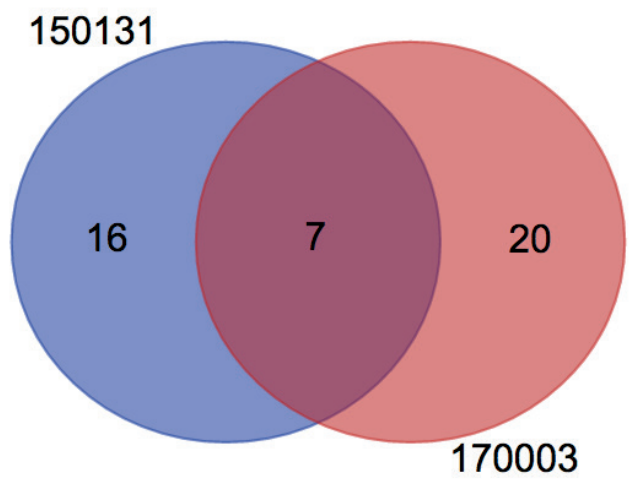

\begin{tabular}{|l|l|l|}
\hline Names & total & elements \\
\hline 150131170003 & 7 & ZNF717 CTDSP2 CDC27 MUC3A NUC16 MUC19 USH2A \\
\hline 150131 & 16 & $\begin{array}{l}\text { ZNF486 TTN SOX2 DYSF CD48 NEPF1 HMCN1 ARL 13B ZNF780A TMEM200C LRP1 } \\
\text { SERPINA1 TECTA ERVW-1 RND2 NT5DC2 }\end{array}$ \\
\hline 170003 & 20 & $\begin{array}{l}\text { ESPL1 MUC17 AHNAK2 ZNF626 ZNF577 LAMA5 OBSCN HLA-DRB1 ZNF736 OTOA } \\
\text { ZNF469 ART5 RABGAP1L COL11A2 PRRC2A PASK NLRP2 CRIPAK MUC21 CTBP2 }\end{array}$ \\
\hline
\end{tabular}

Supplementary Figure 4 I Compound heterozygous gene candidate analysis. 
A
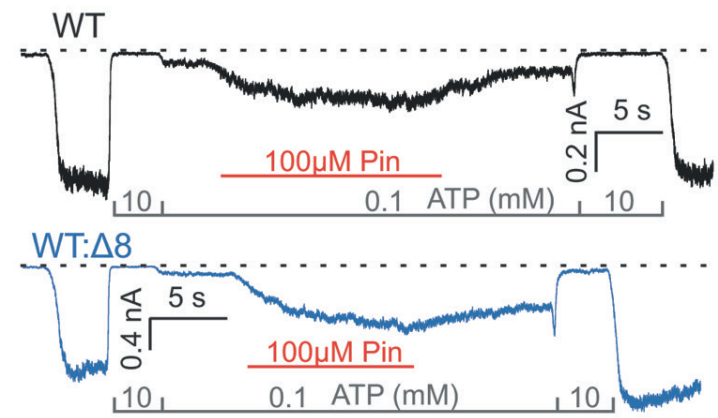

B

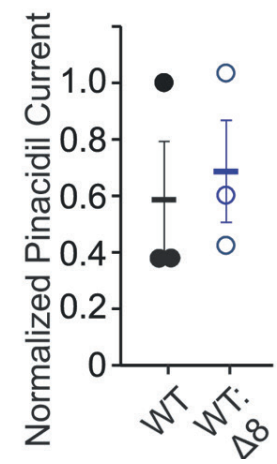

Supplementary Figure 5 | Pinacidil sensitivity is unchanged in recombinant mixed SUR2A-WT/ SUR2A $\mathbf{8}$ channels. (A) Example inside-out patch clamp recordings from Cosm6 cells transfected with Kir6.2 and SUR2A-WT or a 1:1 mix of SUR2A-WT and SUR2A $\triangle 8$. Patches were voltage clamped at $-50 \mathrm{mV}$ and administered ATP (in the presence of $0.5 \mathrm{mM}$ free $\mathrm{Mg}^{2+}$ ) and pinacidil as indicated. (B) Summary of the extent of pinacidil activation. Currents in the presence of pinacidil were normalized to currents in the absence of nucleotides.

A
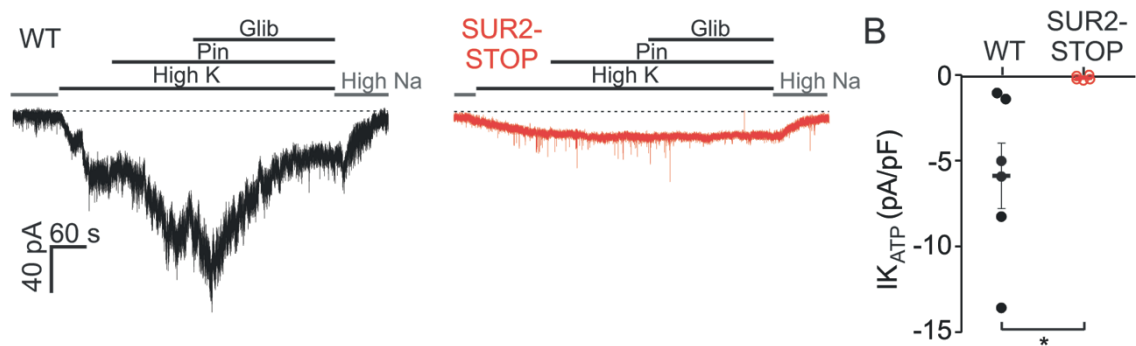

C
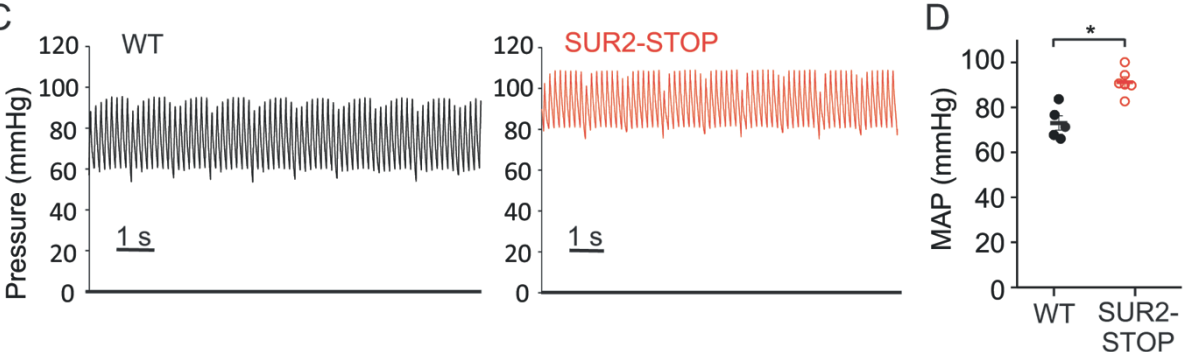

Supplementary Figure 6 I Loss of $\mathrm{K}_{\mathrm{ATP}}$ channel function in aortic smooth muscle, and increased blood pressures in SUR2-STOP mice. (A) Example whole cell patch clamp recordings from acutely isolated aortic smooth muscle cells. Cells were voltage clamped at $-70 \mathrm{mV}$ and KATP channels were activated in high- $\mathrm{K}^{+}$extracellular solution by administration of pinacidil $(100 \mu \mathrm{M})$ as indicated. (B) $I_{\text {KATP }}$ was determined as the glibenclamide-sensitive, pinacidil-activated current. * denotes $p<0.05$ according to students T-test. (C) Example recordings of arterial blood pressures in WT and SUR2STOP mice. (D) Summary of mean arterial pressure (MAP). * denotes $p<0.05$ according to students T-test. 
A

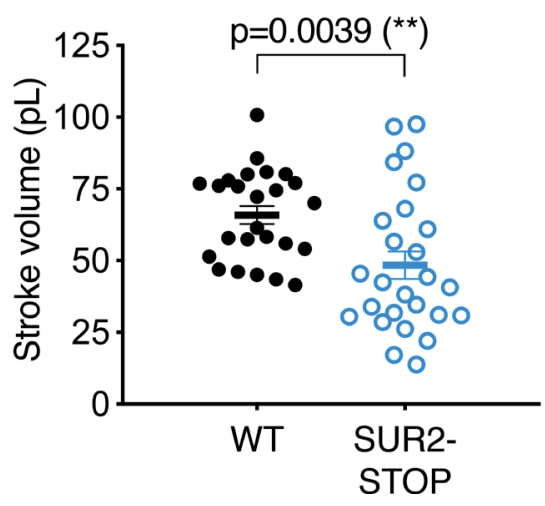

B

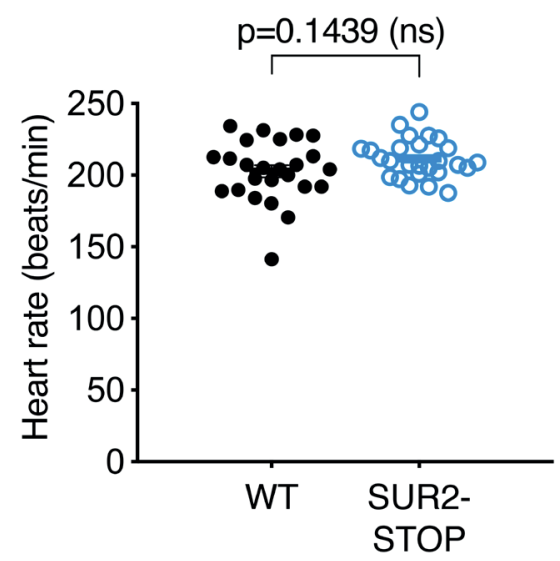

Supplementary Figure 7 | SUR2-STOP mutation induces systolic dysfunction in zebrafish larvae. (A) Quantification of stroke volume and (B) heart beat applying individual characteristic confocal sections from a time series of the embryonic cardiac cycle at $5 \mathrm{dpf}$. For all graphs, significance was determined by two-tailed unpaired Student's t test or Mann-Whitney two-tailed $U$ test. Asterisks indicate statistical significance $(* p \leq 0.05 ; * * p \leq 0.01 ; * * * p \leq 0.001 ; * * * * p \leq 0.0001)$. The black horizontal bar indicates the mean value for each condition. Data shown as mean \pm SEM. Sample size, WT, $n=20$; SUR2-STOP, $n=20$ in. All embryos analysed originated from group matings of adult zebrafish. 
A
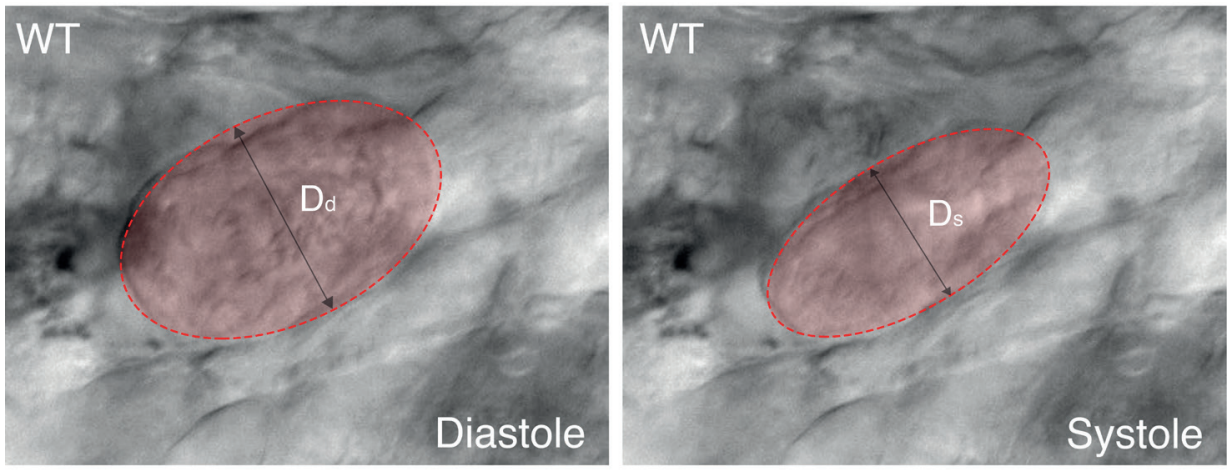

B
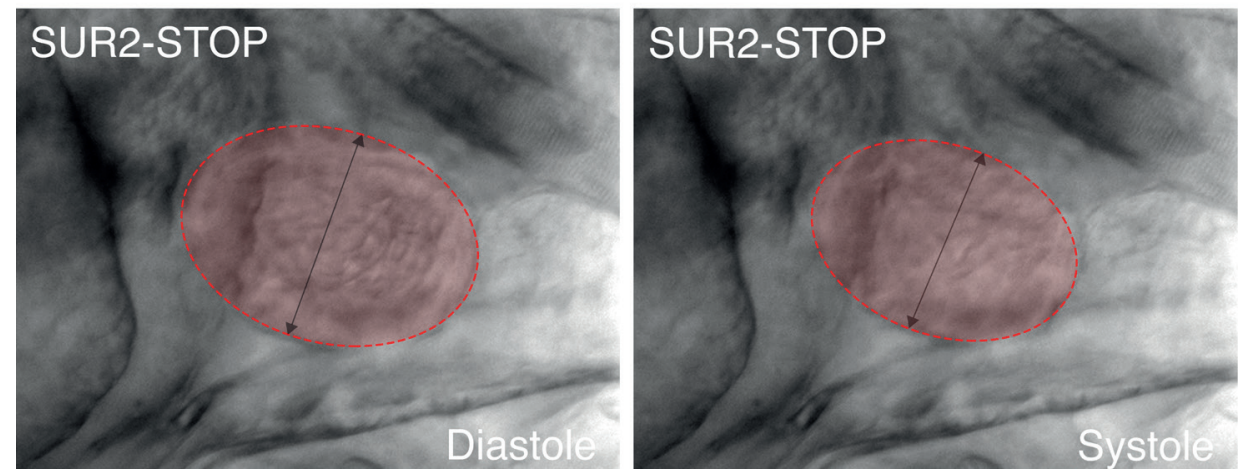

Supplementary Figure 8 | Assessment of ventricular contractility via high-speed video microscopy in zebrafish larvae. Representative images illustrating the morphology of $5 \mathrm{dpf}$ wild-type (A) and SUR2-STOP (B) mutant hearts at diastole and systole as seen from a dorsal view. The ventricular area of the heart is highlighted, with the ventricular diameter $D_{d}$ and $D_{s}$ indicated by a grey arrow. Fractional shortening (FS), a measure of ventricular contractility, was calculated from ventricular diameters at diastole and systole $\left(D_{d}\right.$ and $\left.D_{s}\right)$. 
A

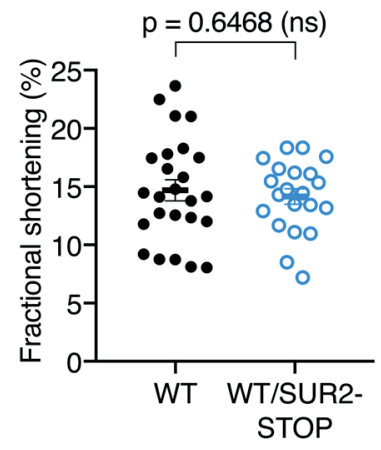

D

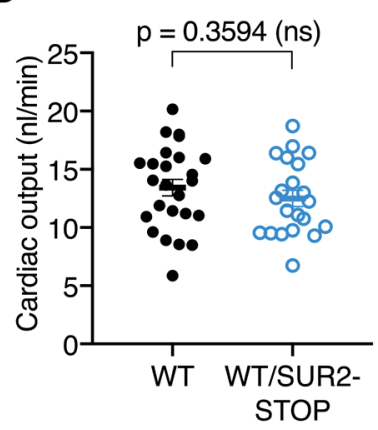

B

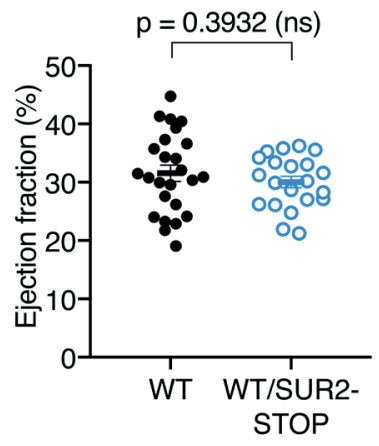

E

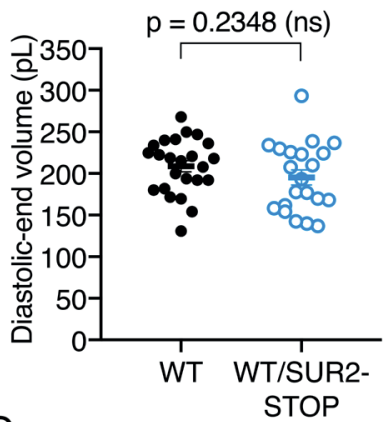

G

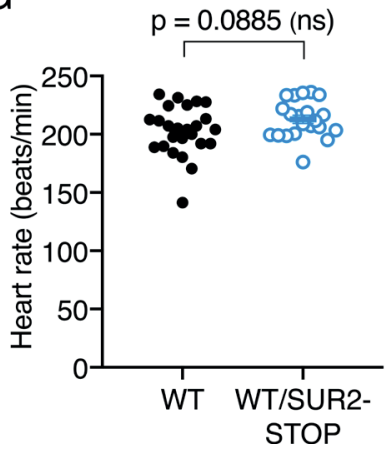

C

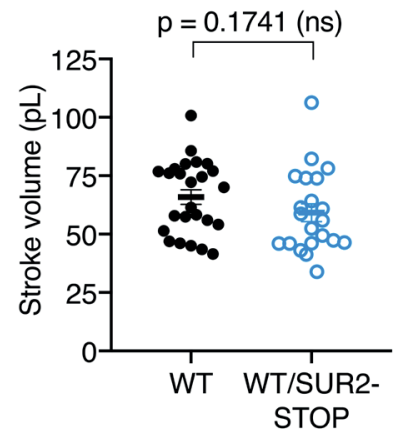

$\mathrm{F}$

$\mathrm{p}=0.3205(\mathrm{~ns})$

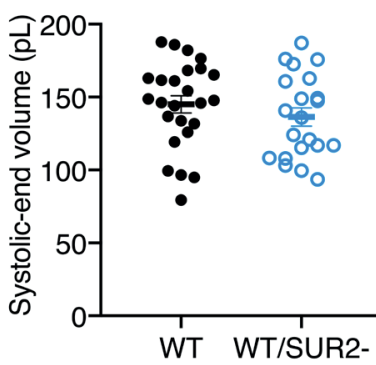

STOP

Supplementary Figure 9 | Zebrafish larvae heterozygous for SUR2-STOP mutation lack cardiac abnormalities. (A)-(G) Quantification of cardiac function in $5 \mathrm{dpf}$ zebrafish larvae heterozygous for SUR2-STOP mutation using individual characteristic confocal sections from a time series of the embryonic cardiac cycle. WT controls are the same as in Fig. 7B-E and Fig. S5A. For all graphs, significance was determined by two-tailed unpaired Student's $t$ test or Mann-Whitney two-tailed $U$ test. The black horizontal bar indicates the mean value for each condition. Data shown as mean \pm SEM. Sample size, WT, $n=25 ;$ WT/SUR2-STOP, $n=21$ in A-G. All embryos analysed originated from group matings of adult zebrafish. 


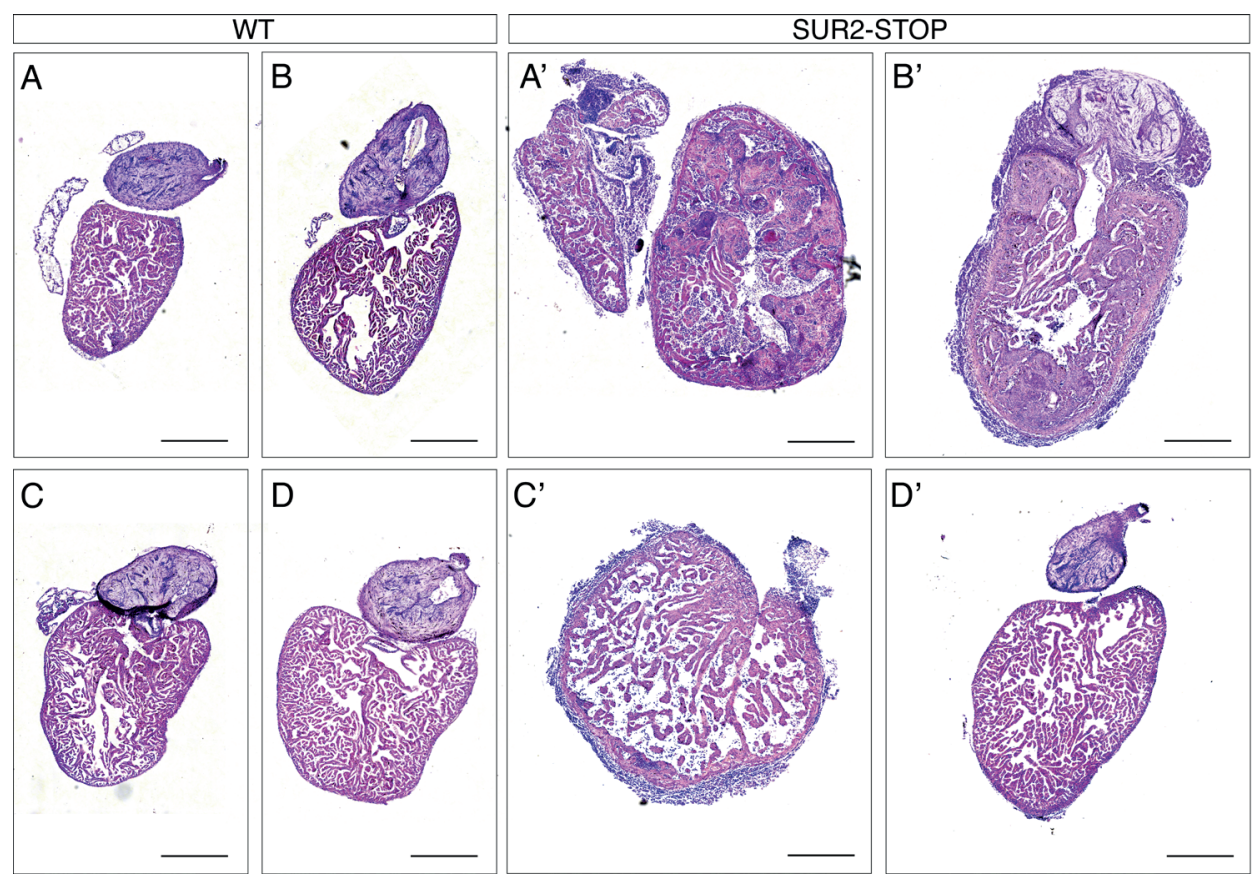

Supplementary Figure 10 | SUR2-STOP mutation induces enlarged heart size in adult zebrafish. Heart histology of WT siblings (A-D) adult SUR2-STOP mutants (A'-D') after H\&E staining. Depiction of four WT and four SUR2-STOP hearts. For assessment of ventricular chamber size, tissue sections showing the largest ventricular area were selected. Sample size, WT, $n=6$, SUR2-STOP, $n=6$. Scale bar, $500 \mathrm{~mm}$. 


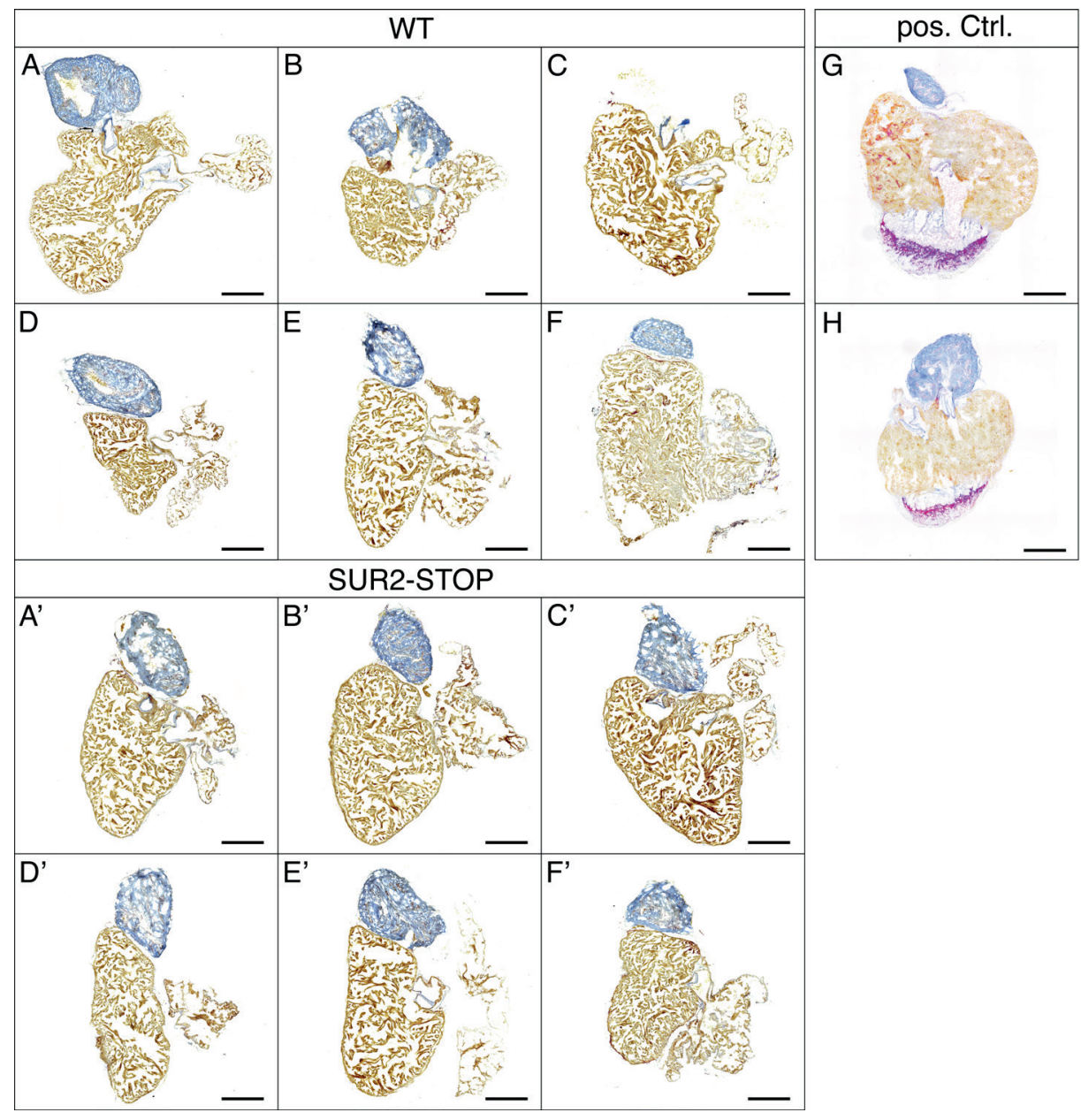

Supplementary Figure 11 | No fibrosis in hearts of adult heart of SUR2-STOP.

Heart histology of adult Wildtype (A-F) and SUR2-STOP mutants ( $\left.\mathbf{A}^{\prime}-\mathbf{F}^{\prime}\right)$ after Acid Fuchsin Orange G (AFOG) staining, which labels myocardium orange, collagen blue and fibrin red. Depiction of 6 WT and 6 SUR2-STOP hearts. Notably, we selected images for presence of atrium and ventricle to show staining of both chambers; not for the largest ventricular area. (G-H) Positive control showing two adult zebrafish hearts 7 days after cryoinjury presenting with red staining to show fibrosis around the apex. Sample size, WT, $n=6$, SUR2-STOP, $n=6$. Scale bar, $500 \mu \mathrm{m}$. 

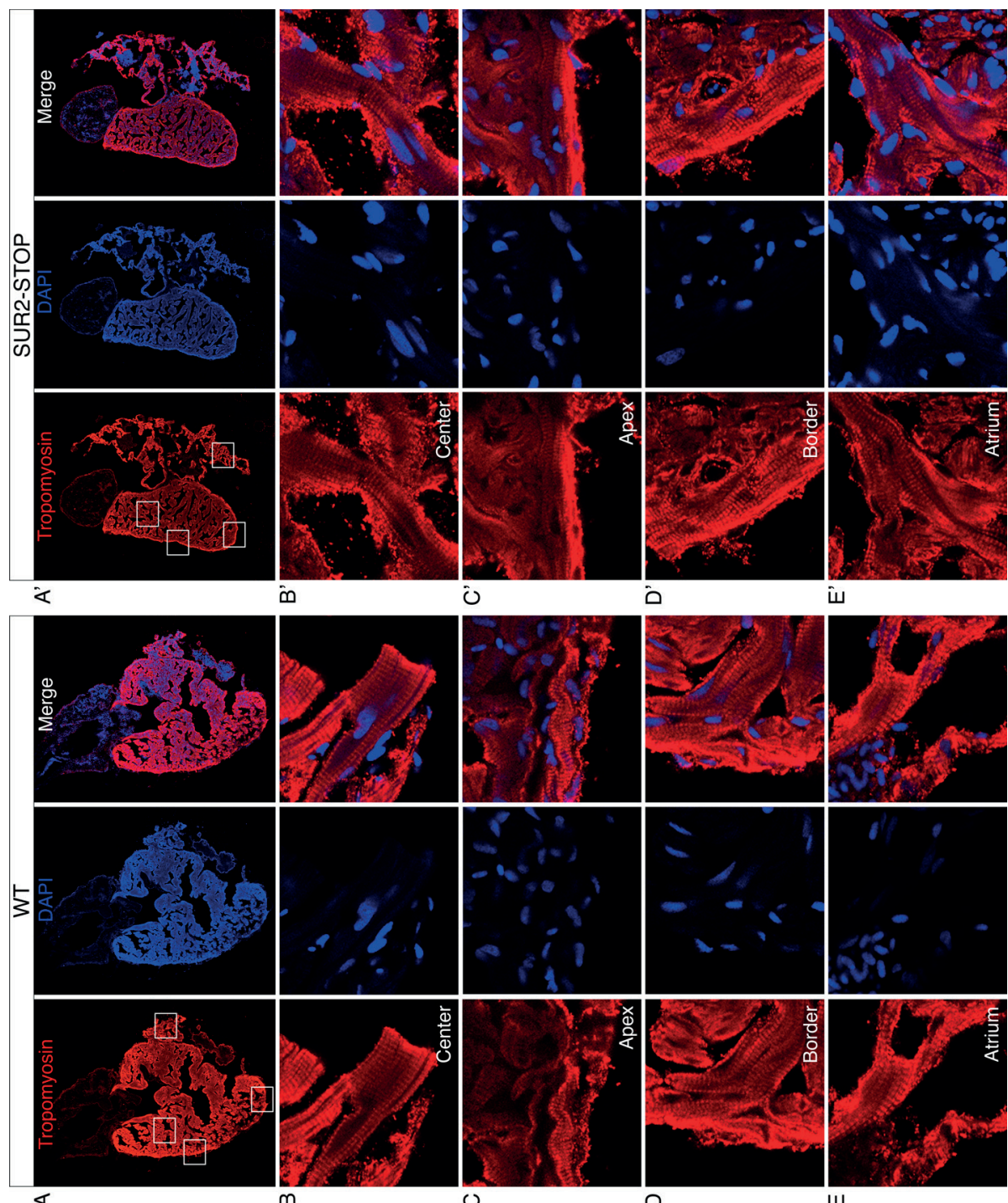

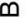

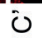

อ

ш
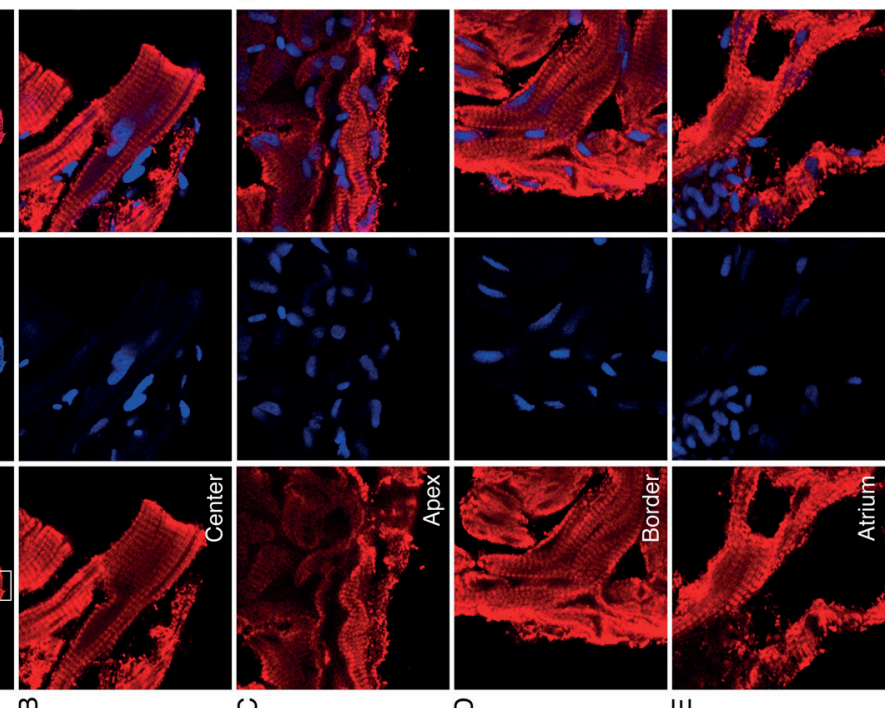

0

ш

Supplementary Figure 12 I SUR2-STOP fish show no abnormalities in myofiber structure. Immunohistochemistry on sagittal sections of adult hearts of WT (A) and SUR2-STOP fish ( $\left.\mathbf{A}^{\prime}\right)$ with antibody against to tropomyosin (red); nuclei are stained with DAPI (blue). To assess structure of myofibers four different areas were assessed: center of ventricle, apex, ventricular border and atrium. Areas are indicated in boxes. (B-E) and (B'-E') Higher magnification views of the boxed areas in $A$ and $A^{\prime}$. Arrowheads indicate stained tropomyosin. Sample size, WT, n=6, SUR2-STOP, $\mathrm{n}=6$. Scale bars, $500 \mu \mathrm{m}$ in $\mathbf{A}$ and $\mathbf{A}^{\prime} ; 100 \mu \mathrm{m}$ in $\mathbf{B}-\mathbf{E}$ and $\mathbf{B}^{\prime}-\mathbf{E}$ '. 
-12
4
4
4

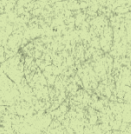

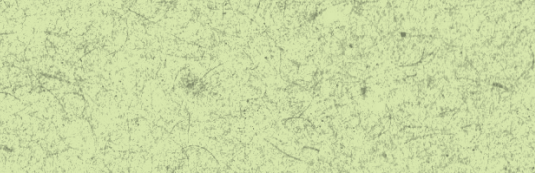

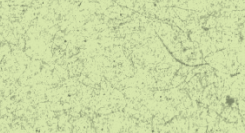

तr.

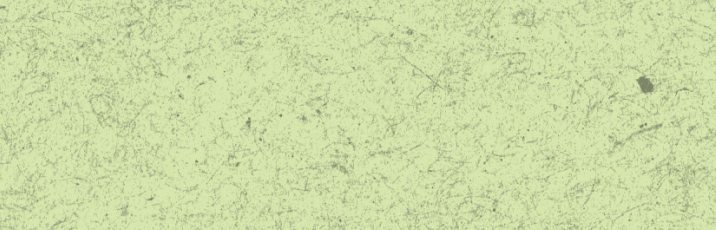

4

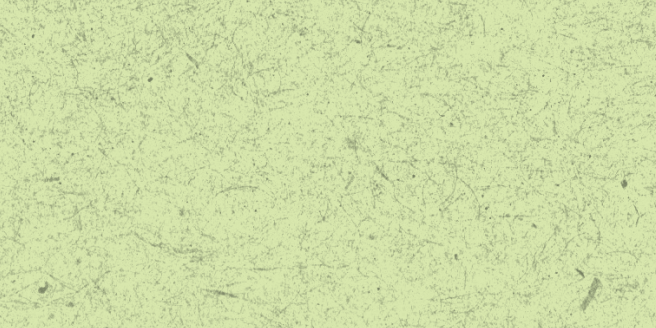




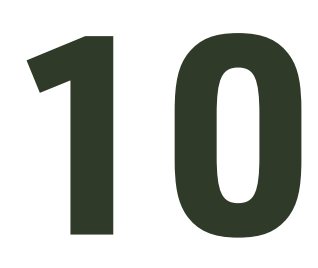

\section{General Discussion: A multi-disciplinary approach to treat rare genetic diseases}

Adapted and expanded from:

Drug repurposing for rare diseases

Helen I. Roessler, Nine V.A.M. Knoers, Mieke M. van Haelst* and Gijs van Haaften*

*These authors contributed equally to this work.

Trends Pharmacol Sci (2021) Epub ahead of print DOI: $10.1016 /$ j.tips.2021.01.003 
The research and patient communities in the field of rare diseases (RDs) are united in the opinion that RDs are in need of novel and optimized therapies. Adequately, the main aim of this thesis was to illustrate that RD research does not stop with diagnosis but should continue until a treatment for these patients has been identified. The path from gene identification to treatment is long and multifaceted requiring the collaboration of various research branches and expertise.

In this chapter, I discuss the results of the studies presented in this thesis and make the case for the establishment of a multi-disciplinary team to accurately diagnose and treat rare genetic diseases.

\subsection{Novel tools for clinical phenotyping of rare disorders}

Although genotype-based clinical diagnosis for genetic disorders has improved drastically with the advances of clinical exome sequencing technologies and accompanying analytical methods, establishing the right diagnosis remains a challenge even for known rare genetic conditions. Thus, next to traditional ways of phenotyping patients and assessing the natural history of a RD, as illustrated in Chapters $\mathbf{3}$ and $\mathbf{4}$, significant effort has been put into computeraided clinical diagnostic systems based on known information of patient phenotypes (J. Chen et al., 2019). For the patient, this promises a shorter clinical path to diagnosis, as well as the possibility to monitor treatment in a non-invasive manner and/or assess risk progressively. As a result, healthcare costs might get reduced. For clinical geneticists and researchers in the field of genetics, such tools facilitate identification of new syndromes applying precise and comprehensive characterization of phenotypic features, improving the detection of novel patterns and similarities. In Chapter 5, we applied 3D facial imaging in order to elucidate distinctive facial features of patients with Cantú syndrome (CS). This study confirmed that 3D-based technology can be applied in clinics to successfully identify CS patients based on their facial morphology avoiding unnecessary misdiagnoses. Despite the many benefits computational phenotyping has to offer, it also raises various ethical and legal issues that should be taken into account. For instance, one has to deal with getting valid consent to use and protect data confidentiality, and addressing threats to privacy, data protection, and copyright. 
Both patients and the RD research communities are also placing increased attention on the establishment and application of patient registries. A significant increase in the amount of registries and requests by patients to take part in such is expected. Information about patients' clinical, demographic, and medical family histories are collected, stored and readily available for retrieval in a standardized and secure way. The data can be applied in the development of biomedical research hypotheses for epidemiology or public health surveillance studies, the recruitment of patients for clinical studies, and the increase of knowledge of RDs. Currently, a wide range of patient registries already exists and further are developed for individual RDs (Chapter 6). In this process, accuracy and quality of information entered by patients, family members and doctors are key and heavily influence the success or failure of such registries.

\subsection{Genome editing technologies}

In recent years, researchers are able to rapidly and efficiently introduce sequence-specific modifications into the genomes of a vast variety of cell types and model organisms due to the establishment of highly versatile genomeediting technologies. The core technologies most commonly applied to facilitate genome editing nowadays are CRISPR/Cas9, TALENs, ZFNs, and homing endonucleases or meganucleases. Much of what we know about gene function in model organisms and their application to humans has come from gene knock-outs - the ability to recapitulate similar phenotypes in model systems upon gene inactivation in animal models (Liu, Petree, Requena, Varshney, \& Varshney, 2019). In Chapter 9, we applied the CRISPR/Cas9 system to knockout the homologues of $A B C C 9$ in both zebrafish and mice resulting in two adequate models for a novel rare genetic condition, ABCC9-related Intellectual disability Myopathy Syndrome (AIMS). This example shows that techniques now considered as standard are still relevant and applied frequently. However, genome editing techniques to validate and further evaluate candidate genes for human disorders are constantly evolving. For instance, in Chapter 7, we developed a novel and effective CRISPR/Cas9-based approach to introduce small patient-specific nucleotide changes into the genome of the zebrafish. Hence, in the following paragraph, I discuss further novel CRISPR/Cas9-mediated genetic tools developed to facilitate human genetic disease research with particular emphasis on applications in the zebrafish. 


\subsubsection{Novel CRISPR/Cas9-based genome editing approaches}

Due to the establishment of RNA-guided CRISPR/Cas9-mediated targeted mutagenesis as a powerful tool, researchers are now able to manipulate the genome to develop novel disease models and elucidate the pathophysiological mechanisms of human disorders. CRISPR-based techniques are being applied for the generation of both knock-out and knock-in alleles (Chapter $\mathbf{7}$ and 9), for transcriptional modulation (Long et al., 2015), epigenome editing (Hilton et al., 2015), and lineage tracing (Spanjaard et al., 2018).

As many genetic diseases are caused by point mutations resulting in amino acid substitutions, multiple approaches have been developed to establish targeted knock-in mutants (Prykhozhij et al., 2018; Y. Zhang, Zhang, \& Ge, 2018). As described in Chapter 7, knock-in is typically achieved via homologous recombination (HR). However, its success rate is still relatively low as nonhomologous end-joining tends to get favored over HR. An alternative approach to introduce point mutations into the genome of zebrafish is CRISPR-mediated base editing resulting in the conversion of G-C base pairs to A-T base pairs or vice versa without promoting a double-strand break (Gaudelli et al., 2017). CRISPR base editors use catalytically inactive Cas9 (dCas9) fused to a cytidine deaminase enzyme resulting in the conversion of cytosine bases to uridine, which are eventually read as thymine during replication (Komor, Kim, Packer, Zuris, \& Liu, 2016). Over time, base editors have been optimized in various ways (Koblan et al., 2018; Rees et al., 2017; Zafra et al., 2018).

Another function of the CRISPR/Cas9 system is the modulation of transcription and the manipulation of the epigenome. To modulate transcription, dCas 9 is fused with transcriptional activators (CRISPRa) or repressors (CRSIPRi). Both have been applied successfully in zebrafish (Long et al., 2015). Moreover, dCas9 has also been fused to various epigenetic effectors (Hilton et al., 2015).

Whilst CRISPR/Cas9 is an effective and straightforward tool for genetic manipulation, it raises multiple concerns regarding its specificity. It has been shown that the CRISPR complex can bind and manipulate unintended targets, so called off-targets, resulting in, amongst others, large deletions (Adikusuma et al., 2018). Notably, in zebrafish genetics such off-target effects can relatively easily be disposed by outcrossing. Studies dealing with off-target effects in zebrafish have observed rather low percentages with one study identifying $1 / 25$ off-target sites in the germline and another showing off-target mutation rates from 1.1 to $2.5 \%$ (Hruscha et al., 2013; Varshney et al., 2015). Recently, multiple Cas9 variants such as Cas9-HF1, eSpCas9, evoCas9, HypaCas9, and others have 
been developed to enhance specificity of the enzyme and as a result decrease off-target effects (Jamal et al., 2018; Liu et al., 2019). Another reason to use novel Cas9 enzymes is to expand the targeting coverage. Researchers have identified additional, naturally occurring CRISPR nucleases with different PAM requirements. In order to recognize other PAM sequences, Cas9 was engineered adequately. This allows to target loci that are lacking a PAM sequence matching to the typically used CRISPR nuclease (Kleinstiver et al., 2015; Liu et al., 2019).

\subsection{Functional tests and the combination of model system}

Model systems research has long contributed to basic biological knowledge and its application to human medicine. Experimental approaches on patient material, cell lines or model organisms reveal abnormal effects of genetic variation and eventually may establish correlations between the patient's genetic variant and the associated disorder (genotype-phenotype correlations). Often a combination of multiple approaches leads to the broadest and most detailed understanding of mutational effects on molecular mechanisms and phenotypic features. This is especially useful when unraveling the pathogenicity of rare genetic disorders involving complex multi-organ system phenotypes that cannot be elucidated with merely one model system. The two examples of RDs described in this thesis, CS (Chapter $\mathbf{7}$ and $\mathbf{8}$ ) and AIMS (Chapter 9), required the application of a range of functional tests and model organisms in order to validate the disease-causing gene variants as well as investigate the underlying pathophysiology and therapeutic options.

In Chapter 9, we first performed cDNA analysis on fibroblasts from patients and healthy controls revealing that the detected variant causes an in-frame deletion of exon 8 of ABCC9. The effect of this deletion on protein function was then investigated using cells transfection experiments, and in vivo modeling of the variant was performed in both mouse and zebrafish which showed similar features as the observed in the patients. This example very well illustrates that researchers should not limit themselves to merely one experimental approach.

As CS has been identified as a clinical entity $\sim 40$ years ago (Cantu, Garcia-Cruz, et al., 1982) and its underlying genetic cause is established since 2012 (Brownstein et al., 2013; Cooper et al., 2014; Harakalova et al., 2012; van Bon et al., 2012), a larger body of research has been performed on this disorder compared to AIMS. Therefore, the following paragraph is entirely dedicated to functional work in the field of CS, showing how all models taken together drastically improved our knowledge of the 
syndrome. Lastly, advantages and limitations of each model system in the context of CS are discussed.

\subsubsection{Functional models for CS-associated cardiovascular features}

An established model for the molecular consequences of CS gene variants is that the missense mutations in ABCC 9 and KCNJ8 lead to enhanced $\mathrm{K}_{\text {ATP }}$ channel activity. This suggestion was first based on an unexpected phenotypic overlap between individuals with CS and patients who had been treated with the drug minoxidil, an antihypertensive vasodilator with potent side effects of hirsutism and pericardial effusions which limited its use (Grange et al., 2006; Houston, McChesney, \& Chatterjee, 1981; Messenger \& Rundegren, 2004; Reichgott, 1981). Minoxidil directly binds to the SUR2 subunit and functions as $K_{\text {ATP }}$ channel agonist (Schwanstecher et al., 1998) Hence, the described similarities between CS phenotypic features and the side effects of minoxidil treatment suggested enhanced channel opening as a result of CS variants. Later on, this hypothesis was confirmed applying functional work in cell-based models, mice and zebrafish. In Chapter $\mathbf{7}$ and $\mathbf{8}$, we describe findings from CS zebrafish models in detail. Hence, the following paragraphs will focus on the contribution of other models to the current level of knowledge of the syndrome.

\section{Cell-based in vitro models for CS}

In order to investigate the suggestion that CS-associated features result from increased $\mathrm{K}_{\text {ATP }}$ channel activity, various ABCC9 and KCNJ8 variants were engineered in the equivalent position in rat SUR2A and each was co-expressed with mouse Kir6.2. Applying macroscopic rubidium $((86) \mathrm{Rb}(+))$ efflux assays and inside-out patch-clamp electrophysiology to measure channel sensitivity to ATP inhibition and to MgADP activation, showed that (i) CS-related variants reveal a gain-of-function (GoF) effect and (ii) that increased $K_{\text {ATP }}$ channel activity can arise by multiple molecular mechanisms such as augmented $\mathrm{Mg}$ nucleotide activation and increased intrinsic stability of the open state due to decreased sensitivity to ATP inhibition (Cooper et al., 2015; Harakalova et al., 2012; McClenaghan et al., 2017). In Chapter 8, we confirm the presence of various molecular mechanisms in zebrafish.

\section{Mouse models for CS}

Mouse models of CS have been developed in which disease-causing mutations were knocked-in the endogenous ABCC9 and KCNJ8 loci using CRISPR/Cas9 genome engineering. CS mice recapitulate many of the key cardiovascular features of CS such as increased vascular dilation and compliance, low blood pressure, cardiac hypertrophy, and increased cardiac output (Huang et al., 2018). 
Interestingly, the most severe CS features were found in Kir6.1[V65M] mice which lack $\mathrm{K}_{\text {ATP }}$ GoF in ventricular cardiomyocytes, suggesting an important role for a none-cardiac muscle origin of the multi-organ pathology of CS. Increased $\mathrm{K}_{\text {ATP }}$ function in vascular smooth muscle (VSM) cells is suggested to result in a drastic decrease of vascular resistance and reduced blood pressure in wildtype (WT) animals (Jahangir \& Terzic, 2005). This would directly lead to lower vessel contractility explaining the reduced blood pressure and vessel dilation observed in CS mice. In contrast to the readily explained vascular anomalies, finding a link between $\mathrm{K}_{\text {ATP }}$ GoF and the CS-associated cardiac phenotypes proves to be more difficult. $\mathrm{K}_{\text {ATP }}$ GoF is expected to shorten action potentials in the heart resulting in reduced cardiac output, whereas GoF animals reveal cardiomegaly and highoutput state. Hence, it is suggested that cardiac enlargement and increased cardiac output arise most likely independently of ventricular $\mathrm{K}_{\text {ATP }}$ activity, and can instead be interpreted as secondary consequences of vascular dysfunction which triggers systemic feedback mechanisms that compensate the lowered blood pressure (Huang et al., 2018).

To directly test whether cardiac remodeling occurs as a secondary response to GoF in VSM K $K_{\text {ATP }}$ channels, CS mice were crossed with animals expressing smooth muscle myosin heavy chain promotor-driven Cre-recombinase and dominant negative KCNJ8 transgenes making inducible suppression of $\mathrm{K}_{\text {ATP }}$ in VSM of WT and CS mice possible. Induction of expression lead to complete loss of $K_{\text {ATP }}$ function eventually resulting in significant rise of mean arterial pressure and complete reversal of cardiac hypertrophy (McClenaghan et al., 2019). These findings not only confirm a principal role for VSM $\mathrm{K}_{\text {ATP }}$ overactivity in the generation of cardiac hypertrophy, but also that it can be reversed once manifested. Therefore, VSM K $\mathrm{K}_{\text {ATP }}$ channels were established as suitable targets for pharmacological therapy of CSrelated cardiovascular abnormalities (Figure 1).

Lastly, to test the potential efficacy of glibenclamide to pharmacologically inhibit overactive VSM $K_{\text {ATP }}$ channels, CS mice were implanted with subcutaneous, slow-release pellets releasing a moderate or high dose of glibenclamide for 4 weeks. Strikingly, cardiac hypertrophy was reversed in a dose-dependent manner and vascular resistance was fully restored, therefore confirming similar results from CS zebrafish presented in Chapter 8. Moreover, blood glucose (BG) measurements during chronic glibenclamide exposure of CS mice revealed a significant decrease of BG on Day 1 post-implantation which returned to normal by Day 2. This observation gives evidence of long-term glycemic stability during high-dose glibenclamide intake (McClenaghan et al., 2019). 


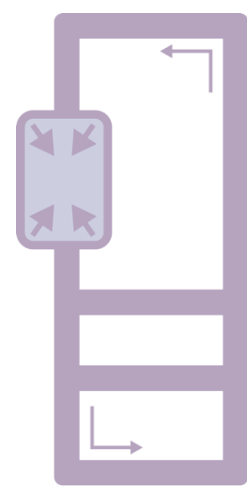

Normal

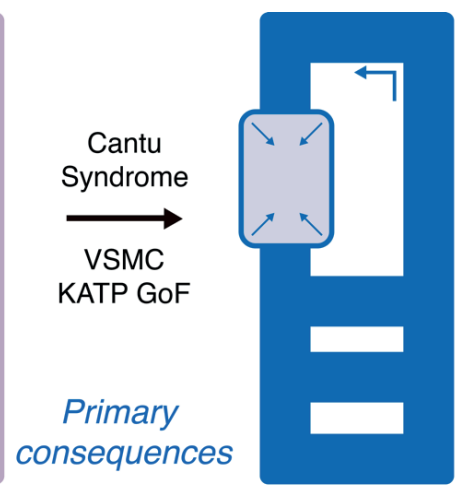

Vasorelaxation

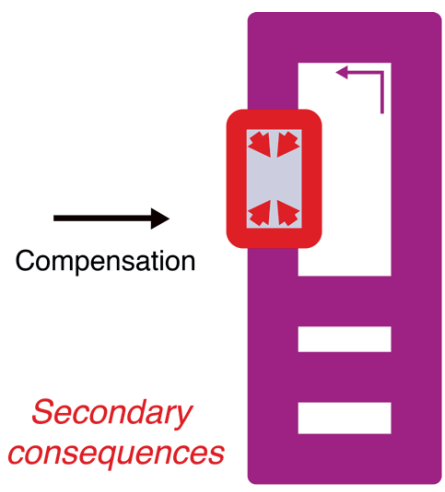

High output, hypertrophic hearts

Figure 1 | Cardiovascular pathophysiology of Cantú syndrome based on findings in model organisms. Primarily, $\mathrm{K}_{\text {ATP }}$ GoF in the vasculature results in reduced excitability, subsequently leading to functional and structural vasodilation, low blood pressure, and under-perfusion. Secondary consequences include compensatory cardiac hypertrophy and hypercontractility. Figure adapted from (McClenaghan 2019).

\subsubsection{Advantages and limitations of model systems}

Choosing an appropriate model organism for functionally studying any given RD requires a gene-by-gene decision based on genetic and anatomical conservation of the gene and phenotype, respectively. Additionally, the biology of model organisms does not always generalize, or is not always applicable to humans.

The structure and functional properties of cardiovascular $\mathrm{K}_{\text {ATP }}$ channels, however, are conserved between zebrafish and mammals making both animal models suitable for studying pathophysiology and possible therapy options for CS (Singareddy et al., manuscript in preparation).

Moreover, long-term consequences of multiple CS features are unknown. For instance, cardiac hypertrophy in CS may predispose to heart failure or arrhythmia. Both clinical manifestations are observed sporadically in the International CS registry (ICSR) patient cohort (Chapter 6). The existing CS animal models provide the opportunity to further study CS pathophysiology, for instance long-term effects like consequences of cardiac hypertrophy.

Due to extensive investigation of underlying molecular mechanisms of CS in model systems mentioned above and the identification of aberrant VSM $\mathrm{K}_{\text {ATP }}$ channels as the origin of CS pathophysiology, suggestions regarding the emergence of 
various CS-related features, other than cardiovascular anomalies, can now be formulated. Patent ductus arteriosus (PDA), which requires correction to prevent complications, is observed in most CS patients and likely arises from vasodilation due to $K_{\text {ATP }}$ overactivity in the dorsal aorta after birth. Similarly, edema could be caused by reduced lymphatic smooth muscle tone, and decreased airway smooth muscle contractility may have an effect on breathing. The underlying molecular basis for hypertrichosis observed in nearly all CS patients remains unclear (Rossi et al., 2012), but is suggested to be associated with elevation of oxygen, blood and nutrient supply in the hair follicle due to dilated blood vessels (Ma et al., 2019). Therefore, CS-related features that can be explained by smooth muscle relaxation are likely to be primary consequences of $\mathrm{K}_{\text {ATP }} \mathrm{GoF}_{\text {, whereas others }}$ can be seen as secondary pathologies. This is an important consideration when approaching therapy options as features arising as secondary consequences may require longer exposure to treatment before an effect is visible.

The discovery of the underlying genetic cause of CS has led to the rapid identification of a large number of additional patients, as well as increased awareness of the syndrome in the field of clinical genetics. To date, genetic testing has identified 110 individuals with heterozygous pathogenic variants in ABCC9, and in three cases a heterozygous pathogenic KCNJ8 variant has been identified. Altogether, there are 35 ABCC9 variants known to alter amino acids in or close to the transmembrane domains of both proteins, but they are nevertheless scattered over the entire length of the molecule (Chapter 6). Interestingly, there are multiple reports of neonatal diabetes-causing mutations in Kir6.2/SUR1 $\mathrm{K}_{\text {ATP }}$ subunits (KCNJ11, ABCC8) that reduce sulfonylurea sensitivity (Koster et al., 2005; Proks, 2013). Similarly, it was demonstrated that a few CS-associated gene mutations also profoundly reduce glibenclamide inhibition of recombinant channels (Cooper et al., 2017; McClenaghan et al., 2017). Therefore, determining glibenclamide sensitivity for specific mutations may be required for future individualized therapy. With advancement of genome editing techniques like CRISPR/Cas9, targeted evaluation of candidate genes has become faster, with research groups capable of generating mouse and zebrafish models for several loci and obtaining homozygous animals in approximately 6 months (Chapter 7) (Kherraf et al., 2018). Nevertheless, the long list of CS-associated gene variants to be functionally tested, requires a system that is capable of high-throughput analysis and rapid genotype-phenotype assessment. For instance, cell-based assays could be utilized to do so. In an attempt to gain more accurate assessment of the potential impact of CS mutations on the pharmacological response, another option would be the study of native channels in human-induced pluripotent stem cell-derived cardiovascular cells. 


\subsection{Pre-clinical approaches for finding rare disease treatments}

In this thesis, we have highlighted one possible approach to drug repurposing in RDs, phenotype-based drug screening in animal models (Chapter 8). However, there are additional techniques that can be applied separately or in parallel to work towards RD therapeutics (Figure 2). Many repurposed drugs in the past have been found serendipitously in the lab or by carefully monitoring the effect of drugs in the clinic and performing retrospective analysis of clinical experiences (Demonaco et al., 2006). As there are more than 7000 RDs in need of treatment, this approach clearly does not meet the requirements for the field. Thus, in the recent years, more systematic, organized and data-oriented searches for candidates have been launched profiting from technological advances like big data analytics, computer models and high-throughput screenings (Ekins et al., 2011; Ferreira \& Andricopulo, 2016; Pushpakom et al., 2019).

\subsubsection{Computational pharmacology and in silico methods}

Nowadays, the capacity to generate data is immense and continues to grow. The unprecedentedly large amounts of data, on both rare and common diseases, have challenged researchers trying to make sense of it (Issa, Byers, \& Dakshanamurthy, 2014). Additionally, data sharing initiatives also open up access to new types of data including patients' medical records and other data ready to be analyzed. The clear need for rational approaches to find alternative indications for existing compounds has resulted in the development of computational or in silico methods for drug repurposing. These methods are appealing because they nominate the most promising candidate drugs for a given indication and apply various direct and indirect evidence to generate a hypothesis, including molecular (Brown, Kong, Kohane, \& Patel, 2016), literature-derived (Patchala \& Jegga, 2015) and clinical data (H. Xu et al., 2015).

Computational techniques, including both target- and ligand-based strategies to systemically design rational repurposing processes and models perfectly complement existing experimental techniques and are therefore widely applied in industry and academia (Ekins, Mestres, \& Testa, 2007a, 2007b). In recent years, as the study of drug repurposing has become a priority in the field of drug discovery some valuable repurposing models have been created and applied to find potential new drug indications (J. Li et al., 2016). These models primarily aim at studying the relationship between drug, target and disease and can be generally divided into two categories: (i) structure-based models including drug/ligand structure and target/receptor domain, and (ii) phenotypic- or 
network-based models (Shameer, Readhead, \& Dudley, 2015; Zhao \& Wei, 2018). To identify candidate compounds, computational approaches are in need of carefully designed multi-step analyses - compound prioritization coupled with well-designed validation experiments.

\subsubsection{Artificial intelligence}

Artificial intelligence ( $\mathrm{Al}$ ), holds great promise in rapidly and efficiently collecting, analyzing and characterizing information and has already being successfully applied to basic research, diagnostics, drug discovery, and clinical trials. Due to the underrepresentation of RDs in research of treatment development, they especially can profit from Al (Brasil et al., 2019).

Al, with an emphasis on deep learning or machine learning $(M L)$, is capable of learning from data. ML algorithms are able to build mathematical models based on sample data, called training data, in order to make predictions or decisions without being explicitly programmed to perform the task. Thus, ML algorithms are able to uncover complex data (Ekins et al., 2019). If models were available for all aspects of drug discovery and development, they could be applied to predict whether a compound is likely to be used for a new indication.

Many studies have already applied ML methods in the field of drug repurposing. Lee et al. applied an ML unified computational framework called URSAHD (unveiling RNA sample annotation for human diseases) to apply genetic and molecular information about thousands of complex disorders, to test drug repurposing (Y. S. Lee et al., 2019).

\subsubsection{Collaborative models}

There is an increasing realization that repurposing drugs requires a collaborative approach combining the strength of academia, industry, governmental bodies and patient organizations. According to Pushpakom et al. there are three key components to successful drug repurposing collaborations: (i) identification of scientific experts with new ideas in RD biology, (ii) alternative funding routes and (iii) enthusiastic engagement among all institutions involved. Recently, multiple repurposing initiatives have been established between pharmaceutical industry, grant funding organizations and academic scientists to address some of the challenges (Pushpakom et al., 2019). One example is the Mechanisms for Human Diseases Initiative (Hayes \& Nutt, 2019). As a result, risks and costs involved in creating a new therapy are drastically reduced. It also has the potential to revolutionize the drug RD pipeline and empower a larger number of patient organizations to drive for treatments for their conditions. 
Pharmaceutical companies not only have the necessary compound libraries of failed or shelved drug candidates but also have immensely valuable knowledge in translational research, drug development and screening technologies that are often too expensive to acquire and maintain for many academic institutions. On the other hand, academic institutions tend to have access to patients and knowledge on disease biology and pathogenesis which can be the basis for highly innovative drugs.

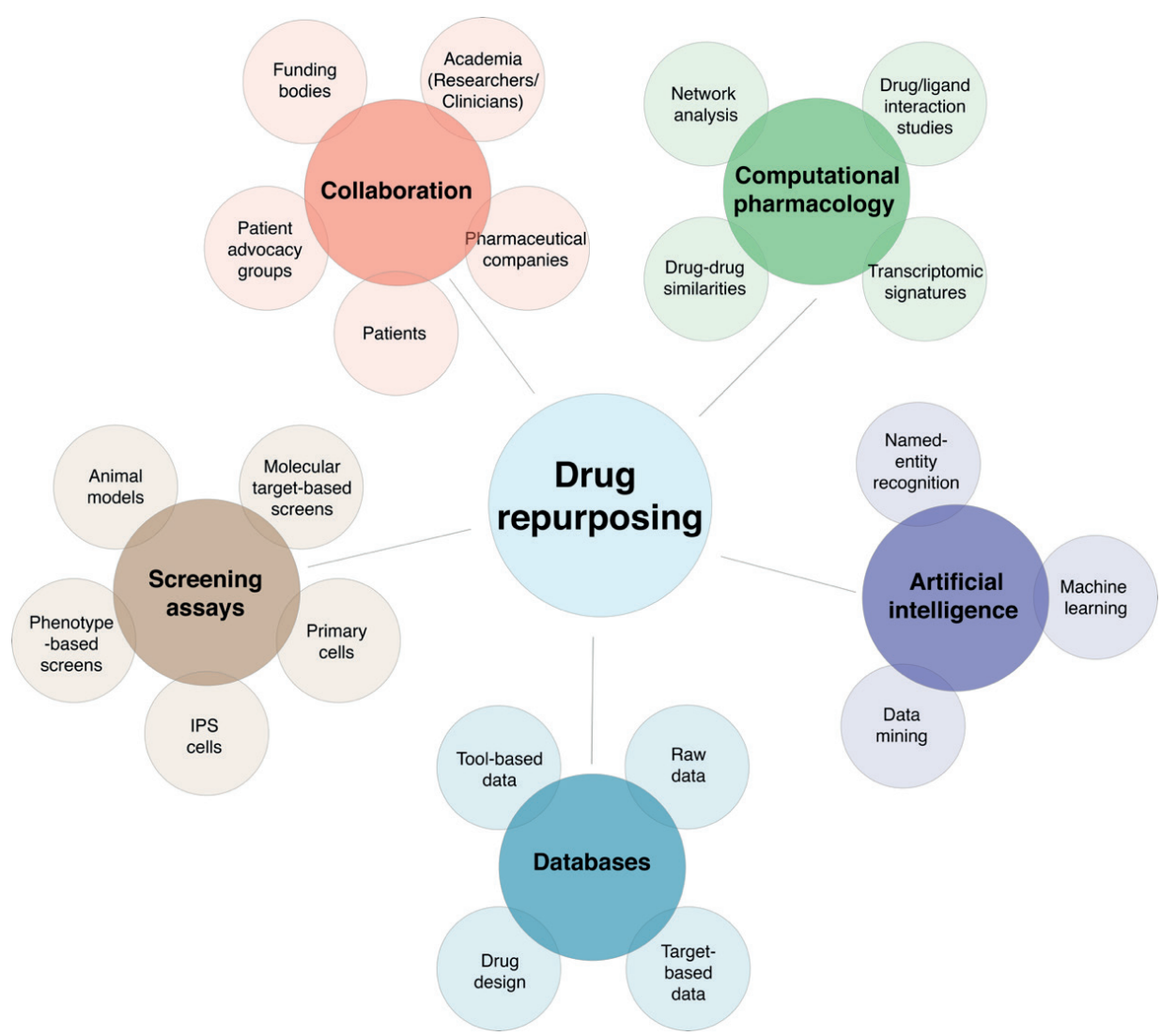

Figure 2 | Approaches applied in drug repurposing for rare diseases. Various approaches can be used individually or in combination to systematically analyze different compounds and diseases for repurposing hypotheses. These include computational and experimental approaches as well as collaboration of various stakeholders involved in the field of rare diseases.

Another aspect of the collaborative approach is working together with patients, their families or patient organizations. The establishment of hundreds of patient advocacy groups (PAG) and charitable foundations to support RD communities have increased public awareness, as well as interest of media and legislative 
bodies. PAGs give valuable insights for clinical trials such as identification of clinical end-points, assisting in patient recruitment or participating in meetings with regulatory agencies to discuss research and development activities.

\subsection{The next step: A clinical trial in rare disease}

In this thesis, we have highlighted the first steps in order to go from a correct genetic RD diagnosis towards therapeutic intervention. By applying two major model organisms, a candidate compound showing a promising efficiency in preventing the establishment of or reversing CS-associated features was identified (Chapter 8) (McClenaghan et al., 2019). The next step will be to test this drug in patients within a clinical trial. However, performing clinical studies for drug development in RDs can be very challenging.

\subsubsection{Challenges of clinical trials in rare disease}

There are several requirements for studying therapeutics for human disorders:

(i) appropriate trial design and analysis to answer the posed research question, (ii) adequate outcome measurements, (iii) selection of correct sample size, (iv) ethical recruitment to participation, ( $v$ ) funds to support the research as well as (vi) knowledgeable study staff (Augustine, Adams, \& Mink, 2013). In particular in RDs, the limitations when it comes to studying a small patient cohort can transform these requirements into considerable challenges.

\section{Statistical power of designed clinical trial}

Typically, the randomized controlled trial is considered as gold standard for establishing efficacy in a research setting (Schulz \& Grimes, 2002). Because of high costs, time-investment and requirement of a large disease cohort, it is usually applied in common diseases, but less in RDs. In the analysis of clinical trial data, RDs show two major disadvantages. Clinical studies for RDs can only enroll a small cohort (due to less patient population). In combination with high variability in phenotype and clinical course observed in a majority of RDs (Rahit \& Tarailo-Graovac, 2020), the study's power decreases drastically. Hence, alternative trial designs and statistical techniques that maximize data from a small and heterogenous group of individuals are necessary. Possible alternative designs could be crossover studies, in which all participants receive the same two or more treatments, but the order in which they receive them depends on the group to which they are randomly assigned, or adaptive design approaches. Such an adaptive design allows adaptations to trial and/or statistical procedures of the trial after its initiation without undermining the validity and integrity of the trial 
(Chow, Chang, \& Pong, 2005). These strategies result in shortening of clinical trial duration and increased success probabilities by dropping inferior treatment arms at interim evaluations or assigning more patients to superior arms by using various randomization schemes in a play-the-winner principle. Notably, due to these measures, drugs for RDs have been approved based on studies that lacked randomization and a placebo-control (Mitsumoto, Dorsey, Beck, Kieburtz, \& Griggs, 2009).

\section{Natural History}

In depth knowledge of the natural history of (rare) disease is significant for trial design as it helps identifying key milestones in disease progression, selecting an appropriate length of the study to monitor change in disease progression, developing adequate inclusion/exclusion criteria and determining a clinically meaningful difference (Chapter 3-6) (Augustine et al., 2013). Due to small patient numbers, geographic dispersion of patients and researchers and small number of researchers with specific expertise, collection of natural history data can be challenging. One possibility to overcome these limitations is multi-center collaboration to strengthen the knowledge of natural history and to establish a patient registry to maintain the collected data as it was done for CS, for instance (Chapter 6).

\section{Outcome Measures}

Validated measures of disease activity or disease progression are often missing in RDs. Due to heterogenous clinical phenotypes, the selection of clinical endpoints that will be informative across the spectrum of disease expression is challenging. Thus, it is essential to keep refining our clinical rating scales to better quantify such versatile disorders and enhance their precision related to small changes. Furthermore, biomarker development can support proof-ofconcept and serve as surrogate outcome.

\section{Subject recruitment}

Another challenge for all RDs is the timely and adequate recruitment of patients meeting all inclusion criteria of the study. Often, researchers would like to focus on a specific aspect of the entire patient population (age, disease stage, etc.), however it may not be feasible to further minimize an already small cohort. In disorders with significant physical impairment, travelling to the closest research center may not be possible. Lastly, for trials investigating repurposed drugs, potential off-label applications can further threaten recruitment. In order to maximize recruited individuals, it is recommended to involve patients during trial design (patient participation in clinical research). According to 
interviews with representatives of RD patients, patients value involvement in trial design and thus have influence on outcomes and measurement instruments, duration of the trial and information that is sent to potential participants (Gaasterland et al., 2019).

\section{Drug Development and Funding}

The process of new drug development is expensive and time-consuming. In instance, pre-approval costs for a new compound may exceed $\$ 800$ million (Morgan, Grootendorst, Lexchin, Cunningham, \& Greyson, 2011). In case of RDs which might be fatal and/or rapidly progressing, there is typically no access to large industry research, large funding opportunities or the luxury of time. Especially when compared to common disorders, RD treatments are expected to have small markets and therefore small economic impact, providing little return on investment. In the current economic climate, funding has become more and more competitive and funding agencies themselves ask for broad public health impact.

\subsubsection{Recent progress in rare disease clinical trials}

Investigation of therapeutic intervention for RDs faces many challenges. Nevertheless, there have been several recent accomplishments. Major achievements in clinical trials of RDs are in three domains: gene therapy, enzyme replacement therapy and therapy with small molecules whose identification can be accelerated by drug repurposing. As outlined before, drug development for RDs is difficult because it is challenging to obtain relevant data from very few patients. I will shortly summarize the current status of clinical trials for drug development in RDs. Sakate et al. outlined and compared clinical trials in RDs in the US, EU and Japan applying data from multiple clinical trial databases, e.g., ClinicalTrials.gov or EU Clinical Trials Register. In total, 28.526 clinical trials were extracted to study $1.535 \mathrm{RDs}$ and 1.539 drugs (orphan and repurposed). From 1999-2016, 84 RDs per year on average were newly targeted. Amongst all diseases, most were studied in only a limited number of trials with $70 \%$ of disease being studied in $>10$ trials and $28 \%$ being studied in only one. Interestingly, most studies involved cancer-related RDs (Sakate, Fukagawa, Takagaki, Okura, \& Matsuyama, 2018). Thus, for most of the RDs which are not associated with cancer, the number of trials and drugs were very small. Especially the amount of phase 3 and 4 trials was rather low indicating that there are still high hurdles for drug development for RDs. 


\subsubsection{What's next for Cantú syndrome: Future clinical studies First-in-human study}

Thus far, a single human CS patient has been treated with glibenclamide ( $\mathrm{Ma}$ et al., 2019). The neonate, born prematurely, had a large PDA, enlarged right ventricle, edema and pulmonary hypertension, amongst other typical CS-related features. After exhausting conventional approaches to manage respiratory problems, a therapeutic glibenclamide trial was started. A small number of hypoglycemic episodes was observed after increase of glibenclamide doses, but these were mild and transient and without associated cardiovascular dysfunction. Pulmonary hypertension improved and cardiac enlargement was no longer visible on echocardiogram.

\section{Proof-of-concept study}

Prompted by observations in both animal models (Chapter 8) (McClenaghan et al., 2019) and the first-in-human study described above (Ma et al., 2019), we intend to test the efficacy of glibenclamide in a small cohort of Dutch patients within a proofof-concept exploratory trial which will, if successful, be followed-up with a multicenter, randomized, phase II/III cross-over study. The principal aim of the proofof-concept exploratory study is to determine whether a full randomized controlled trial to test glibenclamide in a large cohort of CS patients is justifiable and feasible and to optimize its design. Specifically, we intend to assess the efficacy and safety of glibenclamide in individuals with CS. The primary objective will be to determine whether giving glibencamide to CS patients can efficiently reverse hypertrichosis. Secondary objectives involve safety, further evidence of efficacy and collection of experience and data a pivotal study can be based on. The main concern of treating CS patients with glibenclamide is that individuals who do not have diabetes are potentially at risk of developing hypoglycemia as a side effect. In order to determine the efficacy of glibenclamide in reversing further CS-associated features, we will assess (i) cardiovascular abnormalities focusing on cardiomegaly, aortic dilation, tortuosity, high-state output and exercise intolerance, (ii) swelling/edema in the leg, (iii) distinctive facial features as described in Chapter $\mathbf{5}$ and (iv) further skin- and hair-related abnormalities associated with CS (Figure $\mathbf{3}$ ). The collected data will be essential to inform sample size calculations for the primary outcome measures and to estimate treatment period, appropriate outcome measurements and effect size for use in a future full-scale phase II/III trial.

We intend to perform an open-label, single-arm, non-randomized, uncontrolled intervention study for only a limited number of Dutch patients due to the overall rarity of CS. Baseline measurements will be performed before commencement of glibenclamide treatment and patients will be their own controls. The study will 
last for 9 months. The usual starting dose of glibencamide in adults with type 2 diabetes is $2.5 \mathrm{mg}$ once a day. During the exploratory study we propose to start at a lower dose in CS patients as they do not have diabetes and therefore are at risk of developing hypoglycemia. We will start with a run-in period of 2 weeks with $1.25 \mathrm{mg}$ once a day and monitor closely for signs and symptoms of hypoglycemia. In case they tolerate $1.25 \mathrm{mg}$ per day well, we will increase the dose after 2 weeks to $2.5 \mathrm{mg}$ a day.

Patients for the exploratory study will be recruited from our cohort of Dutch CS patients who have attended our annual Cantú syndrome research clinic on a regular basis and are enrolled in the ICSR (Chapter 6).

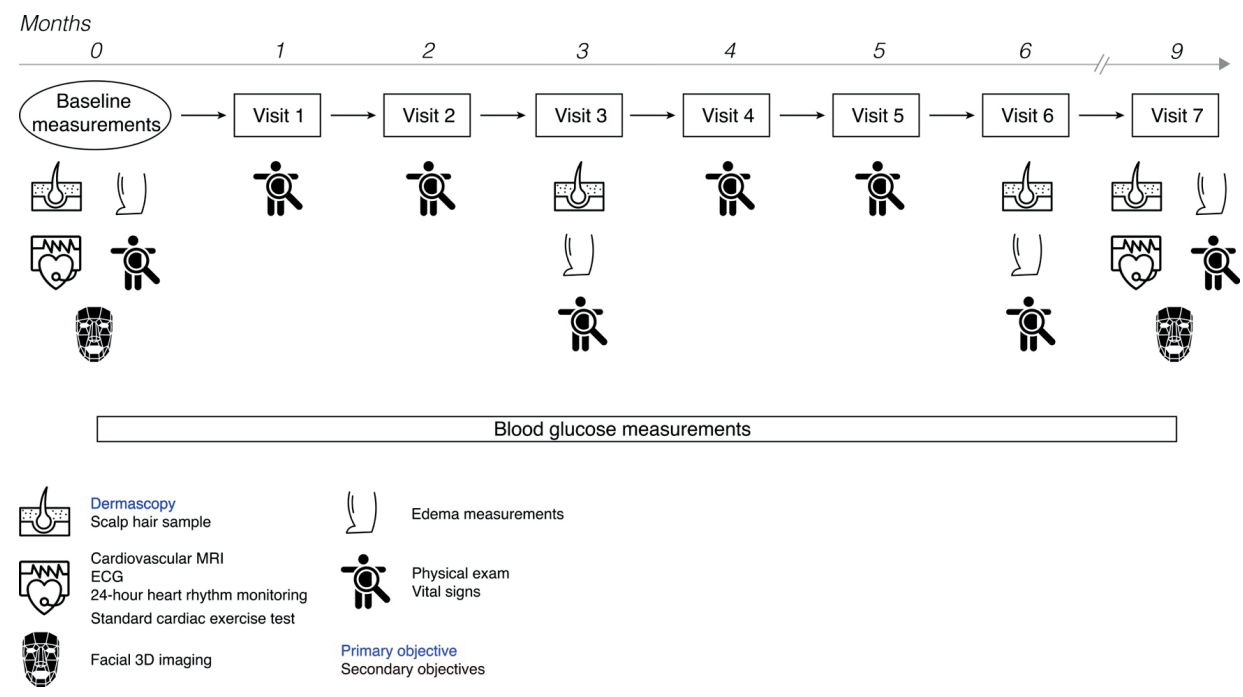

Figure 3 | Flow diagram of proof-of-concept study to test the efficiency and safety of glibenclamide intake in CS patient. The principal aim of this study is to determine whether a full randomized controlled trial to test glibenclamide in CS patients is justifiable and feasible and to optimize its design. Specifically, efficacy and safety of glibenclamide in individuals with CS will be assessed by multiple outcome measurements. After baseline measurements, the subjects will be maintained on glibenclamide for 9 months. CS patients will start on a low dose of glibenclamide $(1.25 \mathrm{mg})$ for 2 weeks and patients will monitor their blood glucose with a glucose sensor for hypoglycemia. In case they tolerate $1.25 \mathrm{mg}$ per day well, the dose will be increased to $2.5 \mathrm{mg}$ a day. If the patient develops hypoglycemia, the dose of glibenclamide will be dropped back down to the last tolerated dose. Patients will be seen monthly for the first 6 months and one more time after another 3 months.

\section{Multi-center cross-over study}

In order to follow-up on the results gained from the proposed proof-of-concept study, we intend to perform a multi-center, randomized, open-label, phase II/ III, 2-period cross-over study in a larger CS cohort. The primary objective of this 
study will be to determine if giving glibenclamide to patients with CS results in further evidence of efficacy in treating CS-associated features. As glibenclamide is an already registered drug, safety is known and will therefore be a secondary objective in this trial. This follow-up study will contain two parts, a dose titration of 4 weeks per patient to reach an optimal glibenclamide dose as well as a cross-over treatment period with sufficient length to show clinical benefits of glibenclamide treatment for CS patients (Figure 4).

Period $1 \quad$ Period 2

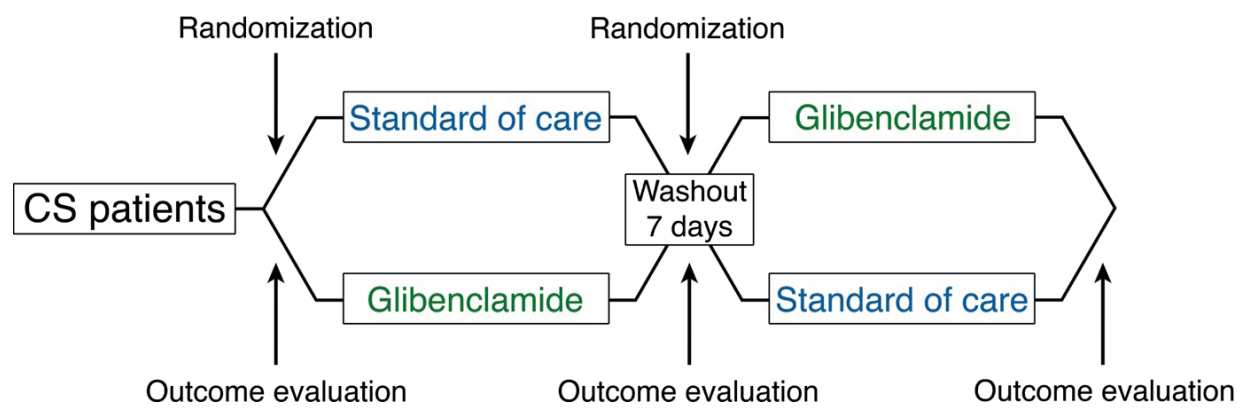

Figure 4: A multi-center, randomized, phase II/III cross-over study in CS patients. If patients are showing benefit from the medication and are not experiencing side effects during the proofof-concept study, we intend to follow up this exploratory study with a European multi-center, randomized, placebo-controlled, phase II/III, 2-period cross-over study in a larger CS cohort. Here, patients will be assigned randomly to a placebo group receiving the best standard of care for CS (e.g., symptomatic treatments with medication, annual ECG/MRI) or a glibenclamide treatment group.

\subsection{Getting connected: The importance of collaboration within the field of rare disorders}

Over the last two decades, important contributions were made at national, European and international levels to improve collaboration into RD research. Due to their low prevalence, challenges associated with RDs are too big for individual countries to tackle by themselves. Additionally, RD research is scattered across laboratories and clinics throughout the world further complicating the general challenges of RDs. Hence, this research field in particular, benefits immensely from coordination on a European (e.g., European Reference Networks) and international scale, as collaboration can enhance the chances of identifying new diagnostics and therapies by increasing the number of available patients for each study and uniting the scattered specialists with complementary expertise, as shown in multiple chapters of this thesis (Chapter 4, 5, 6 and 9). Next to 
collaborative translational research opportunities within different branches of academia, the field of RDs urgently requires extensive partnerships between academia, pharmaceutical industry, funding bodies and patients themselves.

\subsubsection{Translational research networks}

The wide diversity of research disciplines, necessary for an effective understanding, diagnosis and therapy development of any RD, ask for the establishment of translational team strategies on a national and international level. Separately, investigators make valuable, but nonetheless limited contributions. In the current research climate, investigators are challenged to compete for data, even from a scarce patient population in case of RDs, whereas a team effort could result in a more rapid and detailed understanding of various disease-related aspects. Moreover, it is essential that results and data are published for the benefit of the field and patients. This includes data sharing in a responsible manner, for instance by adhering to the FAIR (Findable, Accessible, Interoperable, and Reusable) principles (Boycott, Lau, Cutillo, \& Austin, 2019; Wilkinson et al., 2016).

Chapter 9 is an example of how collaboration within research fields can unite various expertise necessary to investigate and understand a novel RD. Here, the small cohort of patients showing a novel genetic disorder, was identified in Norway. Due to lack of further knowledge about the underlying disease gene $A B C C 9$, our laboratory was contacted with the request to collaborate. Knowing a third research group with expertise and research focus on the molecular mechanisms of ABCC9 mutations and possibilities to investigate this aspect further, the project ended up profiting from three parties whose joint effort led to the discovery of a new RD; AIMS.

A similar approach was applied for the investigation of CS (Chapter 4-6). The E-Rare initiative finances transnational collaborations to improve progress of RD research from bench to bedside, to enable knowledge transfer across countries, and to create vital infrastructures including biobanks and patient registries. A research network emerging from this initiative is the CantúTreat consortium, which was established in 2015 aiming to develop a novel and cost-effective therapeutic approach in management of CS. The consortium brings together an international, multidisciplinary collection of leading researchers and clinicians in the field. Tasks include modeling and experimental testing of specific CS-related variants to further understand the biology of various symptoms and building a global patient registry to generate a critical mass of patients in order to ensure a rapid progression towards clinical application (Figure 5A). 
Monthly online meetings with the consortium members resulted in ongoing discussion about current research or new patients added to the registry. From 2015 till present a yearly meeting at rotating locations is organized for all consortium members and junior researchers. Typically, this was combined with the annual CS research clinic of the hosting partner enabling all members to examine site-specific cohorts. Notably, Chapters 4, 5, 6 and $\mathbf{8}$ of this thesis as well as a wide range of further publications and studies emerged from collaborations between CantúTreat consortium members once again indicating the necessity of intensive networks in the field of RD research (Figure 5B).

A

Establishment of global patient registry

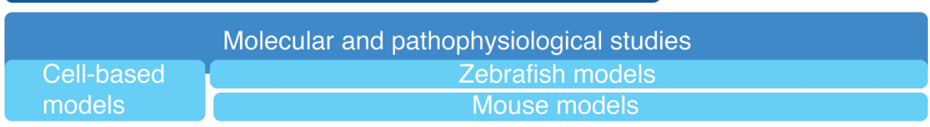

Therapy development in model organisms

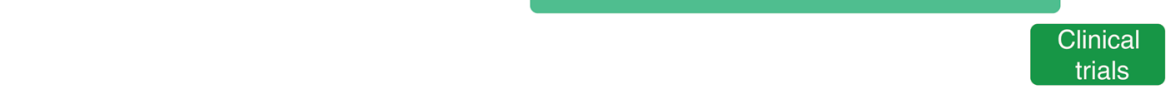

\begin{tabular}{|lllllll}
\hline From gene & $\ldots$ & $\ldots$ & $\ldots$ & $\ldots$ & $\ldots$ & to treatment \\
\hline
\end{tabular}

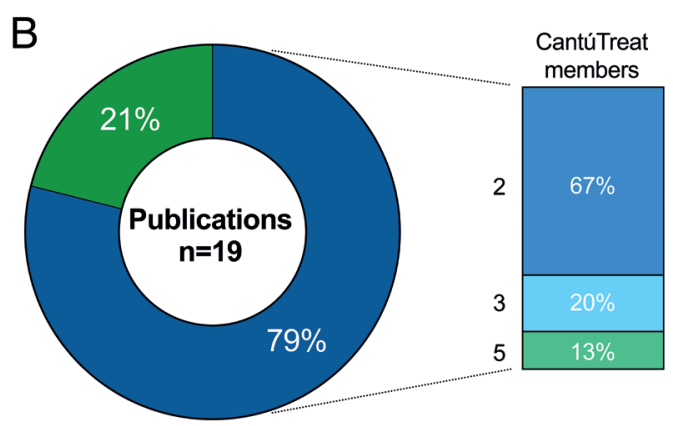

Publications with the involvement of other consortium partners

$\square$ Publications without the involvement of other consortium partners

Figure 5 I Impact of CantúTreat consortium as a practical example for the importance of collaborations in the field of rare disease. (A) Research pipeline of CantúTreat consortium going from gene to treatment. (B) Participation of CantúTreat members in CS-related publications between 2015 and 2020. A majority of all publications derived from at least two CantúTreat consortium members/laboratories. 


\subsubsection{Collaboration between academic institutions and industry}

In academic institutions, progress in genetics and disease mechanisms have resulted in a more detailed scientific understanding of RDs. However, the lack of funding and translational capability majorly limits RDs researchers to bring their biological knowledge from bench to bedside, for instance by initiating clinical trials. As pharmaceutical companies used to only pursue treatments for common disorders with a large number of patients and therefore higher profits, drug development for RDs has mainly occurred in biotech companies or academia who then have to reproduce the same functions and procedures that a large drug company goes through to develop a drug, which is a challenging process. Therefore, it is essential for the field of RD to foster collaborations between academia and industry in order to take advantage of the expertise of both entities. Pharmaceutical companies have screening technologies available that can be applied for the identification of therapeutic compounds as well as significantly more experience and funds for drug development.

Recently, industry-wide trends have revealed that drug companies are changing their approach as the traditional drug discovery research and development model is no longer viable. Hence, they have recently started to shift their focus to RDs.

\subsubsection{Patients as partners}

Another aspect of the collaborative approach, as mentioned above, is working together with patients, their families or patient organizations. RD patients and their caregivers have been significantly enabled by the internet as a tool to connect across the globe and share care and research strategies.

Patient participation in research and drug development is highly encouraged and increasingly implemented, carrying significant weight for the regulatory review process (Chakradhar, 2015; Perfetto, Burke, Oehrlein, \& Epstein, 2015). The formation of patient organizations in the 1990s led to increased data collection and deeper knowledge of RDs in both the US as well as Europe. Additionally, patient representatives are invited to join advisory committee meetings of both the FDA and EMA which allows patients to be heard in matters of benefit-risk balance and innovation in regulatory agencies (Tambuyzer et al., 2020).

Another possibility to increase one's knowledge about RDs is to put effort in capturing and sharing self-reported patient data, in addition to data obtained in a research setting. Nowadays, patients are increasingly involved in reporting their features, outcomes and treatment histories applying social media and patient- 
centric research platforms (Southall et al., 2019). A variety of applications for smartphones are available to assist with tracking health conditions and collecting epidemiological data. This information can be essential for natural history studies - a vital element of drug development and approval. However, it is important to realize that such data collection can vary in quality due to variable data standards, privacy concerns or inaccurate reporting. Data standards are critical to understanding and using data, and to ensure maximal utility in both research and for future product development. Therefore, it is advised to standardize data collection which should be in accordance with regulatory standards and created in consultation with patients and clinicians (Sherman et al., 2016; Southall et al., 2019).

\subsection{Recent developments and challenges in the field of rare diseases}

In recent years, we have witnessed advances in the diagnosis and treatment of RDs which leave us confident that the future of this field is bright, with innovative therapies being developed that have the ability to truly change the life of patients. Nevertheless, we still encounter a variety of challenges and difficulties that need to be addressed before further progress is possible.

\subsubsection{Progress in rare disease research}

The scientific landscape for RDs has been changing rapidly, and this change is expected to accelerate. The combination of various events and activities resulted in a growing interest in RDs. Due to a lengthy diagnostic odyssey, a lack of adequate treatments, and multiple hospitalizations over a lifespan, there is acceptance and public recognition that RDs represent a global public health problem. In particular the expanding role and outreach activities of PAGs have enhanced the awareness of the public, media and legislative bodies in the field of RDs (Groft, 2013). Looking ahead, there is great hope for people living with RDs. The number of patients being enrolled in RD clinical trials is increasing, and people living with RDs are more and more profiting from new treatment options, which are a result from break-through technologies now emerging in medicine.

Biopharmaceutical industry researchers are making considerable progress in the fight against RDs as scientific progress has opened new possibilities. Researchers continue to successfully uncover the molecular and genomic drivers as well as the clinical course of many conditions. Certainly, more attention is 
being paid to RDs in academia highlighted by the drastic increase in publications (Figure 6).

The Orphan Drug Act (ODA) has been essential to increasing RD research in the US. Since its enactment in 1983, more than 770 orphan drugs and biological products have been approved in the US, compared with just 10 in the decade before passage (Haffner, Torrent-Farnell, \& Maher, 2008). Nevertheless, this recent increase has resulted in concerns regarding the Act as some pharmaceutical manufacturers have collected outsized financial benefits while avoiding the regulatory requirements and costs related to non-orphan drug discovery (Gabay, 2019). In Europe, following the adoption of the European Commission (EC) in 2000, 164 orphan medicinal products received marketing authorization by 2018 (Committee for Orphan Medicinal et al., 2011) iv.

Interestingly, in the recent years, there has been an increase in funding for companies focused on RDs, whereas financial support for high prevalence, common diseases has decreased, possibly due to a higher hurdle in developing and gaining approval for medicines that treat these conditions. Successful transition from one phase to the following in clinical studies in between 2005 and 2018 was always higher for RDs compared to groups compiling all disease or chronic high prevalence disorders ${ }^{v i}$. The overall success rate for orphan drugs from Phase I to approval between 2006 and 2015 was about 25\%. The recent FDA drug approvals report for 2019 highlights that 21 of the Centers for Drug Evaluation and Research's (CDER) reported 48 novel drug approvals $(44 \%)$ to treat rare or orphan disease like cystic fibrosis, tenosynovial giant cell tumor, and sickle cell disease. Further approvals were given to help patients with erythropoietic protoporphyria, neuromyelitis optica spectrum disorder, systemic sclerosis-associated interstitial lung disease, and Duchenne muscular dystrophyv.

\subsubsection{Ongoing Challenges}

Despite encouraging progress in the diagnosis of RDs due to recent advance in genomic technologies like NGS, there is still no universal technique available accurate identification and investigation of all genetic variants of clinical interest. It is essential to have basic knowledge of each of the novel genomic techniques available, including their main advantages and drawbacks. Additionally, genetic testing of infants and children with RDs is necessary to make early diagnosis and treatment possible. A lack of knowledge can result in challenges when deciding which type of genetic test is more specific and sensitive for the respective patient. Lastly, the interpretation of genomic variants remains challenging, 
especially when classified as VUS resulting in difficulties during genetic counselling due to uncertainties of their clinical significance. Upcoming fields such as epigenomics, metabolomics and proteomics are progressing rapidly leading to new information that will assist in an accurate interpretation of the large amount of genomic data generated in the laboratories (Ramos-Fuentes, Gonzalez-Meneses, Ars, \& Hernandez-Jaras, 2020).

To ensure that eventually all RD patients can profit from recent scientific progress, we require more efficient and effective models for developing treatments which reveal a clear readout and are sustainable. These models must offer improvement of processes and timelines, promotion of platform approaches and stimulation of innovation in manufacturing.

The large gap between basic research and therapy development is still obvious and can only be bridged by all entities in the RD community working together (Figure 6). Nowadays, a high percentage of quality research in rare conditions never makes it to the clinic or even the pre-clinical phase. Thus, translation of basic research on RDs into therapies needs to become an even higher priority for public and private funding. This will help to decrease the risk of early research results and potentially increase the likelihood of larger industry engagement in bringing new treatments to the markets. Although the number of drugs for RDs is steadily increasing in both the European Union as well as the United States, approved orphan drugs address only a small portion of RDs as only $5 \%$ of them have therapeutic intervention. Additionally, there is a large bias towards specific disease areas. More than a third of the overall number of orphan drug approvals are for oncology-related conditions (Tambuyzer et al., 2020).

Multiple novel techniques are nowadays available to assist and facilitate RD diagnosis as well as care. Currently, various Al-based deep learning algorithms are emerging that support specific diagnostic tasks like the classification of skin conditions (Esteva et al., 2017) or the recognition of facial phenotypes linked to genetic diseases based on 2D and 3D images (Gurovich et al., 2019) (Chapter 5). Video consultations (Ignatowicz et al., 2019), home self-testing kits (Tidy, Shine, Oke, \& Hayward, 2018), remote patient monitoring with sensors (Noah et al., 2018), and electronic prescriptions (Porterfield, Engelbert, \& Coustasse, 2014) are already available. Additionally, positive effects have been observed due to digital tracking of outcome measures (Basch et al., 2016). However, to fully unfold the potential of these new technologies, and at the same time avoid their risks, all parts have to be integrated in an easy-to-use and ethical manner so that both patients and physicians can trust them. In order to ensure that, it will 
be important to trace system processes in order to ensure accountability in case of incorrect diagnosis or treatment complications.

\subsubsection{A hopeful future}

Rare diseases are a very unique category of disorders. Nevertheless, the features of RDs resulting in obvious challenges can also become advantageous for finding new therapeutics. In this thesis, we show that once united, a well-defined patient population, a defined genetic etiology, and an appropriate model system can accelerate therapy development. It is essential to facilitate collaboration between all key stakeholders including academic institutions, government, biopharmaceutical industries, patient advocacies and non-profit organizations to move RD drug discovery forward. Moreover, computational methods can help foster collaborations, add efficiency, build on previous efforts, and eventually drive research in novel directions. The future of RDs is hopeful and promises great advances and even better personalized therapies with the identification of novel treatments and approaches to clinical care.

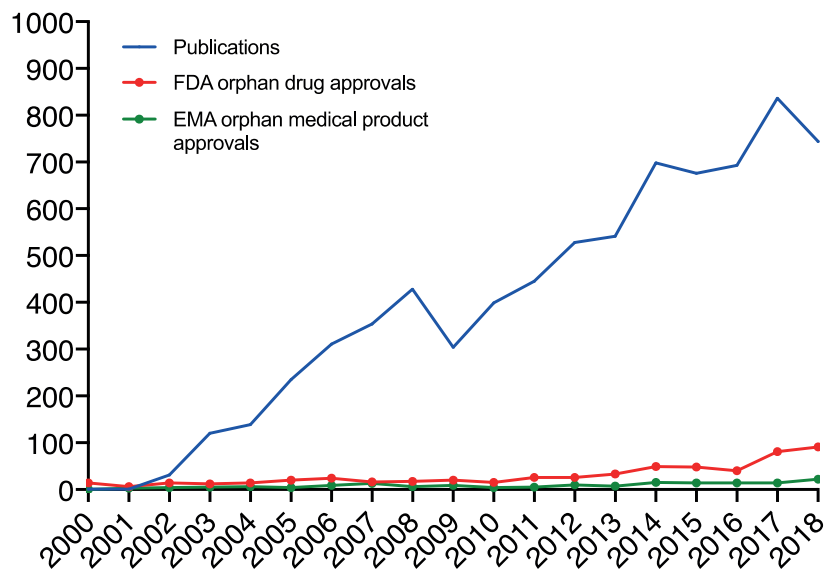

Figure 6 I The gap between science and translation into treatments for rare diseases. Number of publications per year containing the MeSH term "rare disease" in the abstract extracted from PubMed compared to the number of orphan drugs which received approval by the FDA or EMA from 2000 to 2018. Figure adapted from (Tambuyzer 2019). 


\subsection{Take home messages from working in the interface of clinical genetics and disease biology}

This thesis was conducted in the interface of clinical genetics and disease biology combining two pivotal scientific fields essential to move forward in the endeavor of finding new treatment possibilities for rare genetic diseases. In the following paragraph, I would like to discuss various insights I gained while working in the field of translational medicine originating from a strictly biological background without considerable knowledge and experience in clinical research.

Progress in biomedical research is expanding exponentially. Unfortunately, the gap between progress in basic scientific research and translation of clinical research data to real-world settings is still considerable (Butler, 2008). In particular, major hurdles are the performance of "bench-to-bedside" research (Khoury et al., 2007). A possible solution to eliminate these scientific roadblocks is to equip basic scientists with the knowledge and skills to turn novel biological rationales into tools to improve human health. Whereas in the past, many PhD programs have directed scientific researchers towards specialized career paths without providing the possibility to expand their research towards meaningful, clinical findings, nowadays more and more researchers acquire skills essential to successfully address both clinical and basic science aspects of translational research (Costello, 2009). Hence, a primary goal of scientific research nowadays is to train a new generation of researchers in applying the latest biological discoveries to human health to enhance multidisciplinary research teams.

In accordance with the recent development, my PhD reflects a path that incorporated clinical science, public health, and basic biomedical research. While I was able to further deepen and improve my knowledge in the latter, I was for the first time exposed to important concepts, methods and approaches to research with human populations. As a result, I acquired a considerable amount of new and essential skills including protecting patient privacy and consent procedures, knowledge on how to design and run clinical trials, and understanding the regulatory obstacles of drug design and development. Additionally, I was enabled to think critically about how to analyze studies involving human subjects and data compared to research based on model organisms, and subsequently build a translational research project.

Another unique opportunity most basic researchers are typically not exposed to, was the ability to observe and work in clinical settings, and have direct contact with patients and their families. Interacting with patients with disorders relevant 
for pre-clinical research contributes to a more sophisticated understanding of translational science. It solidifies the significance of linking a researcher's own scientific research to their ultimate wish to progress human health. By attending patient consultations and annual CS research clinics, I was able to gain a more realistic perspective on rare genetic diseases by directly talking to patients and their parents about their path to diagnosis and daily challenges. Moreover, patients formulated their expectations of our research work which we were subsequently able to incorporate into our research plans. Such interactions with patients not only inform researchers on how to design their experiments or which question to ask next, it also provides an important frame of reference and positively affects their motivation.

In conclusion, exposing biomedical scientists to clinical research is highly beneficial as it assists them to think more broadly about implications of their research and connections between various disciplines. Lastly, researchers will most likely distinguish themselves from other candidates in their field in future career steps. 



\section{References}

Afifi, H. H., Abdel-Hamid, M. S., Eid, M. M., Mostafa, I. S., \& Abdel-Salam, G. M. H. (2016). De Novo Mutation in ABCC9 Causes Hypertrichosis Acromegaloid Facial Features Disorder. Pediatric Dermatology, 33(2), E109-E113. doi:10.1111/pde.12821

Afrikanova, T., Serruys, A. S., Buenafe, O. E., Clinckers, R., Smolders, I., de Witte, P. A., ... Esguerra, C. V. (2013). Validation of the zebrafish pentylenetetrazol seizure model: locomotor versus electrographic responses to antiepileptic drugs. Plos One, 8(1), e54166. doi:10.1371/journal.pone.0054166

Aldridge, K., Boyadjiev, S. A., Capone, G. T., DeLeon, V. B., \& Richtsmeier, J. T. (2005). Precision and error of three-dimensional phenotypic measures acquired from $3 \mathrm{dMD}$ photogrammetric images. Am J Med Genet A, 138A(3), 247-253. doi:10.1002/ajmg.a.30959

Ardehali, B., Nouraei, S. A., Van Dam, H., Dex, E., Wood, S., \& Nduka, C. (2007). Objective assessment of keloid scars with three-dimensional imaging: quantifying response to intralesional steroid therapy. Plast Reconstr Surg, 119(2), 556-561. doi:10.1097/01.prs.0000252505.52821.76

Armstrong, G. A., Liao, M., You, Z., Lissouba, A., Chen, B. E., \& Drapeau, P. (2016). Homology Directed Knockin of Point Mutations in the Zebrafish tardbp and fus Genes in ALS Using the CRISPR/Cas9 System. Plos One, 11(3), e0150188. doi:10.1371/journal.pone.0150188

Ashcroft, F. M. (2010). New uses for old drugs: neonatal diabetes and sulphonylureas. Cell Metab, 11(3), 179-181. doi:10.1016/j.cmet.2010.02.004

Bedell, V. M., Wang, Y., Campbell, J. M., Poshusta, T. L., Starker, C. G., Krug, R. G., 2nd, . . Ekker, S. C. (2012). In vivo genome editing using a high-efficiency TALEN system. Nature, 491(7422), 114-118. doi:10.1038/nature 11537

Bienengraeber, M., Olson, T. M., Selivanov, V. A., Kathmann, E. C., O'Cochlain, F., Gao, F., .. Terzic, A. (2004). ABCC9 mutations identified in human dilated cardiomyopathy disrupt catalytic KATP channel gating. Nat Genet, 36(4), 382-387. doi:10.1038/ng1329

Brownstein, C. A., Towne, M. C., Luquette, L. J., Harris, D. J., Marinakis, N. S., Meinecke, P., ... Beggs, A. H. (2013). Mutation of KCNJ8 in a patient with Cantu syndrome with unique vascular abnormalities - support for the role of K(ATP) channels in this condition. Eur J Med Genet, 56(12), 678-682. doi:10.1016/j. ejmg.2013.09.009

Cahill, T. J., Ashrafian, H., \& Watkins, H. (2013). Genetic cardiomyopathies causing heart failure. Circ Res, 113(6), 660-675. doi:10.1161/CIRCRESAHA.113.300282

Camison, L., Bykowski, M., Lee, W. W., Carlson, J. C., Roosenboom, J., Goldstein, J. A., . . Weinberg, S. M. (2018). Validation of the Vectra $\mathrm{H} 1$ portable three-dimensional photogrammetry system for facial imaging. Int J Oral Maxillofac Surg, 47(3), 403-410. doi:10.1016/j.ijom.2017.08.008

Cantu, J. M., Garcia-Cruz, D., Sanchez-Corona, J., Hernandez, A., \& Nazara, Z. (1982). A distinct osteochondrodysplasia with hypertrichosis- Individualization of a probable autosomal recessive entity. Human Genetics, 60(1), 36-41. Retrieved from https://www.ncbi.nlm.nih.gov/pubmed/7076246

Carrasco, X., Castillo, S., Aravena, T., Rothhammer, P., \& Aboitiz, F. (2005). Williams syndrome: pediatric, neurologic, and cognitive development. Pediatr Neurol, 32(3), 166-172. doi:10.1016/j. pediatrneurol.2004.09.013

Chari, R., Mali, P., Moosburner, M., \& Church, G. M. (2015). Unraveling CRISPR-Cas9 genome engineering parameters via a library-on-library approach. Nat Methods, 12(9), 823-826. doi:10.1038/nmeth.3473 
Choi, J., Dong, L., Ahn, J., Dao, D., Hammerschmidt, M., \& Chen, J. N. (2007). FoxH1 negatively modulates flk1 gene expression and vascular formation in zebrafish. Developmental Biology, 304(2), 735-744. doi:10.1016/j.ydbio.2007.01.023

Chutkow, W. A., Makielski, J. C., Nelson, D. J., Burant, C. F., \& Fan, Z. (1999). Alternative splicing of sur2 Exon 17 regulates nucleotide sensitivity of the ATP-sensitive potassium channel. J Biol Chem, 274(19), 13656-13665. doi:10.1074/jbc.274.19.13656

Chutkow, W. A., Pu, J., Wheeler, M. T., Wada, T., Makielski, J. C., Burant, C. F., \& McNally, E. M. (2002). Episodic coronary artery vasospasm and hypertension develop in the absence of Sur2 K(ATP) channels. J Clin Invest, 110(2), 203-208. doi:10.1172/JCI15672

Chutkow, W. A., Samuel, V., Hansen, P. A., Pu, J. L., Valdivia, C. R., Makielski, J. C., \& Burant, C. F. (2001). Disruption of Sur2-containing K-ATP channels enhances insulin-stimulated glucose uptake in skeletal muscle. Proceedings of the National Academy of Sciences of the United States of America, 98(20), 11760-11764. doi:DOl 10.1073/pnas.201390398

Colon, C., Ortolano, S., Melcon-Crespo, C., Alvarez, J. V., Lopez-Suarez, O. E., Couce, M. L., \& FernandezLorenzo, J. R. (2017). Newborn screening for Fabry disease in the north-west of Spain. Eur J Pediatr, 176(8), 1075-1081. doi:10.1007/s00431-017-2950-8

Concolino, D., Formicola, S., Camera, G., \& Strisciuglio, P. (2000). Congenital hypertrichosis, cardiomegaly, and osteochondrodysplasia (Cantu syndrome): A new case with unusual radiological findings. American Journal of Medical Genetics, 92(3), 191-194. doi:Doi 10.1002/(Sici)10968628(20000529)92:3<191::Aid-Ajmg6>3.0.C0;2-K

Cooper, P. E., McClenaghan, C., Chen, X., Stary-Weinzinger, A., \& Nichols, C. G. (2017). Conserved functional consequences of disease-associated mutations in the slide helix of Kir6.1 and Kir6.2 subunits of the ATP-sensitive potassium channel. J Biol Chem, 292(42), 17387-17398. doi:10.1074/jbc.M117.804971

Cooper, P. E., Reutter, H., Woelfle, J., Engels, H., Grange, D. K., van Haaften, G., ... Nichols, C. G. (2014). Cantu syndrome resulting from activating mutation in the KCNJ8 gene. Hum Mutat, 35(7), 809-813. doi:10.1002/humu.22555

Cooper, P. E., Sala-Rabanal, M., Lee, S. J., \& Nichols, C. G. (2015). Differential mechanisms of Cantu syndrome-associated gain of function mutations in the ABCC9 (SUR2) subunit of the KATP channel. J Gen Physiol, 146(6), 527-540. doi:10.1085/jgp.201511495

Cox-Brinkman, J., Vedder, A., Hollak, C., Richfield, L., Mehta, A., Orteu, K., .. Hammond, P. (2007). Threedimensional face shape in Fabry disease. Eur J Hum Genet, 15(5), 535-542. doi:10.1038/sj.ejhg.5201798

Czeschik, J. C., Voigt, C., Goecke, T. O., Ludecke, H. J., Wagner, N., Kuechler, A., \& Wieczorek, D. (2013). Wide clinical variability in conditions with coarse facial features and hypertrichosis caused by mutations in ABCC9. Am J Med Genet A, 161A(2), 295-300. doi:10.1002/ajmg.a.35735

Deacon, R. M. (2013). Measuring the strength of mice. J Vis Exp(76). doi:10.3791/2610

Dougherty, J. D., Maloney, S. E., Wozniak, D. F., Rieger, M. A., Sonnenblick, L., Coppola, G., ... Heintz, N. (2013). The disruption of Celf6, a gene identified by translational profiling of serotonergic neurons, results in autism-related behaviors. J Neurosci, 33(7), 2732-2753. doi:10.1523/JNEUROSCI.4762-12.2013

Engels, H., Bosse, K., Ehrbrecht, A., Zahn, S., Hoischen, A., Propping, P., ... Reutter, H. (2002). Further case of Cantu syndrome: Exclusion of cryptic subtelomeric chromosome aberrations. American Journal of Medical Genetics, 111(2), 205-209. doi:10.1002/ajmg.10560

Enright, P. L., \& Sherrill, D. L. (1998). Reference equations for the six-minute walk in healthy adults. Am J Respir Crit Care Med, 158(5 Pt 1), 1384-1387. doi:10.1164/ajrccm.158.5.9710086 
Flagg, T. P., Enkvetchakul, D., Koster, J. C., \& Nichols, C. G. (2010). Muscle KATP channels: recent insights to energy sensing and myoprotection. Physiol Rev, 90(3), 799-829. doi:10.1152/physrev.00027.2009

Foster, M. N., \& Coetzee, W. A. (2016). KATP Channels in the Cardiovascular System. Physiol Rev, 96(1), 177252. doi:10.1152/physrev.00003.2015

Franchignoni, F., Horak, F., Godi, M., Nardone, A., \& Giordano, A. (2010). Using psychometric techniques to improve the Balance Evaluation Systems Test: the mini-BESTest. J Rehabil Med, 42(4), 323-331. doi:10.2340/16501977-0537

Fryssira, H., Psoni, S., Amenta, S., Tsoutsou, E., Sofocleous, C., Manolakos, E., ... Czeschik, J. C. (2017). Cantu Syndrome Associated with Ovarian Agenesis. Mol Syndromol, 8(4), 206-210. doi:10.1159/000471247

Gagnon, J. A., Valen, E., Thyme, S. B., Huang, P., Akhmetova, L., Pauli, A., .. Schier, A. F. (2014). Efficient mutagenesis by Cas9 protein-mediated oligonucleotide insertion and large-scale assessment of singleguide RNAs. Plos One, 9(5), e98186. doi:10.1371/journal.pone.0098186

Gao, J., Xu, D., Sabat, G., Valdivia, H., Xu, W., \& Shi, N. Q. (2014). Disrupting KATP channels diminishes the estrogen-mediated protection in female mutant mice during ischemia-reperfusion. Clin Proteomics, 11(1), 19. doi:10.1186/1559-0275-11-19

Garcia-Cruz, D., Mampel, A., Echeverria, M. I., Vargas, A. L., Castaneda-Cisneros, G., Davalos-Rodriguez, N., . .. Sanchez-Corona, J. (2011). Cantu syndrome and lymphoedema. Clinical Dysmorphology, 20(1), 32-37. doi:10.1097/MCD.0b013e32833d015c

Garcia-Gonzaleza, C. L., Garcia-Cruz, D., Garcia-Cruz, M. O., Castaneda-Cisneros, G., Garcia-Ortiz, J. E., Orozco-Gutierrez, M. H., \& Sanchez-Corona, J. (2012). A familial case of Cantu craniofaciofronto digital syndrome. Clinical Dysmorphology, 21(3), 162-166. doi:10.1097/MCD.0b013e328353a082

GarciaCruz, D., SanchezCorona, J., Nazara, Z., GarciaCruz, M. O., Figuera, L. E., Castaneda, V., \& Cantu, J. M. (1997). Congenital hypertrichosis, osteochondrodysplasia, and cardiomegaly: Further delineation of a new genetic syndrome. American Journal of Medical Genetics, 69(2), 138-151. doi:Doi 10.1002/ (Sici)1096-8628(19970317)69:2<138::Aid-Ajmg5>3.0.C0;2-L

Geiger, R., Strasak, A., Treml, B., Gasser, K., Kleinsasser, A., Fischer, V., .. Stein, J. I. (2007). Six-minute walk test in children and adolescents. J Pediatr, 150(4), 395-399, 399 e391-392. doi:10.1016/j. jpeds.2006.12.052

Gloyn, A. L., Pearson, E. R., Antcliff, J. F., Proks, P., Bruining, G. J., Slingerland, A. S., . . Hattersley, A. T. (2004). Activating mutations in the gene encoding the ATP-sensitive potassium-channel subunit Kir6.2 and permanent neonatal diabetes. N Engl J Med, 350(18), 1838-1849. doi:10.1056/NEJMoa032922

Grady, R. M., Wozniak, D. F., Ohlemiller, K. K., \& Sanes, J. R. (2006). Cerebellar synaptic defects and abnormal motor behavior in mice lacking alpha- and beta-dystrobrevin. J Neurosci, 26(11), 2841-2851. doi:10.1523/JNEUROSCI.4823-05.2006

Grange, D. K., Lorch, S. M., Cole, P. L., \& Singh, G. K. (2006). Cantu syndrome in a woman and her two daughters: Further confirmation of autosomal dominant inheritance and review of the cardiac manifestations. American Journal of Medical Genetics Part A, 140a(15), 1673-1680. doi:10.1002/ajmg.a.31348

Grange, D. K., Nichols, C. G., \& Singh, G. K. (2014). Cantu Syndrome and Related Disorders. In R. A. Pagon, M. P. Adam, H. H. Ardinger, S. E. Wallace, A. Amemiya, L. J. H. Bean, T. D. Bird, N. Ledbetter, H. C. Mefford, R. J. H. Smith, \& K. Stephens (Eds.), GeneReviews(R). Seattle (WA).

Grange, D. K., Roessler, H. I., McClenaghan, C., Duran, K., Shields, K., Remedi, M. S., ... van Haaften, G. (2019). Cantu syndrome: Findings from 74 patients in the International Cantu Syndrome Registry. Am J Med Genet C Semin Med Genet, 181(4), 658-681. doi:10.1002/ajmg.c.31753 
Graziadio, C., Rosa, R. F., Rosa, R. C., Zen, P. R., Flores, J. A., \& Paskulin, G. A. (2011). Short-term followup of a Brazilian patient with Cantu syndrome. Am J Med Genet A, 155A(5), 1184-1188. doi:10.1002/ ajmg.a.33904

Gurovich, Y., Hanani, Y., Bar, O., Nadav, G., Fleischer, N., Gelbman, D., ... Gripp, K. W. (2019). Identifying facial phenotypes of genetic disorders using deep learning. Nat Med, 25(1), 60-64. doi:10.1038/ s41591-018-0279-0

Hajeer, M. Y., Millett, D. T., Ayoub, A. F., \& Siebert, J. P. (2004). Applications of 3D imaging in orthodontics: part I. J Orthod, 31(1), 62-70. doi:10.1179/146531204225011346

Hammond, P. (2007). The use of 3D face shape modelling in dysmorphology. Archives of Disease in Childhood, 92(12), 1120-1126.

Hammond, P., Hutton, T. J., Allanson, J. E., Campbell, L. E., Hennekam, R. C., Holden, S., ... Winter, R. M. (2004). 3D analysis of facial morphology. Am J Med Genet A, 126A(4), 339-348. doi:10.1002/ajmg.a.20665

Hammond, P., \& Suttie, M. (2012). Large-scale objective phenotyping of 3D facial morphology. Hum Mutat, 33(5), 817-825. doi:10.1002/humu.22054

Harakalova, M., van Harssel, J. J., Terhal, P. A., van Lieshout, S., Duran, K., Renkens, I., . . Cuppen, E. (2012). Dominant missense mutations in ABCC9 cause Cantu syndrome. Nat Genet, 44(7), 793-796. doi:10.1038/ng.2324

Hiraki, Y., Miyatake, S., Hayashidani, M., Nishimura, Y., Matsuura, H., Kamada, M., ... Matsumoto, N. (2014). Aortic Aneurysm and Craniosynostosis in a Family With Cantu Syndrome. American Journal of Medical Genetics Part A, 164(1), 231-236. doi:10.1002/ajmg.a.36228

Hoage, T., Ding, Y., \& Xu, X. (2012). Quantifying cardiac functions in embryonic and adult zebrafish. Methods Mol Biol, 843, 11-20. doi:10.1007/978-1-61779-523-7_2

Hopman, S. M., Merks, J. H., Suttie, M., Hennekam, R. C., \& Hammond, P. (2014). Face shape differs in phylogenetically related populations. Eur J Hum Genet, 22(11), 1268-1271. doi:10.1038/ejhg.2013.289

Hu, J. H., Miller, S. M., Geurts, M. H., Tang, W., Chen, L., Sun, N., .. Liu, D. R. (2018). Evolved Cas9 variants with broad PAM compatibility and high DNA specificity. Nature, 556(7699), 57-63. doi:10.1038/ nature 26155

Huang, Y., McClenaghan, C., Harter, T. M., Hinman, K., Halabi, C. M., Matkovich, S. J., ... Nichols, C. G. (2018). Cardiovascular consequences of KATP overactivity in Cantu syndrome. JCI Insight, 3(15). doi:10.1172I jci.insight. 121153

Hutton, T. J., Buxton, B. F., Hammond, P., \& Potts, H. W. (2003). Estimating average growth trajectories in shape-space using kernel smoothing. IEEE Trans Med Imaging, 22(6), 747-753. doi:10.1109/ TMI.2003.814784

Hwang, W. Y., Fu, Y., Reyon, D., Maeder, M. L., Tsai, S. Q., Sander, J. D., .. Joung, J. K. (2013). Efficient genome editing in zebrafish using a CRISPR-Cas system. Nature Biotechnology, 31(3), 227-229. doi:10.1038/ nbt.2501

Inagaki, N., Gonoi, T., Clement, J. P., Wang, C. Z., Aguilar-Bryan, L., Bryan, J., \& Seino, S. (1996). A family of sulfonylurea receptors determines the pharmacological properties of ATP-sensitive $\mathrm{K}+$ channels. Neuron, 16(5), 1011-1017. doi:10.1016/s0896-6273(00)80124-5

Isomoto, S., Kondo, C., Yamada, M., Matsumoto, S., Higashiguchi, O., Horio, Y., ... Kurachi, Y. (1996). A novel sulfonylurea receptor forms with BIR (Kir6.2) a smooth muscle type ATP-sensitive K+ channel. J Biol Chem, 271(40), 24321-24324. doi:10.1074/jbc.271.40.24321 
Jinek, M., Chylinski, K., Fonfara, I., Hauer, M., Doudna, J. A., \& Charpentier, E. (2012). A programmable dual-RNA-guided DNA endonuclease in adaptive bacterial immunity. Science, 337(6096), 816-821. doi:10.1126/science.1225829

Kaler, S. G., Patrinos, M. E., Lambert, G. H., Myers, T. F., Karlman, R., \& Anderson, C. L. (1987). Hypertrichosis and congenital anomalies associated with maternal use of minoxidil. Pediatrics, 79(3), 434-436. Retrieved from https://www.ncbi.nlm.nih.gov/pubmed/3547299

Kane, G. C., Behfar, A., Yamada, S., Perez-Terzic, C., O'Cochlain, F., Reyes, S., ... Terzic, A. (2004). ATPsensitive $\mathrm{K}+$ channel knockout compromises the metabolic benefit of exercise training, resulting in cardiac deficits. Diabetes, 53 Suppl 3, S169-175. doi:10.2337/diabetes.53.suppl_3.s169

Kawano, T., Zoga, V., McCallum, J. B., Wu, H. E., Gemes, G., Liang, M. Y., ... Sarantopoulos, C. D. (2009). ATP-sensitive potassium currents in rat primary afferent neurons: biophysical, pharmacological properties, and alterations by painful nerve injury. Neuroscience, 162(2), 431-443. doi:10.1016/j. neuroscience.2009.04.076

Kini, U., \& Clayton-Smith, J. (2004). Acromegaloid facial appearance syndrome: a further case report. Clinical Dysmorphology, 13(4), 251-253. doi:10.1097/00019605-200410000-00010

Knapik, E. W., Goodman, A., Ekker, M., Chevrette, M., Delgado, J., Neuhauss, S., ... Jacob, H. J. (1998). A microsatellite genetic linkage map for zebrafish (Danio rerio). Nat Genet, 18(4), 338-343. doi:10.1038/ ng0498-338

Kobayashi, D., Cook, A. L., \& Williams, D. A. (2010). Pulmonary Hypertension Secondary to Partial Pulmonary Venous Obstruction in a Child With Cantu Syndrome. Pediatric Pulmonology, 45(7), 727-729. doi:10.1002/ppul.21215

Koh, S. D., Bradley, K. K., Rae, M. G., Keef, K. D., Horowitz, B., \& Sanders, K. M. (1998). Basal activation of ATP-sensitive potassium channels in murine colonic smooth muscle cell. Biophys J, 75(4), 1793-1800. doi:10.1016/S0006-3495(98)77621-0

Koster, J. C., Marshall, B. A., Ensor, N., Corbett, J. A., \& Nichols, C. G. (2000). Targeted overactivity of beta cell K(ATP) channels induces profound neonatal diabetes. Cell, 100(6), 645-654. doi:10.1016/s0092$8674(00) 80701-1$

Kovacs, L., Zimmermann, A., Wawrzyn, H., Schwenzer, K., Seitz, H., Tille, C., .. Biemer, E. (2005). Computer aided surgical reconstruction after complex facial burn injuries -- opportunities and limitations. Burns, 31(1), 85-91. doi:10.1016/j.burns.2004.07.010

Kurban, M., Kim, C. A., Kiuru, M., Fantauzzo, K., Cabral, R., Abbas, 0., .. Christiano, A. M. (2011). Copy Number Variations on Chromosome 4q26-27 Are Associated with Cantu Syndrome. Dermatology, 223(4), 316-320. doi:10.1159/000333800

Lazalde, B., Sanchez-Urbina, R., Nuno-Arana, I., Bitar, W. E., \& de Lourdes Ramirez-Duenas, M. (2000). Autosomal dominant inheritance in Cantu syndrome (congenital hypertrichosis, osteochondrodysplasia, and cardiomegaly). Am J Med Genet, 94(5), 421-427. Retrieved from https://www.ncbi.nlm.nih.gov/ pubmed/11050630

Leon Guerrero, C. R., Pathak, S., Grange, D. K., Singh, G. K., Nichols, C. G., Lee, J. M., \& Vo, K. D. (2016). Neurologic and neuroimaging manifestations of Cantu syndrome: A case series. Neurology, 87(3), 270276. doi:10.1212/WNL.0000000000002861 
Levin, M. D., Singh, G. K., Zhang, H. X., Uchida, K., Kozel, B. A., Stein, P. K., .. Nichols, C. G. (2016). K-ATP channel gain-of-function leads to increased myocardial L-type Ca2+ current and contractility in Cantu syndrome. Proceedings of the National Academy of Sciences of the United States of America, 113(24), 6773-6778. doi:10.1073/pnas.1606465113

Li, H., \& Durbin, R. (2009). Fast and accurate short read alignment with Burrows-Wheeler transform. Bioinformatics, 25(14), 1754-1760. doi:10.1093/bioinformatics/btp324

Liss, B., \& Roeper, J. (2001). Molecular physiology of neuronal K-ATP channels (review). Mol Membr Biol, 18(2), 117-127. Retrieved from https://www.ncbi.nlm.nih.gov/pubmed/11463204

Ma, A., Gurnasinghani, S., Kirk, E. P., McClenaghan, C., Singh, G. K., Grange, D. K., .. Nichols, C. G. (2019). Glibenclamide treatment in a Cantu syndrome patient with a pathogenic $A B C C 9$ gain-of-function variant: Initial experience. Am J Med Genet A. doi:10.1002/ajmg.a.61200

Mannhold, R. (2004). KATP channel openers: structure-activity relationships and therapeutic potential. Med Res Rev, 24(2), 213-266. doi:10.1002/med.10060

Marques, P., Spencer, R., Morrison, P. J., Carr, I. M., Dang, M. N., Bonthron, D. T., ... Korbonits, M. (2018). Cantu syndrome with coexisting familial pituitary adenoma. Endocrine, 59(3), 677-684. doi:10.1007/ s12020-017-1497-9

Martin, G. M., Yoshioka, C., Rex, E. A., Fay, J. F., Xie, Q., Whorton, M. R., .. Shyng, S. L. (2017). Cryo-EM structure of the ATP-sensitive potassium channel illuminates mechanisms of assembly and gating. Elife, 6. doi:10.7554/eLife.24149

McClenaghan, C., Hanson, A., Sala-Rabanal, M., Roessler, H. I., Josifova, D., Grange, D. K., ... Nichols, C. G. (2017). Cantu syndrome-associated SUR2 (ABCC9) mutations in distinct structural domains result in KATP channel gain-of-function by differential mechanisms. J Biol Chem. doi:10.1074/jbc.RA117.000351

McClenaghan, C., Huang, Y., Yan, Z., Harter, T., Halabi, C. M., Chalk, R., .. Nichols, C. G. (2019). Glibenclamide reverses cardiovascular abnormalities of Cantu Syndrome driven by KATP channel overactivity. J Clin Invest. doi:10.1172/JCI130571

McKenna, A., Hanna, M., Banks, E., Sivachenko, A., Cibulskis, K., Kernytsky, A., ... DePristo, M. A. (2010). The Genome Analysis Toolkit: a MapReduce framework for analyzing next-generation DNA sequencing data. Genome Res, 20(9), 1297-1303. doi:10.1101/gr.107524.110

Mehta, P. K., Mamdani, B., Shansky, R. M., Mahurkar, S. D., \& Dunea, G. (1975). Severe hypertension. Treatment with minoxidil. JAMA, 233(3), 249-252. Retrieved from https://www.ncbi.nlm.nih.gov/ pubmed/1173832

Meijering, E., Dzyubachyk, 0., \& Smal, I. (2012). Methods for cell and particle tracking. Methods Enzymol, 504, 183-200. doi:10.1016/B978-0-12-391857-4.00009-4

Menke, L. A., study, D. D. D., Gardeitchik, T., Hammond, P., Heimdal, K. R., Houge, G., ... Hennekam, R. C. (2018). Further delineation of an entity caused by CREBBP and EP300 mutations but not resembling Rubinstein-Taybi syndrome. Am J Med Genet A, 176(4), 862-876. doi:10.1002/ajmg.a.38626

Miki, T., Minami, K., Zhang, L., Morita, M., Gonoi, T., Shiuchi, T., .. Seino, S. (2002). ATP-sensitive potassium channels participate in glucose uptake in skeletal muscle and adipose tissue. Am J Physiol Endocrinol Metab, 283(6), E1178-1184. doi:10.1152/ajpend0.00313.2002

Najmabadi, H., Hu, H., Garshasbi, M., Zemojtel, T., Abedini, S. S., Chen, W., ... Ropers, H. H. (2011). Deep sequencing reveals 50 novel genes for recessive cognitive disorders. Nature, 478(7367), 57-63. doi:10.1038/nature10423 
Nelson, P. T., Estus, S., Abner, E. L., Parikh, I., Malik, M., Neltner, J. H., .. Fardo, D. W. (2014). ABCC9 gene polymorphism is associated with hippocampal sclerosis of aging pathology. Acta Neuropathol, 127(6), 825-843. doi:10.1007/s00401-014-1282-2

Nelson, P. T., Jicha, G. A., Wang, W. X., Ighodaro, E., Artiushin, S., Nichols, C. G., \& Fardo, D. W. (2015). ABCC9/SUR2 in the brain: Implications for hippocampal sclerosis of aging and a potential therapeutic target. Ageing Res Rev, 24(Pt B), 111-125. doi:10.1016/j.arr.2015.07.007

Nelson, P. T., Wang, W. X., Wilfred, B. R., Wei, A., Dimayuga, J., Huang, Q., . . Fardo, D. W. (2015). Novel human ABCC9/SUR2 brain-expressed transcripts and an eQTL relevant to hippocampal sclerosis of aging. J Neurochem, 134(6), 1026-1039. doi:10.1111/jnc.13202

Nevin, N. C., Mulholland, H. C., \& Thomas, P. S. (1996). Congenital hypertrichosis, cardiomegaly and mild osteochondrodysplasia. Am J Med Genet, 66(1), 33-38. doi:10.1002/(SICI)10968628(19961202)66:1<33::AID-AJMG8>3.0.C0;2-X

Nichols, C. G. (2006). KATP channels as molecular sensors of cellular metabolism. Nature, 440(7083), 470 476. doi:10.1038/nature04711

O'brien, J. J., \& Ririe, D. G. (2008). Anesthetic experience in a patient with Cantu syndrome. Pediatric Anesthesia, 18(12), 1255-1257. doi:10.1111/j.1460-9592.2008.02760.x

Olson, T. M., Alekseev, A. E., Moreau, C., Liu, X. K., Zingman, L. V., Miki, T., ... Terzic, A. (2007). KATP channel mutation confers risk for vein of Marshall adrenergic atrial fibrillation. Nat Clin Pract Cardiovasc Med, 4(2), 110-116. doi:10.1038/ncpcardio0792

Park, J. Y., Koo, S. H., Jung, Y. J., Lim, Y. J., \& Chung, M. L. (2014). A Patient With Cantu Syndrome Associated With Fatal Bronchopulmonary Dysplasia and Pulmonary Hypertension. American Journal of Medical Genetics Part A, 164(8), 2118-2120. doi:10.1002/ajmg.a.36563

Pepeu, G. (2004). Mild cognitive impairment: animal models. Dialogues Clin Neurosci, 6(4), 369-377. Retrieved from https://www.ncbi.nlm.nih.gov/pubmed/22034045

Poss, K. D., Wilson, L. G., \& Keating, M. T. (2002). Heart regeneration in zebrafish. Science, 298(5601), 2188-2190. doi:10.1126/science.1077857

Ran, F. A., Hsu, P. D., Wright, J., Agarwala, V., Scott, D. A., \& Zhang, F. (2013). Genome engineering using the CRISPR-Cas9 system. Nature Protocols, 8(11), 2281-2308. doi:10.1038/nprot.2013.143

Razani, B., Zhang, H., Schulze, P. C., Schilling, J. D., Verbsky, J., Lodhi, I. J., . . Semenkovich, C. F. (2011). Fatty acid synthase modulates homeostatic responses to myocardial stress. J Biol Chem, 286(35), 30949-30961. doi:10.1074/jbc.M111.230508

Robertson, S. P., Kirk, E., Bernier, F., Brereton, J., Turner, A., \& Bankier, A. (1999). Congenital hypertrichosis, osteochondrodysplasia, and cardiomegaly: Cantu syndrome. American Journal of Medical Genetics, 85(4), 395-402. doi:Doi 10.1002/(Sici)1096-8628(19990806)85:4<395::Aid-Ajmg17>3.0.Co;2-I

Roessler, H. I., Volker-Touw, C. M. L., Terhal, P. A., van Haaften, G., \& van Haelst, M. M. (2018). Cantu syndrome, the changing phenotype: a report of the two oldest Dutch patients. Clinical Dysmorphology, 27(3), 78-83. doi:10.1097/MCD.0000000000000219

Rosser, E. M., Kaariainen, H., Hurst, J. A., Baraitser, M., Hall, C. M., Clayton, P., \& Leonard, J. V. (1998). Three patients with the osteochondrodysplasia and hypertrichosis syndrome - Cantu syndrome. Clinical Dysmorphology, 7(2), 79-85. doi:Doi 10.1097/00019605-199804000-00001

Schaefer, M. L., Wong, S. T., Wozniak, D. F., Muglia, L. M., Liauw, J. A., Zhuo, M., ... Muglia, L. J. (2000). Altered stress-induced anxiety in adenylyl cyclase type VIII-deficient mice. J Neurosci, 20(13), 48094820. Retrieved from https://www.ncbi.nlm.nih.gov/pubmed/10864938 
Scurr, I., Wilson, L., Lees, M., Robertson, S., Kirk, E., Turner, A., .. Smithson, S. (2011). Cantu syndrome: report of nine new cases and expansion of the clinical phenotype. Am J Med Genet A, 155A(3), 508-518. doi:10.1002/ajmg.a.33885

Shi, N. Q., Ye, B., \& Makielski, J. C. (2005). Function and distribution of the SUR isoforms and splice variants. J Mol Cell Cardiol, 39(1), 51-60. doi:10.1016/j.yjmcc.2004.11.024

Stein, L. R., Wozniak, D. F., Dearborn, J. T., Kubota, S., Apte, R. S., Izumi, Y., ... Imai, S. (2014). Expression of Nampt in hippocampal and cortical excitatory neurons is critical for cognitive function. J Neurosci, 34(17), 5800-5815. doi:10.1523/JNEUROSCI.4730-13.2014

Stoller, D., Kakkar, R., Smelley, M., Chalupsky, K., Earley, J. U., Shi, N. Q., . . McNally, E. M. (2007). Mice lacking sulfonylurea receptor 2 (SUR2) ATP-sensitive potassium channels are resistant to acute cardiovascular stress. J Mol Cell Cardiol, 43(4), 445-454. doi:10.1016/j.yjmcc.2007.07.058

Tajan, M., Paccoud, R., Branka, S., Edouard, T., \& Yart, A. (2018). The RASopathy Family: Consequences of Germline Activation of the RAS/MAPK Pathway. Endocr Rev, 39(5), 676-700. doi:10.1210/er.201700232

Tan, T. Y., Bankier, A., Slater, H. R., Northrop, E. L., Zacharin, M., \& Savarirayan, R. (2005). A patient with monosomy 1p36, atypical features and phenotypic similarities with Cantu Syndrome. American Journal of Medical Genetics Part A, 139a(3), 216-220. doi:10.1002/ajmg.a.31013

Tessadori, F., Roessler, H. I., Savelberg, S. M. C., Chocron, S., Kamel, S. M., Duran, K. J., .. Bakkers, J. (2018). Effective CRISPR/Cas9-based nucleotide editing in zebrafish to model human genetic cardiovascular disorders. Disease Models \& Mechanisms, 11(10). doi:10.1242/dmm.035469

Thabet, M., Miki, T., Seino, S., \& Renaud, J. M. (2005). Treadmill running causes significant fiber damage in skeletal muscle of KATP channel-deficient mice. Physiol Genomics, 22(2), 204-212. doi:10.1152/ physiolgenomics.00064.2005

Thomas, P., Ye, Y., \& Lightner, E. (1996). Mutation of the pancreatic islet inward rectifier Kir6.2 also leads to familial persistent hyperinsulinemic hypoglycemia of infancy. Hum Mol Genet, 5(11), 1809-1812. doi:10.1093/hmg/5.11.1809

Thomas, P. M., Cote, G. J., Wohllk, N., Haddad, B., Mathew, P. M., Rabl, W., .. Bryan, J. (1995). Mutations in the sulfonylurea receptor gene in familial persistent hyperinsulinemic hypoglycemia of infancy. Science, 268(5209), 426-429. doi:10.1126/science. 7716548

Toma, A. M., Zhurov, A., Playle, R., Ong, E., \& Richmond, S. (2009). Reproducibility of facial soft tissue landmarks on 3D laser-scanned facial images. Orthod Craniofac Res, 12(1), 33-42. doi:10.1111/j.16016343.2008.01435.x

Tomona, N., Smith, A. C., Guadagnini, J. P., \& Hart, T. C. (2006). Craniofacial and dental phenotype of SmithMagenis syndrome. Am J Med Genet A, 140(23), 2556-2561. doi:10.1002/ajmg.a.31371

Tricarico, D., Selvaggi, M., Passantino, G., De Palo, P., Dario, C., Centoducati, P., .. Zizzo, N. (2016). ATP Sensitive Potassium Channels in the Skeletal Muscle Function: Involvement of the KCNJ11 (Kir6.2) Gene in the Determination of Mechanical Warner Bratzer Shear Force. Front Physiol, 7, 167. doi:10.3389/ fphys.2016.00167

van Bon, B. W., Gilissen, C., Grange, D. K., Hennekam, R. C., Kayserili, H., Engels, H., .. Hoischen, A. (2012). Cantu syndrome is caused by mutations in ABCC9. American Journal of Human Genetics, 90(6), 10941101. doi:10.1016/j.ajhg.2012.04.014 
Van der Auwera, G. A., Carneiro, M. O., Hartl, C., Poplin, R., Del Angel, G., Levy-Moonshine, A., .. DePristo, M. A. (2013). From FastQ data to high confidence variant calls: the Genome Analysis Toolkit best practices pipeline. Curr Protoc Bioinformatics, 43, 1110 11-33. doi:10.1002/0471250953.bi1110s43

van der Donk, R., Jansen, S., Schuurs-Hoeijmakers, J. H. M., Koolen, D. A., Goltstein, L., Hoischen, A., . . Hehir-Kwa, J. Y. (2019). Next-generation phenotyping using computer vision algorithms in rare genomic neurodevelopmental disorders. Genet Med, 21 (8), 1719-1725. doi:10.1038/s41436-018-0404-y

van der Zwaag, P. A., van Rijsingen, I. A., Asimaki, A., Jongbloed, J. D., van Veldhuisen, D. J., Wiesfeld, A. C., ... van Tintelen, J. P. (2012). Phospholamban R14del mutation in patients diagnosed with dilated cardiomyopathy or arrhythmogenic right ventricular cardiomyopathy: evidence supporting the concept of arrhythmogenic cardiomyopathy. Eur J Heart Fail, 14(11), 1199-1207. doi:10.1093/eurjhf/hfs119

Vidal-Taboada, J. M., Pugliese, M., Salvado, M., Gamez, J., Mahy, N., \& Rodriguez, M. J. (2018). KATP Channel Expression and Genetic Polymorphisms Associated with Progression and Survival in Amyotrophic Lateral Sclerosis. Mol Neurobiol, 55(10), 7962-7972. doi:10.1007/s12035-018-0970-7

Vissers, L. E., \& Veltman, J. A. (2015). Standardized phenotyping enhances Mendelian disease gene identification. Nat Genet, 47(11), 1222-1224. doi:10.1038/ng.3425

von der Weid, P. Y., Lee, S., Imtiaz, M. S., Zawieja, D. C., \& Davis, M. J. (2014). Electrophysiological properties of rat mesenteric lymphatic vessels and their regulation by stretch. Lymphat Res Biol, 12(2), 66-75. doi:10.1089/lrb.2013.0045

Westerfield, M. (1993). The Zebrafish Book: A Guide for the Laboratory Use of Zebrafish

Danio (Brachydanio). University of Oregon Press, Oregon.

Wozniak, D. F., Hartman, R. E., Boyle, M. P., Vogt, S. K., Brooks, A. R., Tenkova, T., ... Muglia, L. J. (2004). Apoptotic neurodegeneration induced by ethanol in neonatal mice is associated with profound learning/ memory deficits in juveniles followed by progressive functional recovery in adults. Neurobiol Dis, 17(3), 403-414. doi:10.1016/j.nbd.2004.08.006

Ye, B., Kroboth, S. L., Pu, J. L., Sims, J. J., Aggarwal, N. T., McNally, E. M., ... Shi, N. Q. (2009). Molecular identification and functional characterization of a mitochondrial sulfonylurea receptor 2 splice variant generated by intraexonic splicing. Circ Res, 105(11), 1083-1093. doi:10.1161/CIRCRESAHA.109.195040

Zhang, H. X., Akrouh, A., Kurata, H. T., Remedi, M. S., Lawton, J. S., \& Nichols, C. G. (2011). HMR 1098 is not an SUR isotype specific inhibitor of heterologous or sarcolemmal K ATP channels. J Mol Cell Cardiol, 50(3), 552-560. doi:10.1016/j.yjmcc.2010.12.011

Zoga, V., Kawano, T., Liang, M. Y., Bienengraeber, M., Weihrauch, D., McCallum, B., ... Sarantopoulos, C. (2010). KATP channel subunits in rat dorsal root ganglia: alterations by painful axotomy. Mol Pain, 6, 6 . doi:10.1186/1744-8069-6-6 
Appendix 1 


\section{Resources}

i https://eur-lex.europa.eu/legal-content/EN/

TXT/?uri=CELEX\%3A32000R0141

ii https://www.fda.gov/media/99546/download

iii https://omim.org/

iv https://www.ema.europa.eu/en/documents/other/orphan-medicinesfigures-2000-2019_en.pdf

$v$ https://www.fda.gov/media/134493/download

vi https://www.bio.org/sites/default/files/legacy/bioorg/docs/Clinical\%20 Development $\% 20$ Success\%20Rates\%202006-2015\%20-\%20BI0,\%20 Biomedtracker, \%20Amplion\%202016.pdf 


\section{Abbreviations}

2D: Two-dimensional

3D: Three-dimensional

6MWT: 6-min walking test

ACE: Angiotensin converting enzyme

ACTH: Adrenocorticotropic hormone

ADHD: Attention deficit hyperactivity disorder

AF: Atrial fibrillation

AFA: Acromegaloid facial appearance

AFF: Acromegaloid facial features

AFOG: Acid Fuchsin Orange

Al: Artificial intelligence

AIMS: ABCC9-related Intellectual disability Myopathy Syndrome

ANOVA: Analysis of variance

APC: Aorto-pulmonary collateral artery

ATP: Adenosine triphosphate

AV: Atrioventricular

BCA: Basal communicating artery

BG: Blood glucose

BMI: Body mass index

BW: Body weight

Cas9: CRISPR associated protein 9

CaV: Voltage-gated calcium channel

cDNA: Complementary deoxyribonucleic acid

CHG: Comparative genomic hybridization

$\mathrm{CHI}$ : Congenital hyperinsulism

CNV: Copy number variant

CRISPR: Clustered regularly interspaced short palindromic repeats

CS: Cantú syndrome

CT: Computed Tomography

DAPI: 4',6-diamidino-2-phenylindole

dCas9: catalytically inactive Cas9

DCM: Dilated cardiomyopathy

DMPK: Drug Metabolism and Pharmacokinetics

DNA: Deoxyribonucleic acid

dpf: days after fertilization

DSM: Dense surface model

ECG: Electrocardiogram

EEG: Electroencephalogram 
EF: Ejection fraction

EMG: Electromyography

ENT: Ears, nose, and throat

ERN: European Reference Network

Exac: Exome Aggregation Consortium

FAC: Fractional area change

FAIR: Findable, Accessible, Interoperable, and Reusable

FD: Fabry disease

FDA: Food drug association

FLAIR: Weighted fluid-attenuated inversion recovery

FMRI: Functional magnetic resonance imaging

fps: frames per seconds

FSH: Follicle-stimulating hormone

FTI: Farnesyltransferase inhibitor

GATK: Genome Analysis Toolkit

GFP: Green fluorescent protein

GI: Gastrointestinal

gnomAD: Genome Aggregation Database

GoF: Gain-of-function

H\&E: Hematoxylin and Eosin

HAFF: Hypertrichosis with acromegaloid facial features

HDR: Homology-directed repair

HET: Heterozygous

HGPS: Hutchinson-Gilford Progeria Syndrome

hpf: hours post fertilization

HR: Homologous recombination

IC50: Half maximal inhibitory concentration

ICSR: International Cantú syndrome Registry

IDT: Integrated DNA Technologies

IL-1: Interleukin-1

ITI: Inter-trial interval

IVIG: Intraveneus immunoglobuline

KATP: ATP-sensitive potassium

$\mathrm{KI}$ : Knock-in

Kir: Inward-rectifier potassium channels

KO: Knock-out

LH: Luteinizing hormone

LMNA: Lamin A/C gene

LoF: Loss-of-function

LV: Left ventricular 
LVIDd: Left ventricular internal dimension in diastole LVM: Left ventricular mass

MAF: Minor allele frequency

MAP: Mean arterial pressure

MCA: Middle cerebral artery

mEDV: Elevated mean end-diastolic

mESV: Elevated mean end-systolic

miniBEST: Mini-Balance Evaluation System Test

ML: Machine learning

MPS: Mucopolysaccharidosis

MR: Magnetic resonance

MRA: Arterial magnetic resonance imaging

$M R I$ : Magnetic resonance imaging

mRNA: messenger RNA

MS222: Tricaine methanesulfonate

MWS: Muckle-Wells syndrome

NBD: Nucleotide-binding domain

NCS: Nerve conduction studies

NGS: Next generation sequencing

NHEJ: Non-homologous end-joining

NS: Noonan syndrome

NT-proBNP: $\mathrm{N}$-terminal pro b-type natriuretic peptide

OCD: Obsessive compulsive disorder

OMIM: Online Mendelian Inheritance in Man

PAG: Patient advocacy groups

PAM: Protospacer adjacent motif

PC1: First principal component

PCA: Principal component analysis

PCR: Polymerase chain reaction

PCS: Posterior communicating segments

PDA: Patent ductus arteriosus

PHN: Pulmonary hypertension

PTU: Phenylthiourea

qPCR: quantitative PCR

RBC: Red blood cell

RD: Rare disease

REDCap: Research Electronic Data Capture

rmANOVA: Repeated-measures ANOVA

RNA: Ribonucleic acid

RTS: Rubinstein-Taybi syndrome 
SD: Standard deviation

SEM: Standard error of mean

SF: Shortening fraction

sgRNA: single-guide RNA

SMS: Smith-Magenis syndrome

SNP: Single-Nucleotide Polymorphism

ssDNA: single-stranded DNA

SSLPS: Simple sequence-length polymorphism

SUR: Sulfonylurea receptor

SV: Stroke volume

TALEN: Transcription activation-like effector nucleases

TBE: Tris-borate-EDTA

Tg: Transgenic

TMD: Transmembrane domaine

TS: Tuberous sclerosis

TSD: Total swimming distance

TSH: Thyroid-stimulating hormone

TST: Total swimming time

TUNEL: TdT-mediated nick end labeling

URSAHD: Unveiling RNA sample annotation for human diseases

UTR: Untranslated regions

VEDV: Ventricular end-diastolic volume

VESV: Ventricular end-systolic volume

VSM: Vascular smooth muscle

VUS: variants of unknown significance

WES: Whole-exome sequencing

WGS: Whole-genome sequencing

WHS: Wolf-Hirschhorn syndrome

WS: Williams syndrome

WT: Wildtype

ZFN: Zinc finger nucleases 


\section{Nederlandse samenvatting}

\section{Onderzoek naar zeldzame erfelijke aandoeningen: zeldzaam uitdagend}

Zeldzame erfelijke aandoeningen omvatten een breed spectrum van fenotypes, waarbij weefsels, orgaansystemen of biologische functies merendeels zijn aangedaan. Deze aandoeningen zijn zeer divers, ongelijk verspreid over de wereld en vaak chronisch, met blijvende invaliditeit of een vroegtijdige dood tot gevolg. Zeldzame aandoeningen zijn een unieke categorie ziekten en de diagnose en behandeling ervan vormt daardoor een ongekende uitdaging. Veel artsen hebben onvoldoende ervaring met het diagnosticeren of behandelen van deze aandoeningen, waardoor de diagnose vaak laat of verkeerd gesteld wordt. Vooral een verkeerde diagnose kan leiden tot extra ingrepen die, wanneer de juiste diagnose eenmaal gesteld is, misplaatst blijken te zijn. De genetische en fenotypische heterogeniteit van veel zeldzame erfelijke aandoeningen is een extra complicerende factor. Dit betekent dat veel (ouders van) patiënten met een zeldzame aandoening na een 'eindeloze' zoektocht pas de juiste diagnose krijgen. Dit kan een lang en frustrerend proces zijn voor patiënten en hun familie.

Om een diagnose te kunnen stellen en ziekten te kunnen voorspellen, is het van essentieel belang om met de ziekte geassocieerde genen en varianten op te sporen. In klinisch-diagnostische laboratoria wordt gebruikgemaakt van nextgeneration sequencing (NGS) om zeldzame aandoeningen met een genetische basis te kunnen analyseren. Om ziekte veroorzakende genen te valideren en in een biologische context te plaatsen, moet voor de meeste zeldzame aandoeningen gewerkt worden met cellulaire en modelorganismen.

Ondanks de wetenschappelijke vooruitgang op het gebied van de moderne genetica en genoomonderzoek, zijn er voor de meeste zeldzame erfelijke aandoeningen nog steeds geen goede behandelmogelijkheden. Vanwege de kleine marktomvang, hoge kosten, lage slaagkansen en het mogelijk lage rendement op investeringen zijn onderzoek en ontwikkeling van weesgeneesmiddelen voor zeldzame aandoeningen niet winstgevend voor de farmaceutische industrie. Een alternatieve tijdbesparende en kosteneffectieve methode die het risico van de ontwikkeling van geneesmiddelen voor zeldzame aandoeningen drastisch kan verminderen, is 'repurposing': het gebruik van geneesmiddelen voor nieuwe indicaties. Deze aanpak heeft tal van voordelen, maar systematische repurposing van geneesmiddelen is niet altijd eenvoudig en kan grote uitdagingen met zich meebrengen. 
Dit proefschrift illustreert verschillende belangrijke aspecten van het onderzoek naar zeldzame aandoeningen, dat het gehele proces van gen tot behandeling omvat. In het bijzonder wordt ingegaan op (1) de noodzaak van gedetailleerde en nauwkeurige klinische fenotypering om de ontstaansgeschiedenis van zeldzame aandoeningen vast te stellen en de diagnose te verbeteren, (2) de voordelen van de zebravis als modelorganisme om de pathofysiologie van zeldzame aandoeningen te valideren en te verhelderen, en (3) een benadering om een bestaand geneesmiddel te gebruiken voor een nieuwe indicatie.

Hoofdstuk 1 behandelt zeldzame aandoeningen en de unieke uitdagingen en gezondheidsproblemen die deze ziekten met zich meebrengen. Het biedt een overzicht van mogelijke stappen en benaderingen om zeldzame aandoeningen met succes te diagnosticeren en te behandelen.

In de wetenschappelijke en klinische wereld vormen zeldzame aandoeningen vaak nog een onderbelicht onderwerp. Daarom is hoofdstuk 2 onderdeel van een naslagwerk over genetische syndromen waarin artsen en onderzoekers informatie vinden over de etiologie, klinische kenmerken, diagnose- en behandelmethoden van het Cantú syndroom (CS), een zeldzame erfelijke aandoening.

Hoofdstuk 3 beschrijft fenotypische en genotypische gegevens van de twee oudste Nederlandse patiënten met het CS en hun kinderen met de ziekte. De consistente klinische kenmerken van het CS op verschillende leeftijden komen aan de orde, en het hoofdstuk bevat informatie over fenotypische veranderingen die in de loop van de tijd optreden. Dit biedt de mogelijkheid van een vroegere klinische en moleculaire diagnose en geoptimaliseerde genetische counseling. Daarnaast benadrukken we de klinische variatie onder patiënten met het CS, zelfs onder familieleden die dezelfde met het CS geassocieerde variant delen.

In het verleden is in enkele klinische rapportages melding gemaakt van mogelijke gedrags- en cognitieve afwijkingen bij personen met het CS. Tot op heden is het gedrag en cognitief functioneren van personen met het CS echter nog niet systematisch beoordeeld. Daarom biedt hoofdstuk 4 de eerste gestandaardiseerde karakterisering van cognitief profiel, sociaal functioneren en psychiatrische symptomen in een groot cohort van onderzoeksdeelnemers bij wie de diagnose CS met moleculair testen gesteld is. Uit resultaten verkregen door afname van verschillende gangbare gestandaardiseerde vragenlijsten blijkt een verminderde non-verbale intelligentie bij kinderen en adolescenten met het CS. Deze verminderde non-verbale intelligentie zou mogelijk kunnen 
leiden tot de lichte leerproblemen die werden waargenomen in eerder gepubliceerde gevallen. Verder tonen de resultaten een sociaal functioneren dat sterk samenhangt met de klinische diagnose autismespectrumstoornis en psychiatrische symptomen zoals angst en ADHD, voornamelijk bij de jongere onderzoeksdeelnemers in dit cohort.

Hoofdstuk 5 beschrijft onderzoek waarin gezichtsdysmorfologie is gekwantificeerd met driedimensionale (3D-) beeldvorming in een cohort van zowel mannelijke als vrouwelijke CS-patiënten met bevestigde varianten van het ABCC9-gen. Uit morfometrische analyse van verschillende delen van het gezicht bleken significante seksespecifieke verschillen in gezichtsvorm. Bovendien blijkt dat 3D-gezichtsfoto's onderscheid kunnen maken tussen het CS en andere erfelijke aandoeningen met specifieke gezichtsdysmorfieën die ten onrechte als CS zijn gediagnosticeerd. Daarom kan klinische toepassing van deze techniek bijdragen aan een vroegere diagnose en het optimaliseren van genetische counseling. Door op deze wijze onnodige misdiagnose te vermijden, ervaren patiënten en ouders minder stress.

Hoofdstuk 6 benadrukt het belang van samenwerking tussen verschillende centra in onderzoek naar zeldzame aandoeningen. Het beschrijft bevindingen uit het International Cantú Syndrome Registry die het resultaat zijn van gezamenlijk onderzoek van vier CS-klinieken in verschillende delen van de wereld. Met 74 onderzoeksdeelnemers is dit het grootste onderzoek naar patiënten met het CS dat tot op heden is gepubliceerd. Het hoofdstuk beschrijft nieuwe met het CS geassocieerde varianten in ABCC9. Daarnaast wordt de genetische basis van de ziekte nader gedefinieerd, en bevat het hoofdstuk een kwantitatieve beoordeling van de penetrantie van de ziektekenmerken.

Hoofdstuk 7 beschrijft een effectieve aanpak om kleine nucleotideveranderingen aan te brengen in het zebravisgenoom. In dit onderzoek zijn vier verschillende knock-in-lijnen met afzonderlijke missense mutaties die menselijke cardiovasculaire ziekten veroorzaken gemaakt in hun aan zebravis orthologe genen door CRISPR/Cas9 te combineren met een kort-template oligonucleotide. Drie van deze lijnen bevatten mutaties in ABCC9 en KCNJ8 die verband houden met het CS. De heterozygote zebravis-knock-in-lijnen in dit onderzoek vertonen essentiële cardiovasculaire kenmerken van de aandoening, waaruit de causaliteit van de geïntroduceerde mutaties voor het CS blijkt. Uit deze resultaten blijkt dat het introduceren van patiënt-allelen in hun zebravisorthologen een brede toepassing belooft voor het modelleren van humane 
erfelijke aandoeningen. Dit maakt de weg vrij voor nieuwe behandelwijzen met behulp van dit modelorganisme.

In hoofdstuk 8 worden deze CS-zebravismodellen verder besproken. Met patchclamping van geïsoleerde cardiomyocyten en vasculaire gladde spiercellen is de aanwezigheid van een aan het CS gerelateerd gain-of-function (GoF)effect op moleculair niveau gevalideerd. Bovendien is aangetoond dat aan het CS gerelateerde varianten resulteren in $\beta$-adrenerge interventie als secundair gevolg van GoF in het hart. Ten slotte is de in vivo-werkzaamheid van verschillende $K_{A T P}$-kanaalblokkers getest door middel van op fenotype gebaseerde screening van geneesmiddelen in zebravissen en zijn twee geneesmiddelen gevonden die cardiale defecten kunnen corrigeren. Deze resultaten geven aan dat de zebravis een geschikt model is om pathofysiologie en behandelmogelijkheden te onderzoeken voor het CS en andere aandoeningen waarin afwijkende cardiovasculaire $\mathrm{K}_{\text {ATP }}$-kanalen een rol spelen.

Hoofdstuk 9 beschrijft een nieuw zeldzaam genetisch syndroom bij zes patiënten homozygoot voor een splice-site mutatie in ABCC9. Met behulp van recombinant-analyse op celniveau wordt aangetoond dat de gevonden variant resulteert in een volledig verlies van eiwitfunctie. Vervolgens is de variant gevalideerd met de muis en zebravis, waaruit blijkt dat functieverlies van dit gen resulteert in vergelijkbare fenotypische kenmerken in beide modelorganismen. Voor deze kanalopathie die het gevolg is van functieverlies van SUR2 bevattende $\mathrm{K}_{\text {ATP }}$-kanalen is in dit onderzoek de term ABCC9-related Intellectual disability Myopathy Syndrome (AIMS) gekozen.

Ter afsluiting wordt in hoofdstuk 10 ingegaan op verdere benaderingen voor de diagnose, modellering en behandeling van zeldzame erfelijke aandoeningen. Hierbij worden recente ontwikkelingen op dit gebied, uitdagingen en mogelijke oplossingen besproken. Ten slotte volgt een samenvatting van wat er de afgelopen jaren is bereikt in het onderzoek naar het Cantú syndroom en een bespreking van toekomstige ontwikkelingen.

De toekomst van onderzoek naar zeldzame aandoeningen ziet er hoopvol uit, maar op dit moment is de toegang tot dit gebied nog beperkt. 


\section{Summary}

\section{Rare genetic disease research and its' challenges}

Rare genetic diseases cover a wide range of phenotypes, affecting the majority of tissue, organ system or biological function. They are heterogeneous in nature, geographically disparate and often chronic, resulting in disability or early death. Rare diseases form a very unique category of disorders and therefore face incomparable challenges when it comes to diagnosis and treatment. Many clinicians have insufficient experience with diagnosing or managing these disorders. Hence, diagnosis is often delayed or incorrect. Especially misdiagnosis can lead to additional interventions that turn out to be inappropriate, once the right diagnosis has been established. The genetic and phenotypic heterogeneity of many rare genetic diseases is an additional complicating factor. Thus, many (parents of) patients with rare disorders experience a "diagnostic odyssey" before finally obtaining the right diagnosis, which can be a long and frustrating journey for both patients and their family. The identification of diseaseassociated genes and variants is essential for diagnosis and disease prediction. Next-generation sequencing (NGS) is an established diagnostic tool in clinical diagnostic laboratories to unravel rare diseases with a genetic background. In order to validate disease-causing genes and put them into a biological context, functional work in cellular and model organisms is necessary in the majority of rare diseases.

Despite scientific advances in modern genetics and genomics, the majority of rare genetic diseases still lack effective treatment options. Due to the small market size, high costs, low success rates and possibly low return on investment, research and development of orphan drugs for rare diseases are not profitable for pharmaceutical companies. An alternative timesaving and cost-efficient method which can drastically reduce the risk of drug development for rare diseases is the process of repurposing drugs for new indications. The advantages of this approach are diverse, nevertheless systematic drug repurposing is not always trivial and can bring along several serious challenges.

The work within this thesis illustrates various important aspects when going from gene to treatment in the field of rare disease research. In particular, I highlight (i) the necessity of detailed and accurate clinical phenotyping to establish natural history of rare diseases and improve diagnosis, (ii) the advantages of the zebrafish as a functional model system to validate and 
elucidate pathophysiology of rare diseases as well as (iii) an approach to repurpose an existing drug for a new indication.

In Chapter 1, I introduce rare diseases and their unique challenges and burdens on human health. I review possible steps and approaches in order to successfully diagnose and treat rare diseases.

The awareness of rare diseases amongst the scientific and clinical community is often still limited. Hence, Chapter $\mathbf{2}$ is part of a genetic syndrome reference guide informing doctors and researchers about the etiology, clinical manifestations, ways of diagnosis and management of Cantú syndrome (CS), a rare genetic disorder.

In Chapter 3, we report phenotypic and genotypic data of the two oldest Dutch CS patients and their affected children. We discuss consistent clinical manifestations of CS at different ages and provide information on phenotypic changes occurring over time enabling earlier clinical and molecular diagnosis and optimized genetic counseling. Additionally, we emphasize clinical variability among CS patients, even among family members sharing the same CS-associated variant.

In the past, a few clinical reports have suggested behavioral and cognitive abnormalities in individuals with CS. However, a systematical assessment of behavioral and cognitive functioning in CS subjects has been missing to this date. Hence, in Chapter 4, we provide the first standardized characterization of cognitive profile, social functioning and psychiatric symptoms in a large cohort of subjects molecularly diagnosed with CS. By applying various common standardized questionnaires, we reveal a decreased nonverbal intelligence in children and adolescents with CS. This decreased nonverbal intelligence could possibly result in the mild learning disabilities that were observed in previously published cases. Additionally, we observe social functioning strongly associated with a clinical diagnosis of autism spectrum disorder and psychiatric symptoms like anxiety and ADHD predominantly in the younger subjects of this cohort.

In Chapter 5, we apply three-dimensional (3D) imaging to quantify facial dysmorphology in a cohort of both male and female CS patients with confirmed ABCC9 variants. Morphometric analysis of different regions of the face revealed gender-specific significant differences in face shape. Moreover, we show that 3D facial photographs can distinguish between CS and other genetic disorders with specific facial dysmorphologies that have been misdiagnosed 
as CS. Hence, when applied in clinics, this technique can assist in an earlier diagnosis, optimize genetic counseling and reduces stress for patients and parents by avoiding unnecessary misdiagnosis.

In Chapter 6, we underline the importance of multi-center collaboration in rare disease research. We report on findings from the International Cantú syndrome Registry which resulted from the coordinated effort of four CS clinics worldwide. With 74 individuals this study represents the largest survey of CS patients published to date. We report multiple novel CS-associated ABCC9 variants, further define the genetic basis of the disease, and provide quantitative assessment of the penetrance of disease features.

In Chapter 7, we present an effective approach to introduce small nucleotide changes in the zebrafish genome. We generated four different knock-in lines carrying distinct human cardiovascular-disorder-causing missense mutations in their zebrafish orthologous genes by combining CRISPR/Cas9 with a short template oligonucleotide. Three of these lines carry mutations in ABCC 9 and KCNJ8 linked to CS. Our heterozygous zebrafish knock-in lines display key cardiovascular features of the disorder, demonstrating the causality of the introduced mutations for CS. These results show that introducing patient alleles in their zebrafish orthologs promises a broad application for modeling human genetic diseases, paving the way for new therapeutic strategies using this model organism.

Chapter 8 further characterizes these CS zebrafish models by applying patchclamping of isolated cardiomyocytes and vascular smooth muscle cells validating the presence of a CS-related gain-of-function (GoF) effect on a molecular level. Moreover, we demonstrate that CS-associated variants result in $\beta$-adrenergic intervention as a secondary consequence to GoF in the heart. Lastly, we test the in vivo efficacy of various $\mathrm{K}_{\mathrm{ATP}}$ channel blockers by phenotype-based drug screening in zebrafish and identify two drugs that are able to correct cardiac defects. These results indicate that zebrafish represent a suitable model to study pathophysiology and therapy options for CS and other disorders involving aberrant cardiovascular $\mathrm{K}_{\mathrm{ATP}}$ channels.

In Chapter 9, we report a novel rare genetic syndrome in six patients homozygous for a splice-site mutation in ABCC9. Applying cell-based recombinant assays, we show that the detected variant results in complete loss of protein function. We then use the mouse and zebrafish to validate the variant and show that loss-of-function of this gene results in similar phenotypic features in both 
model organisms. We term this channelopathy resulting from loss-of-function of SUR2-containing $\mathrm{K}_{\text {ATP }}$ channels, ABCC9-related Intellectual disability Myopathy Syndrome (AIMS).

Finally, in Chapter 10, I discuss further approaches for diagnosing, modeling and treating rare genetic disorders. I highlight recent progress made in the field as well as ongoing challenges and ways to resolve them. Lastly, I summarize the achievements made in Cantú syndrome research in the past years and comment on steps that will be taken in the future.

The future of rare diseases is hopeful, but access today is still limited. 


\section{List of publications}

McClenaghan, C, Hanson, A, Sala-Rabanal, M, Roessler, HI, Josifova, D, Grange, DK, van Haaften, G \& Nichols, CG. Cantu syndrome-associated SUR2 (ABCC9) mutations in distinct structural domains result in KATP channel gain-of- function by differential mechanisms. J Biol Chem. (2017).

Roessler, HI, Volker-Touw, CML, Terhal, PA, van Haaften, G \& van Haelst, MM. Cantú syndrome, the changing phenotype: a report of the two oldest Dutch patients. Clin Dysmorphol. (2018) 27:78-83.

Tessadori, F*, Roessler, HI*, Savelberg, SMC*, Chocron, S, Kamel, SM, Duran, KJ, van Haelst, MM, van Haaften, G \& Bakkers, J. Effective CRISPR/Cas9-based nucleotide editing in zebrafish to model human genetic cardiovascular disorders. Dis Model Mech. (2018) 11.

Smeland, MF*, McClenaghan, C*, Roessler, HI*, Savelberg, S, Hansen, GAM, Hjellnes, H, Arntzen, KA, Muller, KI, Dybesland, AR, Harter, T, Sala-Rabanal, $M$, Emfinger, $\mathrm{CH}$, Huang, $Y$, Singareddy, SS, Gunn, J, Wozniak, DF, Kovacs, A, Massink, M, Tessadori, F, Kamel, SM, Bakkers, J, Remedi, MS, Van Ghelue, M, Nichols, CG \& van Haaften, G. ABCC9-related Intellectual disability Myopathy Syndrome is a $\mathrm{K}_{\text {ATP }}$ channelopathy with loss-of-function mutations in ABCC9. Nat Commun. (2019) 10:4457.

Grange, DK*, Roessler, HI*, McClenaghan, C*, Duran, K, Shields, K, Remedi, MS, Knoers, N, Lee, JM, Kirk, EP, Scurr, I, Smithson, SF, Singh, GK, van Haelst, MM, Nichols, CG \& van Haaften, G. Cantú syndrome: Findings from 74 patients in the International Cantu Syndrome Registry. Am J Med Genet C Semin Med Genet. (2019) 181:658-681.

Roessler, HI, Shields, K, Grange, DK, Knoers, N, van Haaften, G, Hammond, P \& van Haelst, MM. Three-dimensional facial morphology in Cantú syndrome. Am J Med Genet A. (2020). 182(5):1041-1052.

Roessler, HI, Knoers, NVAM, van Haelst, MM\#, van Haaften, G\#. Drug repurposing for rare diseases. Trends Pharmacol Sci (2021). doi:10.1016/j.tips.2021.01.003 (Epub ahead of print).

Roessler, HI, van Haelst, MM. Cantú syndrome. Manuscript in press (2021). 
Roessler, HI, van der Heuvel, LM, Shields, K, Guilliams, KP, Knoers, NVAM, van Haaften, G, Grange, DK, van Haelst, MM. Behavioral and cognitive functioning in Cantú syndrome. Manuscript in press (2021).

\section{Submitted manuscripts}

Roessler, HI, van Haaften, G, van Haelst, MM. The psychosocial impact of rare genetic disorders: A young adult with Cantú syndrome. (Manuscript submitted).

\section{Manuscripts in preparation}

Roessler, HI*, Singareddy, SS*, McClenaghan, C, Savelberg, SMC, Tyron, R, Tessadori, F, P. de Boer, T, Bakkers, J, Knoers, NVAM, van Haelst, MM, Nichols,

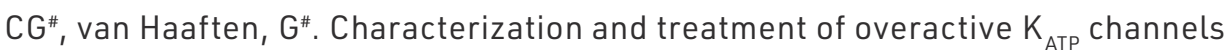
in zebrafish models of Cantú syndrome. (in preparation).

Singareddy, SS, Roessler, HI, McClenaghan, C, van Haaften, G, Nichols, CG. K channels in zebrafish cardiovasculature. (in preparation).

*These first authors contributed equally to this work.

\#These last authors contributed equally to this work. 


\section{Acknowledgements}

This PhD thesis is the output of the effort and support of several people to whom I am extremely grateful. If I have learned anything during this $\mathrm{PhD}$, it is that collaboration and working as a team is what gets you further in science. I could not have done it without the help and contributions of a lot of people and I would like to take this opportunity to give major thanks to everyone involved; either directly by working together and sharing scientific discussion or indirectly by simply motivating me to keep going.

First, I would like to thank the members of my supervisory team, Nine Knoers, Gijs van Haaften and Mieke van Haelst, for their consistent support and guidance during the running of this project.

Nine, I'm very thankful for the continuous support of my PhD study and related research, for your patience, immense knowledge and scientific feedback. You always provided encouragement and were willing and enthusiastic to assist in any way you could throughout the entire project. You made me realize that it is never too early to think about the future and I really appreciate the many fruitful discussions we had regarding mine. Thank you for making the monthly trip to Utrecht for attending our meetings. It is safe to say that I was always looking forward to them in order to get valuable and fresh input for my current work.

Gijs, thank you for your invaluable guidance, support and encouragement throughout this study. Thank you for having so much confidence and trust in me and for giving me the freedom to work so dependently from the very beginning of my project. You taught me the importance and value of collaborations and teamwork in science. You always made me rediscover my, at times lost, enthusiasm for scientific research which eventually led me to re-think my initial decision and not turn my back on academia just yet. Thank you for the opportunity to continue our collaboration in the future.

Mieke, I really appreciate it that you have given me the opportunity to do this PhD considering my minimal knowledge about clinical research four years ago. Thank you for guiding me so positively, teaching me many essential skills in the field of clinical genetics and giving me the time to get my bearings in these new surroundings. You always made me feel confident in my abilities after going back from Amsterdam to Utrecht or coming off the phone with you. I am very glad to have had you as a supervisor throughout these years. 
Thanks a lot also to the members of my supervisory committee, Jeroen Bakkers and Kors van der Ent for their insightful comments and encouragement, but also for the critical question which prompted me to widen my research from various perspectives.

I would also like to thank the members of my reading committee, Madelon Maurice, Mireille Serlie, Kors van der Ent, Toine Egberts and Folkert Asselbergs. Thank you very much for taking the time to critically read my thesis.

To the van Haaften group: Sanne, thank you for teaching me so much about zebrafish, for taking such good care of the Cantú lines when I was busy with something else and for being my go-to person when it comes to translations into Dutch. Federico, thank you for being able to answer almost every question about zebrafish and functional experiments and for always thinking along and being willing to help when I needed guidance or support with an experiment. Karen, thank you for being an expert in the lab, the cloning queen and always giving me advice whenever I was missing bands on a gel. Glen, I am still trying to figure out how you always stay that enthusiastic and motived in the lab, because someday I am hoping to be a little bit more like that myself. Thanks for always being around to talk, listen and give advice on every issue imaginable. Rozemarijn, thank you for always providing a cheery atmosphere to work in and for making me realize that baking can actually be fun. Edith, also a big thanks to you for all the help in the lab and especially the cell culture.

Also a big thank you to the rest of the van Haaften group, former or current members, for your valuable input during work discussions: Laura, Maria, Joline, Christina, Richard, Joe, Kirsten, Albertien and Maarten.

And to all the other roommates, that have not been mentioned so far: Sakshi, Iris, Remi, Marijn; thank you for always maintaining a fun and productive atmosphere in the office.

A big thank you to all members of the Bakkers group for receiving me so well during my times at the Hubrecht. Thank you for your considerate guidance and sharing your expertise in zebrafish research, lab space and materials with me when I was in need.

To all the members of the CantúTreat consortium, thank you very much for the fruitful collaborations and extremely well-planned annual meetings. 
A special thanks to the Nichols group: I'm thankful for the opportunity to have visited you during my PhD which taught me a lot about patch-clamping and electrophysiology.

Thank you to the Cantú syndrome community who were always willing to help us with our research by contributing pictures, filling in questionnaires or coming to our annual Cantú syndrome research clinic. During those clinics, I was able to gain a more realistic perspective on rare genetic diseases by directly hearing from you about your path to diagnosis and daily struggles. Your participation provided an important frame of reference and positively affected my motivation for this project.

Danke an meine Würzburg Crew für die halbjährlichen Treffen und die Möglichkeit sich über die alltäglichen Frustrationen einer Doktorarbeit auszulassen. Öz, danke für die Bereitschaft Kaffee-, Eistee Pfirsich- und Falafelpausen einzulegen wann auch immer sie grade nötig waren.

Last but not the least, möchte ich mich bei meiner Famile bedanken. Daniel, danke auch dir, dass du immer bereit bist mir zuzuhören, egal worum es geht. Ich bin froh, dass wir so nah aneinander wohnen und immer mal wieder die Möglichkeit haben uns zu sehen. Und du wirst schon sehen, irgedwann sehen auch wir Schalke wieder in der Arena gewinnen.

Mama und Papa, vielen Dank für die endlose Unterstützung nicht nur innerhalb dieser vier Jahre, sondern auch davor. Danke, dass ihr immer nur das Beste für mich wollt, auch wenn mich das manchmal weiter weg von zu Hause führt. Die zahlreichen Anrufe, in denen ich mich wieder und wieder darüber beschweren konnte, dass die Fische einfach nicht das tun, was ich gerne möchte, haben einen großen Anteil daran, dass ich diese Doktorarbeit erfolgreich beenden konnte. Vielen Dank für die unerschöpfte Geduld und Ermutigungen, denn wir wissen alle, dass ich manchmal gar nicht so einfach sein kann. 
Acknowledgements

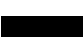

A7 
Appendix 8 


\section{Curriculum vitae}

Helen Isabel Roessler was born on June $20^{\text {th }} 1992$ in Heidelberg, Germany. She graduated from Leibniz Gymanasium Oestringen in 2011. Afterwards, Helen started her study of Biochemistry in at Julius-Maximilians University Wuerzburg and obtained her Bachelor's degree in 2014. During her Masters, she did a semester abroad at the Institute for Molecular Biosciences at University of Queensland, Brisbane, Australia. Helen obtained her Master's degree in Biochemistry in September 2016.

In January 2017 Helen moved to Utrecht in the Netherlands in order to start her doctoral research, which resulted in this thesis, at the department of Genetics of the University Medical Center Utrecht. Here she worked in the interface of clinical genetics and disease biology focusing on a translational approach towards treatment for rare genetic disease. During this time, she was supervised by prof. dr. Nine Knoers, dr. Gijs van Haaften and dr. Mieke van Haelst. Completing her doctoral studies in 2021, Helen currently resides in Utrecht and will start a postdoctoral position with Peter van Hasselt and Gijs van Haaften working on CLN3 disease. 
$x^{2}+x^{2}$
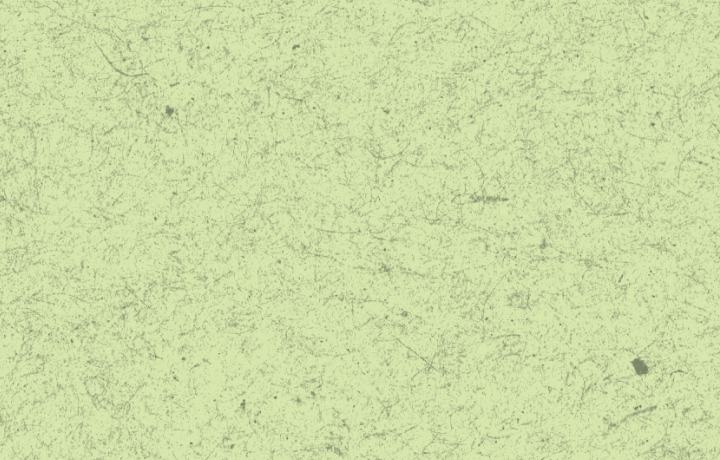

\section{4}
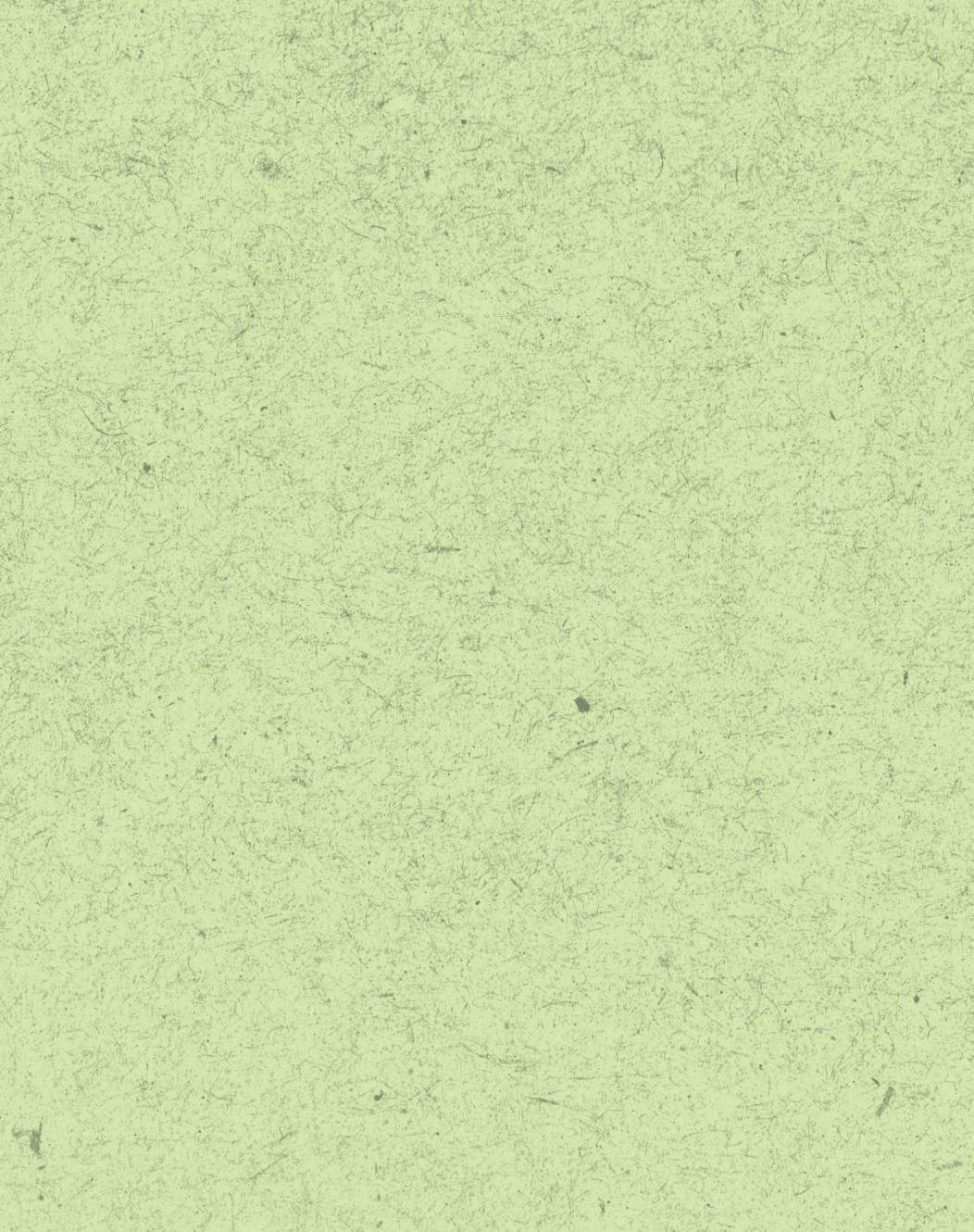

\section{(it}

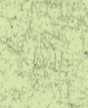

$x+\frac{1}{2}+\frac{1}{2}$

$(x+5,+\infty$
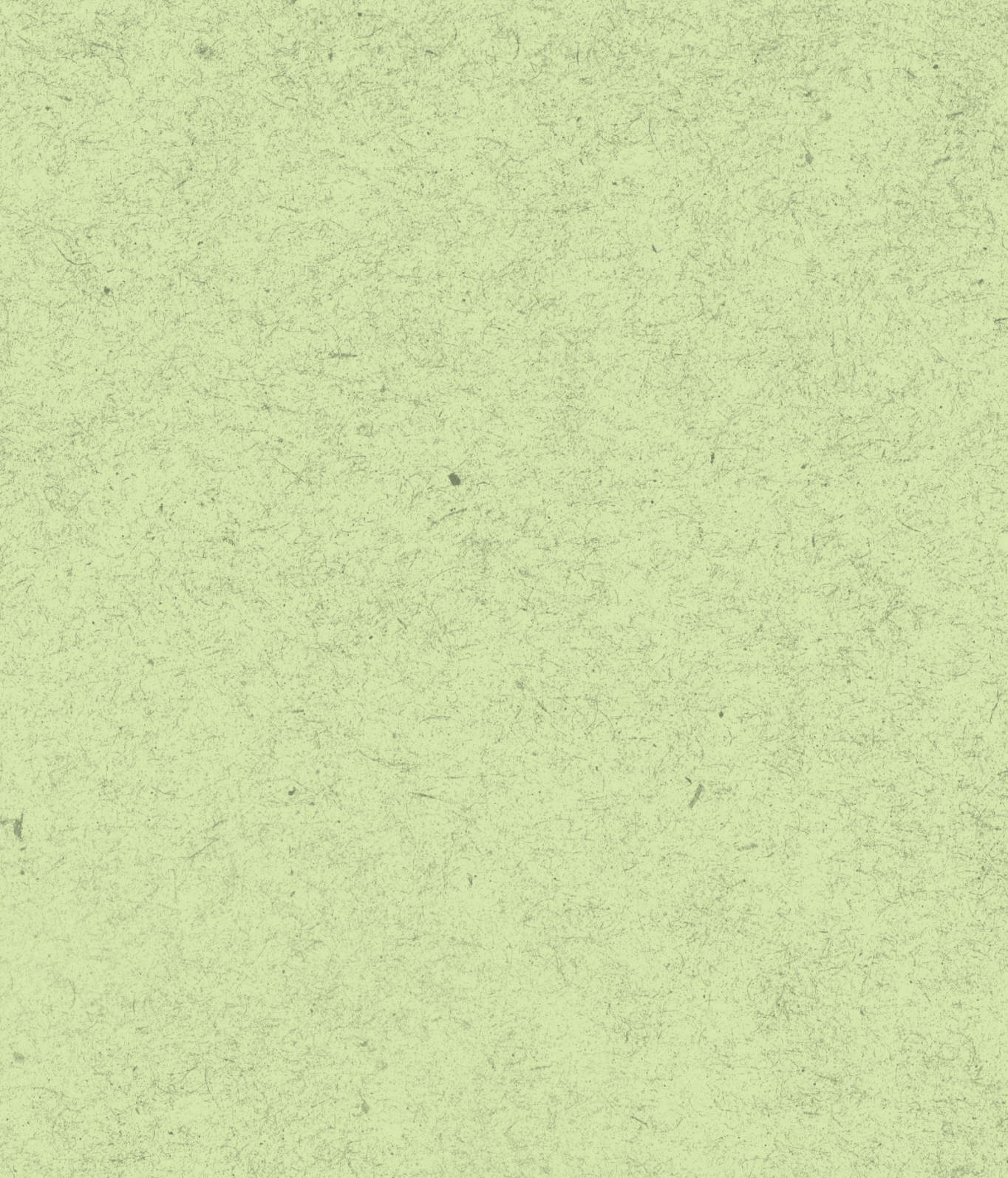